\title{
Convergent Azaspirocyclization of Bromoarenes with $N$-Tosylhydrazones by a Palladium
}

\section{Catalyst}

Aika Yanagimoto, ${ }^{a}$ Yota Uwabe, ${ }^{a}$ Qikun $\mathrm{Wu},{ }^{a}$ Kei Muto, ${ }^{* b}$ and Junichiro Yamaguchi*a

a Department of Applied Chemistry, Waseda University, 513 Wasedatsurumakicho, Shinjuku, Tokyo 162-0041 Japan.

${ }^{b}$ Institute for Advanced Study, Waseda University, 513 Wasedatsurumakicho, Shinjuku, Tokyo 1620041 Japan.

E-mail: keimuto@aoni.waseda.jp (KM),junyamaguchi@waseda.jp (JY)

\section{Table of Contents}

1. General

S2

2. Preparation of Starting Materials

S3-S17

3. Pd-Catalyzed Azaspirocyclization of Bromoarenes with $N$-Tosylhydrazones

4. Acid-Catalyzed Rearrangement of $\mathbf{3}$

S35-S39

5. One-Pot Synthesis of (Z)-2-Benzylidene-6-phenyl-1-thia-6-azaspiro[4.4]non-3-en-7one (3Ha)

6. Sequential Transformation from 1

S40-S42

7. Derivatization of 9

S42-S44

8. Effect of Parameters

S45

9. Limitation of Pd-Catalyzed Azaspirocyclization

S46

10. X-ray Crystal Structure Analysis of 9Aa

11. References

S49-S50

12. ${ }^{1} \mathrm{H}$ NMR, ${ }^{13} \mathrm{C}$ NMR, and ${ }^{19} \mathrm{~F}$ NMR Spectra 


\section{General}

Unless otherwise noted, all reactants or reagents including dry solvents were obtained from commercial suppliers and used as received. $\mathrm{Cs}_{2} \mathrm{CO}_{3}$ was obtained from KANTO Chemical and Iwatani Corporation. $\mathrm{PPh}_{3}$ was obtained from KANTO Chemical. $p$-Toluenesulfonic acid monohydrate was obtained from Tokyo Chemical Industry (TCI). $\mathrm{Pd}_{2}(\mathrm{dba})_{3} \cdot \mathrm{CHCl}_{3}{ }^{[1]}, \quad N^{\top}$-benzylidene-4methylbenzenesulfonohydrazide (2a), ${ }^{[2]}$ 4-methyl- $N$-(4-methylbenzylidene)benzenesulfonohydrazide (2b), ${ }^{[2]} \quad N^{\prime}$-(4-methoxybenzylidene)-4-methylbenzenesulfonohydrazide $\quad(\mathbf{2 e}),{ }^{[2]} \quad N^{\top}$-(4fluorobenzylidene)-4-methylbenzenesulfonohydrazide $\quad(\mathbf{2 f}),{ }^{[2]} \quad N^{n}$-(4-chlorobenzylidene)-4methylbenzenesulfonohydrazide (2g), ${ }^{[3]} N^{\prime}$-(furan-2-ylmethylene)-4-methylbenzenesulfonohydrazide (2i),${ }^{[3]} \quad N^{\prime}$-(2,2-dimethylpropylidene)-4-methylbenzenesulfonohydrazide $\quad(\mathbf{2} \mathbf{j}),{ }^{[4]} \quad N^{\prime}$ (cyclohexylmethylene)-4-methylbenzenesulfonohydrazide $\quad(\mathbf{2 k}),{ }^{[3]}$ and $\quad N^{\prime}$-(diphenylmethylene)-4methylbenzenesulfonohydrazide (2I) ${ }^{[2]}$ were synthesized according to procedures and the spectra matched with those of compounds reported in the literature. Unless otherwise noted, all reactions were performed with dry solvents under an atmosphere of $\mathrm{N}_{2}$ in dried glassware using standard vacuum-line techniques. All dearomative reactions of bromoarenes with $N$-tosylhyrazones were performed in $8-\mathrm{mL}$ glass vessel tubes equipped with a screw cap and heated (IKA Plate RCT digital) in a 16-well aluminum reaction block (IKA DB4.3 Block) unless otherwise noted. All work-up and purification procedures were carried out with reagent-grade solvents in air.

Analytical thin-layer chromatography (TLC) was performed using Silica-gel 70 TLC Plate-Wako $(0.25 \mathrm{~mm})$. The developed chromatogram was analyzed by UV lamp $(254 \mathrm{~nm})$. Flash column chromatography was performed with Biotage Isolera ${ }^{\circledR}$ equipped with Biotage Sfär Cartridge Silica D columns. Preparative thin-layer chromatography (PTLC) was performed using Wakogel B5-F silica coated plates $(0.75 \mathrm{~mm})$ prepared in our laboratory. Preparative recycling gel permeation chromatography (GPC) was performed with a JAI LaboACE LC-5060 instrument equipped with JAIGEL-2HR columns using $\mathrm{CHCl}_{3}$ as an eluent. High-resolution mass spectra were conducted on Thermo Fisher Scientific ExactivePlus Orbitrap (ESI). Nuclear magnetic resonance (NMR) spectra were recorded on a JEOL JNM-ECS-400 $\left({ }^{1} \mathrm{H} 400 \mathrm{MHz},{ }^{13} \mathrm{C} 101 \mathrm{MHz}\right)$ and JNM-ECZ-400 $\left({ }^{1} \mathrm{H} 400 \mathrm{MHz}\right.$, ${ }^{13} \mathrm{C} 101 \mathrm{MHz},{ }^{19} \mathrm{~F} 376 \mathrm{MHz}$ ) spectrometer. Chemical shifts for ${ }^{1} \mathrm{H}$ NMR are expressed in parts per million (ppm) relative to tetramethylsilane $(\delta 0.00 \mathrm{ppm})$ in $\mathrm{CDCl}_{3}$ and $\mathrm{C}_{6} \mathrm{HD}_{5}(\delta 7.16 \mathrm{ppm})$ in benzene$d_{6}$. Chemical shifts for ${ }^{13} \mathrm{C}$ NMR are expressed in ppm relative to $\mathrm{CDCl}_{3}(\delta 77.0 \mathrm{ppm})$ or DMSO- $d_{6}(\delta$ $39.5 \mathrm{ppm})$. Chemical shifts for ${ }^{19} \mathrm{~F}$ NMR are expressed in ppm relative to $\mathrm{PhF}(\delta-113.15 \mathrm{ppm})$. Data are reported as follows: chemical shift, multiplicity $(\mathrm{s}=$ singlet, $\mathrm{d}=$ doublet, $\mathrm{dd}=$ doublet of doublets, $\mathrm{t}$ $=$ triplet, $\mathrm{dt}=$ doublet of triplets, $\mathrm{td}=$ triplet of doublets, $\mathrm{q}=$ quartet, $\mathrm{m}=$ multiplet, brs $=$ broad singlet , coupling constant $(\mathrm{Hz})$, and integration. 


\section{Preparation of Starting Materials}

\section{Procedure 1: Synthesis of 3-(5-Bromofuran-2-yl)- $\mathrm{N}$-arylpropanamides 1}<smiles>[M]C1(C)OC(=O)C(=Cc2ccc(Br)o2)C(=O)O1</smiles>

S1

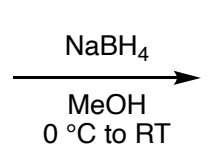

$0^{\circ} \mathrm{C}$ to $\mathrm{RT}$

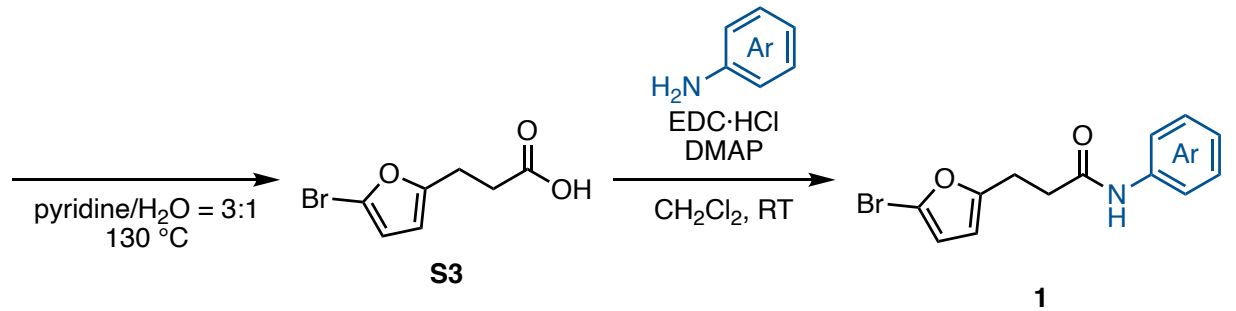

To a round-bottom flask containing a magnetic stirring bar were added 5-bromofuran-2carbaldehyde (1.0 equiv), Meldrum's acid (1.2 equiv), EtOH (0.25 M), and then $\mathrm{NEt}_{3}(7.0 \mathrm{~mol} \%)$ at room temperature. After stirring the mixture for several hours with monitoring reaction progress with TLC, the mixture was concentrated in vacuo to afford a crude mixture of 5-((5-bromofuran-2yl)methylene)-2,2-dimethyl-1,3-dioxane-4,6-dione (S1) as a red oil. This was used for the next step without further purification.

The obtained mixture was dissolved in $\mathrm{MeOH}(0.30 \mathrm{M})$. The mixture was cooled to $0{ }^{\circ} \mathrm{C}$, and then $\mathrm{NaBH}_{4}$ (1.2 equiv) was added portionwise. After stirring the mixture at room temperature for several hours with monitoring reaction progress with TLC, the mixture was concentrated in vacuo. The residue was added $3 \mathrm{M} \mathrm{HCl}$ aq. and extracted three times with EtOAc. The combined organic layer was dried over $\mathrm{Na}_{2} \mathrm{SO}_{4}$, filtrated, and concentrated in vacuo to afford 5-((5-bromofuran-2-yl)methyl)-2,2dimethyl-1,3-dioxane-4,6-dione (S2) as a red oil. This was used for the next step without further purification.

To a crude mixture of $\mathbf{S 2}$ (1.0 equiv) in a round-bottom flask containing a magnetic stirring bar were added pyridine $(1.0 \mathrm{M})$ and $\mathrm{H}_{2} \mathrm{O}(3.0 \mathrm{M})$. After stirring the mixture at $130{ }^{\circ} \mathrm{C}$ for several hours with monitoring reaction progress with TLC, the reaction was quenched with $3 \mathrm{M} \mathrm{HCl}$ aq. The mixture was extracted three times with EtOAc. The combined organic layer was dried over $\mathrm{Na}_{2} \mathrm{SO}_{4}$, filtrated, and concentrated in vacuo to afford 3-(5-bromofuran-2-yl)propanoic acid (S3) as a dark red oil. This was used for the next step without further purification.

To a crude mixture of $\mathbf{S 3}$ (1.0 equiv) in a round-bottom flask containing a magnetic stirring bar were added $\mathrm{EDC} \cdot \mathrm{HCl}\left(1.2\right.$ equiv), DMAP $\left(0.40\right.$ equiv), and $\mathrm{CH}_{2} \mathrm{Cl}_{2}(0.20 \mathrm{M})$. To this mixture, aniline derivative (1.0 equiv) was added. After stirring the mixture at room temperature for several hours with monitoring reaction progress with TLC, the reaction was quenched with $\mathrm{H}_{2} \mathrm{O}$. The mixture was extracted three times with $\mathrm{CH}_{2} \mathrm{Cl}_{2}$. The combined organic layer was dried over $\mathrm{Na}_{2} \mathrm{SO}_{4}$, filtrated, and concentrated in vacuo. The residue was purified by Isolera ${ }^{\circledR}$ to afford amide $\mathbf{1}$. 
<smiles>O=C(CCc1ccc(Br)o1)Nc1ccccc1</smiles>

\section{3-(5-Bromofuran-2-yl)- $N$-phenylpropanamide (1A)}

Purification by Isolera ${ }^{\circledR}$ (hexane/EtOAc $=15: 1$ to $\left.1: 1\right)$ afforded 1A as a white solid $(1.29 \mathrm{~g}, 80 \%$ yield over 4 steps, $5.5 \mathrm{mmol} \mathrm{scale}) .{ }^{1} \mathrm{H}$ NMR $\left(400 \mathrm{MHz}, \mathrm{CDCl}_{3}\right) \delta 7.47(\mathrm{~d}, J=8.0 \mathrm{~Hz}, 2 \mathrm{H}), 7.32(\mathrm{t}, J=$ $8.0 \mathrm{~Hz}, 2 \mathrm{H}), 7.17-7.06(\mathrm{~m}, 2 \mathrm{H}), 6.19$ (d, $J=3.2 \mathrm{~Hz}, 1 \mathrm{H}), 6.07$ (d, $J=3.2 \mathrm{~Hz}, 1 \mathrm{H}), 3.06$ (t, $J=7.6 \mathrm{~Hz}$, 2H), 2.69 (t, $J=7.6 \mathrm{~Hz}, 2 \mathrm{H}) ;{ }^{13} \mathrm{C}$ NMR (101 MHz, DMSO- $\left.d_{6}\right) \delta 169.6,157.2,139.1,128.7,123.1,119.1$, 118.8, 112.2, 108.4, 34.2, 23.4; HRMS (ESI) $\mathrm{m} / z$ calcd for $\mathrm{C}_{13} \mathrm{H}_{12} \mathrm{BrNNaO}_{2}[\mathrm{M}+\mathrm{Na}]^{+}: 315.9944$ found 315.9943.

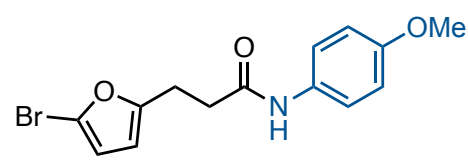

\section{3-(5-Bromofuran-2-yl)- $N$-(4-methoxyphenyl)propanamide (1B)}

Purification by Isolera $^{\circledR}$ (hexane/EtOAc $=9: 1$ to $\left.1: 1\right)$ afforded $1 \mathrm{~B}$ as a white solid $(146.8 \mathrm{mg}, 65 \%$ yield, $0.70 \mathrm{mmol}$ scale from S3). ${ }^{1} \mathrm{H}$ NMR $\left(400 \mathrm{MHz}, \mathrm{CDCl}_{3}\right) \delta 7.37$ (d, $\left.J=8.8 \mathrm{~Hz}, 2 \mathrm{H}\right), 7.08-6.98$ (brs, $1 \mathrm{H}), 6.85(\mathrm{~d}, J=8.8 \mathrm{~Hz}, 2 \mathrm{H}), 6.19(\mathrm{~d}, J=3.2 \mathrm{~Hz}, 1 \mathrm{H}), 6.06(\mathrm{~d}, J=3.2 \mathrm{~Hz}, 1 \mathrm{H}), 3.79(\mathrm{~s}, 3 \mathrm{H}), 3.05(\mathrm{t}, J$ $=7.6 \mathrm{~Hz}, 2 \mathrm{H}), 2.66(\mathrm{t}, J=7.6 \mathrm{~Hz}, 2 \mathrm{H}) ;{ }^{13} \mathrm{C} \mathrm{NMR}\left(101 \mathrm{MHz}, \mathrm{CDCl}_{3}\right) \delta 169.4,156.5,156.3,130.7,122.0$, 119.6, 114.1, 111.9, 108.7, 55.4, 35.5, 24.1; HRMS (ESI) $m / z$ calcd for $\mathrm{C}_{14} \mathrm{H}_{14} \mathrm{BrNO}_{3} \mathrm{Na}[\mathrm{M}+\mathrm{Na}]^{+}$: 346.0049 found 346.0044 .<smiles>O=C(CCc1ccc(Br)o1)Nc1ccc(C(F)(F)F)cc1</smiles>

\section{3-(5-Bromofuran-2-yl)- $N$-(4-(trifluoromethyl)phenyl)propanamide (1C)}

Purification by Isolera $^{\circledR}$ (hexane/EtOAc $=9: 1$ to 1:1) afforded $\mathbf{1 C}$ as a white solid $(136.7 \mathrm{mg}, 60 \%$ yield, $0.70 \mathrm{mmol}$ scale from S3). ${ }^{1} \mathrm{H}$ NMR $\left(400 \mathrm{MHz}, \mathrm{CDCl}_{3}\right) \delta 7.70-7.54(\mathrm{~m}, 4 \mathrm{H}), 6.20(\mathrm{~d}, J=3.2 \mathrm{~Hz}$, $1 \mathrm{H}), 6.07(\mathrm{~d}, J=3.2 \mathrm{~Hz}, 1 \mathrm{H}), 3.06(\mathrm{t}, J=7.6 \mathrm{~Hz}, 2 \mathrm{H}), 2.72(\mathrm{t}, J=7.6 \mathrm{~Hz}, 2 \mathrm{H}) ;{ }^{13} \mathrm{C} \mathrm{NMR}(101 \mathrm{MHz}$, $\left.\mathrm{CDCl}_{3}\right) \delta 169.9,155.9,140.6,126.20\left(\mathrm{q}, J_{\mathrm{C}-\mathrm{F}}=3.9 \mathrm{~Hz}\right), 126.17\left(\mathrm{q}, J_{\mathrm{C}-\mathrm{F}}=32.9 \mathrm{~Hz}\right), 124.0\left(\mathrm{q}, J_{\mathrm{C}-\mathrm{F}}=274\right.$ $\mathrm{Hz}), 119.9,119.5,112.0,108.9,35.7,23.9 ;{ }^{19} \mathrm{~F} \mathrm{NMR}\left(376 \mathrm{MHz}, \mathrm{CDCl}_{3}\right) \delta-62.2 ; \mathrm{HRMS}$ (ESI) $\mathrm{m} / \mathrm{z}$ calcd for $\mathrm{C}_{14} \mathrm{H}_{12} \mathrm{BrF}_{3} \mathrm{NO}_{2}[\mathrm{M}+\mathrm{H}]^{+}: 361.9998$ found 361.9999 .<smiles>O=C(CCc1ccc(Br)o1)Nc1ccc(Br)cc1</smiles>

3-(5-Bromofuran-2-yl)- $N$-(4-bromophenyl)propanamide (1D) 
Purification by Isolera $^{\circledR}$ (hexane/EtOAc $=9: 1$ to $\left.1: 1\right)$ afforded 1D as a white solid $(256.1 \mathrm{mg}, 66 \%$ yield, $1.0 \mathrm{mmol}$ scale from $\mathbf{S 3}) .{ }^{1} \mathrm{H}$ NMR $\left(400 \mathrm{MHz}, \mathrm{CDCl}_{3}\right) \delta 7.47-7.35(\mathrm{~m}, 4 \mathrm{H}), 7.18-7.05(\mathrm{brs}, 1 \mathrm{H})$, $6.19(\mathrm{~d}, J=2.8 \mathrm{~Hz}, 1 \mathrm{H}), 6.06(\mathrm{~d}, J=2.8 \mathrm{~Hz}, 1 \mathrm{H}), 3.05(\mathrm{t}, J=7.2 \mathrm{~Hz}, 2 \mathrm{H}), 2.68(\mathrm{t}, J=7.2 \mathrm{~Hz}, 2 \mathrm{H}) ;{ }^{13} \mathrm{C}$ NMR $\left(101 \mathrm{MHz}, \mathrm{CDCl}_{3}\right) \delta 169.6,156.0,136.6,132.0,121.5,119.8,117.0,112.0,108.8,35.7,23.9$; HRMS (ESI) $m / z$ calcd for $\mathrm{C}_{13} \mathrm{H}_{12} \mathrm{Br}_{2} \mathrm{NO}_{2}[\mathrm{M}+\mathrm{H}]^{+}: 371.9229$ found 371.9230 .

\section{Procedure 2: Synthesis of 1-((5-Bromofuran-2-yl)methyl)-1-methyl-3-phenylurea (1E)}

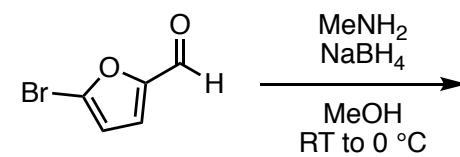<smiles>CNCc1ccc(Br)o1</smiles>

S4

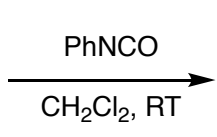

$\mathrm{CH}_{2} \mathrm{Cl}_{2}, \mathrm{RT}$<smiles>CN(Cc1ccc(Br)o1)C(=O)Nc1ccccc1</smiles>

$1 E$

To a round-bottom flask containing a magnetic stirring bar were added 5-bromofuran-2carbaldehyde (525 mg, $3.0 \mathrm{mmol}, 1.0$ equiv) and $\mathrm{MeOH}(15 \mathrm{~mL})$. To this solution was then added $\mathrm{MeNH}_{2}(2.0 \mathrm{M}$ in EtOH, $2.25 \mathrm{~mL}, 4.5 \mathrm{mmol}, 1.5$ equiv) at room temperature. After stirring the mixture for $45 \mathrm{~min}$, the mixture was cooled to $0{ }^{\circ} \mathrm{C}$. To this mixture was added $\mathrm{NaBH}_{4}(114 \mathrm{mg}, 3.0 \mathrm{mmol}, 1.0$ equiv) portionwise. After stirring the mixture at $0{ }^{\circ} \mathrm{C}$ for several hours with monitoring reaction progress with TLC, the reaction was quenched with $\mathrm{H}_{2} \mathrm{O}$. The mixture was extracted three times with $\mathrm{CH}_{2} \mathrm{Cl}_{2}$. The combined organic layer was dried over $\mathrm{Na}_{2} \mathrm{SO}_{4}$, filtrated, and concentrated in vacuo to afford a crude mixture of 1-(5-bromofuran-2-yl)- $N$-methylmethanamine (S4). This was used for the next step without further purification.

To a crude mixture of $\mathbf{S 4}$ (3.0 mmol, 1.0 equiv) in $\mathrm{CH}_{2} \mathrm{Cl}_{2}(15 \mathrm{~mL})$ was added isocyanatobenzene $(0.32 \mathrm{~mL}, 3.00 \mathrm{mmol}, 1.0$ equiv) at room temperature. After stirring the mixture for several hours with monitoring reaction progress with TLC, the reaction was quenched with $\mathrm{H}_{2} \mathrm{O}$. The mixture was extracted three times with $\mathrm{CH}_{2} \mathrm{Cl}_{2}$. The combined organic layer was dried over $\mathrm{Na}_{2} \mathrm{SO}_{4}$, filtrated, and concentrated in vacuo. The residue was purified by Isolera ${ }^{\circledR}$ (hexane/EtOAc $=3: 1$ to EtOAc) to afford $\mathbf{1 E}(720 \mathrm{mg}$, $78 \%$ yield over 2 steps) as a white solid. ${ }^{1} \mathrm{H}$ NMR $\left(400 \mathrm{MHz}, \mathrm{CDCl}_{3}\right) \delta 7.37(\mathrm{~d}, J=8.0 \mathrm{~Hz}, 2 \mathrm{H}), 7.29$ (t, $J=8.0 \mathrm{~Hz}, 2 \mathrm{H}), 7.04$ (t, $J=8.0 \mathrm{~Hz}, 1 \mathrm{H}), 6.53-6.42(\mathrm{brs}, 1 \mathrm{H}), 6.29$ (d, $J=3.2 \mathrm{~Hz}, 1 \mathrm{H}), 6.27$ (d, $J=$ $3.2 \mathrm{~Hz}, 1 \mathrm{H}), 4.50$ (s, 2H), 3.07 (s, 3H); ${ }^{13} \mathrm{C} \mathrm{NMR}\left(101 \mathrm{MHz}, \mathrm{CDCl}_{3}, 333 \mathrm{~K}\right) \delta 155.4,153.6,139.1,128.8$, 123.2, 121.4, 120.2, 112.2, 110.8, 45.4, 34.6; HRMS (ESI) $m / z$ calcd for $\mathrm{C}_{13} \mathrm{H}_{13} \mathrm{BrN}_{2} \mathrm{NaO}_{2}[\mathrm{M}+\mathrm{Na}]^{+}$: 331.0053 found 331.0049 .

\section{Synthesis of (5-Bromofuran-2-yl)methyl phenylcarbamate (1F)}<smiles>CCO[N+](C)(C)OCc1ccc(Br)o1</smiles> 
To a round-bottom flask containing a magnetic stirring bar were added 5-bromofuran-2carbaldehyde (528 mg, $3.0 \mathrm{mmol}, 1.0$ equiv) and THF $(6.0 \mathrm{~mL})$. The solution was cooled to $0{ }^{\circ} \mathrm{C}$, and then $\mathrm{NaBH}_{4}$ (120 mg, 1.0 equiv) was added portionwise. After stirring the mixture at room temperature for3 $\mathrm{h}$, the reaction was quenched with $\mathrm{NH}_{4} \mathrm{Cl}$ aq. The mixture was extracted three times with $\mathrm{Et}_{2} \mathrm{O}$. The combined organic layer was dried over $\mathrm{MgSO}_{4}$, filtrated, and concentrated in vacuo to afford a crude mixture of (5-bromofuran-2-yl)methanol (S5) as a white solid. This was used for the next step without further purification.

To a crude mixture of $\mathbf{S 5}(130.6 \mathrm{mg}, 0.78 \mathrm{mmol}, 1.0$ equiv) in a round-bottom flask containing a magnetic stirring bar were added $\mathrm{CH}_{2} \mathrm{Cl}_{2}(5.0 \mathrm{~mL})$, isocyanatobenzene $(0.80 \mathrm{~mL}, 7.4 \mathrm{mmol}, 10$ equiv), and $\mathrm{NEt}_{3}(0.15 \mathrm{~mL}, 1.1 \mathrm{mmol}, 1.5$ equiv). After stirring the mixture for several hours with monitoring reaction progress with TLC, the reaction was quenched with $\mathrm{H}_{2} \mathrm{O}$. The mixture was extracted three times with $\mathrm{Et}_{2} \mathrm{O}$. The combined organic layer was washed two times with brine. The organic layer was dried over $\mathrm{MgSO}_{4}$, filtrated, and concentrated in vacuo. The residue was purified by Isolera ${ }^{\circledR}$ (hexane/EtOAc $=9: 1$ to $3: 2)$ to afford $\mathbf{1 F}\left(170.3 \mathrm{mg}, 78 \%\right.$ yield) as a white solid. ${ }^{1} \mathrm{H}$ NMR (400 $\left.\mathrm{MHz}, \mathrm{CDCl}_{3}\right) \delta 7.37$ $(\mathrm{d}, J=7.6 \mathrm{~Hz}, 2 \mathrm{H}), 7.31$ (t, $J=7.6 \mathrm{~Hz}, 2 \mathrm{H}), 7.07$ (t, $J=7.6 \mathrm{~Hz}, 1 \mathrm{H}), 6.76-6.65$ (brs, 1H), 6.43 (d, $J=$ $3.6 \mathrm{~Hz}, 1 \mathrm{H}), 6.29(\mathrm{~d}, J=3.6 \mathrm{~Hz}, 1 \mathrm{H}), 5.10(\mathrm{~s}, 2 \mathrm{H}) ;{ }^{13} \mathrm{C} \mathrm{NMR}\left(101 \mathrm{MHz}, \mathrm{CDCl}_{3}\right) \delta 151.6,137.5,129.1$, 123.7, 122.9, 118.7, 113.6, 112.3, 58.3 (one peak was missing due to overlapping); HRMS (ESI) $\mathrm{m} / \mathrm{z}$ calcd for $\mathrm{C}_{12} \mathrm{H}_{10} \mathrm{BrNO}_{3} \mathrm{Na}[\mathrm{M}+\mathrm{Na}]^{+}: 317.9736$ found 317.9738 .

\section{Synthesis of 2-((5-Bromofuran-2-yl)methoxy)- $N$-phenylacetamide (1G)}

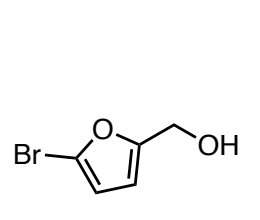

S5

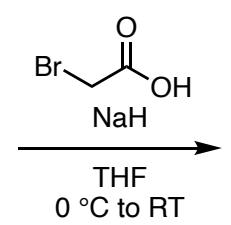

$0^{\circ} \mathrm{C}$ to $\mathrm{RT}$

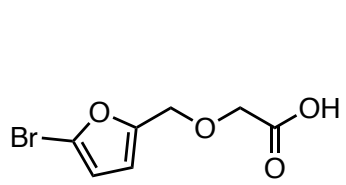

s6
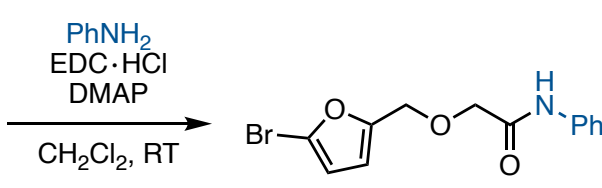

$1 G$

To a solution of $\mathbf{S 5}(353.9 \mathrm{mg}, 2.0 \mathrm{mmol}, 1.0$ equiv) in THF $(4.0 \mathrm{~mL}, 0.50 \mathrm{M})$ in a round-bottom flask was added sodium hydride ( $\mathrm{NaH}$ : 60\% dispersion in paraffin liquid, $251.7 \mathrm{mg}, 6.3 \mathrm{mmol}, 3.2$ equiv) at $0{ }^{\circ} \mathrm{C}$. After stirring the mixture for $30 \mathrm{~min}$, bromoacetic acid (572.2 $\mathrm{mg}, 4.1 \mathrm{mmol}, 2.1$ equiv) was added to the mixture. After stirring the mixture at room temperature for several hours with monitoring reaction progress with TLC, the reaction was quenched with $\mathrm{NH}_{4} \mathrm{Cl}$ aq. The mixture was extracted three times with $\mathrm{Et}_{2} \mathrm{O}$. The combined organic layer was dried over $\mathrm{MgSO}_{4}$, filtrated, and concentrated in vacuo to afford a crude mixture of 2-((5-bromofuran-2-yl)methoxy)acetic acid (S6). This was used for the next step without further purification.

To a crude mixture of $\mathbf{S 6}$ in a round-bottom flask containing a magnetic stirring bar were added $\mathrm{EDC} \cdot \mathrm{HCl}$ (962.9 mg, $5.02 \mathrm{mmol}, 2.5$ equiv), DMAP (101.6 mg, $0.83 \mathrm{mmol}, 42 \mathrm{~mol} \%$ ), aniline (0.40 $\mathrm{mL}, 4.4 \mathrm{mmol}, 2.2$ equiv), and $\mathrm{CH}_{2} \mathrm{Cl}_{2}(10 \mathrm{~mL}, 0.20 \mathrm{M})$. After stirring the mixture at room temperature for several hours with monitoring reaction progress with TLC, the reaction was quenched with $\mathrm{H}_{2} \mathrm{O}$. 
The mixture was extracted three times with $\mathrm{CH}_{2} \mathrm{Cl}_{2}$. The combined organic layer was dried over $\mathrm{Na}_{2} \mathrm{SO}_{4}$, filtrated, and concentrated in vacuo. The residue was purified by Isolera ${ }^{\circledR}$ (hexane/EtOAc $=9: 1$ to 1:1) to afford $\mathbf{1 G}$ as a pale yellow solid (319.1 mg, 51\% yield). ${ }^{1} \mathrm{H}$ NMR (400 $\left.\mathrm{MHz}, \mathrm{CDCl}_{3}\right) \delta 8.35-8.20$ (brs, $1 \mathrm{H}), 7.57$ (d, $J=7.6 \mathrm{~Hz}, 2 \mathrm{H}), 7.34$ (t, $J=7.6 \mathrm{~Hz}, 2 \mathrm{H}), 7.13$ (t, $J=7.6 \mathrm{~Hz}, 1 \mathrm{H}), 6.39$ (d, $J=3.2 \mathrm{~Hz}$, $1 \mathrm{H}), 6.30(\mathrm{~d}, J=3.2 \mathrm{~Hz}, 1 \mathrm{H}), 4.56(\mathrm{~s}, 2 \mathrm{H}), 4.10(\mathrm{~s}, 2 \mathrm{H}) ;{ }^{13} \mathrm{C} \mathrm{NMR}\left(101 \mathrm{MHz}, \mathrm{CDCl}_{3}\right) \delta 167.1,152.0$, 137.0, 129.0, 124.5, 123.2, 119.8, 113.3, 112.2, 69.3, 65.0; HRMS (ESI) $m / z$ calcd for $\mathrm{C}_{13} \mathrm{H}_{13} \mathrm{BrNO}_{3}$ $[\mathrm{M}+\mathrm{H}]^{+}: 310.0073$ found 310.0073 .

\section{Synthesis of 3-(5-Bromothiophen-2-yl)- $N$-arylpropanamides 1}<smiles>O=Cc1ccc(Br)s1</smiles>

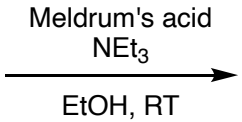<smiles>CC1(C)OC(=O)C(=Cc2ccc(Br)s2)C(=O)O1</smiles>

S7
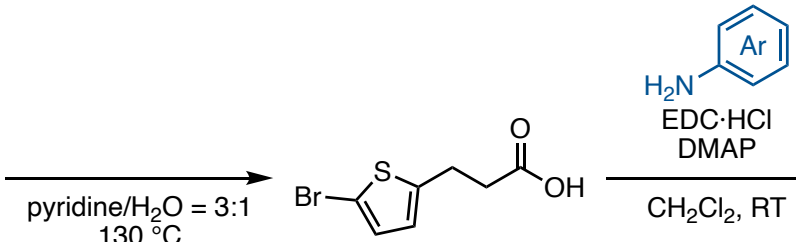
$130^{\circ} \mathrm{C}$

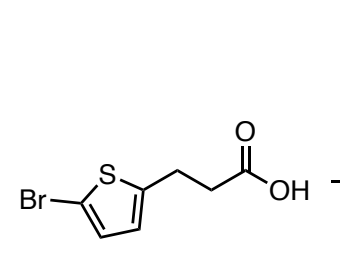

S9

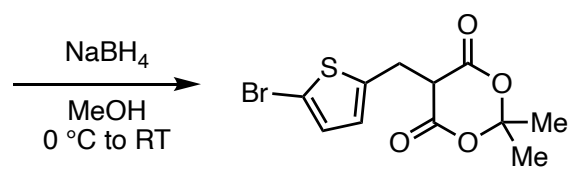

S8

Following a Procedure 1, 3-(5-bromothiophen-2-yl)- $N$-arylpropanamides 1 were synthesized by using 5-bromothiophen-2-carbaldehyde as a starting material.<smiles>O=C(CCc1ccc(Br)s1)Nc1ccccc1</smiles>

\section{3-(5-Bromothiophen-2-yl)- $N$-phenylpropanamide (1H)}

Purification by Isolera ${ }^{\mathbb{}}($ hexane/EtOAc $=19: 1$ to 3:1) afforded $\mathbf{1 H}$ as a white solid (457 $\mathrm{mg}, 2.0$ mmol scale, 79\% yield). ${ }^{1} \mathrm{H}$ NMR $\left(400 \mathrm{MHz}, \mathrm{CDCl}_{3}\right) \delta 7.46(\mathrm{~d}, J=7.6 \mathrm{~Hz}, 2 \mathrm{H}), 7.31(\mathrm{t}, J=7.6 \mathrm{~Hz}$, 2H), 7.24-7.15 (brs, 1H), 7.11 (t, $J=7.6 \mathrm{~Hz}, 1 \mathrm{H}), 6.85$ (d, $J=3.6 \mathrm{~Hz}, 1 \mathrm{H}), 6.61(\mathrm{~d}, J=3.6 \mathrm{~Hz}, 1 \mathrm{H})$, 3.19 (t, $J=7.2 \mathrm{~Hz}, 2 \mathrm{H}), 2.66(\mathrm{t}, J=7.2 \mathrm{~Hz}, 2 \mathrm{H}) ;{ }^{13} \mathrm{C} \mathrm{NMR}\left(101 \mathrm{MHz}, \mathrm{CDCl}_{3}\right) \delta 169.3,144.9,137.5$, 129.6, 129.0, 125.5, 124.5, 119.9, 109.6, 39.0, 25.9; HRMS (ESI) $\mathrm{m} / z$ calcd for $\mathrm{C}_{13} \mathrm{H}_{12} \mathrm{BrNNaOS}$ $[\mathrm{M}+\mathrm{Na}]^{+}: 331.9715$ found 331.9714 .<smiles>Cc1ccc(NC(=O)CCc2ccc(Br)s2)cc1</smiles>

\section{3-(5-Bromothiophen-2-yl)- $N$-( $p$-tolyl)propanamide (1I)}

Purification by Isolera $^{\circledR}($ hexane/EtOAc $=19: 1$ to 3:1) afforded $\mathbf{1 I}$ as a white solid (98.8 $\mathrm{mg}, 0.50$ mmol scale, 61\% yield). ${ }^{1} \mathrm{H}$ NMR $\left(400 \mathrm{MHz}, \mathrm{CDCl}_{3}\right) \delta 7.34(\mathrm{~d}, J=8.0 \mathrm{~Hz}, 2 \mathrm{H}), 7.12(\mathrm{~d}, J=8.0 \mathrm{~Hz}$, 
2H), 7.06-7.00 (brs, 1H), 6.85 (d, $J=3.6 \mathrm{~Hz}, 1 \mathrm{H}), 6.62$ (d, $J=3.6 \mathrm{~Hz}, 1 \mathrm{H}), 3.19$ (t, $J=7.2 \mathrm{~Hz}, 2 \mathrm{H})$, $2.65(\mathrm{t}, J=7.2 \mathrm{~Hz}, 2 \mathrm{H}), 2.31(\mathrm{~s}, 3 \mathrm{H}) ;{ }^{13} \mathrm{C} \mathrm{NMR}\left(101 \mathrm{MHz}, \mathrm{CDCl}_{3}\right) \delta 169.3,145.0,135.0,134.1,129.6$, $129.5,125.4,120.1,109.5,38.9,25.9,20.8$; HRMS (ESI) $\mathrm{m} / z$ calcd for $\mathrm{C}_{14} \mathrm{H}_{15} \mathrm{BrNOS}[\mathrm{M}+\mathrm{H}]^{+}$: 324.0052 found 324.0049 .

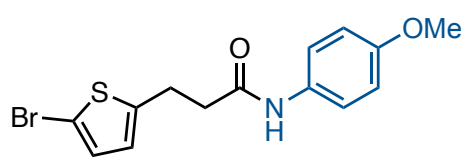

\section{3-(5-Bromothiophen-2-yl)- $N$-(4-methoxyphenyl)propanamide (1J)}

Purification by Isolera ${ }^{\circledR}$ (hexane/EtOAc $=19: 1$ to 3:1) afforded $\mathbf{1 J}$ as a white solid (139.0 $\mathrm{mg}, 0.50$ mmol scale, $81 \%$ yield). ${ }^{1} \mathrm{H}$ NMR (400 MHz, $\left.\mathrm{CDCl}_{3}\right) \delta 7.35$ (d, $\left.J=8.8 \mathrm{~Hz}, 2 \mathrm{H}\right), 7.10-7.02$ (brs, $1 \mathrm{H}$ ), 6.88-6.82 (m, 3H), 6.61 (d, $J=3.6 \mathrm{~Hz}, 1 \mathrm{H}), 3.79$ (s, 3H), 3.18 (t, $J=7.2 \mathrm{~Hz}, 2 \mathrm{H}), 2.64$ (t, $J=7.2 \mathrm{~Hz}$, $2 \mathrm{H}) ;{ }^{13} \mathrm{C} \mathrm{NMR}\left(101 \mathrm{MHz}, \mathrm{CDCl}_{3}\right) \delta 169.5,156.5,145.0,130.6,129.6,125.3,122.1,114.0,109.5,55.4$, 38.6, 26.0 HRMS (ESI) $m / z$ calcd for $\mathrm{C}_{14} \mathrm{H}_{15} \mathrm{BrNO}_{2} \mathrm{~S}[\mathrm{M}+\mathrm{H}]^{+}: 340.0001$ found 340.0001 .<smiles>O=C(CCc1ccc(Br)s1)Nc1ccc(C(F)(F)F)cc1</smiles>

\section{3-(5-Bromothiophen-2-yl)- $N$-(4-(trifluoromethyl)phenyl)propanamide (1K)}

Purification by Isolera ${ }^{\circledR}$ (hexane/EtOAc $=19: 1$ to 3:1) afforded $1 \mathbf{K}$ as a white solid (123.4 mg, 0.50 mmol scale, 66\% yield). ${ }^{1} \mathrm{H}$ NMR (400 MHz, $\left.\mathrm{CDCl}_{3}\right) \delta$ 7.64-7.52 (m, 4H), 7.36-7.28 (brs, $\left.1 \mathrm{H}\right), 6.85$ $(\mathrm{d}, J=3.6 \mathrm{~Hz}, 1 \mathrm{H}), 6.61(\mathrm{~d}, J=3.6 \mathrm{~Hz}, 1 \mathrm{H}), 3.20(\mathrm{t}, J=7.2 \mathrm{~Hz}, 2 \mathrm{H}), 2.70(\mathrm{t}, J=7.2 \mathrm{~Hz}, 2 \mathrm{H}) ;{ }^{13} \mathrm{C} \mathrm{NMR}$ $\left(101 \mathrm{MHz}, \mathrm{CDCl}_{3}\right) \delta 169.8,144.5,140.5,129.7,126.3\left(\mathrm{q}, J_{\mathrm{C}-\mathrm{F}}=3.9 \mathrm{~Hz}\right), 126.2\left(\mathrm{q}, J_{\mathrm{C}-\mathrm{F}}=32.9 \mathrm{~Hz}\right)$, $125.5,124.0\left(\mathrm{q}, J_{\mathrm{C}-\mathrm{F}}=272 \mathrm{~Hz}\right), 119.5,109.8,39.0,25.7 ;{ }^{19} \mathrm{~F} \mathrm{NMR}\left(376 \mathrm{MHz}, \mathrm{CDCl}_{3}\right) \delta-62.2 ; \mathrm{HRMS}$ (ESI) $m / z$ calcd for $\mathrm{C}_{14} \mathrm{H}_{12} \mathrm{BrF}_{3} \mathrm{NOS}[\mathrm{M}+\mathrm{H}]^{+}: 377.9767$ found 377.9768 .<smiles>O=C(CCc1ccc(Br)s1)Nc1ccc(Cl)cc1</smiles>

\section{3-(5-Bromothiophen-2-yl)- $N$-(4-chlorophenyl)propanamide (1L)}

Purification by Isolera ${ }^{\circledR}($ hexane/EtOAc $=19: 1$ to 3:1) afforded $1 \mathrm{~L}$ as a white solid $(115.7 \mathrm{mg}, 0.50$ mmol scale, 67\% yield). ${ }^{1} \mathrm{H}$ NMR (400 MHz, $\left.\mathrm{CDCl}_{3}\right) \delta 7.42(\mathrm{~d}, J=8.8 \mathrm{~Hz}, 2 \mathrm{H}), 7.28$ (d, $J=8.8 \mathrm{~Hz}$, 2H), 7.11-7.01 (brs, 1H), 6.86 (d, $J=3.6 \mathrm{~Hz}, 1 \mathrm{H}), 6.62$ (d, $J=3.6 \mathrm{~Hz}, 1 \mathrm{H}), 3.19$ (t, $J=7.2 \mathrm{~Hz}, 2 \mathrm{H}$ ), $2.66(\mathrm{t}, J=7.2 \mathrm{~Hz}, 2 \mathrm{H}) ;{ }^{13} \mathrm{C} \mathrm{NMR}\left(101 \mathrm{MHz}, \mathrm{CDCl}_{3}\right) \delta 169.7,144.7,136.0,129.6,129.5,129.0,125.4$, 121.3, 109.7, 38.8, 25.8; HRMS (ESI) $\mathrm{m} / z$ calcd for $\mathrm{C}_{13} \mathrm{H}_{12} \mathrm{BrClNOS}[\mathrm{M}+\mathrm{H}]^{+}: 343.9506$ found 343.9504 . 
<smiles>O=C(CCc1ccc(Br)s1)Nc1cccc(Cl)c1</smiles>

\section{3-(5-Bromothiophen-2-yl)- $N$-(3-chlorophenyl)propanamide (1M)}

Purification by Isolera ${ }^{\circledR}($ hexane/EtOAc $=19: 1$ to $3: 1)$ afforded $\mathbf{1 M}$ as a white solid $(123.4 \mathrm{mg}$, $0.50 \mathrm{mmol}$ scale, $66 \%$ yield). ${ }^{1} \mathrm{H}$ NMR (400 MHz, $\left.\mathrm{CDCl}_{3}\right) \delta 7.61(\mathrm{~s}, 1 \mathrm{H}), 7.34-7.15(\mathrm{~m}, 3 \mathrm{H}), 7.09$ (d, $J$ $=7.6 \mathrm{~Hz}, 1 \mathrm{H}), 6.85(\mathrm{~d}, J=3.2 \mathrm{~Hz}, 1 \mathrm{H}), 6.61(\mathrm{~d}, J=3.2 \mathrm{~Hz}, 1 \mathrm{H}), 3.18(\mathrm{t}, J=7.2 \mathrm{~Hz}, 2 \mathrm{H}), 2.66(\mathrm{t}, J=$ $7.2 \mathrm{~Hz}, 2 \mathrm{H}) ;{ }^{13} \mathrm{C}$ NMR $\left(101 \mathrm{MHz}, \mathrm{CDCl}_{3}\right) \delta 170.4,144.5,138.6,134.3,129.8,129.5,125.2,124.5$, 120.4, 118.3, 109.4, 38.3, 25.7; HRMS (ESI) $m / z$ calcd for $\mathrm{C}_{13} \mathrm{H}_{12} \mathrm{BrClNOS}[\mathrm{M}+\mathrm{H}]^{+}: 343.9506$ found 343.9503 .<smiles>O=C(CCc1ccc(Br)s1)Nc1ccccc1Cl</smiles>

\section{3-(5-Bromothiophen-2-yl)- $\mathrm{N}$-(2-chlorophenyl)propanamide (1N)}

Purification by Isolera ${ }^{\circledR}($ hexane/EtOAc $=19: 1$ to $3: 1)$ afforded $1 \mathrm{~N}$ as a white solid $(104.7 \mathrm{mg}, 0.50$ mmol scale, 61\% yield). ${ }^{1} \mathrm{H}$ NMR $\left(400 \mathrm{MHz}, \mathrm{CDCl}_{3}\right) \delta 8.35(\mathrm{~d}, J=8.4 \mathrm{~Hz}, 1 \mathrm{H}), 7.65-7.51$ (brs, $\left.1 \mathrm{H}\right)$, $7.36(\mathrm{dd}, J=8.0,1.2 \mathrm{~Hz}, 1 \mathrm{H}), 7.26(\mathrm{td}, J=8.0,1.2 \mathrm{~Hz}, 1 \mathrm{H}), 7.05(\mathrm{td}, J=8.0,1.2 \mathrm{~Hz}, 1 \mathrm{H}), 6.85(\mathrm{~d}, J=$ $4.0 \mathrm{~Hz}, 1 \mathrm{H}), 6.63(\mathrm{~d}, J=4.0 \mathrm{~Hz}, 1 \mathrm{H}), 3.21(\mathrm{t}, J=7.2 \mathrm{~Hz}, 2 \mathrm{H}), 2.75(\mathrm{t}, J=7.2 \mathrm{~Hz}, 2 \mathrm{H}) ;{ }^{13} \mathrm{C} \mathrm{NMR}(101$ $\left.\mathrm{MHz}, \mathrm{CDCl}_{3}\right) \delta 169.3,144.6,134.2,129.6,128.9,127.6,125.4,124.7,122.7,121.8,109.6,39.0,25.7$; HRMS (ESI) $\mathrm{m} / z$ calcd for $\mathrm{C}_{13} \mathrm{H}_{12} \mathrm{BrClNOS}[\mathrm{M}+\mathrm{H}]^{+}: 343.9506$ found 343.9507.<smiles>O=C(CCc1ccc(Br)s1)Nc1ccc(Br)cc1</smiles>

\section{$N$-(4-Bromophenyl)-3-(5-bromothiophen-2-yl)propanamide (10)}

Purification by Isolera ${ }^{\circledR}($ hexane/EtOAc $=19: 1$ to $3: 1)$ afforded 10 as a white solid $(110.0 \mathrm{mg}, 0.50$ mmol scale, 57\% yield). ${ }^{1} \mathrm{H}$ NMR (400 MHz, $\left.\mathrm{CDCl}_{3}\right) \delta 7.43(\mathrm{~d}, J=8.8 \mathrm{~Hz}, 2 \mathrm{H}), 7.37$ (d, $J=8.8 \mathrm{~Hz}$, 2H), 7.06-7.00 (brs, 1H), 6.86 (d, $J=3.6 \mathrm{~Hz}, 1 \mathrm{H}), 6.62$ (d, $J=3.6 \mathrm{~Hz}, 1 \mathrm{H}), 3.19$ (t, $J=7.2 \mathrm{~Hz}, 2 \mathrm{H})$, $2.66(\mathrm{t}, J=7.2 \mathrm{~Hz}, 2 \mathrm{H}) ;{ }^{13} \mathrm{C} \mathrm{NMR}\left(101 \mathrm{MHz}, \mathrm{CDCl}_{3}\right) \delta 169.5,144.7,136.6,132.0,129.7,125.5,121.5$, 117.1, 109.7, 39.0, 25.8; HRMS (ESI) $m / z$ calcd for $\mathrm{C}_{13} \mathrm{H}_{12} \mathrm{Br}_{2} \mathrm{NOS}[\mathrm{M}+\mathrm{H}]^{+}: 387.9001$ found 387.8999 .

\section{Synthesis of 1-((5-Bromothiophen-2-yl)methyl)-1-methyl-3-phenylurea (1P)}<smiles>O=Cc1ccc(Br)s1</smiles><smiles>C[N+](N)(N)O[Mg]</smiles>

B<smiles>CN(C[Na])Cc1ccc(Br)s1</smiles>

S10<smiles>CC(C)[PH2+]Cc1ccc(Br)s1</smiles>

Following a Procedure 2 using 5-bromothiophen-2-carbaldehyde as a starting material, 1P was obtained as a white solid (789 mg, $2.85 \mathrm{mmol}$ scale, $85 \%$ yield over 2 steps). ${ }^{1} \mathrm{H}$ NMR (400 MHz, 
$\left.\mathrm{CDCl}_{3}\right) \delta 7.38(\mathrm{~d}, J=7.2 \mathrm{~Hz}, 2 \mathrm{H}), 7.30(\mathrm{t}, J=7.2 \mathrm{~Hz}, 2 \mathrm{H}), 7.05(\mathrm{t}, J=7.2 \mathrm{~Hz}, 1 \mathrm{H}), 6.91(\mathrm{~d}, J=3.6 \mathrm{~Hz}$, 1H), $6.76(\mathrm{~d}, J=3.6 \mathrm{~Hz}, 1 \mathrm{H}), 6.42-6.32$ (brs, $1 \mathrm{H}), 4.63$ (s, 2H), $3.02(\mathrm{~s}, 3 \mathrm{H}) ;{ }^{13} \mathrm{C}$ NMR $(101 \mathrm{MHz}$, DMSO- $\left.d_{6}\right) \delta 155.5,143.1,140.3,129.6,128.3,127.3,122.0,120.0,110.7,46.8,34.2 ;$ HRMS (ESI) $\mathrm{m} / \mathrm{z}$ calcd for $\mathrm{C}_{13} \mathrm{H}_{13} \mathrm{BrN}_{2} \mathrm{NaOS}[\mathrm{M}+\mathrm{Na}]^{+}: 346.9824$ found 346.9822 .

\section{Synthesis of 2-(5-Bromothiophen-2-yl)- $N$-phenylbenzamide (1Q)}<smiles>O=Cc1ccccc1-c1cccs1</smiles>

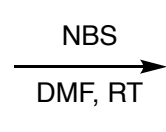<smiles>O=Cc1ccccc1-c1ccc(Br)s1</smiles>

S11

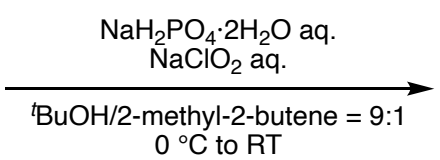
$0{ }^{\circ} \mathrm{C}$ to $\mathrm{RT}$

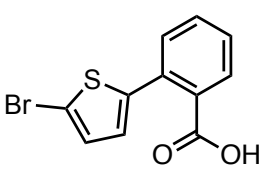

S12

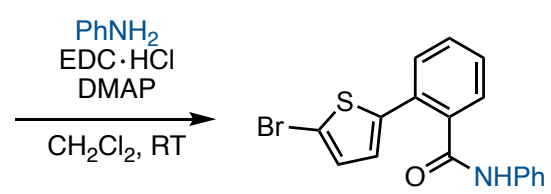

$1 Q$

To a solution of 2-(thiophen-2-yl)benzaldehyde ${ }^{[5]}(848 \mathrm{mg}, 4.50 \mathrm{mmol}, 1.0$ equiv) in DMF (35 mL) was added $N$-bromosuccinimide (NBS: $882 \mathrm{mg}, 4.95 \mathrm{mmol}, 1.1$ equiv). After stirring the mixture for several hours with monitoring reaction progress with TLC, the solvent was evaporated in vacuo. Purification by Isolera ${ }^{\circledR}$ (hexane/EtOAc $=15: 1$ to 2:1) afforded 2-(5-bromothiophen-2-yl)benzaldehyde (S11: $1.11 \mathrm{~g}, 92 \%$ yield).

To a solution of $\mathbf{S 1 1}\left(551 \mathrm{mg}, 2.06 \mathrm{mmol}, 1.0\right.$ equiv) in ${ }^{t} \mathrm{BuOH} / 2$-methyl-2-butene (2.3 mL, 9:1, $0.90 \mathrm{M}$ ) were added $\mathrm{NaClO}_{2}$ aq. (5.5 M, $1.5 \mathrm{~mL}, 8.25 \mathrm{mmol}, 4.0$ equiv) and $\mathrm{NaH}_{2} \mathrm{PO}_{4}$ aq. (5.5 M, 1.5 $\mathrm{mL}, 8.25 \mathrm{mmol}, 4.0$ equiv) at $0{ }^{\circ} \mathrm{C}$. After stirring the mixture at room temperature for several hours with monitoring reaction progress with TLC, the reaction was quenched with $\mathrm{Na}_{2} \mathrm{~S}_{2} \mathrm{O}_{3}$ aq. The mixture was diluted with $\mathrm{Et}_{2} \mathrm{O}$ and extracted with $3.0 \mathrm{M} \mathrm{NaOH}$ aq. The aqueous layer was added $6 \mathrm{M} \mathrm{HCl}$ aq. to adjust $\mathrm{pH}$ 1. The mixture was extracted three times with $\mathrm{Et}_{2} \mathrm{O}$. The combined organic layer was dried over $\mathrm{MgSO}_{4}$, filtrated, and concentrated in vacuo to afford 2-(5-bromothiophen-2-yl)benzoic acid (S12) as a dark red oil. This was used for the next step without further purification.

To a crude mixture of $\mathbf{S 1 2}$ (1.0 equiv) in a round-bottom flask were added EDC $\cdot \mathrm{HCl}$ (474 mg, 2.47 mmol, 1.2 equiv), DMAP (101 mg, $0.825 \mathrm{mmol}, 40 \mathrm{~mol} \%$ ), aniline ( $0.19 \mathrm{~mL}, 2.06 \mathrm{mmol}, 1.0$ equiv), and $\mathrm{CH}_{2} \mathrm{Cl}_{2}(10 \mathrm{~mL})$. After stirring the mixture at room temperature for several hours with monitoring reaction progress with TLC, the reaction was quenched with $\mathrm{H}_{2} \mathrm{O}$. The mixture was extracted three times with $\mathrm{CH}_{2} \mathrm{Cl}_{2}$. The combined organic layer was dried over $\mathrm{Na}_{2} \mathrm{SO}_{4}$, filtrated, and concentrated in vacuo. The residue was purified by Isolera ${ }^{\circledR}$ (hexane/EtOAc $=15: 1$ to $\left.1: 1\right)$ to afford $1 Q$ ( $548 \mathrm{mg}, 74 \%$ yield over 2 steps) as a white solid. ${ }^{1} \mathrm{H}$ NMR $\left(400 \mathrm{MHz}, \mathrm{CDCl}_{3}\right) \delta 7.67(\mathrm{~d}, J=7.6 \mathrm{~Hz}, 1 \mathrm{H}), 7.52-7.40$ (m, $3 \mathrm{H}), 7.40-7.34$ (m, 3H), 7.31 (t, $J=7.6 \mathrm{~Hz}, 2 \mathrm{H}), 7.12$ (t, $J=7.6 \mathrm{~Hz}, 1 \mathrm{H}), 7.00$ (d, $J=3.6 \mathrm{~Hz}, 1 \mathrm{H}), 6.95$ $(\mathrm{d}, J=3.6 \mathrm{~Hz}, 1 \mathrm{H}) ;{ }^{13} \mathrm{C}$ NMR $\left(101 \mathrm{MHz}, \mathrm{CDCl}_{3}, 333 \mathrm{~K}\right) \delta 167.0,142.6,137.7,136.1,131.1,130.7$, 
$130.4,129.0,128.9,128.5,127.5,124.7,120.2,113.2$ (one peak is missing due to overlapping); HRMS (ESI) $m / z$ calcd for $\mathrm{C}_{17} \mathrm{H}_{12} \mathrm{BrNNaOS}[\mathrm{M}+\mathrm{Na}]^{+}: 379.9715$ found 379.9715.

\section{Synthesis of 3-(4,5-Dibromothiophen-2-yl)- $N$-phenylpropanamide (1R)}

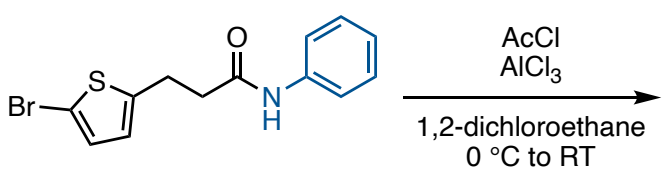

$1 \mathrm{H}$<smiles>O=C(CCc1cc(Br)c(Br)s1)Nc1ccccc1</smiles>

$1 R$

An 8-mL glass tube containing a magnetic stirring bar was dried with a heat-gun in vacuo and filled $\mathrm{N}_{2}$ gas after cooling to room temperature. To this tube were added 1,2-dichloroethane $(0.50 \mathrm{~mL})$, acethyl chloride (26 $\mu \mathrm{L}, 0.36 \mathrm{mmol}, 1.2$ equiv), aluminum chloride (90.3 mg, $0.66 \mathrm{mmol}, 2.3$ equiv) at $0{ }^{\circ} \mathrm{C}$. The reaction mixture was stirred at $0{ }^{\circ} \mathrm{C}$ for $1 \mathrm{~h}$. To this tube was added a solution of amide $\mathbf{1 H}(93.1$ $\mathrm{mg}, 0.30 \mathrm{mmol}, 1.0$ equiv) in 1,2-dichloroethane $(0.44 \mathrm{~mL})$. After stirring the mixture at room temperature for several hours with monitoring reaction progress with TLC, the mixture was passed through a pad of Celite ${ }^{\circledR}$ with $\mathrm{CH}_{2} \mathrm{Cl}_{2}$ as an eluent. The filtrate was added $3.0 \mathrm{M} \mathrm{NaOH}$ aq. and then extracted three times with $\mathrm{CH}_{2} \mathrm{Cl}_{2}$. The combined organic layer was washed with brine. The combined organic layer was dried over $\mathrm{Na}_{2} \mathrm{SO}_{4}$, filtered, and concentrated in vacuo. The residue was purified by Isolera $^{\circledR}$ (hexane/EtOAc $=3: 1$ to $\left.1: 1\right)$ to afford $1 R\left(31.7 \mathrm{mg}, 30 \%\right.$ yield) as a white solid. ${ }^{1} \mathrm{H}$ NMR (400 $\left.\mathrm{MHz}, \mathrm{CDCl}_{3}\right) \delta 7.49(\mathrm{~d}, J=7.6 \mathrm{~Hz}, 2 \mathrm{H}), 7.32$ (t, $\left.J=7.6 \mathrm{~Hz}, 2 \mathrm{H}\right), 7.11(\mathrm{t}, J=7.6 \mathrm{~Hz}, 1 \mathrm{H}), 6.88(\mathrm{~s}, 1 \mathrm{H})$, $3.17(\mathrm{t}, J=7.2 \mathrm{~Hz}, 2 \mathrm{H}), 2.67$ (t, $J=7.2 \mathrm{~Hz}, 2 \mathrm{H}) .{ }^{13} \mathrm{C} \mathrm{NMR}\left(101 \mathrm{MHz}, \mathrm{CDCl}_{3}\right) \delta 169.0,139.1,137.5$, 131.9, 129.0, 124.5, 120.0, 110.1, 108.4, 37.3, 24.9; HRMS (ESI) $m / z$ calcd for $\mathrm{C}_{13} \mathrm{H}_{12} \mathrm{Br}_{2} \mathrm{NOS}[\mathrm{M}+\mathrm{H}]^{+}$: 387.9001 found 387.9002 .

\section{Synthesis of $N$-(3-(5-Bromofuran-2-yl)propyl)aniline (1S)}

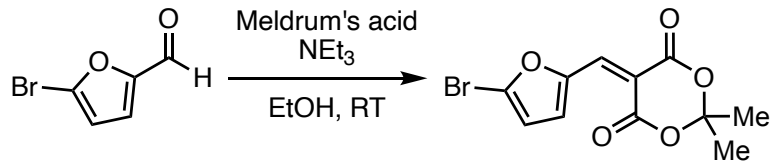

S1

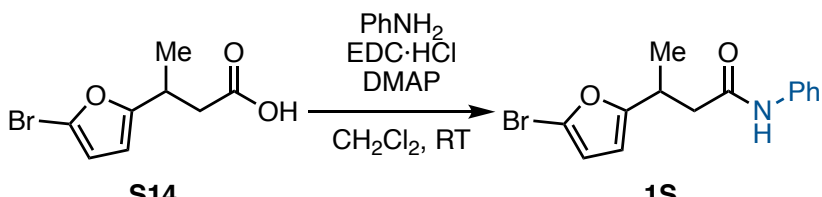

S14

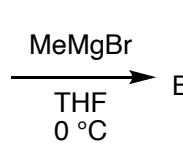

1

s13

To a round-bottom flask containing a magnetic stirring bar were added 5-bromofuran-2carbaldehyde (1.05 g, 6.00 mmol, 1.0 equiv), Meldrum's acid (1.04 g, 7.20 mmol, 1.2 equiv), EtOH (24 $\mathrm{mL})$, and $\mathrm{NEt}_{3}(40 \mu \mathrm{L}, 0.29 \mathrm{mmol}, 5 \mathrm{~mol} \%$ ) at room temperature. After stirring the mixture for several hours with monitoring reaction progress with TLC, the mixture was concentrated in vacuo. The residue was purified by Isolera $^{\circledR}$ (heaxne/EtOAc $=3: 1$ to 1:2) to afford 5-((5-bromofuran-2-yl)methylene)-2,2dimethyl-1,3-dioxane-4,6- dione (S1: $1.61 \mathrm{~g}, 89 \%$ yield) as a yellow solid. 
A $100-\mathrm{mL}$ round-bottom flask containing a magnetic stirring bar was dried with a heat-gun in vacuo and filled $\mathrm{N}_{2}$ after cooling to room temperature. To this flask were added $\mathbf{S 1}$ (753 $\mathrm{mg}, 2.50 \mathrm{mmol}$, 1.0 equiv) and THF (12 mL). The mixture was cooled to $0{ }^{\circ} \mathrm{C}$ and then $\mathrm{MeMgBr}(1.04 \mathrm{M}$ in THF, 2.88 $\mathrm{mL}, 3.00 \mathrm{mmol}, 1.2$ equiv) was added slowly. After stirring the mixture at $0{ }^{\circ} \mathrm{C}$ for $10 \mathrm{~min}$, the reaction was quenched with $1 \mathrm{M} \mathrm{HCl}$ aq. The mixture was extracted three times with EtOAc. The combined organic layer was dried over $\mathrm{Na}_{2} \mathrm{SO}_{4}$, filtrated, and concentrated in vacuo to afford a crude mixture of 5-(1-(5-bromofuran-2-yl)ethyl)-2,2-dimethyl-1,3-dioxane-4,6-dione (S13). This was used for the next step without further purification.

To a crude mixture of $\mathbf{S 1 3}$ (2.5 mmol, 1.0 equiv) in a round-bottom flask were added pyridine (2.5 $\mathrm{mL})$ and $\mathrm{H}_{2} \mathrm{O}(0.83 \mathrm{~mL})$. After stirring the mixture at $130{ }^{\circ} \mathrm{C}$ for several hours with monitoring reaction progress with TLC, the reaction was quenched with $3 \mathrm{M} \mathrm{HCl}$ aq. The mixture was extracted three times with EtOAc. The combined organic layer was dried over $\mathrm{Na}_{2} \mathrm{SO}_{4}$, filtrated, and concentrated in vacuo to afford a crude mixture of 3-(5-bromofuran-2-yl)butanoic acid (S14). This was used for the next step without further purification.

To a crude mixture of $\mathbf{S 1 4}$ (2.5 mmol, 1.0 equiv) in a round-bottom flask were added EDC $\cdot \mathrm{HCl}$ (575 mg, $3.00 \mathrm{mmol}, 1.2$ equiv), DMAP (122 mg, $1.00 \mathrm{mmol}, 40 \mathrm{~mol} \%$ ), aniline (0.23 mL, $2.50 \mathrm{mmol}$, 1.0 equiv), and $\mathrm{CH}_{2} \mathrm{Cl}_{2}(13 \mathrm{~mL})$. After stirring the mixture at room temperature for several hours with monitoring reaction progress with TLC, the reaction was quenched with $\mathrm{H}_{2} \mathrm{O}$. The mixture was extracted three times with $\mathrm{CH}_{2} \mathrm{Cl}_{2}$. The combined organic layer was dried over $\mathrm{Na}_{2} \mathrm{SO}_{4}$, filtrated, and concentrated in vacuo. The residue was purified by Isolera ${ }^{\circledR}$ (hexane/EtOAc $=15: 1$ to $\left.1: 1\right)$ to afford $1 \mathrm{~S}$ (668 $\mathrm{mg}, 87 \%$ yield over 3 steps) as a white solid. ${ }^{1} \mathrm{H}$ NMR $\left(400 \mathrm{MHz}, \mathrm{CDCl}_{3}\right) \delta 7.45(\mathrm{~d}, J=8.0 \mathrm{~Hz}, 2 \mathrm{H}), 7.31(\mathrm{t}, J=$ $8.0 \mathrm{~Hz}, 2 \mathrm{H}), 7.18-7.05(\mathrm{~m}, 2 \mathrm{H}), 6.19$ (d, $J=3.2 \mathrm{~Hz}, 1 \mathrm{H}), 6.05$ (d, $J=3.2 \mathrm{~Hz}, 1 \mathrm{H}), 3.51-3.40(\mathrm{~m}, 1 \mathrm{H})$, $2.74(\mathrm{dd}, J=14.4,3.2 \mathrm{~Hz}, 1 \mathrm{H}), 2.50(\mathrm{dd}, J=14.4,3.2 \mathrm{~Hz}, 1 \mathrm{H}), 1.35(\mathrm{~d}, J=6.8 \mathrm{~Hz}, 3 \mathrm{H}) ;{ }^{13} \mathrm{C} \mathrm{NMR}(101$ MHz, DMSO- $\left.d_{6}\right) \delta 169.1,161.3,139.1,128.7,123.2,119.2,118.9,112.1,107.1,41.9,29.9,18.6$; HRMS (ESI) $m / z$ calcd for $\mathrm{C}_{14} \mathrm{H}_{14} \mathrm{BrNNaO}_{2}[\mathrm{M}+\mathrm{Na}]^{+}: 330.0100$ found 330.0099 .

\section{Synthesis of $N$-(3-(5-Bromofuran-2-yl)propyl)aniline (1T)}
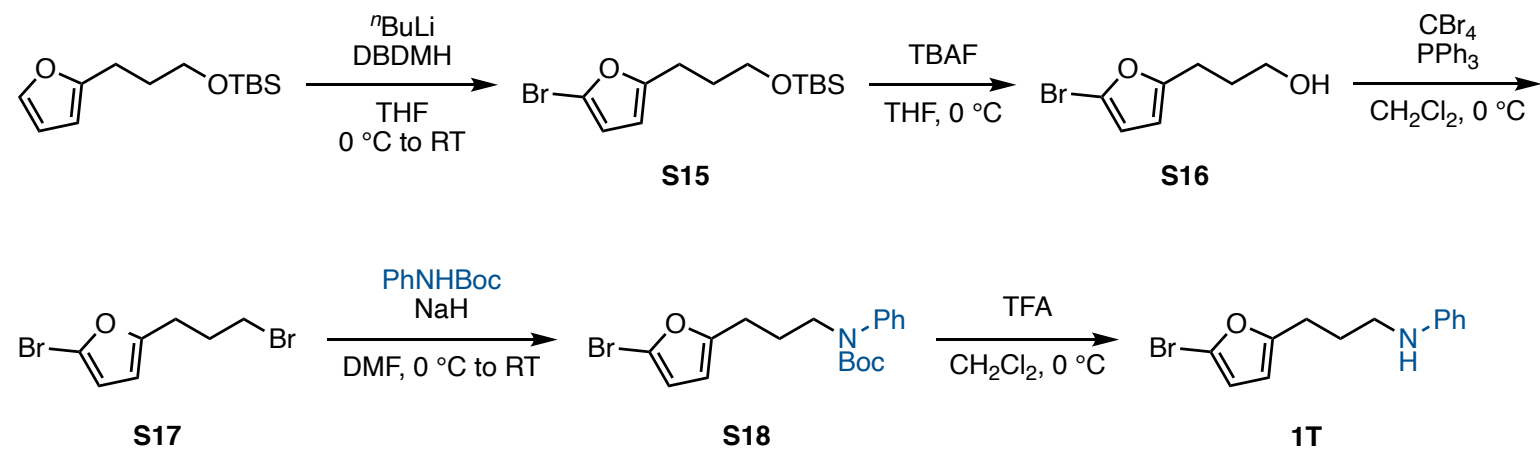

A 100-mL two-necked flask containing a magnetic stirring bar was dried with a heat-gun in vacuo and filled $\mathrm{N}_{2}$ after cooling to room temperature. To this flask was added tert-butyl(3-(furan-2- 
yl)propoxy)dimethylsilane ${ }^{[6-8]}(1.30 \mathrm{~g}, 5.41 \mathrm{mmol}, 1.0$ equiv) in THF $(18 \mathrm{~mL})$. The mixture was cooled to $0{ }^{\circ} \mathrm{C}$ and then $n$-butyllithium ( $1.59 \mathrm{M}$ in hexane, $3.74 \mathrm{~mL}, 5.95 \mathrm{mmol}, 1.1$ equiv) was added slowly. The mixture was stirred at $0{ }^{\circ} \mathrm{C}$ for $45 \mathrm{~min}$. To this mixture was added 1,3-dibromo-5,5dimethylhydantoin (DBDMH: $928 \mathrm{mg}, 3.24 \mathrm{mmol}, 0.60$ equiv) portionwise. The mixture was stirred at room temperature for $30 \mathrm{~min}$. The reaction was quenched with $\mathrm{NaHCO}_{3}$ aq. and $\mathrm{Na}_{2} \mathrm{~S}_{2} \mathrm{O}_{3}$ aq. The mixture was extracted three times with EtOAc. The combined organic layer was dried over $\mathrm{Na}_{2} \mathrm{SO}_{4}$, filtrated, and concentrated in vacuo. The residure was purified by Isolera ${ }^{\circledR}$ (hexane to hexane/EtOAc $=$ 10:1) to afford (3-(5-bromofuran-2-yl)propoxy)(tert-butyl)dimethylsilane (S15: $1.11 \mathrm{~g}, 64 \%$ yield).

To a solution of $\mathbf{S 1 5}(1.11 \mathrm{~g}, 3.49 \mathrm{mmol}, 1.0$ equiv) in THF (12 mL) was added TBAF in THF (1 $\mathrm{M}, 4.19 \mathrm{~mL}, 4.19 \mathrm{mmol}, 1.2$ equiv) at $0{ }^{\circ} \mathrm{C}$. After stirring the mixture at $0{ }^{\circ} \mathrm{C}$ for $1 \mathrm{~h}$ with monitoring reaction progress with TLC, the reaction was quenched with $\mathrm{H}_{2} \mathrm{O}$. The mixture was extracted three times with $\mathrm{Et}_{2} \mathrm{O}$. The combined organic layer was dried over $\mathrm{MgSO}_{4}$, filtrated, and concentrated in vacuo. The resiedure was purified by Isolera ${ }^{\circledR}$ (hexane/EtOAc $=10: 1$ to 2:1) to afford 3-(5-bromofuran-2-yl)propan1-ol (S16: $1.05 \mathrm{~g}$, quant).

To a round-bottom flask containing $\mathbf{S 1 6}$ (1.05 g, 3.49 mmol, 1.0 equiv) and $\mathrm{PPh}_{3}(1.10 \mathrm{~g}, 4.20$ mmol, 1.2 equiv) in $\mathrm{CH}_{2} \mathrm{Cl}_{2}(18 \mathrm{~mL}, 0.19 \mathrm{M})$ was added $\mathrm{CBr}_{4}\left(1.39 \mathrm{~g}, 4.20 \mathrm{mmol}, 1.2\right.$ equiv) at $0{ }^{\circ} \mathrm{C}$. After stirring the mixture at $0{ }^{\circ} \mathrm{C}$ for $1 \mathrm{~h}$ with monitoring reaction progress with TLC, the mixture was passed through a pad of silica-gel with hexane as an eluent, and then concentrated in vacuo to afford 2bromo-5-(3-bromopropyl)furan (S17) as a crude mixture. This was used for the next step without further purification.

To a round-bottom flask containing tert-butyl phenylcarbamate ${ }^{[9]}(809 \mathrm{mg}, 4.19 \mathrm{mmol}, 1.2$ equiv) in DMF (5.0 mL) was added sodium hydride ( $\mathrm{NaH}: 60 \%$ dispersion in paraffin liquid, $195 \mathrm{mg}, 4.89 \mathrm{mmol}$, 1.4 equiv) at $0{ }^{\circ} \mathrm{C}$. After stirring the mixture for $1 \mathrm{~h}, \mathbf{S 1 7}$ (3.49 mmol, 1.0 equiv) in DMF (3.7 mL) was added to this mixture at $0{ }^{\circ} \mathrm{C}$. After stirring the mixture at room temperature for several hours with monitoring reaction progress with TLC, the reaction was quenched with $\mathrm{NH}_{4} \mathrm{Cl}$ aq. The mixture was extracted three times with $\mathrm{Et}_{2} \mathrm{O}$. The combined organic layer was dried over $\mathrm{MgSO}_{4}$, filtrated, and concentrated in vacuo. The residure was purified by Isolera ${ }^{\circledR}$ (hexane/EtOAc $=19: 1$ to 3:1) to afford tert-butyl (3-(5-bromofuran-2-yl)propyl)(phenyl)carbamate (S18: $1.00 \mathrm{~g}, 76 \%$ yield over 3 steps).

To a solution of $\mathbf{S 1 8}$ (1.14 g, $3.00 \mathrm{mmol}, 1.0$ equiv) in $\mathrm{CH}_{2} \mathrm{Cl}_{2}(7.5 \mathrm{~mL})$ was added trifluoroacetic acid (TFA: $2.96 \mathrm{~mL}, 45.1 \mathrm{mmol}, 15$ equiv) at $0{ }^{\circ} \mathrm{C}$. After stirring the mixture at $0{ }^{\circ} \mathrm{C}$ for $1 \mathrm{~h}$ with monitoring reaction progress with TLC, the reaction was quenched with $\mathrm{NaHCO}_{3}$ aq. The mixture was extracted three times with $\mathrm{Et}_{2} \mathrm{O}$. The combined organic layer was dried over $\mathrm{MgSO}_{4}$, filtrated, and concentrated in vacuo. The residue was purified by Isolera ${ }^{\circledR}$ (hexane/EtOAc $=19: 1$ to 3:1) to afford 1 T (737 mg, 88\% yield) as a pale yellow oil. ${ }^{1} \mathrm{H}$ NMR (400 MHz, $\left.\mathrm{CDCl}_{3}\right) \delta 7.18(\mathrm{t}, J=7.6 \mathrm{~Hz}, 2 \mathrm{H}), 6.70$ (t, $J=7.6 \mathrm{~Hz}, 1 \mathrm{H}), 6.59$ (d, $J=7.6 \mathrm{~Hz}, 2 \mathrm{H}), 6.19$ (d, $J=3.2 \mathrm{~Hz}, 1 \mathrm{H}), 6.00(\mathrm{~d}, J=3.2 \mathrm{~Hz}, 1 \mathrm{H}), 3.75-$ 3.57 (brs, $1 \mathrm{H}), 3.17$ (t, $J=7.6 \mathrm{~Hz}, 2 \mathrm{H}), 2.72$ (t, $J=7.6 \mathrm{~Hz}, 2 \mathrm{H}), 1.99-1.89(\mathrm{~m}, 2 \mathrm{H}) ;{ }^{13} \mathrm{C}$ NMR $(101$ 
$\left.\mathrm{MHz}, \mathrm{CDCl}_{3}\right) \delta 157.4,148.1,129.3,119.3,117.3,112.7,111.7,108.1,43.0,27.7,25.7 ;$ HRMS (ESI) $m / z$ calcd for $\mathrm{C}_{13} \mathrm{H}_{15} \mathrm{BrNO}[\mathrm{M}+\mathrm{H}]^{+}: 280.0332$ found 280.0332.

\section{Synthesis of 3-(2-Bromothiophen-3-yl)- $N$-phenylpropanamide (4)}
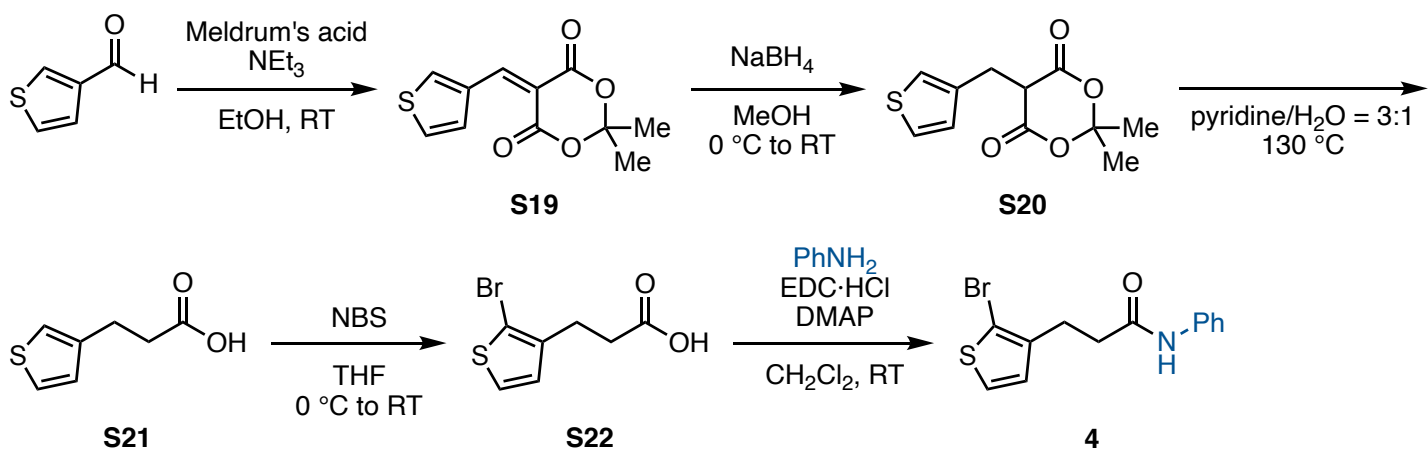

To a round-bottom flask containing thiophene-3-carbaldehyde $(0.18 \mathrm{~mL}, 2.3 \mathrm{mmol}, 1.0$ equiv) and Meldrum's acid (388.3 mg, 2.7 mmol, 1.2 equiv) in EtOH (9.0 mL) was added $\mathrm{NEt}_{3}\left(40 \mu \mathrm{L}, 0.29 \mathrm{mmol}^{\text {, }}\right.$ $12 \mathrm{~mol} \%$ ). After stirring the mixture at room temperature for several hours with monitoring reaction progress with TLC, the mixture was concentrated in vacuo to afford a crude mixture of 2,2-dimethyl-5(thiophen-3-ylmethylene)-1,3-dioxane-4,6-dione (S19) as a yellow oil. This was used for the next step without further purification.

To this crude mixture was added $\mathrm{MeOH}(21 \mathrm{~mL})$. The mixture was cooled to $0{ }^{\circ} \mathrm{C}$, and then $\mathrm{NaBH}_{4}$ (102.2 mg, $2.7 \mathrm{mmol}, 1.2$ equiv) was added portionwise. After stirring the mixture at room temperature for several hours with monitoring reaction progress with TLC, the mixture was concentrated in vacuo. To the mixture was added $1.0 \mathrm{M} \mathrm{HCl}$ aq. and extracted three times with $\mathrm{CH}_{2} \mathrm{Cl}_{2}$. The combined organic layer was dried over $\mathrm{Na}_{2} \mathrm{SO}_{4}$, filtrated, and concentrated in vacuo to afford a crude mixture of 2,2dimethyl-5-(thiophen-3-ylmethyl)-1,3-dioxane-4,6-dione (S20) as a yellow oil. This was used for the next step without further purification.

To a crude mixture of $\mathbf{S 2 0}$ (1.0 equiv) in a round-bottom flask were added pyridine ( $2.3 \mathrm{~mL}$ ) and $\mathrm{H}_{2} \mathrm{O}(0.70 \mathrm{~mL})$. After stirring the mixture at $130^{\circ} \mathrm{C}$ for several hours with monitoring reaction progress with TLC, the reaction was quenched with $6.0 \mathrm{M} \mathrm{HCl}$ aq. The mixture was extracted three times with EtOAc. The combined organic layer was dried over $\mathrm{Na}_{2} \mathrm{SO}_{4}$, filtrated, and concentrated in vacuo. The residue was purified by Isolera ${ }^{\circledR}$ (hexane/EtOAc $=1: 1$ to EtOAc) to afford 3-(thiophen-3-yl)propanoic acid (S21: $311.9 \mathrm{mg}, 89 \%$ yield over 3 steps) as a yellow oil.

To a solution of S21 (312.4 mg, $2.0 \mathrm{mmol}, 1.0$ equiv) in THF (10 mL) was added $N$ bromosuccinimide (NBS: $357.0 \mathrm{mg}, 2.0 \mathrm{mmol}, 1.0$ equiv). After stirring the mixture at room temperature for several hours with monitoring reaction progress with ${ }^{1} \mathrm{H} N M R$, the mixture was concentrated in vacuo. The residue was purified by Isolera ${ }^{\circledR}$ (hexane/EtOAc $=1: 1$ to EtOAc)to afford 3-(2-bromothiophen-3-yl)propanoic acid (S22: $212.1 \mathrm{mg}, 45 \%$ yield). 
To a round-bottom flask containing $\mathbf{S 2 2}(213.0 \mathrm{mg}, 0.90 \mathrm{mmol}, 1.0$ equiv) were added EDC $\cdot \mathrm{HCl}$ (207.5 mg, $1.1 \mathrm{mmol}, 1.2$ equiv), DMAP (44.8 mg, $0.36 \mathrm{mmol}, 40 \mathrm{~mol} \%$ ), aniline ( $0.83 \mathrm{~mL}, 0.90 \mathrm{mmol}$, 1.0 equiv), and $\mathrm{CH}_{2} \mathrm{Cl}_{2}(20 \mathrm{~mL})$. After stirring the mixture at room temperature for several hours with monitoring reaction progress with TLC, the reaction was quenched with $\mathrm{H}_{2} \mathrm{O}$. The mixture was extracted three times with $\mathrm{CH}_{2} \mathrm{Cl}_{2}$. The combined organic layer was dried over $\mathrm{Na}_{2} \mathrm{SO}_{4}$, filtrated, and concentrated in vacuo. The residue was purified by Isolera ${ }^{\circledR}$ (hexane/EtOAc $=15: 1$ to $\left.1: 1\right)$ to afford $4(185.1 \mathrm{mg}, 66 \%$ yield) as a white solid. ${ }^{1} \mathrm{H}$ NMR (400 MHz, $\left.\mathrm{CDCl}_{3}\right) \delta 7.47$ (d, $\left.J=7.6 \mathrm{~Hz}, 2 \mathrm{H}\right), 7.32$ (t, J=7.6 Hz, 2H), 7.21 (d, $J=5.2 \mathrm{~Hz}, 1 \mathrm{H}), 7.11$ (t, $J=7.6 \mathrm{~Hz}, 1 \mathrm{H}), 7.05-6.98$ (brs, 1H), 6.87 (d, $J=5.2 \mathrm{~Hz}, 1 \mathrm{H}), 3.03$ (t, $J=7.2 \mathrm{~Hz}, 2 \mathrm{H}), 2.63(\mathrm{t}, J=7.2 \mathrm{~Hz}, 2 \mathrm{H}) ;{ }^{13} \mathrm{C} \mathrm{NMR}\left(101 \mathrm{MHz}, \mathrm{CDCl}_{3}\right) \delta 170.1,139.7,137.7,128.9$, 128.3, 125.8, 124.3, 120.0, 109.7, 37.2, 25.1. HRMS (ESI) $\mathrm{m} / z$ calcd for $\mathrm{C}_{13} \mathrm{H}_{12} \mathrm{BrNOSNa}[\mathrm{M}+\mathrm{Na}]^{+}$: 331.9715 found 331.9711 .

Synthesis of 3-(4-Bromonaphthalen-1-yl)- $N$-phenylpropanamide (6)<smiles>CCOC(=O)CCc1cccc2ccccc12</smiles>

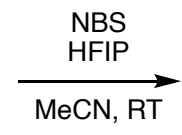<smiles>CCOC(=O)CCc1ccc(Br)c2ccccc12</smiles>

S23

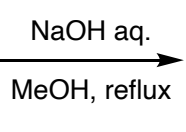

$$
\mathrm{Br}
$$<smiles>O=C(O)CCc1ccc(Br)c2ccccc12</smiles>

S24

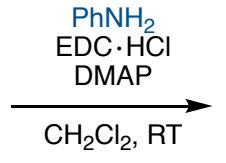<smiles>O=C(CCc1ccc(Br)c2ccccc12)Nc1ccccc1</smiles>

6

To a solution of ethyl 3-(naphthalen-1-yl)propanoate (525.1 mg, $2.3 \mathrm{mmol}, 1.0$ equiv) in MeCN (12 mL) were added $N$-bromosuccinimide (NBS: $452.3 \mathrm{mg}, 2.5 \mathrm{mmol}, 1.1$ equiv) and 1,1,1,3,3,3hexafluoropropan-2-ol (HFIP: $5.0 \mu \mathrm{L}, 48 \mu \mathrm{mol}, 2 \mathrm{~mol} \%$ ). After stirring the mixture at room temperature for several hours with monitoring reaction progress with ${ }^{1} \mathrm{H}$ NMR, the solvent was evaporated in vacuo. The residue was purified by Isolera $^{\circledR}$ (hexane/EtOAc $=19: 1$ to 1:1) to afford 3-ethyl 3-(4bromonaphthalen-1-yl)propanoate (S23: $706.5 \mathrm{mg}$, quant).

To a solution of $\mathbf{S 2 3}$ (706.5 mg, $1.0 \mathrm{mmol}, 1.0$ equiv) in $\mathrm{MeOH}(5.5 \mathrm{~mL})$ was added $6.0 \mathrm{M} \mathrm{NaOH}$ aq. $(5.5 \mathrm{~mL})$. The reaction mixture was refluxed for $4 \mathrm{~h}$. After cooling the reaction mixture to room temperature, the mixture was added $6.0 \mathrm{M} \mathrm{HCl}$ aq. to adjust $\mathrm{pH} \mathrm{1.0.} \mathrm{The} \mathrm{mixture} \mathrm{was} \mathrm{extracted} \mathrm{three}$ times with $\mathrm{CH}_{2} \mathrm{Cl}_{2}$. The combined organic layer was washed with brine. The organic layer was dried over $\mathrm{Na}_{2} \mathrm{SO}_{4}$, filtered, and concentrated in vacuo to afford a crude mixture of 3-(4-bromonaphthalen-1yl)propanoic acid (S24). This was used for the next step without further purification.

To a crude mixture of $\mathbf{S 2 4}$ (1.0 equiv) in a round-bottom flask were added $\mathrm{EDC} \cdot \mathrm{HCl}(523.5 \mathrm{mg}$, $2.7 \mathrm{mmol}, 1.2$ equiv), DMAP (111.2 $\mathrm{mg}, 0.91 \mathrm{mmol}, 40 \mathrm{~mol} \%$ ) aniline ( $0.21 \mathrm{~mL}, 2.3 \mathrm{mmol}, 1.0$ equiv), and $\mathrm{CH}_{2} \mathrm{Cl}_{2}(45 \mathrm{~mL})$. After stirring the mixture at room temperature for several hours with monitoring reaction progress with TLC, the reaction was quenched with $\mathrm{H}_{2} \mathrm{O}$. The mixture was extracted three times 
with $\mathrm{CH}_{2} \mathrm{Cl}_{2}$. The combined organic layer was dried over $\mathrm{Na}_{2} \mathrm{SO}_{4}$, filtrated, and concentrated in vacuo. The residue was purified by Isolera ${ }^{\circledR}$ (hexane/EtOAc $=15: 1$ to $\left.4: 1\right)$ to afford 6 (654.4 $\mathrm{mg}, 81 \%$ yield over 2 steps) as a white solid. ${ }^{1} \mathrm{H}$ NMR $\left(400 \mathrm{MHz}, \mathrm{CDCl}_{3}\right) \delta 8.34-8.28(\mathrm{~m}, 1 \mathrm{H}), 8.12-8.05(\mathrm{~m}, 1 \mathrm{H})$, $7.70(\mathrm{~d}, J=7.2 \mathrm{~Hz}, 1 \mathrm{H}), 7.65-7.56(\mathrm{~m}, 2 \mathrm{H}), 7.43(\mathrm{~d}, J=8.0 \mathrm{~Hz}, 2 \mathrm{H}), 7.34-7.22(\mathrm{~m}, 3 \mathrm{H}), 7.10(\mathrm{t}, J=$ $8.0 \mathrm{~Hz}, 1 \mathrm{H}), 7.02-6.91$ (brs, $1 \mathrm{H}), 3.51(\mathrm{t}, J=7.6 \mathrm{~Hz}, 2 \mathrm{H}), 2.77$ (t, $J=7.6 \mathrm{~Hz}, 2 \mathrm{H}) ;{ }^{13} \mathrm{C} \mathrm{NMR}(101 \mathrm{MHz}$, DMSO- $\left.d_{6}\right) \delta 170.2,139.2,137.9,132.7,131.3,129.7,128.7,127.4,127.1,126.7,124.5,123.6,123.1$, $119.1,37.1,27.7$ (one peak is missing due to overlapping); HRMS (ESI) $\mathrm{m} / z$ calcd for $\mathrm{C}_{19} \mathrm{H}_{17} \mathrm{BrNO}$ $[\mathrm{M}+\mathrm{H}]^{+}: 354.0488$ found 354.0487 .

\section{Synthesis of $N$-Phenyl-3-(thiophen-2-yl)propenamide (8)}<smiles>O=Cc1cccs1</smiles>

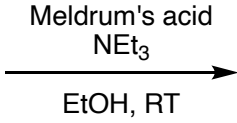
$130^{\circ} \mathrm{C}$

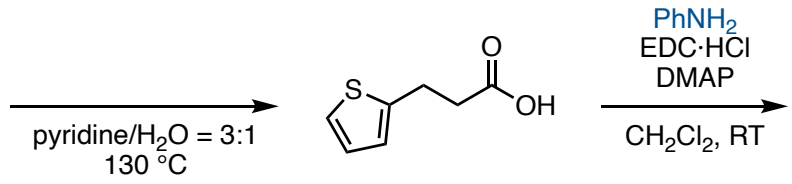<smiles>CC1(C)OC(=O)C(=Cc2cccs2)C(=O)O1</smiles>

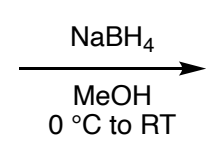<smiles>CC1(C)OC(=O)C(Cc2cccs2)C(=O)O1</smiles>

8

Following the synthetic procedure of $\mathbf{1}$ using 2-thiophenecarboxaldehyde, $\mathbf{8}$ was obtained as a white solid (789.6 mg, $5.0 \mathrm{mmol}$ scale, $66 \%$ yield over 4 steps). The spectra are in accordance with those reported in the literature. ${ }^{[10]}$

\section{Synthesis of $N$-Tosylhydrazones 2}

$$
\int_{\mathrm{O}}^{\mathrm{R}} \stackrel{\mathrm{TsNHNH}_{2}}{\stackrel{\mathrm{MeOH}, 60^{\circ} \mathrm{C}}{\longrightarrow}} \underset{\substack{\mathrm{NNHTS}_{2}^{\mathrm{R}} \\ \mathbf{2}}}{\stackrel{2}{\longrightarrow}}
$$

$N$-Tosylhydrazones were prepared according to literature procedure. ${ }^{[2]}$ A solution of $\mathrm{TsNHNH}_{2}$ (1.0 equiv) in $\mathrm{MeOH}(1.0 \mathrm{M})$ was stirred and heated to $60{ }^{\circ} \mathrm{C}$ until the $\mathrm{TsNHNH}_{2}$ was completely dissolved. To this solution, aldehyde (1.0 equiv) was added dropwise. After stirring the mixture at $60^{\circ} \mathrm{C}$ for several minutes with monitoring reaction progress with TLC, the mixture was concentrated in vacuo. The residue was washed with hexane and dried in vacuo to afford $N$-tosylhydrazones 2.

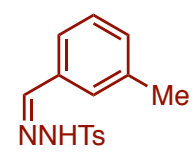

\section{4-Methyl- $N N^{\prime}$-(3-methylbenzylidene)benzenesulfonohydrazide (2c)}

Purification by hexane washing afforded $2 \mathbf{c}(630 \mathrm{mg}$, $73 \%$ yield, $3.0 \mathrm{mmol} \mathrm{scale})$ as a while solid. ${ }^{1} \mathrm{H}$ NMR (400 MHz, $\left.\mathrm{CDCl}_{3}\right) \delta 7.87(\mathrm{~d}, J=8.4 \mathrm{~Hz}, 2 \mathrm{H}), 7.82-7.76$ (brs, 1H), 7.72 (s, 1H), $7.41(\mathrm{~s}, 1 \mathrm{H})$, 
$7.36(\mathrm{~d}, J=7.6 \mathrm{~Hz}, 1 \mathrm{H}), 7.31(\mathrm{~d}, J=8.4 \mathrm{~Hz}, 2 \mathrm{H}), 7.24(\mathrm{t}, J=7.6 \mathrm{~Hz}, 1 \mathrm{H}), 7.18(\mathrm{~d}, J=7.6 \mathrm{~Hz}, 1 \mathrm{H}), 2.41$ (s, 3H), 2.35 (s, 3H); ${ }^{13} \mathrm{C}$ NMR (101 MHz, $\left.\mathrm{CDCl}_{3}\right) \delta 148.1,144.2,138.4,135.2,133.0,131.3,129.7$, 128.5, 127.9, 127.8, 124.7, 21.6, 21.2; HRMS (ESI) $m / z$ calcd for $\mathrm{C}_{15} \mathrm{H}_{17} \mathrm{~N}_{2} \mathrm{O}_{2} \mathrm{~S}[\mathrm{M}+\mathrm{H}]^{+}: 289.1005$ found 289.1003.

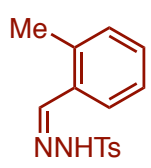

\section{4-Methyl- $N$ '-(2-methylbenzylidene)benzenesulfonohydrazide (2d)}

Purification by hexane washing afforded $\mathbf{2 d}(1.12 \mathrm{~g}, 78 \%$ yield, $5.0 \mathrm{mmol}$ scale) as a while solid. ${ }^{1} \mathrm{H}$ NMR (400 MHz, $\left.\mathrm{CDCl}_{3}\right) \delta 8.00$ (s, 1H), 7.88 (d, $\left.J=8.4 \mathrm{~Hz}, 2 \mathrm{H}\right), 7.84-7.78$ (brs, 1H), 7.66 (d, $J=$ $7.6 \mathrm{~Hz}, 1 \mathrm{H}), 7.32(\mathrm{~d}, J=8.4 \mathrm{~Hz}, 2 \mathrm{H}), 7.26(\mathrm{t}, J=7.6 \mathrm{~Hz}, 2 \mathrm{H}), 7.18$ (t, $J=7.6 \mathrm{~Hz}, 1 \mathrm{H}), 7.14(\mathrm{~d}, J=7.6$ $\mathrm{Hz}, 1 \mathrm{H}), 2.41$ (s, 3H), 2.39 (s, 3H); ${ }^{13} \mathrm{C}$ NMR $\left(101 \mathrm{MHz}, \mathrm{CDCl}_{3}\right) \delta 147.1,144.3,137.1,135.2,131.2$, $130.9,130.1,129.7,128.0,127.4,126.1,21.6,19.8$; HRMS (ESI) $m / z$ calcd for $\mathrm{C}_{15} \mathrm{H}_{17} \mathrm{~N}_{2} \mathrm{O}_{2} \mathrm{~S}[\mathrm{M}+\mathrm{H}]^{+}$: 289.1005 found 289.1003 .

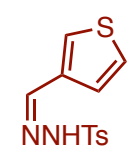

\section{4-Methyl- $N^{\prime}$-(thiophen-3-ylmethylene)benzenesulfonohydrazide (2h)}

Purification by hexane washing afforded $\mathbf{2 h}(696 \mathrm{mg}, 83 \%$ yield, $3.0 \mathrm{mmol}$ scale $)$ as a while solid. ${ }^{1} \mathrm{H}$ NMR (400 MHz, $\left.\mathrm{CDCl}_{3}\right) \delta 7.85(\mathrm{~d}, J=8.0 \mathrm{~Hz}, 2 \mathrm{H}), 7.81$ (s, 1H), 7.63-7.53 (brs, 1H), 7.46 (dd, $J=$ $2.8,0.8 \mathrm{~Hz}, 1 \mathrm{H}), 7.41(\mathrm{dd}, J=5.2,0.8 \mathrm{~Hz}, 1 \mathrm{H}), 7.31$ (d, $J=8.0 \mathrm{~Hz}, 2 \mathrm{H}), 7.29$ (dd, $J=5.2,2.8 \mathrm{~Hz}, 1 \mathrm{H})$, $2.41(\mathrm{~s}, 3 \mathrm{H}) ;{ }^{13} \mathrm{C} \mathrm{NMR}\left(101 \mathrm{MHz}, \mathrm{CDCl}_{3}\right) \delta 144.3,143.4,136.4,135.2,129.7,127.9,127.6,126.6$, 125.1, 21.6; HRMS (ESI) $m / z$ calcd for $\mathrm{C}_{12} \mathrm{H}_{13} \mathrm{~N}_{2} \mathrm{O}_{2} \mathrm{~S}_{2}[\mathrm{M}+\mathrm{H}]^{+}: 281.0413$ found 281.0411.

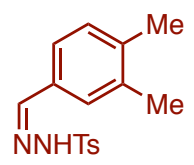

\section{$N^{\prime}$-(3,4-Dimethylbenzylidene)-4-methylbenzenesulfonohydrazide (2m)}

Purification by hexane washing afforded $2 \mathrm{~m}$ ( $375 \mathrm{mg}, 62 \%$ yield, $2.0 \mathrm{mmol}$ scale) as a while solid. ${ }^{1} \mathrm{H}$ NMR (400 MHz, $\left.\mathrm{CDCl}_{3}\right) \delta 7.86(\mathrm{~d}, J=8.4 \mathrm{~Hz}, 2 \mathrm{H}), 7.70(\mathrm{~s}, 1 \mathrm{H}), 7.64(\mathrm{~s}, 1 \mathrm{H}), 7.37(\mathrm{~s}, 1 \mathrm{H}), 7.34-7.27$ $(\mathrm{m}, 3 \mathrm{H}), 7.11(\mathrm{~d}, J=8.0 \mathrm{~Hz}, 1 \mathrm{H}), 2.40(\mathrm{~s}, 3 \mathrm{H}), 2.26(\mathrm{~s}, 6 \mathrm{H}) ;{ }^{13} \mathrm{C} \mathrm{NMR}\left(101 \mathrm{MHz}, \mathrm{CDCl}_{3}\right) \delta 148.5,144.2$, 139.6, 137.0, 135.3, 130.7, 129.9, 129.6, 128.3, 127.9, 125.2, 21.6, 19.8, 19.6; HRMS (ESI) $\mathrm{m} / z$ calcd for $\mathrm{C}_{16} \mathrm{H}_{19} \mathrm{~N}_{2} \mathrm{O}_{2} \mathrm{~S}[\mathrm{M}+\mathrm{H}]^{+}: 303.1162$ found 303.1161 . 


\section{Pd-Catalyzed Azaspirocyclization of Bromoarenes with $\mathrm{N}$-Tosylhydrazones}<smiles>[X]C(=O)NO[Na]</smiles>

1: $0.10 \mathrm{mmol}$

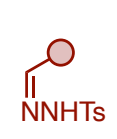

2: 2.0 equiv

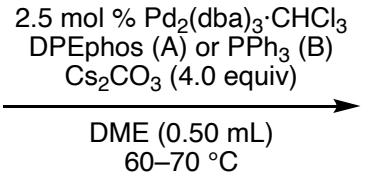

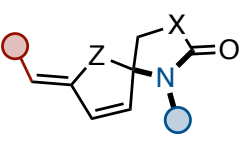

3

\section{General Procedure}

An 8-mL glass tube equipped with a screw cap containing a magnetic stirring bar and $\mathrm{Cs}_{2} \mathrm{CO}_{3}$ (130.3 mg, $0.40 \mathrm{mmol}, 4.0$ equiv) was dried with a heat-gun in vacuo and filled $\mathrm{N}_{2}$ after cooling to room temperature. To this tube were added $\mathrm{Pd}_{2}(\mathrm{dba})_{3} \cdot \mathrm{CHCl}_{3}(2.59 \mathrm{mg}, 2.5 \mu \mathrm{mol}, 2.5 \mathrm{~mol} \%$ ), DPEphos (5.39 $\mathrm{mg}, 10 \mu \mathrm{mol}, 10 \mathrm{~mol} \%$ ) or $\mathrm{PPh}_{3}(5.25 \mathrm{mg}, 20 \mu \mathrm{mol}, 20 \mathrm{~mol} \%)$, bromoarenes 1 ( $0.10 \mathrm{mmol}, 1.0$ equiv), and $N$-tosylhydrazones $2\left(0.20 \mathrm{mmol}, 2.0\right.$ equiv). The tube was placed under vacuum and refilled $\mathrm{N}_{2}$ gas three times. To this tube was added DME $(0.50 \mathrm{~mL})$. The tube was sealed with a screw cap. After stirring the mixture at $60-70{ }^{\circ} \mathrm{C}$ for $12 \mathrm{~h}$ in a 16 -well aluminum reaction block, the mixture was passed through a pad of Celite ${ }^{\circledR}$ with EtOAc as an eluent. The filtrate was concentrated in vacuo. The residue was purified by PTLC to afford the corresponding azaspirocyclic products 3 .

The determination of $Z$ and $E$ isomer was performed by using 3Aa, 3Ka, and 7. The $Z / E$ determination of the other compounds were followed these analyses.
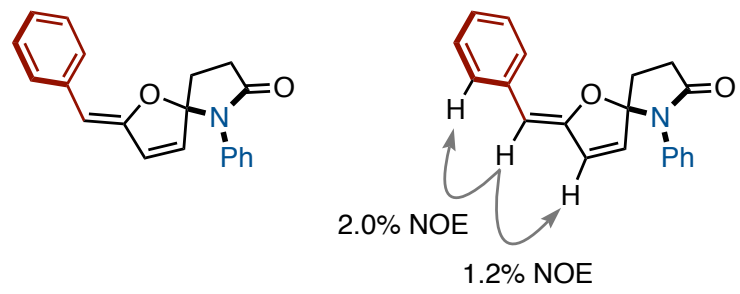

\section{(Z)-2-Benzylidene-6-phenyl-1-oxa-6-azaspiro[4.4]non-3-en-7-one (3Aa)}

The reaction was conducted by using DPEphos at $60^{\circ} \mathrm{C}$. Crude ${ }^{1} \mathrm{H}$ NMR analysis showed $\boldsymbol{Z}$ 3Aa: $\boldsymbol{E - 3 A a}=70: 30 ;{ }^{1} \mathrm{H}$ NMR peaks at $5.43 \mathrm{ppm}(\mathrm{s}, 0.70 \mathrm{H})$ and $6.10 \mathrm{ppm}(\mathrm{s}, 0.30 \mathrm{H})$ were used. Purification by PTLC (hexane/EtOAc $=7: 3)$ afforded 3Aa $(27.3 \mathrm{mg}, 90 \%$ yield, as a mixture of diasteroisomers; $Z: E=74: 26$ ) as a pale yellow solid.

For $Z$ isomer: ${ }^{1} \mathrm{H}$ NMR (400 MHz, $\left.\mathrm{CDCl}_{3}\right) \delta 7.67(\mathrm{dd}, J=8.4,1.2 \mathrm{~Hz}, 2 \mathrm{H}), 7.40-7.12(\mathrm{~m}, 8 \mathrm{H})$, $6.25(\mathrm{~d}, J=5.6 \mathrm{~Hz}, 1 \mathrm{H}), 5.90(\mathrm{~d}, J=5.6 \mathrm{~Hz}, 1 \mathrm{H}), 5.43(\mathrm{~s}, 1 \mathrm{H}), 3.06-2.96(\mathrm{~m}, 1 \mathrm{H}), 2.73-2.58(\mathrm{~m}, 1 \mathrm{H})$, 2.55-2.41 (m, 2H); ${ }^{13} \mathrm{C}$ NMR (101 MHz, $\left.\mathrm{CDCl}_{3}\right) \delta 174.8,155.4,135.6,135.3,130.9,130.7,129.0$, 128.5, 128.1, 127.8, 127.4, 126.2, 109.0, 102.3, 32.2, 29.2; HRMS (ESI) $m / z$ calcd for $\mathrm{C}_{20} \mathrm{H}_{17} \mathrm{NO}_{2} \mathrm{Na}$ $[\mathrm{M}+\mathrm{Na}]^{+}: 326.1152$ found 326.1149 .

For $E$ isomer: ${ }^{1} \mathrm{H}$ NMR $\left(400 \mathrm{MHz}, \mathrm{CDCl}_{3}\right) \delta 7.40-7.12(\mathrm{~m}, 10 \mathrm{H}), 6.69(\mathrm{~d}, J=5.6 \mathrm{~Hz}, 1 \mathrm{H}), 6.10(\mathrm{~s}$, $1 \mathrm{H}), 6.03(\mathrm{dd}, J=5.6,2.0 \mathrm{~Hz}, 1 \mathrm{H}), 2.97-2.86(\mathrm{~m}, 1 \mathrm{H}), 2.73-2.58(\mathrm{~m}, 1 \mathrm{H}), 2.55-2.41(\mathrm{~m}, 2 \mathrm{H})$. 


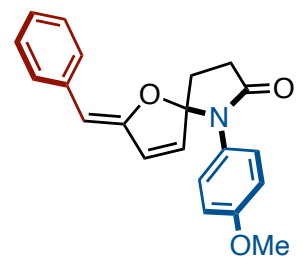

(Z)-2-Benzylidene-6-(4-methoxyphenyl)-1-oxa-6-azaspiro[4.4]non-3-en-7-one (3Ba)

The reaction was conducted by using DPEphos at $60{ }^{\circ} \mathrm{C}$. Crude ${ }^{1} \mathrm{H}$ NMR analysis showed $\boldsymbol{Z}-3 \mathrm{Ba}: \boldsymbol{E}$ 3Ba $=70: 30 ;{ }^{1} \mathrm{H}$ NMR peaks at $5.39 \mathrm{ppm}(\mathrm{s}, 0.70 \mathrm{H})$ and $6.07 \mathrm{ppm}(\mathrm{s}, 0.30 \mathrm{H})$ were used. Purification by PTLC (hexane/EtOAc $=7: 3)$ afforded 3Ba $(28.9 \mathrm{mg}, 87 \%$ yield, as a mixture of diasteroisomers; $Z: E=72: 28)$ as a pale yellow solid.

For $Z$ isomer: ${ }^{1} \mathrm{H}$ NMR $\left(400 \mathrm{MHz}, \mathrm{CDCl}_{3}\right) \delta 7.66(\mathrm{~d}, J=7.2 \mathrm{~Hz}, 2 \mathrm{H}), 7.35(\mathrm{t}, J=7.6 \mathrm{~Hz}, 2 \mathrm{H})$, 7.23-7.13 (m, 1H), 7.12-7.03 (m, 2H), 6.88-6.78 (m, 2H), $6.23(\mathrm{~d}, J=5.6 \mathrm{~Hz}, 1 \mathrm{H}), 5.90(\mathrm{dd}, J=5.6$, $0.8 \mathrm{~Hz}, 1 \mathrm{H}), 5.39(\mathrm{~s}, 1 \mathrm{H}), 3.74(\mathrm{~s}, 3 \mathrm{H}), 3.04-2.84(\mathrm{~m}, 1 \mathrm{H}), 2.70-2.56(\mathrm{~m}, 1 \mathrm{H}), 2.54-2.40(\mathrm{~m}, 2 \mathrm{H}) ;{ }^{13} \mathrm{C}$ NMR (101 MHz, $\left.\mathrm{CDCl}_{3}\right) \delta 175.1,159.0,155.6,130.9,130.8,128.8,128.7,128.5,128.1,127.8,126.2$, 114.3, 109.0, 102.1, 55.3, 31.9, 29.1; HRMS (ESI) $m / z$ calcd for $\mathrm{C}_{21} \mathrm{H}_{20} \mathrm{NO}_{3}[\mathrm{M}+\mathrm{H}]^{+}: 334.1438$ found 334.1436.

For $E$ isomer: ${ }^{1} \mathrm{H}$ NMR (400 MHz, $\left.\mathrm{CDCl}_{3}\right) \delta 7.30-7.25(\mathrm{~m}, 2 \mathrm{H}), 7.23-7.13(\mathrm{~m}, 3 \mathrm{H}), 7.12-7.03(\mathrm{~m}$, 2H), 6.88-6.78 (m, 2H), $6.68(\mathrm{~d}, J=6.0 \mathrm{~Hz}, 1 \mathrm{H}), 6.07$ (s, 1H), 6.03 (dd, $J=6.0,1.6 \mathrm{~Hz}, 1 \mathrm{H}), 3.76$ (s, $3 \mathrm{H}), 3.04-2.84(\mathrm{~m}, 1 \mathrm{H}), 2.70-2.56(\mathrm{~m}, 1 \mathrm{H}), 2.54-2.40(\mathrm{~m}, 2 \mathrm{H})$.

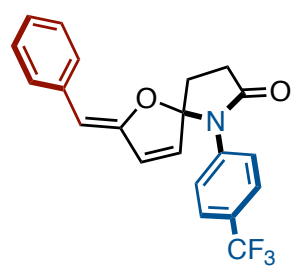

(Z)-2-Benzylidene-6-(4-(trifluoromethyl)phenyl)-1-oxa-6-azaspiro[4.4]non-3-en-7-one (3Ca)

The reaction was conducted by using DPEphos at $60{ }^{\circ} \mathrm{C}$. Crude ${ }^{1} \mathrm{H}$ NMR analysis showed $Z$ 3Ca: $\boldsymbol{E - 3 C a}=71: 29 ;{ }^{1} \mathrm{H}$ NMR peaks at $5.51 \mathrm{ppm}(\mathrm{s}, 0.71 \mathrm{H})$ and $6.15 \mathrm{ppm}(\mathrm{s}, 0.29 \mathrm{H})$ were used. Purification by PTLC (hexane/EtOAc $=7: 3)$ afforded 3Ca $(33.3 \mathrm{mg}, 90 \%$ yield, as a mixture of diasteroisomers; $Z: E=74: 26$ ) as a pale yellow solid.

For $Z$ isomer: ${ }^{1} \mathrm{H}$ NMR (400 MHz, $\left.\mathrm{CDCl}_{3}\right) \delta 7.66(\mathrm{~d}, J=8.4 \mathrm{~Hz}, 2 \mathrm{H}), 7.57(\mathrm{~d}, J=8.4 \mathrm{~Hz}, 2 \mathrm{H})$, 7.40-7.16 (m, 5H), 6.32 (d, $J=5.6 \mathrm{~Hz}, 1 \mathrm{H}), 5.88(\mathrm{~d}, J=5.6 \mathrm{~Hz}, 1 \mathrm{H}), 5.51(\mathrm{~s}, 1 \mathrm{H}), 3.09-2.88(\mathrm{~m}, 1 \mathrm{H})$, 2.77-2.60 (m, 1H), 2.58-2.42 (m, 2H); ${ }^{13} \mathrm{C}$ NMR (101 MHz, $\left.\mathrm{CDCl}_{3}\right) \delta 174.6,155.0,138.8,135.3,131.4$, $130.2,129.5\left(\mathrm{q}, J_{\mathrm{C}-\mathrm{F}}=32.9 \mathrm{~Hz}\right), 128.5,128.2,127.1,126.5,126.0\left(\mathrm{q}, J_{\mathrm{C}-\mathrm{F}}=3.8 \mathrm{~Hz}\right), 123.8\left(\mathrm{q}, J_{\mathrm{C}-\mathrm{F}}=274\right.$ $\mathrm{Hz}), 108.9,103.2,32.4,29.1 ;{ }^{19} \mathrm{~F}$ NMR $\left(376 \mathrm{MHz}, \mathrm{CDCl}_{3}\right) \delta-62.7$; HRMS (ESI) $\mathrm{m} / \mathrm{z}$ calcd for $\mathrm{C}_{21} \mathrm{H}_{16} \mathrm{~F}_{3} \mathrm{NO}_{2} \mathrm{Na}[\mathrm{M}+\mathrm{Na}]^{+}: 394.1025$ found 394.1022 .

For $E$ isomer: $\delta 7.60(\mathrm{~d}, J=8.4 \mathrm{~Hz}, 2 \mathrm{H}), 7.40-7.16(\mathrm{~m}, 7 \mathrm{H}), 6.77(\mathrm{~d}, J=5.6 \mathrm{~Hz}, 1 \mathrm{H}), 6.15(\mathrm{~s}, 1 \mathrm{H})$, $6.01(\mathrm{~d}, J=6.0 \mathrm{~Hz}, 1 \mathrm{H}), 3.09-2.88(\mathrm{~m}, 1 \mathrm{H}), 2.77-2.60(\mathrm{~m}, 1 \mathrm{H}), 2.58-2.42(\mathrm{~m}, 2 \mathrm{H})$ 


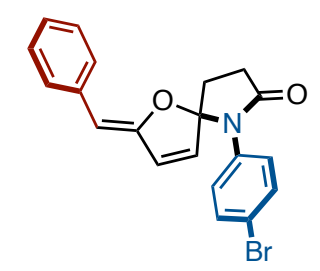

(Z)-2-Benzylidene-6-(4-bromophenyl)-1-oxa-6-azaspiro[4.4]non-3-en-7-one (3Da)

The reaction was conducted by using DPEphos at $60{ }^{\circ} \mathrm{C}$. Crude ${ }^{1} \mathrm{H}$ NMR analysis showed $\boldsymbol{Z}$ 3Da: $\boldsymbol{E - 3 D a}=76: 24 ;{ }^{1} \mathrm{H}$ NMR peaks at $5.45 \mathrm{ppm}(\mathrm{s}, 0.76 \mathrm{H})$ and $6.11 \mathrm{ppm}(\mathrm{s}, 0.24 \mathrm{H})$ were used. Purification by PTLC (hexane/EtOAc $=7: 3)$ afforded 3Da $(25.1 \mathrm{mg}, 66 \%$ yield, as a mixture of diasteroisomers; $Z: E=76: 24)$ as a pale yellow solid.

For $Z$ isomer: ${ }^{1} \mathrm{H}$ NMR $\left(400 \mathrm{MHz}, \mathrm{CDCl}_{3}\right) \delta 7.65(\mathrm{~d}, J=8.4 \mathrm{~Hz}, 2 \mathrm{H}), 7.48-7.39(\mathrm{~m}, 2 \mathrm{H}), 7.38-$ $7.32(\mathrm{t}, J=7.6 \mathrm{~Hz}, 2 \mathrm{H}), 7.24-7.15(\mathrm{~m}, 2 \mathrm{H}), 7.09-7.00(\mathrm{~m}, 1 \mathrm{H}), 6.29(\mathrm{~d}, J=5.6 \mathrm{~Hz}, 1 \mathrm{H}), 5.87$ (dd, $J=$ 5.6, $0.8 \mathrm{~Hz}, 1 \mathrm{H}), 5.45(\mathrm{~s}, 1 \mathrm{H}), 3.05-2.85(\mathrm{~m}, 1 \mathrm{H}), 2.71-2.57(\mathrm{~m}, 1 \mathrm{H}), 2.54-2.40(\mathrm{~m}, 2 \mathrm{H}) ;{ }^{13} \mathrm{C}$ NMR $\left(101 \mathrm{MHz}, \mathrm{CDCl}_{3}\right) \delta 174.7,155.2,135.4,134.4,132.2,131.2,130.4,128.9,128.5,128.2,126.4,121.7$, 108.8, 102.8, 32.2, 29.1; $\mathrm{m} / z$ calcd for $\mathrm{C}_{20} \mathrm{H}_{16} \mathrm{BrNO}_{2} \mathrm{Na}[\mathrm{M}+\mathrm{Na}]^{+}: 404.0257$ found 404.0251.

For $E$ isomer: ${ }^{1} \mathrm{H}$ NMR (400 MHz, $\left.\mathrm{CDCl}_{3}\right) \delta 7.48-7.39(\mathrm{~m}, 2 \mathrm{H}), 7.32-7.27(\mathrm{t}, J=7.6 \mathrm{~Hz}, 2 \mathrm{H})$, $7.24-7.15(\mathrm{~m}, 2 \mathrm{H}), 7.09-7.00(\mathrm{~m}, 3 \mathrm{H}), 6.73(\mathrm{~d}, J=6.0 \mathrm{~Hz}, 1 \mathrm{H}), 6.11(\mathrm{~s}, 1 \mathrm{H}), 6.01(\mathrm{dd}, J=6.0,1.6 \mathrm{~Hz}$, $1 \mathrm{H}), 3.05-2.85(\mathrm{~m}, 1 \mathrm{H}), 2.71-2.57(\mathrm{~m}, 1 \mathrm{H}), 2.54-2.40(\mathrm{~m}, 2 \mathrm{H})$.

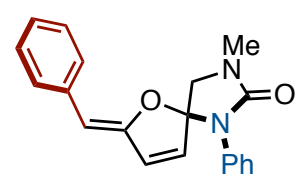

(Z)-7-Benzylidene-3-methyl-1-phenyl-6-oxa-1,3-diazaspiro[4.4]non-8-en-2-one (3Ea)

The reaction was conducted by using $\mathrm{PPh}_{3}$ at $60{ }^{\circ} \mathrm{C}$. Crude ${ }^{1} \mathrm{H}$ NMR analysis showed $\boldsymbol{Z}$-3Ea: $\boldsymbol{E}$ 3Ea $=79: 21 ;{ }^{1} \mathrm{H}$ NMR peaks at $5.44 \mathrm{ppm}(\mathrm{s}, 0.79 \mathrm{H})$ and $6.12 \mathrm{ppm}(\mathrm{s}, 0.21 \mathrm{H})$ were used. Purification by PTLC (hexane/EtOAc $=1: 1)$ afforded 3Ea $(22.6 \mathrm{mg}, 74 \%$ yield, as a mixture of diastereoisomers; $Z: E=79: 21)$ as a pale yellow solid.

For $Z$ isomer: ${ }^{1} \mathrm{H}$ NMR $\left(400 \mathrm{MHz}, \mathrm{CDCl}_{3}\right) \delta 7.67(\mathrm{~d}, J=7.6 \mathrm{~Hz}, 2 \mathrm{H}), 7.34(\mathrm{t}, J=7.6 \mathrm{~Hz}, 2 \mathrm{H})$, $7.31-7.12(\mathrm{~m}, 6 \mathrm{H}), 6.31(\mathrm{~d}, J=5.6 \mathrm{~Hz}, 1 \mathrm{H}), 5.88(\mathrm{~d}, J=5.6 \mathrm{~Hz}, 1 \mathrm{H}), 5.44(\mathrm{~s}, 1 \mathrm{H}), 3.75-3.60(\mathrm{~m}, 2 \mathrm{H})$, $3.01(\mathrm{~s}, 3 \mathrm{H}) ;{ }^{13} \mathrm{C} \mathrm{NMR}\left(101 \mathrm{MHz}, \mathrm{CDCl}_{3}\right) \delta 157.5,155.0,135.7,135.4,131.8,128.9,128.7,128.4$, 128.2, 127.2, 126.8, 126.3, 103.8, 103.0, 56.1, 30.5; HRMS (ESI) $m / z$ calcd for $\mathrm{C}_{20} \mathrm{H}_{19} \mathrm{~N}_{2} \mathrm{O}_{2}[\mathrm{M}+\mathrm{H}]^{+}$: 319.1441 found 319.1440 .

For $E$ isomer: ${ }^{1} \mathrm{H}$ NMR $\left(400 \mathrm{MHz}, \mathrm{CDCl}_{3}\right) \delta 7.31-7.12(\mathrm{~m}, 10 \mathrm{H}), 6.76(\mathrm{~d}, J=5.6 \mathrm{~Hz}, 1 \mathrm{H}), 6.12(\mathrm{~s}$, $1 \mathrm{H}), 6.02(\mathrm{dd}, J=5.6,1.6 \mathrm{~Hz}, 1 \mathrm{H}), 3.75-3.60(\mathrm{~m}, 2 \mathrm{H}), 2.98(\mathrm{~s}, 3 \mathrm{H})$. 


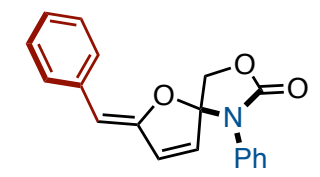

\section{(Z)-7-Benzylidene-1-phenyl-3,6-dioxa-1-azaspiro[4.4]non-8-en-2-one (3Fa)}

The reaction was conducted by using DPEphos at $60^{\circ} \mathrm{C}$. Crude ${ }^{1} \mathrm{H}$ NMR analysis showed $\boldsymbol{Z}-\mathbf{3 F a}: \boldsymbol{E}$ 3Fa $=68: 32 ;{ }^{1} \mathrm{H}$ NMR peaks at $5.52 \mathrm{ppm}(\mathrm{s}, 0.68 \mathrm{H})$ and $6.21 \mathrm{ppm}(\mathrm{s}, 0.32 \mathrm{H})$ were used. Purification by PTLC (hexane/EtOAc $=7: 3)$ afforded 3Fa $(13.1 \mathrm{mg}$, 43\% yield, as a mixture of diastereoisomers; Z:E $=73: 27$ ) as a pale yellow solid.

For $Z$ isomer: ${ }^{1} \mathrm{H}$ NMR (400 MHz, $\left.\mathrm{CDCl}_{3}\right) \delta 7.69(\mathrm{~d}, J=7.6 \mathrm{~Hz}, 2 \mathrm{H}), 7.44-7.16(\mathrm{~m}, 8 \mathrm{H}), 6.39(\mathrm{~d}$, $J=6.0 \mathrm{~Hz}, 1 \mathrm{H}), 5.89(\mathrm{~d}, J=6.0 \mathrm{~Hz}, 1 \mathrm{H}), 5.52(\mathrm{~s}, 1 \mathrm{H}), 4.72-4.46(\mathrm{~m}, 2 \mathrm{H}) ;{ }^{13} \mathrm{C} \mathrm{NMR}\left(101 \mathrm{MHz}, \mathrm{CDCl}_{3}\right)$ $\delta$ 155.4, 154.4, 134.9, 133.9, 133.0, 129.14, 129.08, 128.6, 128.4, 126.9, 126.8, 105.3, 104.3, 71.8 (one peak was missing due to overlapping); HRMS (ESI) $m / z$ calcd for $\mathrm{C}_{19} \mathrm{H}_{16} \mathrm{NO}_{3}[\mathrm{M}+\mathrm{H}]^{+}: 306.1125$ found 306.1125 .

For $E$ isomer: $1 \mathrm{H} \mathrm{NMR}\left(400 \mathrm{MHz}, \mathrm{CDCl}_{3}\right) \delta 7.44-7.16(\mathrm{~m}, 10 \mathrm{H}), 6.81(\mathrm{~d}, J=6.0 \mathrm{~Hz}, 1 \mathrm{H}), 6.21(\mathrm{~s}$, $1 \mathrm{H}), 6.03(\mathrm{dd}, J=6.0,2.0 \mathrm{~Hz}, 1 \mathrm{H}), 4.72-4.46(\mathrm{~m}, 2 \mathrm{H})$.

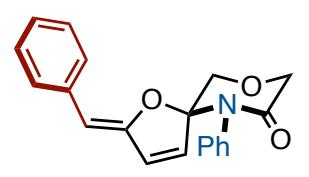

\section{(Z)-2-Benzylidene-6-phenyl-1,9-dioxa-6-azaspiro[4.5] dec-3-en-7-one (3Ga)}

The reaction was conducted by using DPEphos at $60{ }^{\circ} \mathrm{C}$. Crude ${ }^{1} \mathrm{H}$ NMR analysis showed $\boldsymbol{Z}$ 3Ga: $\boldsymbol{E - 3 G a}=70: 30 ;{ }^{1} \mathrm{H}$ NMR peaks at $5.33 \mathrm{ppm}(\mathrm{s}, 0.70 \mathrm{H})$ and $6.16 \mathrm{ppm}(\mathrm{s}, 0.30 \mathrm{H})$ were used. Purification by PTLC (hexane/EtOAc $=1: 1)$ afforded 3Ga $(30.0 \mathrm{mg}, 94 \%$ yield, as a mixture of diasteroisomers; $Z: E=70: 30$ ) as a pale yellow solid.

For $Z$ isomer: $\delta 7.71(\mathrm{~d}, J=7.2 \mathrm{~Hz}, 2 \mathrm{H}), 7.41-7.11(\mathrm{~m}, 8 \mathrm{H}), 6.12(\mathrm{~d}, J=5.6 \mathrm{~Hz}, 1 \mathrm{H}), 5.87(\mathrm{~d}, J=$ $5.6 \mathrm{~Hz}, 1 \mathrm{H}), 5.33(\mathrm{~s}, 1 \mathrm{H}), 4.63-4.50(\mathrm{~m}, 1 \mathrm{H}), 4.45-4.35(\mathrm{~m}, 1 \mathrm{H}), 4.13-4.00(\mathrm{~m}, 2 \mathrm{H}) ;{ }^{13} \mathrm{C}$ NMR $(101$ $\left.\mathrm{MHz}, \mathrm{CDCl}_{3}\right) \delta 167.3,155.4,135.9,135.2,131.6,129.2,129.0,128.5,128.3,128.0,127.8,126.4,103.0$, 101.6, 71.0, 68.6; HRMS (ESI) $m / z$ calcd for $\mathrm{C}_{20} \mathrm{H}_{18} \mathrm{NO}_{3}[\mathrm{M}+\mathrm{H}]^{+}: 320.1281$ found 320.1283.

For $E$ isomer: $\delta 7.41-7.11(\mathrm{~m}, 8 \mathrm{H}), 7.11-7.05(\mathrm{~m}, 2 \mathrm{H}), 6.55(\mathrm{~d}, J=6.0 \mathrm{~Hz}, 1 \mathrm{H}), 6.16(\mathrm{~s}, 1 \mathrm{H}), 5.98$ (dd, $J=6.0,1.6 \mathrm{~Hz}, 1 \mathrm{H}), 4.63-4.50(\mathrm{~m}, 1 \mathrm{H}), 4.45-4.35(\mathrm{~m}, 1 \mathrm{H}), 4.13-4.00(\mathrm{~m}, 2 \mathrm{H})$.

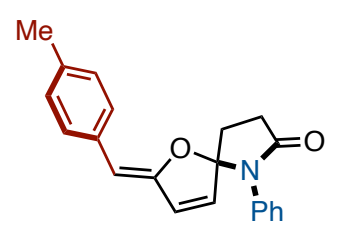

(Z)-2-(4-Methylbenzylidene)-6-phenyl-1-oxa-6-azaspiro[4.4]non-3-en-7-one (3Ab) 
The reaction was conducted by using $\mathrm{PPh}_{3}$ at $60{ }^{\circ} \mathrm{C}$. Crude ${ }^{1} \mathrm{H}$ NMR analysis showed $\boldsymbol{Z}-\mathbf{3 A b}: \boldsymbol{E}$ $\mathbf{3 A b}=78: 22 ;{ }^{1} \mathrm{H}$ NMR peaks at $5.40 \mathrm{ppm}(\mathrm{s}, 0.78 \mathrm{H})$ and $6.07 \mathrm{ppm}(\mathrm{s}, 0.22 \mathrm{H})$ were used. Purification by PTLC (hexane/EtOAc $=7: 3)$ afforded $\mathbf{3 A b}(30.2 \mathrm{mg}, 95 \%$ yield, as a mixture of diasteroisomers; $Z: E=81: 19)$ as a pale yellow solid.

For $Z$ isomer: ${ }^{1} \mathrm{H}$ NMR (400 MHz, $\left.\mathrm{CDCl}_{3}\right) \delta 7.56(\mathrm{~d}, J=8.0 \mathrm{~Hz}, 2 \mathrm{H}), 7.36-7.05(\mathrm{~m}, 7 \mathrm{H}), 6.23(\mathrm{~d}$, $J=5.6 \mathrm{~Hz}, 1 \mathrm{H}), 5.85(\mathrm{~d}, J=5.6 \mathrm{~Hz}, 1 \mathrm{H}), 5.40(\mathrm{~s}, 1 \mathrm{H}), 3.05-2.94(\mathrm{~m}, 1 \mathrm{H}), 2.70-2.57(\mathrm{~m}, 1 \mathrm{H}), 2.52-$ $2.41(\mathrm{~m}, 2 \mathrm{H}), 2.36(\mathrm{~s}, 3 \mathrm{H}) ;{ }^{13} \mathrm{C} \mathrm{NMR}\left(101 \mathrm{MHz}, \mathrm{CDCl}_{3}\right) \delta 174.8,154.9,136.1,135.4,132.7,130.9$, 130.2, 129.2, 128.9, 128.0, 127.8, 127.4, 108.9, 102.3, 32.3, 29.2, 21.2; HRMS (ESI) $\mathrm{m} / \mathrm{z}$ calcd for $\mathrm{C}_{21} \mathrm{H}_{20} \mathrm{NO}_{2}[\mathrm{M}+\mathrm{H}]^{+}: 318.1489$ found 318.1487 .

For $E$ isomer: ${ }^{1} \mathrm{H}$ NMR $\left(400 \mathrm{MHz}, \mathrm{CDCl}_{3}\right) \delta 7.36-7.05(\mathrm{~m}, 9 \mathrm{H}), 6.68(\mathrm{~d}, J=5.6 \mathrm{~Hz}, 1 \mathrm{H}), 6.07(\mathrm{~s}$, $1 \mathrm{H}), 5.99(\mathrm{dd}, J=5.6,1.6 \mathrm{~Hz}, 1 \mathrm{H}), 2.96-2.86(\mathrm{~m}, 1 \mathrm{H}), 2.70-2.57(\mathrm{~m}, 1 \mathrm{H}), 2.52-2.41(\mathrm{~m}, 2 \mathrm{H}), 2.31(\mathrm{~s}$, $3 \mathrm{H})$.

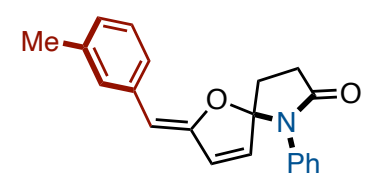

(Z)-2-(3-Methylbenzylidene)-6-phenyl-1-oxa-6-azaspiro[4.4]non-3-en-7-one (3Ac)

The reaction was conducted by using $\mathrm{PPh}_{3}$ at $60{ }^{\circ} \mathrm{C}$. Crude ${ }^{1} \mathrm{H}$ NMR analysis showed $\boldsymbol{Z}$-3Ac: $\boldsymbol{E}$ 3Ac $=75: 25 ;{ }^{1} \mathrm{H}$ NMR peaks at $5.39 \mathrm{ppm}(\mathrm{s}, 0.75 \mathrm{H})$ and $6.07 \mathrm{ppm}(\mathrm{s}, 0.25 \mathrm{H})$ were used. Purification by PTLC (hexane/EtOAc = 7:3) afforded 3Ac $(15.6 \mathrm{mg}, 49 \%$ yield, as a mixture of diasteroisomers; $Z: E=77: 23)$ as a pale yellow solid.

For $Z$ isomer: ${ }^{1} \mathrm{H}$ NMR $\left(400 \mathrm{MHz}, \mathrm{CDCl}_{3}\right) \delta 7.53(\mathrm{~d}, J=7.6 \mathrm{~Hz}, 1 \mathrm{H}), 7.41(\mathrm{~s}, 1 \mathrm{H}), 7.36-7.21(\mathrm{~m}$, 2H), 7.20-7.12 (m, 3H), $7.02(\mathrm{~d}, J=7.6 \mathrm{~Hz}, 1 \mathrm{H}), 6.98(\mathrm{~d}, J=7.6 \mathrm{~Hz}, 1 \mathrm{H}), 6.23(\mathrm{~d}, J=5.6 \mathrm{~Hz}, 1 \mathrm{H})$, $5.87(\mathrm{~d}, J=5.6 \mathrm{~Hz}, 1 \mathrm{H}), 5.39(\mathrm{~s}, 1 \mathrm{H}), 3.06-2.94(\mathrm{~m}, 1 \mathrm{H}), 2.73-2.57(\mathrm{~m}, 1 \mathrm{H}), 2.55-2.41(\mathrm{~m}, 2 \mathrm{H}), 2.37$ (s, 3H); ${ }^{13} \mathrm{C}$ NMR $\left(101 \mathrm{MHz}, \mathrm{CDCl}_{3}\right) \delta 174.9,155.3,138.0,135.5,135.4,131.0,130.6,129.0,128.8$, $128.4,127.8,127.4,127.1,125.3,109.0,102.5,32.3,29.2,21.6$; HRMS (ESI) $m / z$ calcd for $\mathrm{C}_{21} \mathrm{H}_{20} \mathrm{NO}_{2}$ $[\mathrm{M}+\mathrm{H}]^{+}: 318.1489$ found 318.1487 .

For $E$ isomer: ${ }^{1} \mathrm{H}$ NMR (400 MHz, $\left.\mathrm{CDCl}_{3}\right) \delta 7.36-7.21(\mathrm{~m}, 9 \mathrm{H}), 6.70(\mathrm{~d}, J=6.0 \mathrm{~Hz}, 1 \mathrm{H}), 6.07(\mathrm{~s}$, $1 \mathrm{H}), 6.01(\mathrm{dt}, J=6.0,1.6 \mathrm{~Hz}, 1 \mathrm{H}), 2.97-2.86(\mathrm{~m}, 1 \mathrm{H}), 2.73-2.57(\mathrm{~m}, 1 \mathrm{H}), 2.55-2.41(\mathrm{~m}, 2 \mathrm{H}), 2.31(\mathrm{~s}$, $3 \mathrm{H})$.

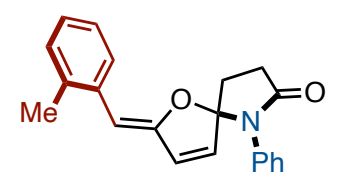

(Z)-2-(2-Methylbenzylidene)-6-phenyl-1-oxa-6-azaspiro[4.4]non-3-en-7-one (3Ad) 
The reaction was conducted by using DPEphos at $70{ }^{\circ} \mathrm{C}$. Crude ${ }^{1} \mathrm{H}$ NMR analysis showed $\boldsymbol{Z}$ 3Ad: $\boldsymbol{E - 3 A d}=70: 30 ;{ }^{1} \mathrm{H}$ NMR peaks at $5.54 \mathrm{ppm}(\mathrm{s}, 0.70 \mathrm{H})$ and $6.09 \mathrm{ppm}(\mathrm{s}, 0.30 \mathrm{H})$ were used. Purification by PTLC (hexane/EtOAc $=7: 3)$ afforded 3Ad $(14.3 \mathrm{mg}, 45 \%$ yield, as a mixture of diastereoisomers; $Z: E=73: 27$ ) as a pale yellow solid.

For $Z$ isomer: ${ }^{1} \mathrm{H}$ NMR $\left(400 \mathrm{MHz}, \mathrm{CDCl}_{3}\right) \delta 8.13(\mathrm{~d}, J=8.0 \mathrm{~Hz}, 1 \mathrm{H}), 7.37-7.02(\mathrm{~m}, 8 \mathrm{H}), 6.29(\mathrm{~d}$, $J=5.6 \mathrm{~Hz}, 1 \mathrm{H}), 5.89(\mathrm{~d}, J=5.6 \mathrm{~Hz}, 1 \mathrm{H}), 5.54(\mathrm{~s}, 1 \mathrm{H}), 3.04-2.88(\mathrm{~m}, 1 \mathrm{H}), 2.72-2.59(\mathrm{~m}, 1 \mathrm{H}), 2.55-$ $2.41(\mathrm{~m}, 2 \mathrm{H}), 2.33$ (s, 3H); ${ }^{13} \mathrm{C} \mathrm{NMR}\left(101 \mathrm{MHz}, \mathrm{CDCl}_{3}\right): 174.9,155.4,135.4,135.1,133.9,131.2,130.6$, 130.1, 129.0, 128.1 127.8, 127.4, 126.3, 126.1, 109.0, 99.0, 32.2, 29.2, 20.2; HRMS (ESI) $\mathrm{m} / \mathrm{z}$ calcd for $\mathrm{C}_{21} \mathrm{H}_{20} \mathrm{NO}_{2}[\mathrm{M}+\mathrm{H}]^{+}: 318.1489$ found 318.1487 .

For $E$ isomer: ${ }^{1} \mathrm{H}$ NMR $\left(400 \mathrm{MHz}, \mathrm{CDCl}_{3}\right) \delta 7.37-7.02(\mathrm{~m}, 9 \mathrm{H}), 6.48(\mathrm{~d}, J=5.6 \mathrm{~Hz}, 1 \mathrm{H}), 6.09(\mathrm{~s}$, $1 \mathrm{H}), 5.97(\mathrm{dd}, J=5.6,2.0 \mathrm{~Hz}, 1 \mathrm{H}), 3.04-2.88(\mathrm{~m}, 1 \mathrm{H}), 2.72-2.59(\mathrm{~m}, 1 \mathrm{H}), 2.55-2.41(\mathrm{~m}, 2 \mathrm{H}), 2.27$ (s, $3 \mathrm{H})$.

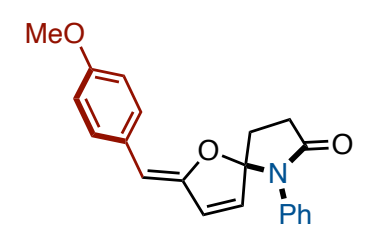

\section{(Z)-2-(4-Methoxybenzylidene)-6-phenyl-1-oxa-6-azaspiro[4.4]non-3-en-7-one (3Ae)}

The reaction was conducted by using $\mathrm{PPh}_{3}$ at $60{ }^{\circ} \mathrm{C}$. Crude ${ }^{1} \mathrm{H}$ NMR analysis showed $\boldsymbol{Z}$-3Ae: $\boldsymbol{E}$ 3Ae $=79: 21 ;{ }^{1} \mathrm{H}$ NMR peaks at $5.83 \mathrm{ppm}(\mathrm{d}, J=5.6 \mathrm{~Hz}, 0.79 \mathrm{H})$ and $5.98 \mathrm{ppm}(\mathrm{dd}, J=6.0,2.0 \mathrm{~Hz}$, $0.21 \mathrm{H})$ were used. Purification by PTLC (hexane/EtOAc $=7: 3)$ afforded $3 \mathrm{Ae}(20.7 \mathrm{mg}, 62 \%$ yield, as a mixture of diastereoisomers; $Z: E=84: 16)$ as a pale yellow solid.

For $Z$ isomer: ${ }^{1} \mathrm{H}$ NMR $\left(400 \mathrm{MHz}, \mathrm{CDCl}_{3}\right) \delta 7.61(\mathrm{~d}, J=8.8 \mathrm{~Hz}, 2 \mathrm{H}), 7.36-7.22(\mathrm{~m}, 3 \mathrm{H}), 7.21-$ $7.13(\mathrm{~m}, 2 \mathrm{H}), 6.90(\mathrm{~d}, J=8.8 \mathrm{~Hz}, 2 \mathrm{H}), 6.23(\mathrm{~d}, J=5.6 \mathrm{~Hz}, 1 \mathrm{H}), 5.83(\mathrm{~d}, J=5.6 \mathrm{~Hz}, 1 \mathrm{H}), 5.38(\mathrm{~s}, 1 \mathrm{H})$, $3.84(\mathrm{~s}, 3 \mathrm{H}), 3.05-2.86(\mathrm{~m}, 1 \mathrm{H}), 2.73-2.58(\mathrm{~m}, 1 \mathrm{H}), 2.53-2.41(\mathrm{~m}, 2 \mathrm{H}) ;{ }^{13} \mathrm{C} \mathrm{NMR}\left(101 \mathrm{MHz}, \mathrm{CDCl}_{3}\right)$ $\delta 174.8,158.1,154.1,135.4,130.9,129.6,129.4,129.0,128.4,127.8,127.4,114.0,108.9,102.0,55.3$, 32.4, 29.2; HRMS (ESI) $\mathrm{m} / z$ calcd for $\mathrm{C}_{21} \mathrm{H}_{20} \mathrm{NO}_{3}[\mathrm{M}+\mathrm{H}]^{+}: 334.1438$ found 334.1436.

For $E$ isomer: ${ }^{1} \mathrm{H}$ NMR $\left(400 \mathrm{MHz}, \mathrm{CDCl}_{3}\right) \delta$ 7.36-7.22 (m, 3H), 7.21-7.13 (m, 2H), $7.10(\mathrm{~d}, J=$ $9.2 \mathrm{~Hz}, 2 \mathrm{H}), 6.83(\mathrm{~d}, J=9.2 \mathrm{~Hz}, 2 \mathrm{H}), 6.65(\mathrm{~d}, J=6.0 \mathrm{~Hz}, 1 \mathrm{H}), 6.05(\mathrm{~s}, 1 \mathrm{H}), 5.98$ (dd, $J=6.0,2.0 \mathrm{~Hz}$, $1 \mathrm{H}), 3.79(\mathrm{~s}, 3 \mathrm{H}), 3.05-2.86(\mathrm{~m}, 1 \mathrm{H}), 2.73-2.58(\mathrm{~m}, 1 \mathrm{H}), 2.53-2.41(\mathrm{~m}, 2 \mathrm{H})$.

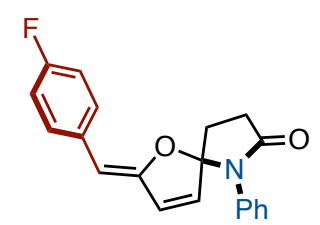

(Z)-2-(4-Fluorobenzylidene)-6-phenyl-1-oxa-6-azaspiro[4.4]non-3-en-7-one (3Af) 
The reaction was conducted by using DPEphos at $60^{\circ} \mathrm{C}$. Crude ${ }^{1} \mathrm{H}$ NMR analysis showed $\boldsymbol{Z}$-3Af: $\boldsymbol{E}$ 3Af $=76: 24 ;{ }^{1} \mathrm{H}$ NMR peaks at $6.23 \mathrm{ppm}(\mathrm{d}, J=6.0 \mathrm{~Hz}, 0.76 \mathrm{H})$ and $6.61 \mathrm{ppm}(\mathrm{d}, J=5.6 \mathrm{~Hz}, 0.24 \mathrm{H})$ were used. Purification by PTLC (hexane/EtOAc $=7: 3)$ afforded $\mathbf{3 A f}(15.1 \mathrm{mg}, 47 \%$ yield, as a mixture of diastereoisomers; $Z: E=80: 20$ ) as a pale yellow solid.

For $Z$ isomer: ${ }^{1} \mathrm{H}$ NMR (400 MHz, $\left.\mathrm{CDCl}_{3}\right) \delta$ 7.66-7.60 (m, 2H), 7.37-7.09 (m, 5H), 7.07-7.00 (m, 2H), $6.23(\mathrm{~d}, J=6.0 \mathrm{~Hz}, 1 \mathrm{H}), 5.89(\mathrm{~d}, J=6.0 \mathrm{~Hz}, 1 \mathrm{H}), 5.39(\mathrm{~s}, 1 \mathrm{H}), 3.05-2.86(\mathrm{~m}, 1 \mathrm{H}), 2.74-2.58(\mathrm{~m}$, $1 \mathrm{H}), 2.56-2.39(\mathrm{~m}, 2 \mathrm{H}) ;{ }^{13} \mathrm{C} \mathrm{NMR}\left(101 \mathrm{MHz}, \mathrm{CDCl}_{3}\right) \delta 174.8,161.1\left(\mathrm{~d}, J_{\mathrm{C}-\mathrm{F}}=247 \mathrm{~Hz}\right), 155.0\left(\mathrm{~d}, J_{\mathrm{C}-\mathrm{F}}\right.$ $=1.9 \mathrm{~Hz}), 135.3,131.8\left(\mathrm{~d}, J_{\mathrm{C}-\mathrm{F}}=2.9 \mathrm{~Hz}\right), 130.73,130.67,129.6\left(\mathrm{~d}, J_{\mathrm{C}-\mathrm{F}}=7.7 \mathrm{~Hz}\right), 129.0,127.9,127.4$, $115.4\left(\mathrm{~d}, J_{\mathrm{C}-\mathrm{F}}=21.2 \mathrm{~Hz}\right), 109.0,101.2,32.2,29.1 ;{ }^{19} \mathrm{~F}$ NMR $\left(376 \mathrm{MHz}, \mathrm{CDCl}_{3}\right) \delta-115.2$; HRMS (ESI) $m / z$ calcd for $\mathrm{C}_{20} \mathrm{H}_{17} \mathrm{FNO}_{2}[\mathrm{M}+\mathrm{H}]^{+}: 322.1238$ found 322.1238 .

For $E$ isomer: ${ }^{1} \mathrm{H}$ NMR $\left(400 \mathrm{MHz}, \mathrm{CDCl}_{3}\right) \delta$ 7.37-7.09 (m, 7H), 7.00-6.93 (m, 2H), $6.61(\mathrm{~d}, J=$ $5.6 \mathrm{~Hz}, 1 \mathrm{H}), 6.06-6.02(\mathrm{~m}, 2 \mathrm{H}), 3.05-2.86(\mathrm{~m}, 1 \mathrm{H}), 2.74-2.58(\mathrm{~m}, 1 \mathrm{H}), 2.56-2.39(\mathrm{~m}, 2 \mathrm{H}) ;{ }^{19} \mathrm{~F} \mathrm{NMR}$ $\left(376 \mathrm{MHz}, \mathrm{CDCl}_{3}\right) \delta-116.2$.

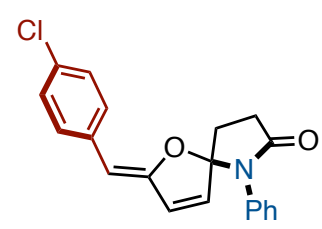

\section{(Z)-2-(4-Chlorobenzylidene)-6-phenyl-1-oxa-6-azaspiro[4.4]non-3-en-7-one (3Ag)}

The reaction was conducted by using DPEphos at $60{ }^{\circ} \mathrm{C}$. Crude ${ }^{1} \mathrm{H}$ NMR analysis showed $\boldsymbol{Z}$ 3Ag: $\boldsymbol{E - 3 A g}=70: 30 ;{ }^{1} \mathrm{H}$ NMR peaks at $5.37 \mathrm{ppm}(\mathrm{s}, 0.70 \mathrm{H})$ and $6.03 \mathrm{ppm}(\mathrm{s}, 0.30 \mathrm{H})$ were used. Purification by PTLC (hexane/EtOAc $=7: 3)$ afforded 3Ag $(10.1 \mathrm{mg}, 30 \%$ yield, as a mixture of diastereoisomers; $Z: E=78: 22)$ as a pale yellow solid.

For $Z$ isomer: ${ }^{1} \mathrm{H}$ NMR $\left(400 \mathrm{MHz}, \mathrm{CDCl}_{3}\right) \delta 7.57(\mathrm{~d}, J=8.8 \mathrm{~Hz}, 2 \mathrm{H}), 7.37-7.07(\mathrm{~m}, 7 \mathrm{H}), 6.24(\mathrm{~d}$, $J=5.6 \mathrm{~Hz}, 1 \mathrm{H}), 5.93(\mathrm{~d}, J=5.6 \mathrm{~Hz}, 1 \mathrm{H}), 5.37(\mathrm{~s}, 1 \mathrm{H}), 3.05-2.86(\mathrm{~m}, 1 \mathrm{H}), 2.73-2.59(\mathrm{~m}, 1 \mathrm{H}), 2.56-$ $2.42(\mathrm{~m}, 2 \mathrm{H}) ;{ }^{13} \mathrm{C}$ NMR $\left(101 \mathrm{MHz}, \mathrm{CDCl}_{3}\right) \delta 174.8,155.8,135.3,134.1,131.3,130.7,129.2,129.03$, $128.98,128.6,127.9,127.4,109.2,101.2,32.2,29.1$; HRMS (ESI) $m / z$ calcd for $\mathrm{C}_{20} \mathrm{H}_{17} \mathrm{ClNO}_{2}[\mathrm{M}+\mathrm{H}]^{+}$: 338.0942 found 338.0941 .

For $E$ isomer: ${ }^{1} \mathrm{H}$ NMR (400 MHz, $\left.\mathrm{CDCl}_{3}\right) \delta 7.37-7.07(\mathrm{~m}, 9 \mathrm{H}), 6.63(\mathrm{~d}, J=5.6 \mathrm{~Hz}, 1 \mathrm{H}), 6.06(\mathrm{dd}$, $J=5.6,1.6 \mathrm{~Hz}, 1 \mathrm{H}), 6.03(\mathrm{~s}, 1 \mathrm{H}), 3.05-2.86(\mathrm{~m}, 1 \mathrm{H}), 2.73-2.59(\mathrm{~m}, 1 \mathrm{H}), 2.56-2.42(\mathrm{~m}, 2 \mathrm{H})$.

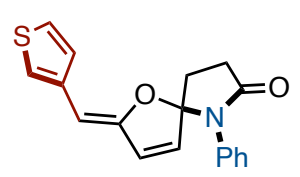

(Z)-6-Phenyl-2-(thiophen-3-ylmethylene)-1-oxa-6-azaspiro[4.4] non-3-en-7-one (3Ah)

The reaction was conducted by using $\mathrm{PPh}_{3}$ at $60{ }^{\circ} \mathrm{C}$. Crude ${ }^{1} \mathrm{H}$ NMR analysis showed $\boldsymbol{Z}-\mathbf{3 A h}: \boldsymbol{E}$ 3Ah $=77: 23 ;{ }^{1} \mathrm{H}$ NMR peaks at $5.86 \mathrm{ppm}(\mathrm{d}, J=5.6 \mathrm{~Hz}, 0.77 \mathrm{H})$ and $6.00 \mathrm{ppm}(\mathrm{dd}, J=5.6,1.6 \mathrm{~Hz}$, 
$0.23 \mathrm{H})$ were used. Purification by PTLC (hexane/EtOAc $=7: 3$ ) afforded $\mathbf{3 A h}(25.2 \mathrm{mg}, 81 \%$ yield, as a mixture of diastereoisomers; $Z: E=78: 22$ ) as a pale yellow solid.

For $Z$ isomer: ${ }^{1} \mathrm{H}$ NMR $\left(400 \mathrm{MHz}, \mathrm{CDCl}_{3}\right) \delta 7.47(\mathrm{~d}, J=2.0 \mathrm{~Hz}, 1 \mathrm{H}), 7.39-7.21(\mathrm{~m}, 5 \mathrm{H}), 7.19$ $7.09(\mathrm{~m}, 2 \mathrm{H}), 6.22(\mathrm{~d}, J=5.6 \mathrm{~Hz}, 1 \mathrm{H}), 5.86(\mathrm{~d}, J=5.6 \mathrm{~Hz}, 1 \mathrm{H}), 5.54(\mathrm{~s}, 1 \mathrm{H}), 3.02-2.85(\mathrm{~m}, 1 \mathrm{H}), 2.72-$ $2.56(\mathrm{~m}, 1 \mathrm{H}), 2.53-2.39(\mathrm{~m}, 2 \mathrm{H}) ;{ }^{13} \mathrm{C} \mathrm{NMR}\left(101 \mathrm{MHz}, \mathrm{CDCl}_{3}\right) \delta$ 174.7, 154.6, 136.4, 135.4, 130.6, 130.3, 129.0, 128.2, 127.8, 127.3, 125.0, 121.9, 108.7, 96.8, 32.3, 29.2; HRMS (ESI) $\mathrm{m} / \mathrm{z}$ calcd for $\mathrm{C}_{18} \mathrm{H}_{16} \mathrm{NO}_{2} \mathrm{~S}[\mathrm{M}+\mathrm{H}]^{+}: 310.0896$ found 310.0894 .

For $E$ isomer: ${ }^{1} \mathrm{H}$ NMR $\left(400 \mathrm{MHz}, \mathrm{CDCl}_{3}\right) \delta 7.39-7.21(\mathrm{~m}, 4 \mathrm{H}), 7.19-7.09(\mathrm{~m}, 2 \mathrm{H}), 6.99-6.95(\mathrm{~m}$, $2 \mathrm{H}), 6.69(\mathrm{~d}, J=5.6 \mathrm{~Hz}, 1 \mathrm{H}), 6.04(\mathrm{~s}, 1 \mathrm{H}), 6.00(\mathrm{dd}, J=5.6,1.6 \mathrm{~Hz}, 1 \mathrm{H}), 3.02-2.85(\mathrm{~m}, 1 \mathrm{H}), 2.72-2.56$ (m, 1H), 2.53-2.39 (m, 2H).

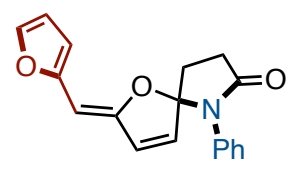

\section{(Z)-2-(Furan-2-ylmethylene)-6-phenyl-1-oxa-6-azaspiro[4.4]non-3-en-7-one (3Ai)}

The reaction was conducted by using $\mathrm{PPh}_{3}$ at $60{ }^{\circ} \mathrm{C}$. Crude ${ }^{1} \mathrm{H}$ NMR analysis showed $\boldsymbol{Z}-\mathbf{3 A i}: \boldsymbol{E}-\mathbf{3 A i}$ $=45: 55 ;{ }^{1} \mathrm{H}$ NMR peaks at $5.51 \mathrm{ppm}(\mathrm{s}, 0.45 \mathrm{H})$ and $5.83 \mathrm{ppm}(\mathrm{s}, 0.55 \mathrm{H})$ were used. Purification by PTLC (hexane/EtOAc = 7:3) afforded 3Ai $(21.1 \mathrm{mg}, 72 \%$ yield, as a mixture of diastereoisomers; Z:E $=45: 55)$ as a brown solid.

For $Z$ isomer: ${ }^{1} \mathrm{H}$ NMR (400 MHz, $\left.\mathrm{CDCl}_{3}\right) \delta 7.38-7.09(\mathrm{~m}, 6 \mathrm{H}), 6.70(\mathrm{~d}, J=3.2 \mathrm{~Hz}, 1 \mathrm{H}), 6.48(\mathrm{dd}$, $J=3.2,2.0 \mathrm{~Hz}, 1 \mathrm{H}), 6.22(\mathrm{~d}, J=5.6 \mathrm{~Hz}, 1 \mathrm{H}), 5.90(\mathrm{~d}, J=5.6 \mathrm{~Hz}, 1 \mathrm{H}), 5.51(\mathrm{~s}, 1 \mathrm{H}), 3.03-2.83(\mathrm{~m}, 1 \mathrm{H})$, 2.72-2.56 (m, 1H), 2.55-2.35 (m, 2H); HRMS (ESI) $m / z$ calcd for $\mathrm{C}_{18} \mathrm{H}_{16} \mathrm{NO}_{3}[\mathrm{M}+\mathrm{H}]^{+}: 294.1125$ found 294.1123.

For $E$ isomer: ${ }^{1} \mathrm{H}$ NMR (400 MHz, $\left.\mathrm{CDCl}_{3}\right) \delta 7.38-7.09(\mathrm{~m}, 6 \mathrm{H}), 7.05(\mathrm{~d}, J=6.0 \mathrm{~Hz}, 1 \mathrm{H}), 6.35(\mathrm{dd}$, $J=3.2,2.0 \mathrm{~Hz}, 1 \mathrm{H}), 6.09(\mathrm{~d}, J=3.2 \mathrm{~Hz}, 1 \mathrm{H}), 6.01(\mathrm{dd}, J=6.0,1.6 \mathrm{~Hz}, 1 \mathrm{H}), 5.83(\mathrm{~s}, 1 \mathrm{H}), 3.03-2.83$ (m, $1 \mathrm{H}), 2.72-2.56(\mathrm{~m}, 1 \mathrm{H}), 2.55-2.35(\mathrm{~m}, 2 \mathrm{H})$.

${ }^{13} \mathrm{C}$ NMR of a mixture of $\boldsymbol{Z}-\mathbf{3 A i}$ and $\boldsymbol{E - 3 A i}$ (45:55) (101 MHz, $\left.\mathrm{CDCl}_{3}\right): \delta 174.5,174.4,156.0,154.0$, 151.4, 151.2, 141.2, 140.8, 133.9, 131.5, 129.6, 129.0, 128.9, 128.2, 127.8, 127.73, 127.65, 127.5, 111.9, $111.4,108.9,108.5,107.1,106.5,92.1,92.0,32.3,32.0,29.2$ (four peaks are missing due to overlapping).

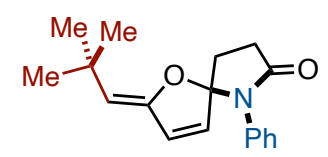

\section{(Z)-2-(2,2-Dimethylpropylidene)-6-phenyl-1-oxa-6-azaspiro[4.4]non-3-en-7-one (3Aj)}

The reaction was conducted by using $\mathrm{PPh}_{3}$ at $70{ }^{\circ} \mathrm{C}$. Crude ${ }^{1} \mathrm{H}$ NMR analysis showed $\boldsymbol{Z}-\mathbf{3 A j}: \boldsymbol{E}-\mathbf{3 A j}$ $=76: 24 ;{ }^{1} \mathrm{H}$ NMR peaks at $4.42 \mathrm{ppm}(\mathrm{s}, 0.76 \mathrm{H})$ and $5.10 \mathrm{ppm}(\mathrm{d}, J=1.2 \mathrm{~Hz}, 0.24 \mathrm{H})$ were used. Purification by PTLC (hexane/EtOAc $=7: 3)$ afforded $\mathbf{3 A j}(17.8 \mathrm{mg}, 63 \%$ yield, as a mixture of 
diastereoisomers; $Z: E=79: 21$ ) as a pale yellow solid. Further purification by PTLC (hexane/EtOAc $=$ 1:1) for the characterization was performed to give pure $Z$ isomer and $E$ isomer.

For $Z$ isomer: ${ }^{1} \mathrm{H}$ NMR (400 MHz, $\left.\mathrm{CDCl}_{3}\right) \delta$ 7.36-7.30 (m, 2H), 7.30-7.24 (m, 1H), 7.18-7.13 (m, $2 \mathrm{H}), 6.00(\mathrm{~d}, J=5.6 \mathrm{~Hz}, 1 \mathrm{H}), 5.67$ (dd, $J=5.6,0.8 \mathrm{~Hz}, 1 \mathrm{H}), 4.42$ (s, 1H), 2.89 (dt, $J=17.2,9.6 \mathrm{~Hz}$, $1 \mathrm{H}), 2.66-2.53(\mathrm{~m}, 1 \mathrm{H}), 2.42-2.33(\mathrm{~m}, 2 \mathrm{H}), 1.18(\mathrm{~s}, 9 \mathrm{H}) ;{ }^{13} \mathrm{C} \mathrm{NMR}\left(101 \mathrm{MHz}, \mathrm{CDCl}_{3}\right) \delta 174.8,153.3$, 135.5, 130.9, 128.8, 128.6, 127.6, 127.5, 112.6, 107.8, 32.3, 31.6, 30.5, 29.3; HRMS (ESI) $\mathrm{m} / \mathrm{z}$ calcd for $\mathrm{C}_{18} \mathrm{H}_{22} \mathrm{NO}_{2}[\mathrm{M}+\mathrm{H}]^{+}: 284.1645$ found 284.1644.

For $E$ isomer: ${ }^{1} \mathrm{H}$ NMR $\left(400 \mathrm{MHz}, \mathrm{CDCl}_{3}\right) \delta 7.35-7.28(\mathrm{~m}, 2 \mathrm{H}), 7.28-7.23(\mathrm{~m}, 1 \mathrm{H}), 7.20-7.13(\mathrm{~m}$, $2 \mathrm{H}), 6.55(\mathrm{~d}, J=6.0 \mathrm{~Hz}, 1 \mathrm{H}), 5.82(\mathrm{dd}, J=6.0,1.6 \mathrm{~Hz}, 1 \mathrm{H}), 5.10(\mathrm{~d}, J=1.2 \mathrm{~Hz}, 1 \mathrm{H}), 2.86(\mathrm{dt}, J=16.8$, $9.6 \mathrm{~Hz}, 1 \mathrm{H}), 2.64-2.51(\mathrm{~m}, 1 \mathrm{H}), 2.42-2.32(\mathrm{~m}, 2 \mathrm{H}), 1.10(\mathrm{~s}, 9 \mathrm{H}) ;{ }^{13} \mathrm{C} \mathrm{NMR}\left(101 \mathrm{MHz}, \mathrm{CDCl}_{3}\right) \delta 174.5$, $153.4,135.7,132.0,128.7,127.5,127.3,126.8,113.4,104.2,32.0,31.8,31.6,29.3$.

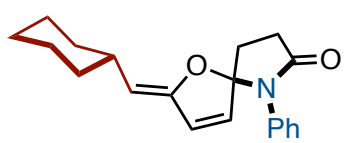

\section{(Z)-2-(Cyclohexylmethylene)-6-phenyl-1-oxa-6-azaspiro[4.4]non-3-en-7-one (3Ak)}

The reaction was conducted by using DPEphos at $70{ }^{\circ} \mathrm{C}$. Crude ${ }^{1} \mathrm{H}$ NMR analysis showed $\boldsymbol{Z}$ 3Ak: $\boldsymbol{E - 3 A k}=54: 46 ;{ }^{1} \mathrm{H}$ NMR peaks at $4.35 \mathrm{ppm}(\mathrm{d}, J=9.6 \mathrm{~Hz}, 0.54 \mathrm{H})$ and $4.84 \mathrm{ppm}(\mathrm{dd}, J=9.6,1.2$ $\mathrm{Hz}, 0.46 \mathrm{H})$ were used. Purification by PTLC (hexane/EtOAc $=7: 3$ ) afforded 3Ak $(16.2 \mathrm{mg}, 53 \%$ yield, as a mixture of diastereoisomers; $Z: E=64: 36)$ as a pale yellow solid.

For $Z$ isomer: ${ }^{1} \mathrm{H}$ NMR (400 MHz, $\left.\mathrm{CDCl}_{3}\right) \delta 7.35-7.11(\mathrm{~m}, 5 \mathrm{H}), 6.04(\mathrm{~d}, J=5.2 \mathrm{~Hz}, 1 \mathrm{H}), 5.70(\mathrm{~d}$, $J=5.2 \mathrm{~Hz}, 1 \mathrm{H}), 4.35(\mathrm{~d}, J=9.2 \mathrm{~Hz}, 1 \mathrm{H}), 2.96-2.81(\mathrm{~m}, 1 \mathrm{H}), 2.64-2.53(\mathrm{~m}, 1 \mathrm{H}), 2.53-2.42(\mathrm{~m}, 1 \mathrm{H})$, 2.42-2.34 (m, 2H), 1.80-1.02 (m, 10H). ${ }^{13} \mathrm{C}$ NMR (101 MHz, $\left.\mathrm{CDCl}_{3}\right) \delta 174.7,153.7,135.6,131.1$, 129.8, 129.6, 128.8, 128.7, 127.4, 108.5, 107.1, 34.7, 33.4, 33.3, 32.2, 29.3, 26.1, 25.9; HRMS (ESI) $\mathrm{m} / z$ calcd for $\mathrm{C}_{20} \mathrm{H}_{24} \mathrm{NO}_{2}[\mathrm{M}+\mathrm{H}]^{+}: 310.1802$ found 310.1799 .

For $E$ isomer: ${ }^{1} \mathrm{H}$ NMR $\left(400 \mathrm{MHz}, \mathrm{CDCl}_{3}\right) \delta 7.35-7.11(\mathrm{~m}, 5 \mathrm{H}), 6.40(\mathrm{~d}, J=6.0 \mathrm{~Hz}, 1 \mathrm{H}), 5.77(\mathrm{dd}$, $J=6.0,2.0 \mathrm{~Hz}, 1 \mathrm{H}), 4.84(\mathrm{dd}, J=8.8,1.2 \mathrm{~Hz}, 1 \mathrm{H}), 2.96-2.81(\mathrm{~m}, 1 \mathrm{H}), 2.64-2.53(\mathrm{~m}, 1 \mathrm{H}), 2.42-2.34$ (m, 2H), 2.08-1.97 (m, 1H), 1.80-1.02 (m, 10H).

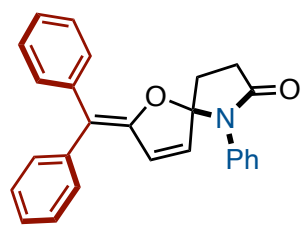

\section{2-(Diphenylmethylene)-6-phenyl-1-oxa-6-azaspiro[4.4]non-3-en-7-one (3Al)}

The reaction was conducted by using $\mathrm{PPh}_{3}$ and $\mathrm{KF}$ (2.0 equiv) at $60{ }^{\circ} \mathrm{C}$ for $36 \mathrm{~h}$. Purification by PTLC (hexane/EtOAc $=7: 3$, and then hexane/EtOAc $=1: 1)$ afforded $\mathbf{3 A l}(13.8 \mathrm{mg}, 36 \%$ yield) as a white solid. ${ }^{1} \mathrm{H}$ NMR $\left(400 \mathrm{MHz}, \mathrm{CDCl}_{3}\right) \delta$ 7.50-7.45 (m, 2H), 7.38-7.27 (m, 8H), 7.25-7.18 (m, 3H), 
7.14-7.09 (m, 2H), 6.17 (d, $J=6.0 \mathrm{~Hz}, 1 \mathrm{H}), 5.87$ (d, $J=6.0 \mathrm{~Hz}, 1 \mathrm{H}), 3.01-2.89$ (m, 1H), 2.69-2.59 (m, 1H), 2.58-2.42 (m, 2H); ${ }^{13} \mathrm{C} \mathrm{NMR}\left(101 \mathrm{MHz}, \mathrm{CDCl}_{3}\right) \delta 174.8,153.8,139.7,138.6,135.5,131.2,131.1$, $129.9,129.4,128.9,128.2,128.0,127.8,127.5,127.0,126.5,115.6,108.3,32.2,29.3$; HRMS (ESI) $m / z$ calcd for $\mathrm{C}_{26} \mathrm{H}_{22} \mathrm{NO}_{2}[\mathrm{M}+\mathrm{H}]^{+}: 380.1645$ found 380.1646 .

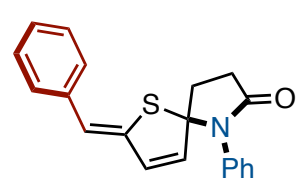

\section{(Z)-2-Benzylidene-6-phenyl-1-thia-6-azaspiro[4.4]non-3-en-7-one (3Ha)}

The reaction was conducted by using DPEphos at $70{ }^{\circ} \mathrm{C}$. Crude ${ }^{1} \mathrm{H}$ NMR analysis showed $\boldsymbol{Z}$ 3Ha: $\boldsymbol{E - 3 H a}=78: 22 ;{ }^{1} \mathrm{H}$ NMR peaks at $6.50 \mathrm{ppm}(\mathrm{s}, 0.78 \mathrm{H})$ and $6.48 \mathrm{ppm}(\mathrm{s}, 0.22 \mathrm{H})$ were used. Purification by PTLC (hexane/EtOAc $=7: 3)$ afforded $3 \mathbf{H a ~}(25.6 \mathrm{mg}, 80 \%$ yield, as a mixture of diasteroisomers; $Z: E=84: 16$ ) as a pale yellow solid.

For $Z$ isomer: ${ }^{1} \mathrm{H}$ NMR (400 MHz, $\left.\mathrm{CDCl}_{3}\right) \delta 7.43(\mathrm{~d}, J=7.6 \mathrm{~Hz}, 2 \mathrm{H}), 7.39-7.14(\mathrm{~m}, 8 \mathrm{H}), 6.50(\mathrm{~s}$, $1 \mathrm{H}), 6.34(\mathrm{~d}, J=6.0 \mathrm{~Hz}, 1 \mathrm{H}), 5.88(\mathrm{~d}, J=6.0 \mathrm{~Hz}, 1 \mathrm{H}), 2.91-2.62(\mathrm{~m}, 4 \mathrm{H}) ;{ }^{13} \mathrm{C} \mathrm{NMR}\left(101 \mathrm{MHz}, \mathrm{CDCl}_{3}\right)$ $\delta 173.9,139.9,136.4,136.0,134.2,128.8,128.5,128.3,128.1,127.9,127.8,127.1,122.1,92.0,37.4$, 30.5; HRMS (ESI) $m / z$ calcd for $\mathrm{C}_{20} \mathrm{H}_{18} \mathrm{NOS}[\mathrm{M}+\mathrm{H}]^{+}: 320.1104$ found 320.1101 .

For $E$ isomer: ${ }^{1} \mathrm{H}$ NMR $\left(400 \mathrm{MHz}, \mathrm{CDCl}_{3}\right) \delta 7.39-7.14(\mathrm{~m}, 10 \mathrm{H}), 6.76(\mathrm{dd}, J=6.0,0.8 \mathrm{~Hz}, 1 \mathrm{H})$, $6.48(\mathrm{~s}, 1 \mathrm{H}), 6.00(\mathrm{dd}, J=6.0,1.6 \mathrm{~Hz}, 1 \mathrm{H}), 2.91-2.62(\mathrm{~m}, 4 \mathrm{H})$.

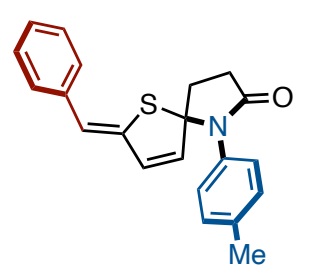

\section{(Z)-2-Benzylidene-6-(p-tolyl)-1-thia-6-azaspiro[4.4]non-3-en-7-one (3Ia)}

The reaction was conducted by using $\mathrm{PPh}_{3}$ at $70{ }^{\circ} \mathrm{C}$. Crude ${ }^{1} \mathrm{H}$ NMR analysis showed $\boldsymbol{Z}$-3Ia:E-3Ia $=79: 21 ;{ }^{1} \mathrm{H}$ NMR peaks at $5.88 \mathrm{ppm}(\mathrm{d}, J=6.0 \mathrm{~Hz}, 0.79 \mathrm{H})$ and $6.00 \mathrm{ppm}(\mathrm{dd}, J=6.0,1.2 \mathrm{~Hz}, 0.21 \mathrm{H})$ were used. Purification by PTLC (hexane/EtOAc $=7: 3$, and then hexane/EtOAc $=1: 1$ ) afforded 3Ia (16.8 $\mathrm{mg}, 50 \%$ yield, as a mixture of diasteroisomers; $Z / E=83: 17)$ as a pale yellow solid.

For $Z$ isomer: ${ }^{1} \mathrm{H}$ NMR $\left(400 \mathrm{MHz}, \mathrm{CDCl}_{3}\right) \delta 7.42(\mathrm{~d}, J=7.6 \mathrm{~Hz}, 2 \mathrm{H}), 7.36(\mathrm{t}, J=7.6 \mathrm{~Hz}, 2 \mathrm{H})$, $7.31-7.10(\mathrm{~m}, 5 \mathrm{H}), 6.49$ (s, 1H), $6.32(\mathrm{~d}, J=6.0 \mathrm{~Hz}, 1 \mathrm{H}), 5.88$ (d, $J=6.0 \mathrm{~Hz}, 1 \mathrm{H}), 2.88-2.63(\mathrm{~m}, 4 \mathrm{H})$, $2.30(\mathrm{~s}, 3 \mathrm{H}) ;{ }^{13} \mathrm{C} \mathrm{NMR}\left(101 \mathrm{MHz}, \mathrm{CDCl}_{3}\right) \delta 174.1,140.1,137.8,136.4,135.8,134.3,132.9,129.5$, $128.5,128.3,127.9,127.1,122.0,92.1,37.2,30.4,21.1$; HRMS (ESI) $m / z$ calcd for $\mathrm{C}_{21} \mathrm{H}_{20} \mathrm{NOS}[\mathrm{M}+\mathrm{H}]^{+}$: 334.1260 found 334.1258 .

For $E$ isomer: ${ }^{1} \mathrm{H}$ NMR (400 MHz, $\left.\mathrm{CDCl}_{3}\right) \delta 7.31-7.10(\mathrm{~m}, 9 \mathrm{H}), 6.75(\mathrm{~d}, J=6.0 \mathrm{~Hz}, 1 \mathrm{H}), 6.47(\mathrm{~s}$, $1 \mathrm{H}), 6.00(\mathrm{dd}, J=6.0,1.2 \mathrm{~Hz}, 1 \mathrm{H}), 2.88-2.63(\mathrm{~m}, 4 \mathrm{H}), 2.31(\mathrm{~s}, 3 \mathrm{H})$. 


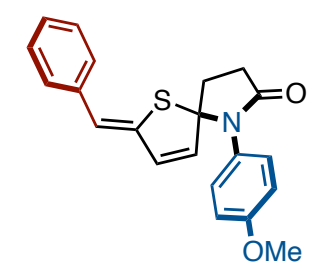

(Z)-2-Benzylidene-6-(4-methoxyphenyl)-1-thia-6-azaspiro[4.4]non-3-en-7-one (3Ja)

The reaction was conducted by using $\mathrm{PPh}_{3}$ at $70{ }^{\circ} \mathrm{C}$. Crude ${ }^{1} \mathrm{H}$ NMR analysis showed $\boldsymbol{Z}$-3Ja:E-3Ja $=77: 23) ;{ }^{1} \mathrm{H}$ NMR peaks at $5.88 \mathrm{ppm}(\mathrm{d}, J=6.0 \mathrm{~Hz}, 0.77 \mathrm{H})$ and $6.00 \mathrm{ppm}(\mathrm{d}, J=6.0,0.23 \mathrm{H})$ were used. Purification by PTLC (hexane/EtOAc $=7: 3$ ) and further purification by TLC (hexane/EtOAc $=1: 1$ ) afforded 3Ja (17.5 mg, 50\% yield, as a mixture of diasteroisomers; $Z / E=81: 19)$ as a pale yellow solid.

For $Z$ isomer: ${ }^{1} \mathrm{H}$ NMR $\left(400 \mathrm{MHz}, \mathrm{CDCl}_{3}\right) \delta 7.42(\mathrm{~d}, J=7.6 \mathrm{~Hz}, 2 \mathrm{H}), 7.37(\mathrm{t}, J=7.6 \mathrm{~Hz}, 2 \mathrm{H})$, 7.31-7.14 (m, 3H), 6.90-6.82 (m, 2H), $6.49(\mathrm{~s}, 1 \mathrm{H}), 6.33(\mathrm{~d}, J=6.0 \mathrm{~Hz}, 1 \mathrm{H}), 5.88(\mathrm{~d}, J=6.0 \mathrm{~Hz}, 1 \mathrm{H})$, 3.76 (s, 3H), 2.88-2.62 (m, 4H); ${ }^{13} \mathrm{C}$ NMR (101 MHz, $\left.\mathrm{CDCl}_{3}\right) \delta 174.2,159.0,140.1,136.4,135.9,134.3$, $129.5,128.5,128.3,127.1,122.0,114.2,92.2,55.3,37.0,30.4$ (one peak is missing due to overlapping); HRMS (ESI) $m / z$ calcd for $\mathrm{C}_{21} \mathrm{H}_{20} \mathrm{NO}_{2} \mathrm{~S}[\mathrm{M}+\mathrm{H}]^{+}: 350.1209$ found 350.1208 .

For $E$ isomer: ${ }^{1} \mathrm{H}$ NMR $\left(400 \mathrm{MHz}, \mathrm{CDCl}_{3}\right) \delta 7.31-7.14(\mathrm{~m}, 7 \mathrm{H}), 6.90-6.82(\mathrm{~m}, 2 \mathrm{H}), 6.75(\mathrm{~d}, J=$ $6.0 \mathrm{~Hz}, 1 \mathrm{H}), 6.47(\mathrm{~s}, 1 \mathrm{H}), 6.00(\mathrm{~d}, J=6.0 \mathrm{~Hz}, 1 \mathrm{H}), 3.78(\mathrm{~s}, 3 \mathrm{H}), 2.88-2.62(\mathrm{~m}, 4 \mathrm{H})$.
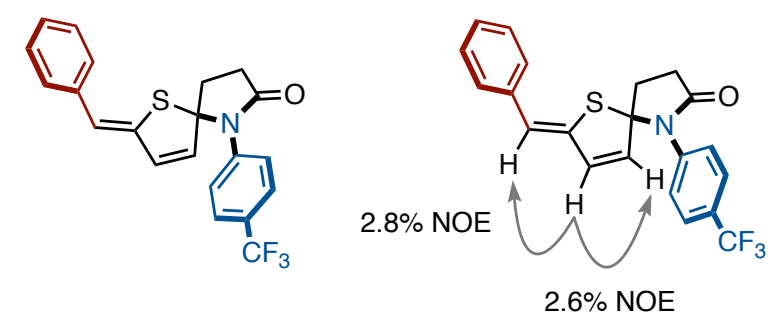

\section{(Z)-2-Benzylidene-6-(4-(trifluoromethyl)phenyl)-1-thia-6-azaspiro[4.4] non-3-en-7-one (3Ka)}

The reaction was conducted by using $\mathrm{PPh}_{3}$ at $70{ }^{\circ} \mathrm{C}$. Crude ${ }^{1} \mathrm{H}$ NMR analysis showed $\boldsymbol{Z}-3 \mathrm{Ka}: \boldsymbol{E}$ 3Ka $=83: 17 ;{ }^{1} \mathrm{H}$ NMR peaks at $5.85 \mathrm{ppm}(\mathrm{d}, J=6.4 \mathrm{~Hz}, 0.83 \mathrm{H})$ and $5.97 \mathrm{ppm}(\mathrm{dd}, J=6.4,0.8 \mathrm{~Hz}$, $0.17 \mathrm{H}$ ) were used. Purification by PTLC (hexane/EtOAc $=7: 3$, and then hexane/EtOAc $=1: 1$ ) afforded $3 \mathrm{Ka}(25.5 \mathrm{mg}, 66 \%$ yield, as a mixture of diasteroisomers; $Z / E=84: 16)$ as a pale yellow solid.

For $Z$ isomer: ${ }^{1} \mathrm{H}$ NMR (400 MHz, $\left.\mathrm{CDCl}_{3}\right) \delta$ 7.64-7.55 (m, 2H), 7.49-7.35 (m, 5H), 7.28-7.16 (m, 2H), $6.58(\mathrm{~s}, 1 \mathrm{H}), 6.41(\mathrm{~d}, J=6.4 \mathrm{~Hz}, 1 \mathrm{H}), 5.85(\mathrm{~d}, J=6.4 \mathrm{~Hz}, 1 \mathrm{H}), 2.93-2.65(\mathrm{~m}, 4 \mathrm{H}) ;{ }^{13} \mathrm{C} \mathrm{NMR}(101$ $\left.\mathrm{MHz}, \mathrm{CDCl}_{3}\right) \delta 173.8,139.3,136.5,136.1,133.6,129.3\left(\mathrm{q}, J_{\mathrm{C}-\mathrm{F}}=32.9 \mathrm{~Hz}\right), 128.6,128.5,128.3,127.4$, $127.3,125.8\left(\mathrm{q}, J_{\mathrm{C}-\mathrm{F}}=2.9 \mathrm{~Hz}\right), 123.8\left(\mathrm{q}, J_{\mathrm{C}-\mathrm{F}}=274 \mathrm{~Hz}\right), 122.9,91.7,37.7,30.4 ;{ }^{19} \mathrm{~F}$ NMR $(376 \mathrm{MHz}$, $\left.\mathrm{CDCl}_{3}\right) \delta-62.7$; HRMS (ESI) $m / z$ calcd for $\mathrm{C}_{21} \mathrm{H}_{17} \mathrm{~F}_{3} \mathrm{NOS}[\mathrm{M}+\mathrm{H}]^{+}: 388.0978$ found 388.0977.

For $E$ isomer: ${ }^{1} \mathrm{H}$ NMR (400 MHz, $\left.\mathrm{CDCl}_{3}\right) \delta 7.64-7.55(\mathrm{~m}, 2 \mathrm{H}), 7.49-7.35(\mathrm{~m}, 5 \mathrm{H}), 7.31(\mathrm{t}, J=$ $8.0 \mathrm{~Hz}, 2 \mathrm{H}), 6.83(\mathrm{dd}, J=6.4,0.8 \mathrm{~Hz}, 1 \mathrm{H}), 6.54(\mathrm{~s}, 1 \mathrm{H}), 5.97$ (dd, $J=6.4,0.8 \mathrm{~Hz}, 1 \mathrm{H}), 2.93-2.65$ (m, $4 \mathrm{H})$. 


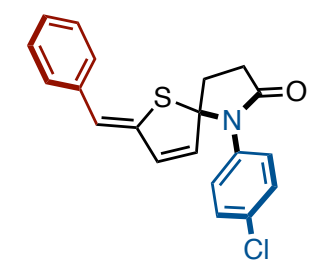

(Z)-2-Benzylidene-6-(4-chlorophenyl)-1-thia-6-azaspiro[4.4]non-3-en-7-one (3La)

The reaction was conducted by using $\mathrm{PPh}_{3}$ at $70{ }^{\circ} \mathrm{C}$. Crude ${ }^{1} \mathrm{H}$ NMR analysis showed $\boldsymbol{Z}$-3La: $\boldsymbol{E}$ 3La $=84: 16 ;{ }^{1} \mathrm{H}$ NMR peaks at $5.85 \mathrm{ppm}(\mathrm{d}, J=6.0 \mathrm{~Hz}, 0.84 \mathrm{H})$ and $5.97 \mathrm{ppm}(\mathrm{dd}, J=6.0,1.6 \mathrm{~Hz}$, $0.16 \mathrm{H}$ ) were used. Purification by PTLC (hexane/EtOAc $=7: 3$ ) and further purification by TLC (hexane/EtOAc $=1: 1)$ afforded $3 \mathbf{L a}(20.5 \mathrm{mg}, 58 \%$ yield, as a mixture of diasteroisomers; $Z / E=84: 16)$ as a pale yellow solid.

For $Z$ isomer: ${ }^{1} \mathrm{H}$ NMR $\left(400 \mathrm{MHz}, \mathrm{CDCl}_{3}\right) \delta 7.43(\mathrm{~d}, J=7.2 \mathrm{~Hz}, 2 \mathrm{H}), 7.40-7.34(\mathrm{~m}, 2 \mathrm{H}), 7.34$ $7.27(\mathrm{~m}, 2 \mathrm{H}), 7.26-7.16(\mathrm{~m}, 3 \mathrm{H}), 6.54(\mathrm{~s}, 1 \mathrm{H}), 6.36(\mathrm{~d}, J=6.0 \mathrm{~Hz}, 1 \mathrm{H}), 5.85(\mathrm{~d}, J=6.0 \mathrm{~Hz}, 1 \mathrm{H}), 2.88-$ $2.61(\mathrm{~m}, 4 \mathrm{H}) ;{ }^{13} \mathrm{C}$ NMR $\left(101 \mathrm{MHz}, \mathrm{CDCl}_{3}\right) \delta 173.9,139.6,136.3,134.3,133.8,133.6,129.1,129.0$, 128.6, 128.3 127.3, 122.5, 91.8, 37.3, 30.4 (one peak is missing due to overlapping); HRMS (ESI) $\mathrm{m} / \mathrm{z}$ calcd for $\mathrm{C}_{20} \mathrm{H}_{17} \mathrm{ClNOS}[\mathrm{M}+\mathrm{H}]^{+}: 354.0714$ found 354.0711.

For $E$ isomer: ${ }^{1} \mathrm{H}$ NMR (400 MHz, $\left.\mathrm{CDCl}_{3}\right) \delta$ 7.34-7.27 (m, 4H), 7.26-7.16 (m, 5H), 6.79 (dd, $J=$ 6.0, $0.8 \mathrm{~Hz}, 1 \mathrm{H}), 6.50(\mathrm{~s}, 1 \mathrm{H}), 5.97(\mathrm{dd}, J=6.0,1.6 \mathrm{~Hz}, 1 \mathrm{H}), 2.88-2.61(\mathrm{~m}, 4 \mathrm{H})$.

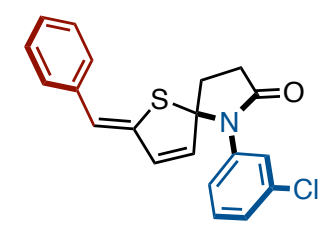

(Z)-2-Benzylidene-6-(3-chlorophenyl)-1-thia-6-azaspiro[4.4]non-3-en-7-one (3Ma)

The reaction was conducted by using $\mathrm{PPh}_{3}$ at $70{ }^{\circ} \mathrm{C}$. Crude ${ }^{1} \mathrm{H}$ NMR analysis showed $\boldsymbol{Z}$-3Ma: $\boldsymbol{E}$ 3Ma $=80: 20 ;{ }^{1} \mathrm{H}$ NMR peaks at $5.87 \mathrm{ppm}(\mathrm{d}, J=6.0 \mathrm{~Hz}, 0.80 \mathrm{H})$ and $5.97 \mathrm{ppm}(\mathrm{dd}, J=6.0,1.6 \mathrm{~Hz}$, $0.20 \mathrm{H}$ ) were used. Purification by PTLC (hexane/EtOAc $=7: 3$ ) and further purification by TLC (hexane/EtOAc $=1: 1)$ afforded $\mathbf{3 M a}(21.3 \mathrm{mg}, 60 \%$ yield, as a mixture of diasteroisomers; $Z / E=81: 19)$ as a pale yellow solid.

For $Z$ isomer: ${ }^{1} \mathrm{H}$ NMR $\left(400 \mathrm{MHz}, \mathrm{CDCl}_{3}\right) \delta 7.43(\mathrm{~d}, J=7.6 \mathrm{~Hz}, 2 \mathrm{H}), 7.37(\mathrm{t}, J=7.6 \mathrm{~Hz}, 2 \mathrm{H})$, $7.33-7.26(\mathrm{~m}, 2 \mathrm{H}), 7.25-7.16(\mathrm{~m}, 3 \mathrm{H}), 6.55(\mathrm{~s}, 1 \mathrm{H}), 6.39(\mathrm{~d}, J=6.0 \mathrm{~Hz}, 1 \mathrm{H}), 5.87(\mathrm{~d}, J=6.0 \mathrm{~Hz}, 1 \mathrm{H})$, 2.88-2.61 (m, 4H); ${ }^{13} \mathrm{C}$ NMR (101 MHz, $\left.\mathrm{CDCl}_{3}\right) \delta 173.8,139.5,136.9,136.32,136.25,134.2,133.7$, 129.7, 128.6, 128.3, 128.2, 128.0, 127.3, 125.9, 122.6, 91.8, 37.4, 30.4; HRMS (ESI) $\mathrm{m} / \mathrm{z}$ calcd for $\mathrm{C}_{20} \mathrm{H}_{17} \mathrm{ClNOS}[\mathrm{M}+\mathrm{H}]^{+}: 354.0714$ found 354.0711.

For $E$ isomer: ${ }^{1} \mathrm{H}$ NMR (400 MHz, $\left.\mathrm{CDCl}_{3}\right) \delta 7.33-7.16(\mathrm{~m}, 9 \mathrm{H}), 6.80(\mathrm{~d}, J=6.0 \mathrm{~Hz}, 1 \mathrm{H}), 6.52(\mathrm{~s}$, $1 \mathrm{H}), 5.97(\mathrm{dd}, J=6.0,1.6 \mathrm{~Hz}, 1 \mathrm{H}), 2.88-2.61(\mathrm{~m}, 4 \mathrm{H})$. 


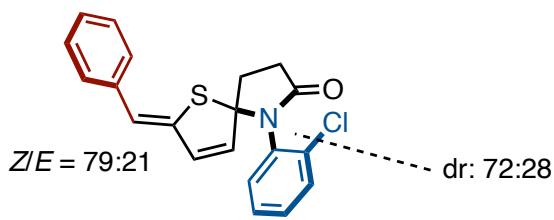

60:20:13:7

\section{(Z)-2-Benzylidene-6-(2-chlorophenyl)-1-thia-6-azaspiro[4.4]non-3-en-7-one (3Na)}

The reaction was conducted by using $\mathrm{PPh}_{3}$ at $70{ }^{\circ} \mathrm{C}$. Crude ${ }^{1} \mathrm{H}$ NMR analysis showed $\boldsymbol{Z}-3 \mathrm{Na}: \boldsymbol{E}$ $3 \mathrm{Na}=79: 21, \mathrm{dr}=72: 28 ;{ }^{1} \mathrm{H}$ NMR peaks at $6.05 \mathrm{ppm}(\mathrm{d}, J=6.0 \mathrm{~Hz}, 0.60 \mathrm{H}), 6.11 \mathrm{ppm}(\mathrm{d}, J=6.0 \mathrm{~Hz}$, $0.20 \mathrm{H}), 6.73 \mathrm{ppm}(\mathrm{dd}, J=6.0,1.6 \mathrm{~Hz}, 0.13 \mathrm{H})$ and $6.22 \mathrm{ppm}(\mathrm{dd}, J=6.0,1.6 \mathrm{~Hz}, 0.07 \mathrm{H})$, were used. Purification by PTLC (hexane/EtOAc $=7: 3$ ) and further purification by TLC (hexane/EtOAc $=1: 1$ ) afforded $\mathbf{3 N a}$ (50.2 $\mathrm{mg}, 79 \%$ yield, $0.18 \mathrm{mmol}$ scale, as an inseparable mixture of diastereoisomers; $Z / E$ $=82: 18, \mathrm{dr}=72: 28)$ as a pale yellow solid.

For a major isomer: ${ }^{1} \mathrm{H}$ NMR $\left(400 \mathrm{MHz}, \mathrm{CDCl}_{3}\right) \delta 7.47-7.42(\mathrm{~m}, 2 \mathrm{H}), 7.39(\mathrm{t}, J=8.4 \mathrm{~Hz}, 2 \mathrm{H})$, 7.35-7.17 (m, 5H), $6.49(\mathrm{~s}, 1 \mathrm{H}), 6.27(\mathrm{~d}, J=6.0 \mathrm{~Hz}, 1 \mathrm{H}), 6.05(\mathrm{~d}, J=6.0 \mathrm{~Hz}, 1 \mathrm{H}), 2.92-2.65(\mathrm{~m}, 4 \mathrm{H})$; ${ }^{13} \mathrm{C}$ NMR $\left(101 \mathrm{MHz}, \mathrm{CDCl}_{3}\right) \delta 173.4,139.7,136.4,135.3,133.8,133.3,132.8,130.5,130.0,129.9$, 128.6, 128.3, 127.6, 127.2, 122.0, 92.6, 37.6, 30.3; HRMS (ESI) $m / z$ calcd for $\mathrm{C}_{20} \mathrm{H}_{17} \mathrm{ClNOS}[\mathrm{M}+\mathrm{H}]^{+}$: 354.0714 found 354.0712 .

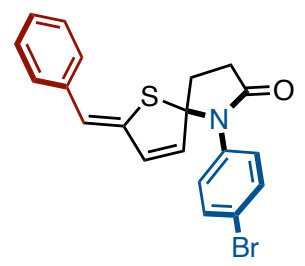

(Z)-2-Benzylidene-6-(4-bromophenyl)-1-thia-6-azaspiro[4.4] non-3-en-7-one (3Oa)

The reaction was conducted by using $\mathrm{PPh}_{3}$ at $70{ }^{\circ} \mathrm{C}$. Crude ${ }^{1} \mathrm{H}$ NMR analysis showed $\boldsymbol{Z}-3 \mathrm{Oa}: \boldsymbol{E}$ 30a $=83: 17 ;{ }^{1} \mathrm{H}$ NMR peaks at $5.85 \mathrm{ppm}(\mathrm{d}, J=6.0 \mathrm{~Hz}, 0.83 \mathrm{H})$ and $5.96 \mathrm{ppm}(\mathrm{dd}, J=6.0,1.6 \mathrm{~Hz}$, $0.17 \mathrm{H}$ ) were used. Purification by PTLC (hexane/EtOAc $=7: 3$, and then hexane/EtOAc $=1: 1$ ) afforded 30 a (20.3 mg, $56 \%$ yield, as a mixture of diasteroisomers; $Z / E=84: 16)$ as a pale yellow solid.

For $Z$ isomer: ${ }^{1} \mathrm{H}$ NMR $\left(400 \mathrm{MHz}, \mathrm{CDCl}_{3}\right) \delta 7.52-7.27(\mathrm{~m}, 6 \mathrm{H}), 7.25-7.12(\mathrm{~m}, 3 \mathrm{H}), 6.54(\mathrm{~s}, 1 \mathrm{H})$, $6.37(\mathrm{~d}, J=6.0 \mathrm{~Hz}, 1 \mathrm{H}), 5.85(\mathrm{~d}, J=6.0 \mathrm{~Hz}, 1 \mathrm{H}), 2.88-2.62(\mathrm{~m}, 4 \mathrm{H}) ;{ }^{13} \mathrm{C} \mathrm{NMR}\left(101 \mathrm{MHz}, \mathrm{CDCl}_{3}\right) \delta$ $173.8,139.5,136.3,136.2,134.8,133.8,132.0,129.4,128.6,128.3,127.3,122.6,121.6,91.7,37.3$, 30.4; HRMS (ESI) $m / z$ calcd for $\mathrm{C}_{20} \mathrm{H}_{17} \mathrm{BrNOS}[\mathrm{M}+\mathrm{H}]^{+}: 398.0209$ found 398.0208 .

For $E$ isomer: ${ }^{1} \mathrm{H}$ NMR $\left(400 \mathrm{MHz}, \mathrm{CDCl}_{3}\right) \delta$ 7.52-7.27 (m, 6H), 7.25-7.12 (m, 3H), $6.79(\mathrm{~d}, J=$ $6.0 \mathrm{~Hz}, 1 \mathrm{H}), 6.51(\mathrm{~s}, 1 \mathrm{H}), 5.96(\mathrm{dd}, J=6.0,1.6 \mathrm{~Hz}, 1 \mathrm{H}), 2.88-2.62(\mathrm{~m}, 4 \mathrm{H})$.

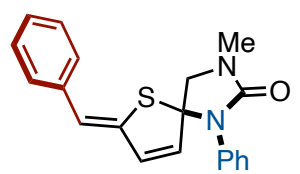




\section{(Z)-7-Benzylidene-3-methyl-1-phenyl-6-thia-1,3-diazaspiro[4.4]non-8-en-2-one (3Pa)}

The reaction was conducted by using $\mathrm{PPh}_{3}$ at $70{ }^{\circ} \mathrm{C}$. Crude ${ }^{1} \mathrm{H}$ NMR analysis showed $\boldsymbol{Z}-3 P a: E$ $3 \mathbf{P a}=87: 13 ;{ }^{1} \mathrm{H}$ NMR peaks at $6.52 \mathrm{ppm}(\mathrm{s}, 0.87 \mathrm{H})$ and $6.47 \mathrm{ppm}(\mathrm{s}, 0.13 \mathrm{H})$ were used. Purification by PTLC (hexane/EtOAc $=1: 1)$ afforded 3Pa $(23.4 \mathrm{mg}, 70 \%$ yield, as a mixture of diastereoisomers; Z:E $=85: 15)$ as a pale yellow solid.

For $Z$ isomer: ${ }^{1} \mathrm{H}$ NMR (400 MHz, $\left.\mathrm{CDCl}_{3}\right) \delta 7.41(\mathrm{~d}, J=7.6 \mathrm{~Hz}, 2 \mathrm{H}), 7.37-7.13(\mathrm{~m}, 8 \mathrm{H}), 6.52(\mathrm{~s}$, $1 \mathrm{H}), 6.42(\mathrm{~d}, J=6.0 \mathrm{~Hz}, 1 \mathrm{H}), 5.95(\mathrm{~d}, J=6.0 \mathrm{~Hz}, 1 \mathrm{H}), 3.91(\mathrm{~d}, J=10.0 \mathrm{~Hz}, 1 \mathrm{H}), 3.81(\mathrm{~d}, J=10.0 \mathrm{~Hz}$, 1H), $2.96(\mathrm{~s}, 3 \mathrm{H}) ;{ }^{13} \mathrm{C} \mathrm{NMR}\left(101 \mathrm{MHz}, \mathrm{CDCl}_{3}\right) \delta 158.1,139.7,137.1,136.3,136.2,132.2,128.52$, $128.50,128.3,127.5,127.2,126.7,122.6,87.6,60.4,31.1$; HRMS (ESI) $m / z$ calcd for $\mathrm{C}_{20} \mathrm{H}_{18} \mathrm{~N}_{2} \mathrm{NaOS}$ $[\mathrm{M}+\mathrm{Na}]^{+}: 357.1032$ found 357.1033 .

For $E$ isomer: ${ }^{1} \mathrm{H}$ NMR $\left(400 \mathrm{MHz}, \mathrm{CDCl}_{3}\right) \delta 7.37-7.13(\mathrm{~m}, 10 \mathrm{H}), 6.83(\mathrm{~d}, J=6.0 \mathrm{~Hz}, 1 \mathrm{H}), 6.47(\mathrm{~s}$, $1 \mathrm{H}), 6.07(\mathrm{dd}, J=6.0,1.2 \mathrm{~Hz}, 1 \mathrm{H}), 3.91(\mathrm{~d}, J=10.0 \mathrm{~Hz}, 1 \mathrm{H}), 3.81$ (d, $J=10.0 \mathrm{~Hz}, 1 \mathrm{H}), 2.95(\mathrm{~s}, 3 \mathrm{H})$.

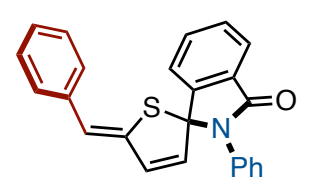

\section{(Z)-5'-Benzylidene-2-phenyl-5' $H$-spiro[isoindoline-1,2'-thiophen]-3-one (3Qa)}

The reaction was conducted by using $\mathrm{PPh}_{3}$ at $70{ }^{\circ} \mathrm{C}$. Crude ${ }^{1} \mathrm{H}$ NMR analysis showed $\boldsymbol{Z}-3 \mathbf{Q}$ a: $\boldsymbol{E}$ 3Qa $=75: 25 ;{ }^{1} \mathrm{H}$ NMR peaks at $5.92 \mathrm{ppm}(\mathrm{d}, J=6.0 \mathrm{~Hz}, 0.75 \mathrm{H})$ and $6.02 \mathrm{ppm}(\mathrm{dd}, J=6.0,1.6 \mathrm{~Hz}$, $0.25 \mathrm{H}$ ) were used. Purification by PTLC (hexane/EtOAc $=7: 3$ ) afforded 3Qa $(26.9 \mathrm{mg}, 73 \%$ yield, as a mixture of diastereoisomers; $Z / E=76: 24)$ as a pale yellow solid.

For $Z$ isomer: ${ }^{1} \mathrm{H}$ NMR (400 MHz, $\left.\mathrm{CDCl}_{3}\right) \delta 7.95-7.91(\mathrm{~m}, 1 \mathrm{H}), 7.69-7.28(\mathrm{~m}, 11 \mathrm{H}), 7.21(\mathrm{t}, J=$ $6.8 \mathrm{~Hz}, 2 \mathrm{H}), 6.60(\mathrm{~s}, 1 \mathrm{H}), 6.55(\mathrm{~d} . J=6.0 \mathrm{~Hz}, 1 \mathrm{H}), 5.92(\mathrm{~d}, J=6.0 \mathrm{~Hz}, 1 \mathrm{H}) ; \delta 167.0,146.1,140.3,137.0$, 136.2, 135.4, 133.0, 132.6, 129.5, 128.9, 128.6, 128.4, 128.2, 127.9, 127.3, 123.9, 123.7, 122.8, 90.0 (one peak is missing due to overlapping); HRMS (ESI) $m / z$ calcd for $\mathrm{C}_{24} \mathrm{H}_{17} \mathrm{NNaOS}[\mathrm{M}+\mathrm{Na}]^{+}: 390.0923$ found 390.0925 .

For $E$ isomer: ${ }^{1} \mathrm{H}$ NMR (400 MHz, $\left.\mathrm{CDCl}_{3}\right) \delta 7.95-7.91(\mathrm{~m}, 1 \mathrm{H}), 7.69-7.28(\mathrm{~m}, 13 \mathrm{H}), 6.96(\mathrm{dd}, J$ $=6.0,1.6 \mathrm{~Hz}, 1 \mathrm{H}), 6.55(\mathrm{~d} . J=6.0 \mathrm{~Hz}, 1 \mathrm{H}), 6.02(\mathrm{dd}, J=6.0,1.6 \mathrm{~Hz}, 1 \mathrm{H})$.

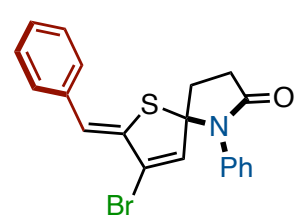

\section{(Z)-2-Benzylidene-3-bromo-6-phenyl-1-thia-6-azaspiro[4.4]non-3-en-7-one (3Ra)}

The reaction was conducted by using $\mathrm{PPh}_{3}$ at $70{ }^{\circ} \mathrm{C}$. Crude ${ }^{1} \mathrm{H}$ NMR analysis showed $\boldsymbol{Z}$-3Ra: $\boldsymbol{E}$ 3Ra $=85: 15 ;{ }^{1} \mathrm{H}$ NMR peaks at $6.48 \mathrm{ppm}(\mathrm{s}, 0.85 \mathrm{H})$ and $6.44 \mathrm{ppm}(\mathrm{s}, 0.15 \mathrm{H})$ were used. Purification by PTLC (hexane/EtOAc $=7: 3$ ) and further purification by TLC (hexane/EtOAc $=1: 1$ ) afforded 3Ra 
$(15.9 \mathrm{mg}, 40 \%$ yield, as a mixture of diasteroisomers; ratio $=86: 14)$ as a pale yellow solid. $Z / E$ structure of the major compound could not be determined by NOE.

For a major isomer: ${ }^{1} \mathrm{H}$ NMR $\left(400 \mathrm{MHz}, \mathrm{CDCl}_{3}\right) \delta 7.42-7.21(\mathrm{~m}, 10 \mathrm{H}), 6.50(\mathrm{~s}, 1 \mathrm{H}), 6.48(\mathrm{~s}, 1 \mathrm{H})$, 3.01-2.91 (m, 1H), 2.89-2.66 (m, 3H); $\left.{ }^{13} \mathrm{C} \mathrm{NMR} \mathrm{(101} \mathrm{MHz,} \mathrm{CDCl}_{3}\right) \delta$ 174.0, 137.6, 135.9, 135.8, 135.2, $128.8,128.7,128.3,128.2,128.1,127.5,127.0,122.6,90.3,35.2,29.9 . ;$ HRMS (ESI) $\mathrm{m} / \mathrm{z}$ calcd for $\mathrm{C}_{20} \mathrm{H}_{16} \mathrm{BrNNaOS}[\mathrm{M}+\mathrm{Na}]^{+}: 420.0028$ found 420.0027.

For a minor isomer: ${ }^{1} \mathrm{H}$ NMR $\left(400 \mathrm{MHz}, \mathrm{CDCl}_{3}\right) \delta 7.42-7.21(\mathrm{~m}, 8 \mathrm{H}), 7.11(\mathrm{~d}, J=7.6 \mathrm{~Hz}, 2 \mathrm{H})$, $6.90(\mathrm{~s}, 1 \mathrm{H}), 6.44(\mathrm{~s}, 1 \mathrm{H}), 3.01-2.91(\mathrm{~m}, 1 \mathrm{H}), 2.89-2.66(\mathrm{~m}, 3 \mathrm{H})$.

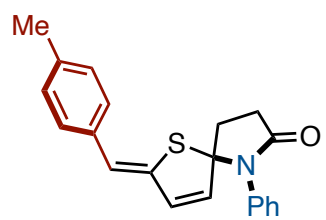

\section{(Z)-2-(4-Methylbenzylidene)-6-phenyl-1-thia-6-azaspiro[4.4]non-3-en-7-one (3Hb)}

The reaction was conducted by using $\mathrm{PPh}_{3}$ at $70{ }^{\circ} \mathrm{C}$. Crude ${ }^{1} \mathrm{H}$ NMR analysis showed $\boldsymbol{Z}-\mathbf{3 H b}: \boldsymbol{E}$ $3 \mathrm{Hb}=81: 19 ;{ }^{1} \mathrm{H}$ NMR peaks at $6.48 \mathrm{ppm}(\mathrm{s}, 0.81 \mathrm{H})$ and $6.44 \mathrm{ppm}(\mathrm{s}, 0.19 \mathrm{H})$ were used. Purification by PTLC (hexane/EtOAc $=7: 3)$ afforded $\mathbf{3 H b}(27.6 \mathrm{mg}$, 83\% yield, as a mixture of diastereoisomers; $Z: E=82: 18)$ as a pale yellow solid.

For $Z$ isomer: ${ }^{1} \mathrm{H}$ NMR (400 MHz, $\left.\mathrm{CDCl}_{3}\right) \delta 7.38-7.23(\mathrm{~m}, 7 \mathrm{H}), 7.17(\mathrm{~d}, J=8.0 \mathrm{~Hz}, 2 \mathrm{H}), 6.48(\mathrm{~s}$, $1 \mathrm{H}), 6.32(\mathrm{~d}, J=6.0 \mathrm{~Hz}, 1 \mathrm{H}), 5.84(\mathrm{~d}, J=6.0 \mathrm{~Hz}, 1 \mathrm{H}), 2.90-2.62(\mathrm{~m}, 4 \mathrm{H}), 2.35(\mathrm{~s}, 3 \mathrm{H}) ;{ }^{13} \mathrm{C} \mathrm{NMR}(101$ $\left.\mathrm{MHz}, \mathrm{CDCl}_{3}\right) \delta 173.9,138.9,137.1,136.0,135.7,133.64,133.58,129.3,128.8,128.2,127.9,127.7$, 122.1, 92.0, 37.5, 30.5, 21.3; HRMS (ESI) $m / z$ calcd for $\mathrm{C}_{21} \mathrm{H}_{19} \mathrm{NNaOS}[\mathrm{M}+\mathrm{Na}]^{+}: 356.1080$ found 356.1077 .

For $E$ isomer: ${ }^{1} \mathrm{H}$ NMR (400 MHz, $\left.\mathrm{CDCl}_{3}\right) \delta 7.38-7.23(\mathrm{~m}, 5 \mathrm{H}), 7.10(\mathrm{~d}, J=8.0 \mathrm{~Hz}, 2 \mathrm{H}), 7.05(\mathrm{~d}$, $J=8.0 \mathrm{~Hz}, 2 \mathrm{H}), 6.75(\mathrm{~d}, J=6.0 \mathrm{~Hz}, 1 \mathrm{H}), 6.44(\mathrm{~s}, 1 \mathrm{H}), 5.97(\mathrm{~d}, J=6.0 \mathrm{~Hz}, 1 \mathrm{H}), 2.90-2.62(\mathrm{~m}, 4 \mathrm{H}), 2.31$ (s, 3H).

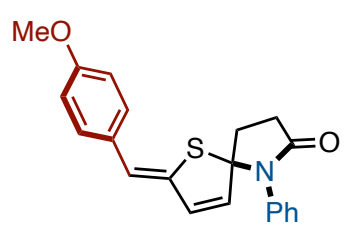

\section{(Z)-2-(4-Methoxybenzylidene)-6-phenyl-1-thia-6-azaspiro[4.4]non-3-en-7-one (3He)}

The reaction was conducted by using $\mathrm{PPh}_{3}$ at $70{ }^{\circ} \mathrm{C}$. Crude ${ }^{1} \mathrm{H}$ NMR analysis showed $\boldsymbol{Z}$-3He: $\boldsymbol{E}$ $3 \mathrm{He}=84: 16 ;{ }^{1} \mathrm{H}$ NMR peaks at $5.81 \mathrm{ppm}(\mathrm{d}, J=6.0 \mathrm{~Hz}, 0.84 \mathrm{H})$ and $5.95 \mathrm{ppm}(\mathrm{dd}, J=6.0,1.2 \mathrm{~Hz}$, $0.16 \mathrm{H})$ were used. Purification by PTLC (hexane/EtOAc $=7: 3$ ) afforded $\mathbf{3 H e}(24.0 \mathrm{mg}, 69 \%$ yield, as a mixture of diastereoisomers; $Z: E=82: 18$ ) as a pale yellow solid. 
For $Z$ isomer: ${ }^{1} \mathrm{H}$ NMR (400 MHz, $\left.\mathrm{CDCl}_{3}\right) \delta 7.40-7.22(\mathrm{~m}, 7 \mathrm{H}), 6.90(\mathrm{~d}, J=9.2 \mathrm{~Hz}, 2 \mathrm{H}), 6.46(\mathrm{~s}$, $1 \mathrm{H}), 6.32(\mathrm{~d}, J=6.0 \mathrm{~Hz}, 1 \mathrm{H}), 5.81(\mathrm{~d}, J=6.0 \mathrm{~Hz}, 1 \mathrm{H}), 3.82(\mathrm{~s}, 3 \mathrm{H}), 2.90-2.60(\mathrm{~m}, 4 \mathrm{H}) ;{ }^{13} \mathrm{C} \mathrm{NMR}(101$ $\left.\mathrm{MHz} \mathrm{CDCl}_{3}\right) \delta 174.0,158.7,137.6,136.0,135.8,133.0,129.7,129.2,128.8,127.9,127.7,121.7,114.0$, 92.0, 55.3, 37.6, 30.5; HRMS (ESI) $m / z$ calcd for $\mathrm{C}_{21} \mathrm{H}_{19} \mathrm{NNaO}_{2} \mathrm{~S}[\mathrm{M}+\mathrm{Na}]^{+}: 372.1029$ found 372.1028 .

For $E$ isomer: ${ }^{1} \mathrm{H}$ NMR $\left(400 \mathrm{MHz}, \mathrm{CDCl}_{3}\right) \delta 7.40-7.22(\mathrm{~m}, 5 \mathrm{H}), 7.09(\mathrm{~d}, J=8.8 \mathrm{~Hz}, 2 \mathrm{H}), 6.81(\mathrm{~d}$, $J=8.8 \mathrm{~Hz}, 2 \mathrm{H}), 6.73(\mathrm{dd}, J=6.0,1.2 \mathrm{~Hz}, 1 \mathrm{H}), 6.41(\mathrm{~s}, 1 \mathrm{H}), 5.95(\mathrm{dd}, J=6.0,1.2 \mathrm{~Hz}, 1 \mathrm{H}), 3.78(\mathrm{~s}, 3 \mathrm{H})$, $2.90-2.60(\mathrm{~m}, 4 \mathrm{H})$.

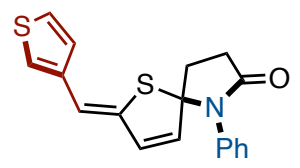

(Z)-6-Phenyl-2-(thiophen-3-ylmethylene)-1-thia-6-azaspiro[4.4]non-3-en-7-one (3Hh)

The reaction was conducted by using $\mathrm{PPh}_{3}$ at $70{ }^{\circ} \mathrm{C}$. Crude ${ }^{1} \mathrm{H}$ NMR analysis showed $\boldsymbol{Z}-\mathbf{3 H h}: \boldsymbol{E}$ $3 \mathrm{Hh}=76: 24 ;{ }^{1} \mathrm{H}$ NMR peaks at $6.55 \mathrm{ppm}(\mathrm{s}, 0.76 \mathrm{H})$ and $6.39 \mathrm{ppm}(\mathrm{s}, 0.24 \mathrm{H})$ were used. Purification by PTLC (hexane/EtOAc $=7: 3)$ afforded $\mathbf{3 H h}(26.0 \mathrm{mg}, 80 \%$ yield, as a mixture of diastereoisomers; $Z: E=78: 22)$ as a pale yellow solid.

For $Z$ isomer: ${ }^{1} \mathrm{H}$ NMR (400 MHz, $\left.\mathrm{CDCl}_{3}\right) \delta 7.40-7.23(\mathrm{~m}, 7 \mathrm{H}), 7.19(\mathrm{dd}, J=4.8,1.2 \mathrm{~Hz}, 1 \mathrm{H})$, $6.55(\mathrm{~s}, 1 \mathrm{H}), 6.31(\mathrm{~d}, J=6.0 \mathrm{~Hz}, 1 \mathrm{H}), 5.86(\mathrm{~d}, J=6.0 \mathrm{~Hz}, 1 \mathrm{H}), 2.91-2.62(\mathrm{~m}, 4 \mathrm{H}) ;{ }^{13} \mathrm{C} \mathrm{NMR}(101 \mathrm{MHz}$, $\left.\mathrm{CDCl}_{3}\right) \delta 173.9,139.3,138.5,138.2,135.3,134.2,128.8,127.92,127.86,127.8,125.6,123.2,115.9$, 91.9, 37.5, 30.4; HRMS (ESI) $m / z$ calcd for $\mathrm{C}_{18} \mathrm{H}_{15} \mathrm{NNaOS}_{2}[\mathrm{M}+\mathrm{Na}]^{+}: 348.0485$ found 348.0485.

For $E$ isomer: ${ }^{1} \mathrm{H}$ NMR (400 MHz, $\left.\mathrm{CDCl}_{3}\right) \delta 7.40-7.23(\mathrm{~m}, 6 \mathrm{H}), 7.05-7.01(\mathrm{~m}, 1 \mathrm{H}), 6.98(\mathrm{dd}, J=$ 4.8, $0.8 \mathrm{~Hz}, 1 \mathrm{H}), 6.79$ (dd, $J=6.0,1.2 \mathrm{~Hz}, 1 \mathrm{H}), 6.39$ (s, 1H), 5.99 (dd, $J=6.0,1.2 \mathrm{~Hz}, 1 \mathrm{H}), 2.91-2.62$ $(\mathrm{m}, 4 \mathrm{H})$.

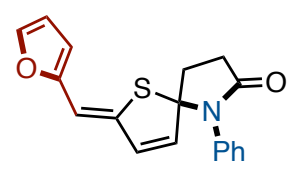

\section{(Z)-2-(Furan-2-ylmethylene)-6-phenyl-1-thia-6-azaspiro[4.4]non-3-en-7-one (3Hi)}

The reaction was conducted by using $\mathrm{PPh}_{3}$ at $70{ }^{\circ} \mathrm{C}$. Crude ${ }^{1} \mathrm{H}$ NMR analysis showed $\boldsymbol{Z}-\mathbf{3 H i}: \boldsymbol{E}-\mathbf{3 H i}$ $=65: 35 ;{ }^{1} \mathrm{H}$ NMR peaks at $5.87 \mathrm{ppm}(\mathrm{d}, J=6.0 \mathrm{~Hz}, 0.65 \mathrm{H})$ and $6.00 \mathrm{ppm}(\mathrm{dd}, J=6.0,1.6 \mathrm{~Hz}, 0.35 \mathrm{H})$ were used. Purification by PTLC (hexane/EtOAc $=7: 3$, and then hexane/EtOAc $=1: 1$ ) afforded 3Hi (13.1 $\mathrm{mg}, 42 \%$ yield, as a mixture of diastereoisomers; $Z: E=73: 27$ ) as a brown solid.

For $Z$ isomer: ${ }^{1} \mathrm{H}$ NMR (400 MHz, $\left.\mathrm{CDCl}_{3}\right) \delta 7.45(\mathrm{~d}, J=1.6 \mathrm{~Hz}, 1 \mathrm{H}), 7.38-7.23(\mathrm{~m}, 5 \mathrm{H}), 6.46(\mathrm{dd}$, $J=3.2,1.6 \mathrm{~Hz}, 1 \mathrm{H}), 6.41-6.38(\mathrm{~m}, 2 \mathrm{H}), 6.29$ (d, $J=6.0 \mathrm{~Hz}, 1 \mathrm{H}), 5.87$ (d, $J=6.0 \mathrm{~Hz}, 1 \mathrm{H}), 2.92-2.63$ (m, 4H); ${ }^{13} \mathrm{C}$ NMR $\left(101 \mathrm{MHz}, \mathrm{CDCl}_{3}\right) \delta 174.0,152.1,142.2,138.4,135.7,135.0,134.7,128.8,127.9$, 127.8, 112.0, 109.8, 109.7, 91.6, 37.4, 30.5; HRMS (ESI) $\mathrm{m} / z$ calcd for $\mathrm{C}_{18} \mathrm{H}_{15} \mathrm{NNaO}_{2} \mathrm{~S}[\mathrm{M}+\mathrm{Na}]^{+}$: 332.0716 found 332.0714 . 
For $E$ isomer: ${ }^{1} \mathrm{H}$ NMR $\left(400 \mathrm{MHz}, \mathrm{CDCl}_{3}\right) \delta 7.38-7.23(\mathrm{~m}, 7 \mathrm{H}), 6.35(\mathrm{dd}, J=3.2,2.0 \mathrm{~Hz}, 1 \mathrm{H})$, 6.14-6.11 (m, 2H), $6.00(\mathrm{dd}, J=6.0,1.6 \mathrm{~Hz}, 1 \mathrm{H}), 2.90-2.60(\mathrm{~m}, 4 \mathrm{H})$.

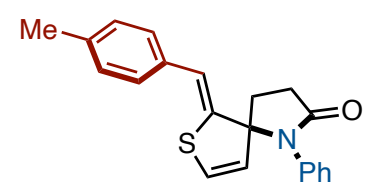

\section{(Z)-6-(4-Methylbenzylidene)-1-phenyl-7-thia-1-azaspiro[4.4]non-8-en-2-one (5b)}

Purification by PTLC (hexane/EtOAc $=7: 3)$ afforded $\mathbf{5 b}(20.3 \mathrm{mg}, 0.10 \mathrm{mmol} \mathrm{scale}, 67 \%$ yield) as a pale yellow solid. ${ }^{1} \mathrm{H}$ NMR $\left(400 \mathrm{MHz}, \mathrm{CDCl}_{3}\right) \delta 7.31-7.14(\mathrm{~m}, 9 \mathrm{H}), 6.73(\mathrm{~s}, 1 \mathrm{H}), 6.39(\mathrm{~d}, J=6.4$ $\mathrm{Hz}, 1 \mathrm{H}), 5.74$ (d, $J=6.4 \mathrm{~Hz}, 1 \mathrm{H}), 3.00-2.90$ (m, 1H), 2.76-2.66 (m, 1H), 2.49-2.40 (m, 1H), 2.39-2.29 $(\mathrm{m}, 4 \mathrm{H}) ;{ }^{13} \mathrm{C}$ NMR $\left(101 \mathrm{MHz}, \mathrm{CDCl}_{3}\right) \delta 174.3,139.6,137.4,136.9,133.0,129.3,128.7,128.3,126.5$, 126.2, 125.6, 125.0, 122.3, 82.1, 35.3, 29.5, 21.2; HRMS (ESI) $\mathrm{m} / z$ calcd for $\mathrm{C}_{21} \mathrm{H}_{19} \mathrm{NNaOS}[\mathrm{M}+\mathrm{Na}]^{+}$: 356.1080 found 356.1074 .

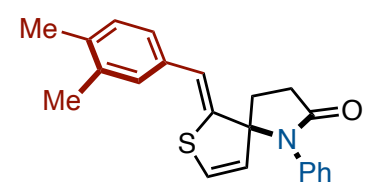

(Z)-6-(3,4-Dimethylbenzylidene)-1-phenyl-7-thia-1-azaspiro[4.4]non-8-en-2-one (5m)

Purification by PTLC (hexane/EtOAc = 7:3) afforded 5m (20.3 mg, $0.10 \mathrm{mmol} \mathrm{scale}, 58 \%$ yield) as a pale yellow solid. ${ }^{1} \mathrm{H}$ NMR $\left(400 \mathrm{MHz}, \mathrm{CDCl}_{3}\right) \delta$ 7.31-7.21 (m, 4H), 7.20-7.10 (m, 4H), 6.71 (s, $1 \mathrm{H}), 6.38(\mathrm{~d}, J=6.0 \mathrm{~Hz}, 1 \mathrm{H}), 5.73(\mathrm{~d}, J=6.0 \mathrm{~Hz}, 1 \mathrm{H}), 3.00-2.90(\mathrm{~m}, 1 \mathrm{H}), 2.76-2.64(\mathrm{~m}, 1 \mathrm{H}), 2.50-2.40$ (m, 1H), 2.40-2.29 (m, 1H), 2.27 (s, 3H), $\left.2.26(\mathrm{~s}, 3 \mathrm{H}) ;{ }^{13} \mathrm{C} \mathrm{NMR} \mathrm{(101} \mathrm{MHz,} \mathrm{CDCl}_{3}\right) \delta 174.3,139.3$, $136.9,136.8,136.2,133.4,129.8,129.7,128.7,126.5,126.1,125.7,125.6,125.0,122.3,82.0,35.3$, 29.5, 19.8, 19.5; HRMS (ESI) $m / z$ calcd for $\mathrm{C}_{22} \mathrm{H}_{22} \mathrm{NOS}[\mathrm{M}+\mathrm{H}]^{+}: 348.1417$ found 348.1418 .
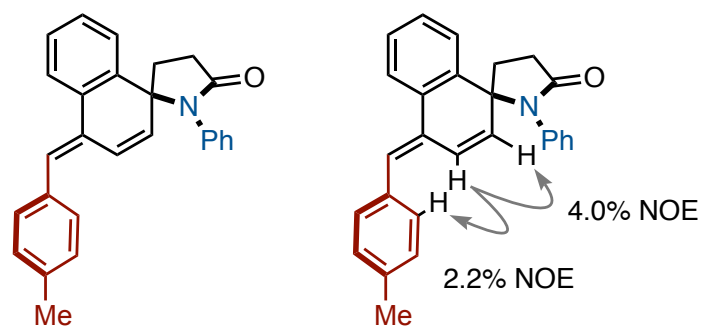

(E)-4-(4-Methylbenzylidene)-1'-phenyl-4H-spiro[naphthalene-1,2'-pyrrolidin]-5'-one (7)

Purification by PTLC (hexane/EtOAc $=7: 3)$ afforded $7(35.0 \mathrm{mg}, 0.10 \mathrm{mmol} \mathrm{scale}, 93 \%$ yield) as a pale yellow solid. ${ }^{1} \mathrm{H}$ NMR $\left(400 \mathrm{MHz}, \mathrm{CDCl}_{3}\right) \delta 7.91(\mathrm{dd}, J=8.0,2.0 \mathrm{~Hz}, 1 \mathrm{H}), 7.42(\mathrm{dd}, J=8.0,1.6$ Hz, 1H), 7.38-7.29 (m, 3H), 7.28 (d, J=7.6 Hz, 2H), 7.20 (d, $J=7.6 \mathrm{~Hz}, 2 \mathrm{H}), 7.18-7.10(\mathrm{~m}, 4 \mathrm{H}), 7.06$ (t, $J=8.4 \mathrm{~Hz}, 1 \mathrm{H}), 6.97$ (d, $J=10.0 \mathrm{~Hz}, 1 \mathrm{H}), 6.06$ (d, $J=10.0 \mathrm{~Hz}, 1 \mathrm{H}), 2.91-2.70(\mathrm{~m}, 2 \mathrm{H}), 2.43-2.32$ $(\mathrm{m}, 5 \mathrm{H}) ;{ }^{13} \mathrm{C} \mathrm{NMR}\left(101 \mathrm{MHz}, \mathrm{CDCl}_{3}\right) \delta 174.7,137.9,137.4,137.2,133.8,133.5,132.3,129.4,129.1$, 
$128.8,128.4,128.0,127.7,126.4,125.9,124.9,124.7,123.1,66.6,37.8,30.5,21.3$ (one peak is missing due to overlapping); HRMS (ESI) $m / z$ calcd for $\mathrm{C}_{27} \mathrm{H}_{24} \mathrm{NO}[\mathrm{M}+\mathrm{H}]^{+}: 378.1852$ found 378.1850 .

\section{Acid-Catalyzed Rearrangement of 3}

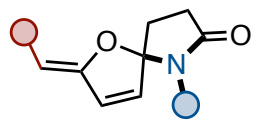

3

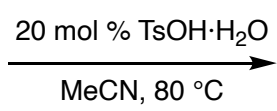

$\mathrm{MeCN}, 80^{\circ} \mathrm{C}$

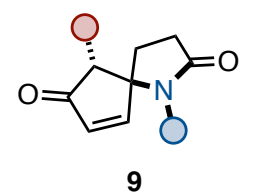

\section{General Procedure}

To an 8-mL glass tube containing a magnetic stirring bar were added azaspirocycle 3 (1.0 equiv), $p$ toluenesulfonic acid monohydrate $\left(\mathrm{TsOH} \cdot \mathrm{H}_{2} \mathrm{O}: 20 \mathrm{~mol} \%\right)$, and $\mathrm{MeCN}(0.25 \mathrm{M})$. The tube was sealed with a screw cap. After stirring the mixture at $80{ }^{\circ} \mathrm{C}$ for several hours with monitoring reaction progress with TLC, the reaction was quenched with $\mathrm{NaHCO}_{3}$ aq. The mixture was extracted three times with EtOAc. The combined organic layer was dried over $\mathrm{Na}_{2} \mathrm{SO}_{4}$, filtrated, and concentrated in vacuo. The residue was purified by PTLC to afford the corresponding azaspiro-cyclopentenone 9.
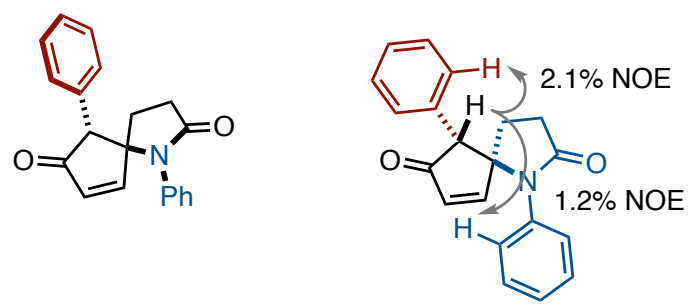

$\left(5 S^{*}, 6 R^{*}\right)-1,6-$ Diphenyl-1-azaspiro[4.4]non-8-ene-2,7-dione (9Aa)

Purification by PTLC (hexane/EtOAc = 1:1) afforded 9Aa (40.3 mg, $141 \mu \mathrm{mol} \mathrm{scale,} \mathrm{93 \%} \mathrm{yield)}$ as a pale yellow solid. ${ }^{1} \mathrm{H} \mathrm{NMR}\left(400 \mathrm{MHz}, \mathrm{CDCl}_{3}\right) \delta 7.74(\mathrm{~d}, J=5.6 \mathrm{~Hz}, 1 \mathrm{H}), 7.49(\mathrm{t}, J=8.0 \mathrm{~Hz}, 2 \mathrm{H})$, 7.44-7.29 (m, 6H), 7.13 (d, $J=8.0 \mathrm{~Hz}, 2 \mathrm{H}), 6.37$ (d, $J=5.6 \mathrm{~Hz}, 1 \mathrm{H}), 3.76(\mathrm{~s}, 1 \mathrm{H}), 2.41-2.29$ (m, 1H), 2.16-1.96 (m, 2H), 1.93-1.81 (m, 1H); ${ }^{13} \mathrm{C}$ NMR (101 MHz, $\left.\mathrm{CDCl}_{3}\right) \delta$ 204.0, 174.6, 163.2, 135.2, 134.0, $130.1,129.7,129.0,128.5,128.1,128.0,75.2,58.5,29.6,28.6$ (one peak is missing due to overlapping); HRMS (ESI) $m / z$ calcd for $\mathrm{C}_{20} \mathrm{H}_{18} \mathrm{NO}_{2}[\mathrm{M}+\mathrm{H}]^{+}: 304.1332$ found 304.1330 .

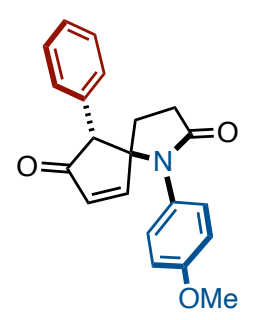

$\left(5 S^{*}, 6 R^{*}\right)-1-(4-M e t h o x y p h e n y l)-6-p h e n y l-1-a z a s p i r o[4.4]$ non-8-ene-2,7-dione (9Ba)

Purification by PTLC (hexane/EtOAc $=1: 1)$ afforded 9Ba (16.8 mg, $63 \mu \mathrm{mol} \mathrm{scale,} 80 \%$ yield) as a brown solid. ${ }^{1} \mathrm{H}$ NMR $\left(400 \mathrm{MHz}, \mathrm{CDCl}_{3}\right) \delta 7.68(\mathrm{~d}, J=6.0 \mathrm{~Hz}, 1 \mathrm{H}), 7.45-7.32(\mathrm{~m}, 3 \mathrm{H}), 7.25-7.19$ 
(m, 2H), 7.16-7.10 (m, 2H), 7.02-6.95 (m, 2H), $6.35(\mathrm{~d}, J=6.0 \mathrm{~Hz}, 1 \mathrm{H}), 3.83(\mathrm{~s}, 3 \mathrm{H}), 3.76(\mathrm{~s}, 1 \mathrm{H})$, 2.38-2.26 (m, 1H), 2.14-1.94 (m, 2H), 1.92-1.78 (m, 1H); $\left.{ }^{13} \mathrm{C} \mathrm{NMR} \mathrm{(101} \mathrm{MHz,} \mathrm{CDCl}_{3}\right) \delta 204.1,174.8$, 163.1, 159.5, 134.2, 134.1, 130.1, 129.6, 129.0, 128.0, 127.5, 115.0, 75.2, 58.5, 55.5, 29.5, 28.4; HRMS (ESI) $m / z$ calcd for $\mathrm{C}_{21} \mathrm{H}_{20} \mathrm{NO}_{3}[\mathrm{M}+\mathrm{H}]^{+}: 334.1438$ found 334.1436 .

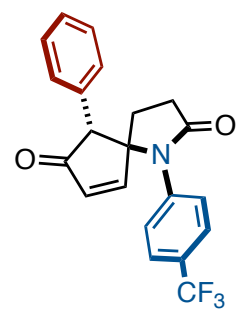

$\left(5 S^{*}, 6 R^{*}\right)$-6-Phenyl-1-(4-(trifluoromethyl)phenyl)-1-azaspiro[4.4]non-8-ene-2,7-dione (9Ca)

Purification by PTLC (hexane/EtOAc = 1:1) afforded 9Ca (52.9 mg, $0.19 \mathrm{mmol}$ scale, $75 \%$ yield) as a brown solid. ${ }^{1} \mathrm{H}$ NMR $\left(400 \mathrm{MHz}, \mathrm{CDCl}_{3}\right) \delta 7.77(\mathrm{~d}, J=6.0 \mathrm{~Hz}, 1 \mathrm{H}), 7.75(\mathrm{~d}, J=8.4 \mathrm{~Hz}, 2 \mathrm{H}), 7.48$ (d, $J=8.0 \mathrm{~Hz}, 2 \mathrm{H}), 7.44-7.33(\mathrm{~m}, 3 \mathrm{H}), 7.11-7.05(\mathrm{~m}, 2 \mathrm{H}), 6.43$ (d, $J=6.0 \mathrm{~Hz}, 1 \mathrm{H}), 3.71(\mathrm{~s}, 1 \mathrm{H}), 2.40$ $2.30(\mathrm{~m}, 1 \mathrm{H}), 2.17-1.97(\mathrm{~m}, 2 \mathrm{H}), 1.92-1.80(\mathrm{~m}, 1 \mathrm{H}) ;{ }^{13} \mathrm{C} \mathrm{NMR}\left(101 \mathrm{MHz}, \mathrm{CDCl}_{3}\right) \delta 203.2,174.6,162.8$, $138.8,134.3,133.6,130.1\left(\mathrm{q}, J_{\mathrm{C}-\mathrm{F}}=33.0 \mathrm{~Hz}\right), 130.0,129.1,128.2,127.7,126.8$ (q, $\left.J_{\mathrm{C}-\mathrm{F}}=3.9 \mathrm{~Hz}\right), 123.6$ $\left(\mathrm{q}, J_{\mathrm{C}-\mathrm{F}}=274 \mathrm{~Hz}\right) 75.2,58.7,29.5,28.8 ;{ }^{19} \mathrm{~F}$ NMR $\left(376 \mathrm{MHz}, \mathrm{CDCl}_{3}\right) \delta-62.8$; HRMS (ESI) $\mathrm{m} / \mathrm{z}$ calcd for $\mathrm{C}_{21} \mathrm{H}_{17} \mathrm{~F}_{3} \mathrm{NO}_{2}[\mathrm{M}+\mathrm{H}]^{+}: 372.1205$ found 372.1207.

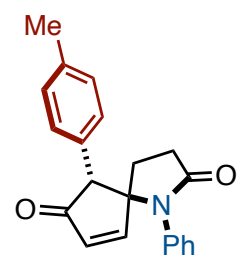

$\left(5 S^{*}, 6 R^{*}\right)$-1-Phenyl-6-( $p$-tolyl)-1-azaspiro[4.4] non-8-ene-2,7-dione (9Ab)

Purification by PTLC (hexane/EtOAc = 1:1) afforded 9Ab (17.5 mg, $57.0 \mu \mathrm{mol}$ scale, 97\% yield) as a pale yellow solid. ${ }^{1} \mathrm{H} \mathrm{NMR}\left(400 \mathrm{MHz}, \mathrm{CDCl}_{3}\right) \delta 7.72(\mathrm{~d}, J=6.0 \mathrm{~Hz}, 1 \mathrm{H}), 7.48(\mathrm{t}, J=7.6 \mathrm{~Hz}, 2 \mathrm{H})$, $7.39(\mathrm{t}, J=7.6 \mathrm{~Hz}, 1 \mathrm{H}), 7.33-7.27(\mathrm{~m}, 2 \mathrm{H}), 7.20(\mathrm{~d}, J=7.6 \mathrm{~Hz}, 2 \mathrm{H}), 7.00(\mathrm{~d}, J=7.6 \mathrm{~Hz}, 2 \mathrm{H}), 6.35(\mathrm{~d}$, $J=6.0 \mathrm{~Hz}, 1 \mathrm{H}), 3.72(\mathrm{~s}, 1 \mathrm{H}), 2.39-2.28(\mathrm{~m}, 4 \mathrm{H}), 2.13-1.97(\mathrm{~m}, 2 \mathrm{H}), 1.94-1.84(\mathrm{~m}, 1 \mathrm{H}) ;{ }^{13} \mathrm{C} \mathrm{NMR}(101$ $\left.\mathrm{MHz}, \mathrm{CDCl}_{3}\right) \delta 204.2,174.6,163.1,137.8,135.2,134.0,130.9,129.9,129.7,128.5,128.1,75.2,58.2$, 29.6, 28.6, 21.1 (one peak is missing due to overlapping); HRMS (ESI) $m / z$ calcd for $\mathrm{C}_{21} \mathrm{H}_{20} \mathrm{NO}_{2}$ $[\mathrm{M}+\mathrm{H}]^{+}: 318.1489$ found 318.1488 .

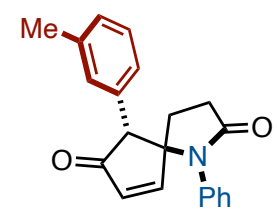

$\left(5 S^{*}, 6 R^{*}\right)-1-P h e n y l-6-(m-t o l y l)-1-a z a s p i r o[4.4]$ non-8-ene-2,7-dione (9Ac) 
Purification by PTLC (hexane/EtOAc $=1: 1)$ afforded 9Ac $(15.6 \mathrm{mg}, 49.2 \mu \mathrm{mol}$ scale, quant.) as a pale yellow solid. ${ }^{1} \mathrm{H}$ NMR $\left(400 \mathrm{MHz}, \mathrm{CDCl}_{3}\right) \delta 7.73(\mathrm{~d}, J=5.6 \mathrm{~Hz}, 1 \mathrm{H}), 7.48(\mathrm{t}, J=7.6 \mathrm{~Hz}, 2 \mathrm{H}), 7.39$ (t, $J=7.6 \mathrm{~Hz}, 1 \mathrm{H}), 7.33-7.24(\mathrm{~m}, 3 \mathrm{H}), 7.16(\mathrm{~d}, J=7.6 \mathrm{~Hz}, 1 \mathrm{H}), 6.93-6.89(\mathrm{~m}, 2 \mathrm{H}), 6.36$ (d, $J=5.6 \mathrm{~Hz}$, $1 \mathrm{H}), 3.72(\mathrm{~s}, 1 \mathrm{H}), 2.40-2.29(\mathrm{~m}, 4 \mathrm{H}), 2.13-1.98(\mathrm{~m}, 2 \mathrm{H}), 1.96-1.86(\mathrm{~m}, 1 \mathrm{H}) ;{ }^{13} \mathrm{C}$ NMR $(101 \mathrm{MHz}$, $\left.\mathrm{CDCl}_{3}\right) \delta 204.3,174.6,163.3,138.6,135.2,134.1,134.0,130.6,129.7,128.82,128.80,128.5,128.2$, 127.2, 75.2, 58.5, 29.6, 28.6, 21.5; HRMS (ESI) $m / z$ calcd for $\mathrm{C}_{21} \mathrm{H}_{20} \mathrm{NO}_{2}[\mathrm{M}+\mathrm{H}]^{+}: 318.1489$ found 318.1488 .

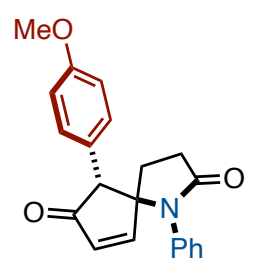

$\left(5 S^{*}, 6 R^{*}\right)-6-(4-M e t h o x y p h e n y l)-1-p h e n y l-1-a z a s p i r o[4.4]$ non-8-ene-2,7-dione (9Ae)

Purification by PTLC (hexane/EtOAc = 1:1) afforded 9Ae (12.8 mg, $42.3 \mu \mathrm{mol}$ scale, 91\% yield) as a pale yellow solid. ${ }^{1} \mathrm{H}$ NMR $\left(400 \mathrm{MHz}, \mathrm{CDCl}_{3}\right) \delta 7.72(\mathrm{~d}, J=5.6 \mathrm{~Hz}, 1 \mathrm{H}), 7.48(\mathrm{t}, J=7.6 \mathrm{~Hz}, 2 \mathrm{H})$, 7.39 (t, $J=7.6 \mathrm{~Hz}, 1 \mathrm{H}), 7.33-7.28(\mathrm{~m}, 2 \mathrm{H}), 7.04$ (d, $J=7.2 \mathrm{~Hz}, 2 \mathrm{H}), 6.93$ (d, $J=7.2 \mathrm{~Hz}, 2 \mathrm{H}), 6.35$ (d, $J=5.6 \mathrm{~Hz}, 1 \mathrm{H}), 3.82(\mathrm{~s}, 3 \mathrm{H}), 3.71(\mathrm{~s}, 1 \mathrm{H}), 2.39-2.29(\mathrm{~m}, 1 \mathrm{H}), 2.14-1.96(\mathrm{~m}, 2 \mathrm{H}), 1.95-1.84(\mathrm{~m}, 1 \mathrm{H})$; ${ }^{13} \mathrm{C}$ NMR $\left(101 \mathrm{MHz}, \mathrm{CDCl}_{3}\right) \delta$ 204.2, 174.6, 163.0, 159.3, 135.2, 134.0, 131.1, 129.7, 128.5, 128.1, 125.8, 114.4, 75.2, 57.9, 55.3, 29.6, 28.6; HRMS (ESI) $\mathrm{m} / z$ calcd for $\mathrm{C}_{21} \mathrm{H}_{20} \mathrm{NO}_{3}[\mathrm{M}+\mathrm{H}]^{+}: 334.1437$ found 334.1436 .

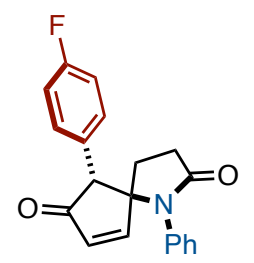

$\left(5 S^{*}, 6 R^{*}\right)-6-(4-F l u o r o p h e n y l)-1-p h e n y l-1-a z a s p i r o[4.4]$ non-8-ene-2,7-dione (9Af)

Purification by PTLC (hexane/EtOAc $=1: 1)$ afforded 9Af (7.4 mg, $24.9 \mu \mathrm{mol} \mathrm{scale,} \mathrm{93 \%} \mathrm{yield)} \mathrm{as}$ a pale yellow solid. ${ }^{1} \mathrm{H}$ NMR $\left(400 \mathrm{MHz}, \mathrm{CDCl}_{3}\right) \delta 7.75(\mathrm{~d}, J=6.0 \mathrm{~Hz}, 1 \mathrm{H}), 7.49(\mathrm{t}, J=7.2 \mathrm{~Hz}, 2 \mathrm{H})$, $7.40(\mathrm{t}, J=7.2 \mathrm{~Hz}, 1 \mathrm{H}), 7.32-7.28(\mathrm{~m}, 2 \mathrm{H}), 7.13-7.08(\mathrm{~m}, 4 \mathrm{H}), 6.37(\mathrm{~d}, J=6.0 \mathrm{~Hz}, 1 \mathrm{H}), 3.75(\mathrm{~s}, 1 \mathrm{H})$, 2.42-2.33 (m, 1H), 2.16-2.05 (m, 1H), 2.00-1.85 (m, 2H); $\left.{ }^{13} \mathrm{C} \mathrm{NMR} \mathrm{(101} \mathrm{MHz,} \mathrm{CDCl}_{3}\right) \delta$ 203.6, 174.4, $163.2,162.4\left(\mathrm{~d}, J_{\mathrm{C}-\mathrm{F}}=249 \mathrm{~Hz}\right), 135.1,133.9,131.7\left(\mathrm{~d}, J_{\mathrm{C}-\mathrm{F}}=7.7 \mathrm{~Hz}\right), 129.8,128.6,127.9,116.1\left(\mathrm{~d}, J_{\mathrm{C}-}\right.$ ${ }_{\mathrm{F}}=22.3 \mathrm{~Hz}$ ), 75.0, 57.8, 29.5, 28.5 (one peak is missing due to overlapping); ${ }^{19} \mathrm{~F}$ NMR (376 MHz, $\left.\mathrm{CDCl}_{3}\right) \delta-113.4$; HRMS (ESI) $\mathrm{m} / z$ calcd for $\mathrm{C}_{20} \mathrm{H}_{17} \mathrm{FNO}_{2}[\mathrm{M}+\mathrm{H}]^{+}: 322.1238$ found 322.1239. 


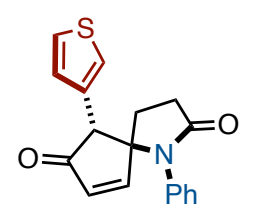

$\left(5 S^{*}, 6 R^{*}\right)-1-P h e n y l-6-(t h i o p h e n-3-y l)-1-a z a s p i r o[4.4]$ non-8-ene-2,7-dione (9Ah)

Purification by PTLC (hexane/EtOAc = 1:1) afforded 9Ah (18.2 mg, $58.8 \mu \mathrm{mol} \mathrm{scale}$, quant.) as a pale yellow solid. ${ }^{1} \mathrm{H}$ NMR (400 MHz, $\left.\mathrm{CDCl}_{3}\right) \delta 7.72(\mathrm{~d}, J=6.0 \mathrm{~Hz}, 1 \mathrm{H}), 7.48(\mathrm{t}, J=8.0 \mathrm{~Hz}, 2 \mathrm{H}), 7.44$ $7.37(\mathrm{~m}, 2 \mathrm{H}), 7.32-7.27(\mathrm{~m}, 2 \mathrm{H}), 7.20(\mathrm{dd}, J=2.8,1.2 \mathrm{~Hz}, 1 \mathrm{H}), 6.94(\mathrm{dd}, J=5.2,1.2 \mathrm{~Hz}, 1 \mathrm{H}), 6.33(\mathrm{~d}$, $J=6.0 \mathrm{~Hz}, 1 \mathrm{H}), 3.87$ (s, 1H), 2.45-2.33 (m, 1H), 2.16-2.06 (m, 1H), 2.06-1.93 (m, 2H); ${ }^{13} \mathrm{C}$ NMR (101 $\left.\mathrm{MHz}, \mathrm{CDCl}_{3}\right) \delta 203.0,174.6,162.7,135.1,133.5,133.2,129.7,128.5,128.0,127.8,126.7,125.0,74.8$, 54.2, 29.6, 28.6; HRMS (ESI) $m / z$ calcd for $\mathrm{C}_{18} \mathrm{H}_{16} \mathrm{NO}_{2} \mathrm{~S}[\mathrm{M}+\mathrm{H}]^{+}: 310.0896$ found 310.0895 .

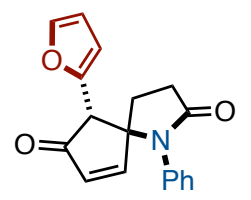

$\left(5 S^{*}, 6 S^{*}\right)-6-($ Furan-2-yl)-1-phenyl-1-azaspiro[4.4]non-8-ene-2,7-dione (9Ai)

Purification by PTLC (hexane/EtOAc = 1:1) afforded 9Ai (10.0 mg, $56.9 \mu \mathrm{mol}$ scale, $60 \%$ yield) as a brown solid. ${ }^{1} \mathrm{H}$ NMR $\left(400 \mathrm{MHz}, \mathrm{CDCl}_{3}\right) \delta 7.66(\mathrm{~d}, J=6.0 \mathrm{~Hz}, 1 \mathrm{H}), 7.50-7.44(\mathrm{~m}, 3 \mathrm{H}), 7.40(\mathrm{t}, J$ $=7.2 \mathrm{~Hz}, 1 \mathrm{H}), 7.32-7.27(\mathrm{~m}, 2 \mathrm{H}), 6.45(\mathrm{dd}, J=3.2,2.0 \mathrm{~Hz}, 1 \mathrm{H}), 6.38(\mathrm{~d}, J=3.2 \mathrm{~Hz}, 1 \mathrm{H}), 6.27(\mathrm{~d}, J=$ $6.0 \mathrm{~Hz}, 1 \mathrm{H}), 3.86(\mathrm{~s}, 1 \mathrm{H}), 2.48-2.39(\mathrm{~m}, 1 \mathrm{H}), 2.22-2.11(\mathrm{~m}, 1 \mathrm{H}), 2.10-1.94(\mathrm{~m}, 2 \mathrm{H}) ;{ }^{13} \mathrm{C}$ NMR $(101$ $\left.\mathrm{MHz}, \mathrm{CDCl}_{3}\right) \delta 200.2,174.9,162.4,147.8,142.9,135.1,133.5,129.7,128.8,111.0,110.4,74.5,52.6$, 29.7, 28.7 (one peak is missing due to overlapping); HRMS (ESI) $m / z$ calcd for $\mathrm{C}_{18} \mathrm{H}_{16} \mathrm{NO}_{3}[\mathrm{M}+\mathrm{H}]^{+}$: 294.1125 found 294.1123.

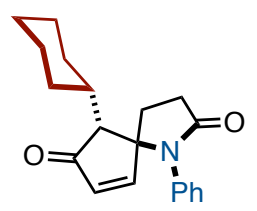

$\left(5 S^{*}, 6 R^{*}\right)-6-C y c l o h e x y l-1-p h e n y l-1-a z a s p i r o[4.4]$ non-8-ene-2,7-dione (9Ak)

Purification by PTLC (hexane/EtOAc = 1:1) afforded 9Ak (2.3 mg, $23.9 \mu \mathrm{mol} \mathrm{scale,} \mathrm{31 \%} \mathrm{yield)}$ as a pale yellow solid ${ }^{1} \mathrm{H}$ NMR $\left(400 \mathrm{MHz}, \mathrm{CDCl}_{3}\right) \delta 7.61(\mathrm{~d}, J=6.0 \mathrm{~Hz}, 1 \mathrm{H}), 7.41-7.30(\mathrm{~m}, 3 \mathrm{H}), 7.04$ (d, $J=7.2 \mathrm{~Hz}, 2 \mathrm{H}), 6.14$ (d, $J=6.0 \mathrm{~Hz}, 1 \mathrm{H}), 2.82-2.54(\mathrm{~m}, 3 \mathrm{H}), 2.25$ (d, $J=3.2 \mathrm{~Hz}, 1 \mathrm{H}), 2.20-2.12$ (m, $1 \mathrm{H}), 1.82-1.13(\mathrm{~m}, 11 \mathrm{H}) ;{ }^{13} \mathrm{C} \mathrm{NMR}\left(101 \mathrm{MHz}, \mathrm{CDCl}_{3}\right) \delta 205.8,174.2,162.9,135.2,134.2,129.5,128.5$, 128.4 , 74.4, 56.1, 37.2, 32.5, 30.1, 29.1, 27.4, 27.1, 26.5, 25.9; HRMS (ESI) $m / z$ calcd for $\mathrm{C}_{20} \mathrm{H}_{24} \mathrm{NO}_{2}$ $[\mathrm{M}+\mathrm{H}]^{+}: 310.1802$ found 310.1799 . 


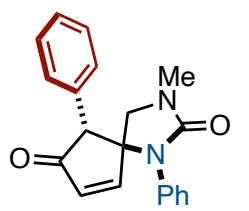

$\left(5 S^{*}, 6 R^{*}\right)-3-M e t h y l-1,6-d i p h e n y l-1,3-d i a z a s p i r o[4.4]$ non-8-ene-2,7-dione (9Ea)

Purification by PTLC (hexane/EtOAc = 1:2) afforded 9Ea (10.9 mg, $51.2 \mu \mathrm{mol}$ scale, 67\% yield) as a white solid. ${ }^{1} \mathrm{H}$ NMR $\left(400 \mathrm{MHz}, \mathrm{CDCl}_{3}\right) \delta 7.81(\mathrm{~d}, J=6.0 \mathrm{~Hz}, 1 \mathrm{H}), 7.45-7.27(\mathrm{~m}, 8 \mathrm{H}), 7.06(\mathrm{~d}, J$ $=7.2 \mathrm{~Hz}, 2 \mathrm{H}), 6.43(\mathrm{~d}, J=6.0 \mathrm{~Hz}, 1 \mathrm{H}), 3.82(\mathrm{~s}, 1 \mathrm{H}), 3.26(\mathrm{~d}, J=5.6 \mathrm{~Hz}, 1 \mathrm{H}), 3.05(\mathrm{~d}, J=5.6 \mathrm{~Hz}, 1 \mathrm{H})$, 2.55 (s, 3H); ${ }^{13} \mathrm{C}$ NMR $\left(101 \mathrm{MHz}, \mathrm{CDCl}_{3}\right) \delta 204.4,162.2,158.2,136.0,135.1,134.1,129.7,129.4$, $128.8,127.9,127.3,70.8,57.3,51.9,30.5$ (one peak is missing due to overlapping); HRMS (ESI) $\mathrm{m} / z$ calcd for $\mathrm{C}_{20} \mathrm{H}_{19} \mathrm{~N}_{2} \mathrm{O}_{2}[\mathrm{M}+\mathrm{H}]^{+}: 319.1441$ found 319.1439 . 
5. One-Pot Synthesis of (Z)-2-Benzylidene-6-phenyl-1-thia-6-azaspiro[4.4]non-3-en-7-one (3Ha)

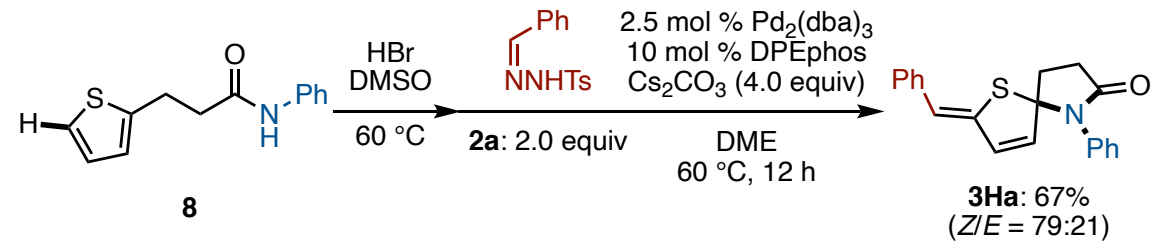

Following Jiao's procedure, ${ }^{[11]}$ this bromination was performed. To an $8-\mathrm{mL}$ glass tube equipped with a screw cap containing a magnetic stirring bar were added $N$-phenyl-3-(thiophen-2-yl)propanamide (8: $23.1 \mathrm{mg}, 0.10 \mathrm{mmol}, 1.0$ equiv), EtOAc $(0.40 \mathrm{~mL})$. To this solution were then added DMSO (7.8 $\mu \mathrm{L}$, $0.11 \mathrm{mmol}, 1.1$ equiv) and $\mathrm{HBr}$ aq. (10.0 M, $12.0 \mu \mathrm{L}, 0.12 \mathrm{mmol}, 1.2$ equiv) at room temperature. The tube was sealed with a screw cap. After stirring the mixture at $60{ }^{\circ} \mathrm{C}$ for $2 \mathrm{~h}$, the mixture was concentrated in vacuo and filled $\mathrm{N}_{2}$. To the same tube were added $\mathrm{Cs}_{2} \mathrm{CO}_{3}(135.5 \mathrm{mg}, 0.40 \mathrm{mmol}, 4.0$ equiv), $\mathrm{Pd}_{2}(\mathrm{dba})_{3} \cdot \mathrm{CHCl}_{3}(2.60 \mathrm{mg}, 2.5 \mu \mathrm{mol}, 2.5 \mathrm{~mol} \%$ ), DPEphos (5.40 mg, $10.0 \mu \mathrm{mol}, 10 \mathrm{~mol} \%$ ), and $2 \mathrm{a}$ (55.0 $\mathrm{mg}, 0.20 \mathrm{mmol}, 2.0$ equiv). The tube was placed under vacuum and refilled $\mathrm{N}_{2}$ gas three times. To this mixture was added DME $(0.50 \mathrm{~mL})$. The tube was sealed with a screw cap. After stirring the mixture at $60{ }^{\circ} \mathrm{C}$ for $12 \mathrm{~h}$ in a 16 -well aluminum reaction block, the mixture was passed through a pad of Celite ${ }^{\circledR}$ with EtOAc as an eluent. The filtrate was concentrated in vacuo and the residue was purified by PTLC (hexane/EtOAc $=7: 3)$ afforded $3 \mathbf{H a}(21.4 \mathrm{mg}, 67 \%$ yield, as a mixture of diasteroisomers; $Z / E=79: 21$ ) as a pale yellow solid.

\section{Sequential Transformation from 1}

Synthesis of $\left(5 S^{*}, 6 R^{*}\right)$-1,6-diphenyl-1-azaspiro[4.4]non-8-ene-2,7-dione (9Aa)

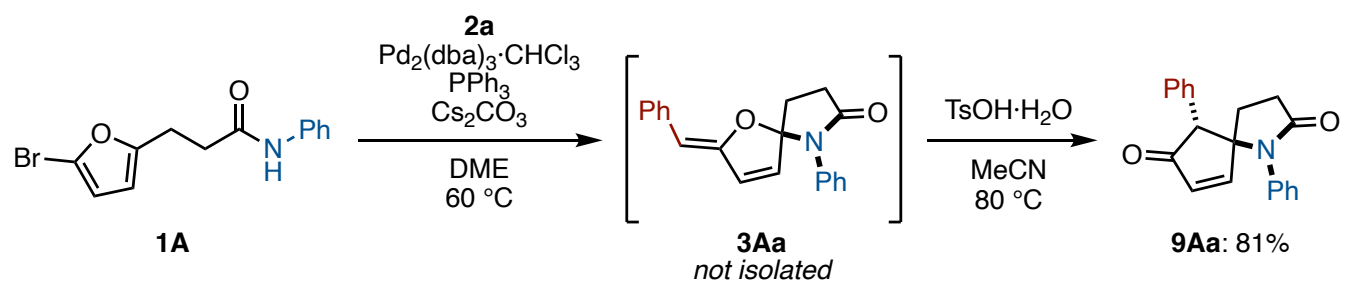

An 8-mL glass tube equipped with a screw cap containing a magnetic stirring bar and $\mathrm{Cs}_{2} \mathrm{CO}_{3}$ (524.3 mg, $1.6 \mathrm{mmol}, 4.0$ equiv) was dried with a heat-gun in vacuo and filled $\mathrm{N}_{2}$ after cooling to room temperature. To this tube were added $\mathrm{Pd}_{2}(\mathrm{dba})_{3} \cdot \mathrm{CHCl}_{3}(10.3 \mathrm{mg}, 10 \mu \mathrm{mol}, 2.5 \mathrm{~mol} \%), \mathrm{PPh}_{3}(21.0 \mathrm{mg}$, $80 \mu \mathrm{mol}, 20 \mathrm{~mol} \%)$, bromofuran $\mathbf{1 A}(117.7 \mathrm{mg}, 0.40 \mathrm{mmol}, 1.0$ equiv) and 2a (219.5 mg, $0.80 \mathrm{mmol}$, 2.0 equiv). The tube was placed under vacuum and refilled $\mathrm{N}_{2}$ gas three times. To this tube was added DME $(2.0 \mathrm{~mL})$. The tube was sealed with a screw cap. After stirring the mixture at $60^{\circ} \mathrm{C}$ for $12 \mathrm{~h}$, the mixture was passed through a pad of Celite ${ }^{\circledR}$ pad with EtOAc as an eluent. The filtrate was concentrated in vacuo. To the mixture were added $p$-toluenesulfonic acid monohydrate $\left(\mathrm{TsOH} \cdot \mathrm{H}_{2} \mathrm{O}: 13.0 \mathrm{mg}, 0.068\right.$ $\mathrm{mmol}, 17 \mathrm{~mol} \%$ ), and $\mathrm{MeCN}(1.6 \mathrm{~mL})$. After stirring the mixture at $80^{\circ} \mathrm{C}$ for $30 \mathrm{~min}$, the reaction was quenched with $\mathrm{NaHCO}_{3}$ aq. The mixture was extracted three times with EtOAc. The combined organic 
layer was dried over $\mathrm{Na}_{2} \mathrm{SO}_{4}$, filtrated, and concentrated in vacuo. The residue was purified by Isolera ${ }^{\circledR}$ (hexane/EtOAc $=4: 1$ to EtOAc) to afford 9Aa $(98.3 \mathrm{mg}, 81 \%$ yield) as a pale yellow solid.

\section{Synthesis of $\left(4 R^{*}, 5 S^{*}, 6 R^{*}\right)-4-m e t h y l-1,6-d i p h e n y l-1-a z a s p i r o[4.4]$ non-8-ene-2,7-dione (9Sa)}
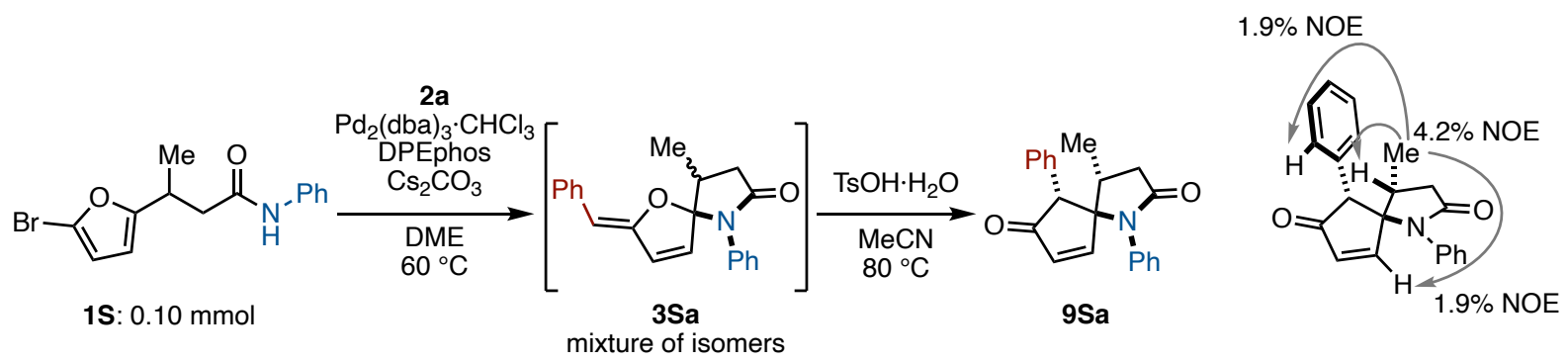

An 8-mL glass tube equipped with a screw cap containing a magnetic stirring bar and $\mathrm{Cs}_{2} \mathrm{CO}_{3}$ (133.8 mg, $0.40 \mathrm{mmol}, 4.0$ equiv) was dried with a heat-gun in vacuo and filled $\mathrm{N}_{2}$ after cooling to room temperature. To this tube were added $\mathrm{Pd}_{2}(\mathrm{dba})_{3} \cdot \mathrm{CHCl}_{3}(2.6 \mathrm{mg}, 2.5 \mu \mathrm{mol}, 2.5 \mathrm{~mol} \%$ ), DPEphos (5.4 $\mathrm{mg}, 10 \mu \mathrm{mol}, 10 \mathrm{~mol} \%$ ), bromofuran $1 \mathrm{~S}$ (31.4 mg, $0.10 \mathrm{mmol}, 1.0$ equiv), and $2 \mathbf{a}$ (54.0 mg, $0.20 \mathrm{mmol}$, 2.0 equiv). The tube was placed under vacuum and refilled $\mathrm{N}_{2}$ gas three times. To this tube was added DME $(0.50 \mathrm{~mL})$. The tube was sealed with a screw cap. After stirring the mixture at $60{ }^{\circ} \mathrm{C}$ for $12 \mathrm{~h}$, the mixture was passed through a pad of Celite ${ }^{\circledR}$ with EtOAc as an eluent. The filtrate was concentrated in vacuo. The residue was purified by PTLC to afford the corresponding azaspirocyclic product 3Sa as a mixture of isomers (37:29:19:15). To an 8-mL glass tube were added 3Sa (1.0 equiv), $\mathrm{TsOH} \cdot \mathrm{H}_{2} \mathrm{O}(4.0$ $\mathrm{mg}, 20 \mu \mathrm{mol}, 20 \mathrm{~mol} \%)$, and $\mathrm{MeCN}(0.40 \mathrm{~mL})$. The tube was sealed with a screw cap. After stirring the mixture at $80^{\circ} \mathrm{C}$ for $13 \mathrm{~h}$, the reaction was quenched with $\mathrm{NaHCO}_{3}$ aq. The mixture was extracted three times with EtOAc. The combined organic layer was dried over $\mathrm{Na}_{2} \mathrm{SO}_{4}$, filtrated, and concentrated in vacuo. The residue was purified by PTLC $\left(\mathrm{CHCl}_{3} / \mathrm{MeOH}=29: 1\right.$ and then $\left.\mathrm{CHCl}_{3} / \mathrm{EtOAc}=7: 1\right)$ to afford $9 \mathrm{Sa}\left(21.6 \mathrm{mg}, 68 \%\right.$ yield) as a pale yellow solid. ${ }^{1} \mathrm{H}$ NMR $\left(400 \mathrm{MHz}, \mathrm{CDCl}_{3}\right) \delta 7.74(\mathrm{~d}, J=6.0$ $\mathrm{Hz}, 1 \mathrm{H}), 7.48$ (t, $J=7.6 \mathrm{~Hz}, 2 \mathrm{H}), 7.43-7.24(\mathrm{~m}, 6 \mathrm{H}), 7.06(\mathrm{~d}, J=6.8 \mathrm{~Hz}, 2 \mathrm{H}), 6.53(\mathrm{~d}, J=6.0 \mathrm{~Hz}, 1 \mathrm{H})$, $3.83(\mathrm{~s}, 1 \mathrm{H}), 2.44-2.31(\mathrm{~m}, 1 \mathrm{H}), 2.27-2.12(\mathrm{~m}, 2 \mathrm{H}), 0.94(\mathrm{~d}, J=6.4 \mathrm{~Hz}, 3 \mathrm{H}) ;{ }^{13} \mathrm{C}$ NMR $(101 \mathrm{MHz}$, $\left.\mathrm{CDCl}_{3}\right) \delta 203.3,173.9,161.3,135.6,135.4,133.5,130.0,129.7,128.9,128.4,127.9,127.5,78.4,58.4$, 38.2, 34.4, 16.9; HRMS (ESI) $m / z$ calcd for $\mathrm{C}_{21} \mathrm{H}_{20} \mathrm{NO}_{2}[\mathrm{M}+\mathrm{H}]^{+}: 318.1489$ found 318.1487.

\section{Synthesis of $\left(5 S^{*}, 6 R^{*}\right)-1,6$-diphenyl-1-azaspiro[4.4]non-8-en-7-one (9Ta)}

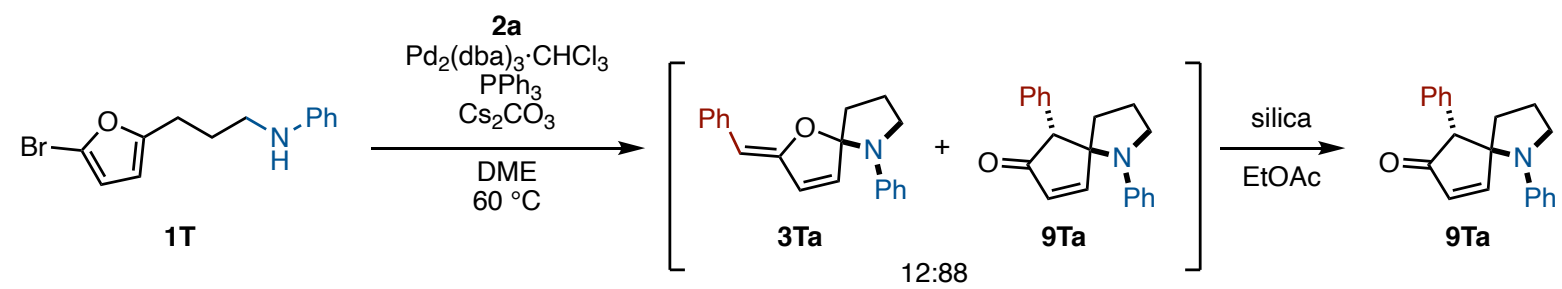

An 8-mL glass tube equipped with a screw cap containing a magnetic stirring bar and $\mathrm{Cs}_{2} \mathrm{CO}_{3}$ (134.0 mg, $0.41 \mathrm{mmol}, 4.1$ equiv) was dried with a heat-gun in vacuo and filled $\mathrm{N}_{2}$ after cooling to room 
temperature. To this tube were added $\mathrm{Pd}_{2}(\mathrm{dba})_{3} \cdot \mathrm{CHCl}_{3}(2.6 \mathrm{mg}, 2.5 \mu \mathrm{mol}, 2.5 \mathrm{~mol} \%), \mathrm{PPh}_{3}:(5.3 \mathrm{mg}$, $20 \mu \mathrm{mol}, 20 \mathrm{~mol} \%$ ), and $2 \mathrm{a}$ ( $55.5 \mathrm{mg}, 0.20 \mathrm{mmol}, 2.0$ equiv). The tube was placed under vacuum and refilled $\mathrm{N}_{2}$ gas three times. To this tube was added a solution of bromofuran $1 \mathrm{~T}(28.0 \mathrm{mg}, 0.10 \mathrm{mmol}$, 1.0 equiv) in DME $(0.50 \mathrm{~mL})$. The tube was sealed with a screw cap. After stirring the mixture at $60^{\circ} \mathrm{C}$ for $12 \mathrm{~h}$, the mixture was passed through a pad of Celite ${ }^{\circledR}$ with EtOAc as an eluent. The filtrate was concentrated in vacuo (3Ta and 9Ta were generated in a ratio of 12:88, judged by ${ }^{1} \mathrm{H}$ NMR analysis). This crude mixture was treated with silica-gel in EtOAc $(3.0 \mathrm{~mL})$ for $30 \mathrm{~min}$. After removing the solvent in vacuo, the residue was purified by Isolera ${ }^{\circledR}$ (hexane/EtOAc $=15: 1$ to $\left.3: 1\right)$ to afford 9Ta $(23.1 \mathrm{mg}$, $80 \%$ yield over 2 steps) as a white solid. ${ }^{1} \mathrm{H}$ NMR $\left(400 \mathrm{MHz}, \mathrm{CDCl}_{3}\right) \delta 7.76(\mathrm{~d}, J=6.0 \mathrm{~Hz}, 1 \mathrm{H}), 7.35-$ $7.21(\mathrm{~m}, 5 \mathrm{H}), 7.10-7.05(\mathrm{~m}, 2 \mathrm{H}), 6.82-6.75(\mathrm{~m}, 3 \mathrm{H}), 6.41(\mathrm{~d}, J=6.0 \mathrm{~Hz}, 1 \mathrm{H}), 4.63(\mathrm{~s}, 1 \mathrm{H}), 3.43-3.33$ (m, 2H), 2.09-1.97 (m, 1H), 1.90-1.81 (m, 1H), 1.67-1.55 (m, 1H), 0.94-0.80 (m, 1H); ${ }^{13} \mathrm{C} \mathrm{NMR}(101$ $\left.\mathrm{MHz}_{2} \mathrm{CDCl}_{3}\right) \delta 204.4,169.6,145.3,135.1,131.9,130.4,129.5,128.4,127.3,117.2,112.4,74.0,58.2$, 49.8, 38.3, 21.4; HRMS (ESI) $m / z$ calcd for $\mathrm{C}_{20} \mathrm{H}_{20} \mathrm{NO}[\mathrm{M}+\mathrm{H}]^{+}: 290.1539$ found 290.1538.

\section{Derivatization of 9}

Synthesis of $\left(5 S^{*}, 6 S^{*}\right)$-6-allyl-1,6-diphenyl-1-azaspiro[4.4]non-8-ene-2,7-dione (10)

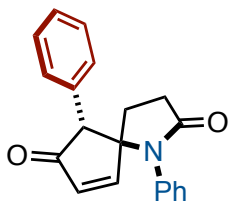

9Aa

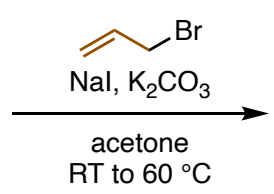

$\mathrm{RT}$ to $60^{\circ} \mathrm{C}$

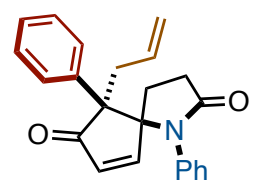

10

To an 8 -mL glass tube with a magnetic stirring bar were added 9Aa $(20.5 \mathrm{mg}, 66 \mu \mathrm{mol})$, acetone (0.50 mL), $\mathrm{K}_{2} \mathrm{CO}_{3}(45.2 \mathrm{mg}, 0.33 \mathrm{mmol}, 5.0$ equiv), $\mathrm{NaI}(13.1 \mathrm{mg}, 87 \mu \mathrm{mol}, 1.3$ equiv), and allyl bromide (17 $\mu \mathrm{L}, 0.20 \mathrm{mmol}, 3.0$ equiv). After stirring the mixture at room temperature for $6.5 \mathrm{~h}$, to the mixture were further added $\mathrm{K}_{2} \mathrm{CO}_{3}(42.0 \mathrm{mg}, 0.30 \mathrm{mmol}, 4.5$ equiv), $\mathrm{NaI}$ (12.2 mg, $81 \mu \mathrm{mol}, 1.2$ equiv) and allyl bromide ( $17 \mu \mathrm{L}, 0.20 \mathrm{mmol}, 3.0$ equiv). After stirring the mixture for $15 \mathrm{~h}$ at $60{ }^{\circ} \mathrm{C}$, the reaction was quenched with $\mathrm{H}_{2} \mathrm{O}$. The mixture was extracted three times with EtOAc. Combined organic layer was dried over $\mathrm{Na}_{2} \mathrm{SO}_{4}$, filtrated, and concentrated in vacuo. The residue was purified by PTLC (hexane/EtOAc $=1: 1)$ to afford $10(12.9 \mathrm{mg}, 57 \%$ yield $)$ as a brown solid. ${ }^{1} \mathrm{H}$ NMR $\left(400 \mathrm{MHz}, \mathrm{CDCl}_{3}\right)$ $\delta 7.62(\mathrm{~d}, J=6.0 \mathrm{~Hz}, 1 \mathrm{H}), 7.22-7.15(\mathrm{~m}, 1 \mathrm{H}), 7.12(\mathrm{t}, J=7.2 \mathrm{~Hz}, 2 \mathrm{H}), 7.10-7.05(\mathrm{~m}, 2 \mathrm{H}), 7.02(\mathrm{t}, J=$ $7.2 \mathrm{~Hz}, 1 \mathrm{H}), 6.92(\mathrm{t}, J=7.2 \mathrm{~Hz}, 2 \mathrm{H}), 6.39(\mathrm{~d}, J=6.0 \mathrm{~Hz}, 1 \mathrm{H}), 6.12(\mathrm{~d}, J=7.6 \mathrm{~Hz}, 2 \mathrm{H}), 5.48-5.34(\mathrm{~m}$, $1 \mathrm{H}), 4.96-4.84(\mathrm{~m}, 2 \mathrm{H}), 3.10-2.97(\mathrm{~m}, 1 \mathrm{H}), 2.96-2.70(\mathrm{~m}, 3 \mathrm{H}), 2.58-2.44(\mathrm{~m}, 2 \mathrm{H}) ;{ }^{13} \mathrm{C} \mathrm{NMR}(101 \mathrm{MHz}$, $\left.\mathrm{CDCl}_{3}\right) \delta 206.2,174.4,160.2,137.7,135.6,133.9,132.7,128.6,128.2,128.0,127.8,126.91,126.86$, 118.6, 78.0, 59.5, 45.6, 29.3, 27.4; HRMS (ESI) $m / z$ calcd for $\mathrm{C}_{23} \mathrm{H}_{22} \mathrm{NO}_{2}[\mathrm{M}+\mathrm{H}]^{+}: 344.1645$ found 344.1646.

Synthesis of $\left(5 S^{*}, 8 S^{*}, 9 R^{*}\right)-8$-hydroxy-8-methyl-1,9-diphenyl-1-azaspiro[4.4]non-6-en-2-one (11) 


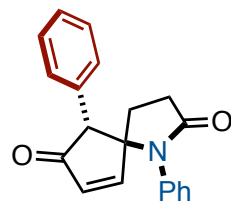

9Aa

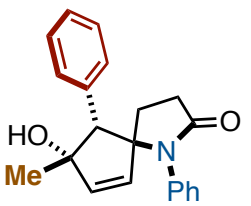

11

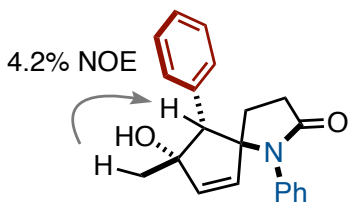

An 8-mL glass tube with a magnetic stirring bar was dried up by a heat-gun in vacuo and filled $\mathrm{N}_{2}$ after cooling to room temperature. To this tube were added 9Aa $(30.3 \mathrm{mg}, 0.10 \mathrm{mmol})$ and THF (0.70 $\mathrm{mL})$ under the stream of $\mathrm{N}_{2}$ gas. To the solution was added $\mathrm{MeMgBr}(1.0 \mathrm{M}$ in THF, $0.15 \mathrm{~mL}, 0.15$ mmol, 1.5 equiv) dropwise at $-78{ }^{\circ} \mathrm{C}$. After stirring the mixture at $-78{ }^{\circ} \mathrm{C}$ for $3 \mathrm{~h}$, the reaction was quenched with $\mathrm{NH}_{4} \mathrm{Cl}$ aq. The mixture was extracted three times with EtOAc. Combined organic layer was dried over $\mathrm{Na}_{2} \mathrm{SO}_{4}$, filtrated, and concentrated in vacuo. The mixture was purified by PTLC $\left(\mathrm{CHCl}_{3} / \mathrm{MeOH}=9: 1\right)$ to afford $11(23.4 \mathrm{mg}, 73 \%$ yield, $\mathrm{dr}=98: 2)$ as a brown solid. ${ }^{1} \mathrm{H} \mathrm{NMR}(400 \mathrm{MHz}$, $\left.\mathrm{CDCl}_{3}\right) \delta 7.49-7.42(\mathrm{~m}, 4 \mathrm{H}), 7.40-7.32(\mathrm{~m}, 4 \mathrm{H}), 7.27-7.23(\mathrm{~m}, 3 \mathrm{H}), 6.26(\mathrm{~d}, J=6.0 \mathrm{~Hz}, 1 \mathrm{H}), 5.97(\mathrm{~d}$, $J=6.0 \mathrm{~Hz}, 1 \mathrm{H}), 2.95(\mathrm{~s}, 1 \mathrm{H}), 2.68-2.59$ (m, 1H), 2.32-2.13 (m, 2H), 1.91-1.80 (m, 1H), 1.55-1.49 (brs, $1 \mathrm{H}), 1.14(\mathrm{~s}, 3 \mathrm{H}) ;{ }^{13} \mathrm{C} \mathrm{NMR}\left(101 \mathrm{MHz}, \mathrm{CDCl}_{3}\right) \delta 175.3,139.9,137.8,136.2,135.2,131.6,129.1,128.6$, 128.5, 127.7, 127.6, 81.3, 79.9, 58.6, 30.2, 29.7, 27.4; HRMS (ESI) $m / z$ calcd for $\mathrm{C}_{21} \mathrm{H}_{22} \mathrm{NO}_{2}[\mathrm{M}+\mathrm{H}]^{+}$: 320.1645 found 320.1643 .

\section{Synthesis of $\left(5 S^{*}, 6 R^{*}\right)-6$-phenyl-1-azaspiro[4.4]non-8-ene-2,7-dione (12)}

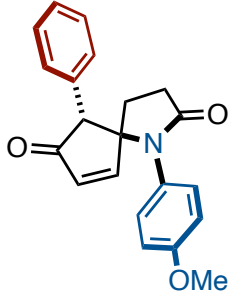

9Ba

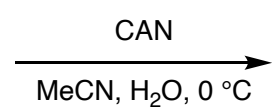

To a $20-\mathrm{mL}$ glass tube with a magnetic stirring bar were added $\mathbf{9 B a}(33.9 \mathrm{mg}, 0.10 \mathrm{mmol}, 1.0$ equiv), $\mathrm{MeCN}\left(1.5 \mathrm{~mL}\right.$ ), and then a solution of ammonium cerium(IV) nitrate in $\mathrm{H}_{2} \mathrm{O}$ (CAN: $0.15 \mathrm{M}$, $2.2 \mathrm{~mL}, 0.33 \mathrm{mmol}, 3.3$ equiv) at $0{ }^{\circ} \mathrm{C}$. After stirring the mixture at $0{ }^{\circ} \mathrm{C}$ for $1 \mathrm{~h}$, the mixture was diluted with $\mathrm{H}_{2} \mathrm{O}$ and then was extracted three times with EtOAc. To combined organic layer was added $\mathrm{Na}_{2} \mathrm{~S}_{2} \mathrm{O}_{3}$ aq. The mixture was extracted three times with EtOAc. Combined organic layer was dried over $\mathrm{Na}_{2} \mathrm{SO}_{4}$, filtrated, and concentrated in vacuo. The mixture was purified by GPC to afford $\mathbf{1 2}(16.7 \mathrm{mg}$, $73 \%$ yield) as a brown solid. ${ }^{1} \mathrm{H}$ NMR $\left(400 \mathrm{MHz}, \mathrm{CDCl}_{3}\right) \delta 7.48(\mathrm{~d}, J=6.0 \mathrm{~Hz}, 1 \mathrm{H}), 7.42-7.32(\mathrm{~m}, 3 \mathrm{H})$, 7.24-7.10 (m, 3H), 6.37 (dd, $J=6.0,1.2 \mathrm{~Hz}, 1 \mathrm{H}), 3.74(\mathrm{~s}, 1 \mathrm{H}), 2.18-2.06(\mathrm{~m}, 1 \mathrm{H}), 2.06-1.94(\mathrm{~m}, 1 \mathrm{H})$, $1.92-1.82(\mathrm{~m}, 1 \mathrm{H}), 1.82-1.61(\mathrm{~m}, 1 \mathrm{H}) ;{ }^{13} \mathrm{C}$ NMR $\left(101 \mathrm{MHz}, \mathrm{CDCl}_{3}\right) \delta 204.3,178.0,163.1,134.2,133.8$, $129.8,128.9,128.0,69.1,63.2,29.6$ (one peak is missing due to overlapping); HRMS (ESI) $\mathrm{m} / z$ calcd for $\mathrm{C}_{14} \mathrm{H}_{14} \mathrm{NO}_{2}[\mathrm{M}+\mathrm{H}]^{+}: 228.1019$ found 228.1019. Note: Compound 12 was unstable on both silica-gel and basic alumina. 


\section{Synthesis of $\left(2 R^{*}, 5 S^{*}, 6 R^{*}, 7 S^{*}\right)$-2-allyl-1,6-diphenyl-1-azaspiro[4.4]non-8-en-7-ol (13)}

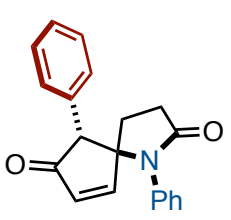

9Aa

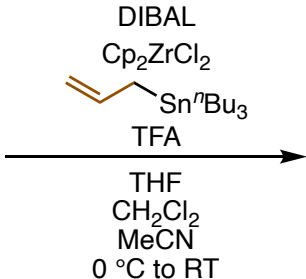

$0{ }^{\circ} \mathrm{C}$ to $\mathrm{RT}$
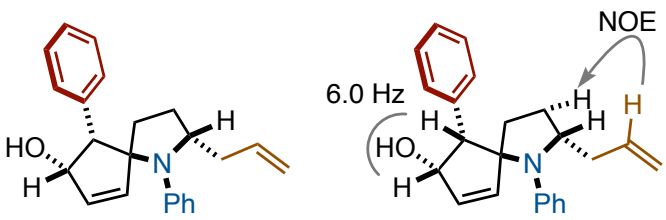

13

Following Sato and Chida's procedure ${ }^{[12]}$ with a slight modification, this reductive allylation was performed. An 8-mL glass tube with a magnetic stirring bar was dried up by a heat-gun in vacuo and filled $\mathrm{N}_{2}$ after cooling to room temperature. To this tube were added $\mathrm{Cp}_{2} \mathrm{ZrCl}_{2}(44.0 \mathrm{mg}, 0.15 \mathrm{mmol}, 3.0$ equiv) and THF (0.26 mL). To the solution was added DIBAL (1.0 M in $n$-hexane, $90 \mu \mathrm{L}, 90 \mu \mathrm{mol}, 1.8$ equiv) dropwise at room temperature and stirred for $6 \mathrm{~h}$ at room temperature. To this mixture was added a solution of $9 \mathrm{Aa}$ ( $15.1 \mathrm{mg}, 50 \mu \mathrm{mol}, 1.0$ equiv) in $\mathrm{CH}_{2} \mathrm{Cl}_{2}(0.26 \mathrm{~mL})$. After stirring this mixture at room temperature for $10 \mathrm{~min}$, the reaction was cooled to $0{ }^{\circ} \mathrm{C}$. To this mixture were added $\mathrm{MeCN}(0.26 \mathrm{~mL})$, allyltributyltin ( $48.6 \mathrm{mg}, 0.15 \mathrm{mmol}, 3.0$ equiv), and TFA ( $11.7 \mathrm{mg}, 0.10 \mathrm{mmol}, 2.1$ equiv) at $0{ }^{\circ} \mathrm{C}$. After stirring the mixture at room temperature for $6 \mathrm{~h}$, the reaction was quenched with $\mathrm{NaHCO}_{3}$ aq. The mixture was extracted three times with EtOAc. Combined organic layer was dried over $\mathrm{Na}_{2} \mathrm{SO}_{4}$, filtrated, and concentrated in vacuo. The residue was purified by PTLC (hexane/EtOAc $=9: 1$ ) to afford 13 (8.8 $\mathrm{mg}, 53 \%$ yield, as an inseparable mixture of diastereoisomers; $\mathrm{dr}=89: 11$ ) as a pale yellow oil. A relative stereochemistry of $\mathbf{1 3}$ was determined by NOESY and coupling constant. ${ }^{[13]}$

For a major isomer: ${ }^{1} \mathrm{H}$ NMR $\left(400 \mathrm{MHz}, \mathrm{CDCl}_{3}\right) \delta$ 7.42-7.36 (m, 2H), 7.25-7.17 (m, 5H), $6.91(\mathrm{~d}$, $J=8.0 \mathrm{~Hz}, 2 \mathrm{H}), 6.72(\mathrm{t}, J=8.0 \mathrm{~Hz}, 1 \mathrm{H}), 6.43(\mathrm{~d}, J=6.0 \mathrm{~Hz}, 1 \mathrm{H}), 6.07(\mathrm{dd}, J=6.0,2.8 \mathrm{~Hz}, 1 \mathrm{H}), 5.76-$ $5.63(\mathrm{~m}, 1 \mathrm{H}), 5.02-4.94(\mathrm{~m}, 3 \mathrm{H}), 3.91(\mathrm{~d}, J=6.0 \mathrm{~Hz}, 1 \mathrm{H}), 3.65(\mathrm{t}, J=8.8 \mathrm{~Hz}, 1 \mathrm{H}), 2.43(\mathrm{dd}, J=14.0$, $6.8 \mathrm{~Hz}, 1 \mathrm{H}), 2.24-2.18$ (m, 1H), 2.10-1.91 (m, 2H), 1.85-1.63 (brs, 1H), 1.40-1.20 (m, 1H), 0.80-0.68 $(\mathrm{m}, 1 \mathrm{H}) ;{ }^{1} \mathrm{H}$ NMR $\left(400 \mathrm{MHz}\right.$, benzene- $\left.d_{6}, 333 \mathrm{~K}\right) \delta 7.51-7.47(\mathrm{~m}, 2 \mathrm{H}), 7.24(\mathrm{t}, J=8.0 \mathrm{~Hz}, 2 \mathrm{H}), 7.14-$ $7.07(\mathrm{~m}, 3 \mathrm{H}), 7.04(\mathrm{~d}, J=8.8 \mathrm{~Hz}, 2 \mathrm{H}), 6.79(\mathrm{t}, J=7.2 \mathrm{~Hz}, 1 \mathrm{H}), 6.18(\mathrm{~d}, J=6.0 \mathrm{~Hz}, 1 \mathrm{H}), 5.69$ (dd, $J=$ $6.0,2.8 \mathrm{~Hz}, 1 \mathrm{H}), 5.63-5.50(\mathrm{~m}, 1 \mathrm{H}), 4.95-4.83(\mathrm{~m}, 2 \mathrm{H}), 4.52$ (d, $J=6.0,2.8 \mathrm{~Hz}, 1 \mathrm{H}), 3.94$ (d, $J=6.0$ $\mathrm{Hz}, 1 \mathrm{H}), 3.66$ (t, $J=8.8 \mathrm{~Hz}, 1 \mathrm{H}), 2.37$ (dd, $J=13.6,7.2 \mathrm{~Hz}, 1 \mathrm{H}), 2.30-2.18(\mathrm{~m}, 1 \mathrm{H}), 1.99-1.83(\mathrm{~m}, 2 \mathrm{H})$, $1.24-1.15(\mathrm{~m}, 1 \mathrm{H}), 1.04-0.83(\mathrm{~m}, 2 \mathrm{H}) ;{ }^{13} \mathrm{C} \mathrm{NMR}\left(101 \mathrm{MHz}, \mathrm{CDCl}_{3}\right) \delta 148.4,145.0,138.3,135.5,130.8$, 130.3, 129.1, 128.0, 126.7, 116.8, 116.6, 115.5, 78.7, 76.7, 58.2, 48.8, 37.3, 35.8, 25.8; HRMS (ESI) $m / z$ calcd for $\mathrm{C}_{23} \mathrm{H}_{26} \mathrm{NO}[\mathrm{M}+\mathrm{H}]^{+}: 332.2009$ found 332.2008. 


\section{Effect of Parameters}

\section{Effect of ligand}

\begin{tabular}{|c|c|c|c|c|}
\hline & $\mathrm{N}^{-P r}$ & + & $\begin{array}{c}5.0 \mathrm{~mol} \% \mathrm{Pd}(\mathrm{OAc}) \\
\text { ligand } \\
\mathrm{K}_{2} \mathrm{CO}_{3}(4.0 \text { equiv })\end{array}$ & \\
\hline & & NNHTs & $\begin{array}{c}\text { THF }(0.20 \mathrm{M}) \\
60^{\circ} \mathrm{C}, 12 \mathrm{~h}\end{array}$ & \\
\hline & $\begin{array}{c}1 \mathrm{~T} \\
10 \mathrm{mmol})\end{array}$ & $\begin{array}{c}\mathbf{2 a} \\
\text { (2.0 equiv) }\end{array}$ & & 3Ta \\
\hline entry & ligand (a mol \%) & recovery of $1 \mathrm{~T}^{\mathrm{a}}(\%)$ & yield of $\mathbf{3} \mathrm{Ta}^{\mathrm{a}}(\%)$ & yield of $9 \mathrm{Ta}^{\mathrm{a}}(\%)$ \\
\hline 1 & L1 (20) & 47 & 1 & 47 \\
\hline 2 & $\mathrm{PPh}_{3}(20)$ & 58 & 3 & 43 \\
\hline 3 & $\mathrm{P}(\text { o-tolyl })_{3}(20)$ & 92 & trace & 3 \\
\hline 4 & $\mathrm{P}(m \text {-tolyl })_{3}(20)$ & 57 & 1 & 40 \\
\hline 5 & $\mathrm{P}(p \text {-tolyl })_{3}(20)$ & 55 & 1 & 40 \\
\hline 6 & $\mathrm{P}(p \text {-anisy })_{3}(20)$ & 57 & 2 & 36 \\
\hline 7 & L2 (20) (20) & 73 & 2 & 30 \\
\hline 8 & $\mathrm{P}(2 \text {-furyl })_{3}(20)$ & 65 & 2 & 28 \\
\hline 9 & $\mathrm{P}\left(\mathrm{C}_{6} \mathrm{~F}_{5}\right)_{3}(20)$ & 98 & 0 & 0 \\
\hline 10 & $\mathrm{IPr} \cdot \mathrm{HCl}(20)$ & 100 & 0 & 0 \\
\hline 11 & IMes. $\mathrm{HCl}(20)$ & 100 & 0 & 0 \\
\hline 12 & dppb (10) & 86 & 3 & 26 \\
\hline 13 & DPÉphos (10) & 59 & 3 & 35 \\
\hline 14 & none & 91 & 0 & 0 \\
\hline
\end{tabular}

[a] Recoveries and yields were determined by ${ }^{1} \mathrm{H}$ NMR using $\mathrm{CH}_{2} \mathrm{Br}_{2}$ as an internal standard<smiles>O=C1C=CCC1(c1ccccc1)N1CCCC1c1ccccc1</smiles>

9Ta<smiles>CN(C)c1ccc(P)cc1</smiles>

L1<smiles>COc1cc(C)cc(OC)c1</smiles>

L2

\section{Effect of Pd source}<smiles>Brc1ccc(CCCN[PH2+]c2ccccc2)o1</smiles>

$1 \mathrm{~T}$

$(0.10 \mathrm{mmol})$

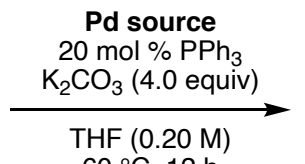

$60^{\circ} \mathrm{C}, 12 \mathrm{~h}$<smiles>C1=CC(N2CCC2)OC1=Cc1ccccc1</smiles>

3Ta<smiles>O=C1C=CC2(CCCN2Pc2ccccc2)[PH+]1</smiles>

9Ta

\begin{tabular}{ccccc}
\hline entry & $\mathbf{P d}$ source (a mol \%) & recovery of $\mathbf{1 \mathbf { T } ^ { \mathrm { a } } ( \% )}$ & yield of $\mathbf{3} \mathbf{T a}^{\mathrm{a}}(\%)$ & yield of $\mathbf{9 T a} \mathbf{T a}^{\mathrm{a}}(\%)$ \\
\hline 1 & $\mathrm{Pd}(\mathrm{OAc})_{2}(5.0)$ & 58 & 3 & 43 \\
2 & $\mathrm{Pd}(\mathrm{PPh})_{4}(5.0)$ & 71 & 2 & 47 \\
3 & $\mathrm{Pd}_{2}(\mathrm{dba})_{3} \cdot \mathrm{CHCl}_{3}(2.5)$ & 52 & 2 & 51 \\
\hline
\end{tabular}

[a] Recoveries and yields were determined by ${ }^{1} \mathrm{H}$ NMR using $\mathrm{CH}_{2} \mathrm{Br}_{2}$ as an internal standard

\section{Effect of solvent and base}

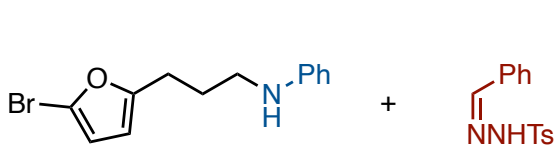

$1 \mathrm{~T}$

$(0.10 \mathrm{mmol})$

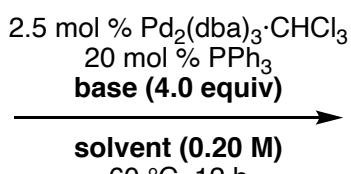

$60^{\circ} \mathrm{C}, 12 \mathrm{~h}$

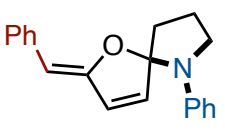

3Ta<smiles>O=C1C=CC2(c3ccccc3)CCCN12</smiles>

9Ta

\begin{tabular}{|c|c|c|c|c|c|}
\hline entry & base & solvent & recovery of $1 \mathrm{~T}^{\mathrm{a}}(\%)$ & yield of $3 \mathrm{Ta}^{\mathrm{a}}(\%)$ & yield of $9 \mathrm{Ta}^{\mathrm{a}}(\%)$ \\
\hline 1 & $\mathrm{~K}_{2} \mathrm{CO}_{3}$ & THF & 52 & 2 & 51 \\
\hline 2 & $\mathrm{~K}_{3} \mathrm{PO}_{4}$ & THF & 47 & 3 & 47 \\
\hline 3 & $\mathrm{Cs}_{2} \mathrm{CO}_{3}$ & THF & 16 & 22 & 44 \\
\hline 4 & $\mathrm{NaH}$ & THF & 0 & 0 & 23 \\
\hline 5 & $\mathrm{KOH}$ & THF & 34 & 0 & 65 \\
\hline 6 & $\mathrm{KOH}$ & DME & 0 & 0 & 1 \\
\hline 7 & $\mathrm{Cs}_{2} \mathrm{CO}_{3}$ & DME & 0 & 10 & 72 \\
\hline 8 & $\mathrm{Cs}_{2} \mathrm{CO}_{3}$ & DCE & 35 & 0 & 60 \\
\hline
\end{tabular}

[a] Recoveries and yields were determined by ${ }^{1} \mathrm{H}$ NMR using $\mathrm{CH}_{2} \mathrm{Br}_{2}$ as an internal standard 


\section{Limitation of Pd-Catalyzed Azaspirocyclization}<smiles>NC(=O)OCCCc1ccc(Br)o1</smiles>

1.0 equiv<smiles>O=C(CCc1ccc(Br)cc1)Nc1ccccc1</smiles>

1.0 equiv<smiles></smiles>

1.0 equiv<smiles>[R]NC(=O)CCc1ccc(Br)s1</smiles>

1.0 equiv

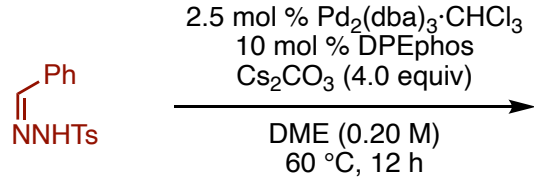

2a: 2.0 equiv

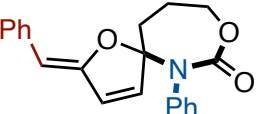

$0 \%$<smiles>O=CC=C1C=CC2(C=C1)CCC(=O)N2c1ccccc1</smiles>

$6 \%$<smiles>CC(C)(C)OC(=O)N1C=CC(=CC=[Te])C12CCC(=O)N2c1ccccc1</smiles>

$0 \%$

$2.5 \mathrm{~mol} \% \mathrm{Pd}_{2}(\mathrm{dba})_{3} \cdot \mathrm{CHCl}_{3}$<smiles>C1=N[Si]C=[Sn]C1</smiles>
$20 \mathrm{~mol}_{3} \mathrm{PPh}_{3}$

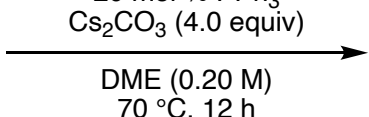<smiles>[R]N1C(=O)CCC12C=C/C(=C/c1ccccc1)S2</smiles>

2a: 2.0 equiv<smiles>CCN1C(=O)CCC12C=C/C(=C/c1ccccc1)S2</smiles>

$0 \%$<smiles>CC(C)(C)N1C(=O)CCC12C=C/C(=C\c1ccccc1)S2</smiles>

$0 \%$<smiles>O=C1CCC2(C=C/C(=C\c3ccccc3)S2)N1C(=O)c1ccccc1</smiles>

$0 \%$ 


\section{X-ray Crystal Structure Analysis of 9Aa}

A crystal of $9 \mathrm{Aa}$ was grown from a $\mathrm{CHCl}_{3} /$ hexane solution (vapor diffusion). A suitable crystal was mounted with Paratone oil on a MiTeGen MicroMounts and transferred to the 3-axis Eulerian Goniometer of a Rigaku R-AXIS RAPID II system with Ultrax $18 \mathrm{~kW}$ rotating anode X-ray generator using graphite-monochromated $\mathrm{Cu}-\mathrm{K}_{\alpha}$ radiation and imaging plate area detector. Cell parameters were determined and refined, and raw frame data were integrated using RAPID-AUTO (RIGAKU, 1998). The structures were solved by direct methods with (SHELXT) ${ }^{[14]}$ and refined by full-matrix leastsquares techniques against $F^{2}$ (SHELXL-2018/3) ${ }^{[15]}$ by using Olex2 software package. ${ }^{[16]}$ The intensities were corrected for Lorentz and polarization effects. The non-hydrogen atoms were refined anisotropically. Hydrogen atoms were placed using AFIX instructions.

Table S1. Crystallographic Data and Structure Refinement Details for 9Aa

\begin{tabular}{|c|c|}
\hline Compound & 9Aa \\
\hline CCDC number & 2086786 \\
\hline Empirical formula & $\mathrm{C}_{20} \mathrm{H}_{17} \mathrm{NO}_{2}$ \\
\hline Formula weight & 303.35 \\
\hline$T / \mathrm{K}$ & $173(2)$ \\
\hline Crystal system & orthorhombic \\
\hline Space group & Pbca \\
\hline$a / \AA$ & $11.5675(11)$ \\
\hline$b / \AA$ & $15.2858(15)$ \\
\hline$c / \AA$ & $18.2662(17)$ \\
\hline$\alpha /{ }^{\circ}$ & 90 \\
\hline$\beta /{ }^{\circ}$ & 90 \\
\hline$\gamma / \circ$ & 90 \\
\hline$V / \AA^{3}$ & $3229.8(5)$ \\
\hline$Z$ & 8 \\
\hline $\mathrm{D}_{\text {calc }}, / \mathrm{g} \mathrm{cm}^{-3}$ & 1.248 \\
\hline$\mu / \mathrm{mm}^{-1}$ & 0.642 \\
\hline $\mathrm{F}(000)$ & 1280.0 \\
\hline Crystal size / mm & $0.4 \times 0.2 \times 0.1$ \\
\hline$\lambda / \AA$ & 1.5418 \\
\hline $2 \theta$ range $/^{\circ}$ & 9.684 to 136.484 \\
\hline Reflns collected & 34384 \\
\hline Indep reflns $/ R_{\text {int }}$ & $2946 / 0.0234$ \\
\hline Params & 208 \\
\hline GOF on $F^{2}$ & 1.070 \\
\hline$R_{1}, \mathrm{w} R_{2}[I>2 \sigma(I)]$ & $0.0368,0.0887$ \\
\hline$R_{1}, \mathrm{w} R_{2}$ [all data] & $0.0402,0.0908$ \\
\hline Max./Mini. Peak / e $\AA^{-3}$ & $0.17 /-0.24$ \\
\hline
\end{tabular}




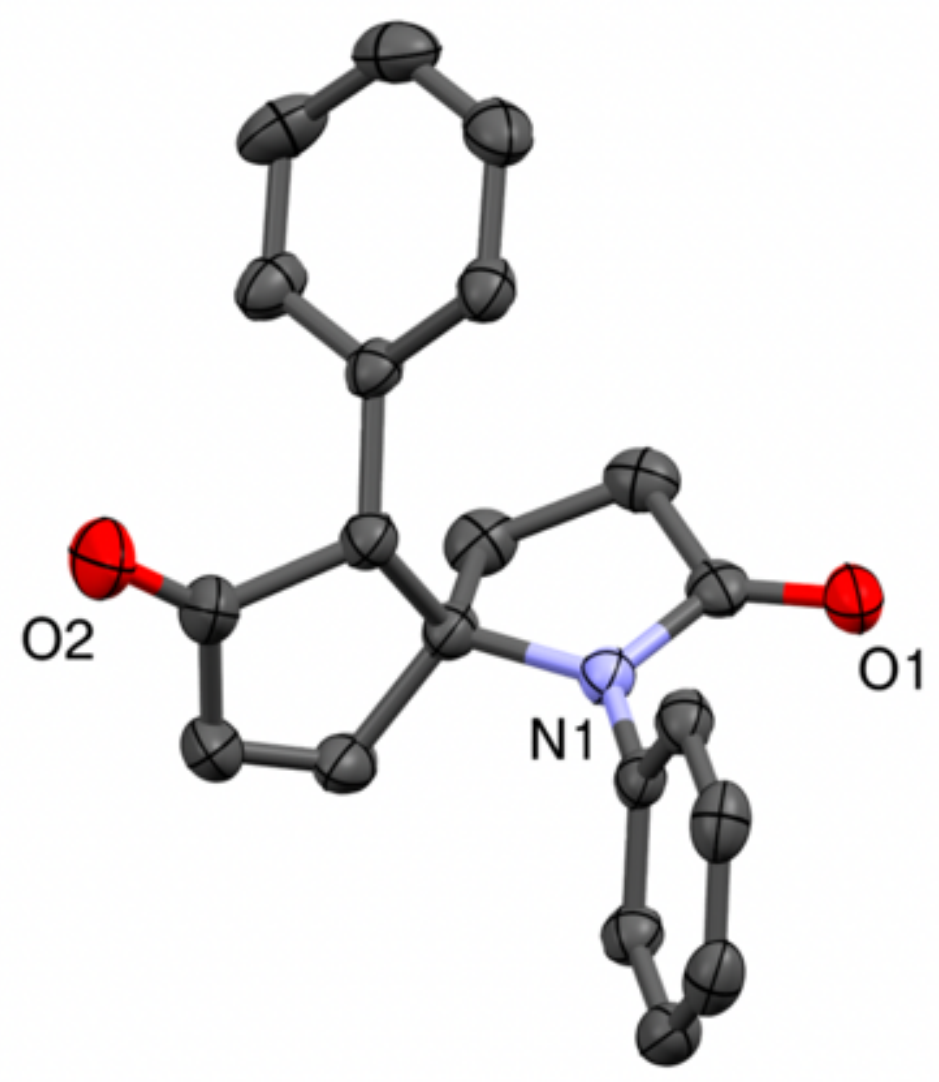

Figure S1. ORTEP drawing of 9Aa with 50\% thermal ellipsoid. All hydrogen atoms are omitted for clarity. 


\section{References}

[1] Zalesskiy, S. S.; Ananikov, V. P. $\mathrm{Pd}_{2}(\mathrm{dba})_{3}$ as a Precursor of Soluble Metal Complexes and Nanoparticles: Determination of Palladium Active Species for Catalysis and Synthesis. Organometallics 2012, 31, 2302-2309.

[2] Bzeih, T.; Zhang, K.; Khalaf, A.; Hachem, A.; Alami, M.; Hamze, A. One-Pot Reaction between NTosylhydrazones and 2-Nitrobenzyl Bromide: Route to NH-Free C2-Arylindoles. J. Org. Chem. 2019, $84,228-238$.

[3] Liu, P.; Xu, Q.-Q.; Dong, C.; Lei, X.; Lin, G.-Q. A Complementary Approach to 3,5-Substituted Pyrazoles with Tosylhydrazones and Terminal Alkynes Mediated by TfOH. Synlett 2012, 23, 20872092.

[4] Allwood, D. M.; Blakemore, D. C.; Ley, S. V. Preparation of Unsymmetrical Ketones from Tosylhydrazones and Aromatic Aldehydes via Formyl C-H Bond Insertion. Org. Lett. 2014, 16, 3064 3067.

[5] Yamada, N.; Kamatani, J.; Saitoh, A. Novel Naphthothiophene Compound and Organic LightEmitting Device Including the Same. Patent WO2011132623A1. 2011-10-27.

[6] Bober, A. E.; Proto, J. T.; Brummond, K. M. Intramolecular Didehydro-Diels-Alder Reaction for the Synthesis of Benzo- and Dihydrobenzo-Fused Heterocycles. Org. Lett. 2017, 19, 1500-1503.

[7] Boullosa, S.; Gándara, Z.; Pérez, M.; Gómez, G.; Fall, Y. The Furan Approach to Thiacyclic Compounds. Stereoselective Synthesis of 2,3-Disubstituted Tetrahydrothiopyrans. Tetrahedron Lett. 2008, 49, 4040-4042.

[8] Zhu, L.; Song, L.; Tong, R. Diastereoselective Reductive Ring Expansion of Spiroketal Dihydropyranones to $c i s$-Fused Bicyclic Ethers. Org. Lett. 2012, 14, 5892-5895.

[9] Youn, S. W.; Kim, Y. H. Pd(II)/Ag(I)-Promoted One-Pot Synthesis of Cyclic Ureas from (Hetero)Aromatic Amines and Isocyanates. Org. Lett. 2016, 18, 6140-6143.

[10] Yang, P.; Wang, X.; Ma, Y.; Sun, Y.; Zhang, L.; Yue, J.; Fu, K.; Zhou, J. S.; Tang, B. NickelCatalyzed $C$-Alkylation of Thioamide, Amides and Esters by Primary Alcohols through a Hydrogen Autotransfer Strategy. Chem. Commun. 2020, 56, 14083-14086.

[11] Song, S.; Sun, X.; Li, X.; Yuan, Y.; Jiao, N. Efficient and Practical Oxidative Bromination and Iodination of Arenes and Heteroarenes with DMSO and Hydrogen Halide: A Mild Protocol for LateStage Functionalization. Org. Lett. 2015, 17, 2886-2889.

[12] Nakajima, M.; Oda, Y.; Wada, T.; Minamikawa, R.; Shirokane, K.; Sato, T.; Chida, N. Chemoselective Reductive Nucleophilic Addition to Tertiary Amides, Secondary Amides, and $\mathrm{N}$ Methoxyamides. Chem. Eur. J. 2014, 20, 17565-17571. 
[13] Nakata, K.; Kiyotsuka, Y.; Kitazume, T.; Kobayashi, Y. Realization of Anti-S $\mathrm{S}_{\mathrm{N}} 2^{\prime}$ Selective Allylation of 4-Cyclopentene-1,3-Diol Monoester with Aryl- and Alkenyl-Zinc Reagents. Org. Lett. 2008, 10, 1345-1348.

[14] Sheldrick, G. M. SHTLXT - Integrated Space-Group and Crystal-Structure Determination. Acta Cryst. 2015, A71, 3-8.

[15] Sheldrick, G. M. Crystal Structure Refinement with SHELXL. Acta Cryst. 2015, C71, 3-8.

[16] Dolomanov, O. V.; Bourhis, L. J.; Gildea, R. J.; Howard, J. A. K.; Puschmann, H. OLEX2: A Complete Structure Solution, Refinement and Analysis Program. J. Appl. Crystallogr. 2009, 42, 339341. 
12. ${ }^{1} \mathrm{H}$ NMR, ${ }^{13} \mathrm{C}$ NMR, and ${ }^{19} \mathrm{~F}$ NMR Spectra

${ }^{1} \mathrm{H}$ NMR of $1 \mathrm{~A}\left(400 \mathrm{MHz}, \mathrm{CDCl}_{3}\right)$

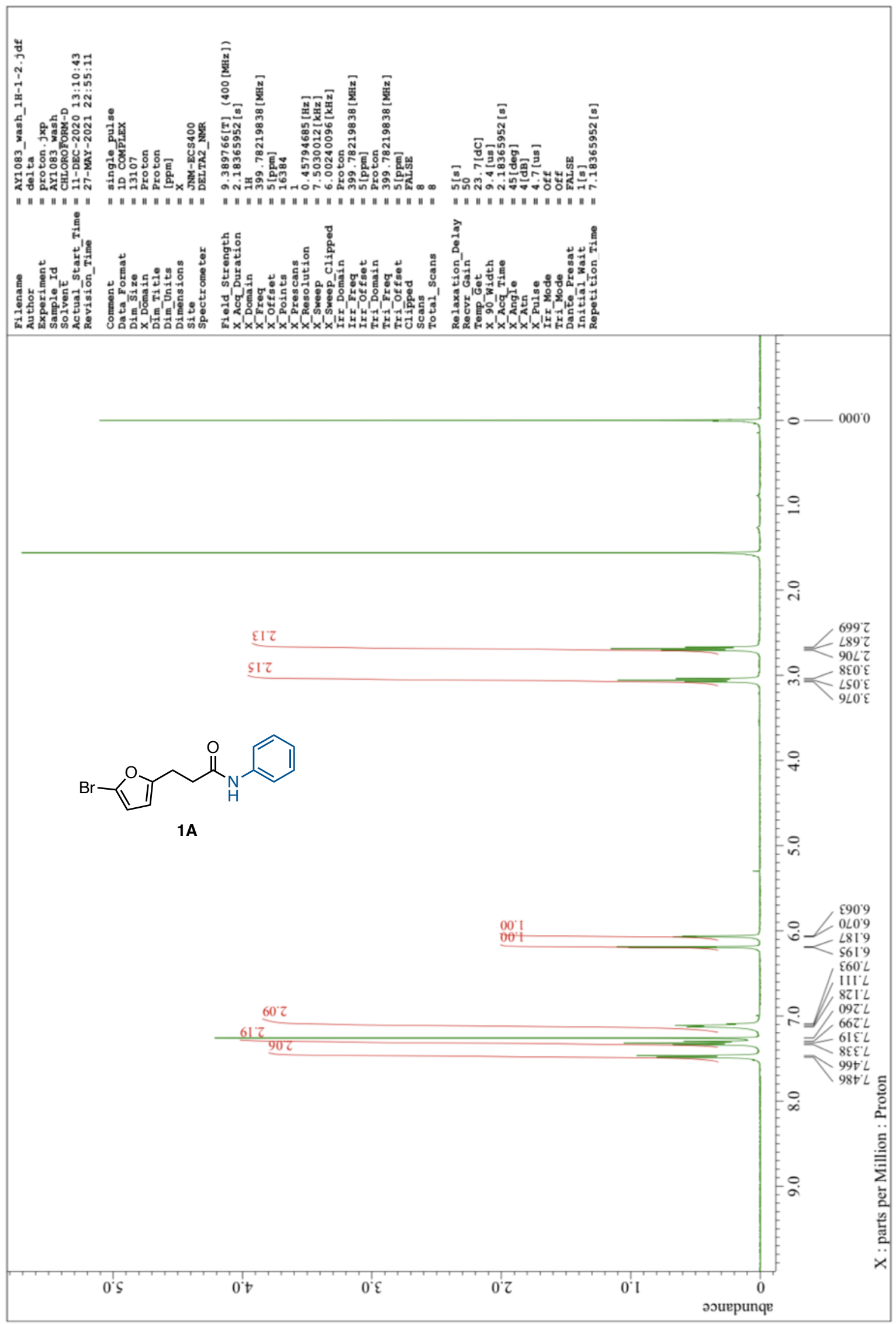


${ }^{13}$ C NMR of $1 \mathrm{~A}\left(101 \mathrm{MHz}\right.$, DMSO- $\left.d_{6}\right)$

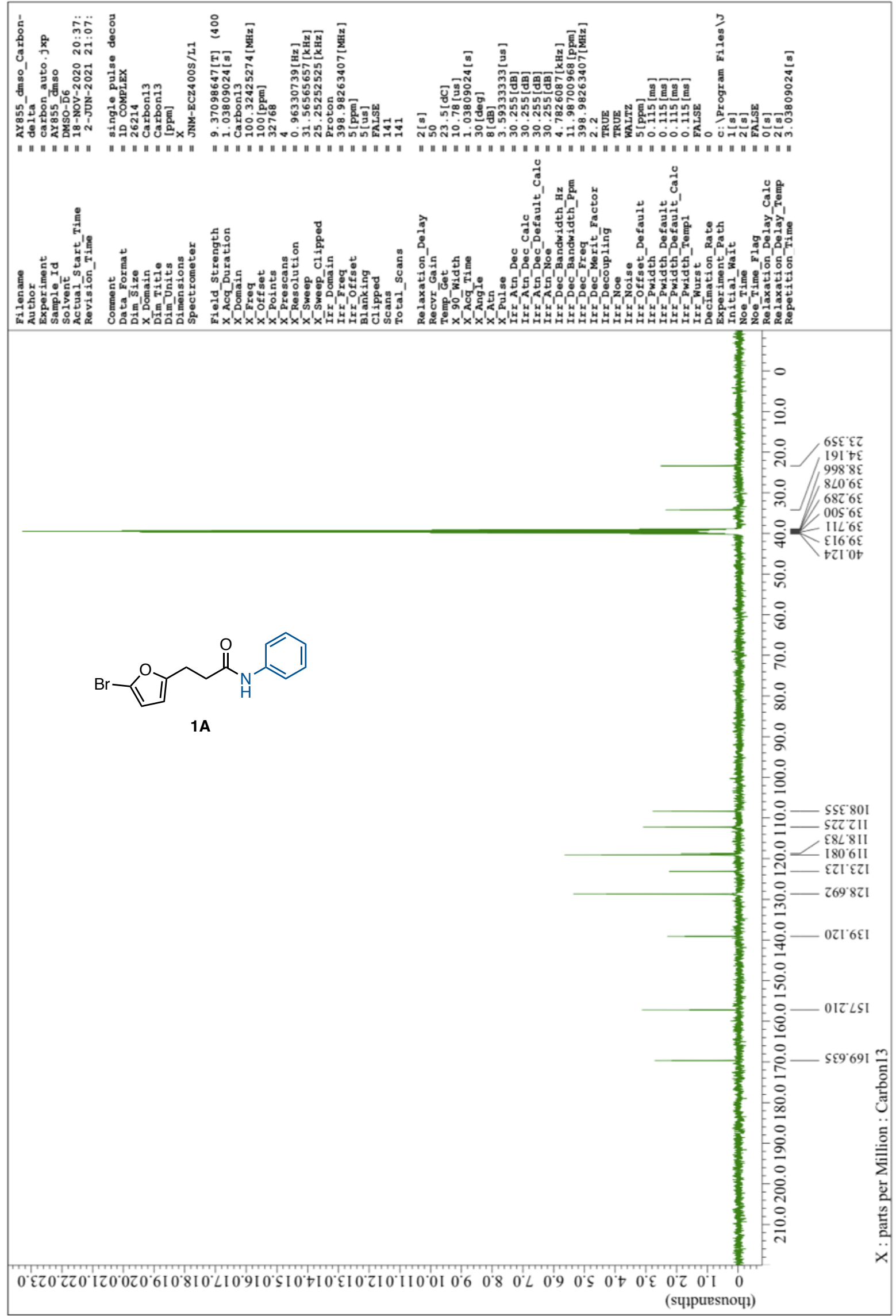


${ }^{1} \mathrm{H}$ NMR of $1 \mathrm{~B}\left(400 \mathrm{MHz}, \mathrm{CDCl}_{3}\right)$

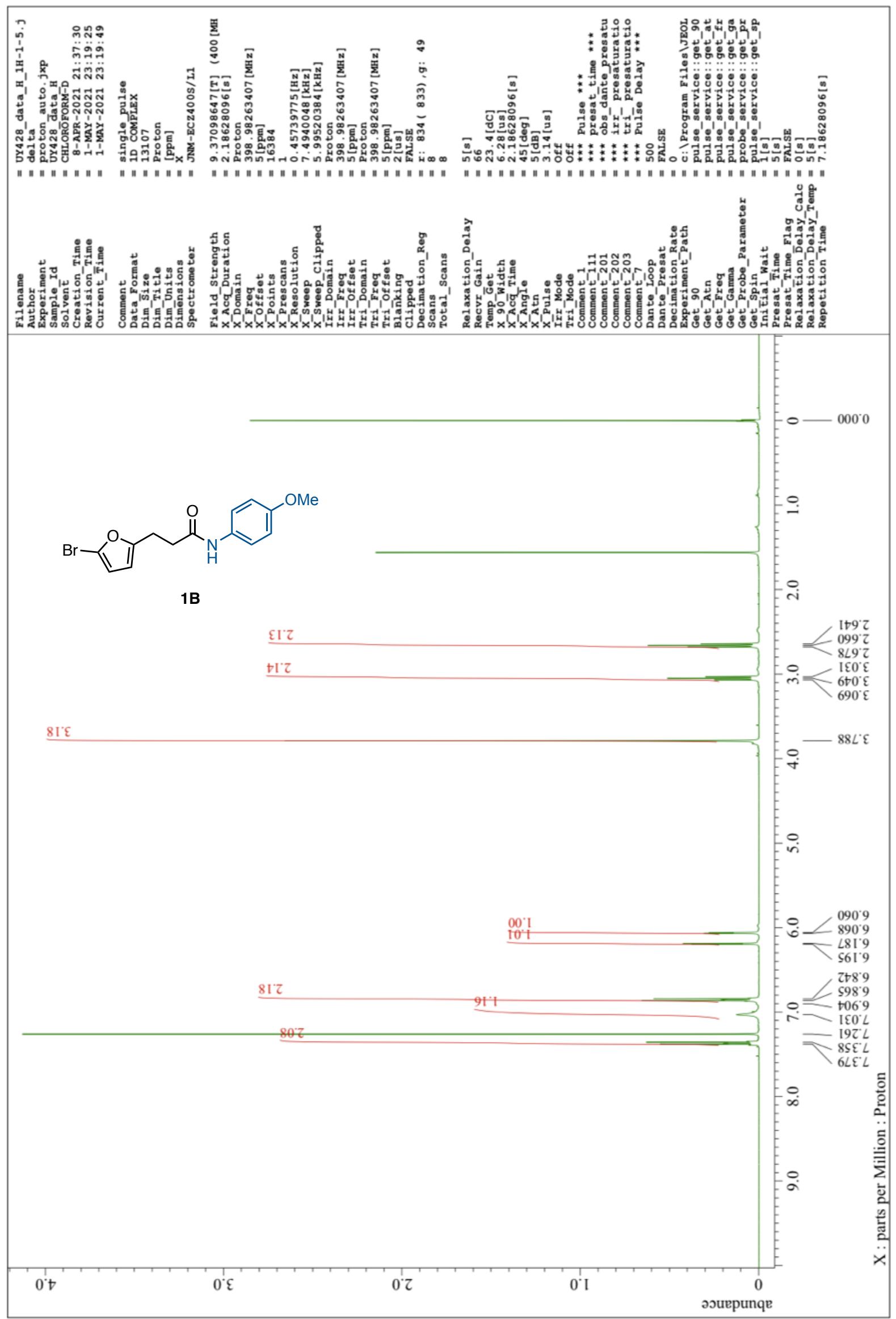


${ }^{13} \mathrm{C}$ NMR of $1 \mathrm{~B}\left(101 \mathrm{MHz}, \mathrm{CDCl}_{3}\right)$

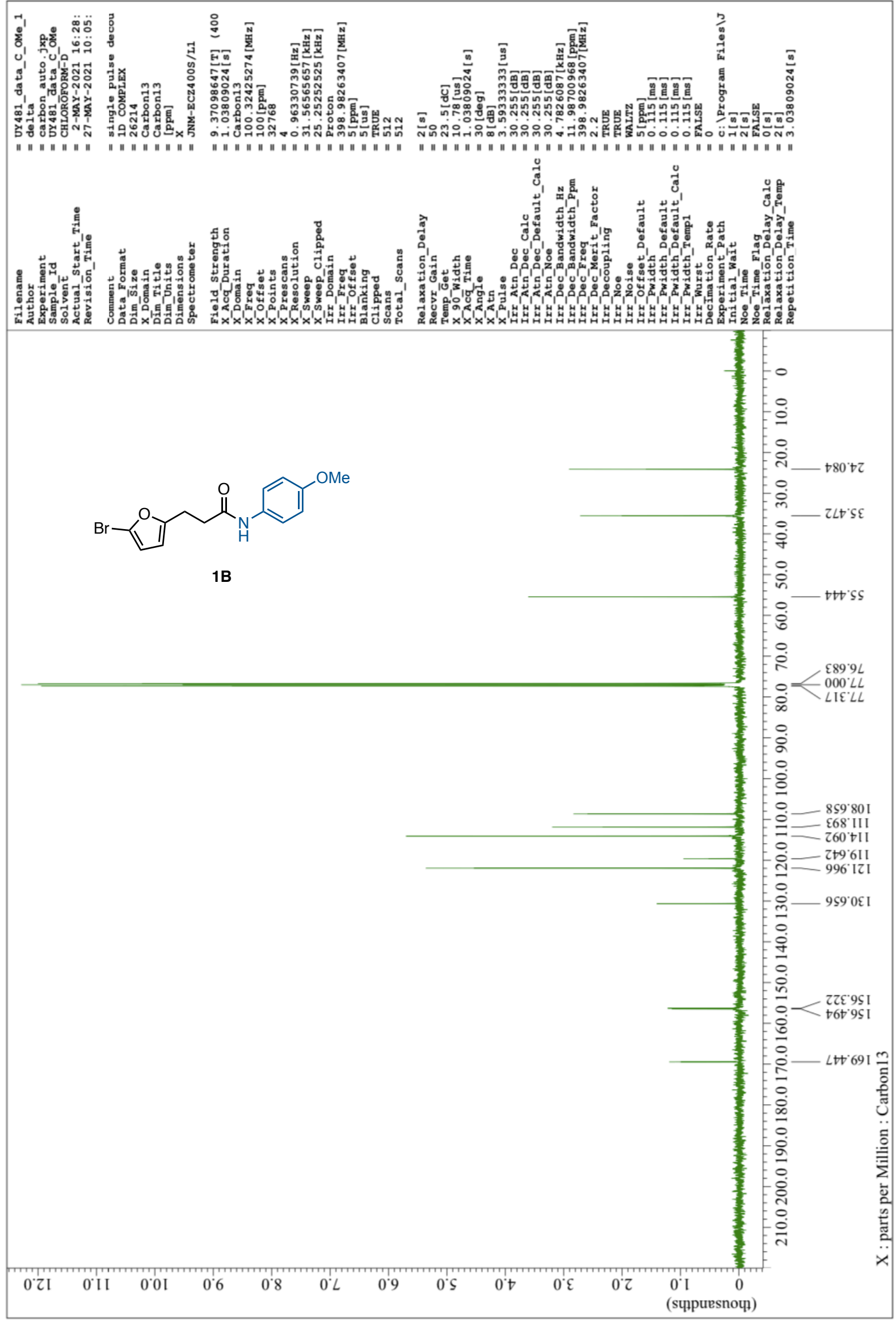


${ }^{1} \mathrm{H}$ NMR of $1 \mathrm{C}\left(400 \mathrm{MHz}, \mathrm{CDCl}_{3}\right)$

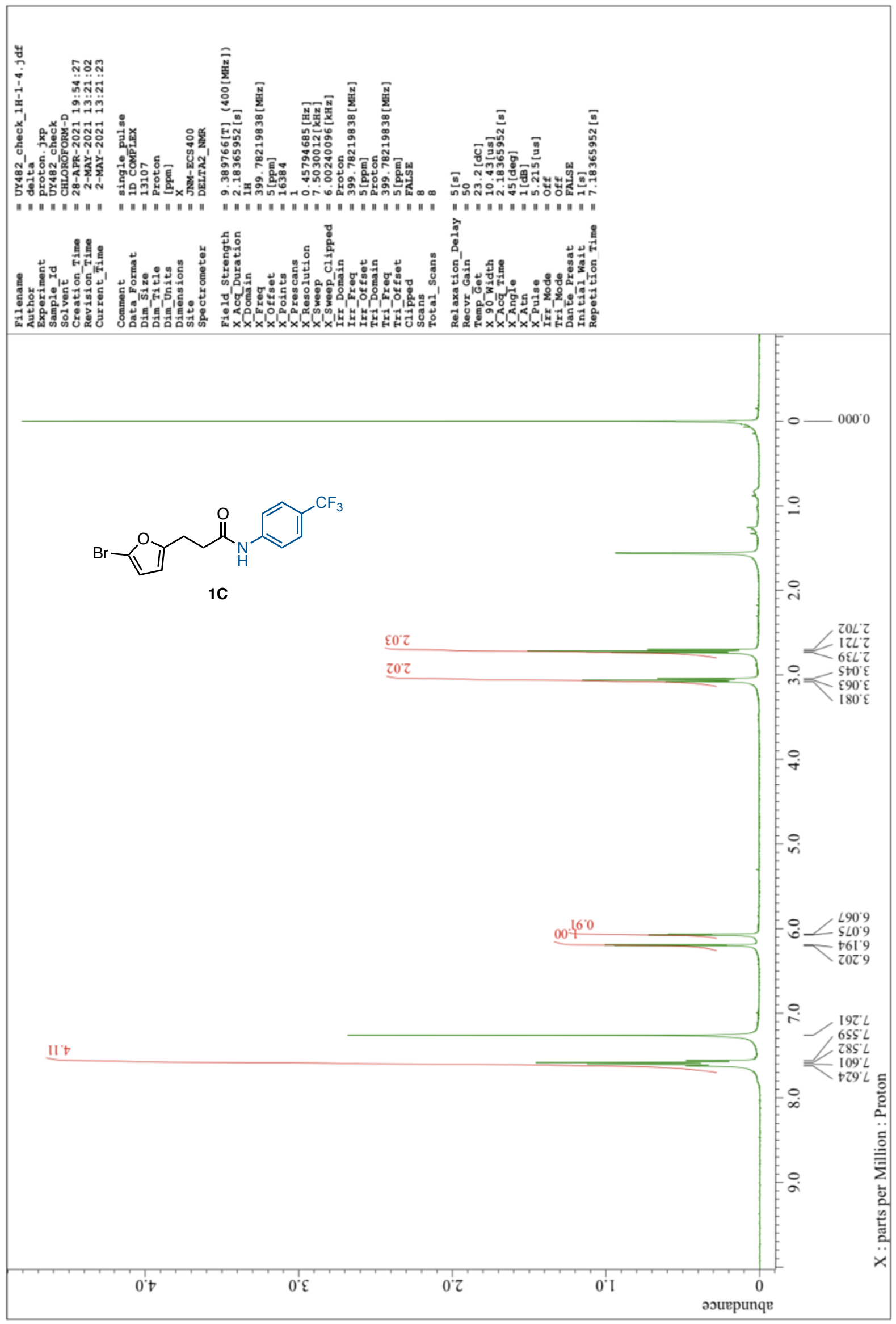


${ }^{13} \mathrm{C}$ NMR of $1 \mathrm{C}\left(101 \mathrm{MHz}, \mathrm{CDCl}_{3}\right)$

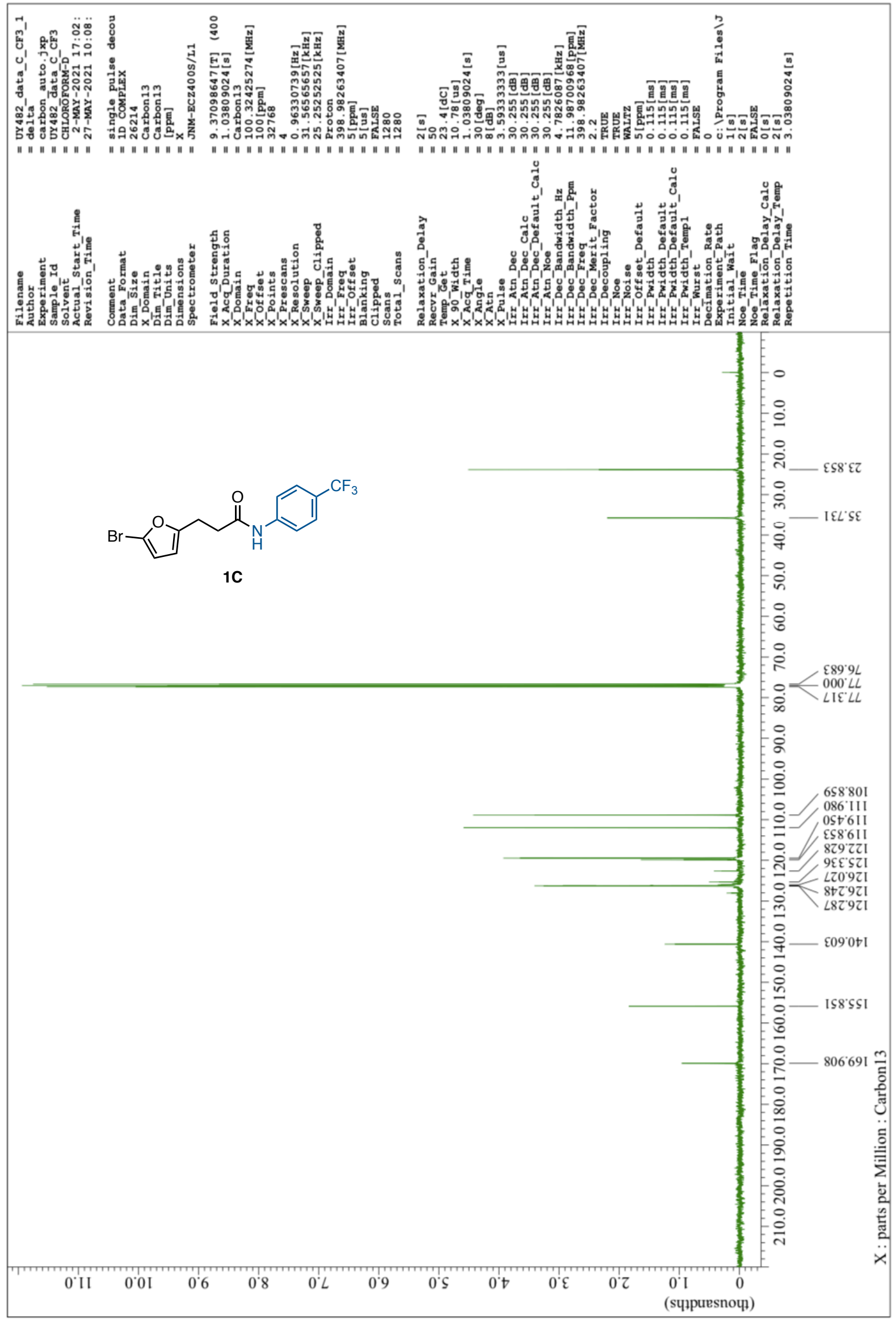


19F NMR of $1 \mathrm{C}\left(376 \mathrm{MHz}, \mathrm{CDCl}_{3}\right)$

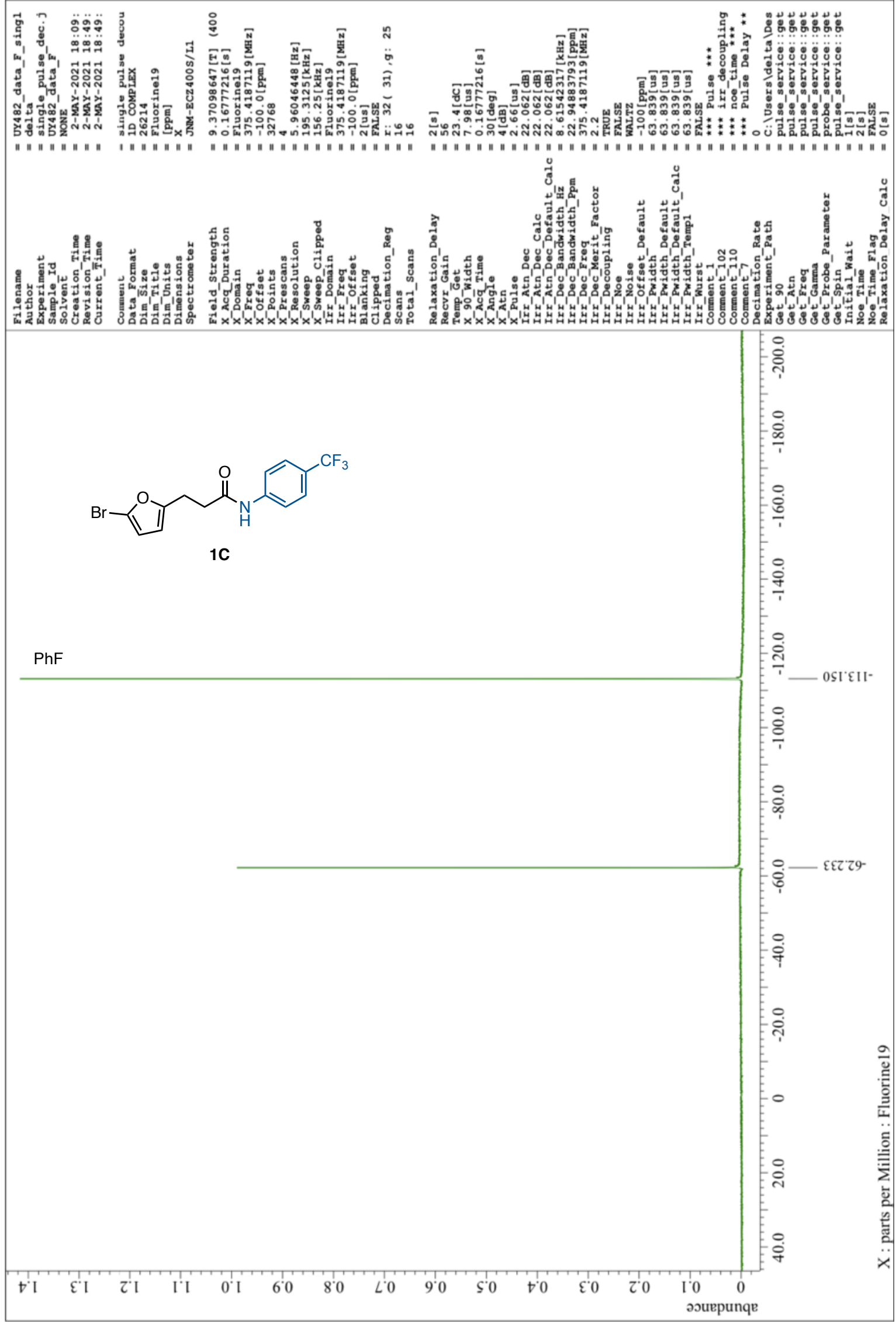


${ }^{1} \mathrm{H}$ NMR of $1 \mathrm{D}\left(400 \mathrm{MHz}, \mathrm{CDCl}_{3}\right)$

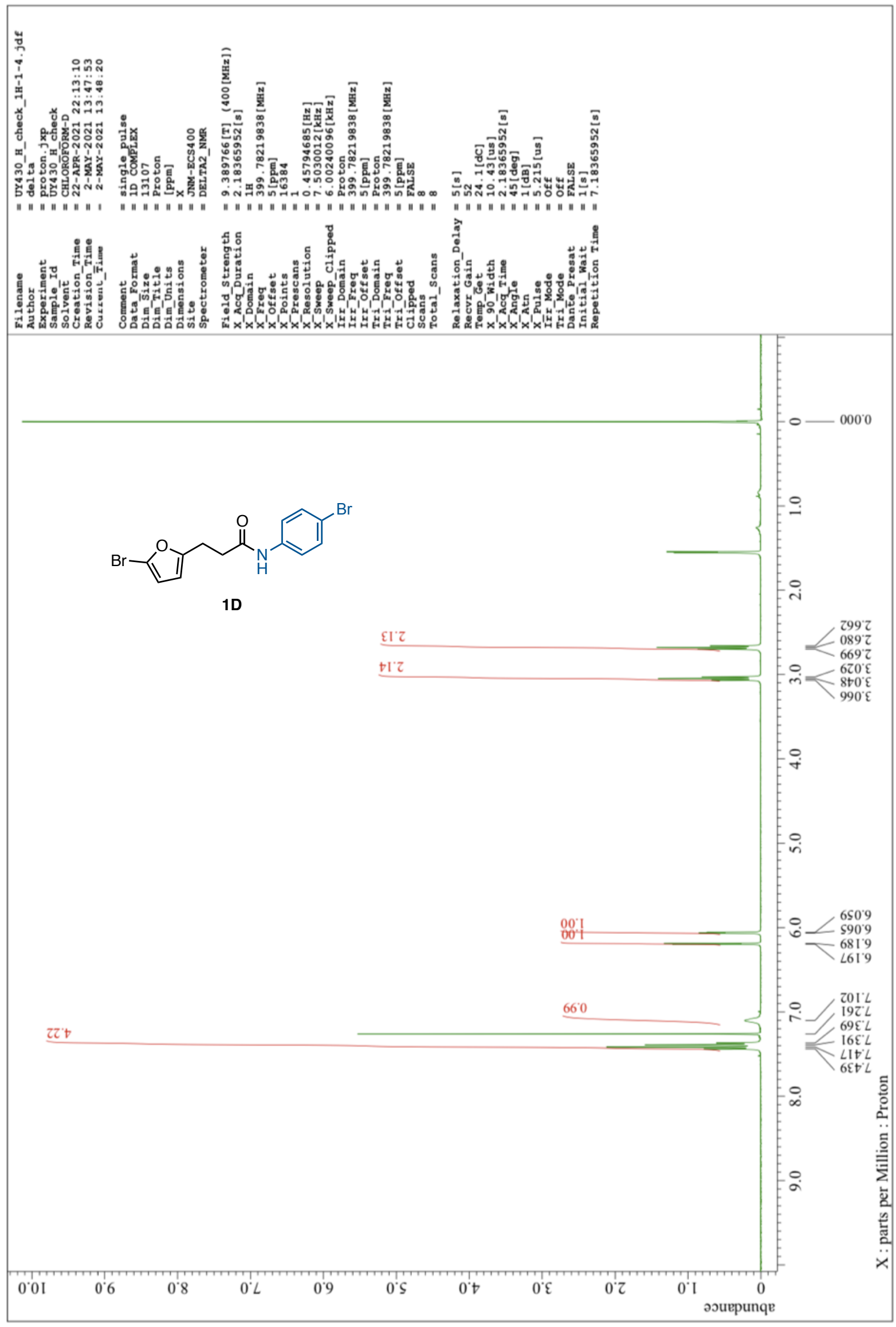


${ }^{13} \mathrm{C}$ NMR of $1 \mathrm{D}\left(101 \mathrm{MHz}, \mathrm{CDCl}_{3}\right)$

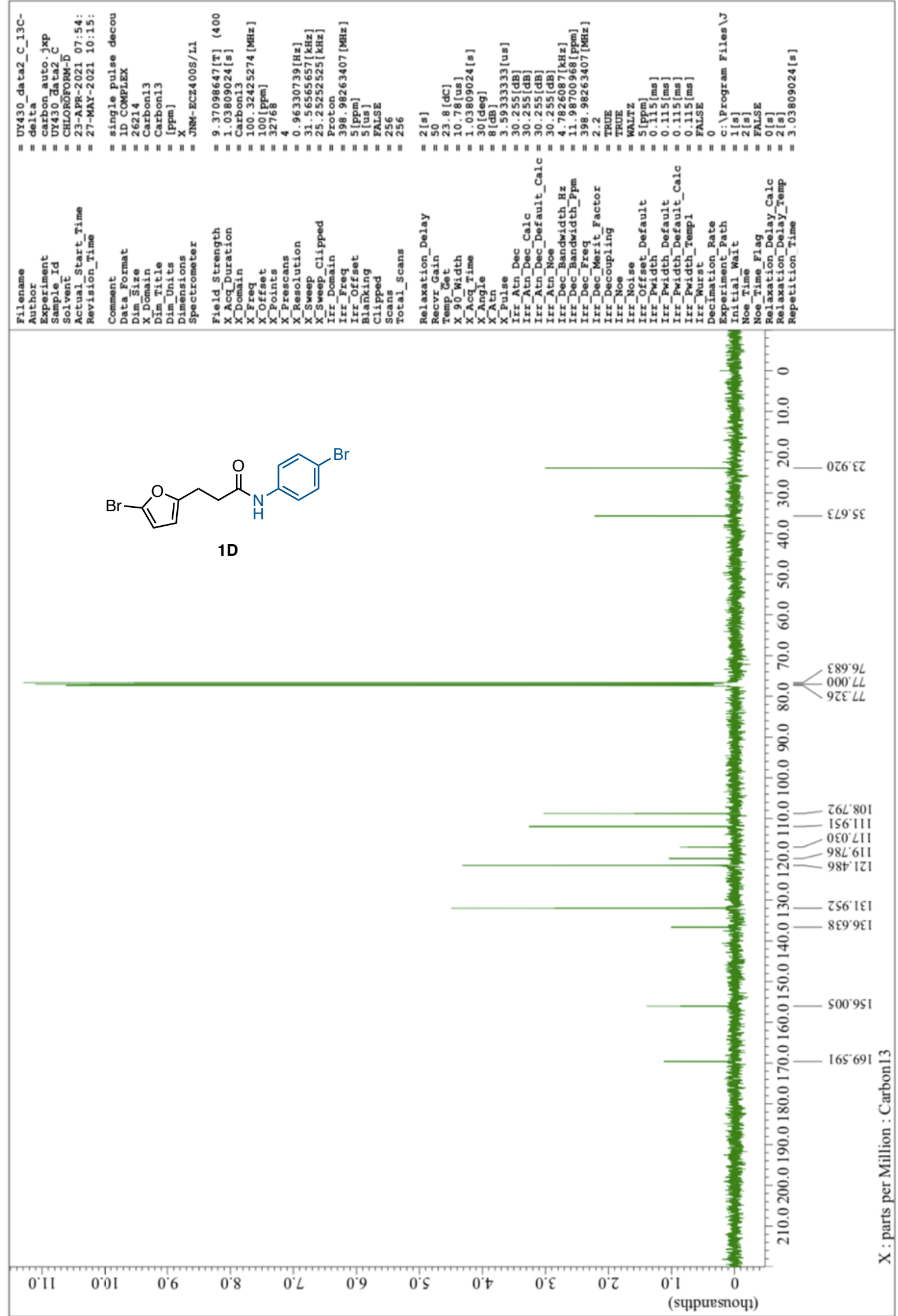


${ }^{1} \mathrm{H}$ NMR of $1 \mathrm{E}$ (400 MHz, $\mathrm{CDCl}_{3}$ )

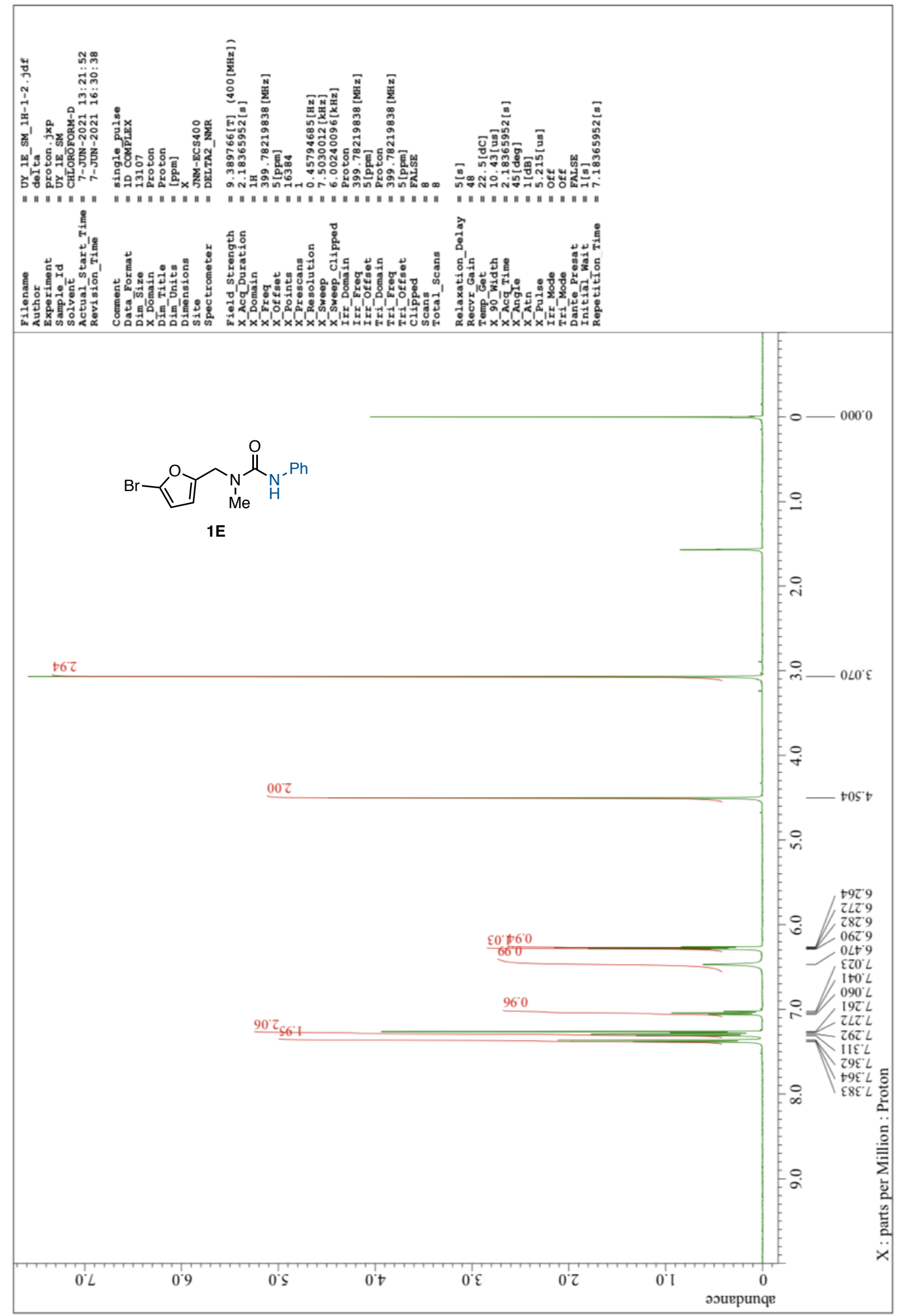


${ }^{13} \mathrm{C}$ NMR of 1E (101 MHz, $\left.\mathrm{CDCl}_{3}, 333 \mathrm{~K}\right)$

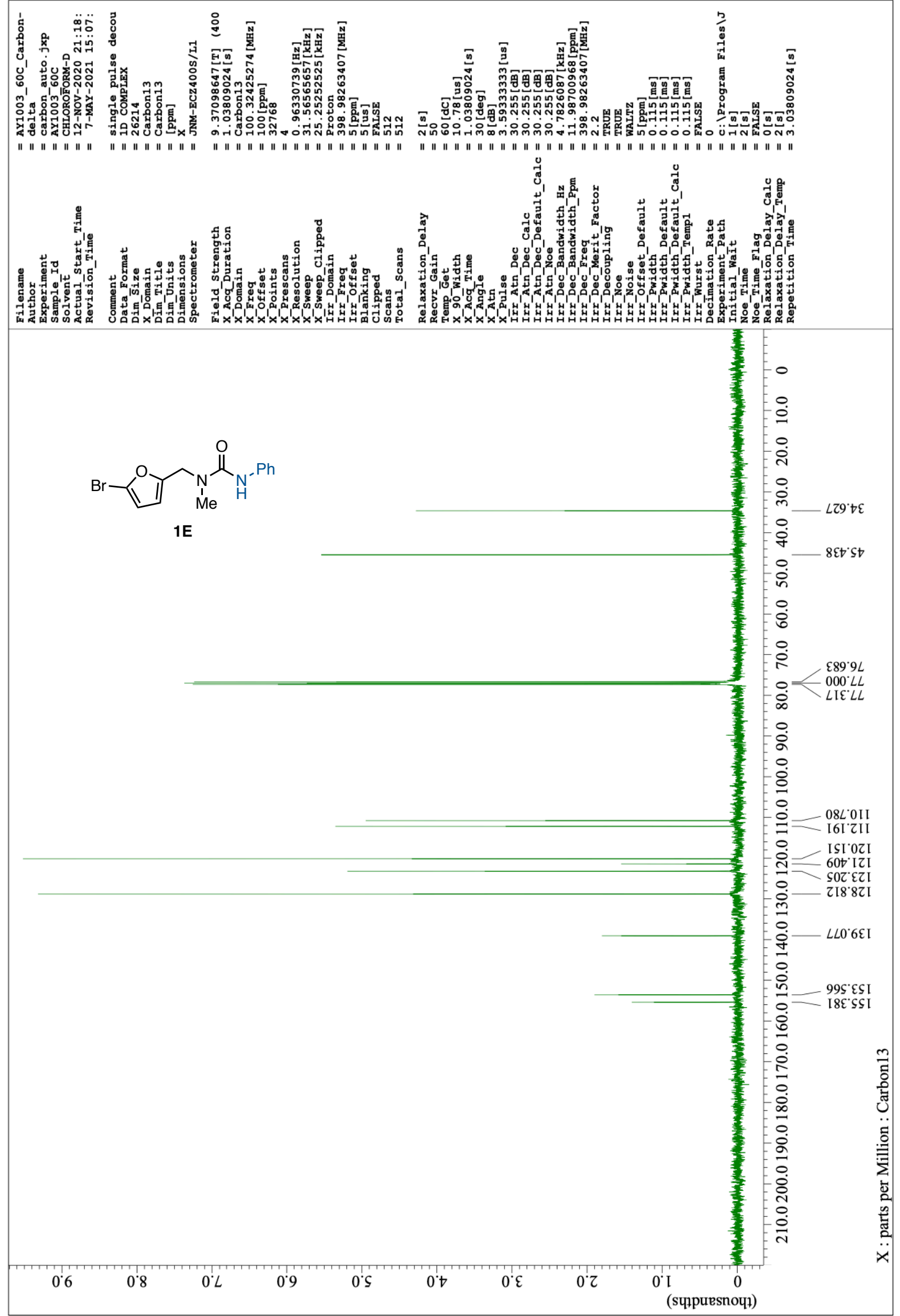


${ }^{1} \mathrm{H}$ NMR of $1 \mathrm{~F}\left(400 \mathrm{MHz}, \mathrm{CDCl}_{3}\right)$

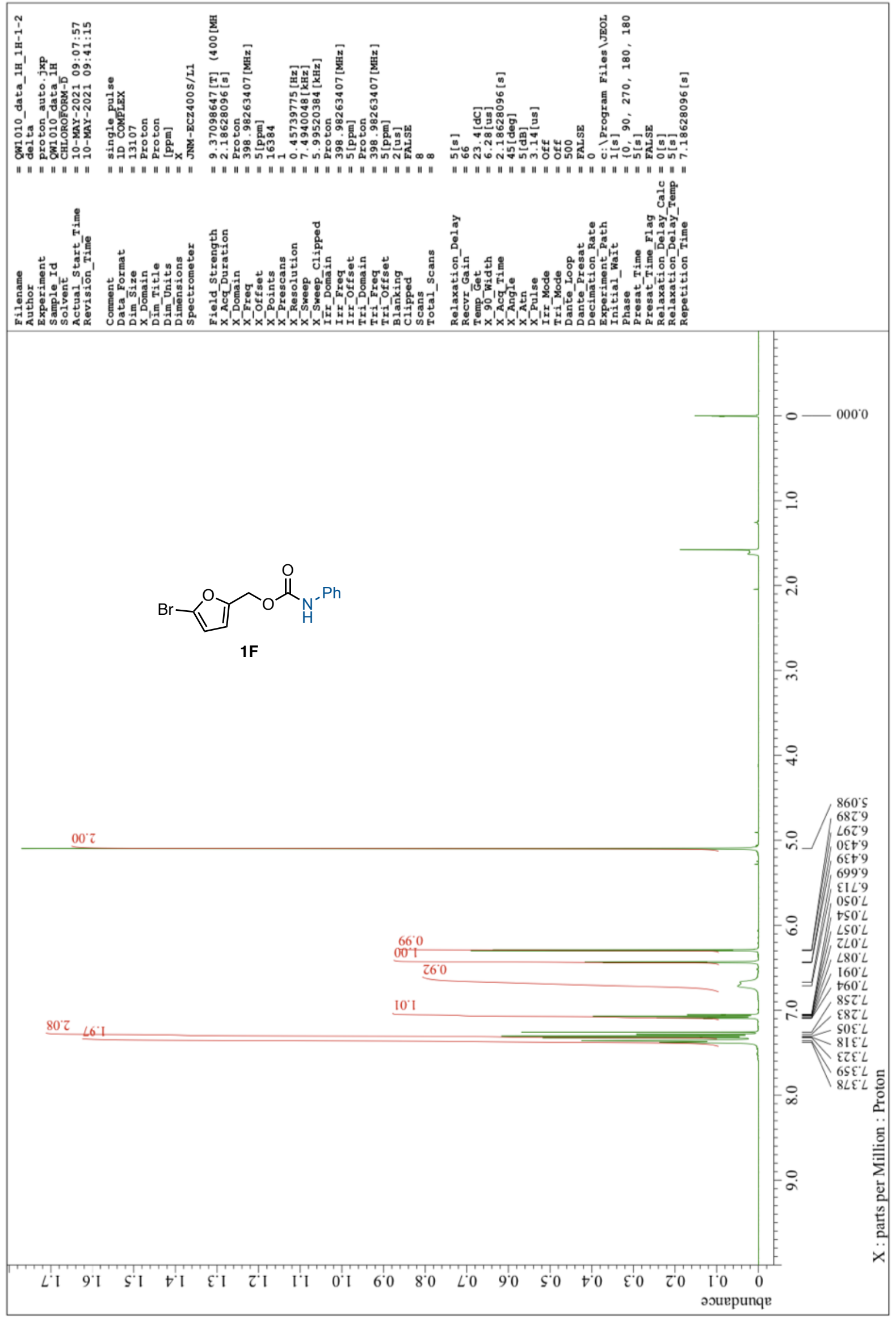


${ }^{13} \mathrm{C}$ NMR of 1F (101 MHz, $\left.\mathrm{CDCl}_{3}\right)$

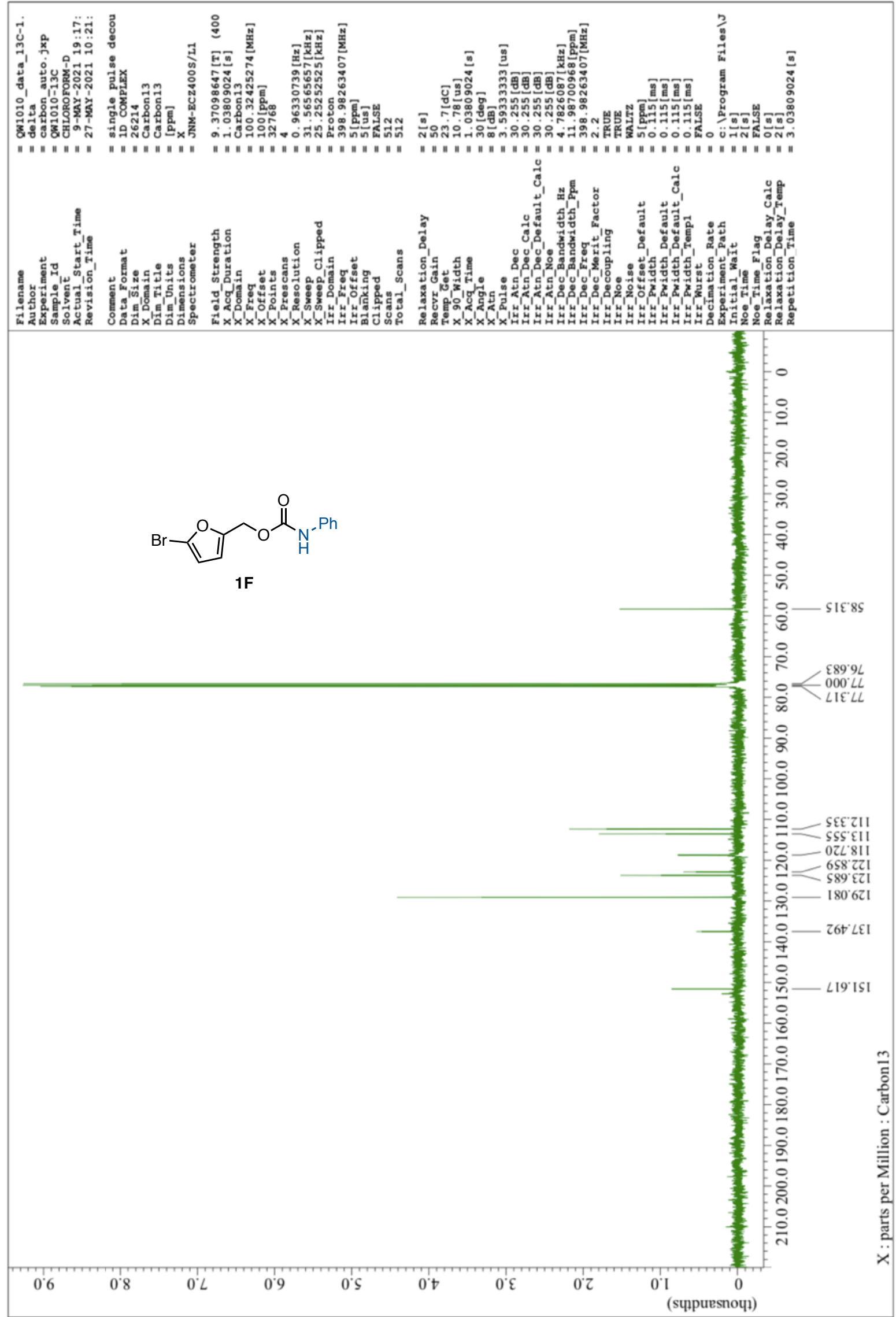


${ }^{1} \mathrm{H}$ NMR of $1 \mathrm{G}\left(400 \mathrm{MHz}, \mathrm{CDCl}_{3}\right)$

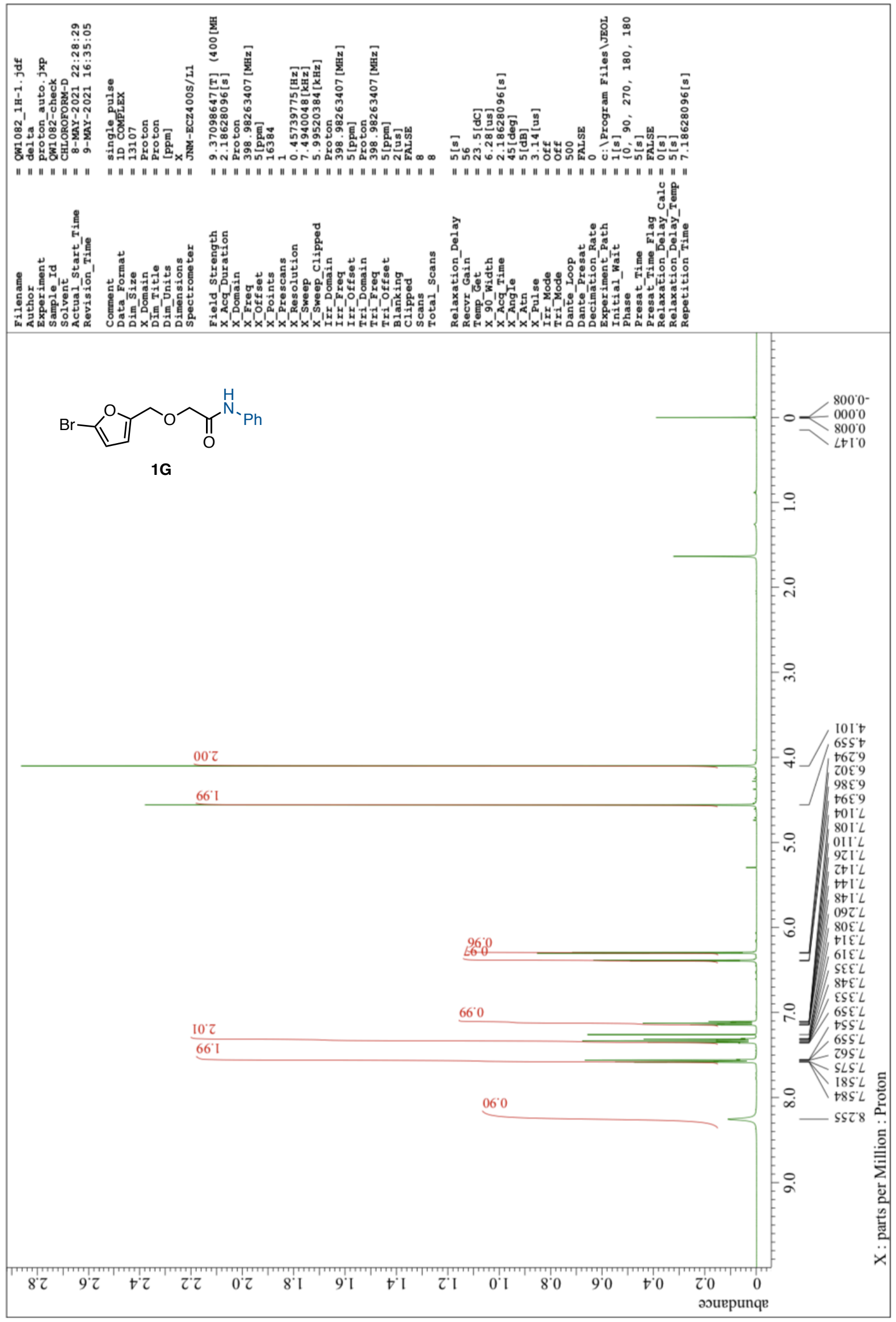


${ }^{13} \mathrm{C}$ NMR of $1 \mathrm{G}\left(101 \mathrm{MHz}, \mathrm{CDCl}_{3}\right)$

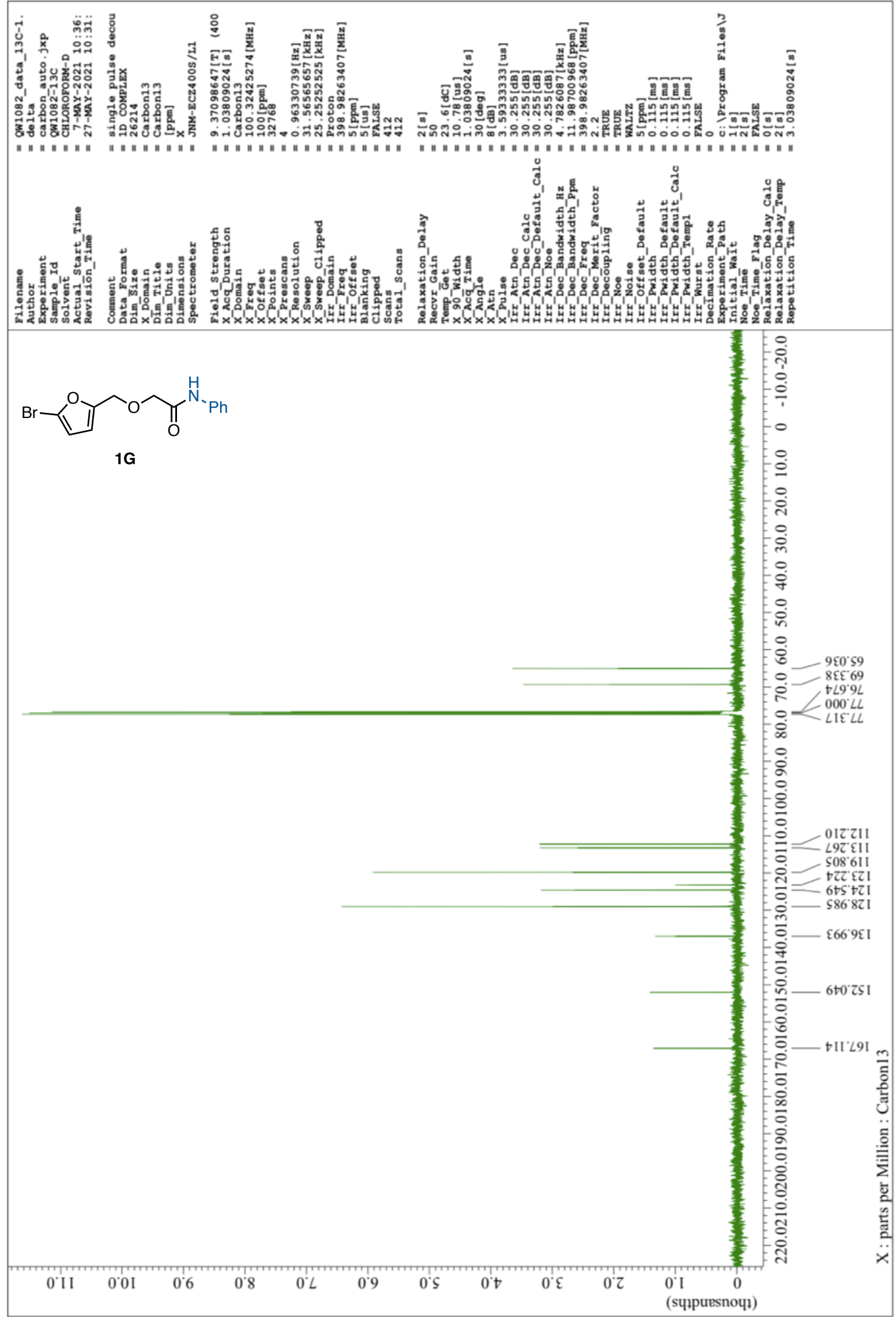


${ }^{1} \mathrm{H}$ NMR of $1 \mathrm{H}\left(400 \mathrm{MHz}, \mathrm{CDCl}_{3}\right)$

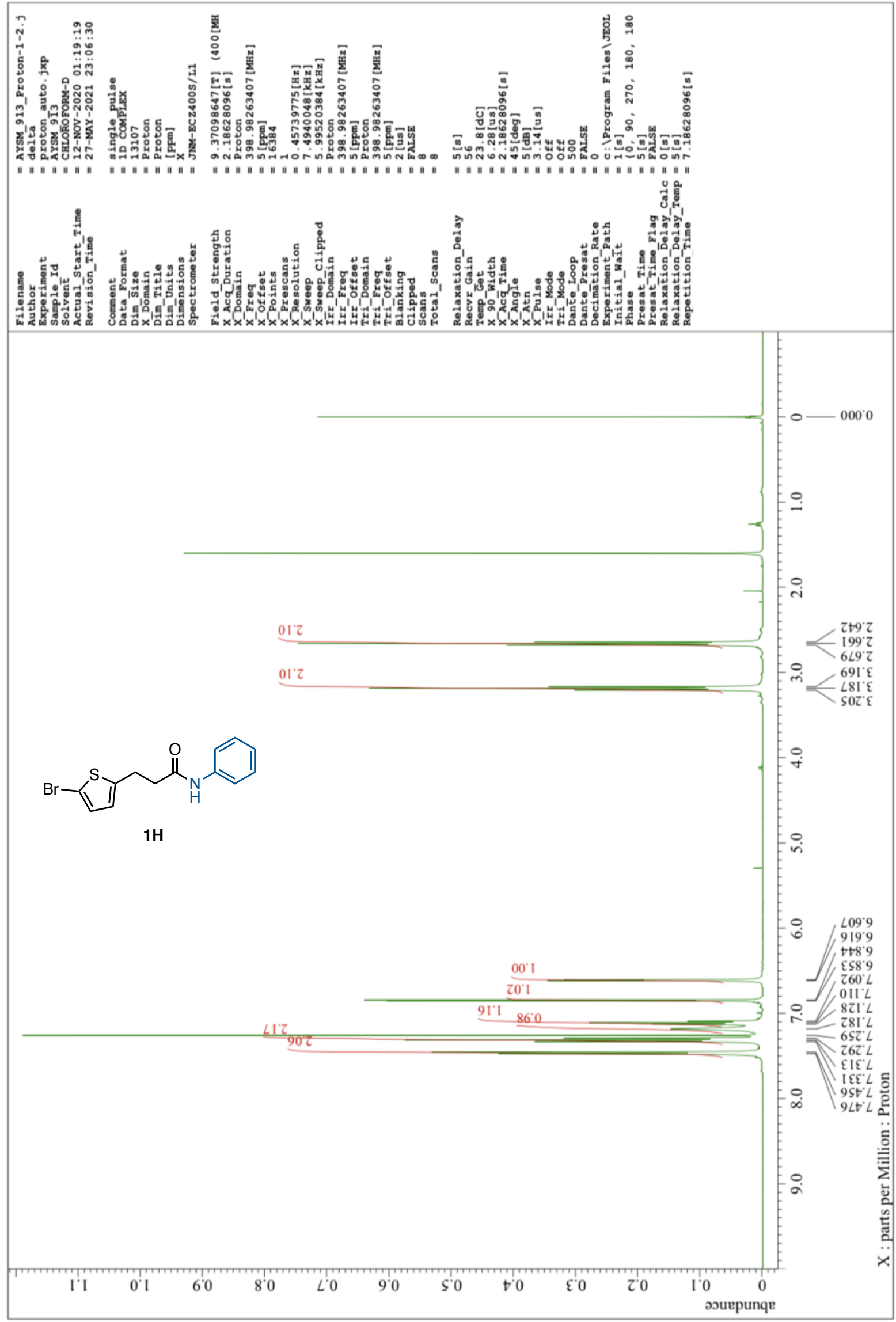


${ }^{13} \mathrm{C}$ NMR of $1 \mathrm{H}\left(101 \mathrm{MHz}, \mathrm{CDCl}_{3}\right)$

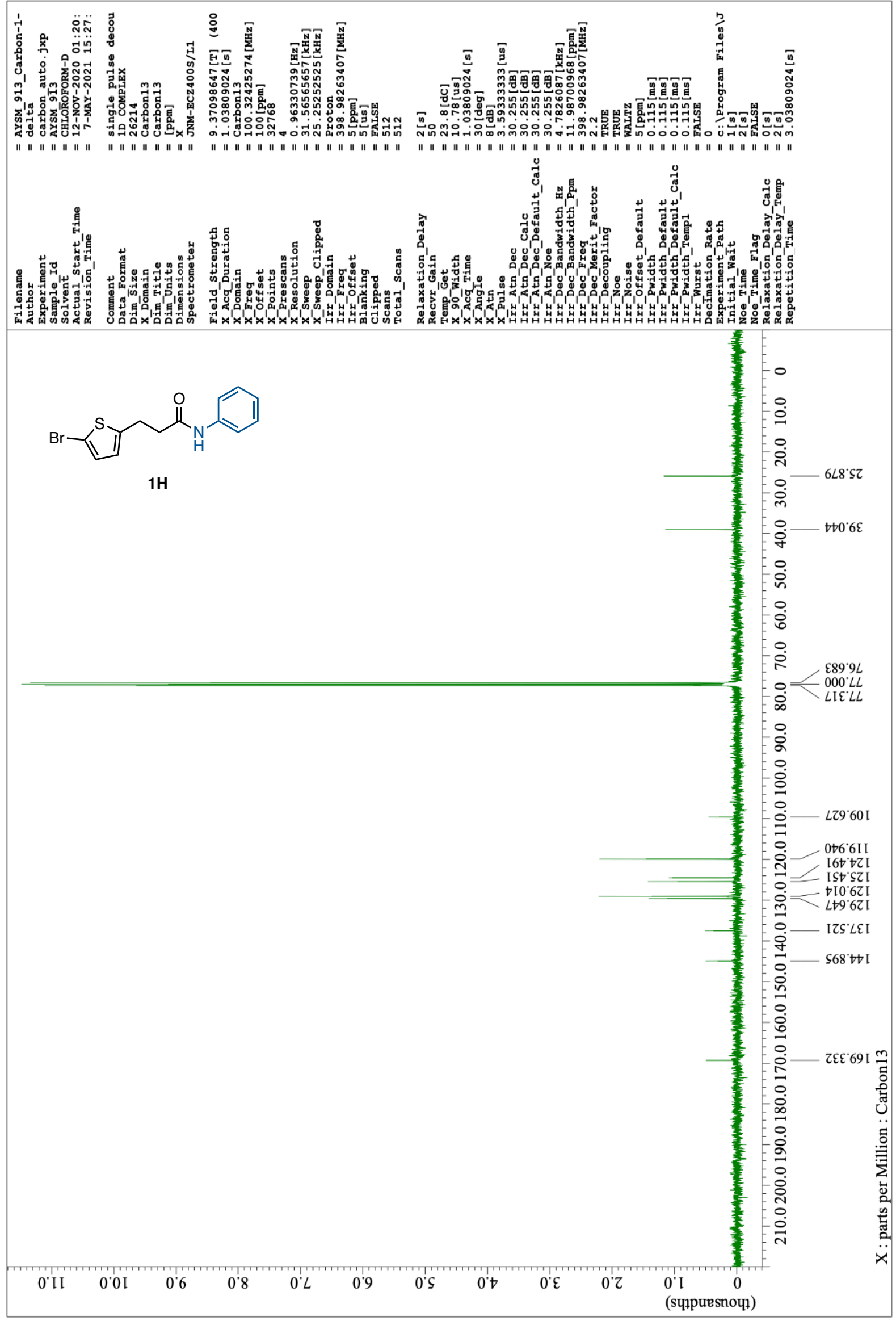


${ }^{1} \mathrm{H}$ NMR of 1I (400 MHz, $\left.\mathrm{CDCl}_{3}\right)$

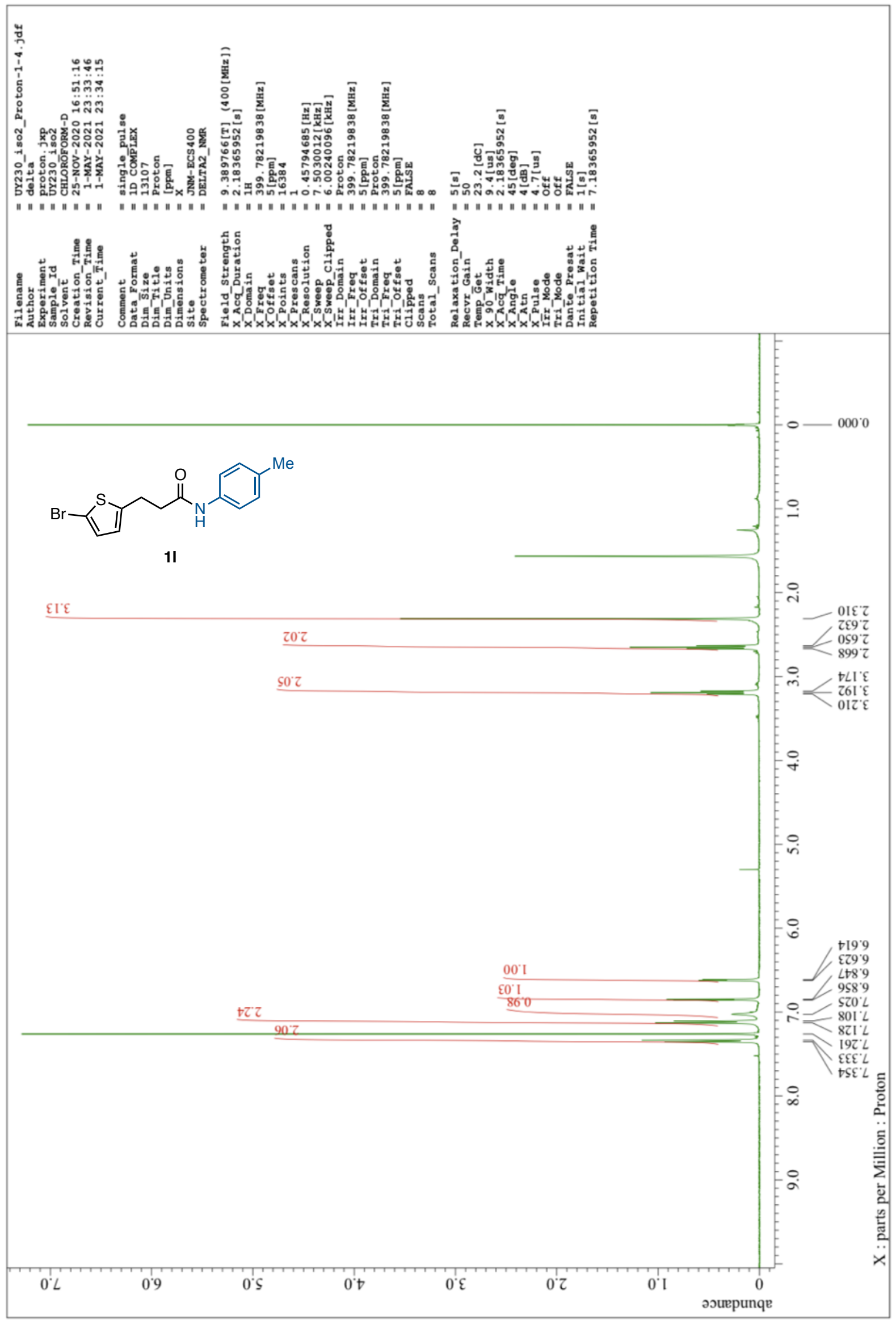


${ }^{13} \mathrm{C}$ NMR of $1 \mathrm{I}\left(101 \mathrm{MHz}, \mathrm{CDCl}_{3}\right)$

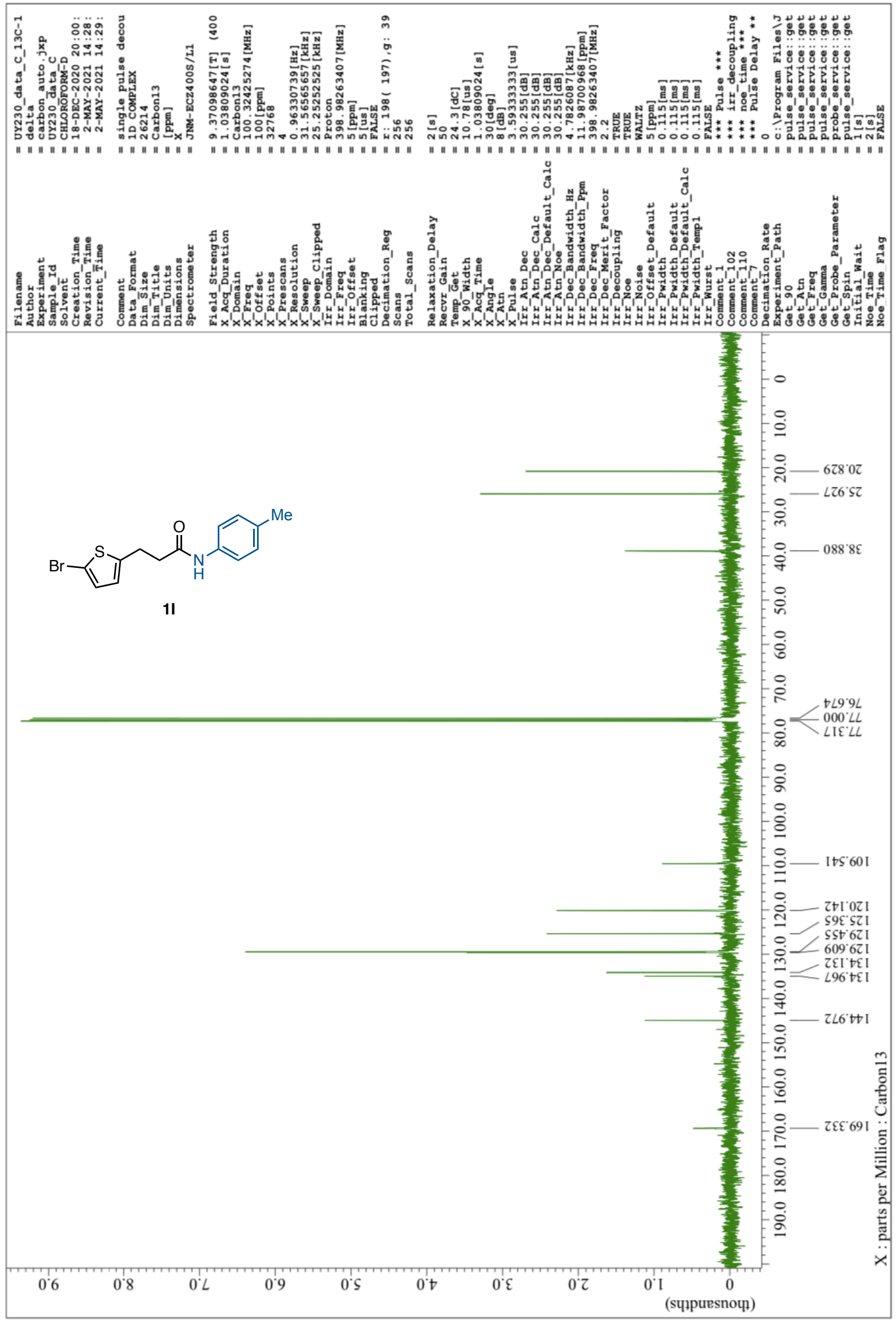


${ }^{1} \mathrm{H}$ NMR of $1 \mathrm{~J}\left(400 \mathrm{MHz}, \mathrm{CDCl}_{3}\right)$

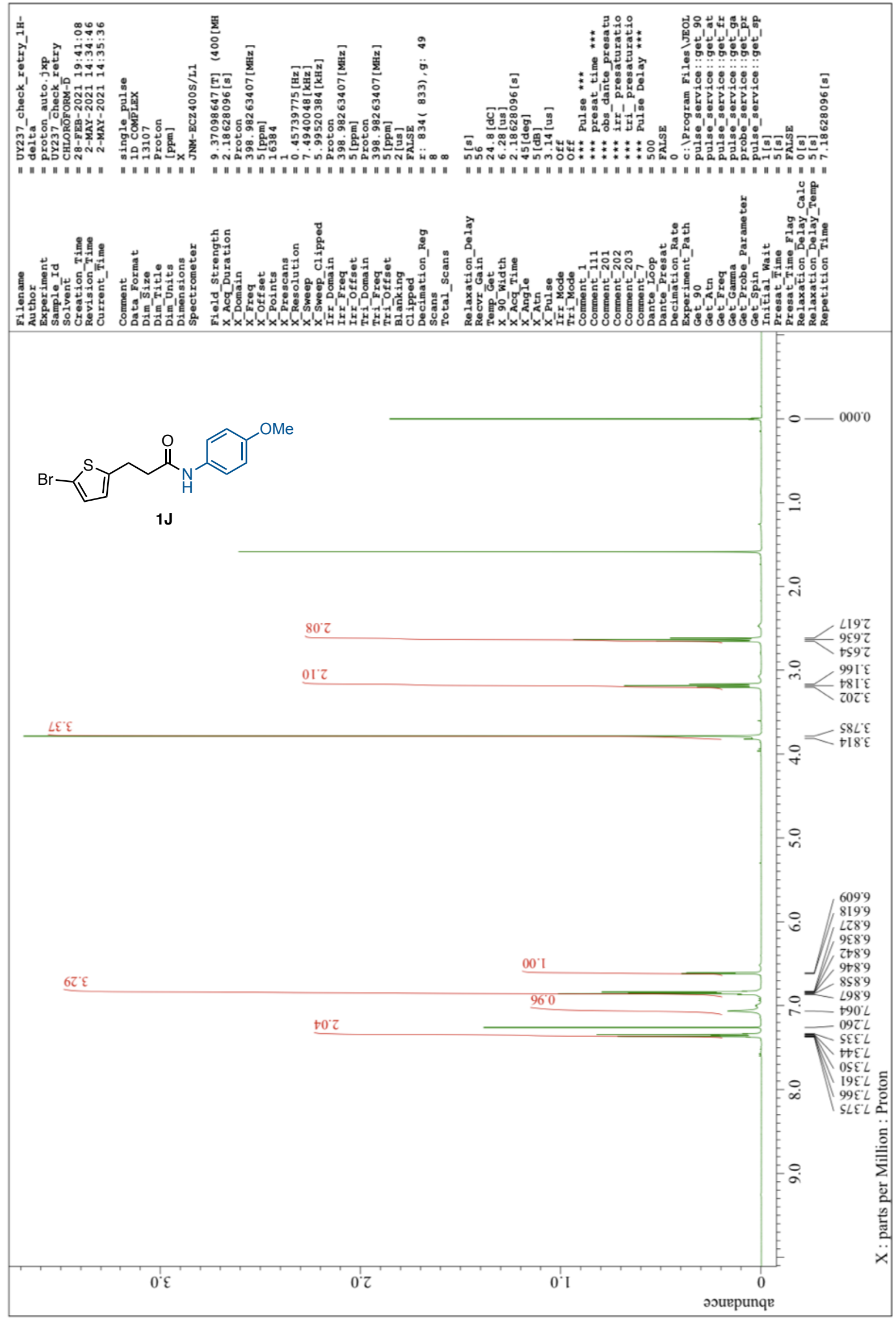


${ }^{13} \mathrm{C}$ NMR of $1 \mathrm{~J}\left(101 \mathrm{MHz}, \mathrm{CDCl}_{3}\right)$

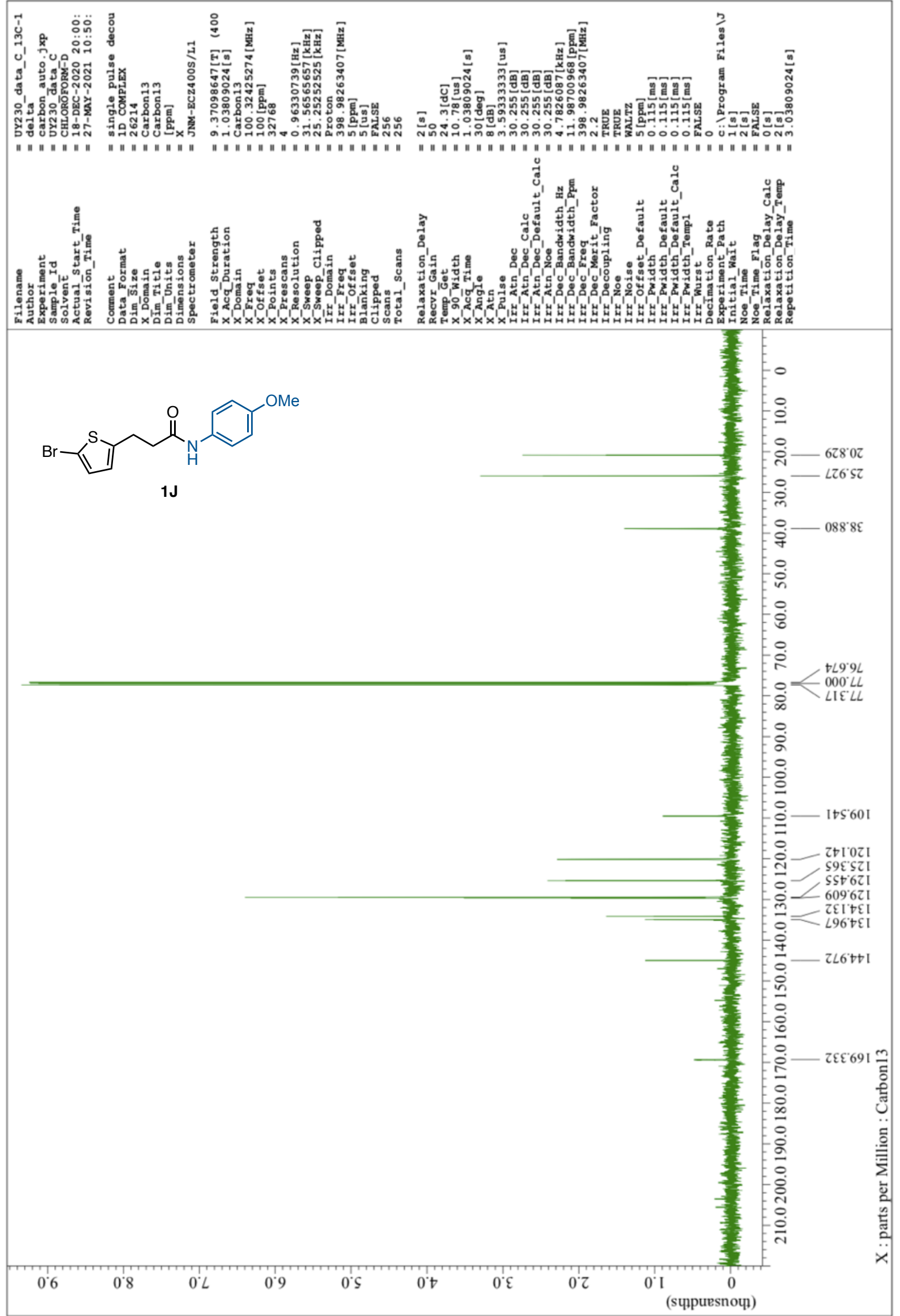


${ }^{1} \mathrm{H}$ NMR of $1 \mathrm{~K}\left(400 \mathrm{MHz}, \mathrm{CDCl}_{3}\right)$

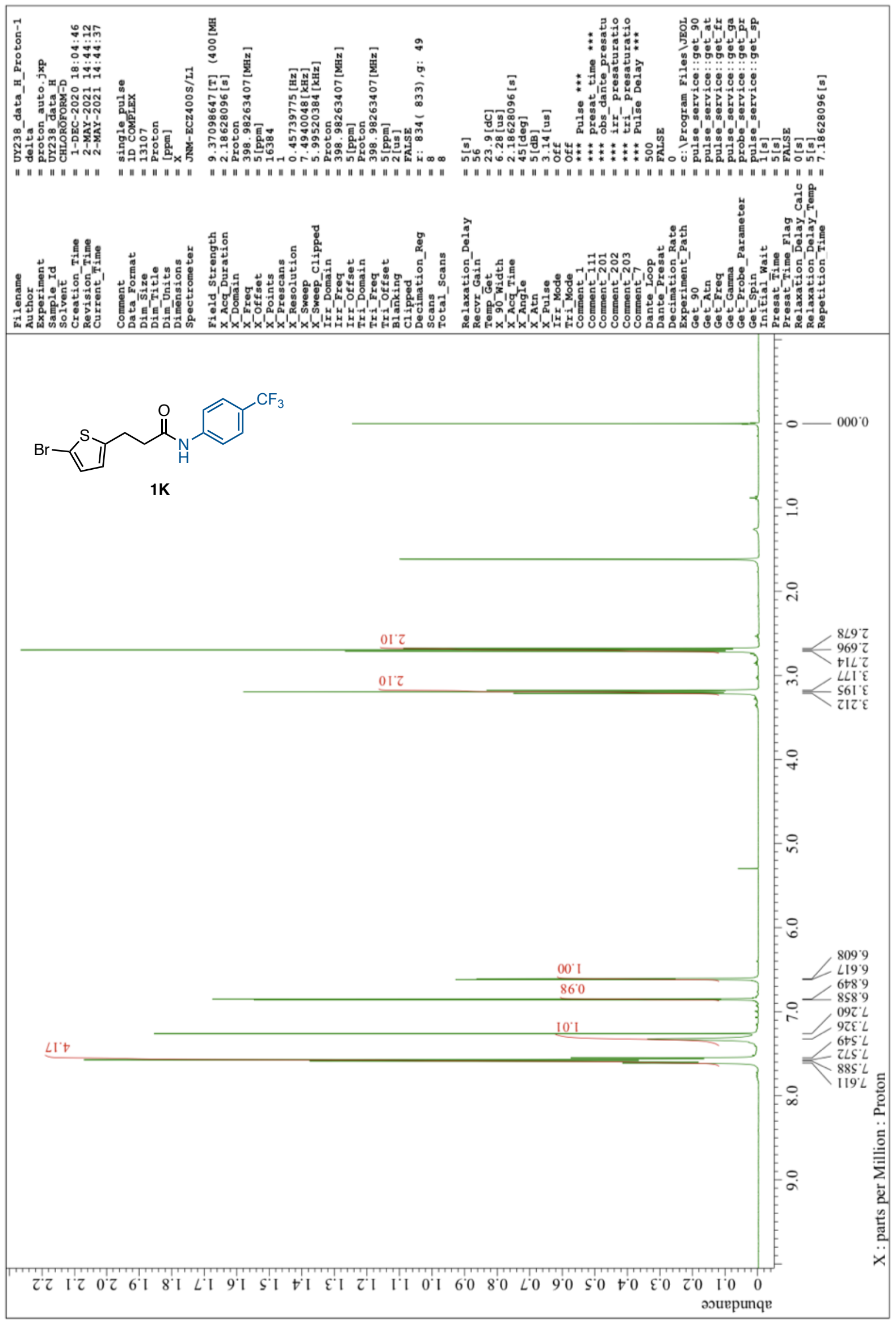


${ }^{13} \mathrm{C}$ NMR of $1 \mathrm{~K}\left(101 \mathrm{MHz}, \mathrm{CDCl}_{3}\right)$

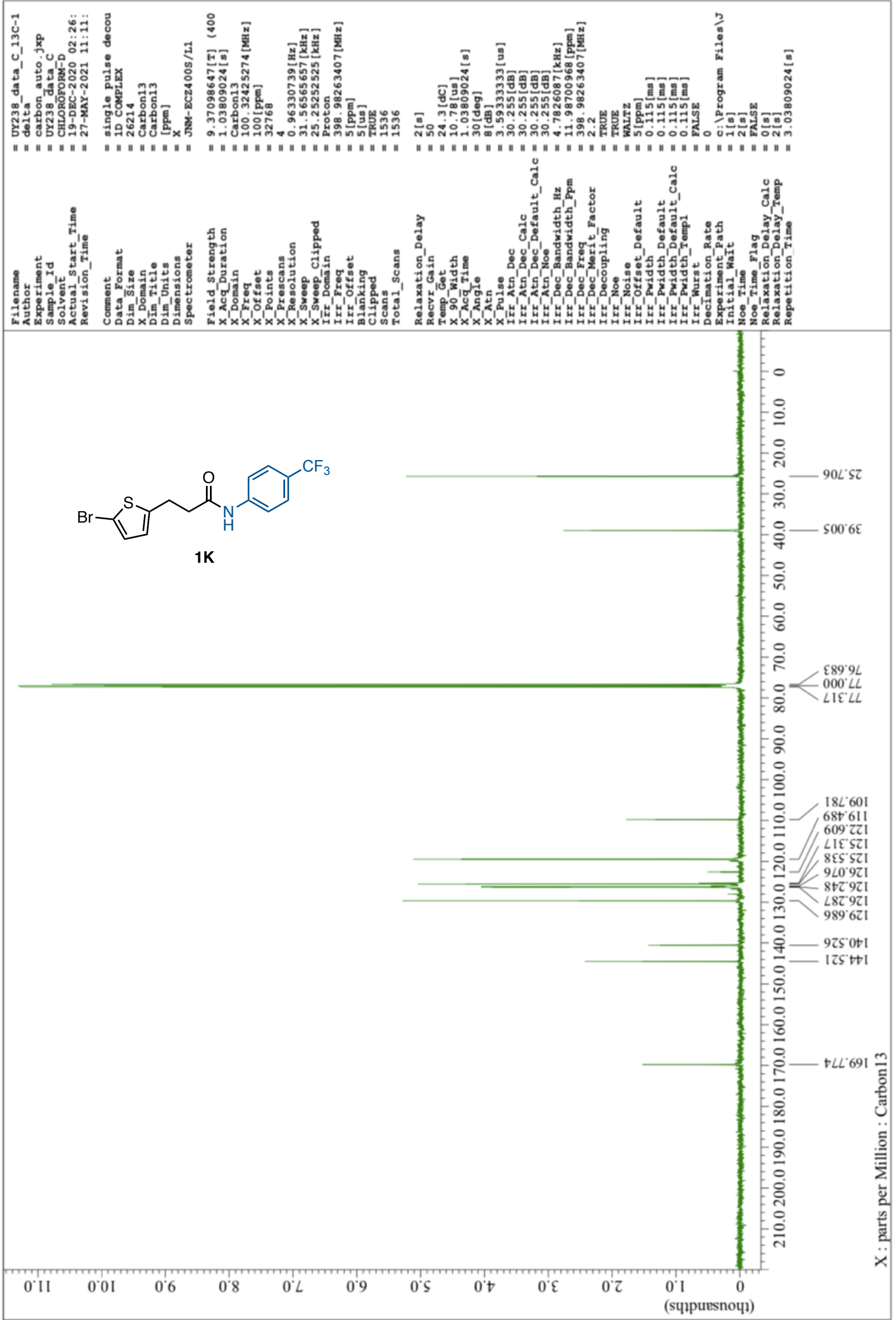


19F NMR of $1 \mathrm{~K}\left(376 \mathrm{MHz}, \mathrm{CDCl}_{3}\right)$

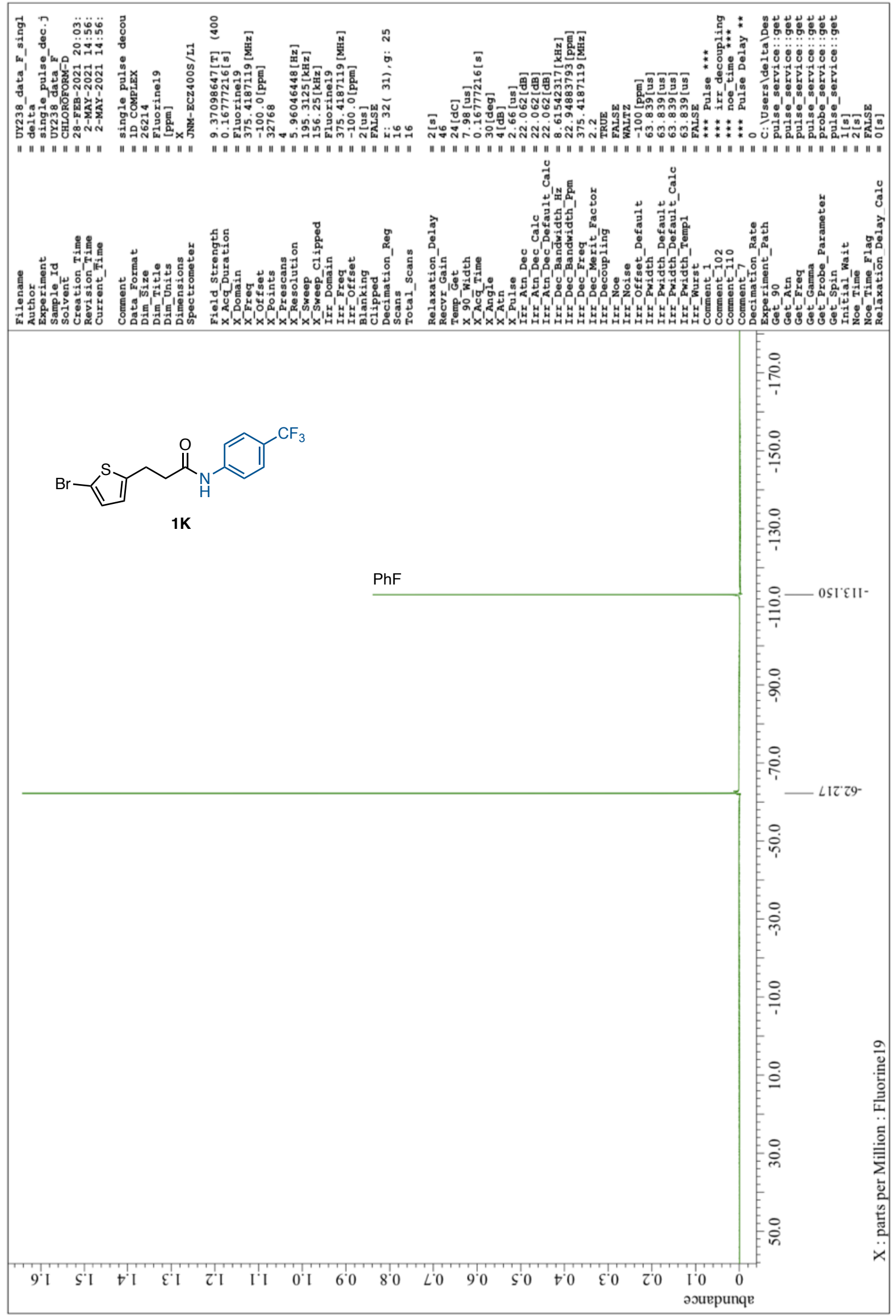


${ }^{1} \mathrm{H}$ NMR of $1 \mathrm{~L}\left(400 \mathrm{MHz}, \mathrm{CDCl}_{3}\right)$

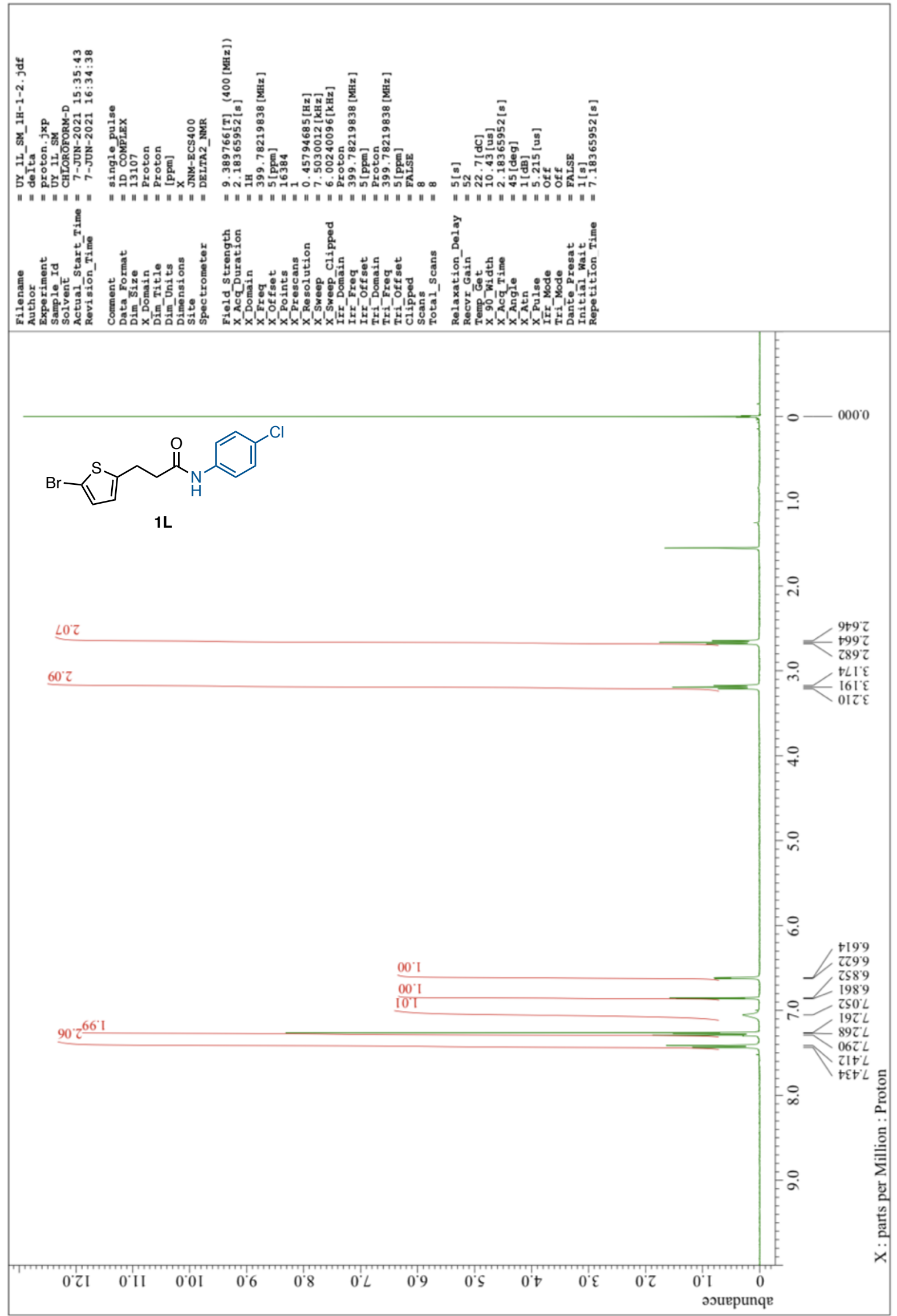


${ }^{13} \mathrm{C}$ NMR of 1L (101 MHz, $\left.\mathrm{CDCl}_{3}\right)$

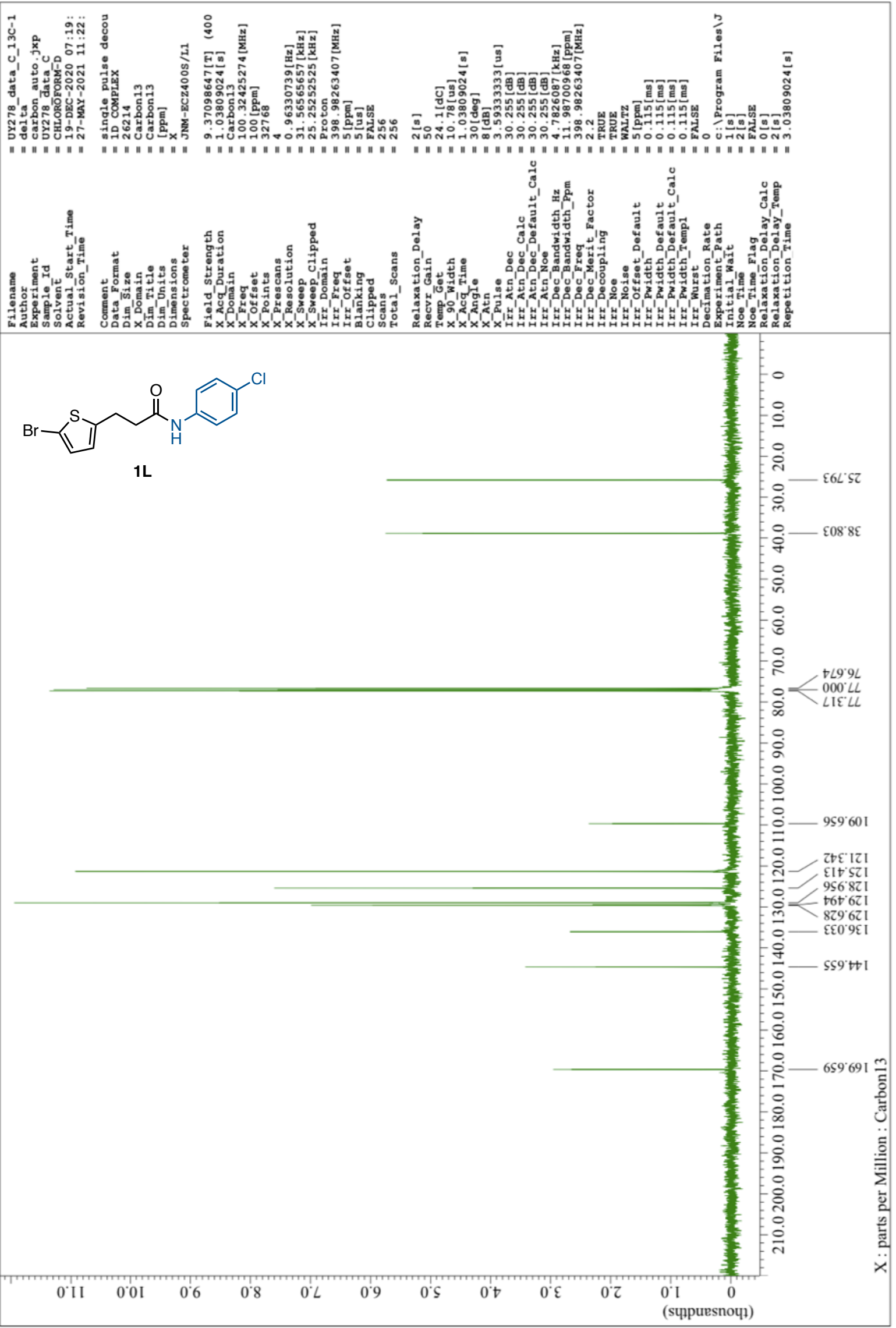


${ }^{1} \mathrm{H}$ NMR of $1 \mathrm{M}\left(400 \mathrm{MHz}, \mathrm{CDCl}_{3}\right)$

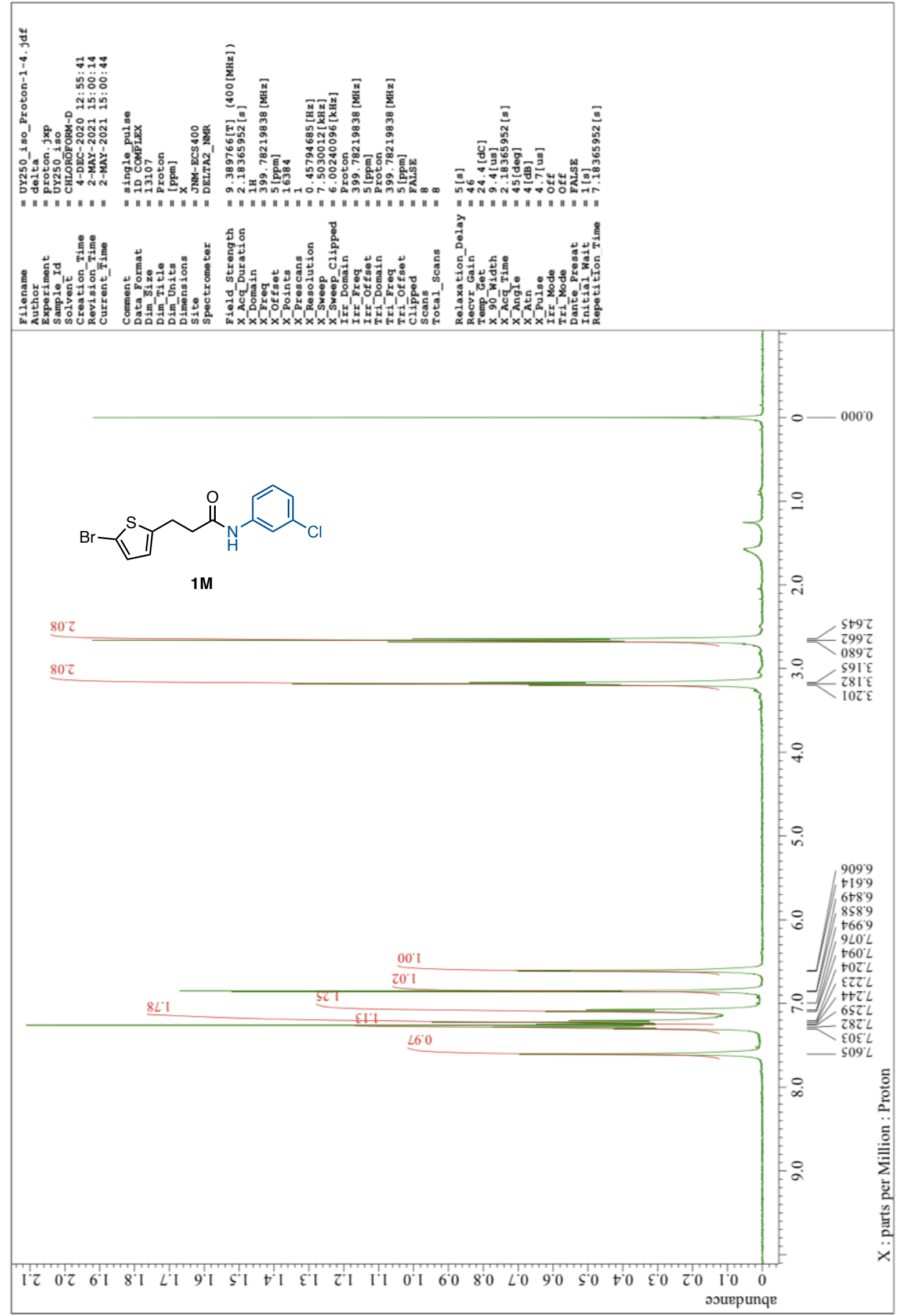


${ }^{13} \mathrm{C}$ NMR of $1 \mathrm{M}\left(101 \mathrm{MHz}, \mathrm{CDCl}_{3}\right)$

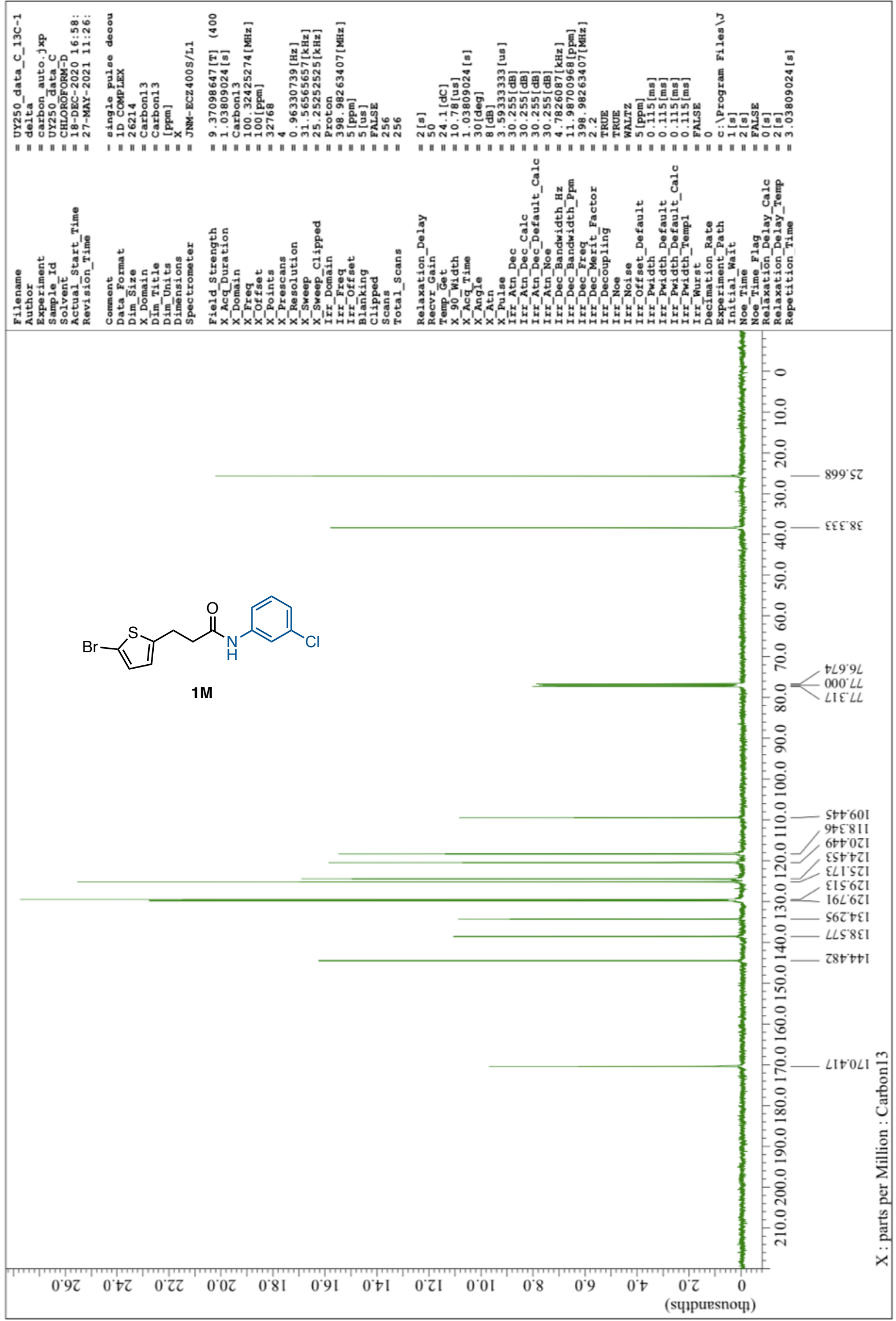


${ }^{1} \mathrm{H}$ NMR of $1 \mathrm{~N}\left(400 \mathrm{MHz}, \mathrm{CDCl}_{3}\right)$

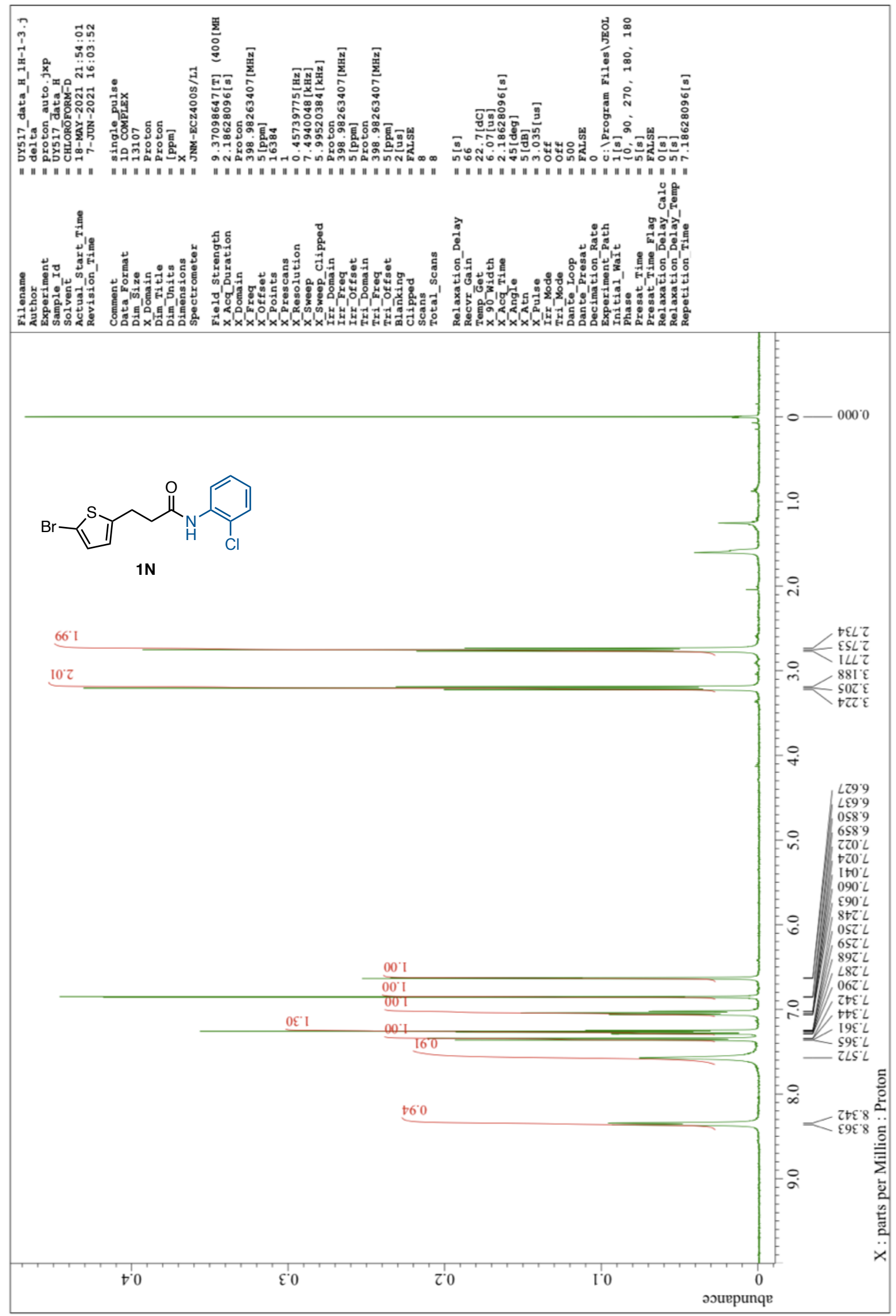


${ }^{13} \mathrm{C}$ NMR of $1 \mathrm{~N}\left(101 \mathrm{MHz}, \mathrm{CDCl}_{3}\right)$

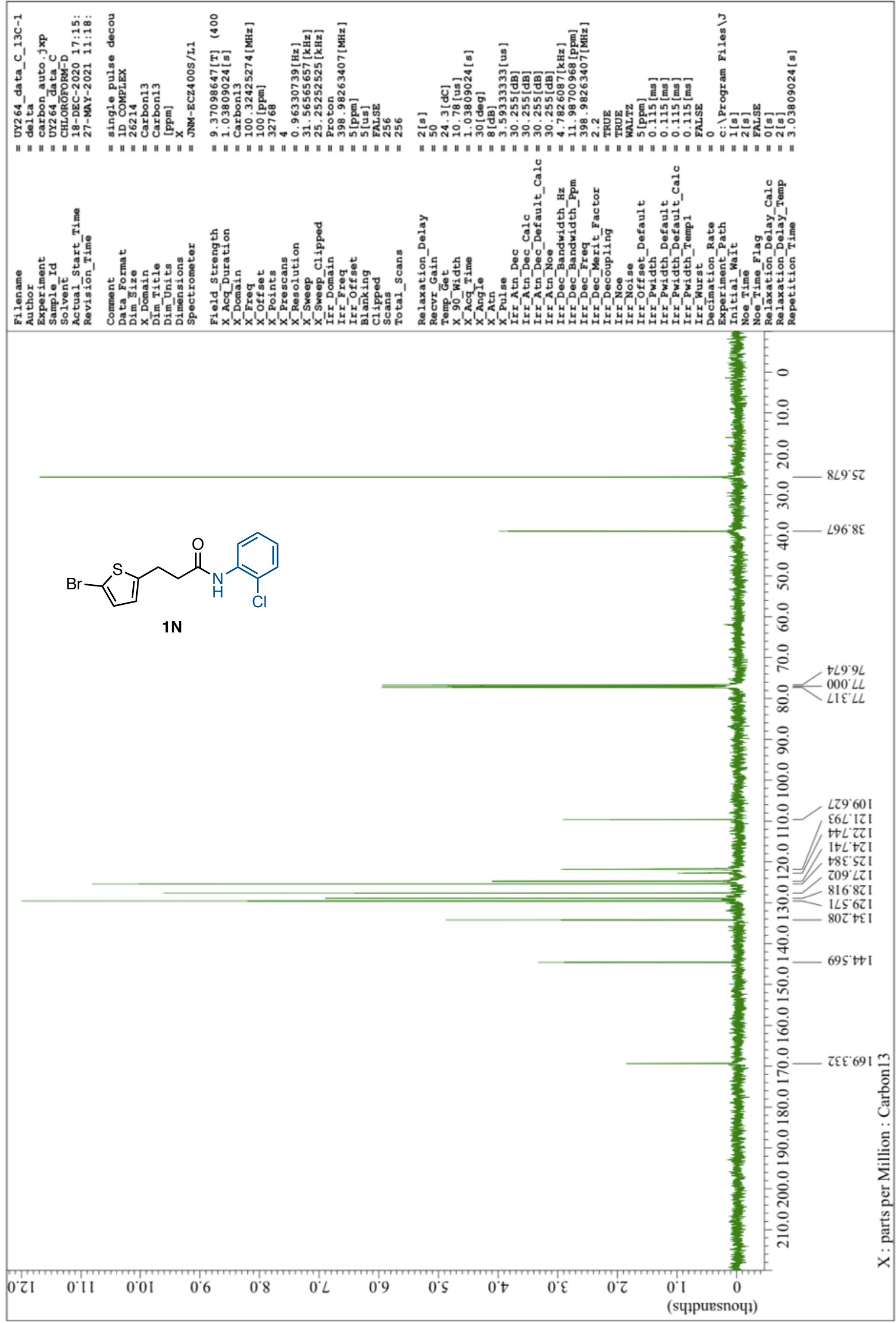


${ }^{1} \mathrm{H}$ NMR of $10\left(400 \mathrm{MHz}, \mathrm{CDCl}_{3}\right)$

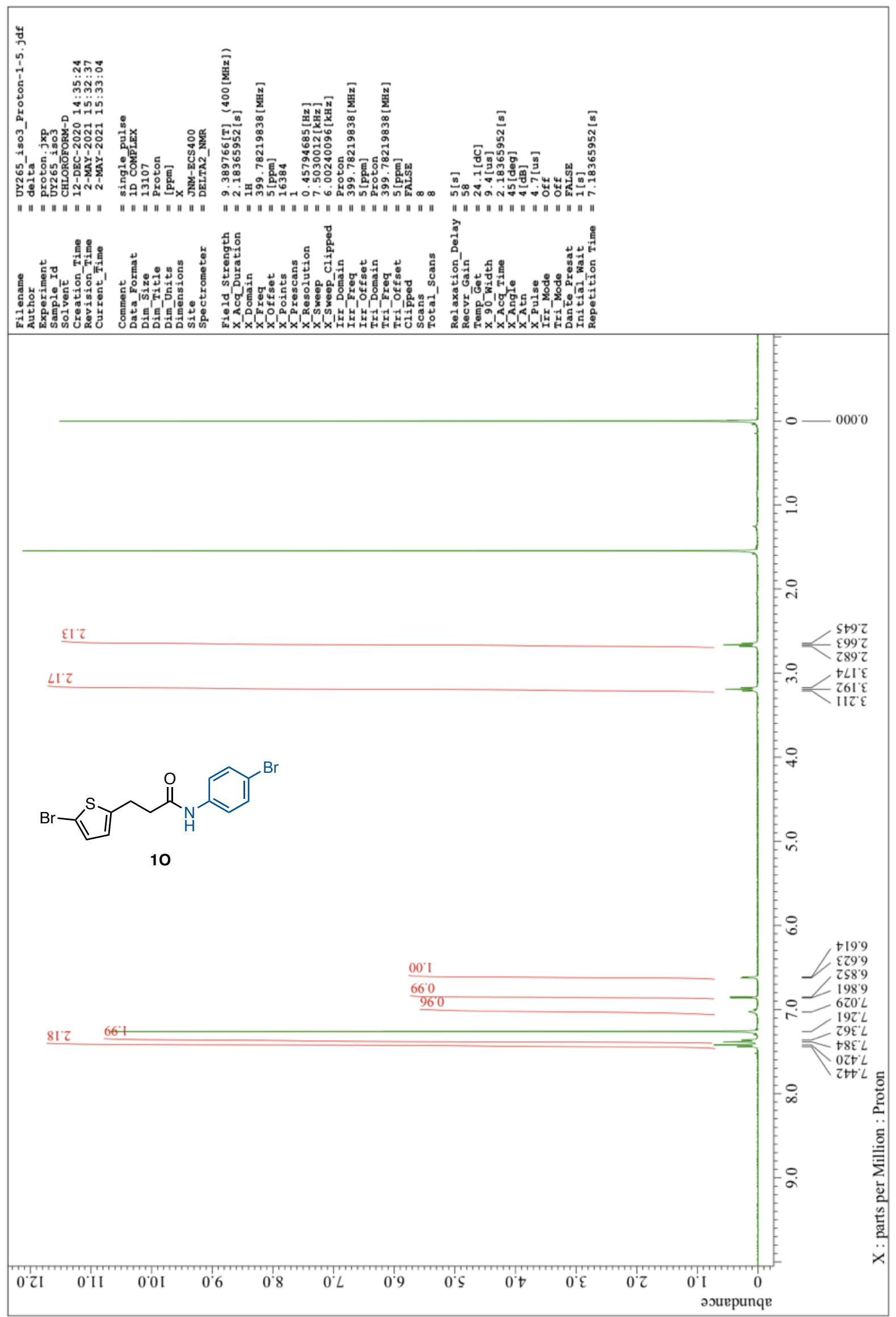


${ }^{13} \mathrm{C}$ NMR of $10\left(101 \mathrm{MHz}, \mathrm{CDCl}_{3}\right)$

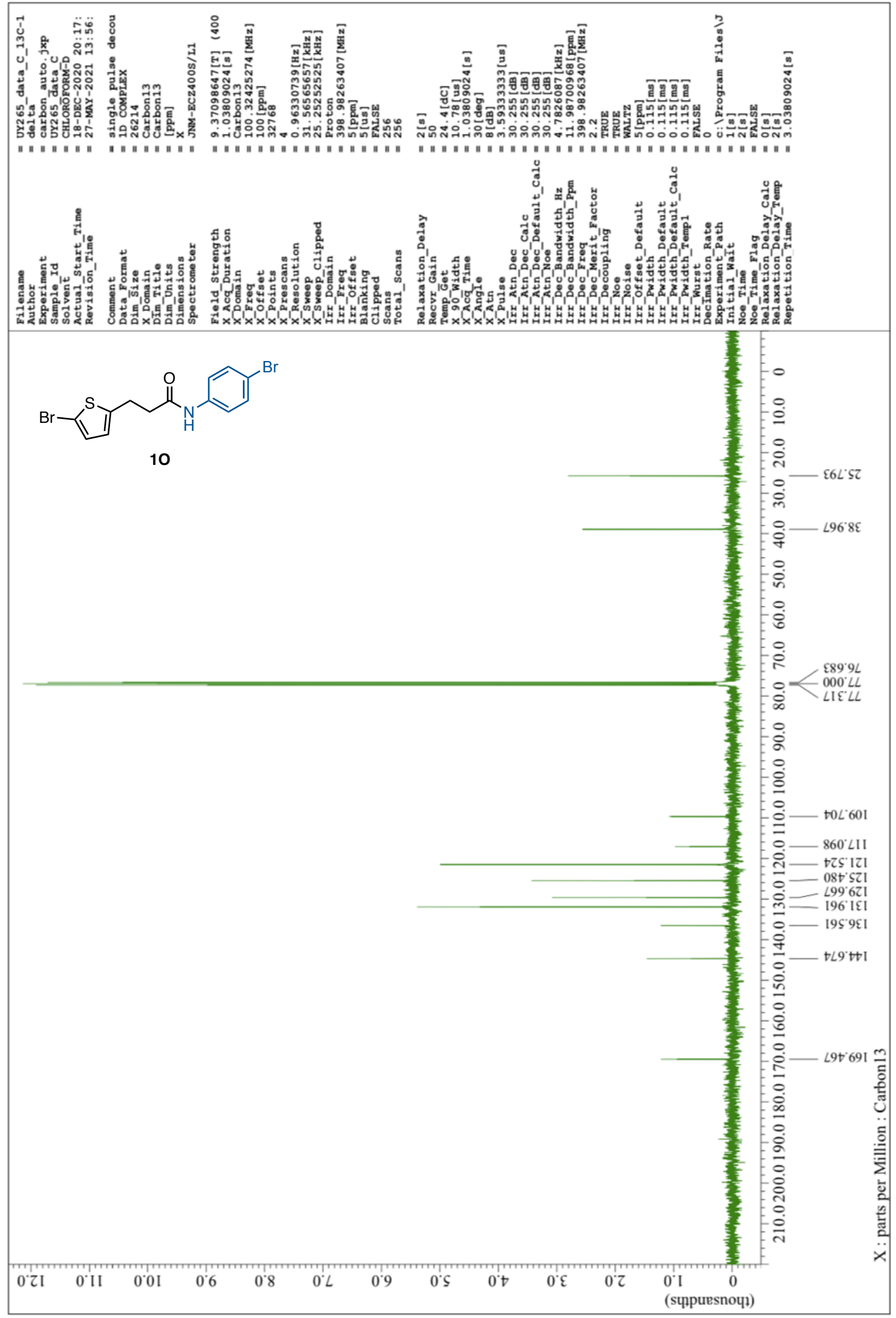


${ }^{1} \mathrm{H}$ NMR of 1P (400 MHz, $\left.\mathrm{CDCl}_{3}\right)$

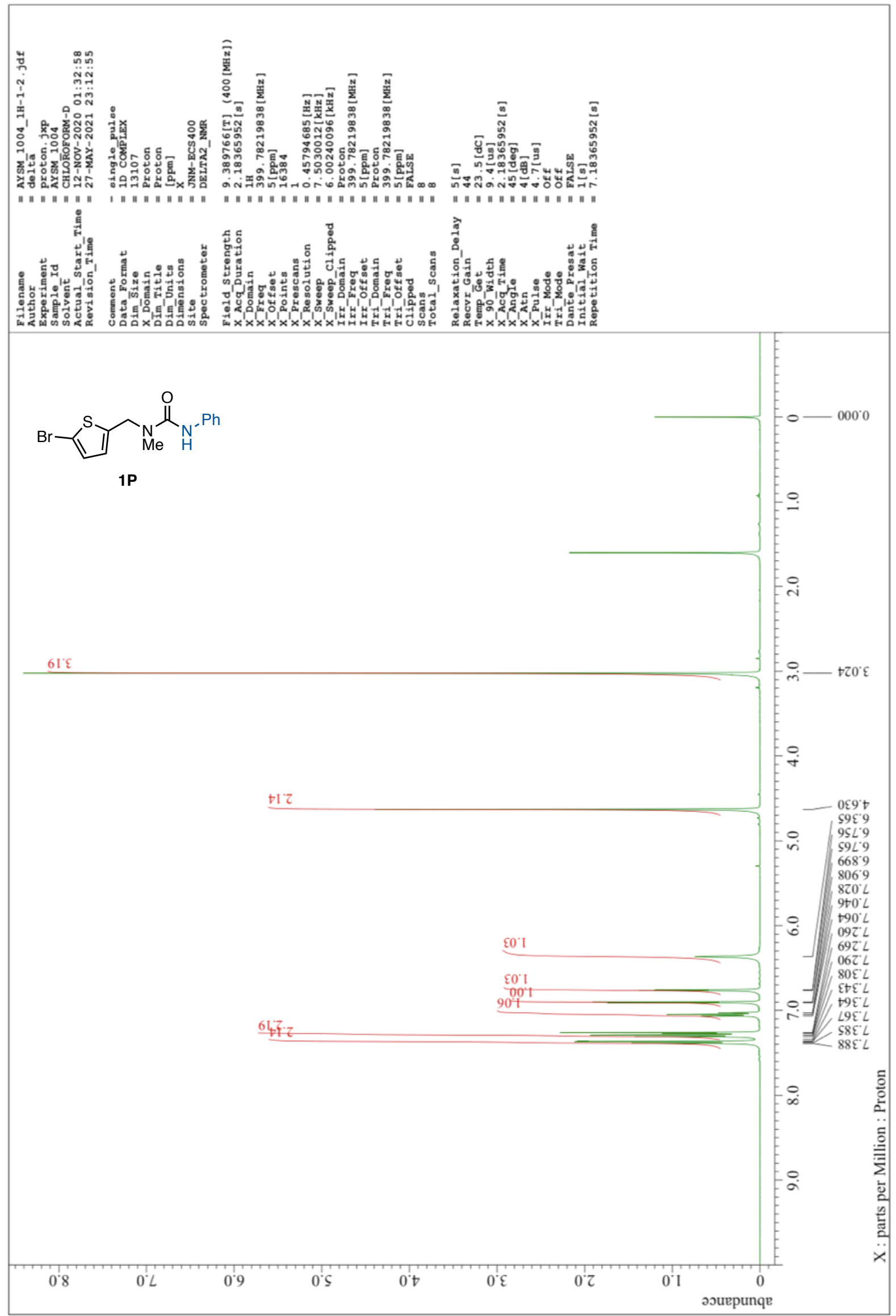


${ }^{13}$ C NMR of 1P (101 MHz, DMSO- $\left.d_{6}\right)$

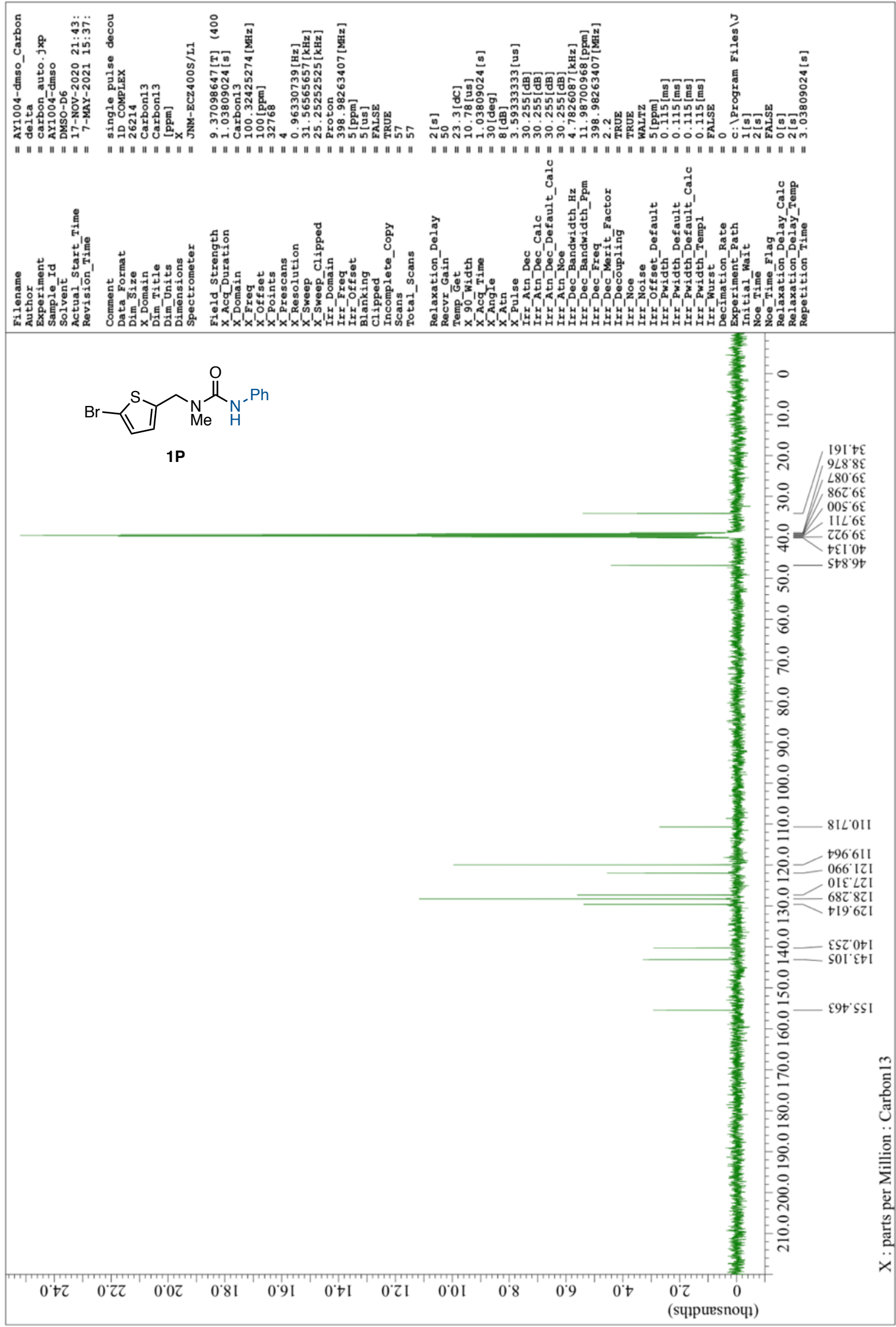


${ }^{1} \mathrm{H}$ NMR of $1 \mathrm{Q}\left(400 \mathrm{MHz}, \mathrm{CDCl}_{3}\right)$

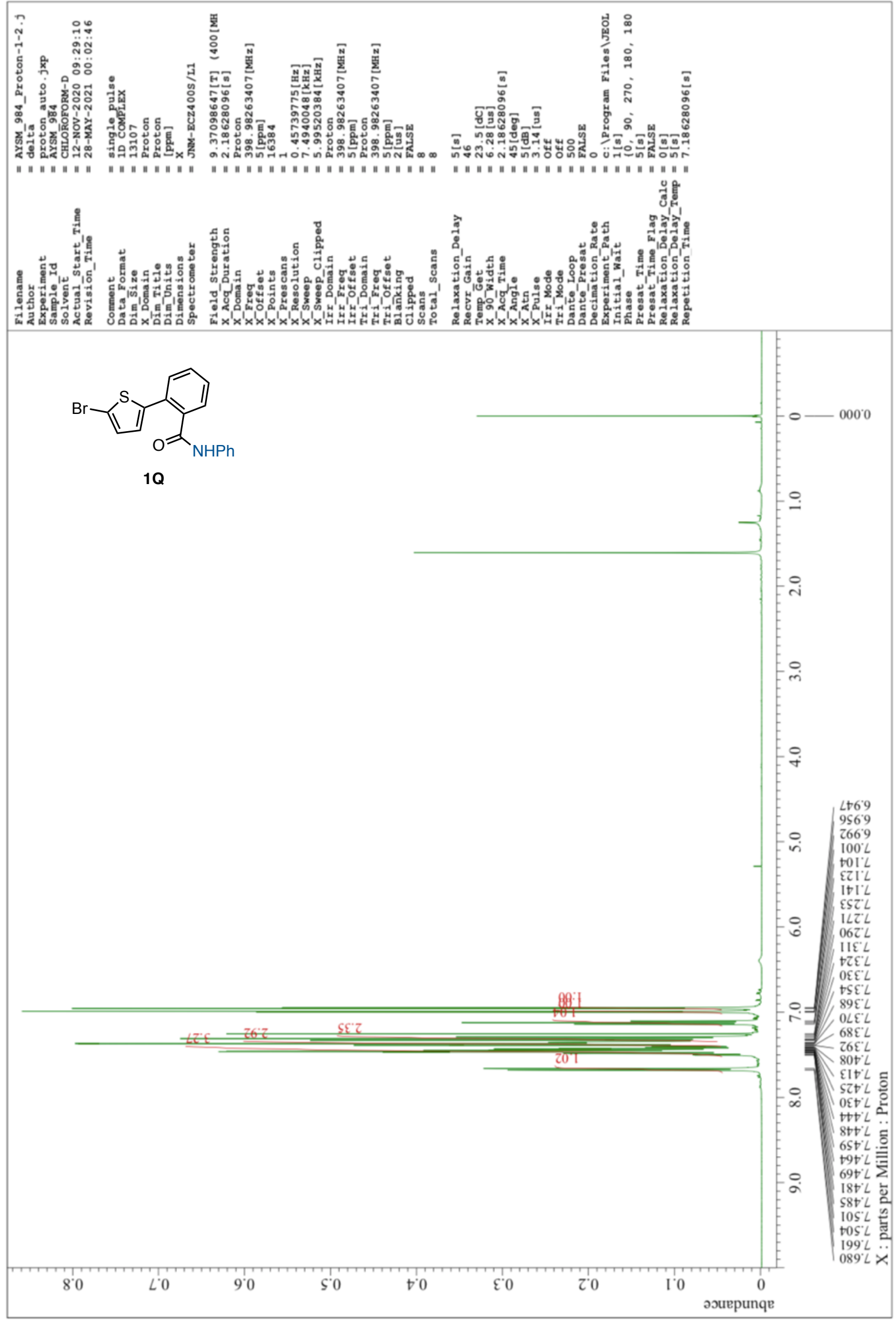


${ }^{13} \mathrm{C}$ NMR of $1 \mathrm{Q}\left(101 \mathrm{MHz}, \mathrm{CDCl}_{3}, 333 \mathrm{~K}\right)$

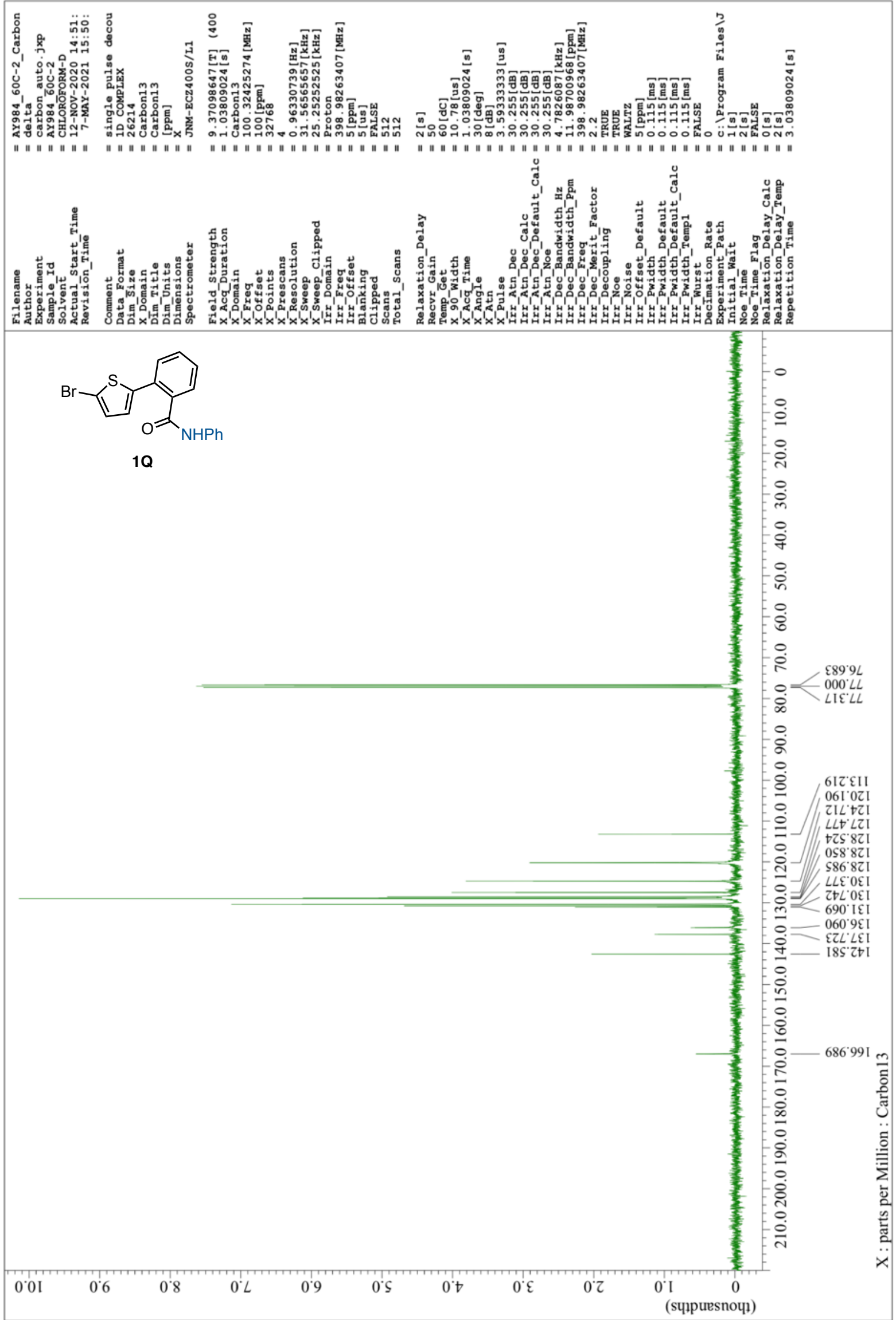


${ }^{1} \mathrm{H}$ NMR of $1 \mathrm{R}\left(400 \mathrm{MHz}, \mathrm{CDCl}_{3}\right)$

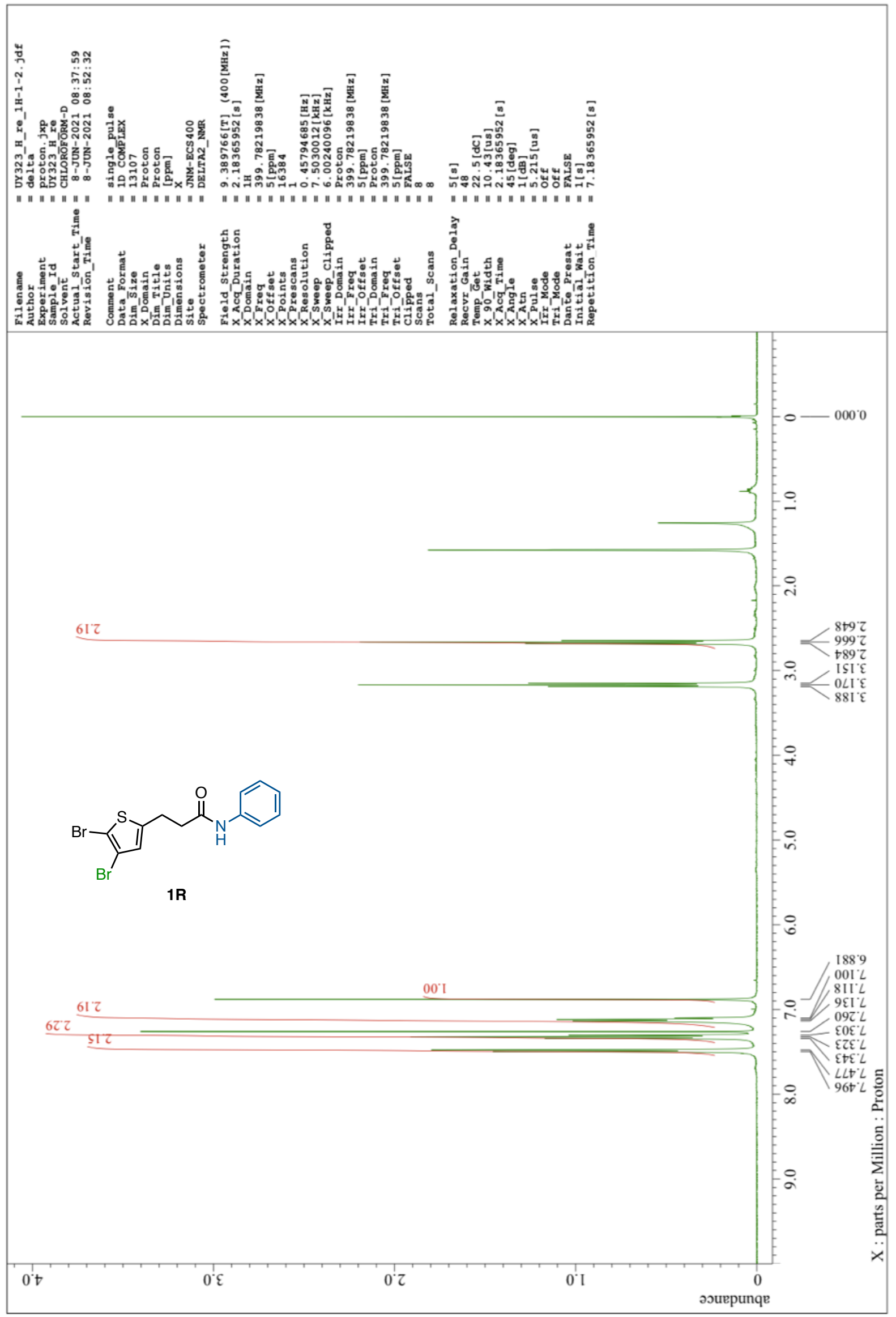


${ }^{13} \mathrm{C}$ NMR of $1 \mathrm{R}\left(101 \mathrm{MHz}, \mathrm{CDCl}_{3}\right)$

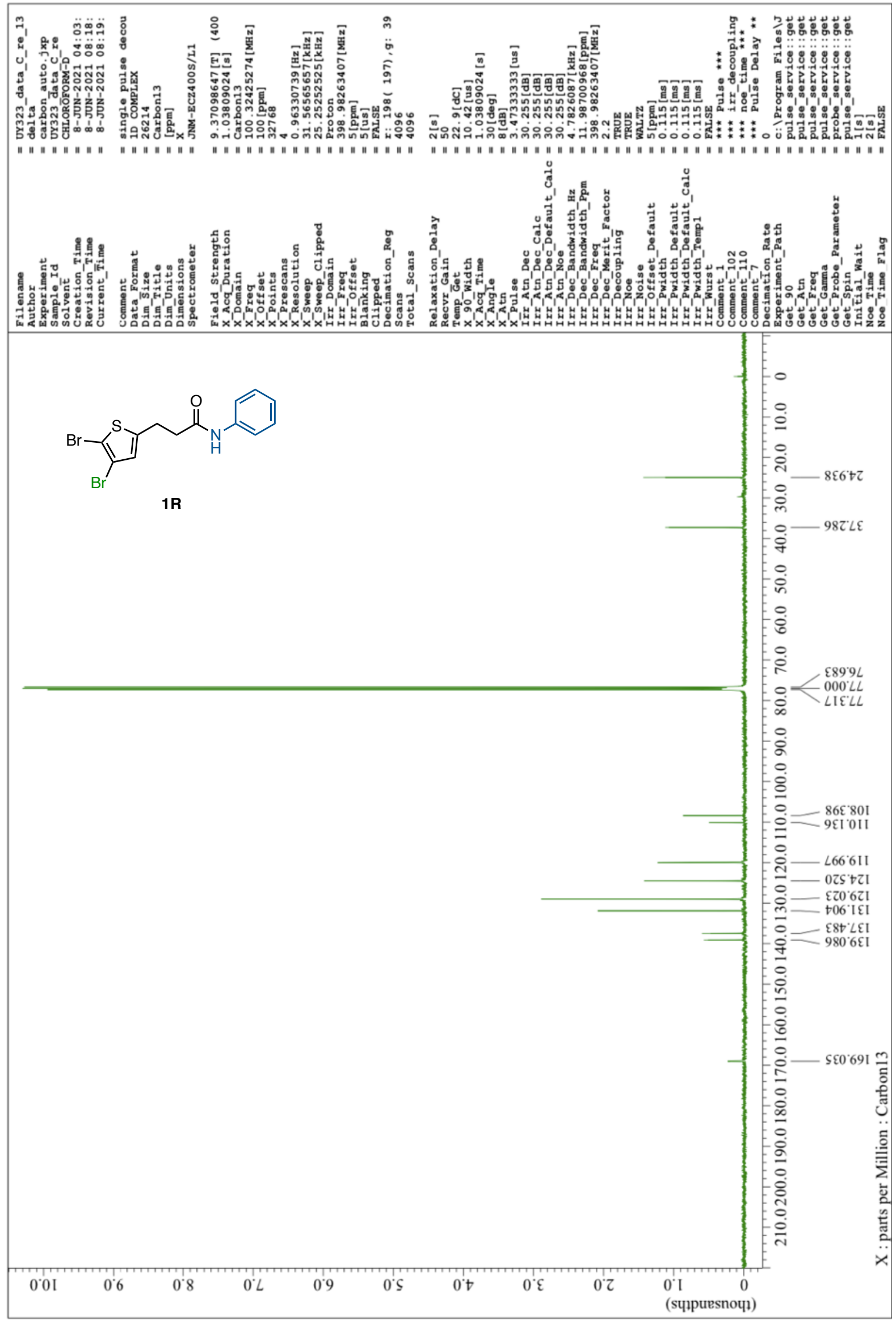


${ }^{1} \mathrm{H}$ NMR of $1 \mathrm{~S}\left(400 \mathrm{MHz}, \mathrm{CDCl}_{3}\right)$

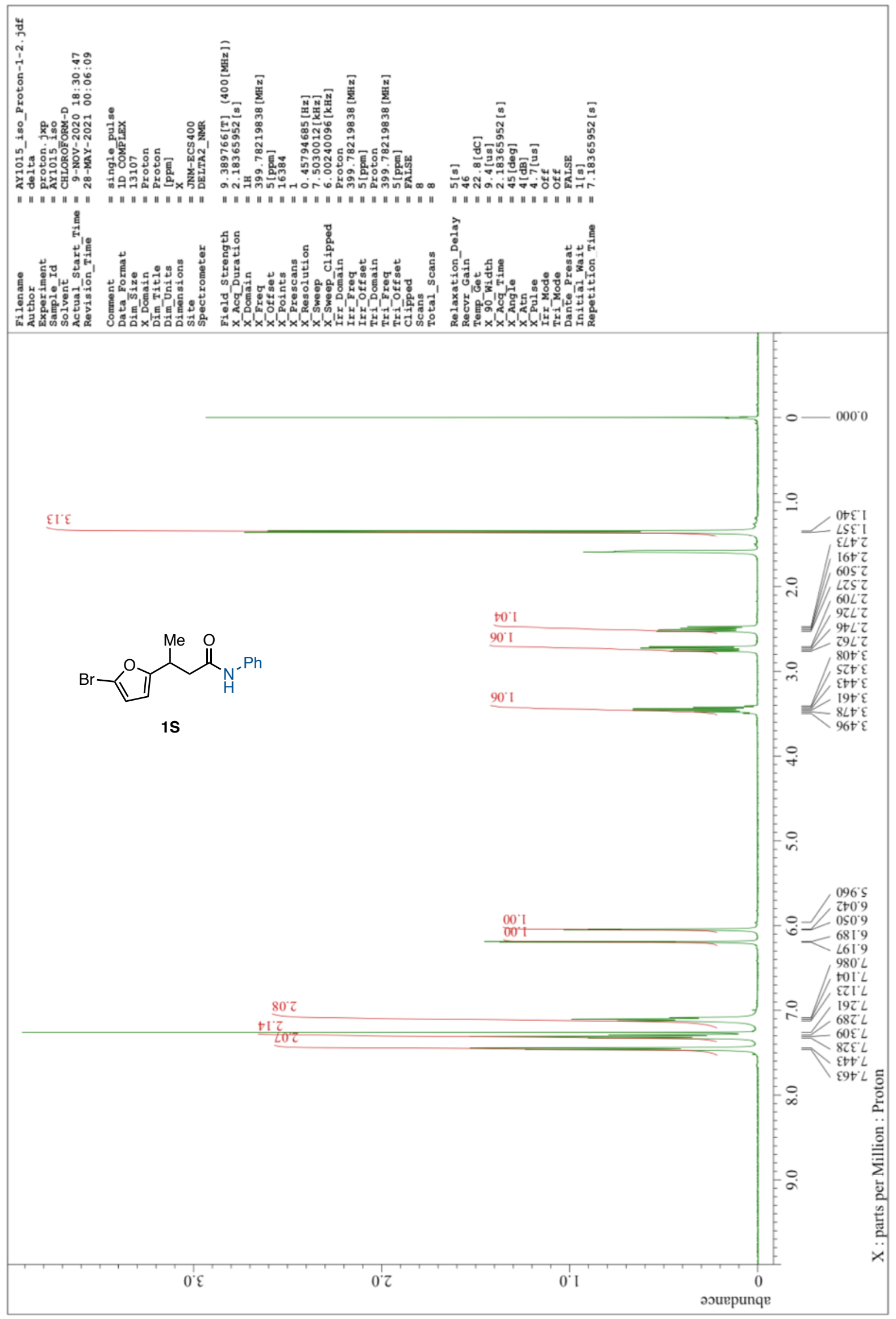


${ }^{13}$ C NMR of $1 S$ (101 MHz, DMSO- $\left.d_{6}\right)$

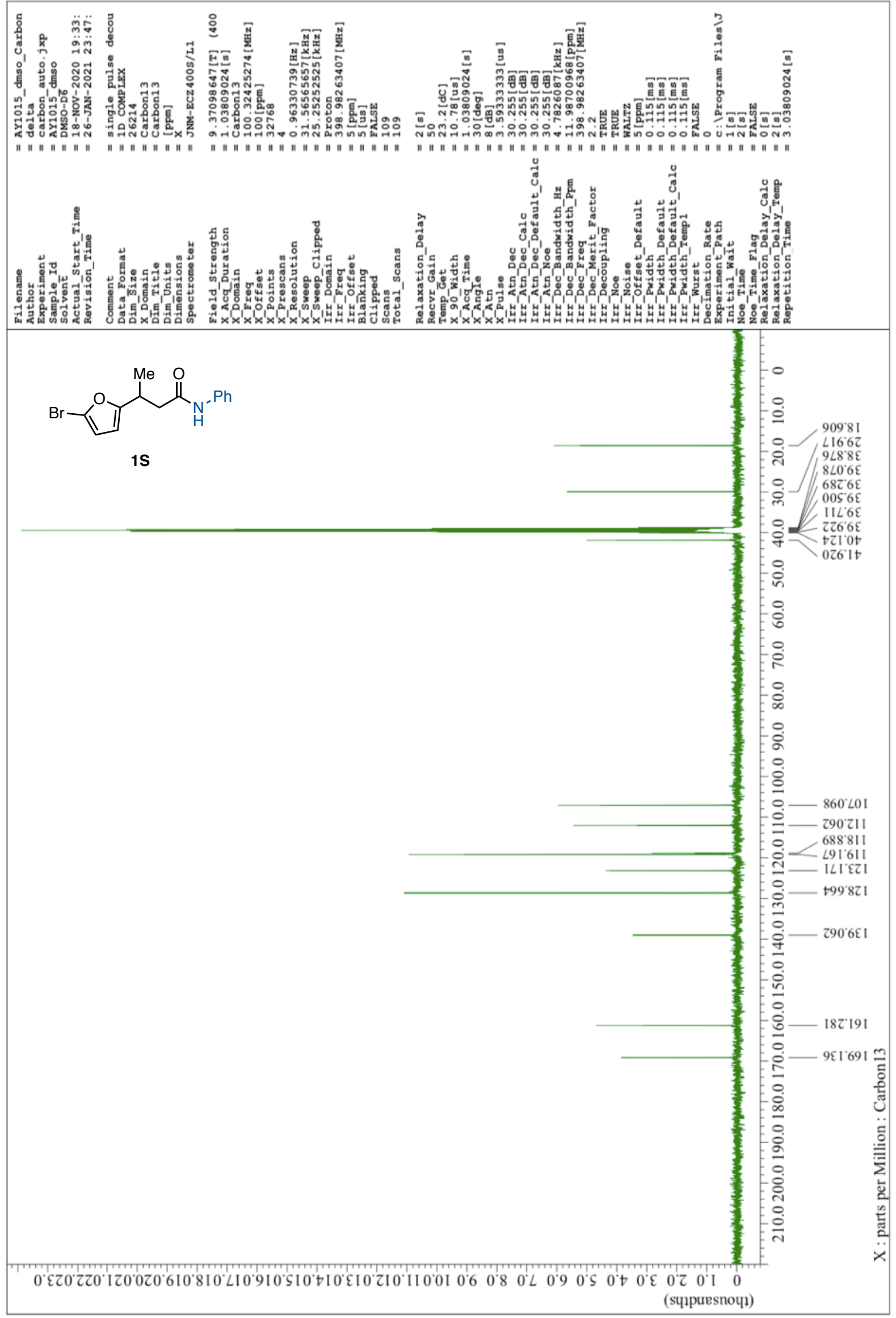


${ }^{1} \mathrm{H}$ NMR of $1 \mathrm{~T}$ (400 MHz, $\mathrm{CDCl}_{3}$ )

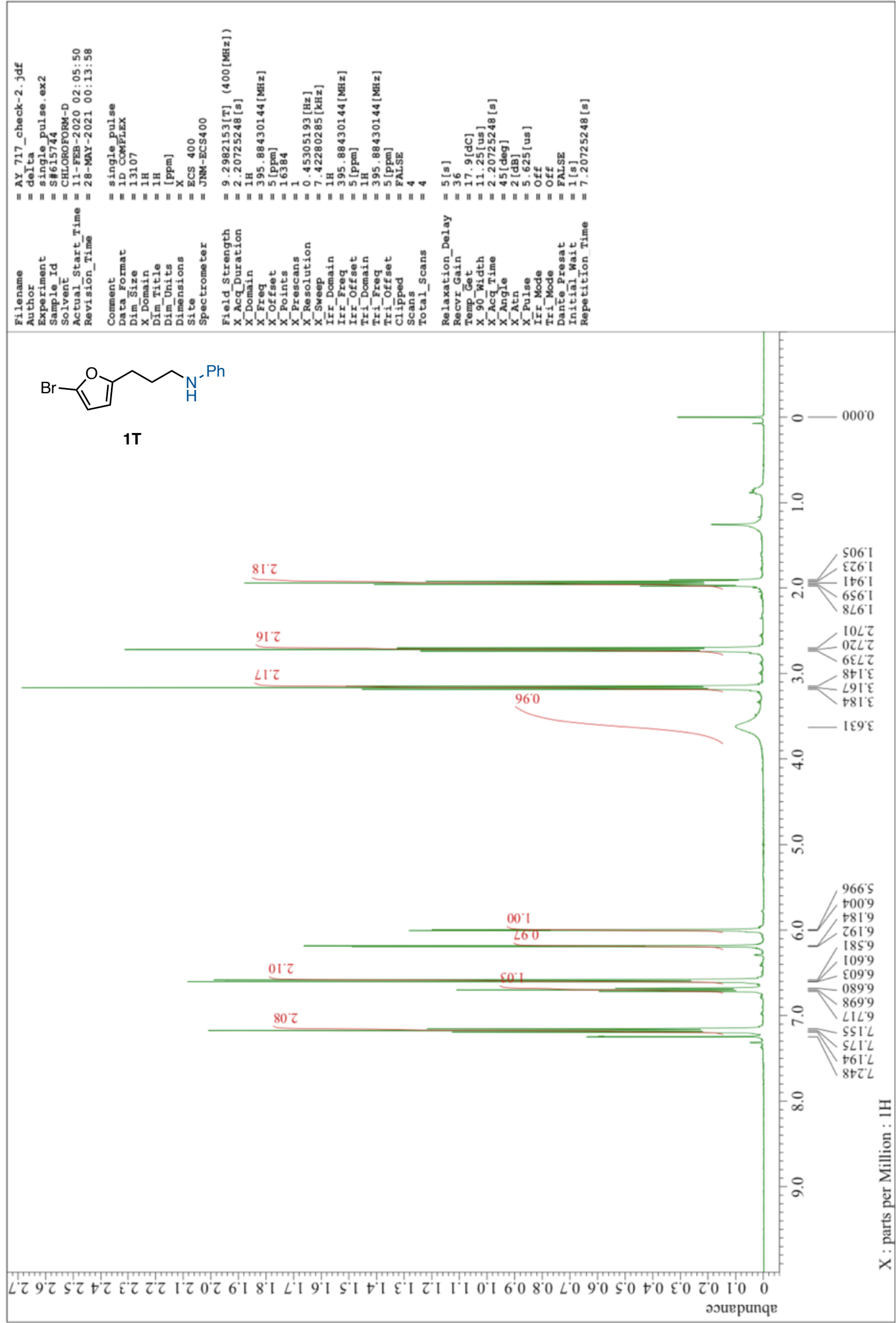


${ }^{13} \mathrm{C}$ NMR of $1 \mathrm{~T}\left(101 \mathrm{MHz}, \mathrm{CDCl}_{3}\right)$

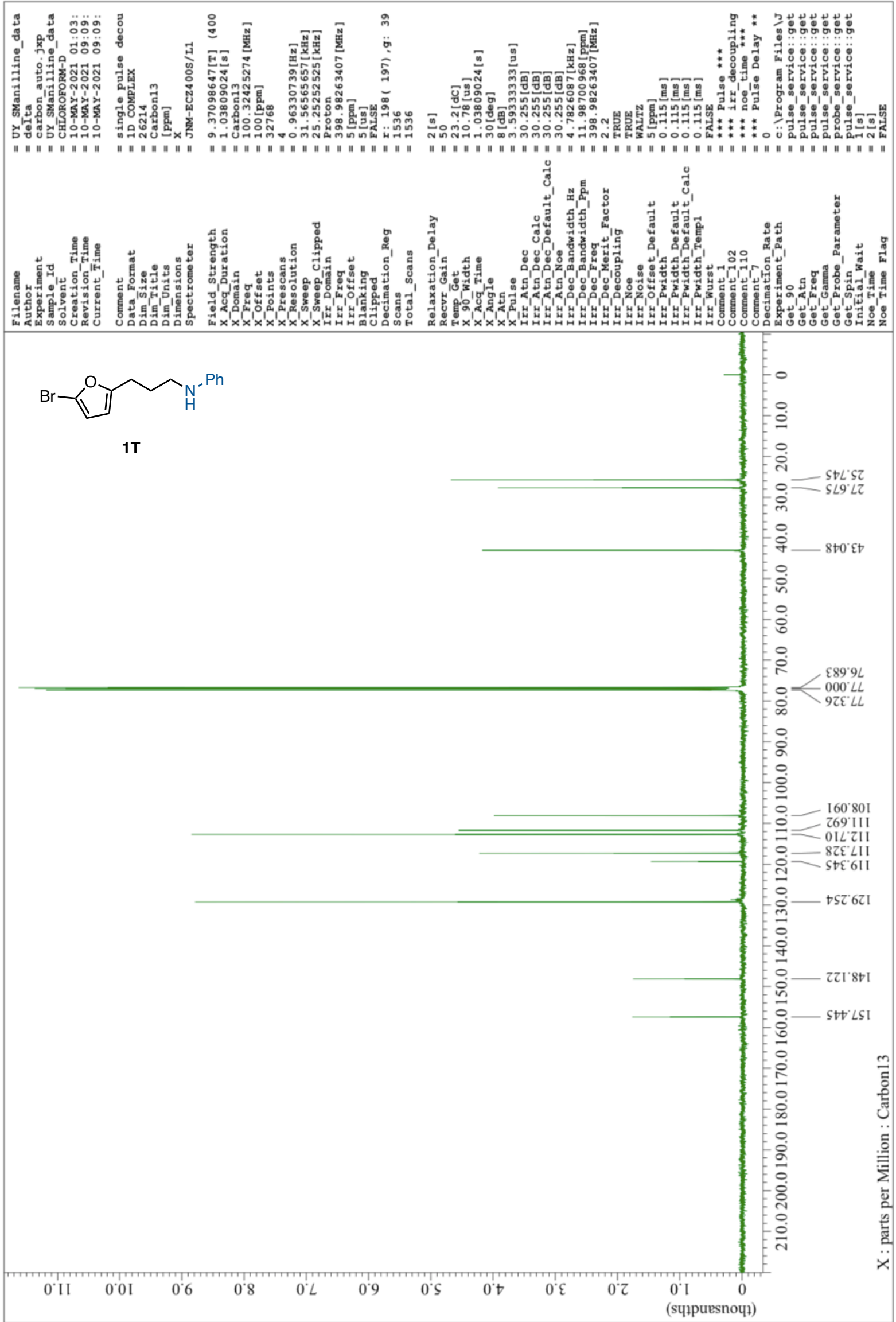


${ }^{1} \mathrm{H}$ NMR of $4\left(400 \mathrm{MHz}, \mathrm{CDCl}_{3}\right)$

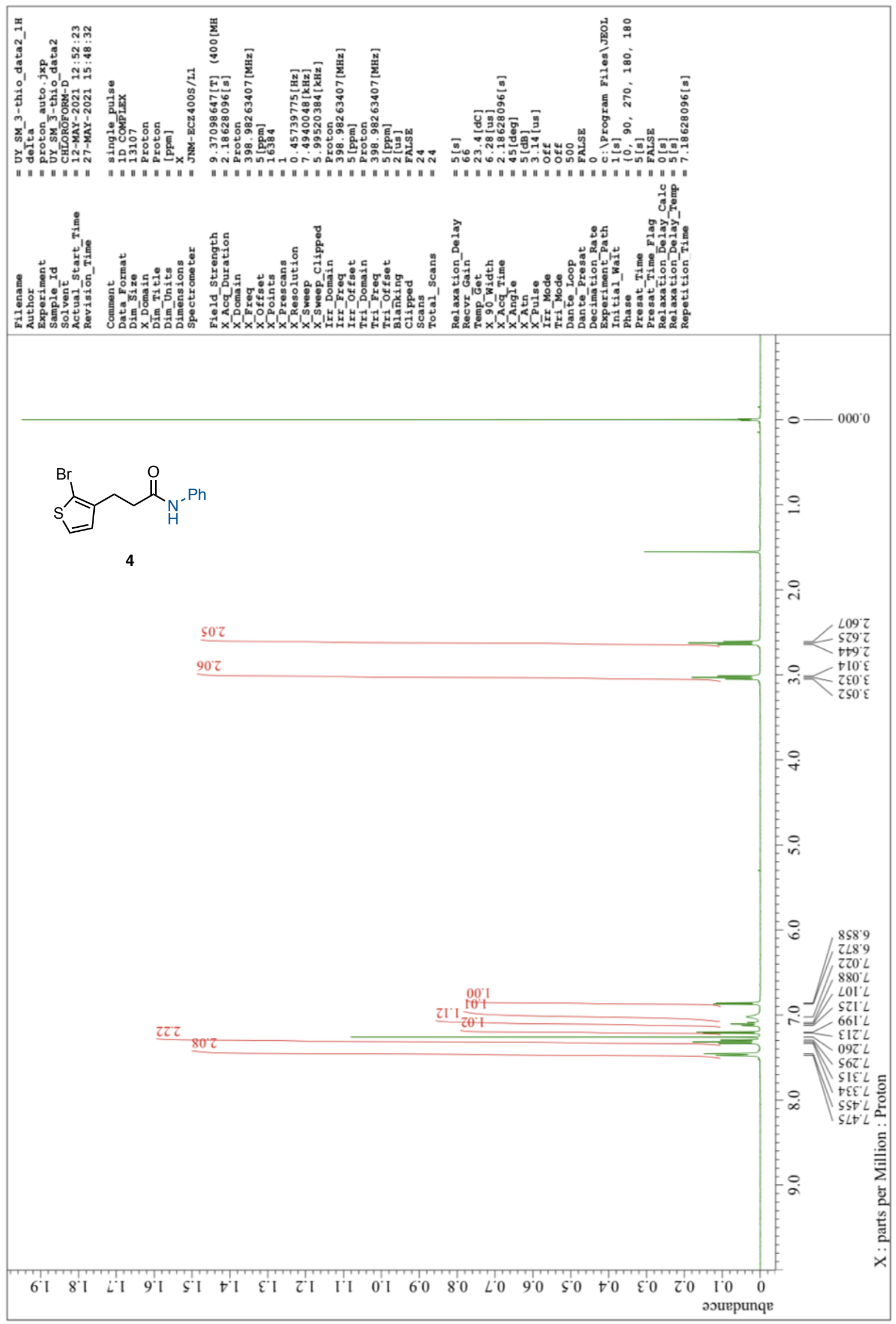


${ }^{13} \mathrm{C}$ NMR of $4\left(101 \mathrm{MHz}, \mathrm{CDCl}_{3}\right)$

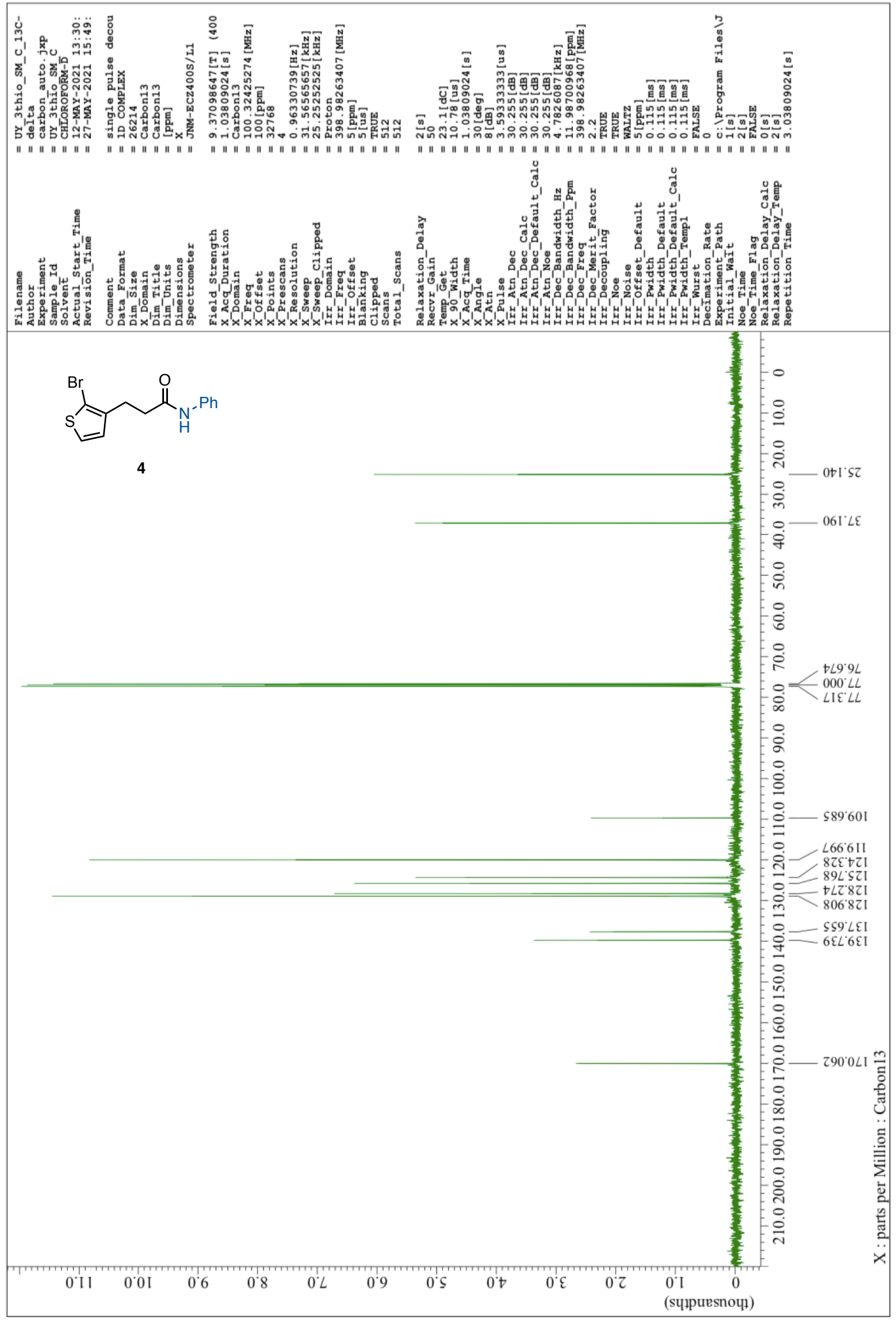


${ }^{1} \mathrm{H}$ NMR of $6\left(400 \mathrm{MHz}, \mathrm{CDCl}_{3}\right)$

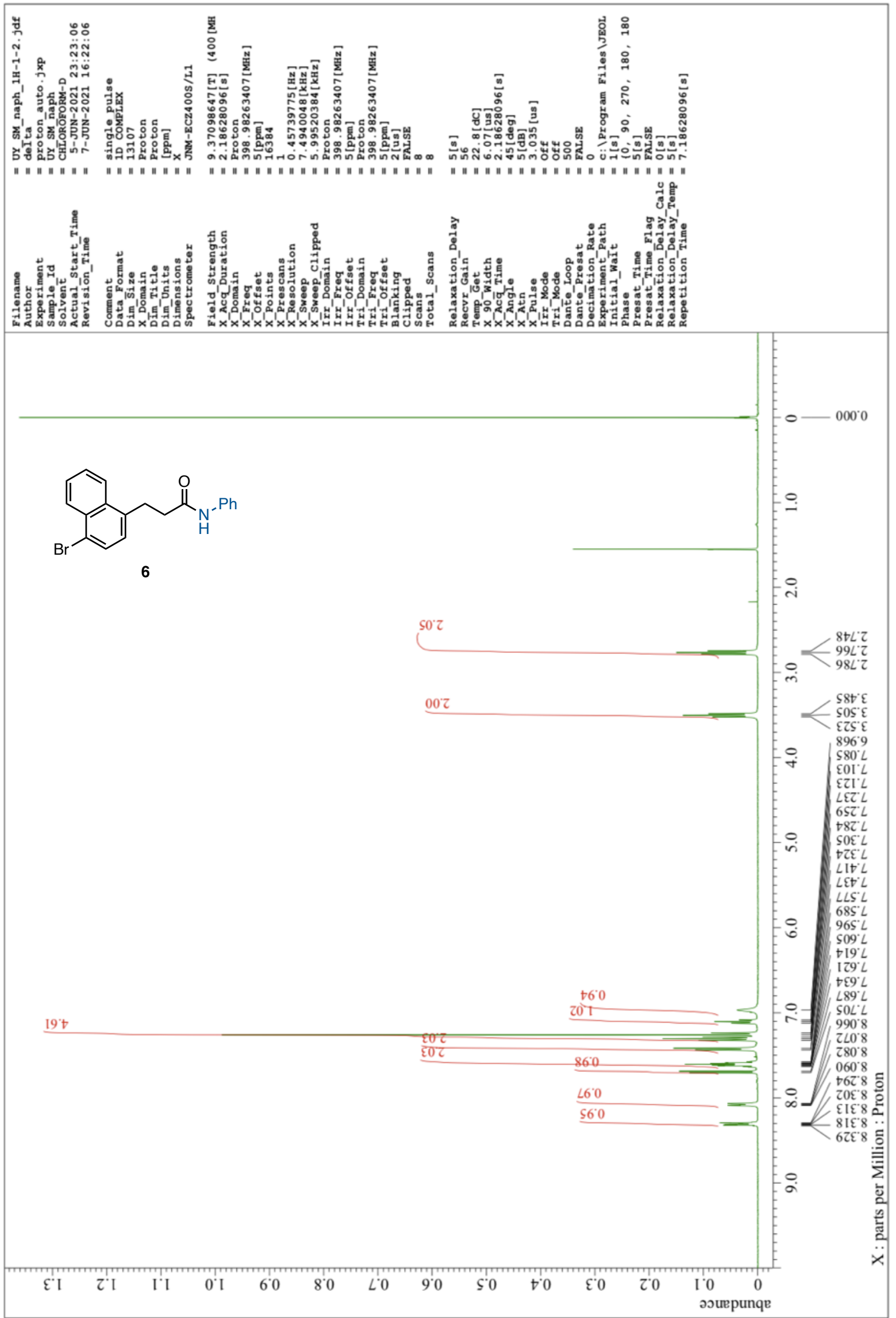


${ }^{13}$ C NMR of 6 (101 MHz, DMSO- $\left.d_{6}\right)$

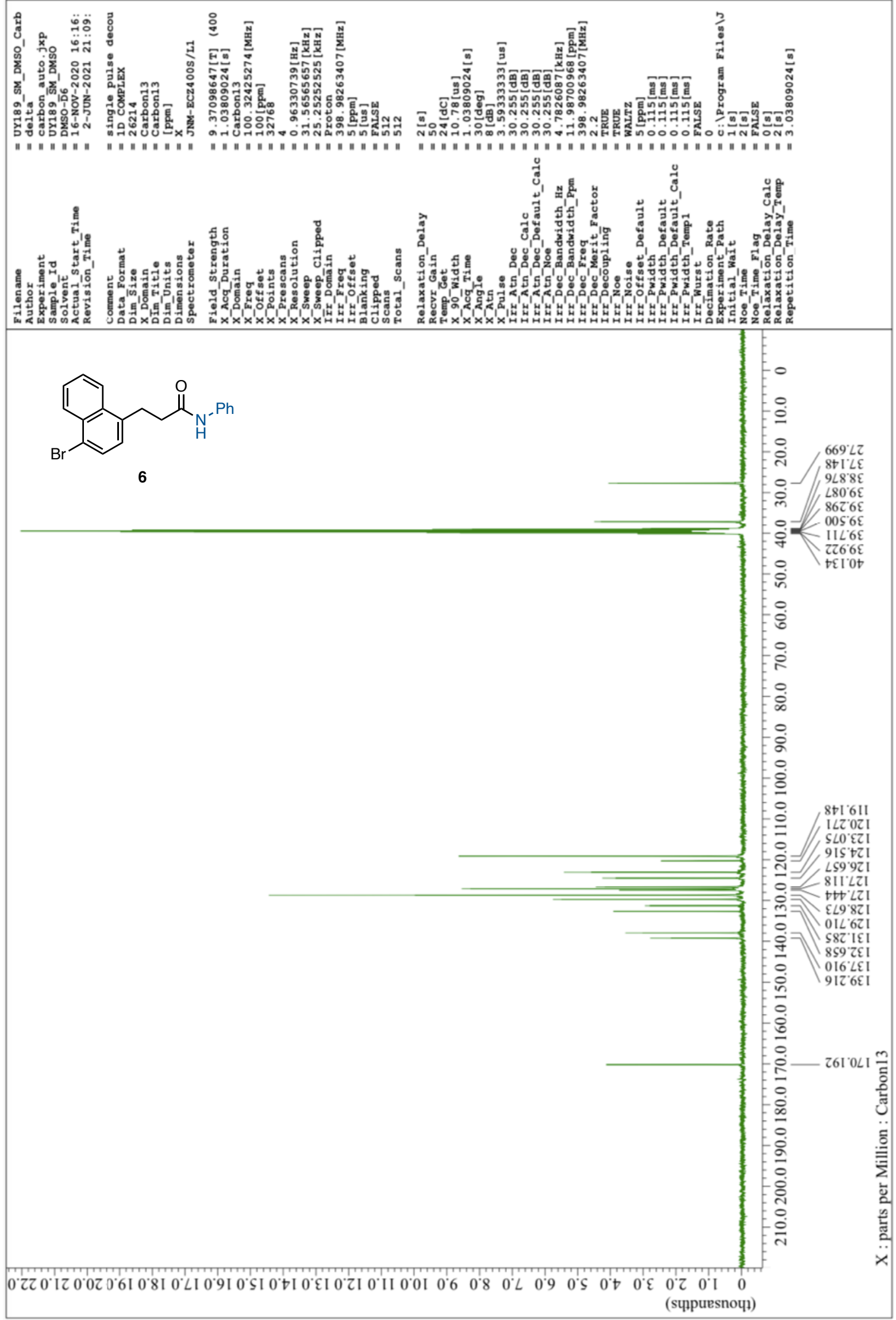


${ }^{1} \mathrm{H}$ NMR of $2 \mathrm{c}\left(400 \mathrm{MHz}, \mathrm{CDCl}_{3}\right)$

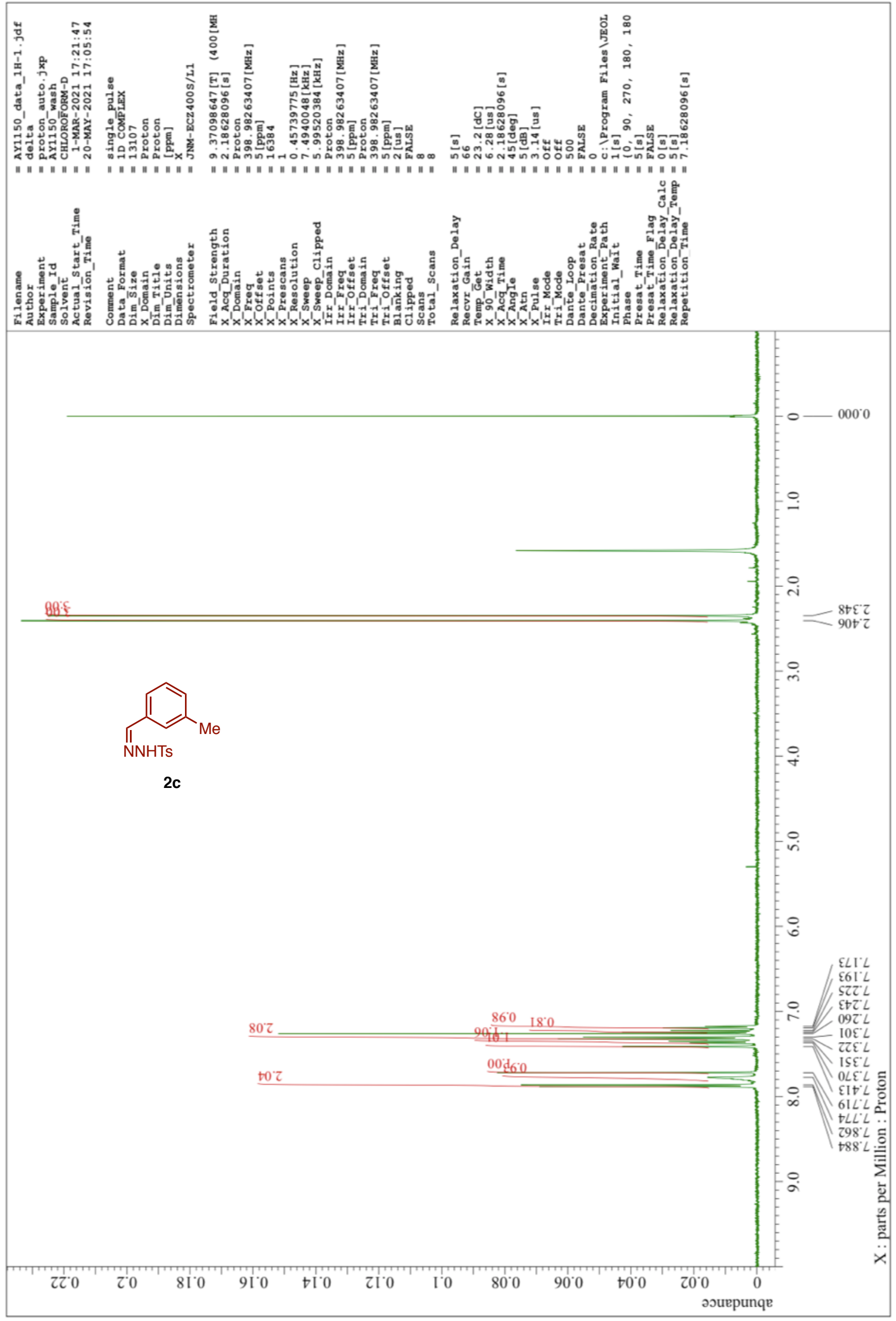


${ }^{13} \mathrm{C}$ NMR of $2 \mathrm{c}\left(101 \mathrm{MHz}, \mathrm{CDCl}_{3}\right)$

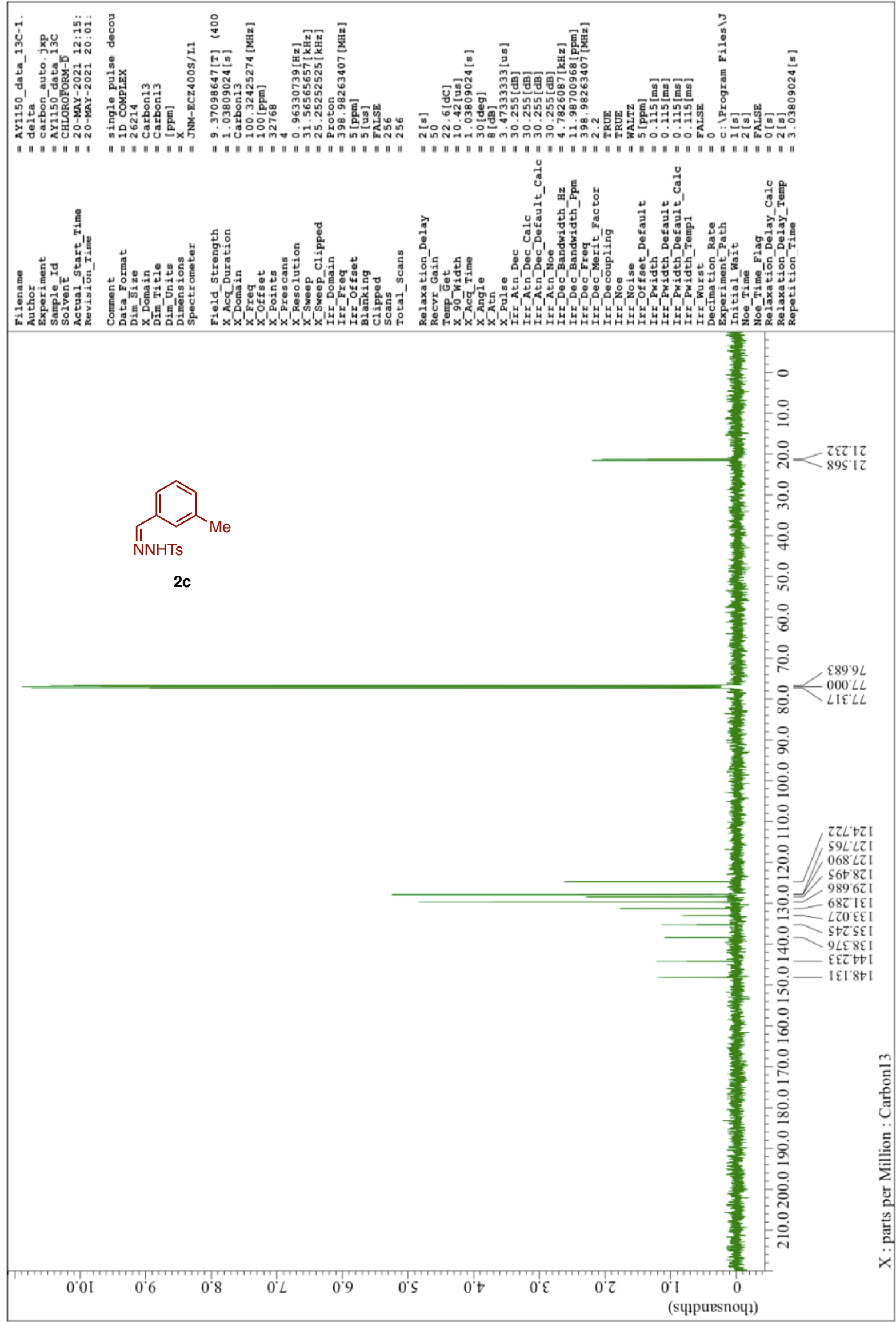


${ }^{1} \mathrm{H}$ NMR of 2d (400 MHz, $\left.\mathrm{CDCl}_{3}\right)$

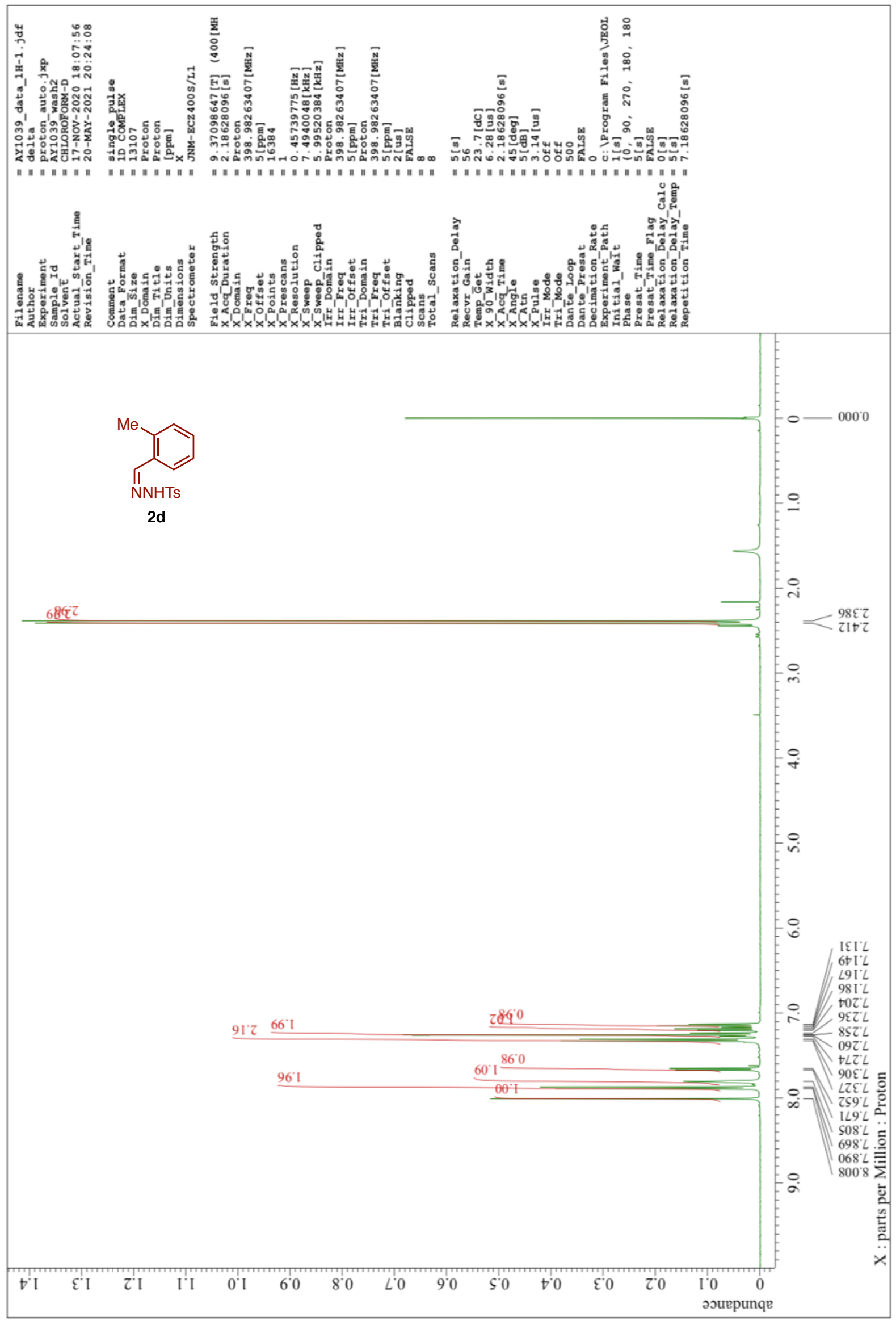


${ }^{13} \mathrm{C}$ NMR of 2d (101 MHz, $\left.\mathrm{CDCl}_{3}\right)$

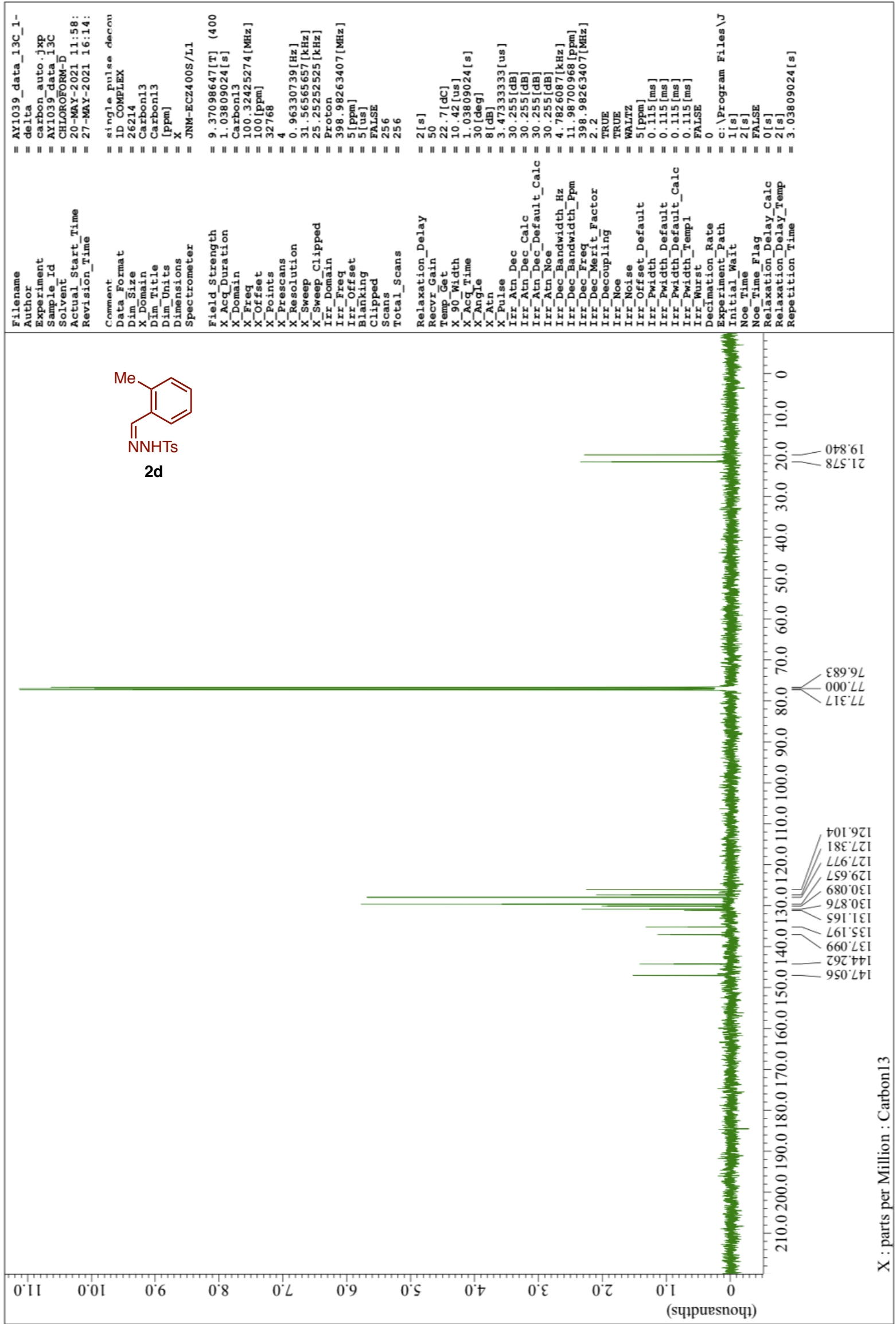


${ }^{1} \mathrm{H}$ NMR of $2 \mathrm{~h}\left(400 \mathrm{MHz}, \mathrm{CDCl}_{3}\right)$

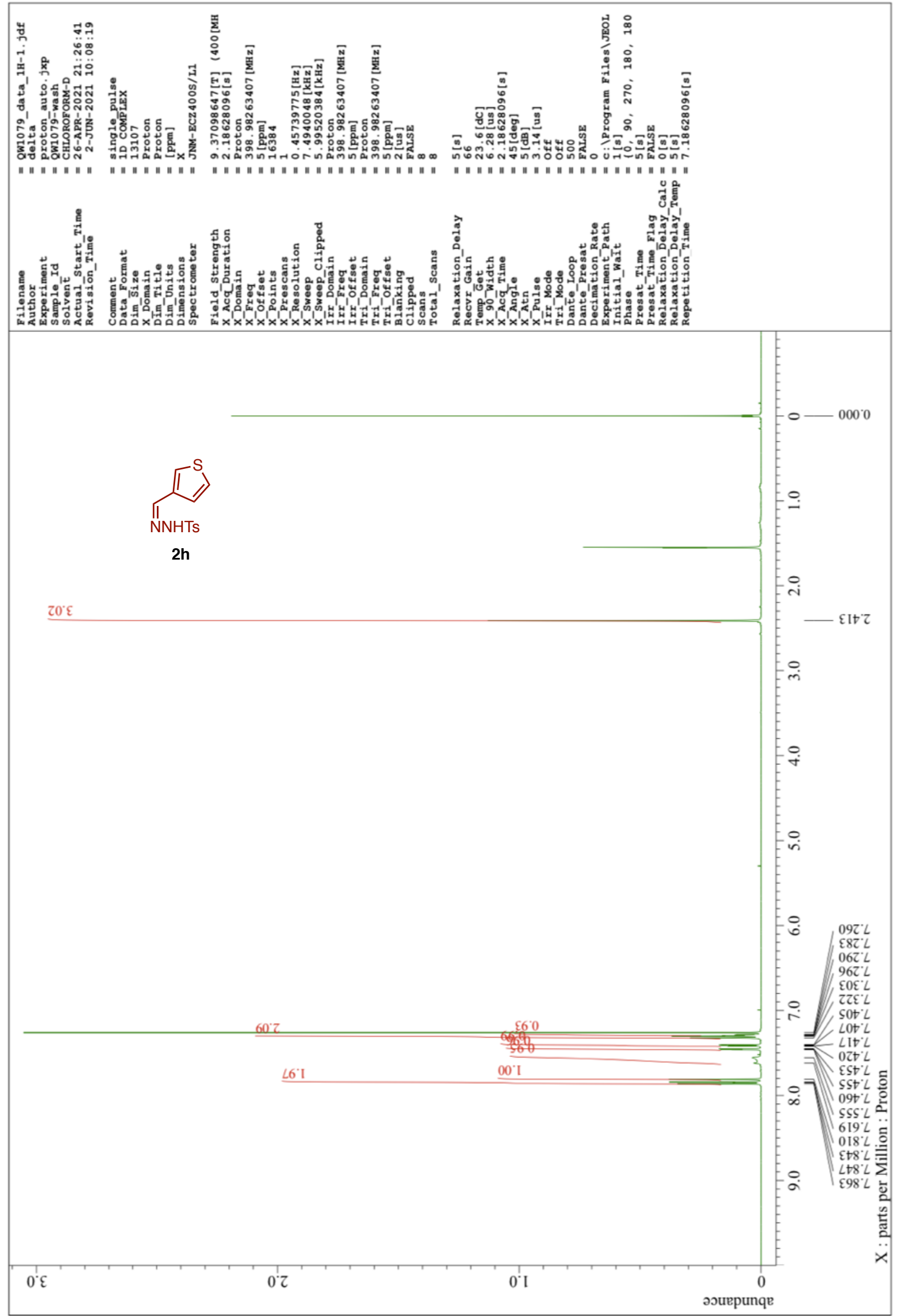


${ }^{13} \mathrm{C}$ NMR of $2 \mathrm{~h}\left(101 \mathrm{MHz}, \mathrm{CDCl}_{3}\right)$

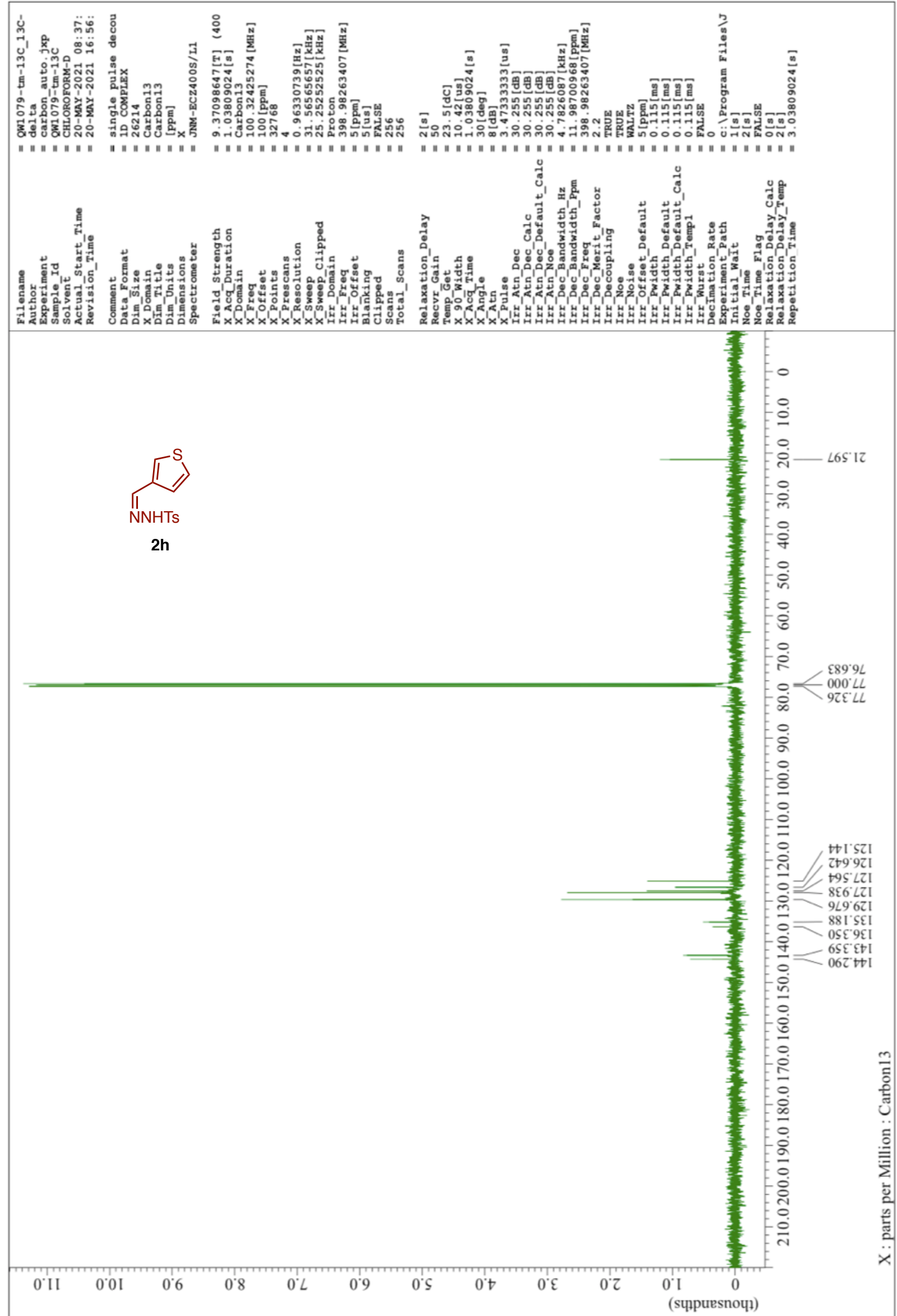


${ }^{1} \mathrm{H}$ NMR of $2 \mathrm{~m}\left(400 \mathrm{MHz}, \mathrm{CDCl}_{3}\right)$

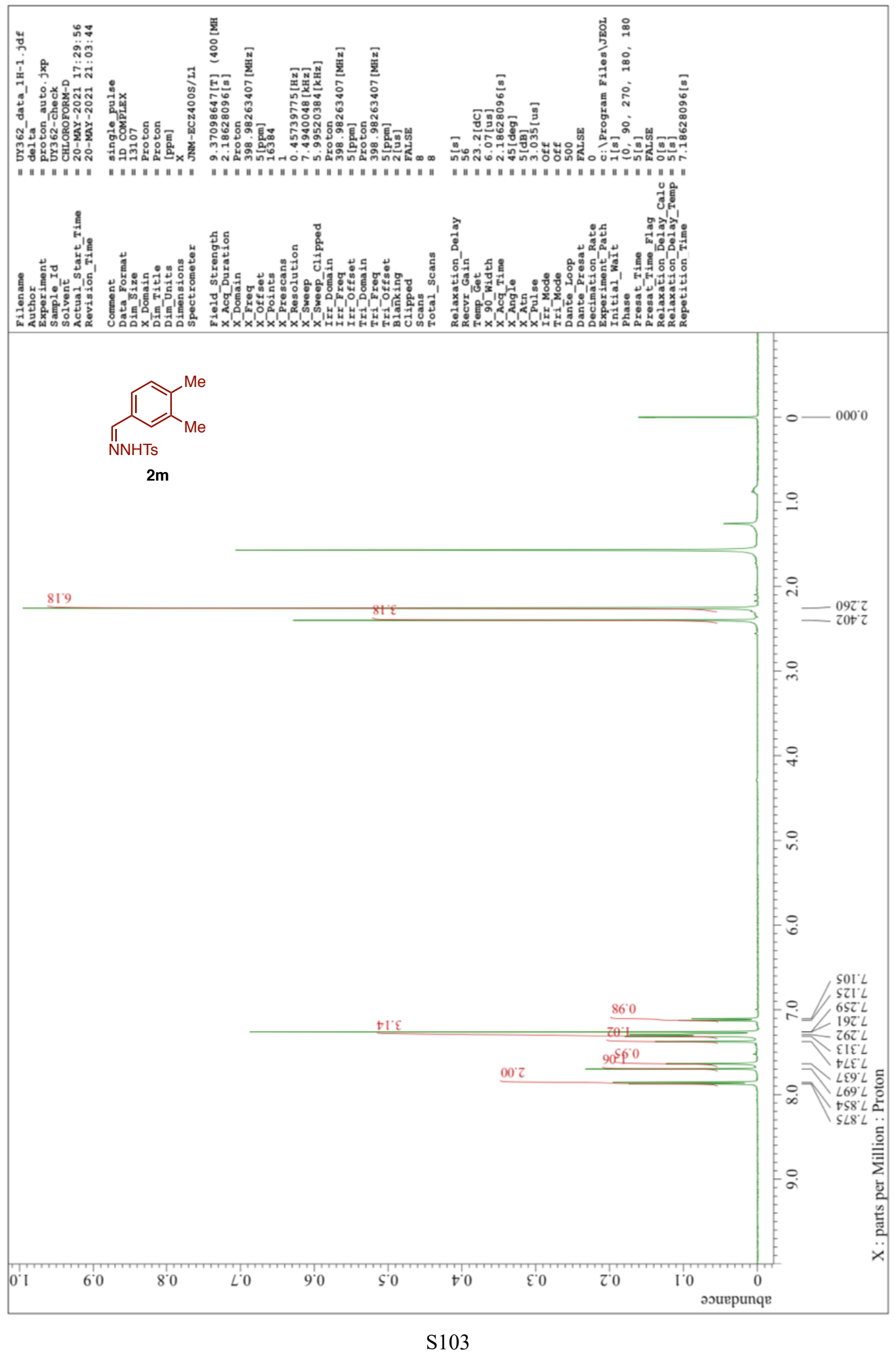


${ }^{13} \mathrm{C}$ NMR of $2 \mathrm{~m}\left(101 \mathrm{MHz}, \mathrm{CDCl}_{3}\right)$

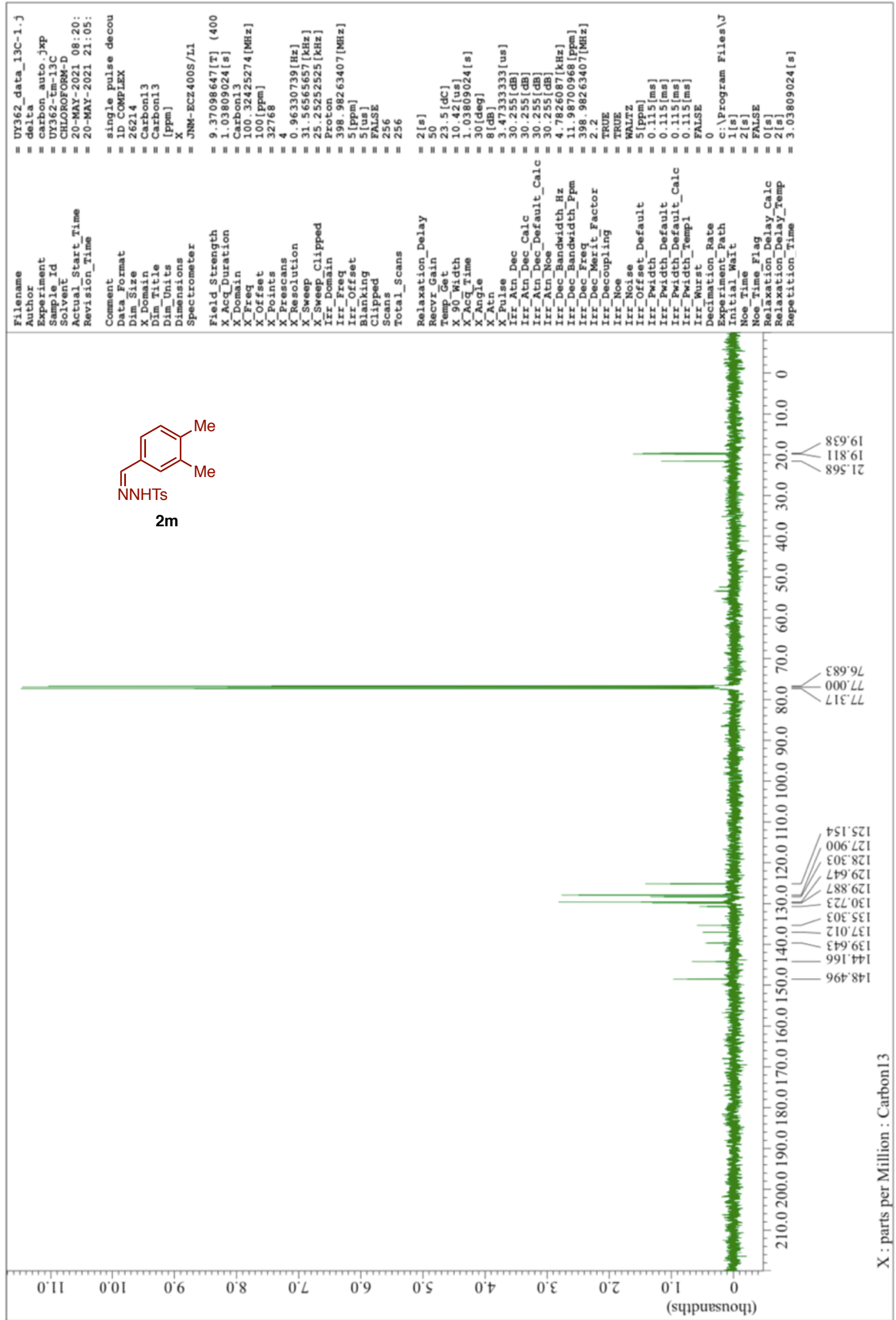


${ }^{1} \mathrm{H}$ NMR of $3 \mathrm{Aa}$ (400 $\left.\mathrm{MHz}, \mathrm{CDCl}_{3}\right)$

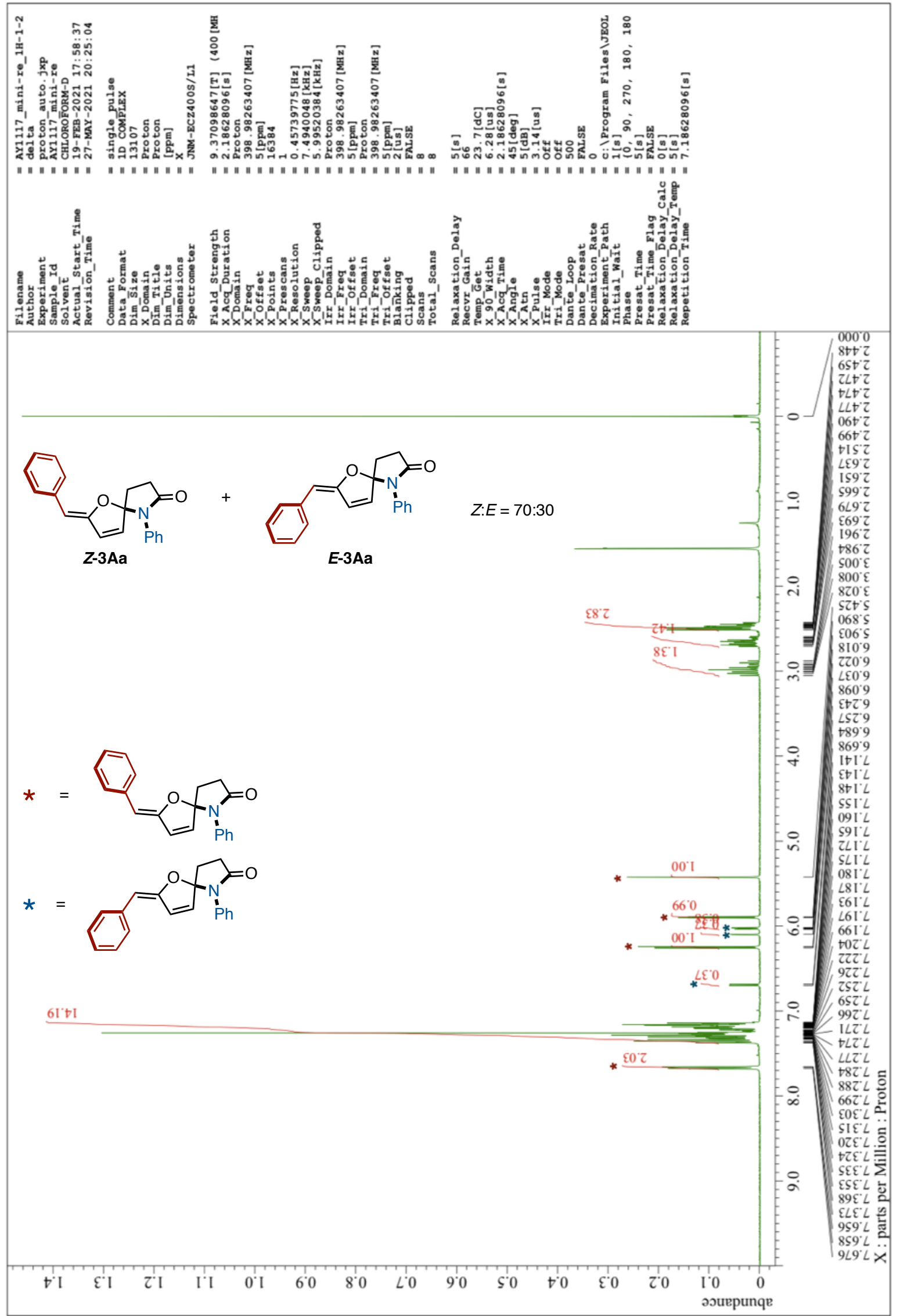


${ }^{13} \mathrm{C}$ NMR of $3 \mathrm{Aa}\left(101 \mathrm{MHz}, \mathrm{CDCl}_{3}\right)$

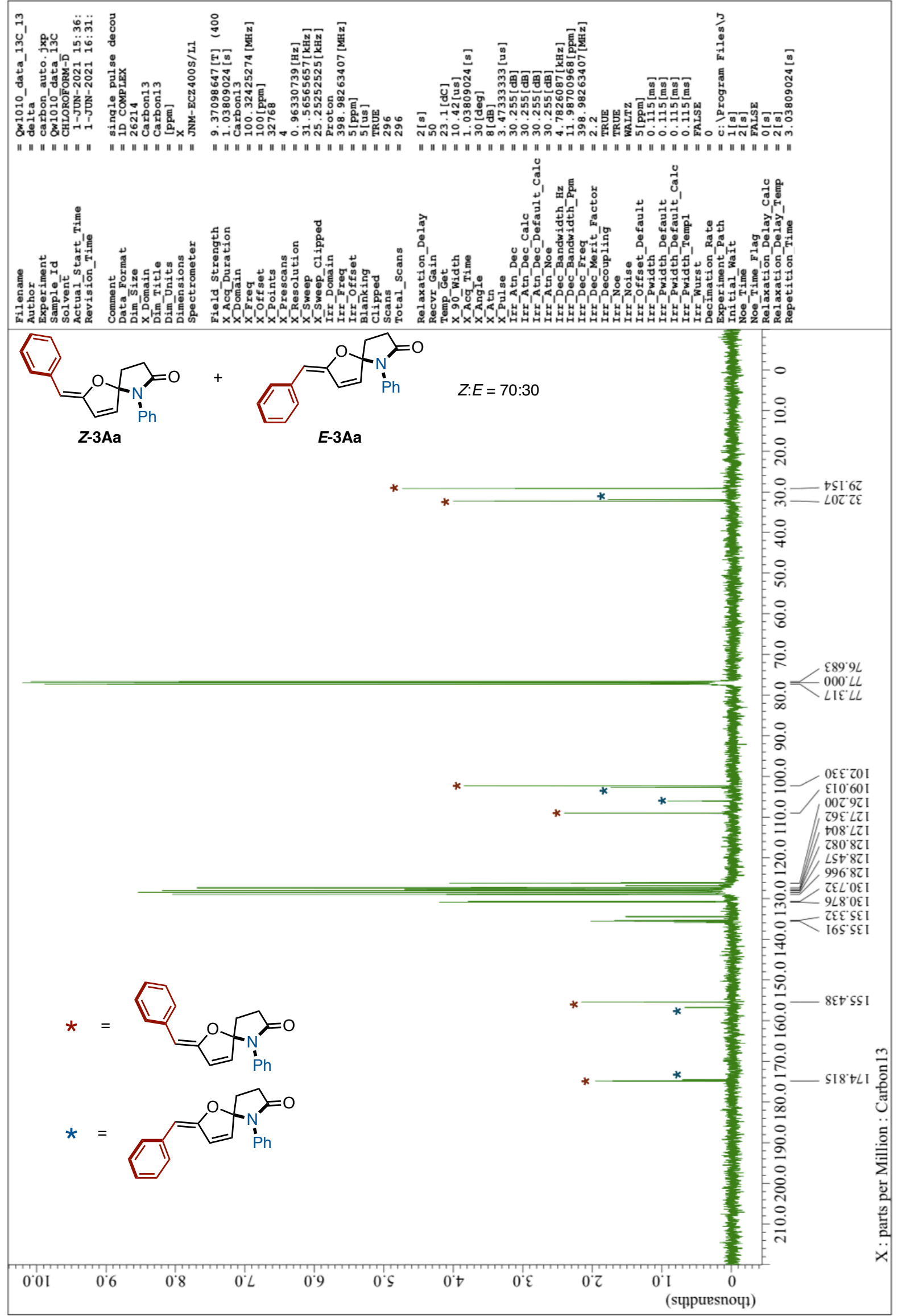


${ }^{1} \mathrm{H}$ NMR of 3Ba (400 $\left.\mathrm{MHz}, \mathrm{CDCl}_{3}\right)$

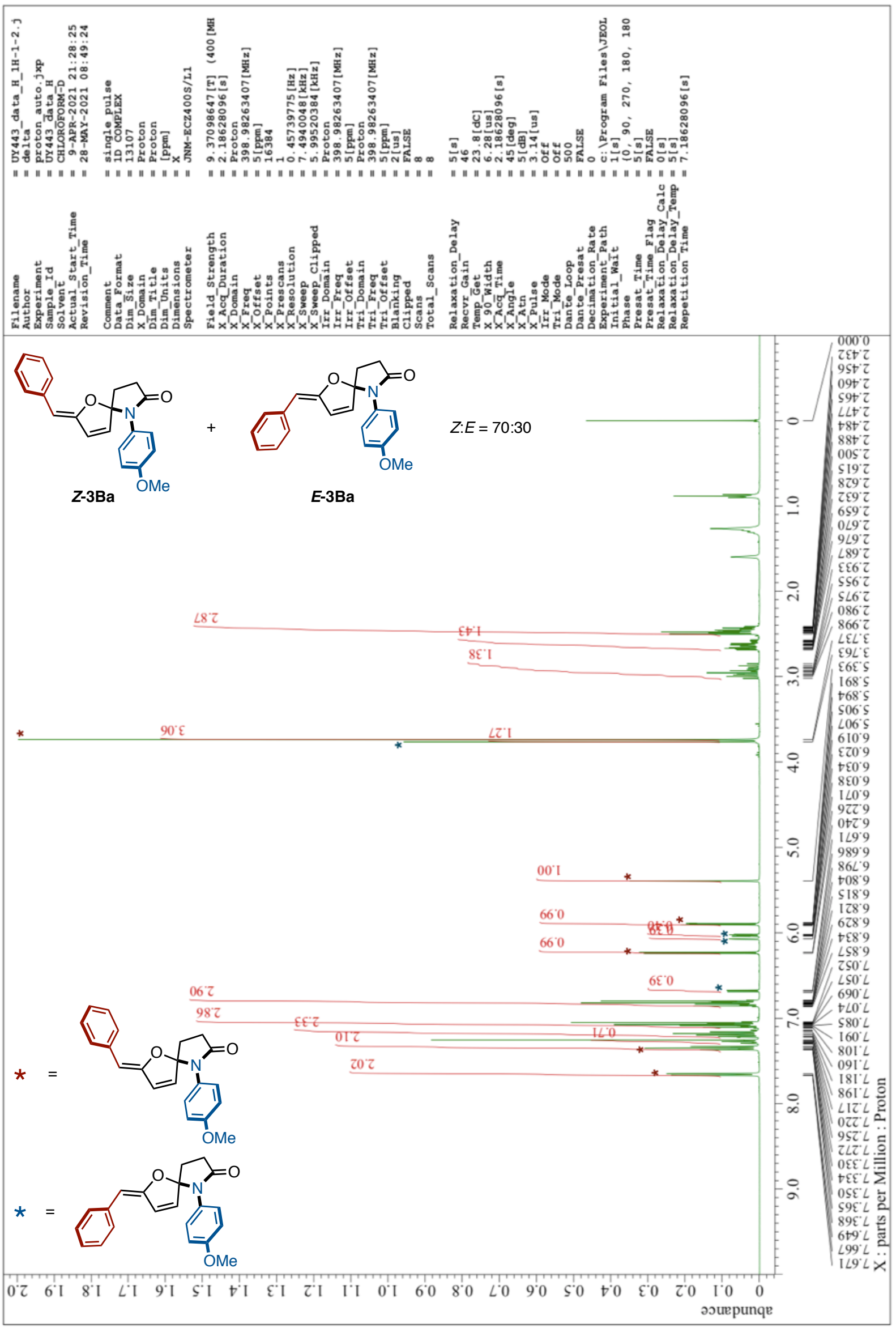


${ }^{13} \mathrm{C}$ NMR of 3Ba (101 MHz, $\left.\mathrm{CDCl}_{3}\right)$

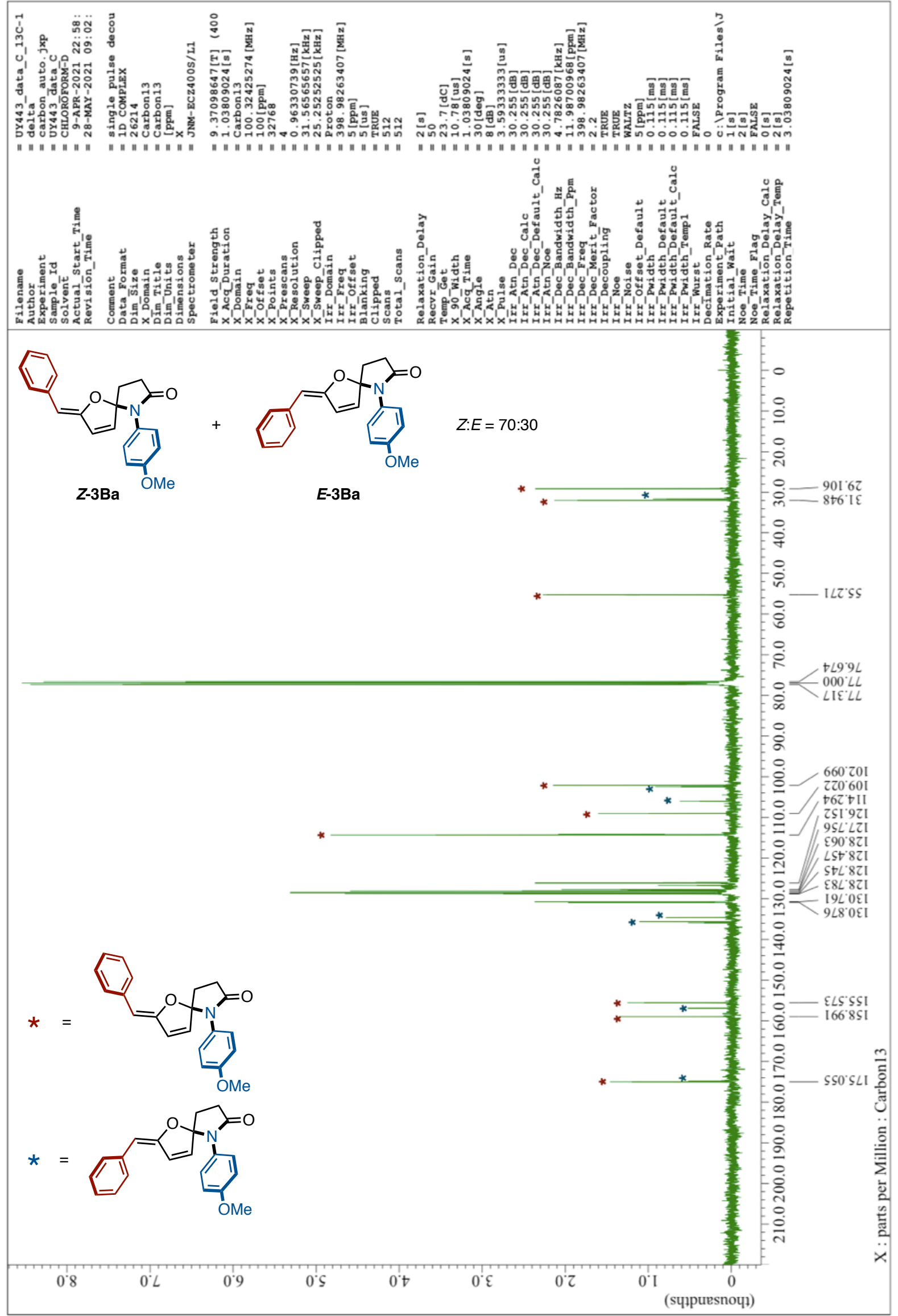


${ }^{1} \mathrm{H}$ NMR of 3Ca (400 $\left.\mathrm{MHz}, \mathrm{CDCl}_{3}\right)$

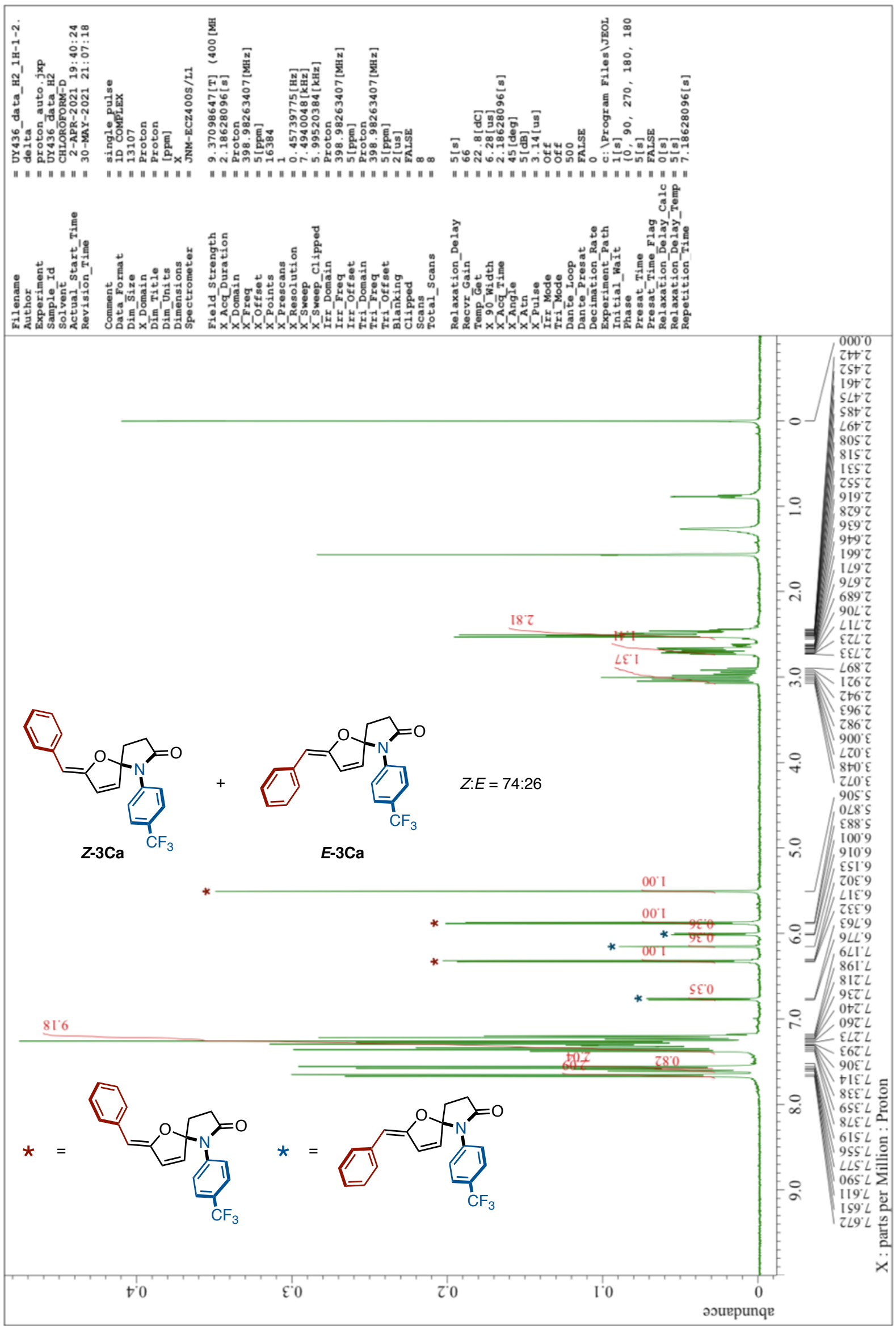

S109 
${ }^{13} \mathrm{C}$ NMR of $3 \mathrm{Ca}\left(101 \mathrm{MHz}, \mathrm{CDCl}_{3}\right)$

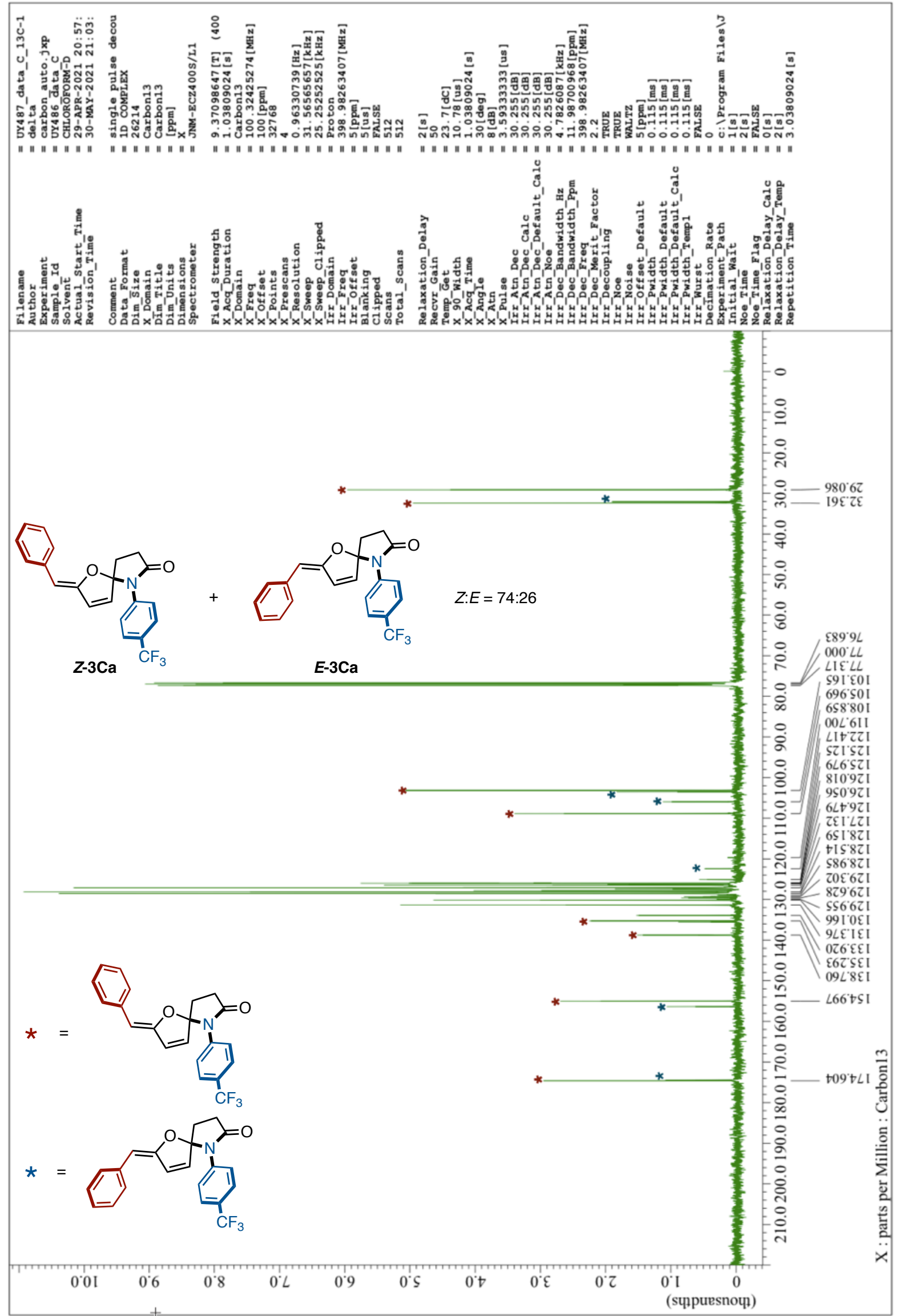


19F NMR of $3 \mathrm{Ca}\left(376 \mathrm{MHz}, \mathrm{CDCl}_{3}\right)$

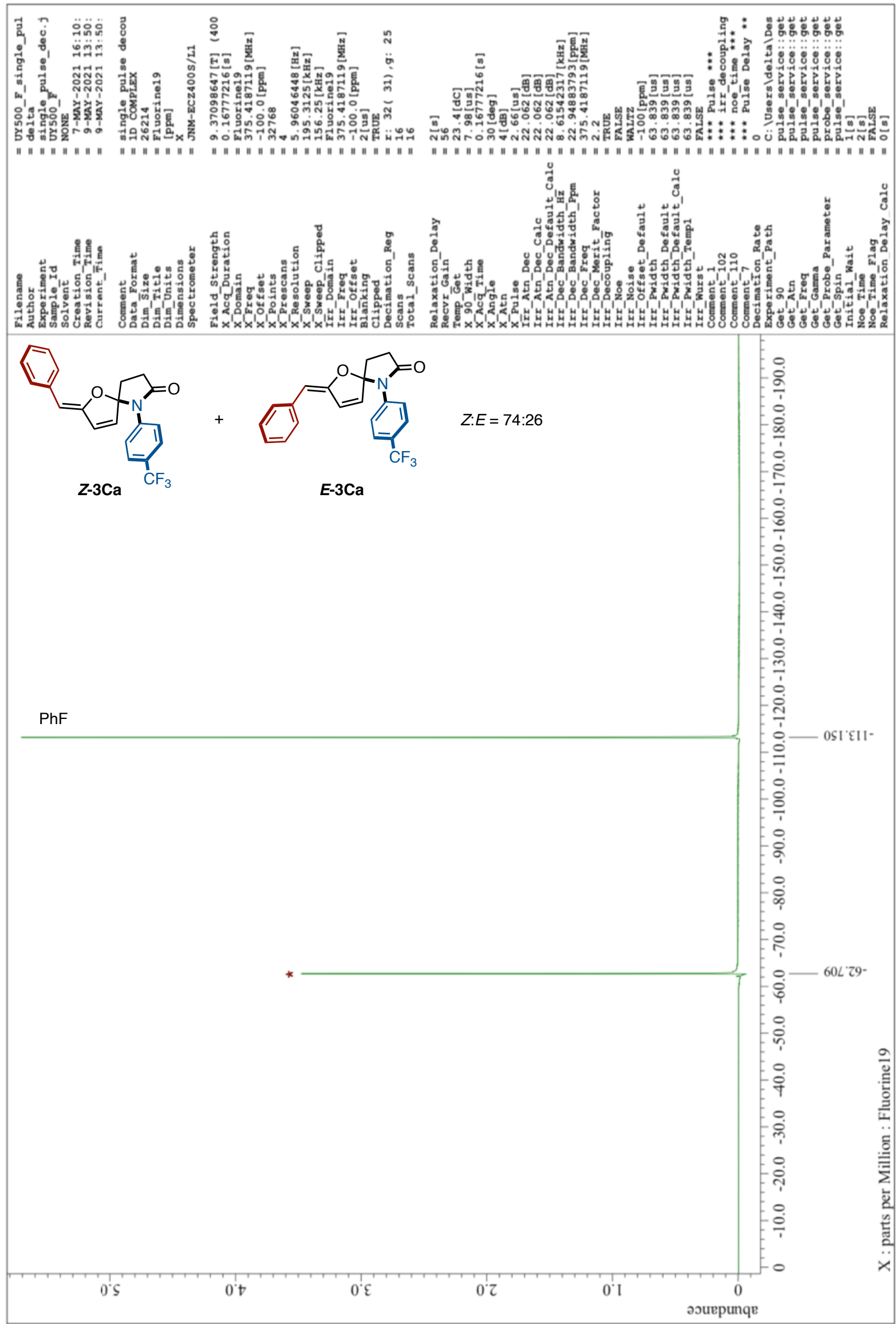


${ }^{1} \mathrm{H}$ NMR of 3Da (400 MHz, $\left.\mathrm{CDCl}_{3}\right)$

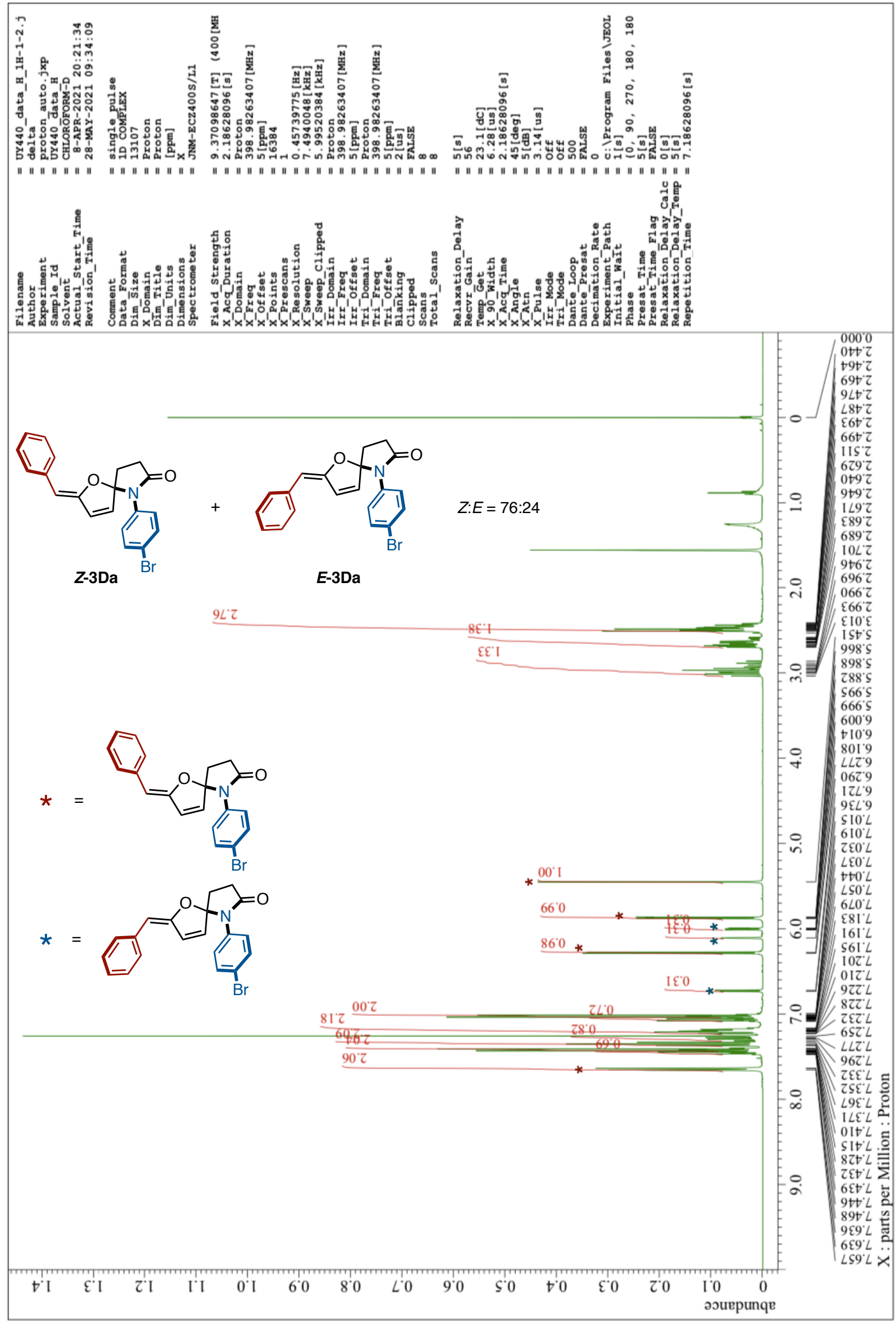


${ }^{13} \mathrm{C}$ NMR of 3Da (101 MHz, $\left.\mathrm{CDCl}_{3}\right)$

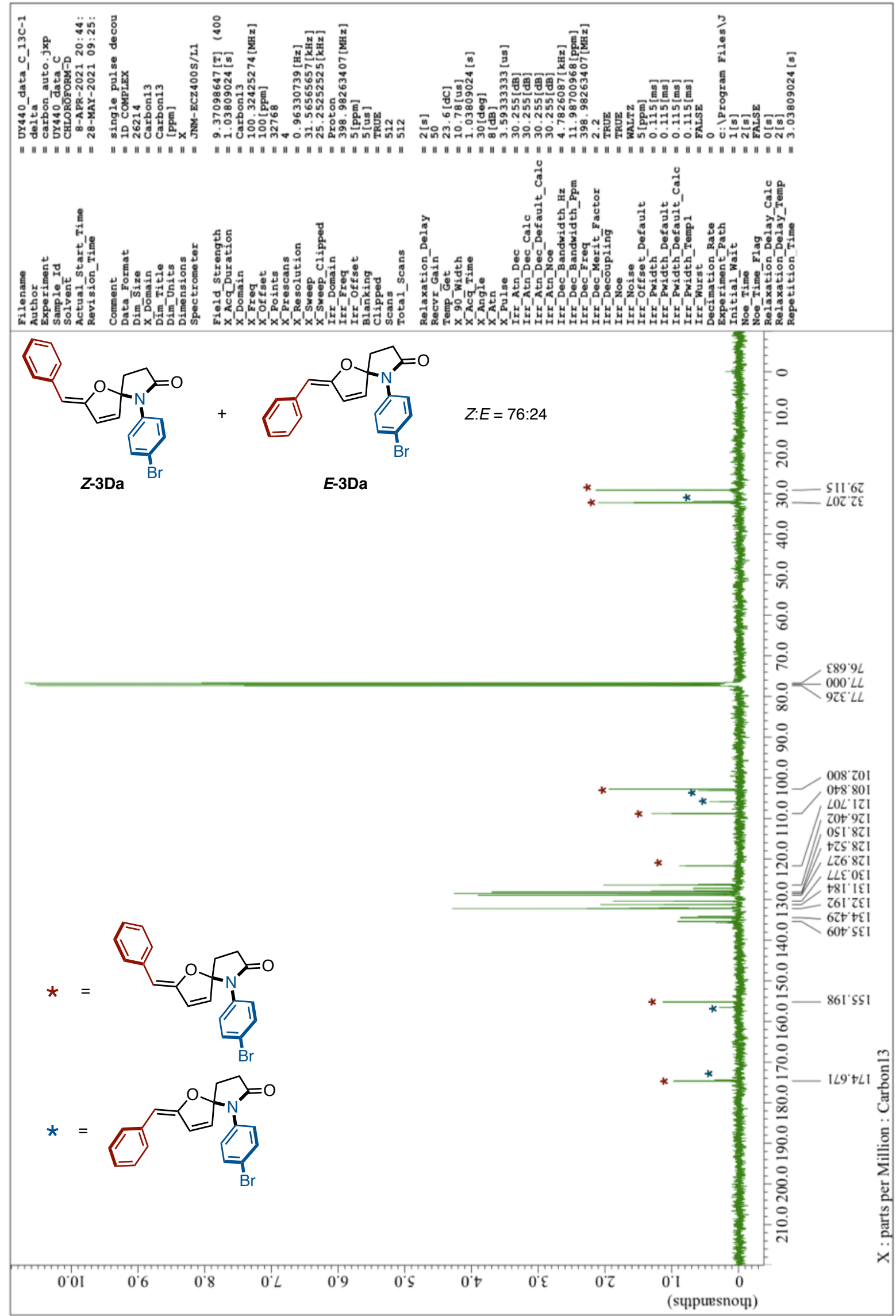


${ }^{1} \mathrm{H}$ NMR of 3Ea (400 $\left.\mathrm{MHz}, \mathrm{CDCl}_{3}\right)$

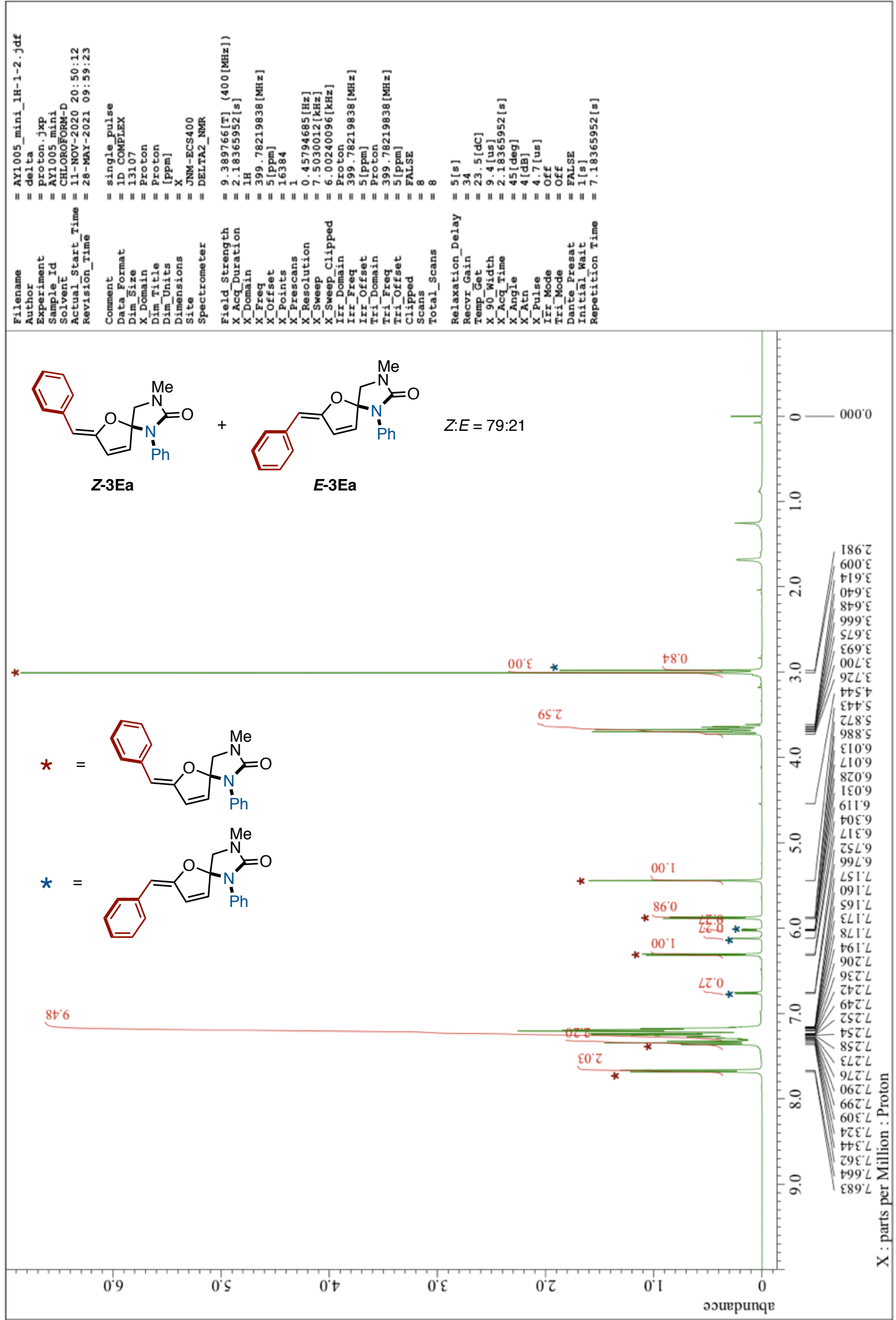


${ }^{13} \mathrm{C}$ NMR of 3Ea (101 MHz, $\left.\mathrm{CDCl}_{3}\right)$

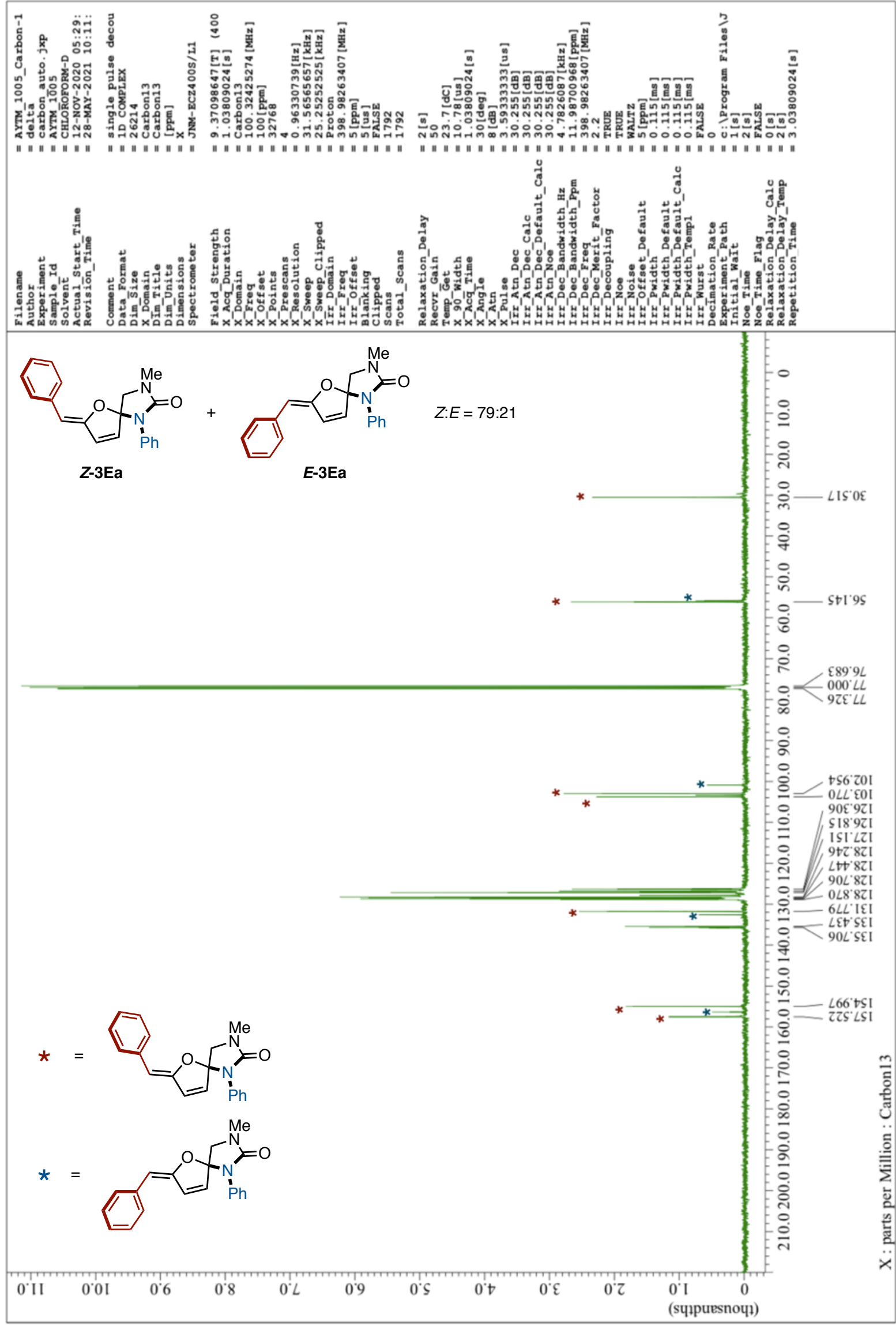


${ }^{1} \mathrm{H}$ NMR of 3Fa (400 $\left.\mathrm{MHz}, \mathrm{CDCl}_{3}\right)$

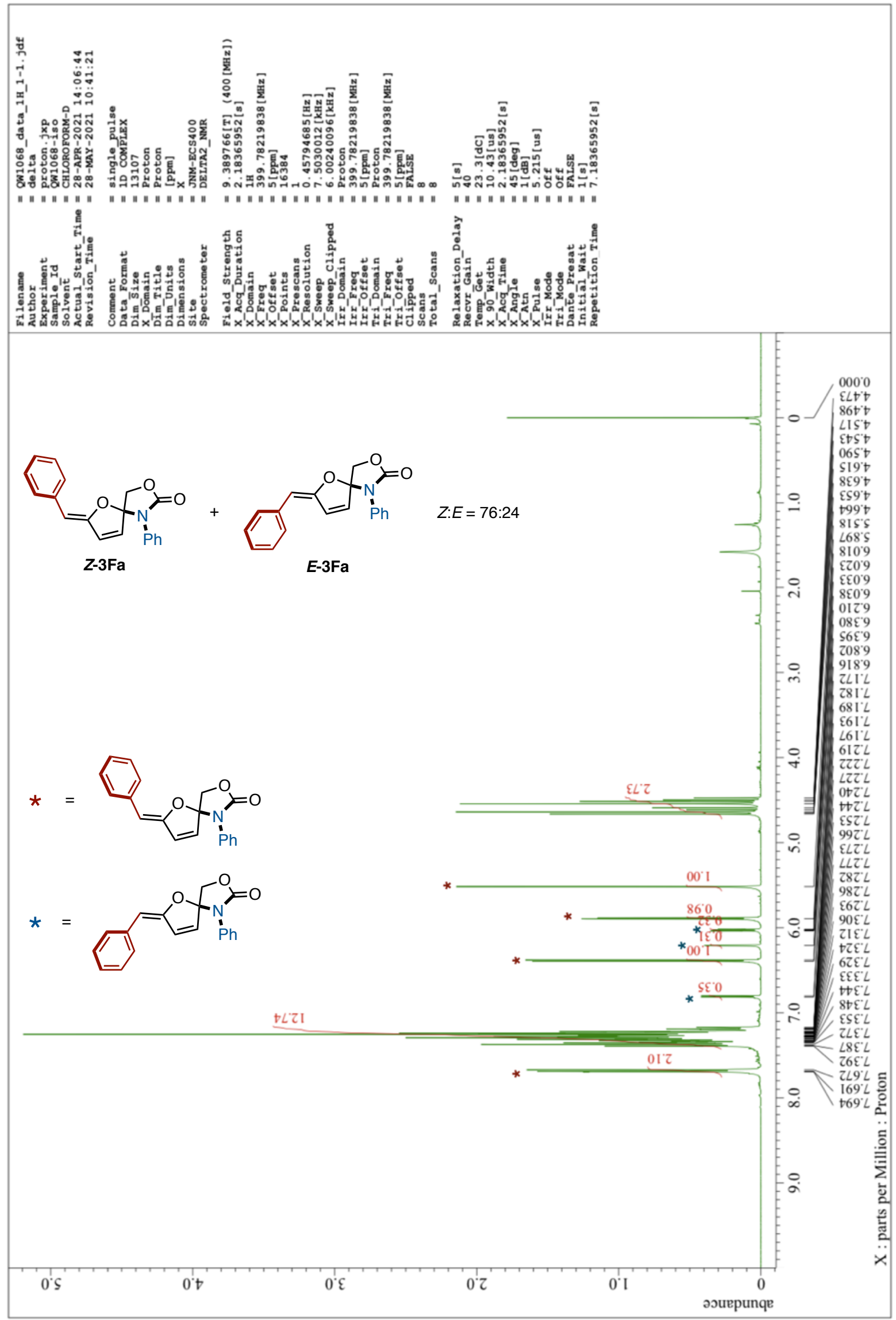


${ }^{13} \mathrm{C}$ NMR of $3 \mathrm{Fa}\left(101 \mathrm{MHz}, \mathrm{CDCl}_{3}\right)$

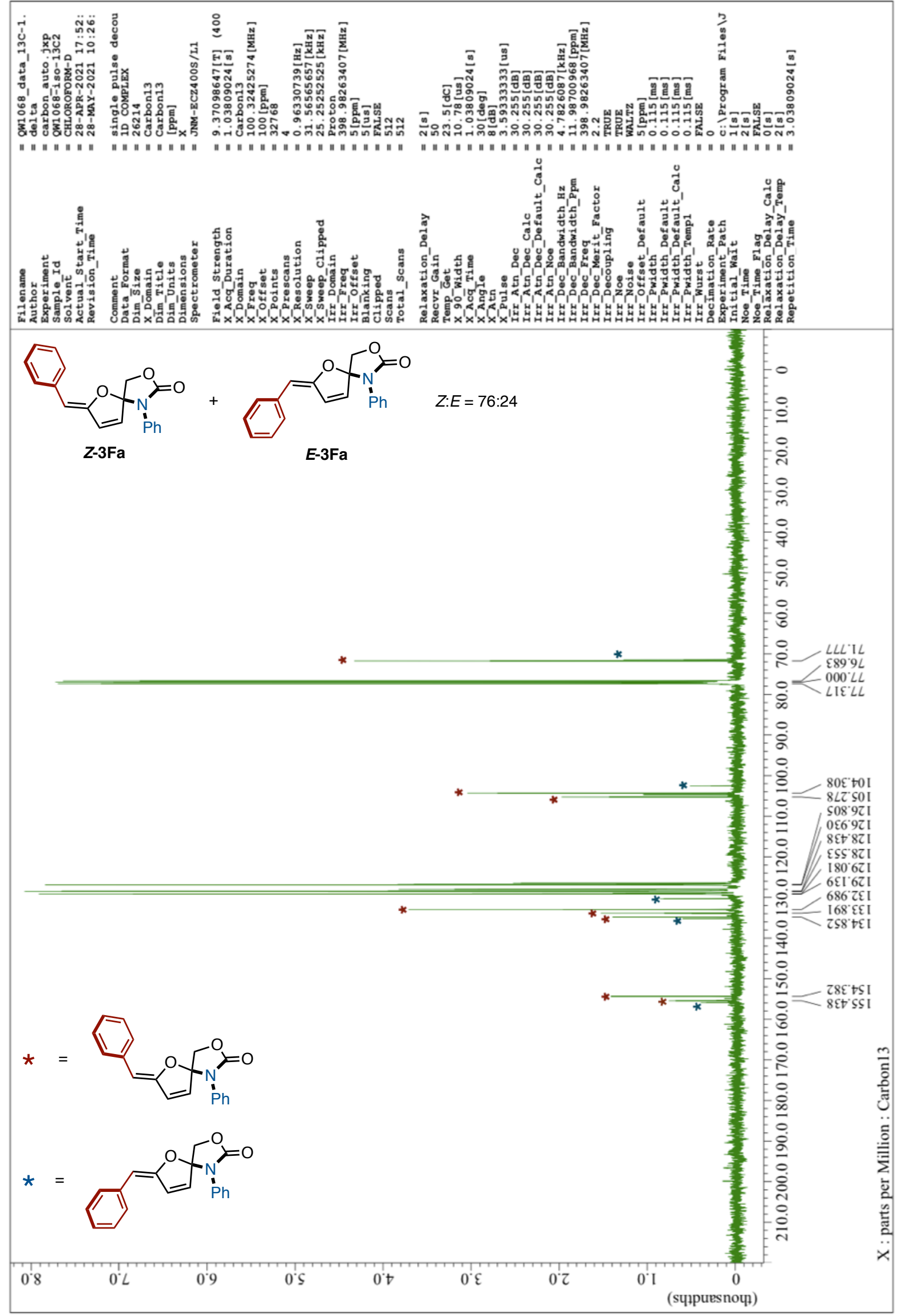


${ }^{1} \mathrm{H}$ NMR of $3 \mathrm{Ga}\left(400 \mathrm{MHz}, \mathrm{CDCl}_{3}\right)$

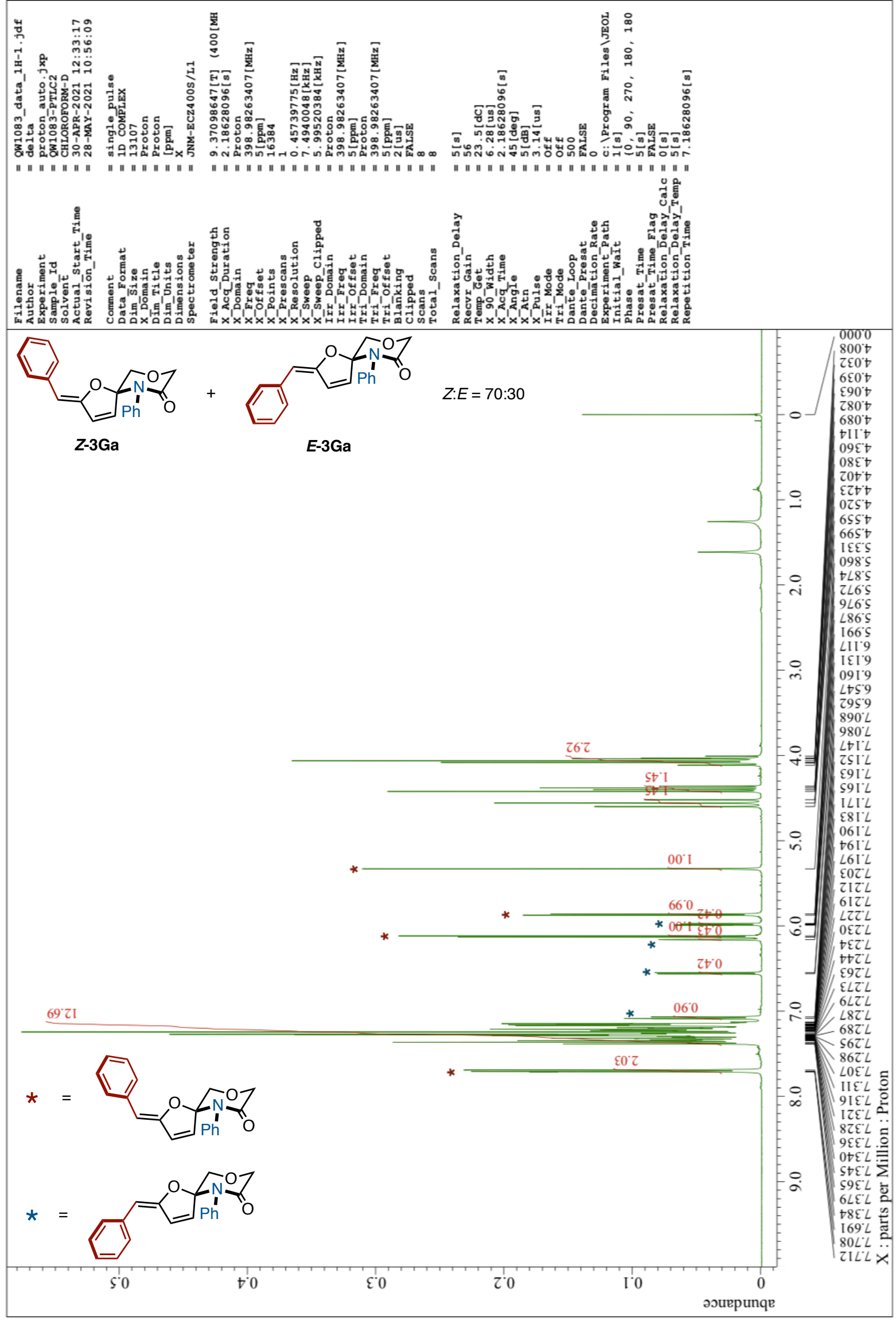


${ }^{13} \mathrm{C}$ NMR of $3 \mathrm{Ga}\left(101 \mathrm{MHz}, \mathrm{CDCl}_{3}\right)$

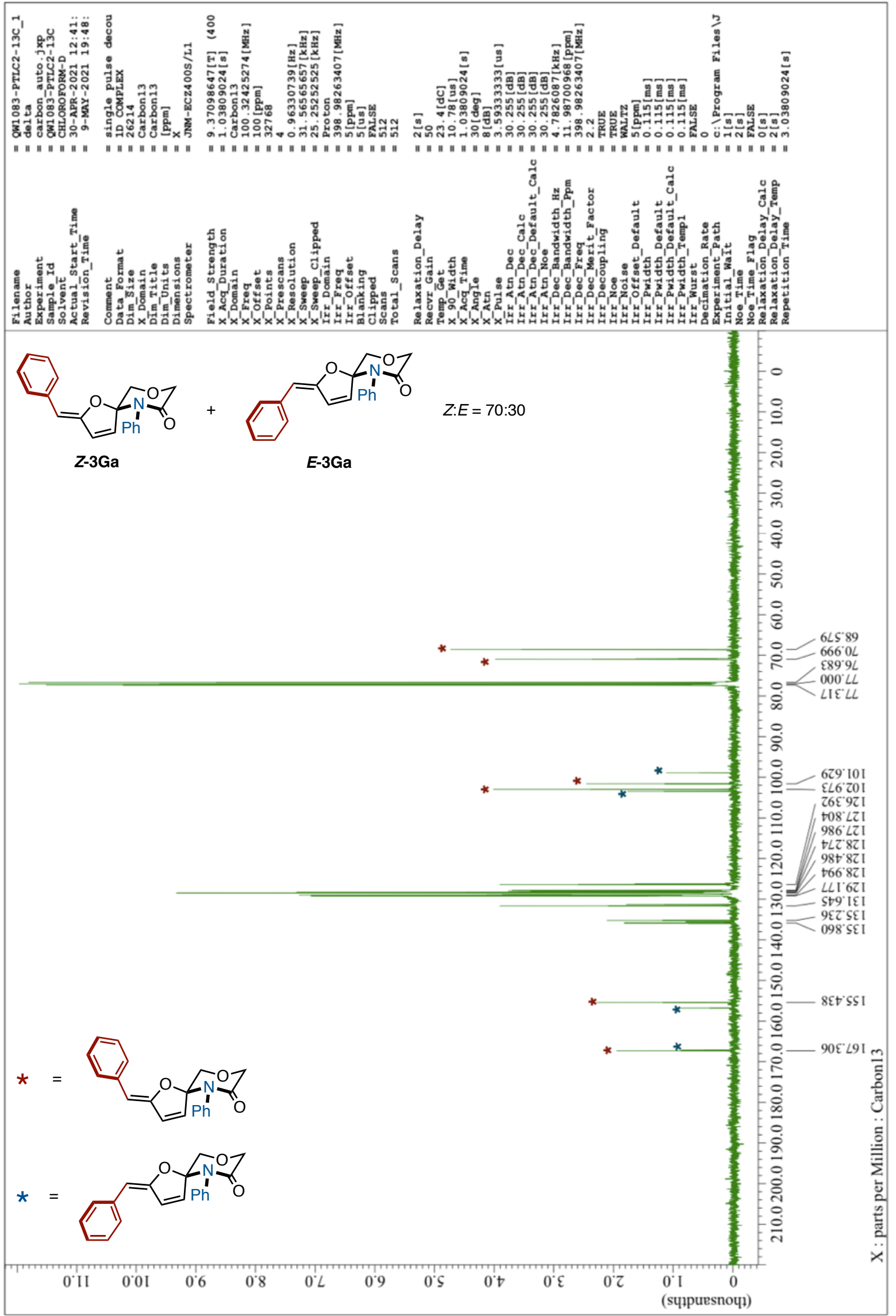


${ }^{1} \mathrm{H}$ NMR of $3 \mathrm{Ab}\left(400 \mathrm{MHz}, \mathrm{CDCl}_{3}\right)$

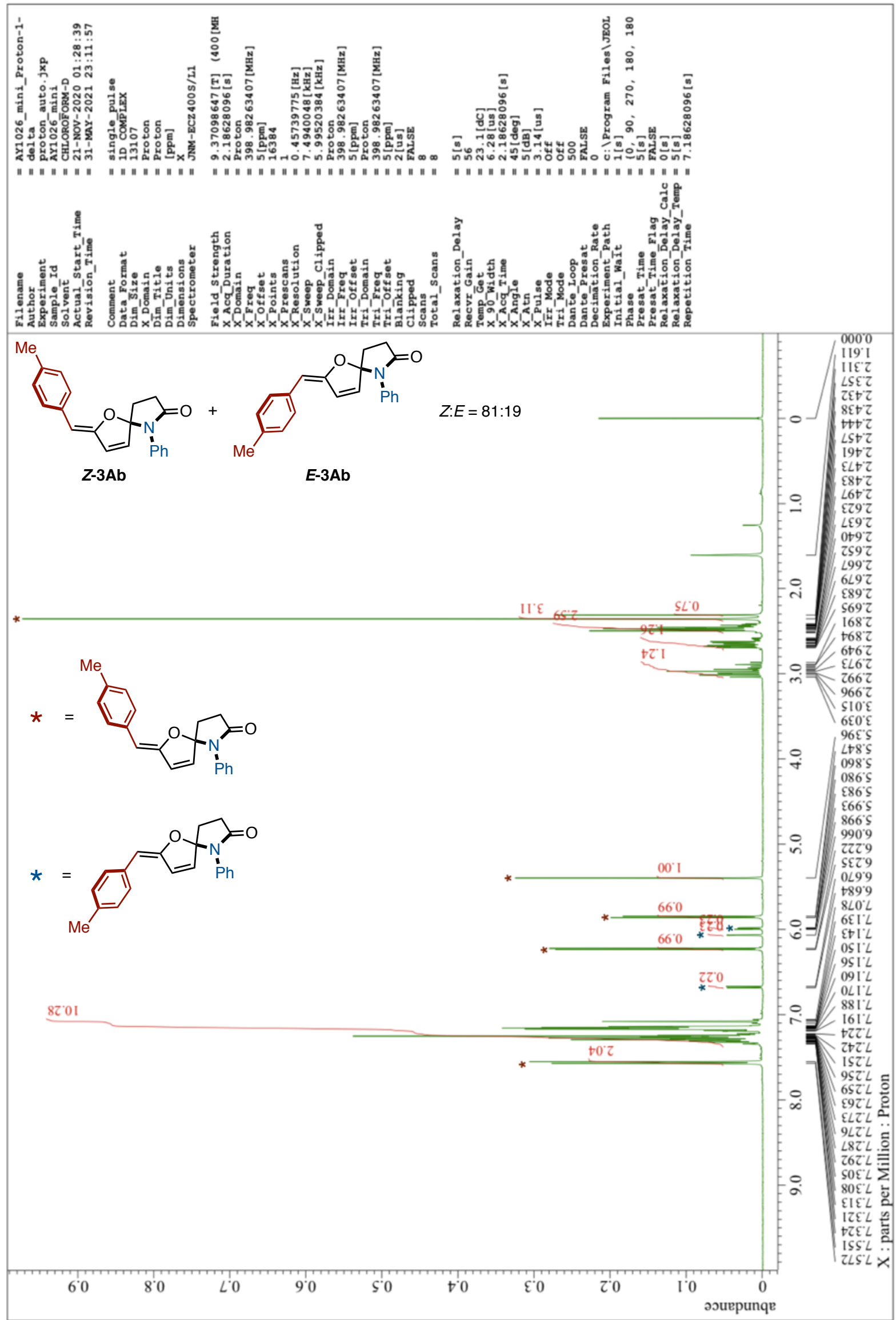


${ }^{13} \mathrm{C}$ NMR of $3 \mathrm{Ab}\left(101 \mathrm{MHz}, \mathrm{CDCl}_{3}\right)$

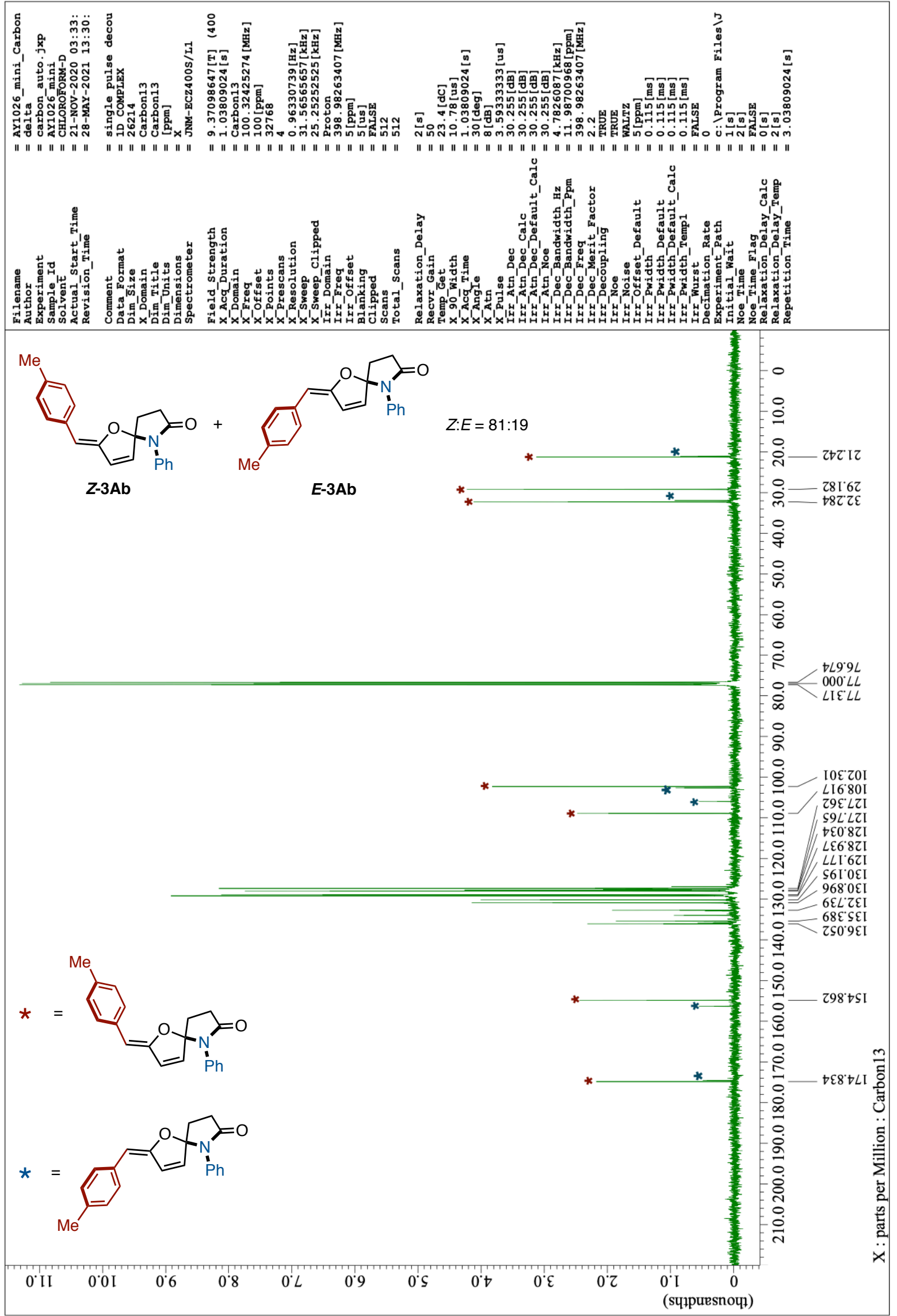


${ }^{1} \mathrm{H}$ NMR of $3 \mathrm{Ac}\left(400 \mathrm{MHz}, \mathrm{CDCl}_{3}\right)$

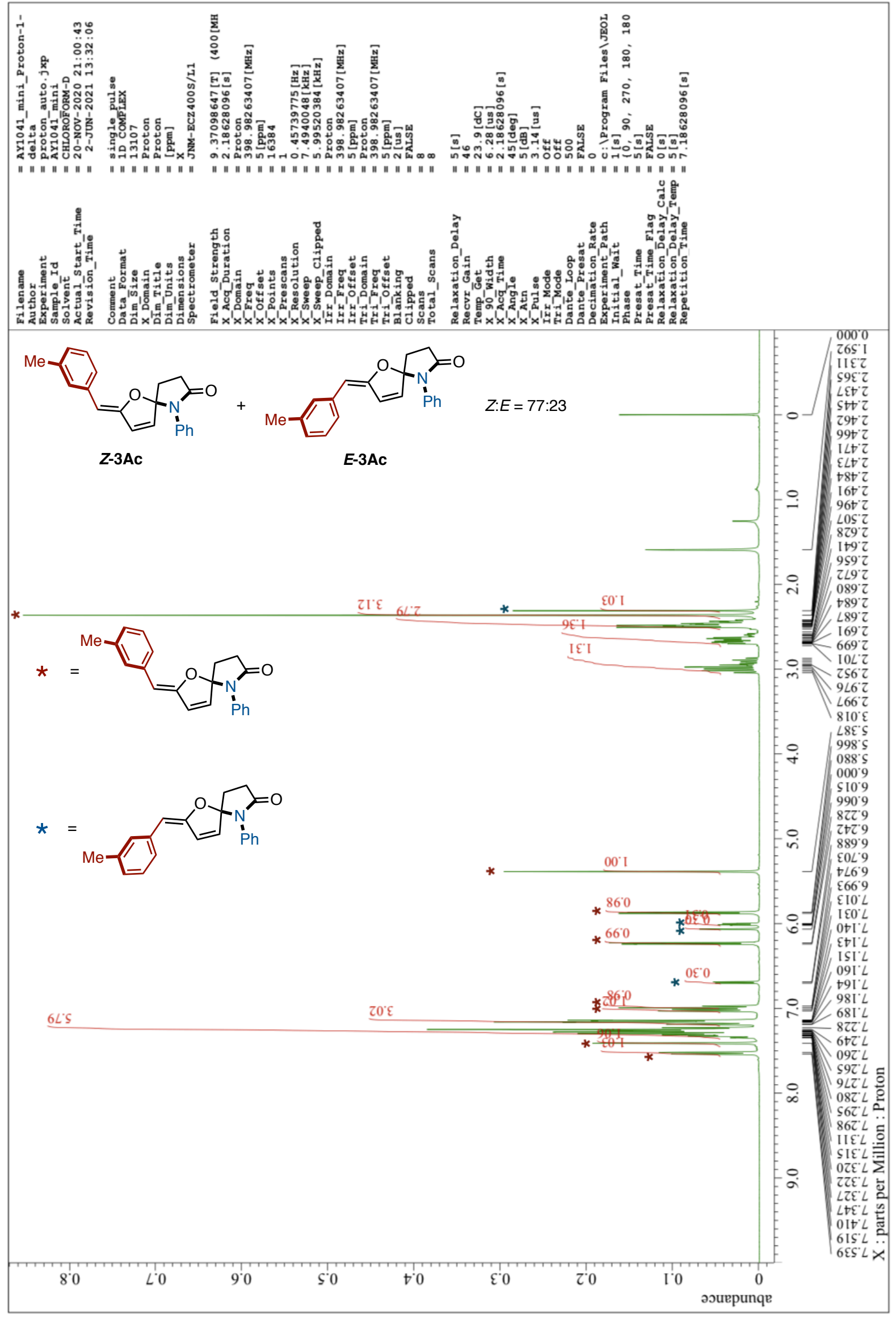


${ }^{13} \mathrm{C}$ NMR of $3 \mathrm{Ac}\left(101 \mathrm{MHz}, \mathrm{CDCl}_{3}\right)$

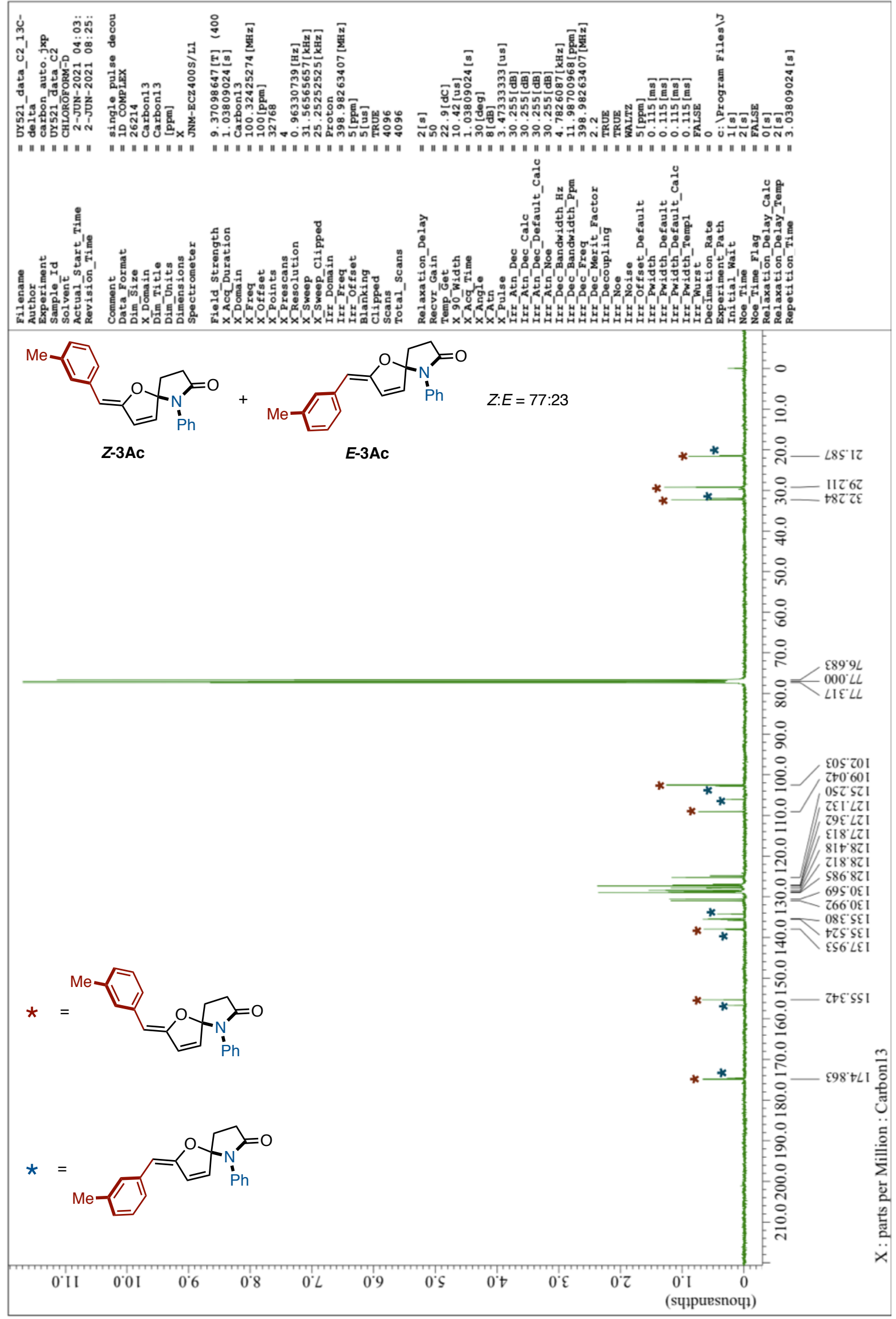


${ }^{1} \mathrm{H}$ NMR of 3Ad (400 $\left.\mathrm{MHz}, \mathrm{CDCl}_{3}\right)$

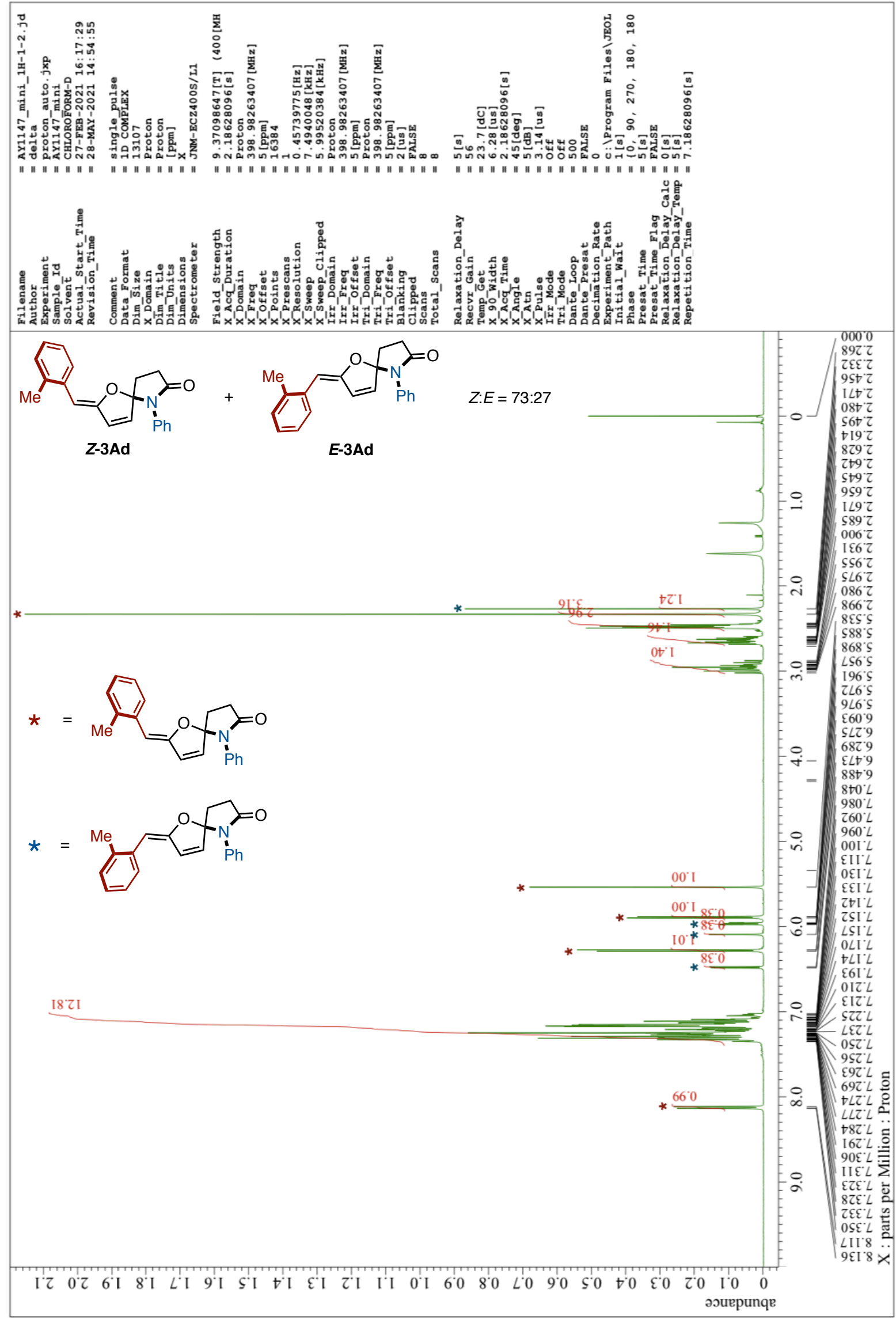


${ }^{13} \mathrm{C}$ NMR of 3Ad (101 MHz, $\left.\mathrm{CDCl}_{3}\right)$

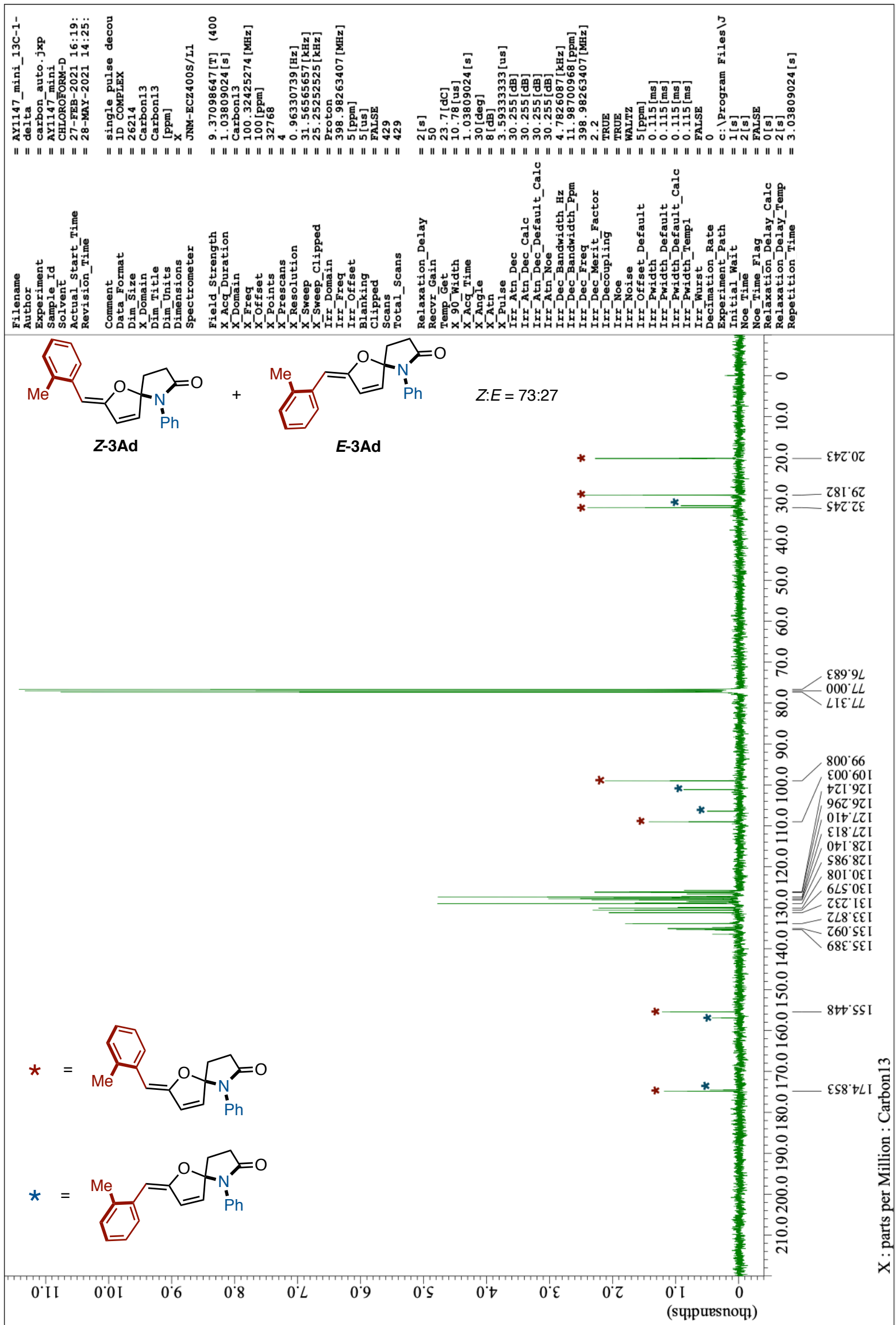


${ }^{1} \mathrm{H}$ NMR of $3 \mathrm{Ae}\left(400 \mathrm{MHz}, \mathrm{CDCl}_{3}\right)$

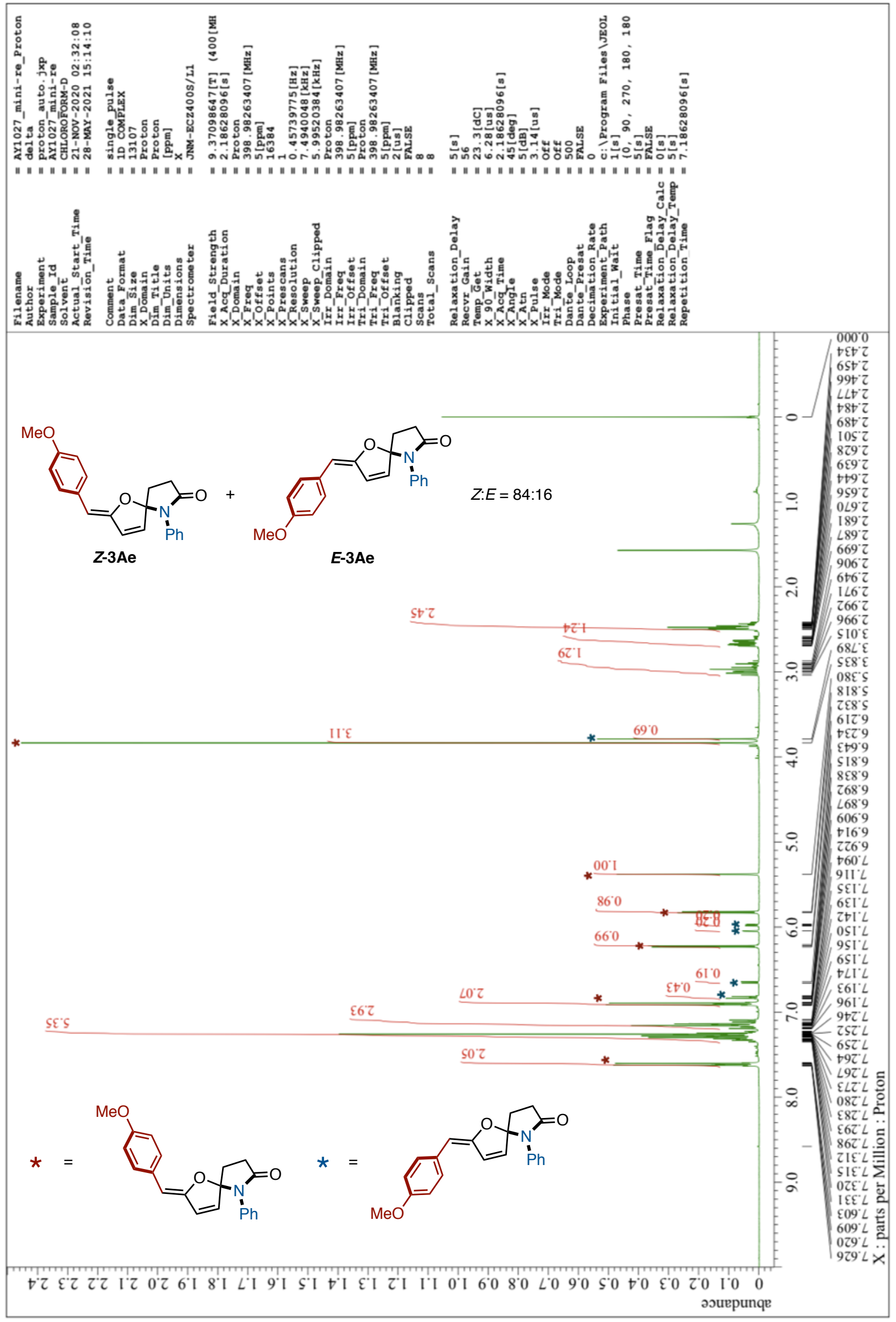


${ }^{13} \mathrm{C}$ NMR of $3 \mathrm{Ae}\left(101 \mathrm{MHz}, \mathrm{CDCl}_{3}\right)$

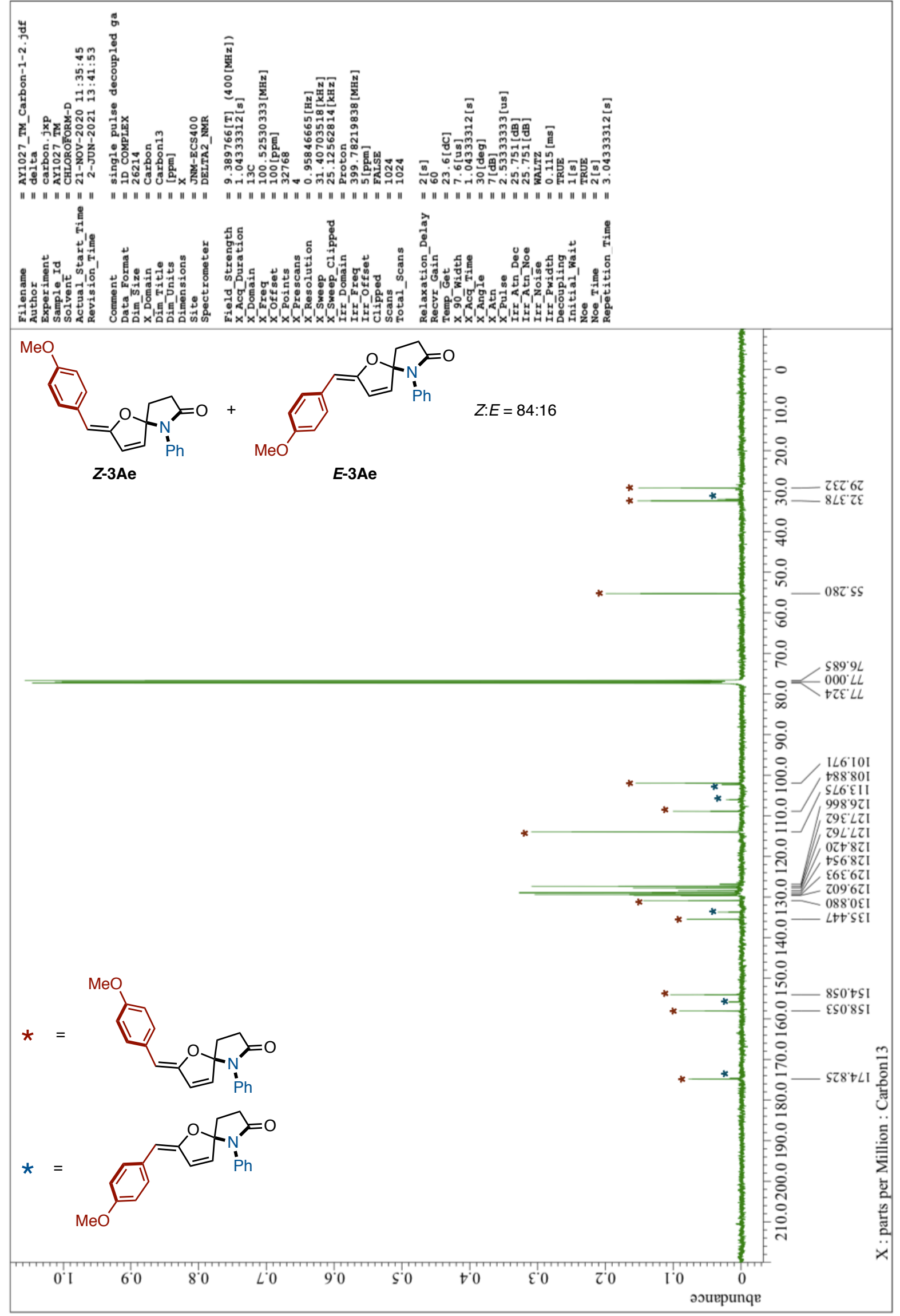


${ }^{1} \mathrm{H}$ NMR of 3Af (400 MHz, $\left.\mathrm{CDCl}_{3}\right)$

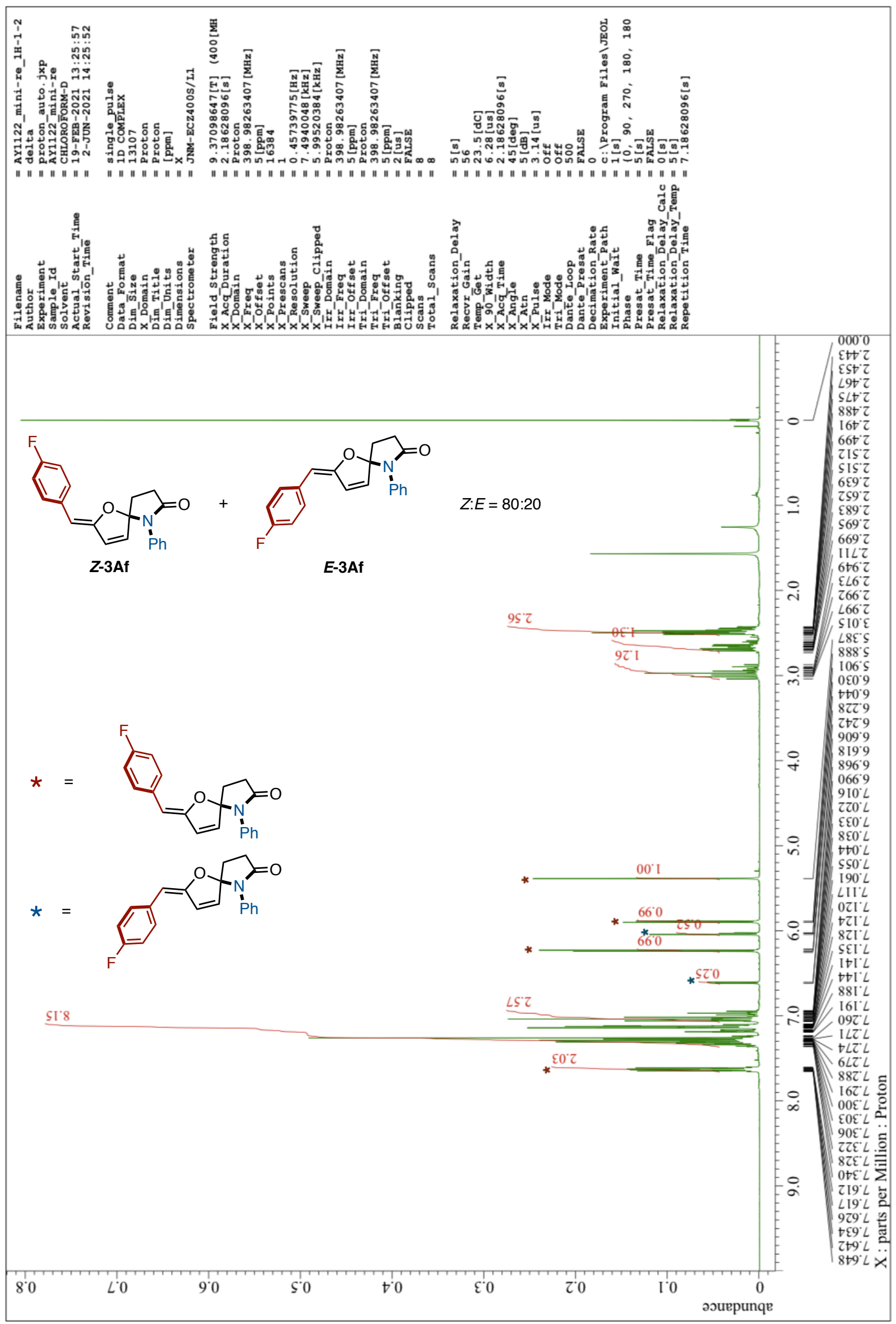


${ }^{13} \mathrm{C}$ NMR of $3 \mathrm{Af}\left(101 \mathrm{MHz}, \mathrm{CDCl}_{3}\right)$

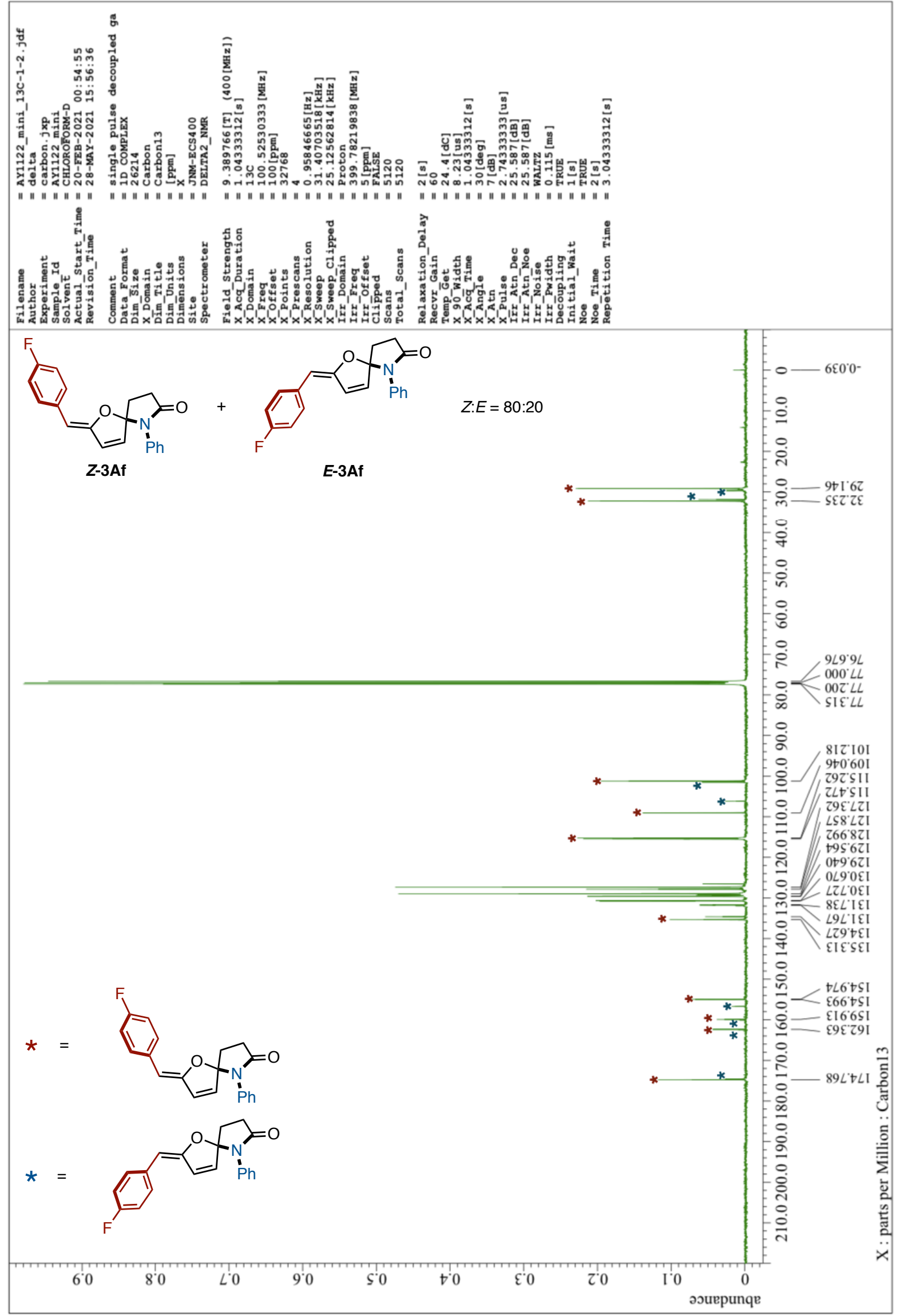


19F NMR of $3 \mathrm{Af}\left(376 \mathrm{MHz}, \mathrm{CDCl}_{3}\right)$

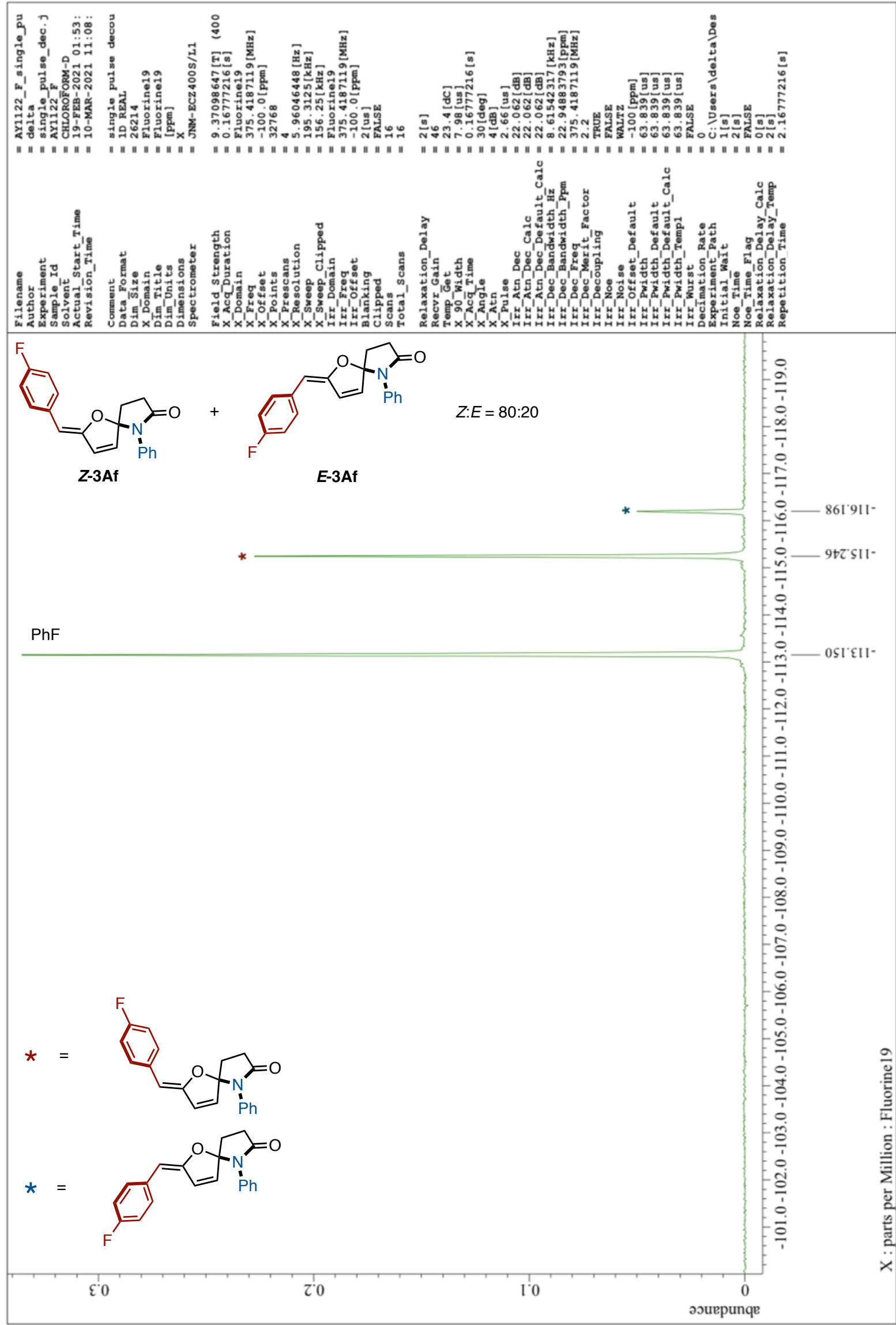


${ }^{1} \mathrm{H}$ NMR of $3 \mathrm{Ag}\left(400 \mathrm{MHz}, \mathrm{CDCl}_{3}\right)$

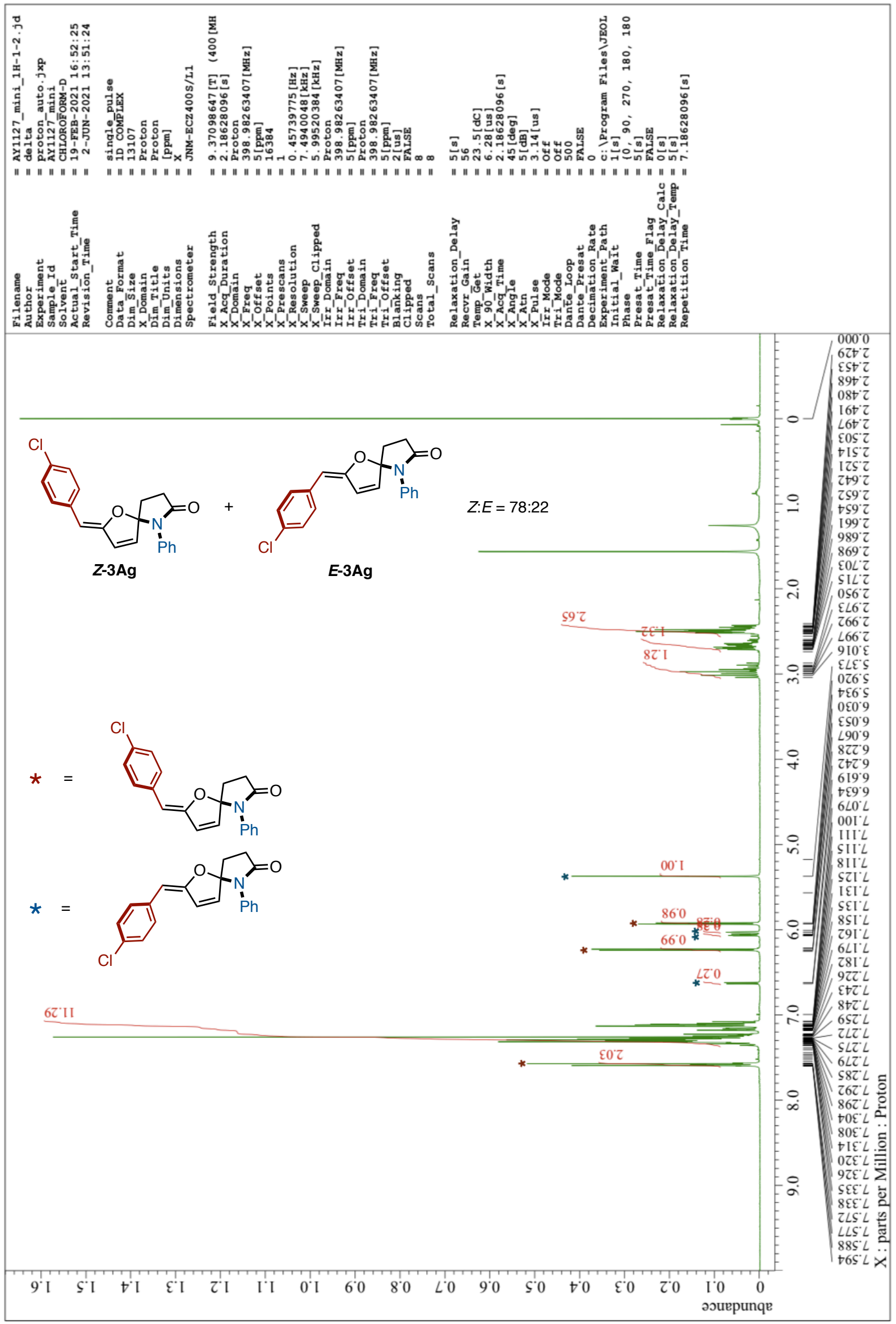


${ }^{13} \mathrm{C}$ NMR of $3 \mathrm{Ag}\left(101 \mathrm{MHz}, \mathrm{CDCl}_{3}\right)$

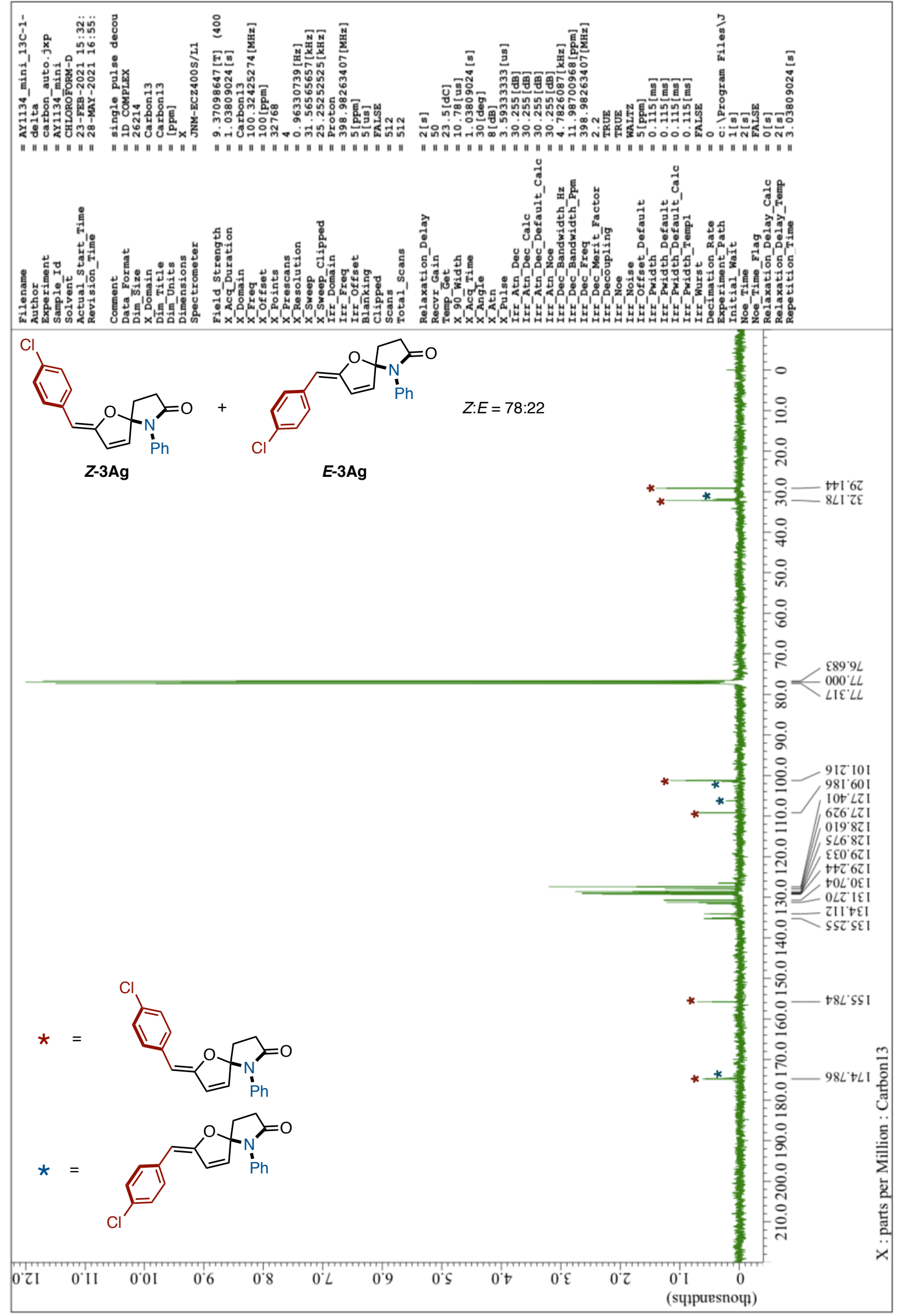


${ }^{1} \mathrm{H}$ NMR of $3 \mathrm{Ah}\left(400 \mathrm{MHz}, \mathrm{CDCl}_{3}\right)$

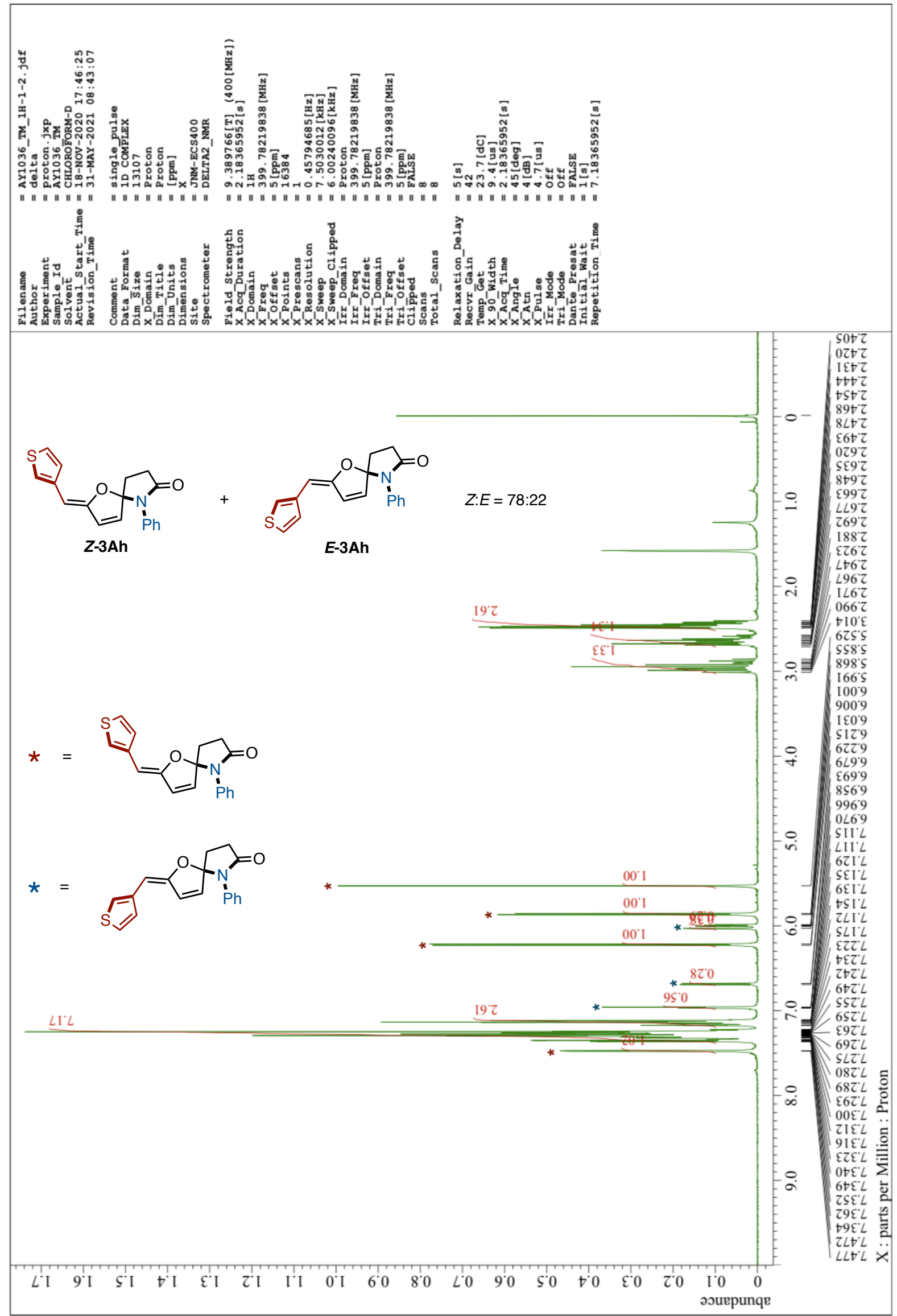


${ }^{13} \mathrm{C}$ NMR of $3 \mathrm{Ah}\left(101 \mathrm{MHz}, \mathrm{CDCl}_{3}\right)$

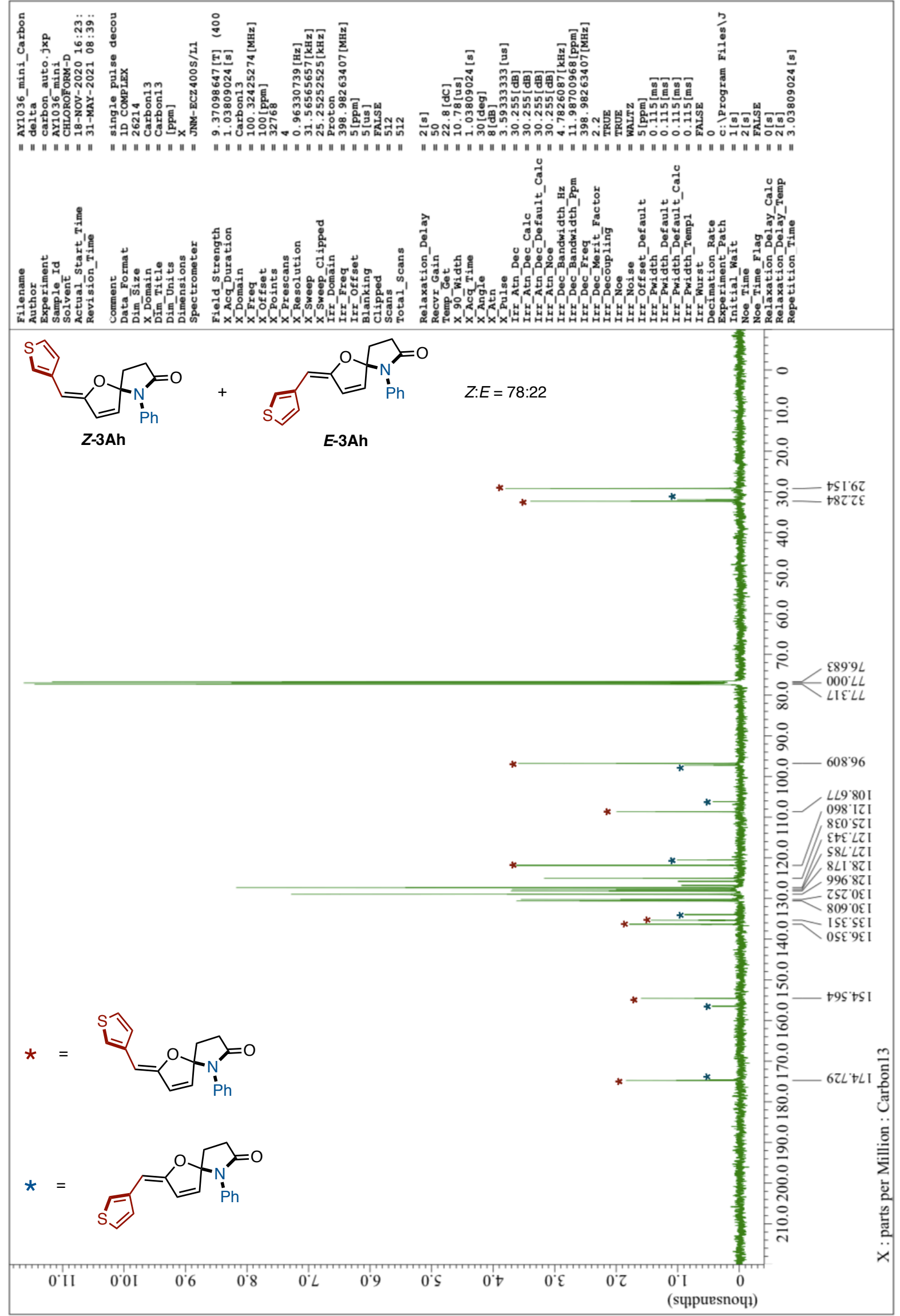


${ }^{1} \mathrm{H}$ NMR of 3Ai (400 MHz, $\left.\mathrm{CDCl}_{3}\right)$

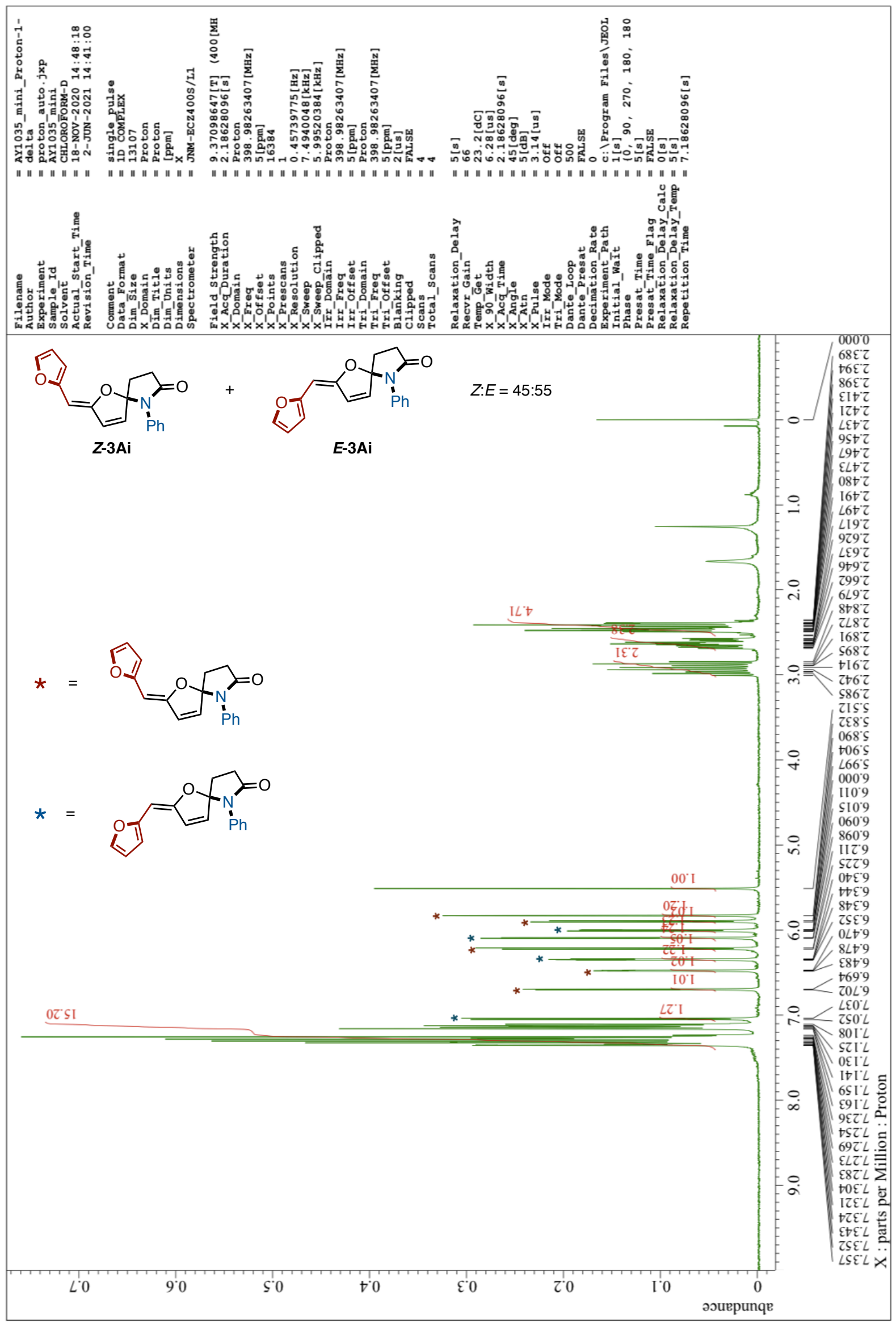


${ }^{13} \mathrm{C}$ NMR of 3Ai (101 MHz, $\left.\mathrm{CDCl}_{3}\right)$

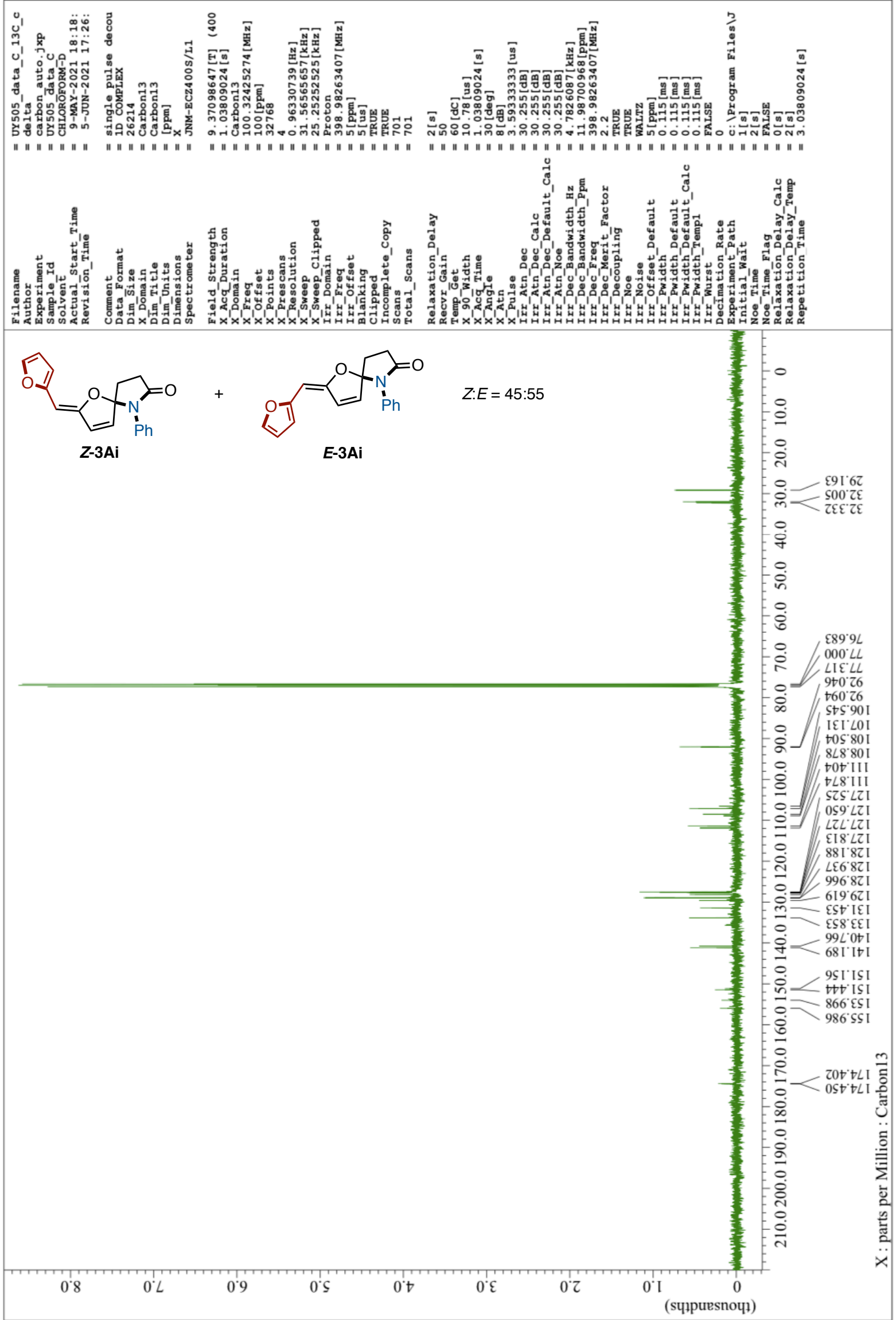


${ }^{1} \mathrm{H}$ NMR of 3Aj (400 $\left.\mathrm{MHz}, \mathrm{CDCl}_{3}\right)$

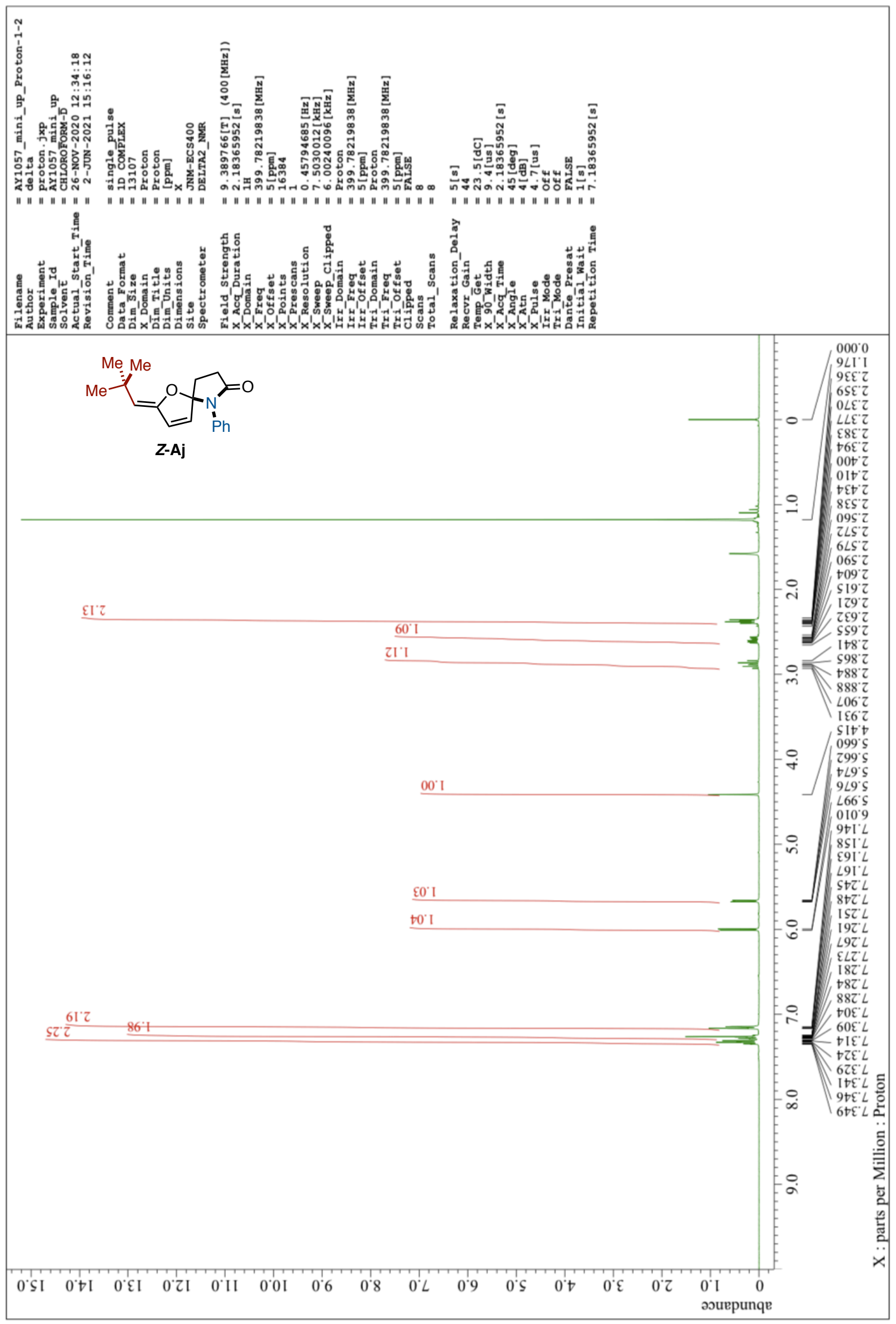


${ }^{13} \mathrm{C}$ NMR of $3 \mathrm{Aj}\left(101 \mathrm{MHz}, \mathrm{CDCl}_{3}\right)$

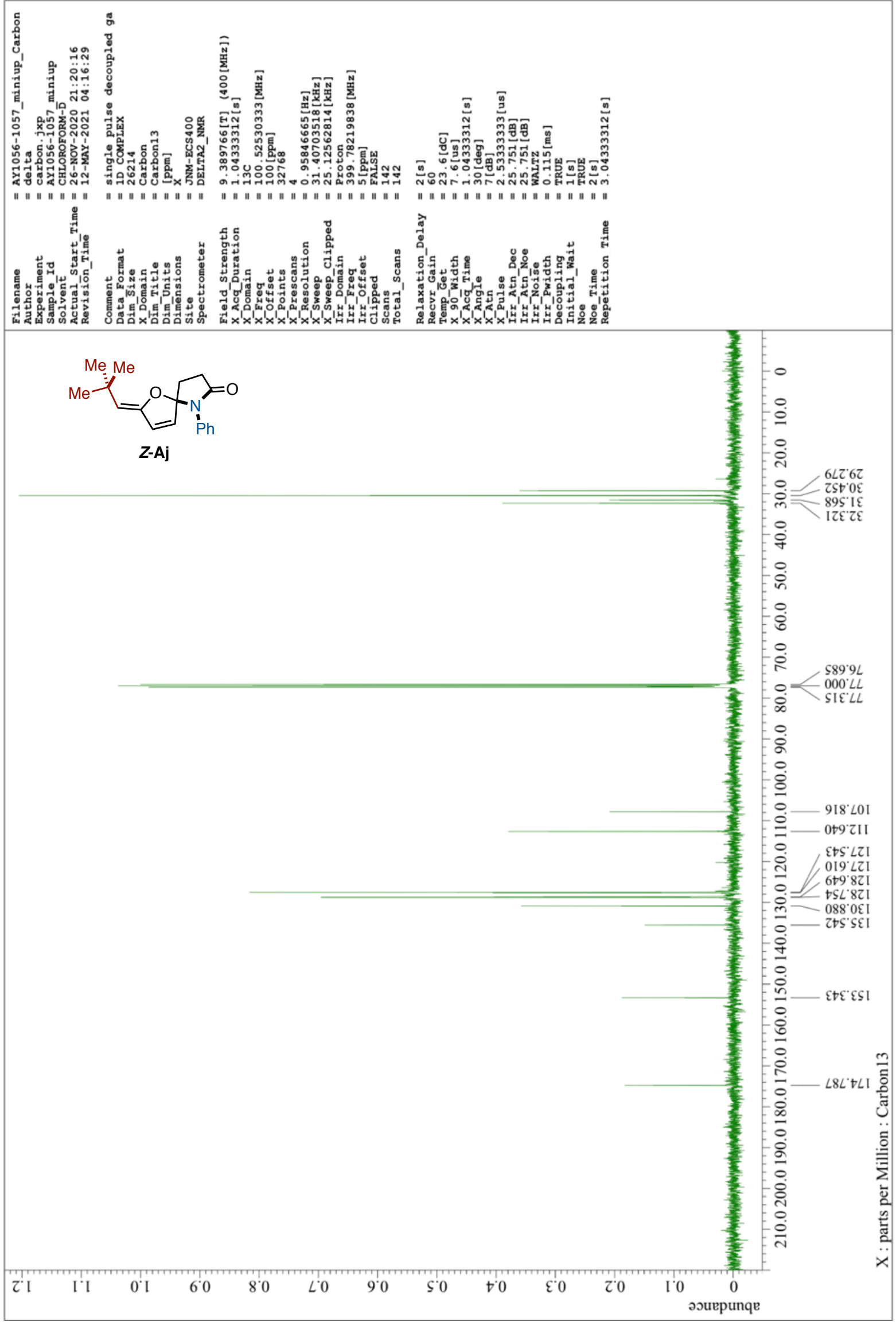


${ }^{1} \mathrm{H}$ NMR of 3Aj (400 MHz, $\left.\mathrm{CDCl}_{3}\right)$

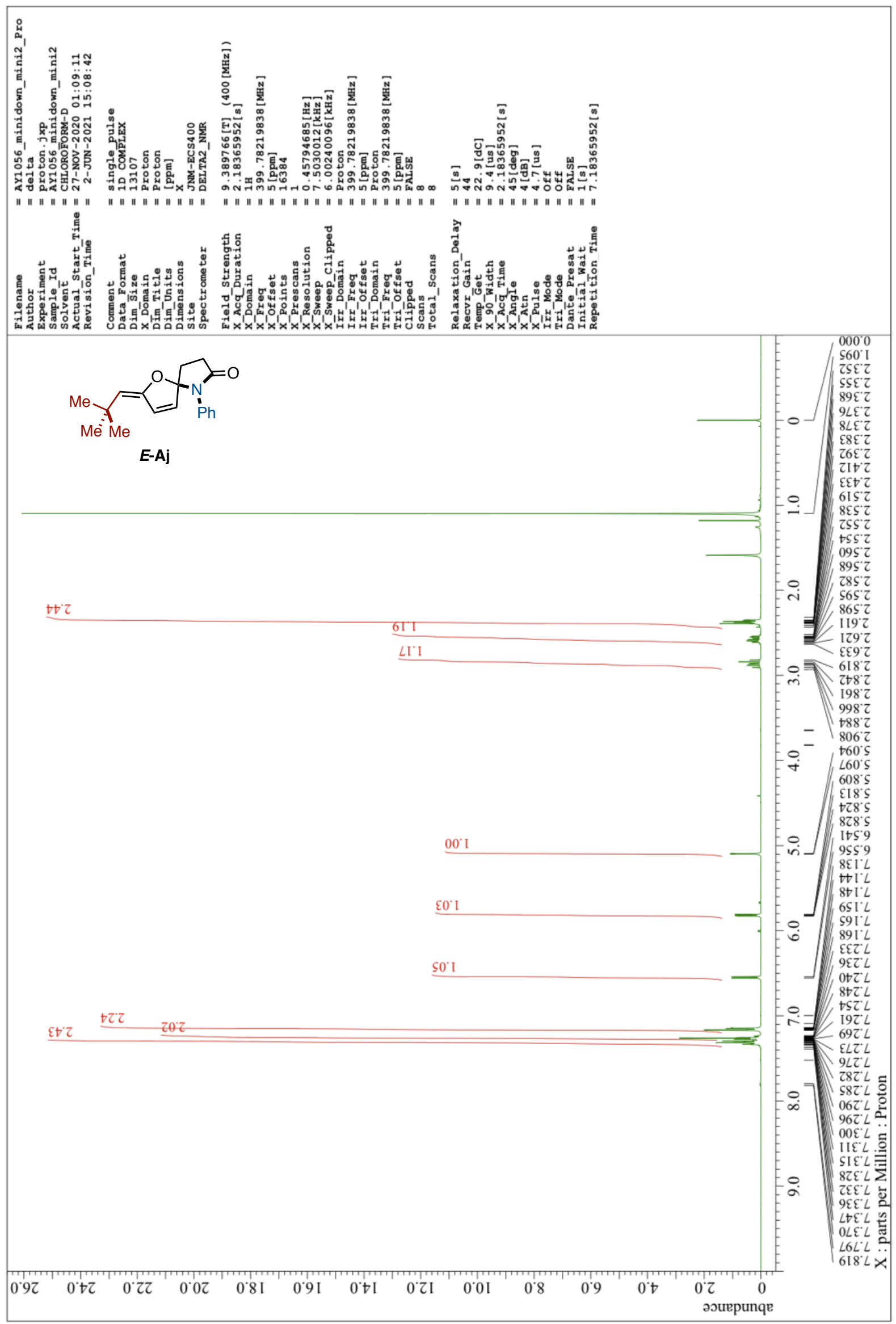


${ }^{13} \mathrm{C}$ NMR of 3Aj (101 MHz, $\left.\mathrm{CDCl}_{3}\right)$

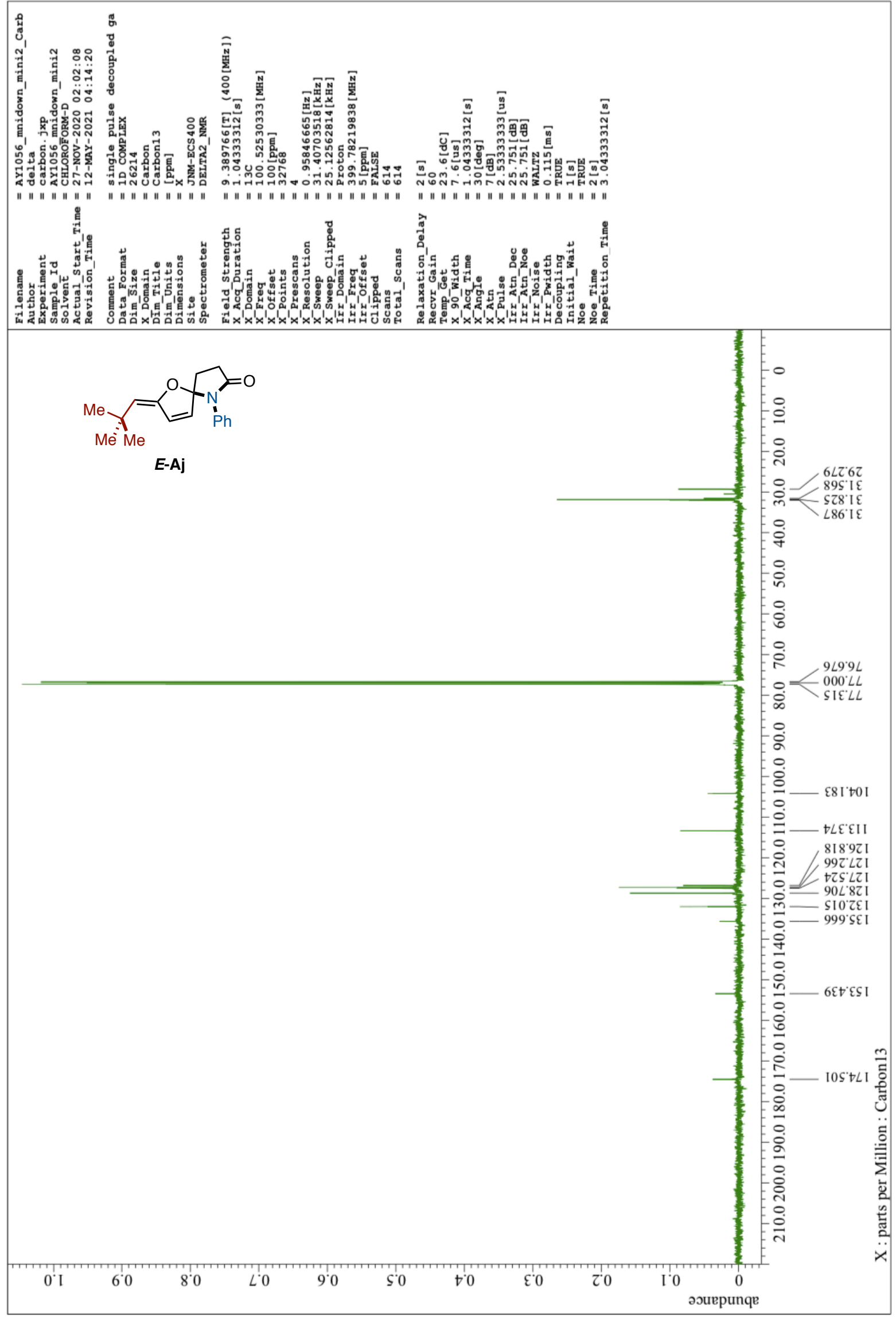


${ }^{1} \mathrm{H}$ NMR of 3Ak (400 $\left.\mathrm{MHz}, \mathrm{CDCl}_{3}\right)$

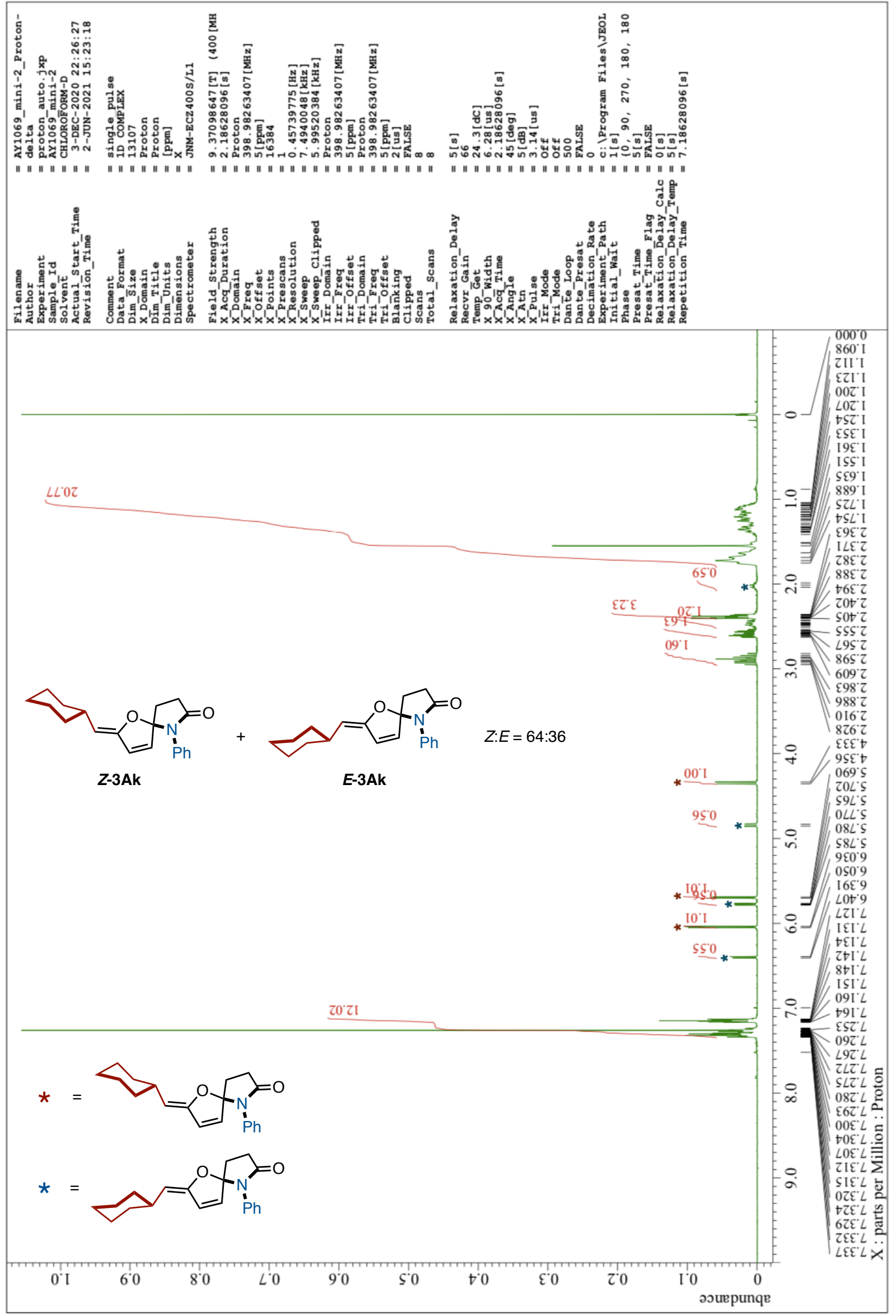


${ }^{13} \mathrm{C}$ NMR of 3Ak (101 MHz, $\left.\mathrm{CDCl}_{3}\right)$

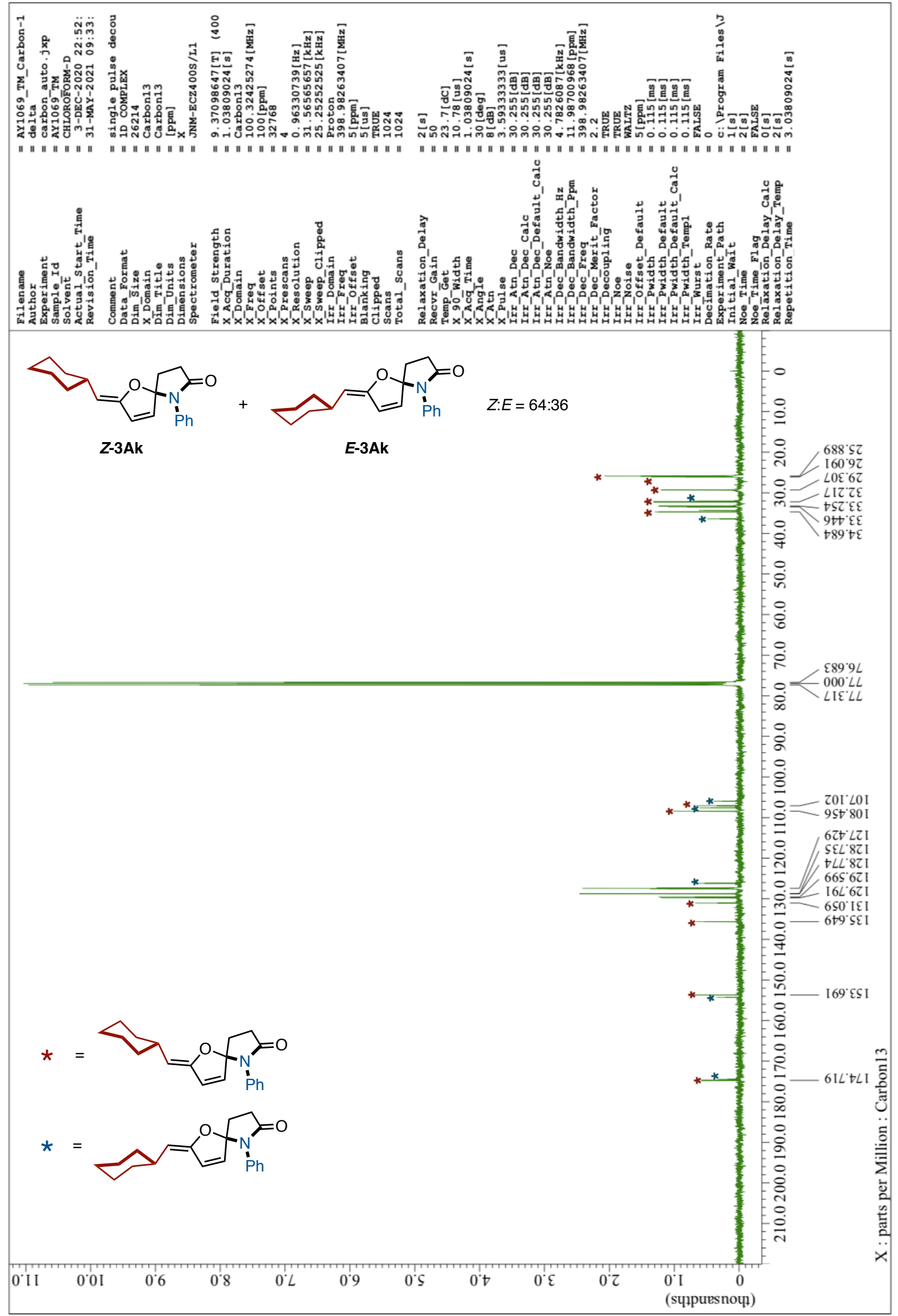


${ }^{1} \mathrm{H}$ NMR of 3Al (400 MHz, $\left.\mathrm{CDCl}_{3}\right)$

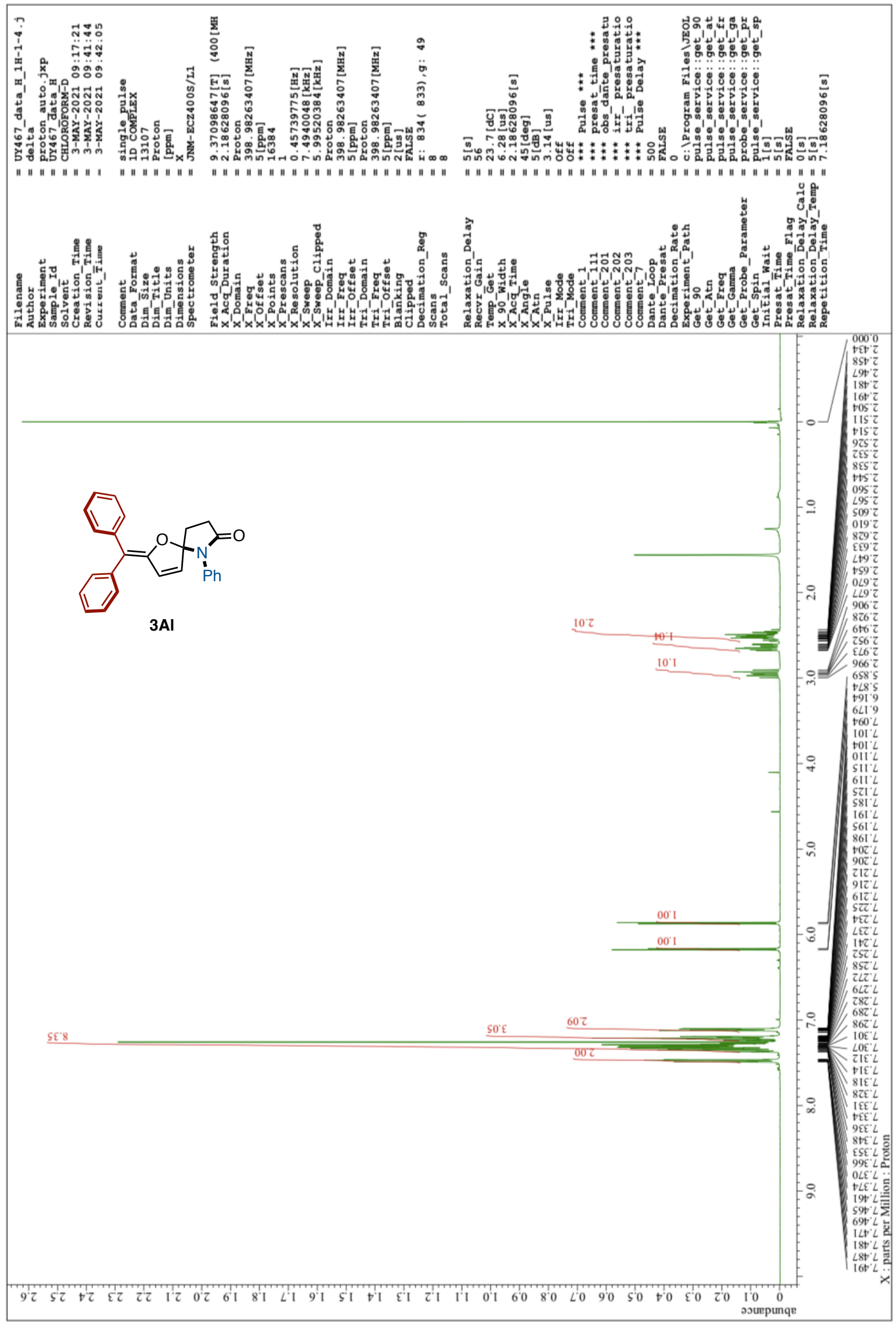


${ }^{13} \mathrm{C}$ NMR of 3Al (101 MHz, $\left.\mathrm{CDCl}_{3}\right)$

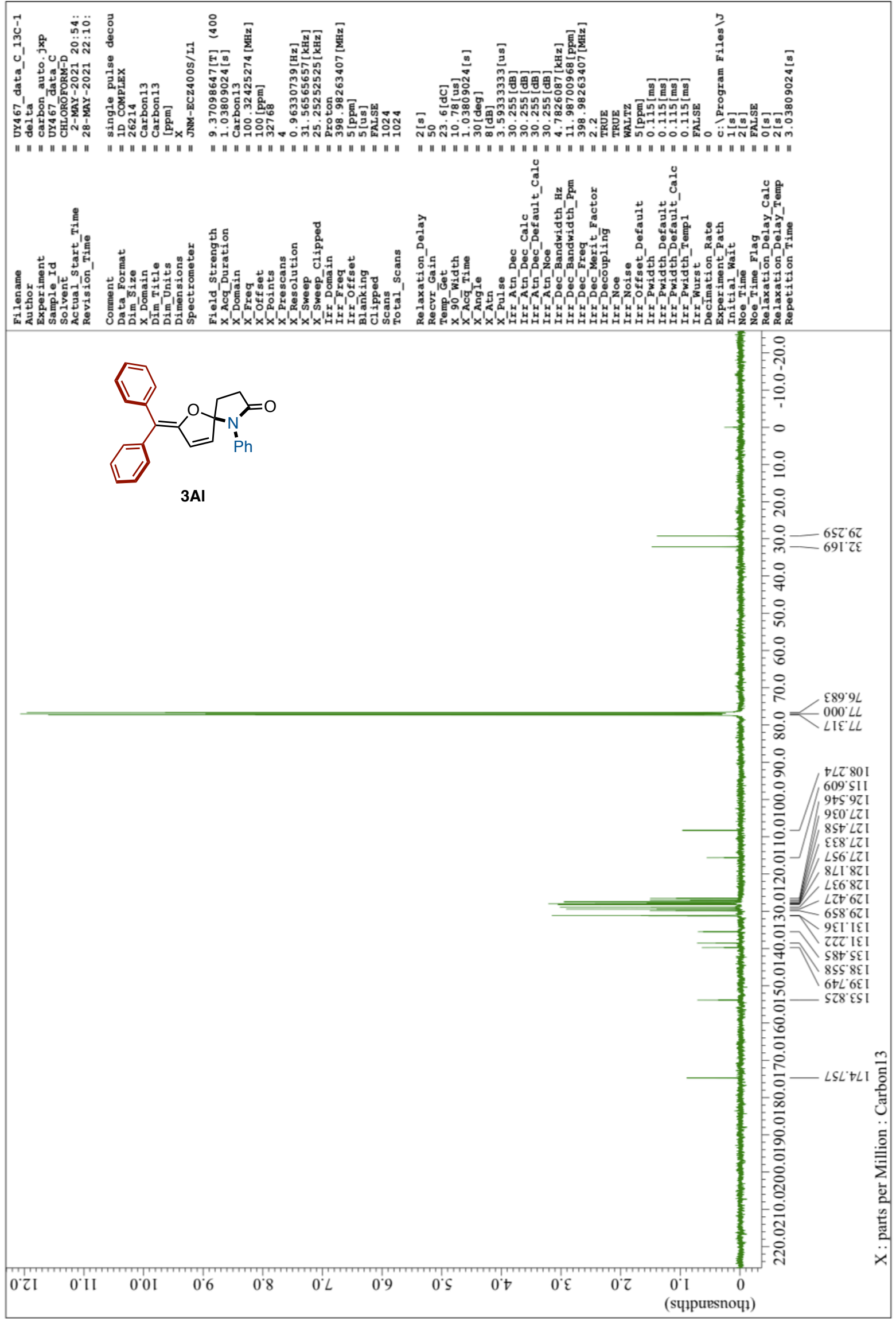


${ }^{1} \mathrm{H}$ NMR of $3 \mathrm{Ha}\left(400 \mathrm{MHz}, \mathrm{CDCl}_{3}\right)$

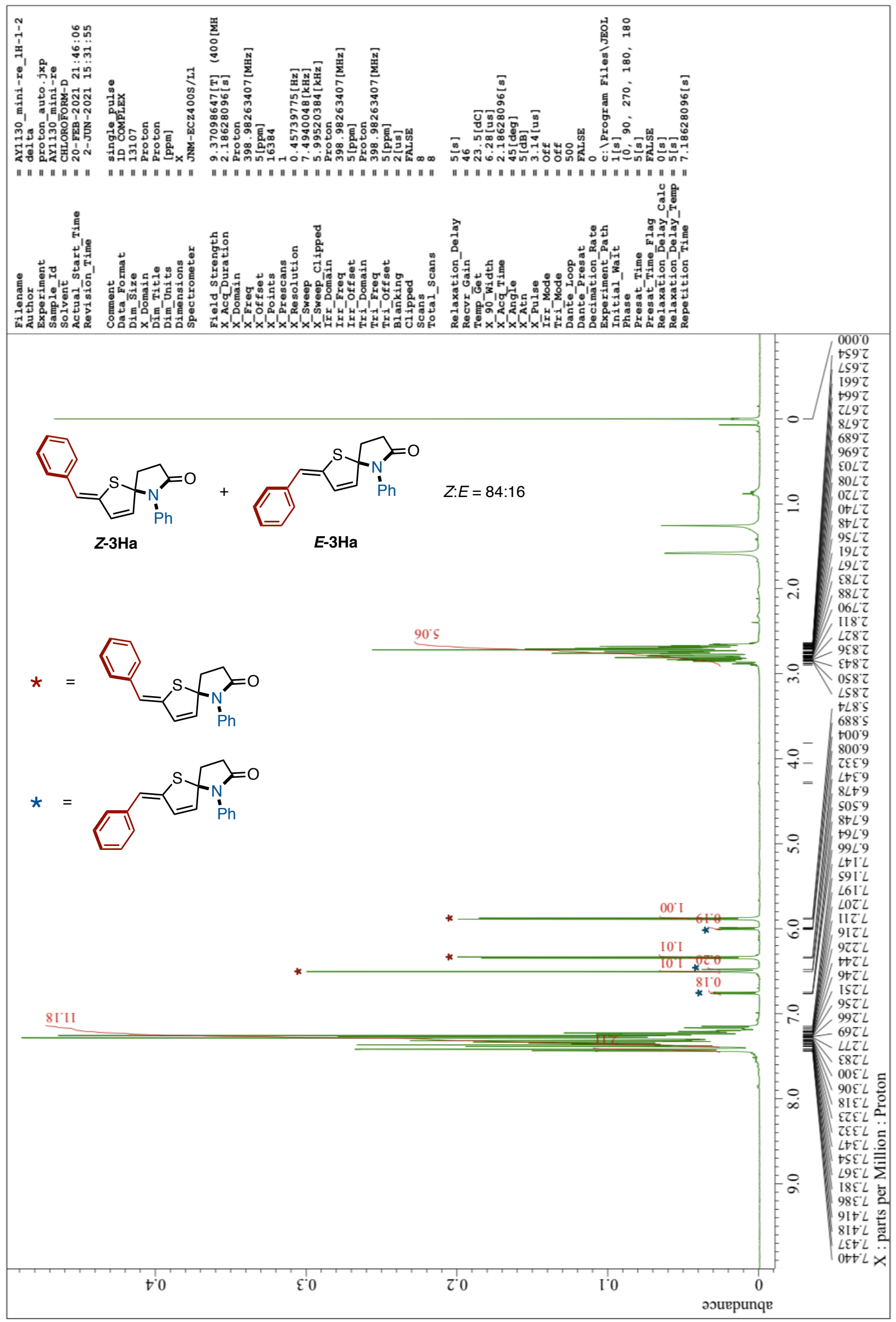


${ }^{13} \mathrm{C}$ NMR of $3 \mathrm{Ha}\left(101 \mathrm{MHz}, \mathrm{CDCl}_{3}\right)$

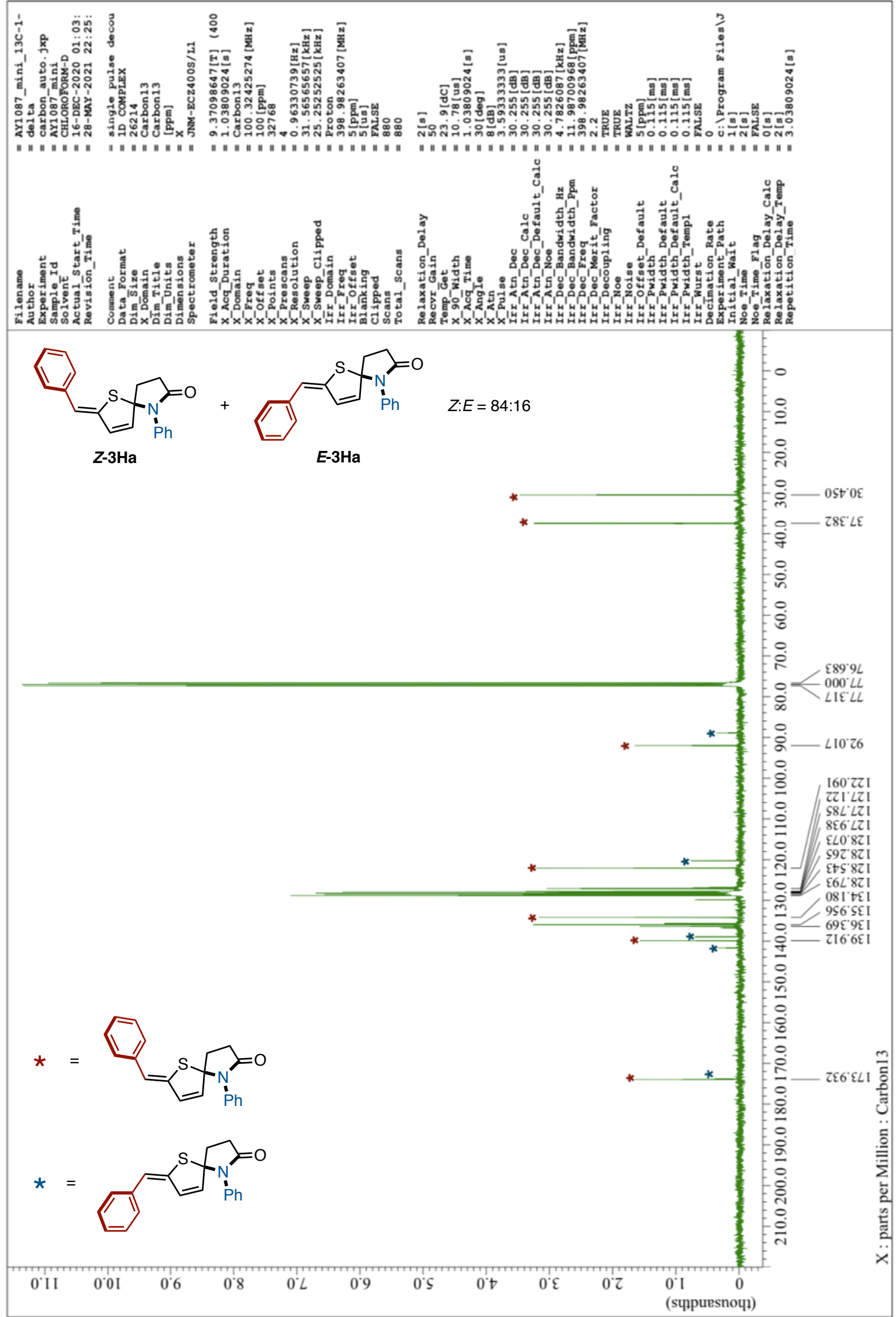


${ }^{1} \mathrm{H}$ NMR of 3Ia (400 MHz, $\left.\mathrm{CDCl}_{3}\right)$

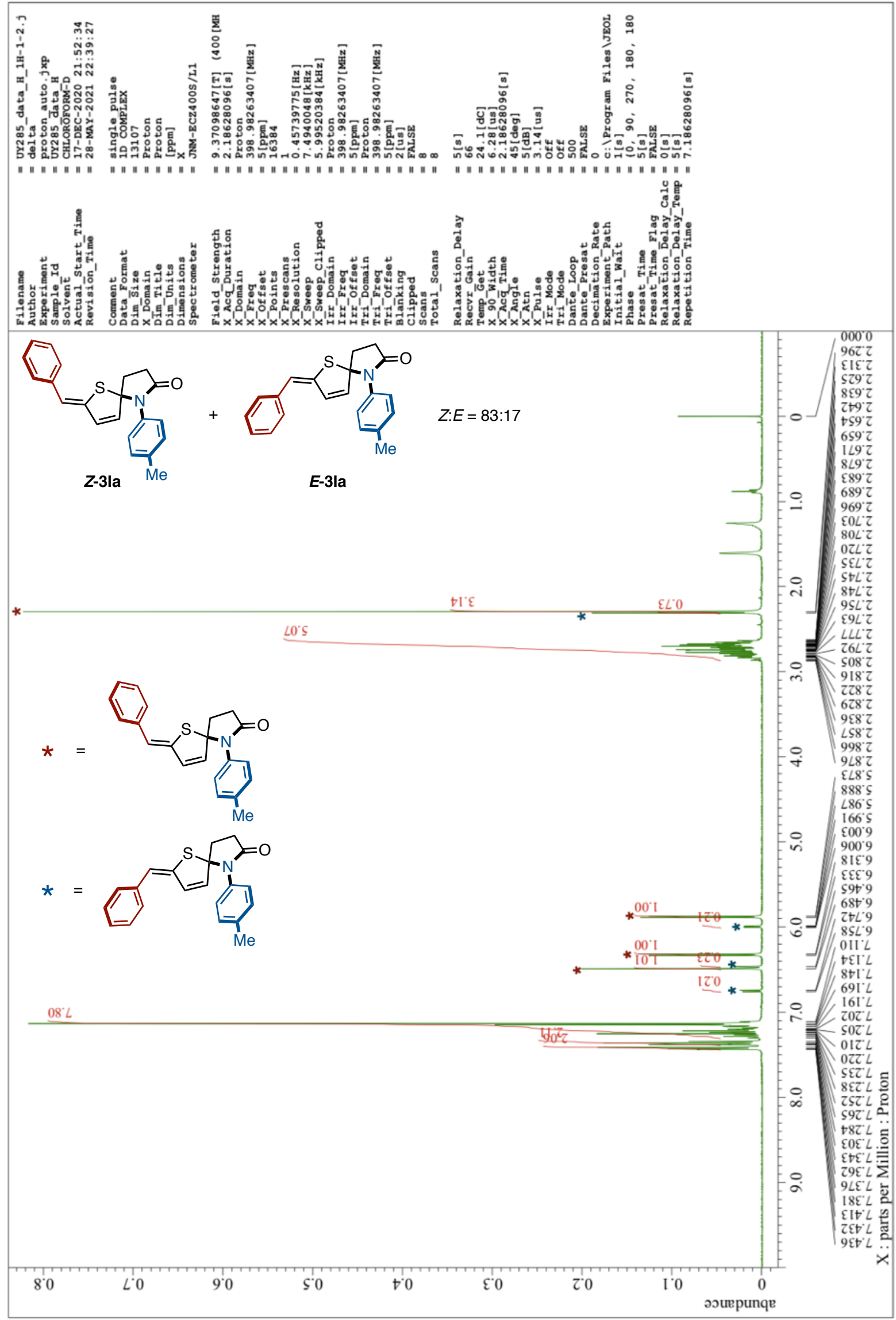


${ }^{13} \mathrm{C}$ NMR of 3Ia (101 $\left.\mathrm{MHz}, \mathrm{CDCl}_{3}\right)$

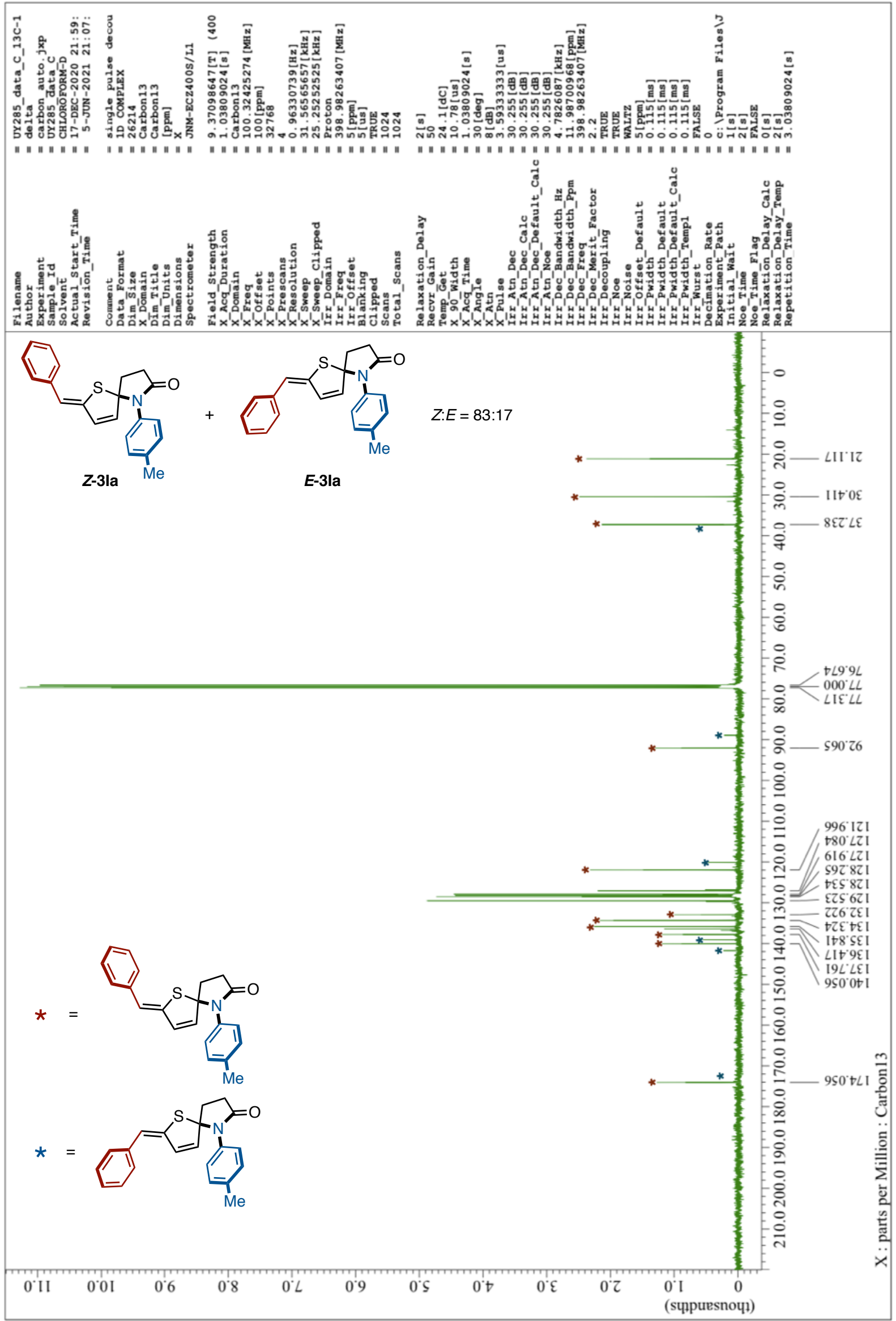

S148 
${ }^{1} \mathrm{H}$ NMR of 3Ja (400 MHz, $\left.\mathrm{CDCl}_{3}\right)$

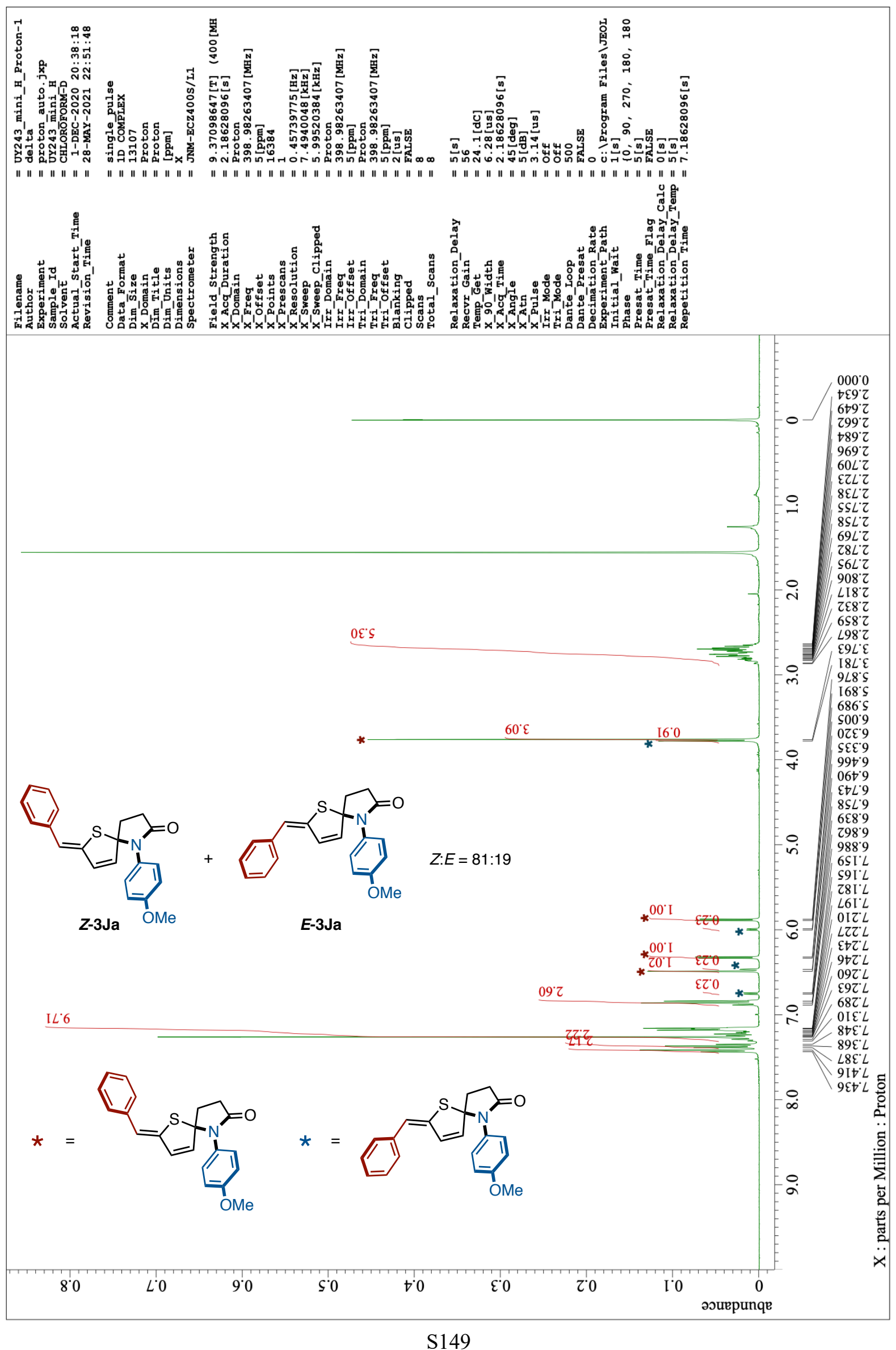


${ }^{13} \mathrm{C}$ NMR of 3Ja (101 MHz, $\left.\mathrm{CDCl}_{3}\right)$

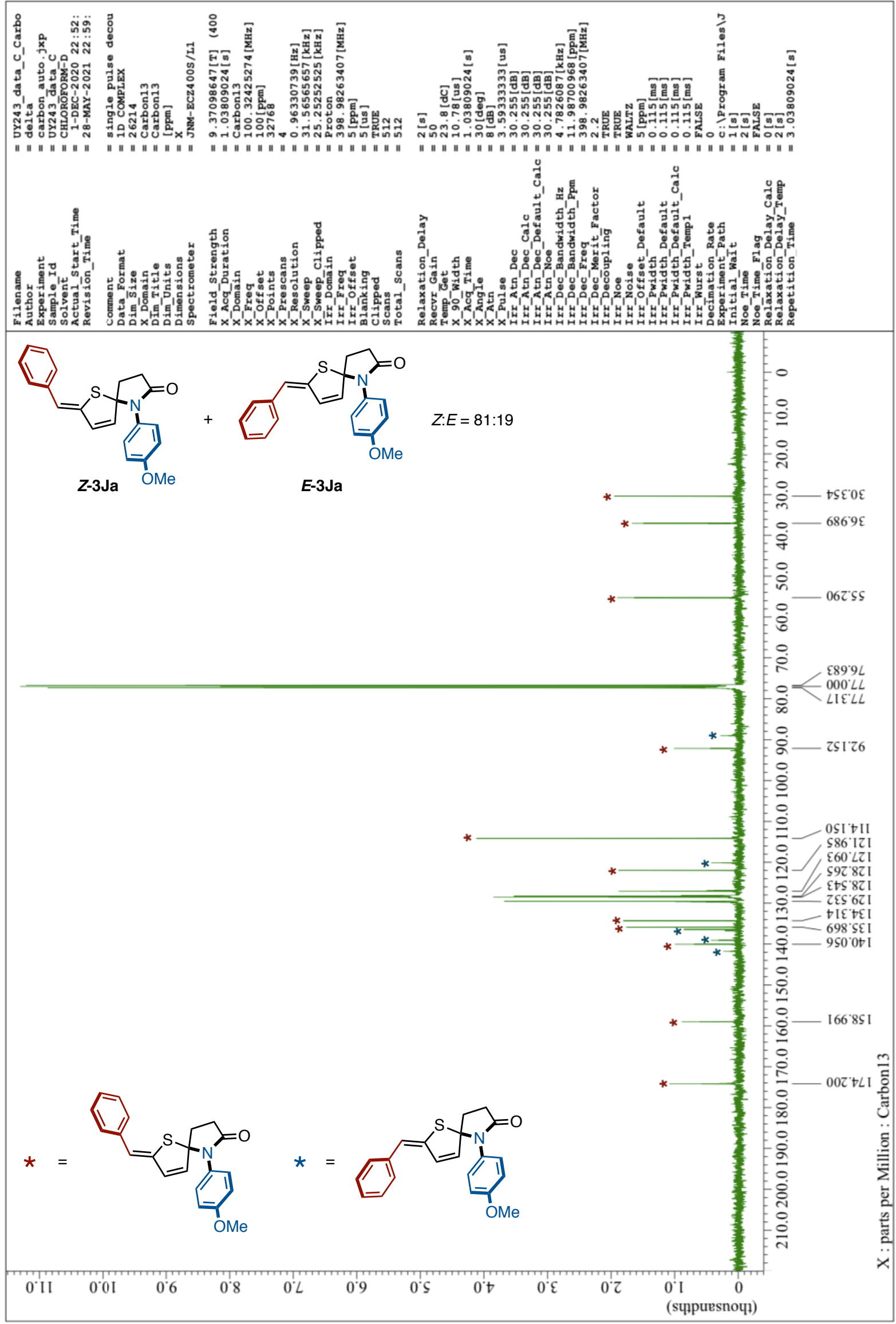


${ }^{1} \mathrm{H}$ NMR of $3 \mathrm{Ka}\left(400 \mathrm{MHz}, \mathrm{CDCl}_{3}\right)$

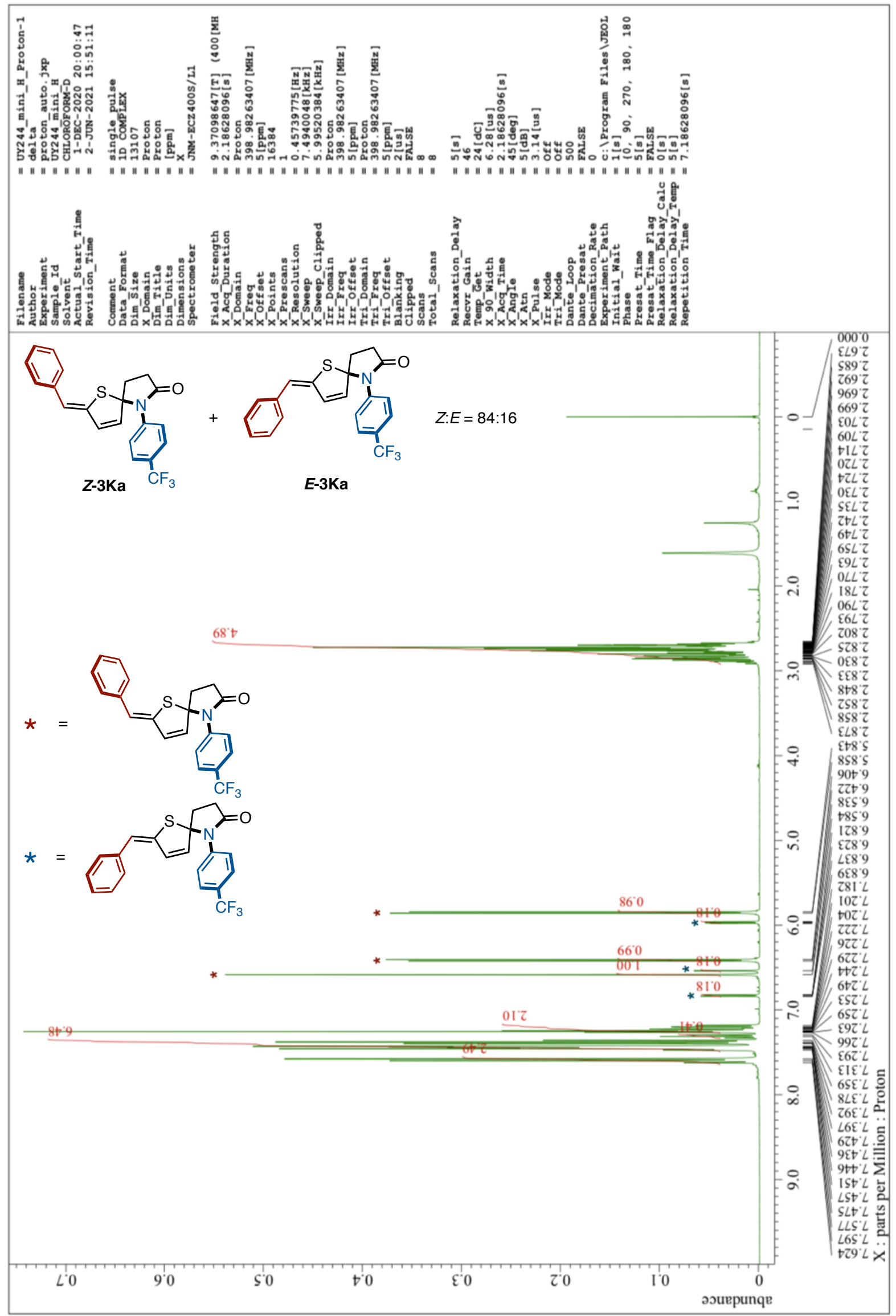


${ }^{13} \mathrm{C}$ NMR of $3 \mathrm{Ka}\left(101 \mathrm{MHz}, \mathrm{CDCl}_{3}\right)$

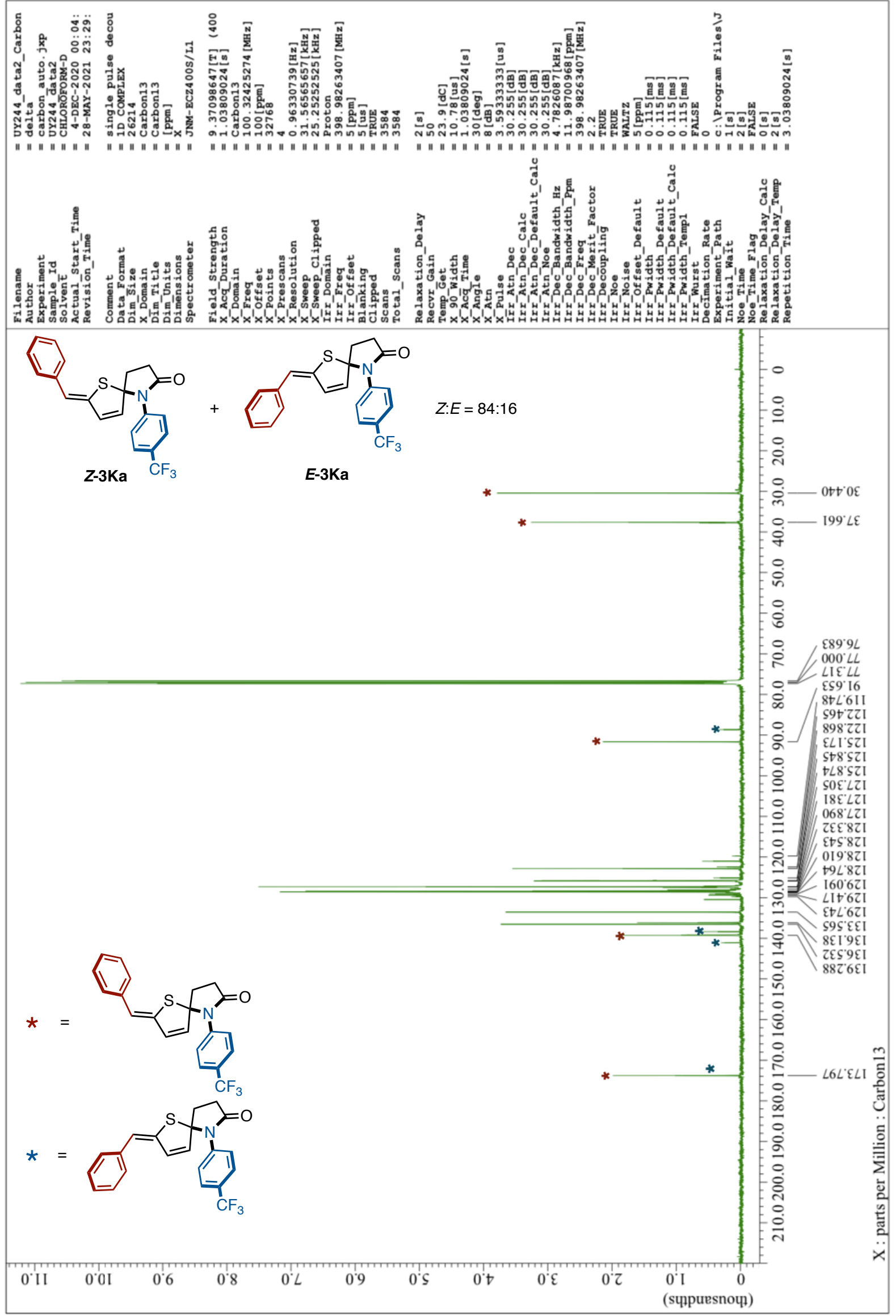


19F NMR of $3 \mathrm{Ka}\left(376 \mathrm{MHz}, \mathrm{CDCl}_{3}\right)$

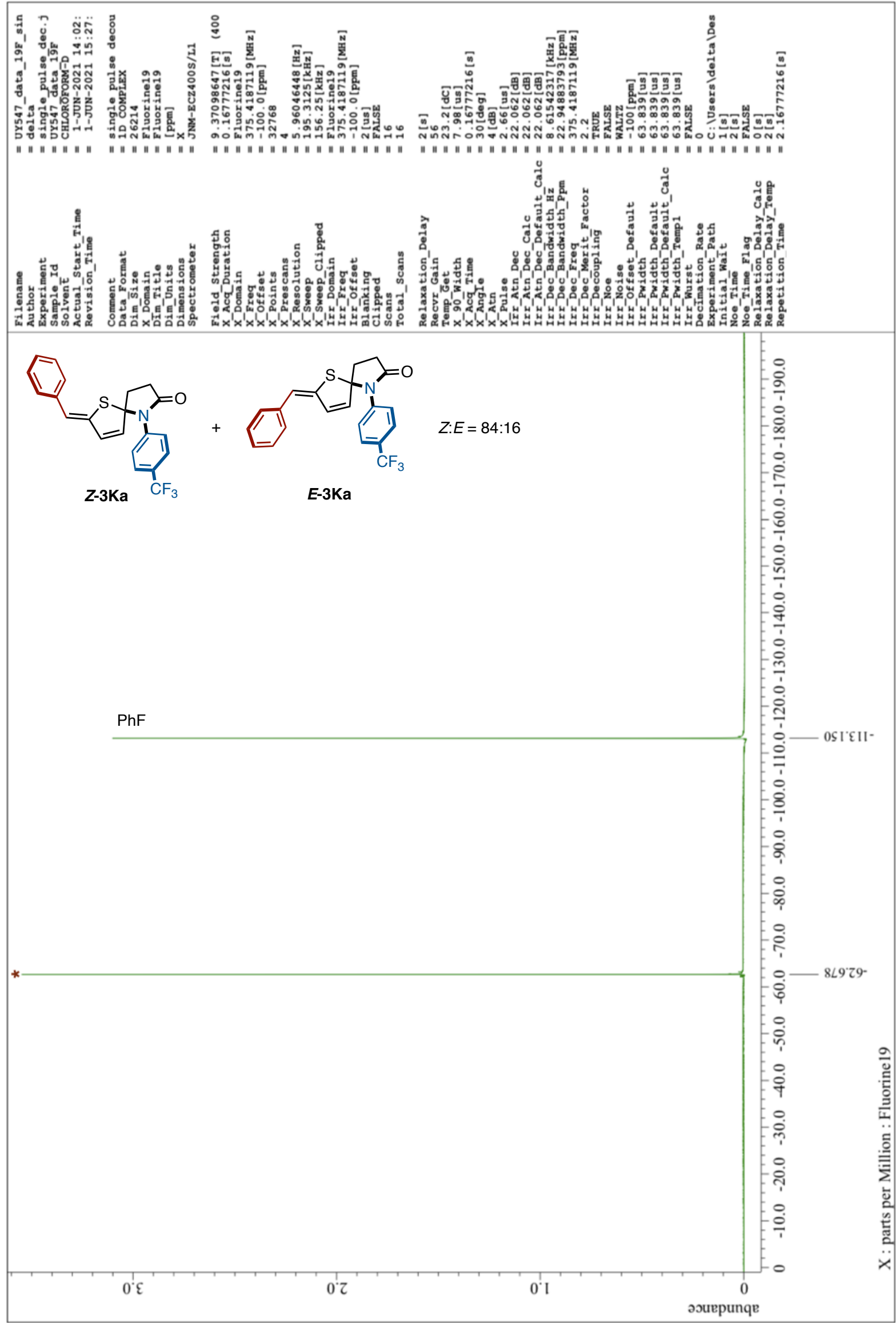


${ }^{1} \mathrm{H}$ NMR of 3La (400 $\left.\mathrm{MHz}, \mathrm{CDCl}_{3}\right)$

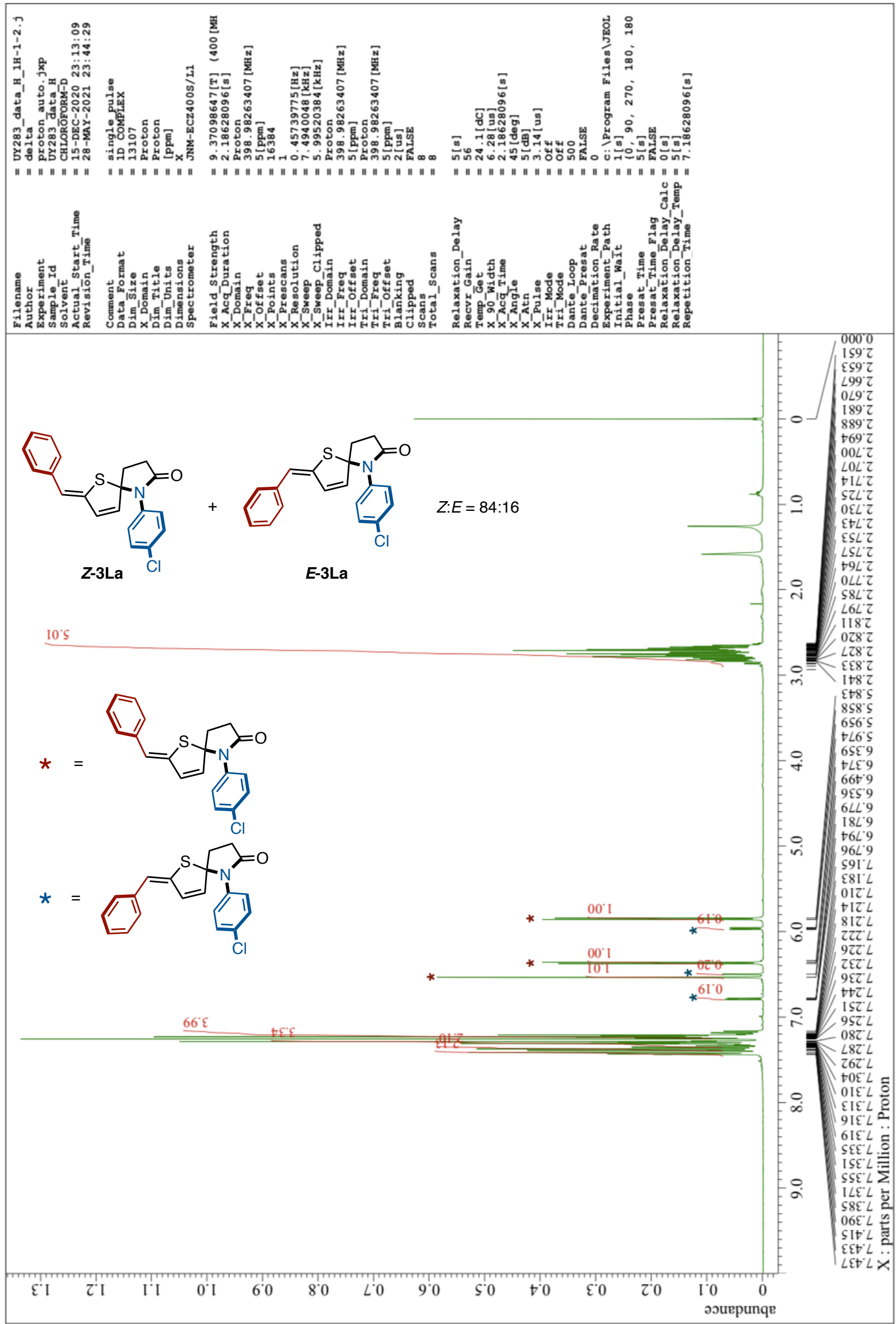


${ }^{13} \mathrm{C}$ NMR of 3La (101 MHz, $\left.\mathrm{CDCl}_{3}\right)$

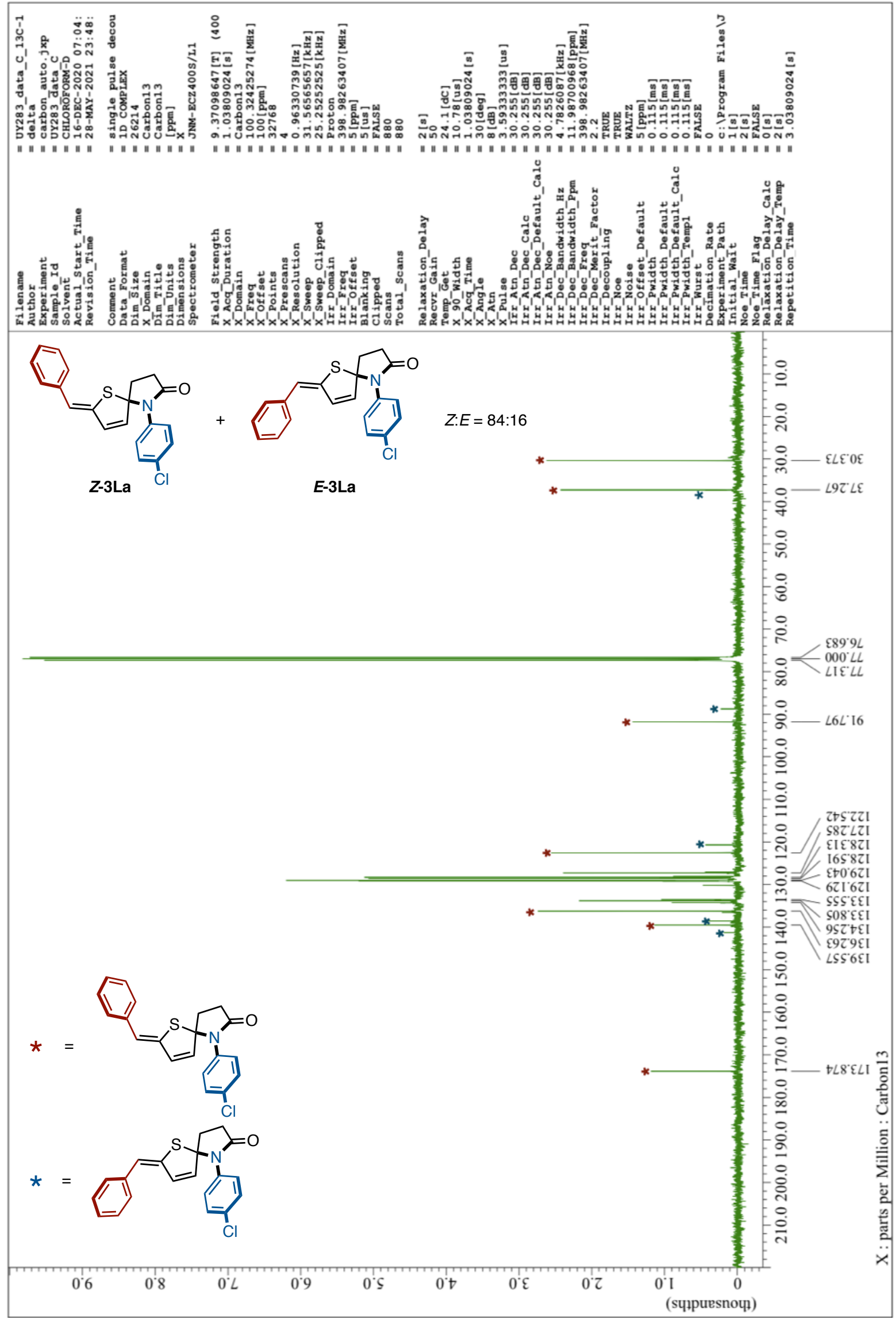


${ }^{1} \mathrm{H}$ NMR of 3Ma (400 $\left.\mathrm{MHz}, \mathrm{CDCl}_{3}\right)$

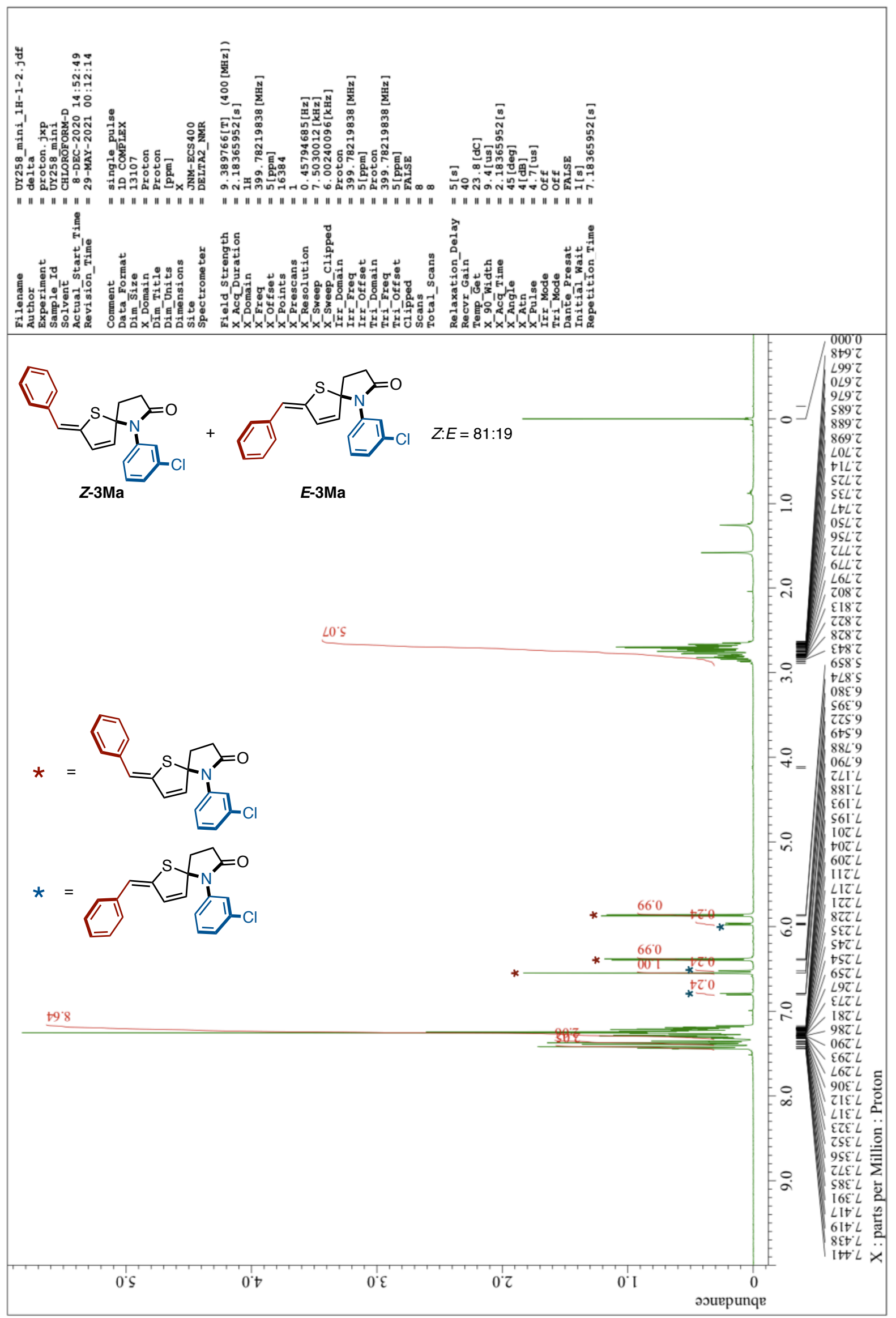


${ }^{13} \mathrm{C}$ NMR of 3Ma (101 MHz, $\left.\mathrm{CDCl}_{3}\right)$

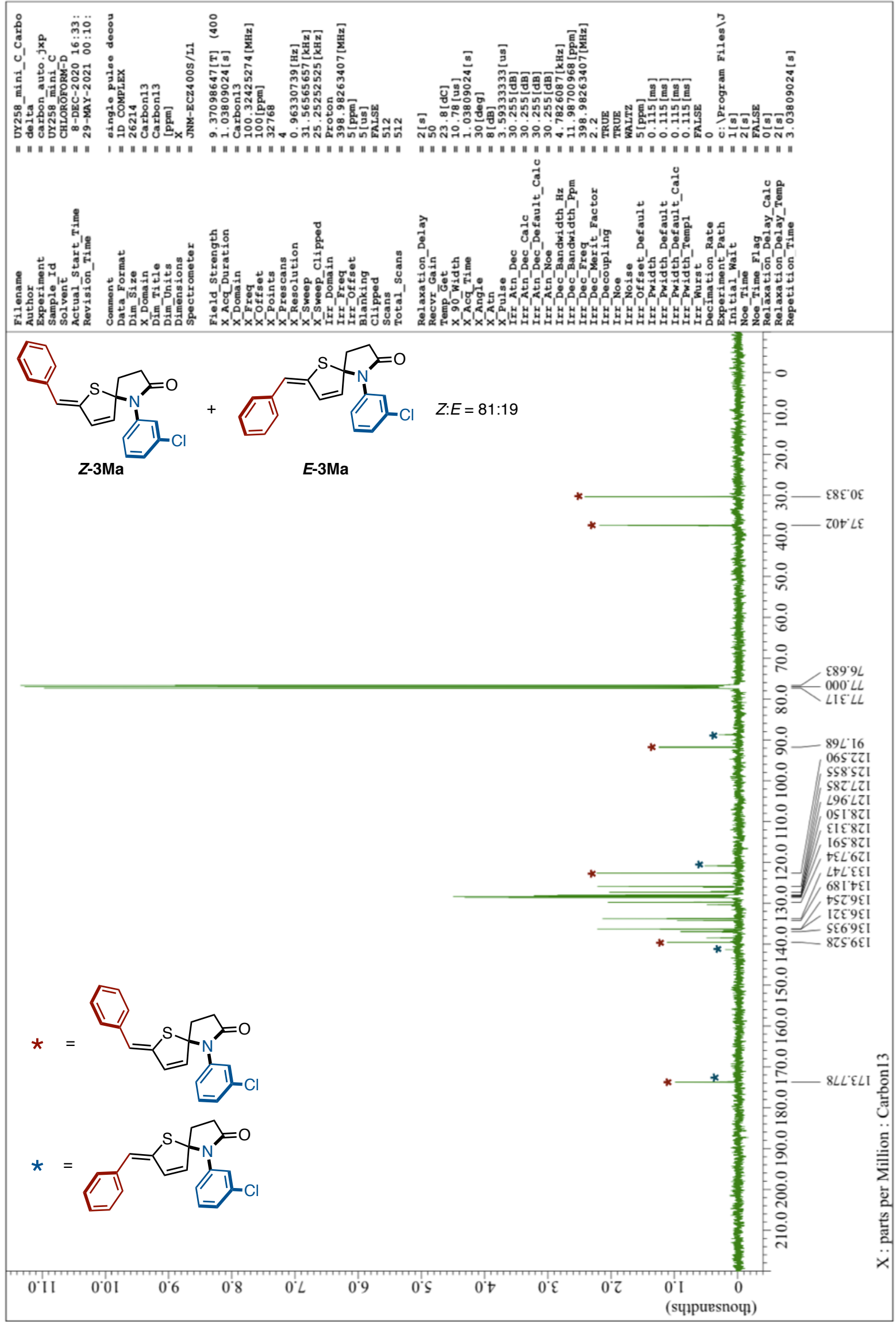


${ }^{1} \mathrm{H}$ NMR of $3 \mathrm{Na}\left(400 \mathrm{MHz}, \mathrm{CDCl}_{3}\right)$

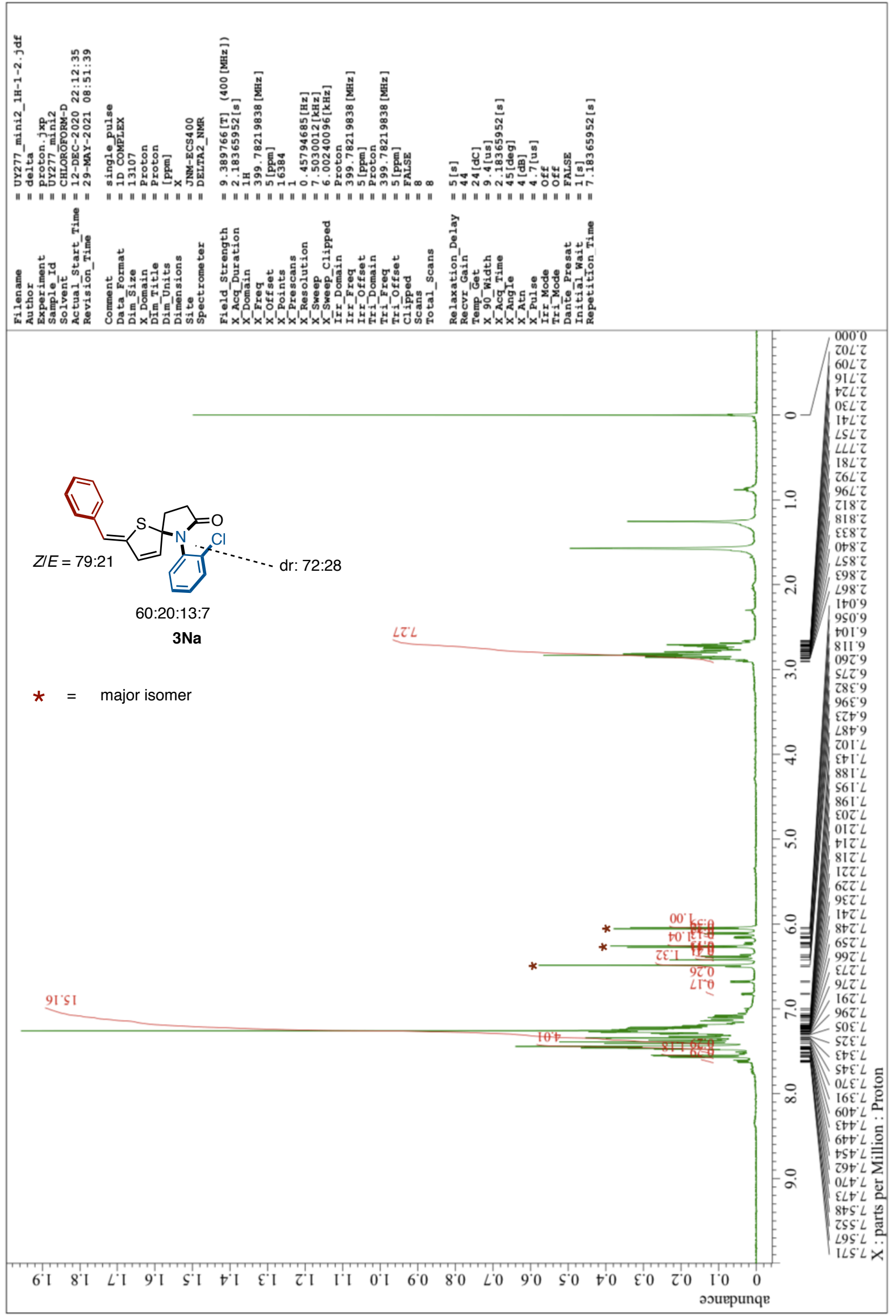


${ }^{13} \mathrm{C}$ NMR of $3 \mathrm{Na}\left(101 \mathrm{MHz}, \mathrm{CDCl}_{3}\right)$

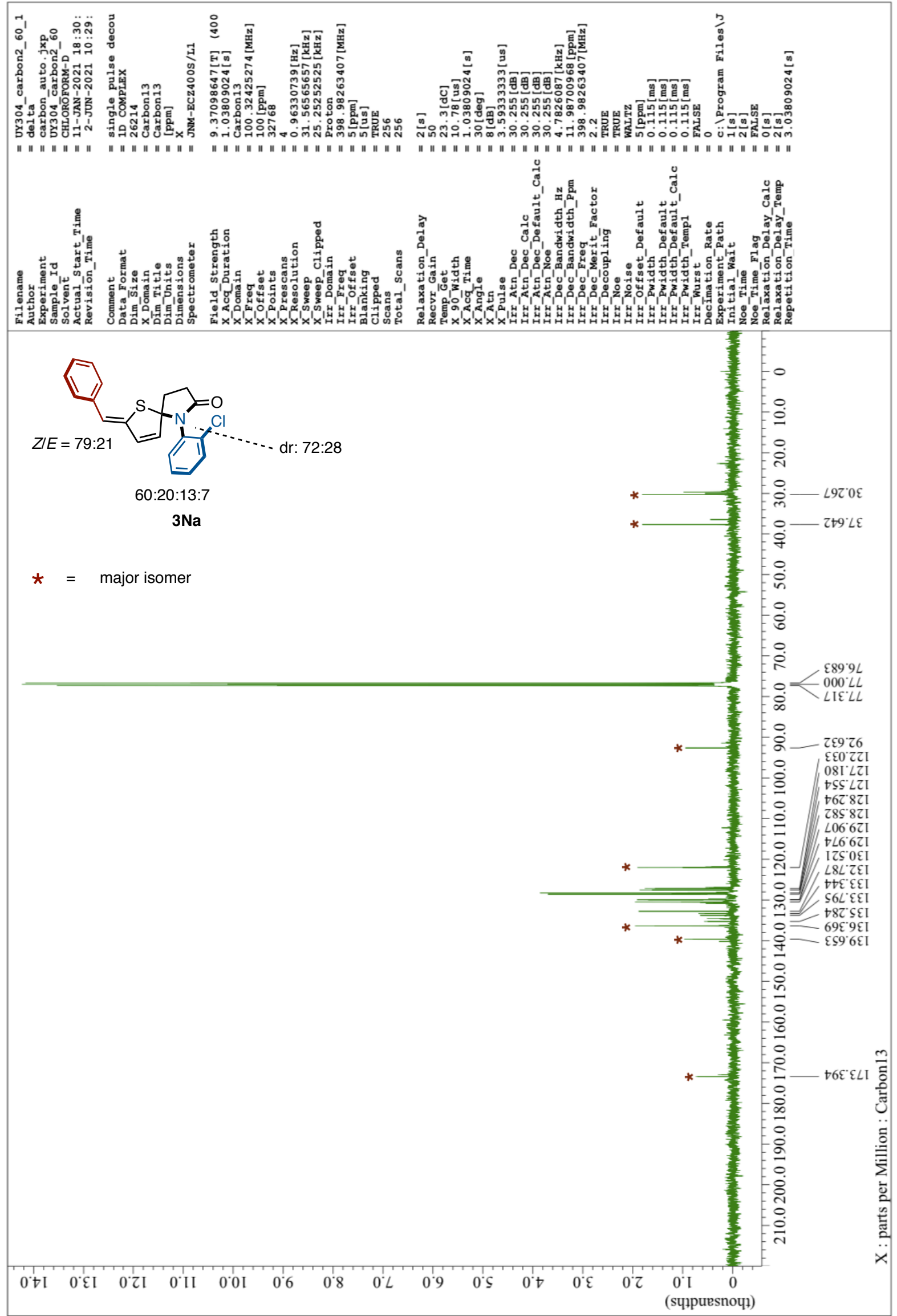


${ }^{1} \mathrm{H}$ NMR of $3 \mathrm{Oa}\left(400 \mathrm{MHz}, \mathrm{CDCl}_{3}\right)$

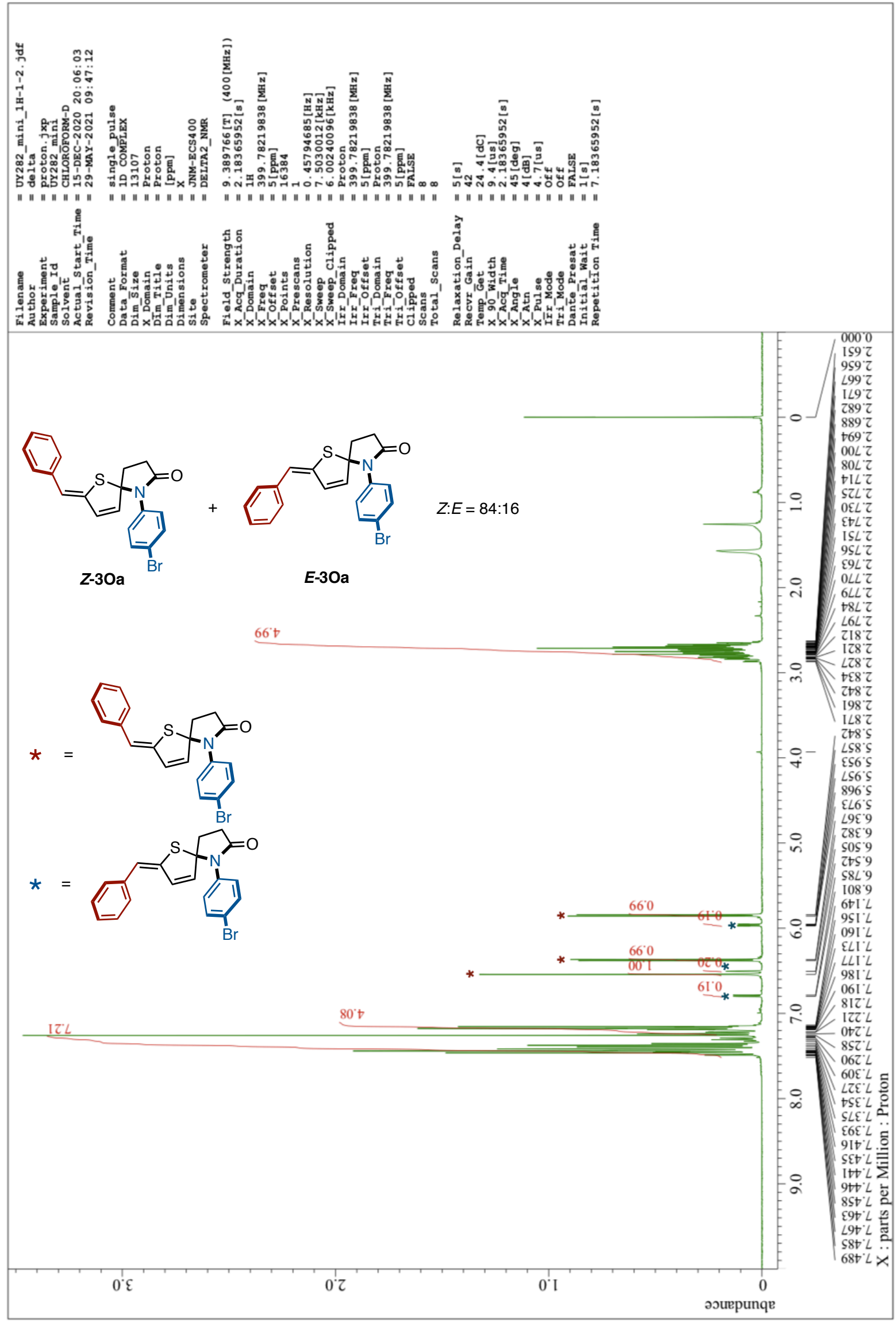


${ }^{13} \mathrm{C}$ NMR of $3 \mathrm{Oa}\left(101 \mathrm{MHz}, \mathrm{CDCl}_{3}\right)$

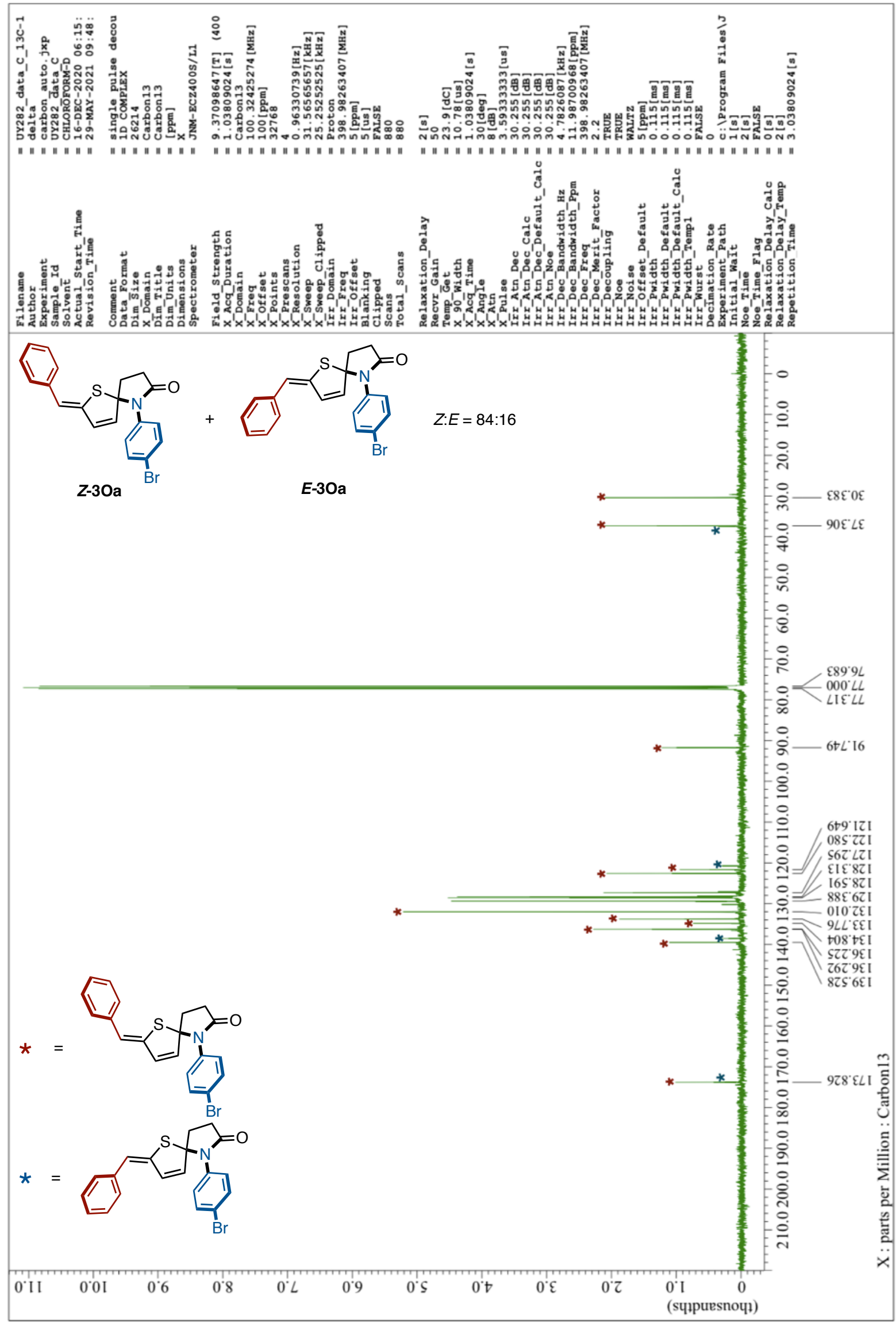


${ }^{1} \mathrm{H}$ NMR of 3Pa (400 $\left.\mathrm{MHz}, \mathrm{CDCl}_{3}\right)$

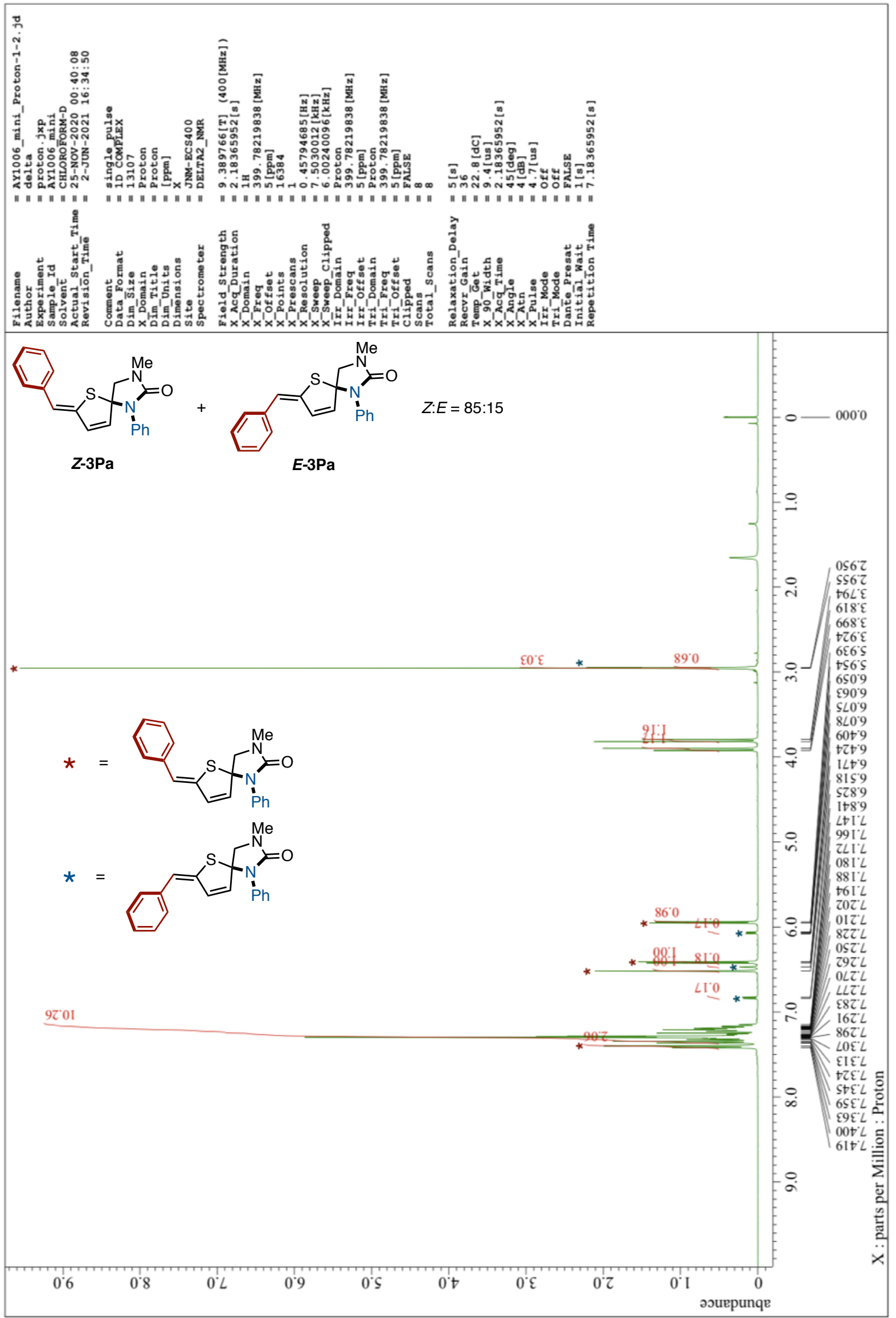


${ }^{13} \mathrm{C}$ NMR of 3Pa (101 MHz, $\left.\mathrm{CDCl}_{3}\right)$

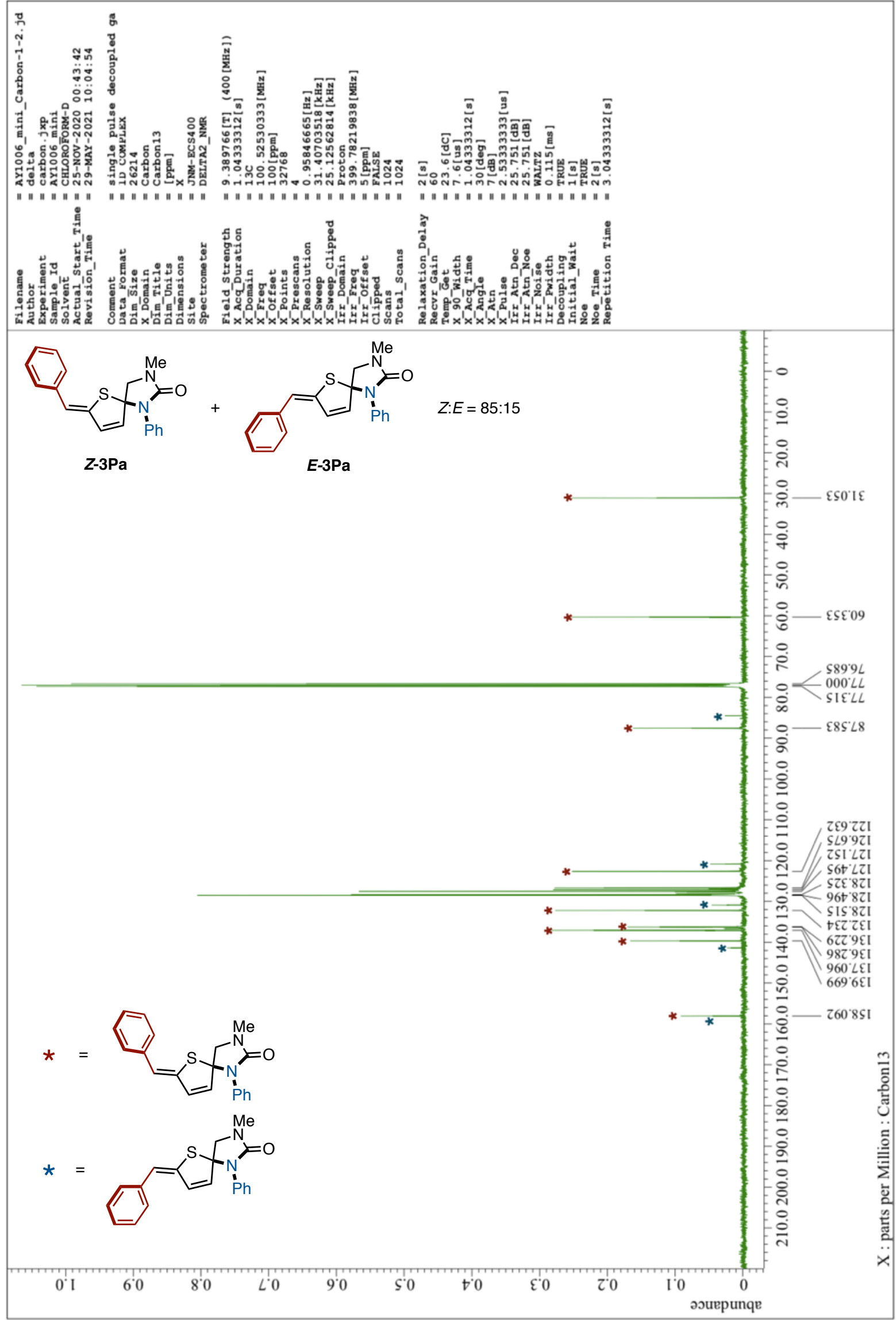


${ }^{1} \mathrm{H}$ NMR of 3Qa (400 $\left.\mathrm{MHz}, \mathrm{CDCl}_{3}\right)$

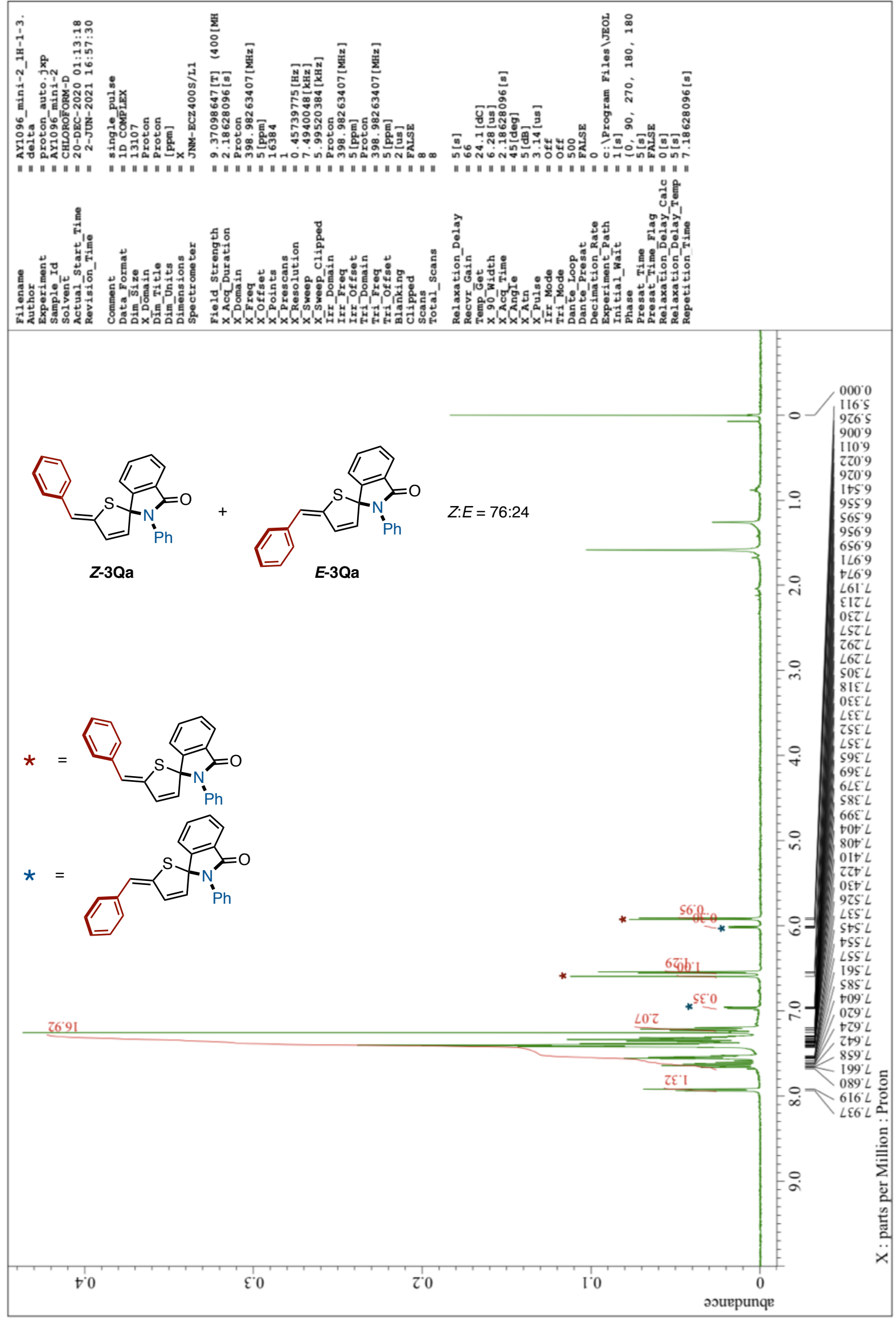


${ }^{13} \mathrm{C}$ NMR of 3Qa (101 MHz, $\left.\mathrm{CDCl}_{3}\right)$

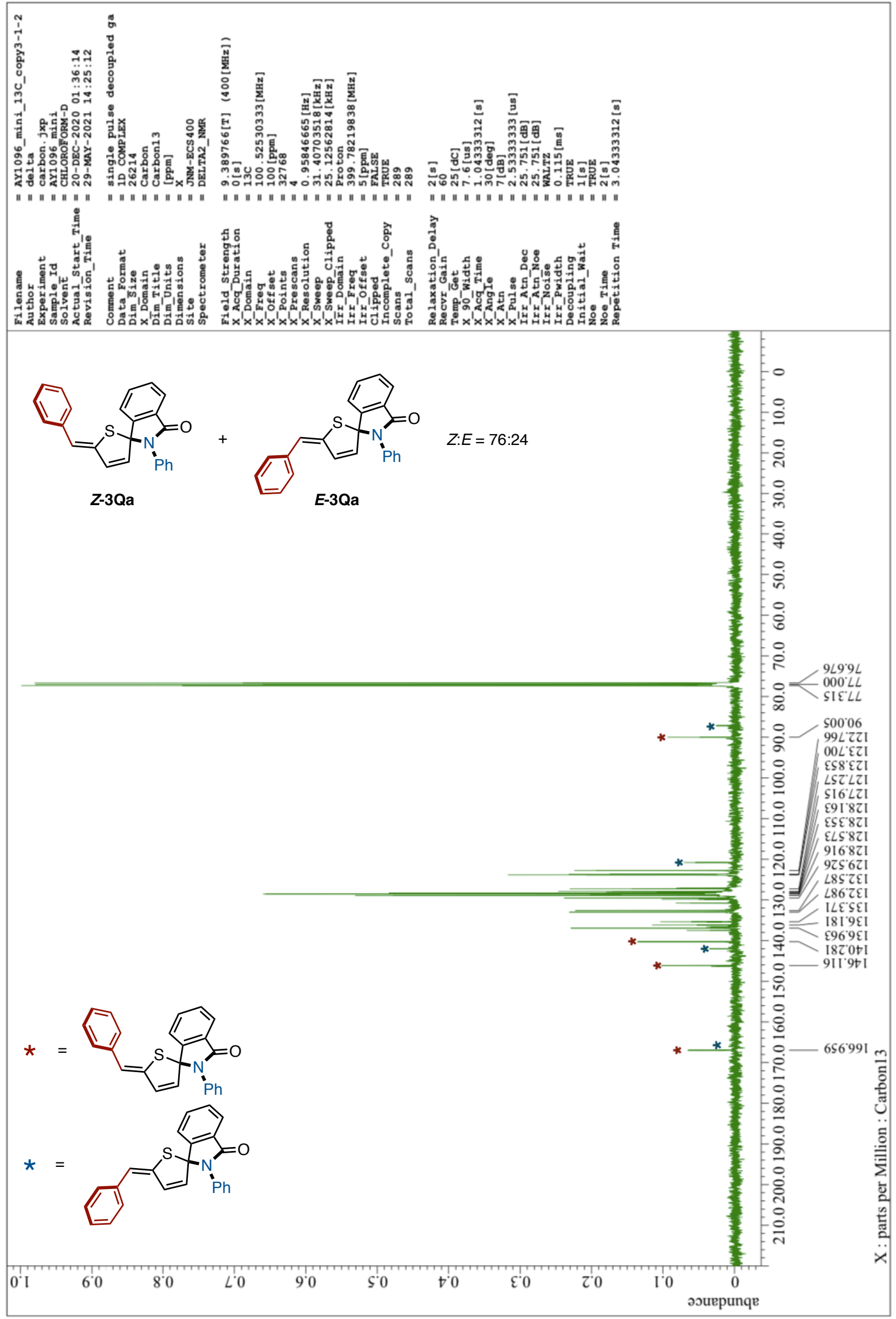


${ }^{1} \mathrm{H}$ NMR of 3Ra (400 MHz, $\left.\mathrm{CDCl}_{3}\right)$

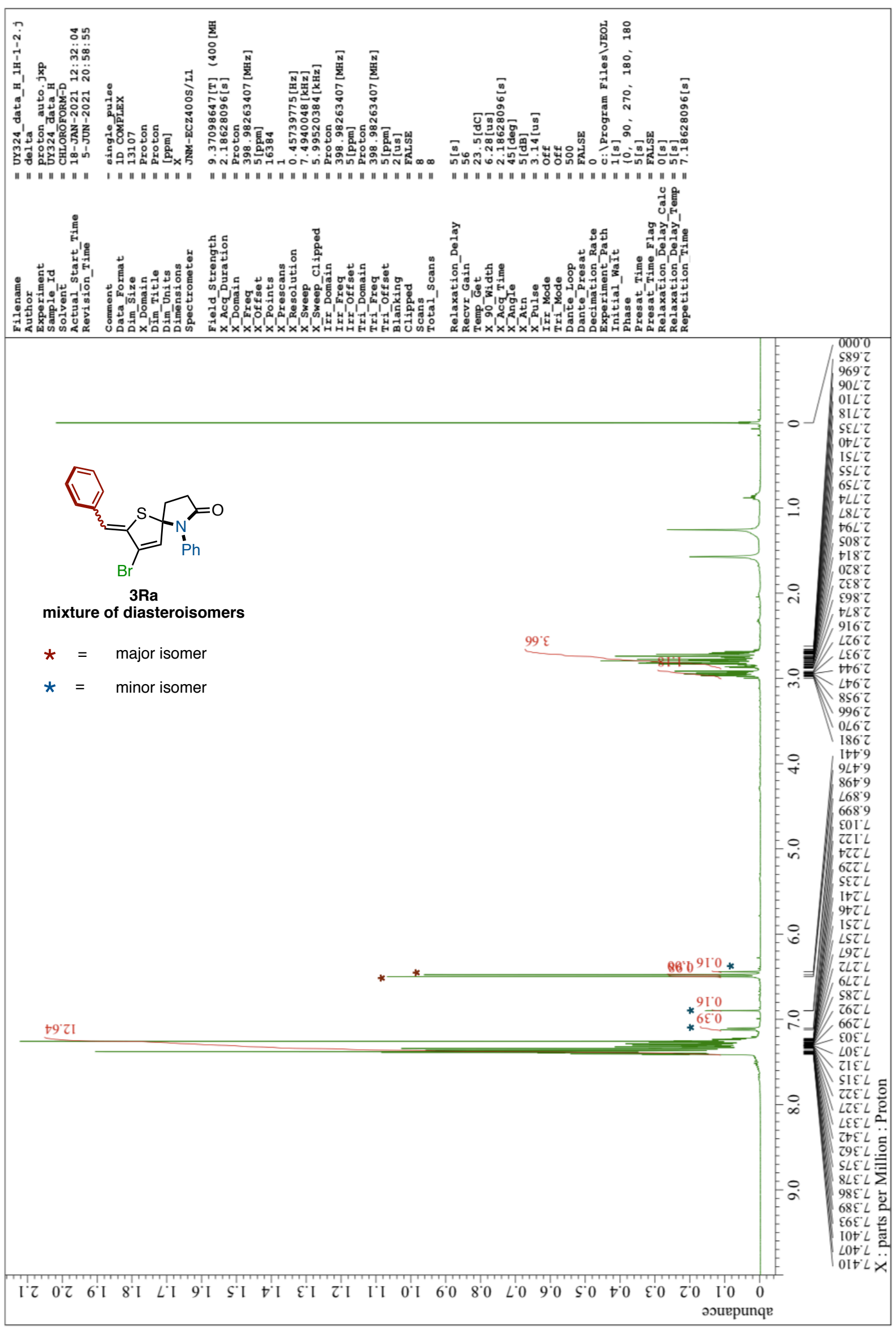


${ }^{13} \mathrm{C}$ NMR of 3Ra (101 MHz, $\left.\mathrm{CDCl}_{3}\right)$

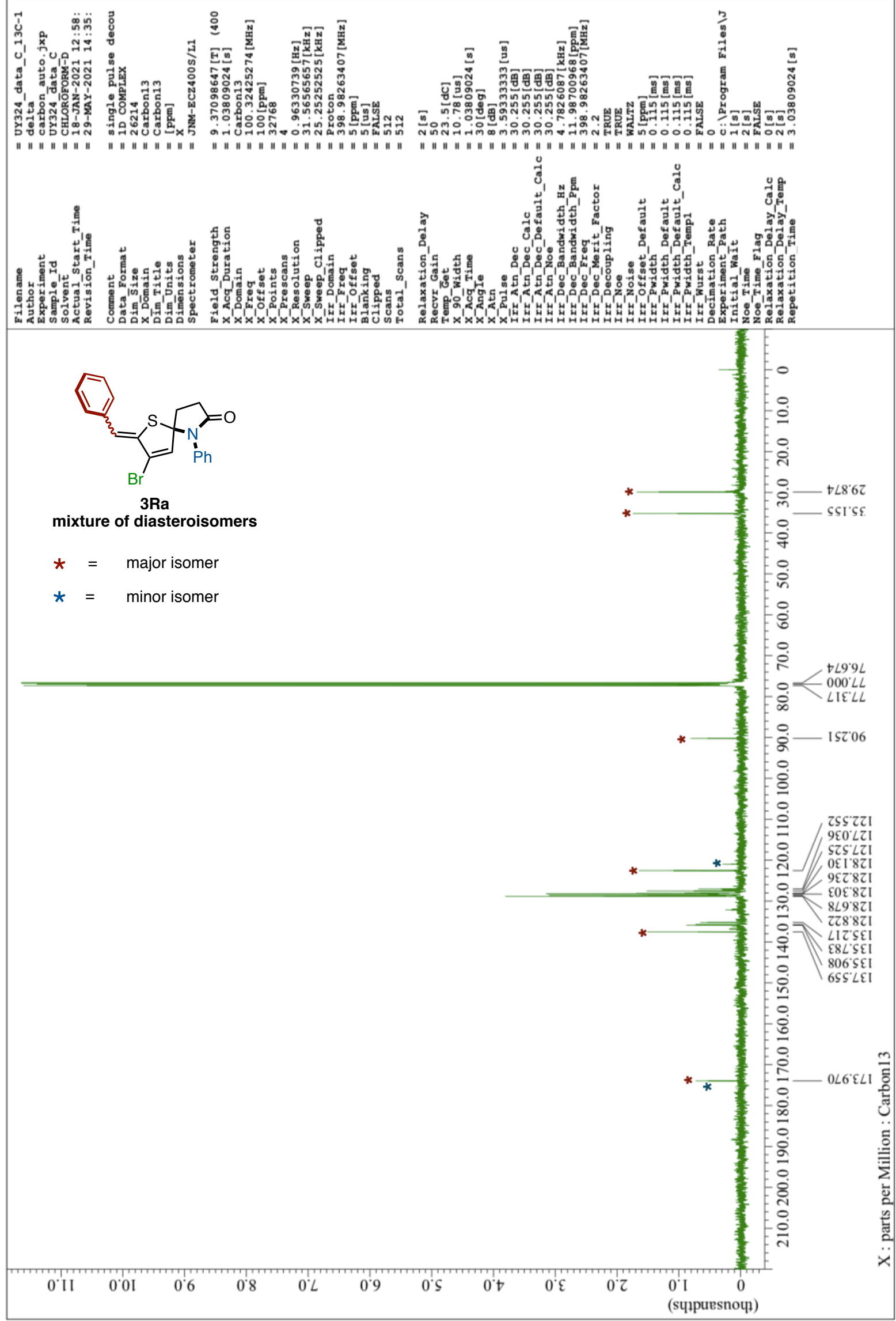


${ }^{1} \mathrm{H}$ NMR of $3 \mathrm{Hb}\left(400 \mathrm{MHz}, \mathrm{CDCl}_{3}\right)$

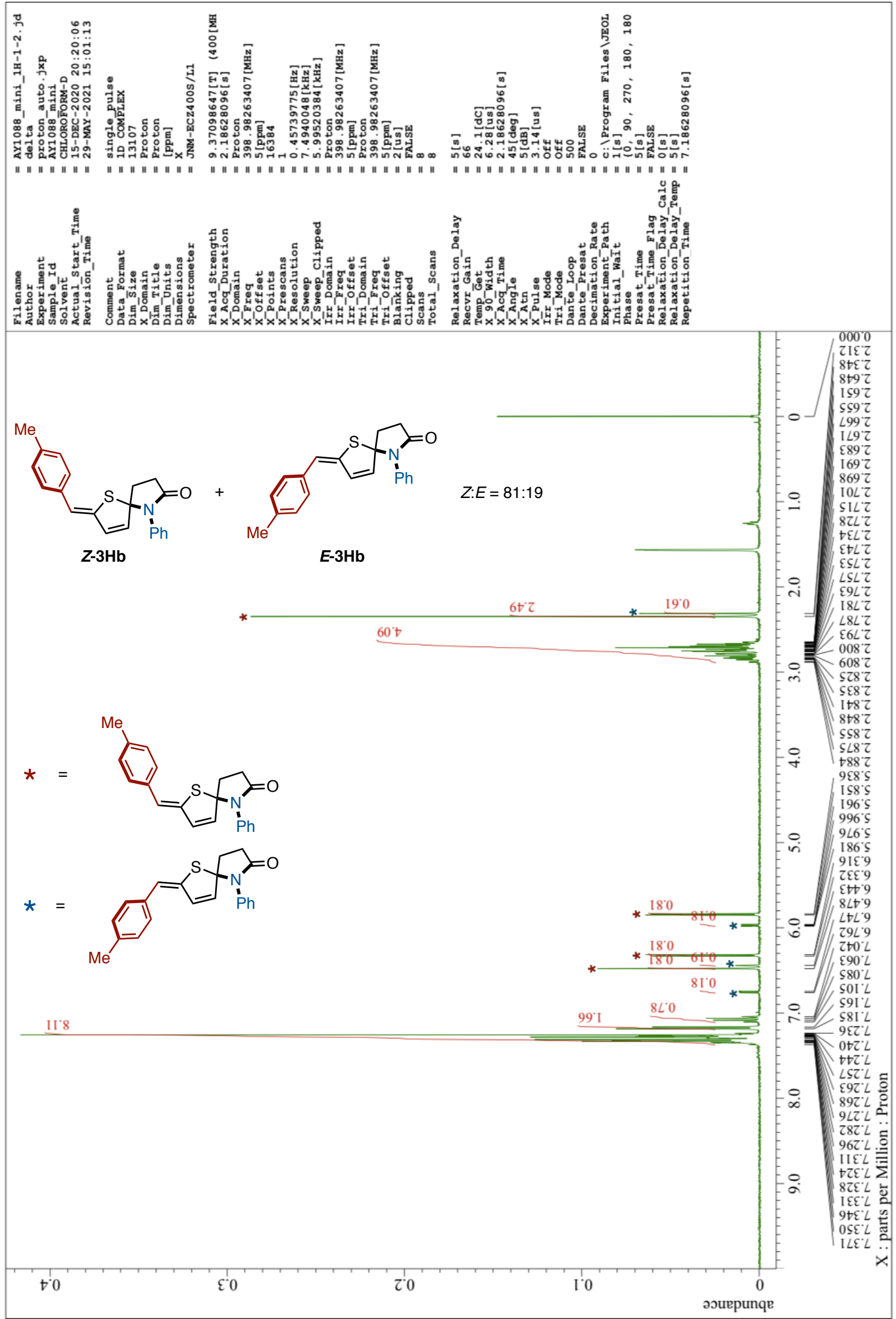


${ }^{13} \mathrm{C}$ NMR of $3 \mathrm{Hb}\left(101 \mathrm{MHz}, \mathrm{CDCl}_{3}\right)$

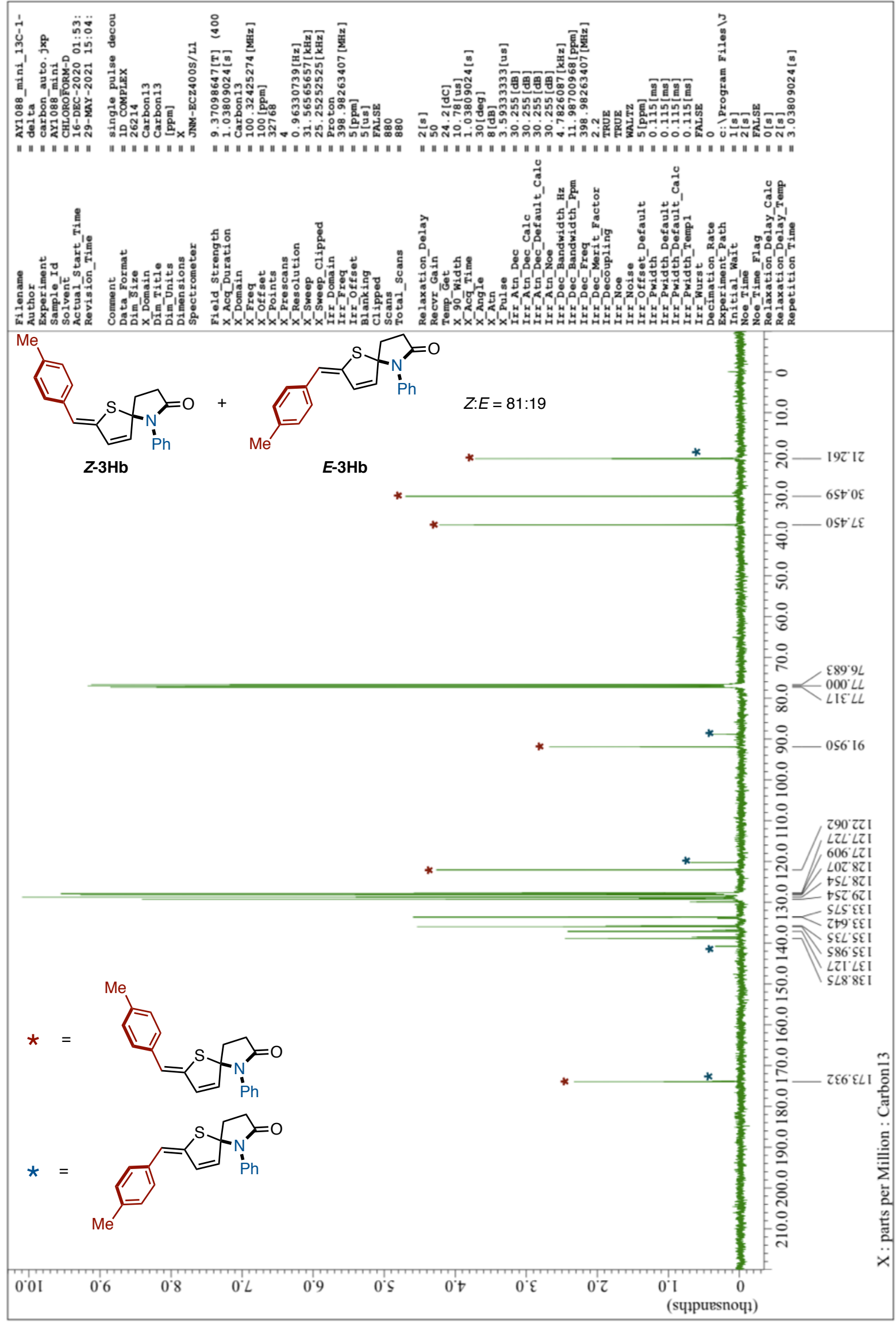


${ }^{1} \mathrm{H}$ NMR of $3 \mathrm{He}\left(400 \mathrm{MHz}, \mathrm{CDCl}_{3}\right)$

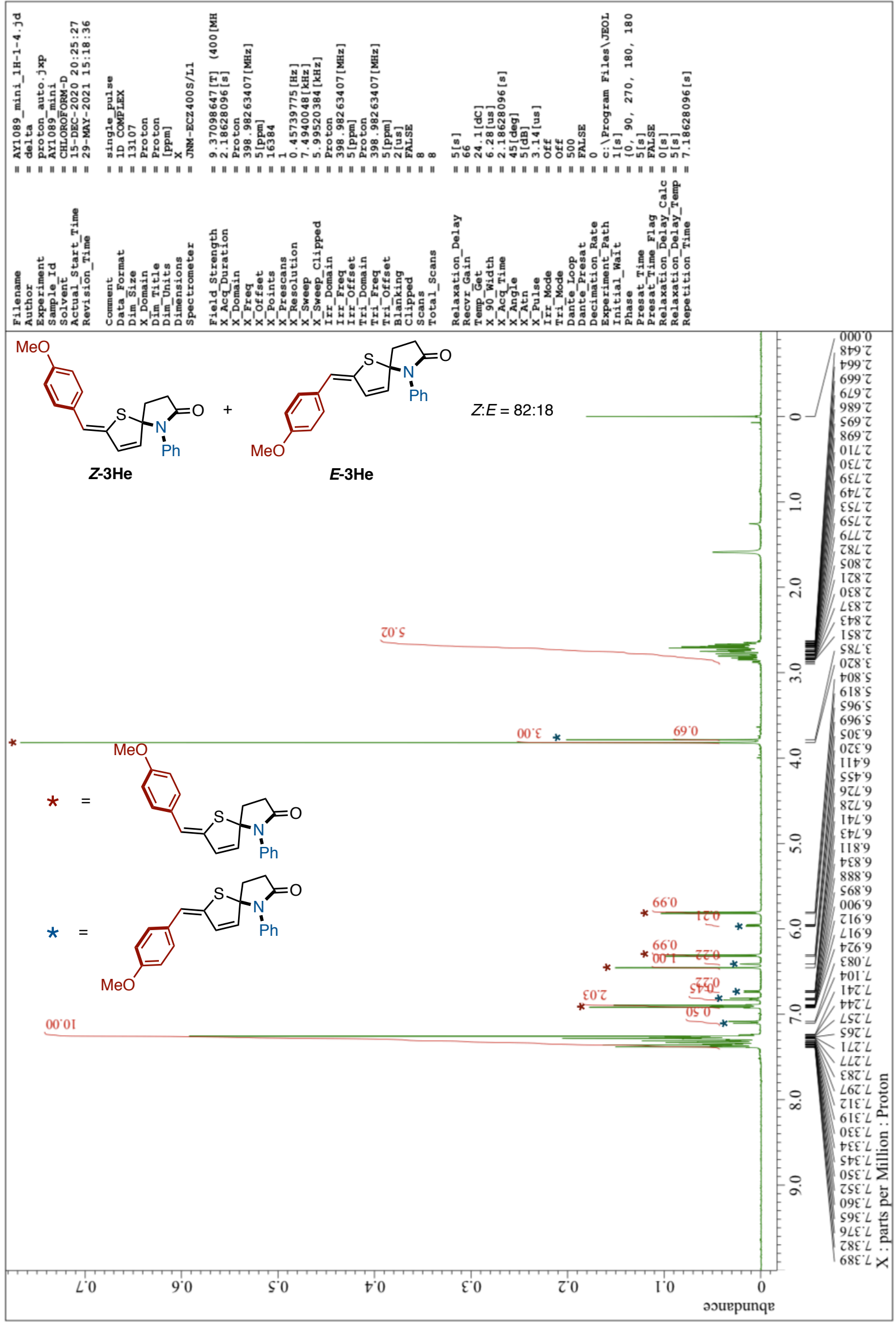


${ }^{13} \mathrm{C}$ NMR of $3 \mathrm{He}\left(101 \mathrm{MHz}, \mathrm{CDCl}_{3}\right)$

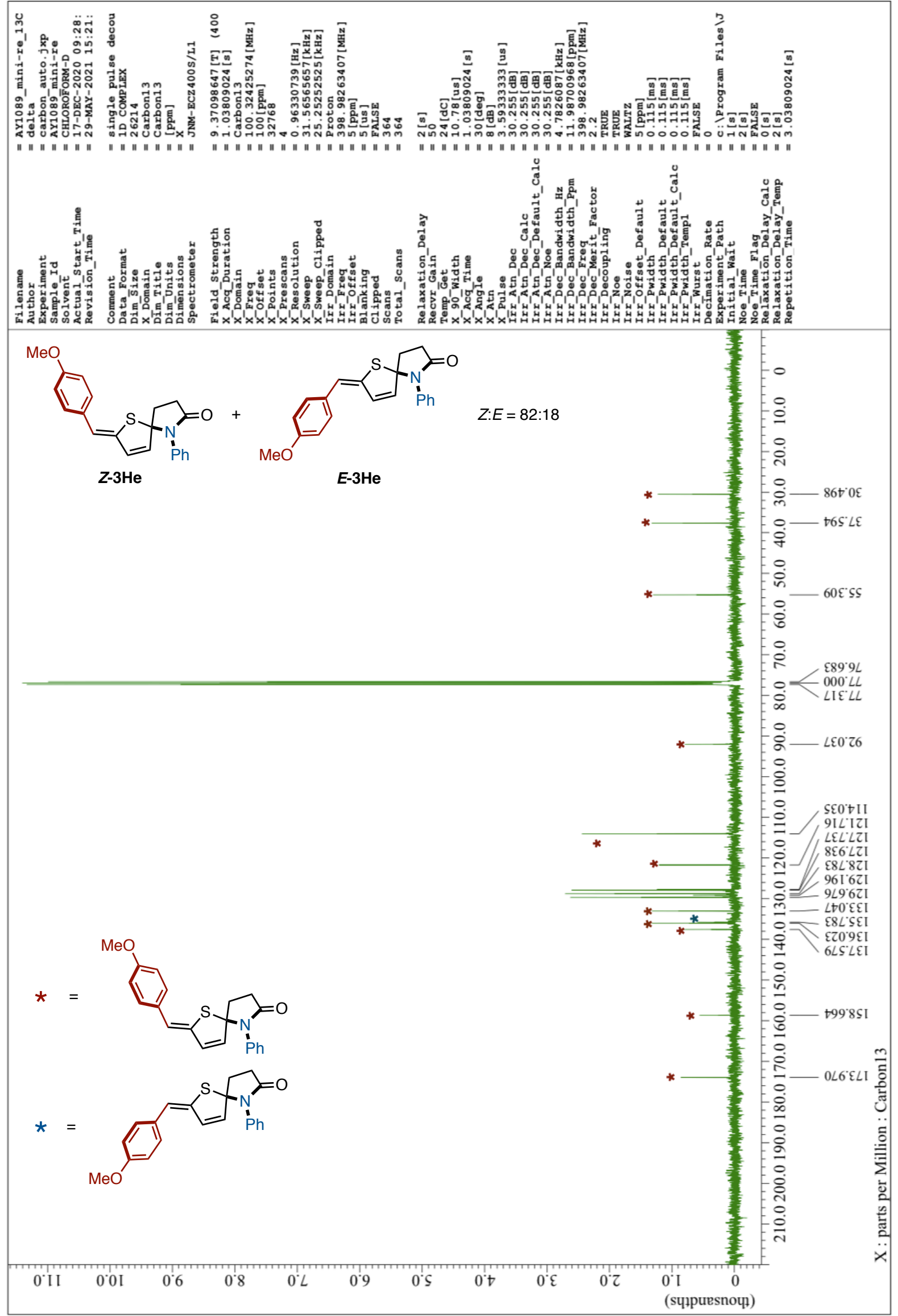


${ }^{1} \mathrm{H}$ NMR of $3 \mathrm{Hh}\left(400 \mathrm{MHz}, \mathrm{CDCl}_{3}\right)$

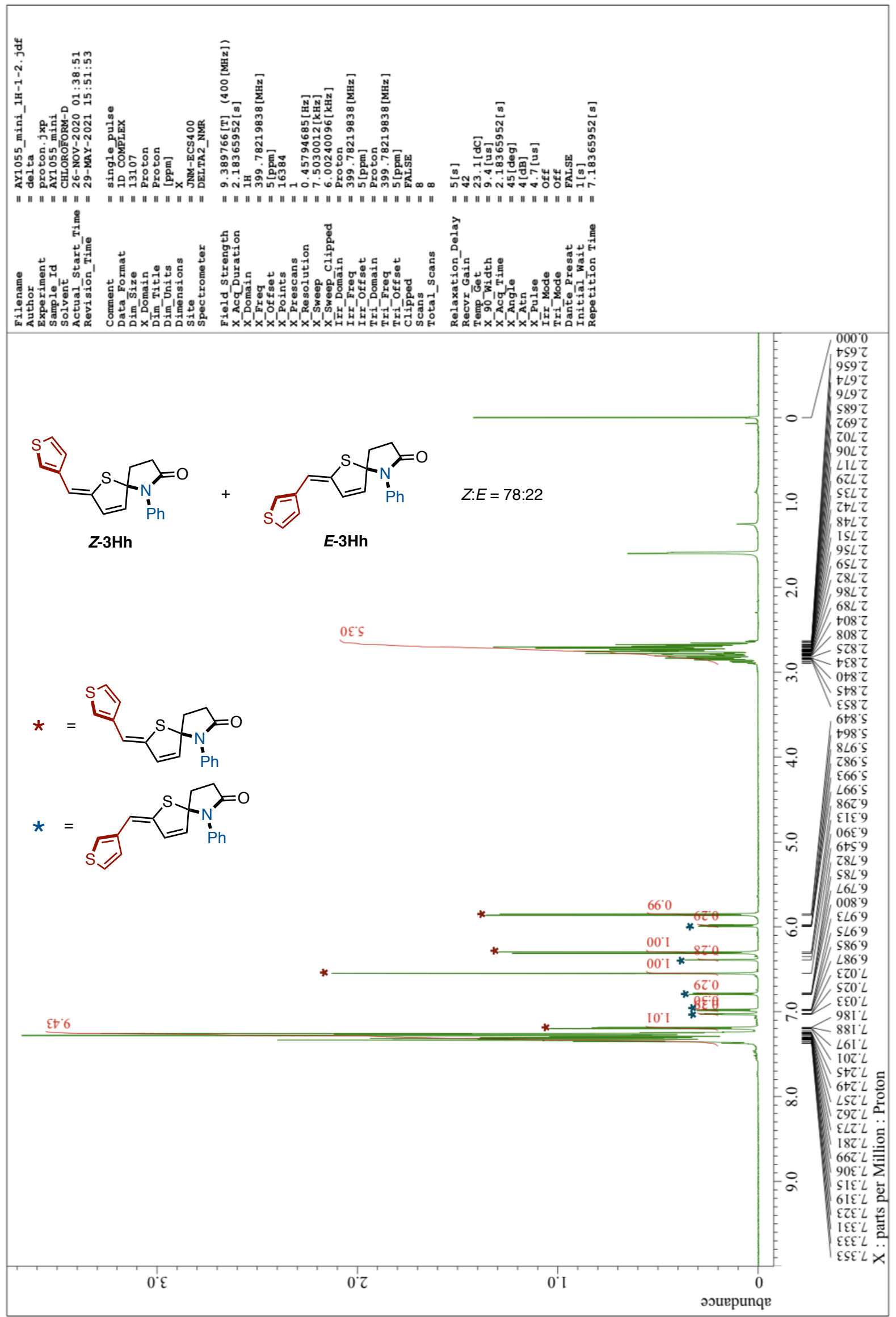


${ }^{13} \mathrm{C}$ NMR of $3 \mathrm{Hh}\left(101 \mathrm{MHz}, \mathrm{CDCl}_{3}\right)$

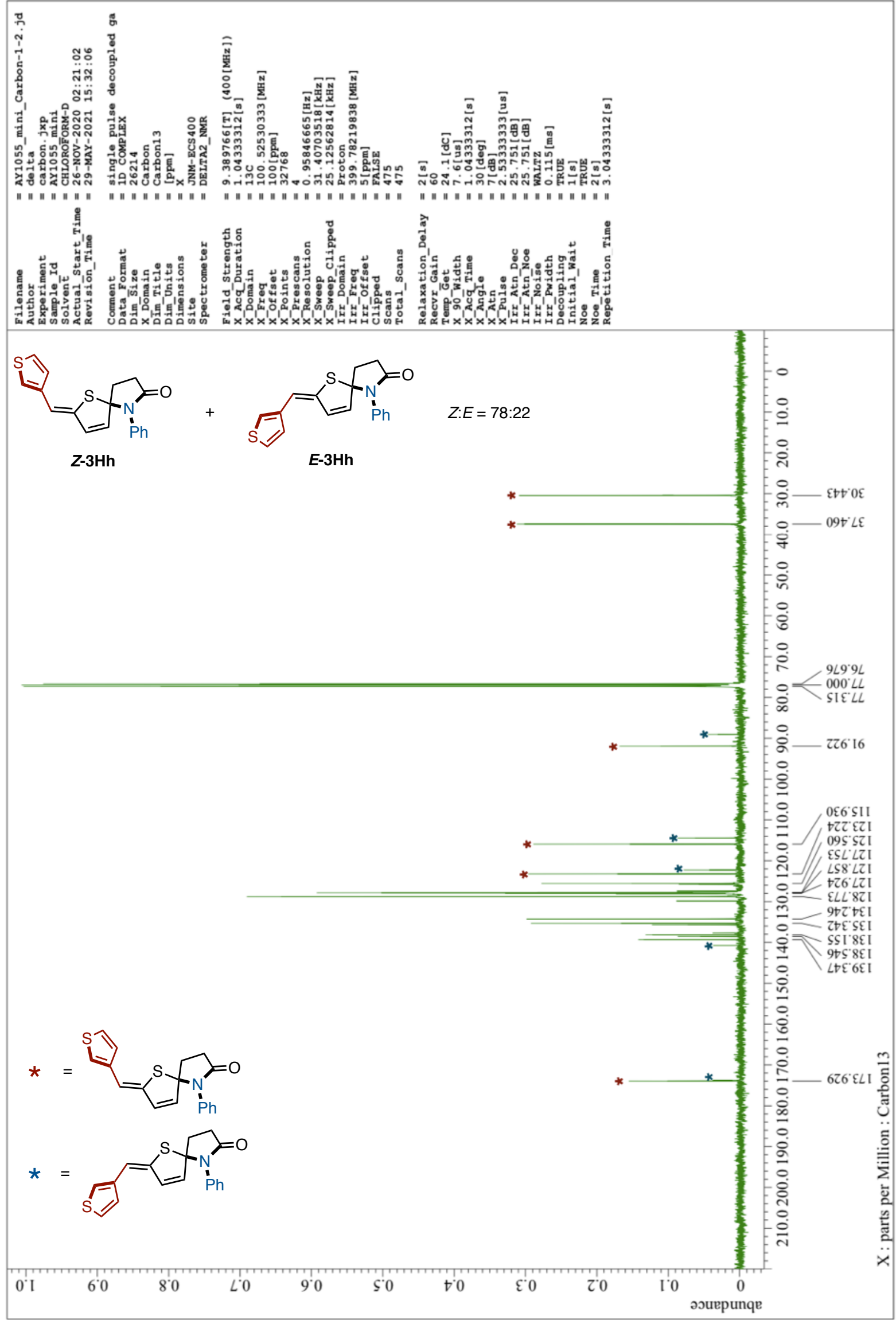


${ }^{1} \mathrm{H}$ NMR of $3 \mathrm{Hi}\left(400 \mathrm{MHz}, \mathrm{CDCl}_{3}\right)$

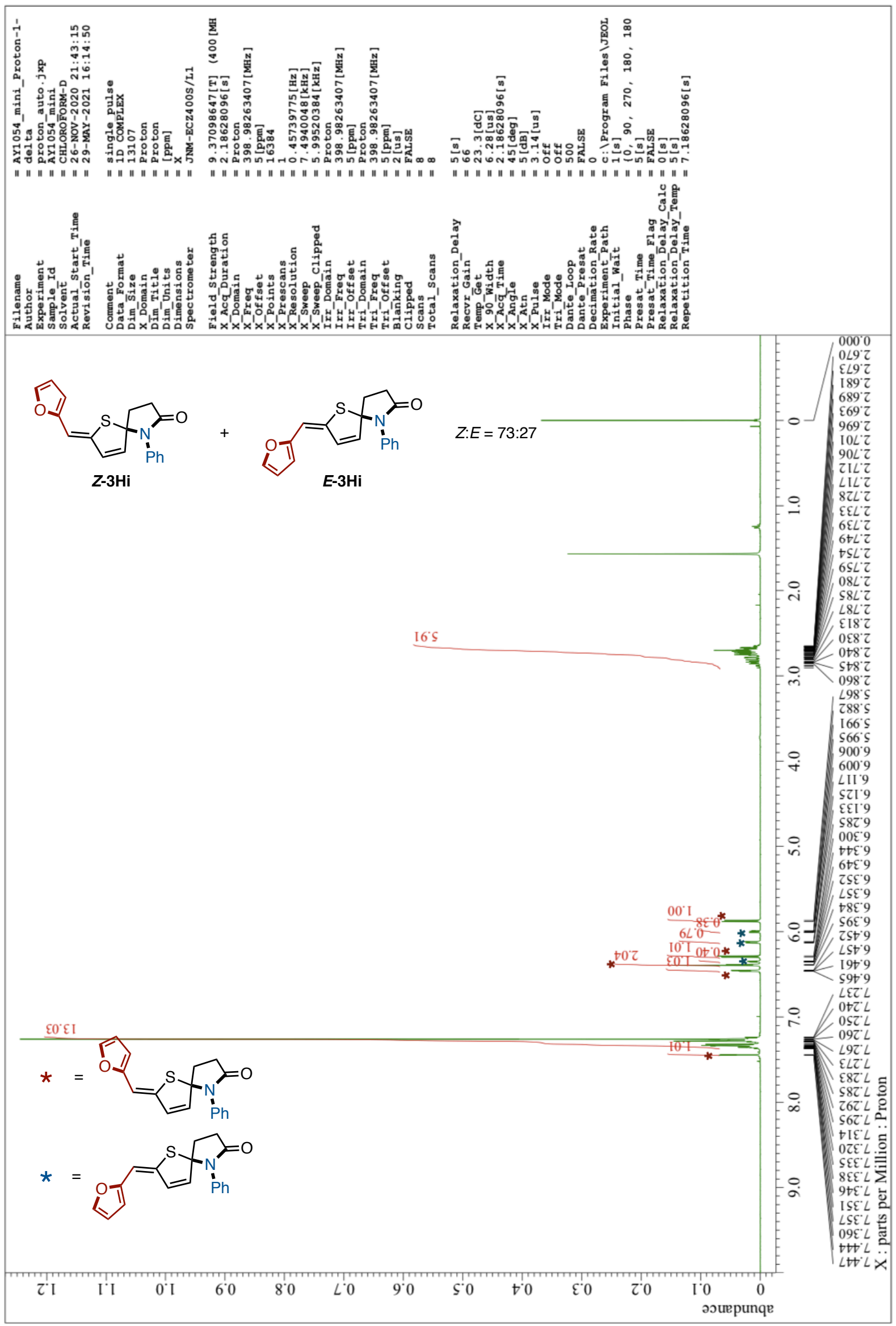


${ }^{13} \mathrm{C}$ NMR of $3 \mathrm{Hi}\left(101 \mathrm{MHz}, \mathrm{CDCl}_{3}\right)$

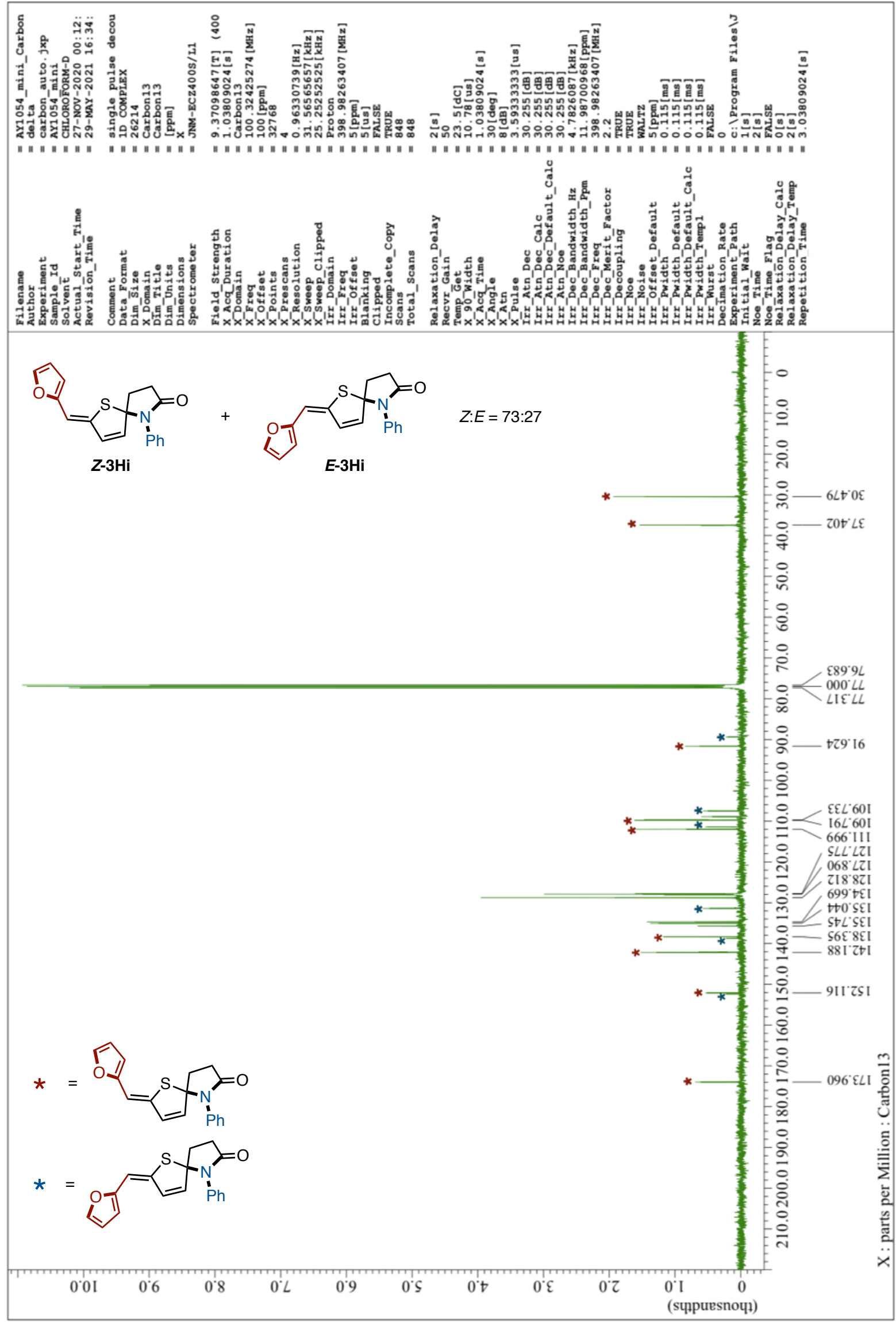


${ }^{1} \mathrm{H}$ NMR of $5 \mathrm{~b}\left(400 \mathrm{MHz}, \mathrm{CDCl}_{3}\right)$

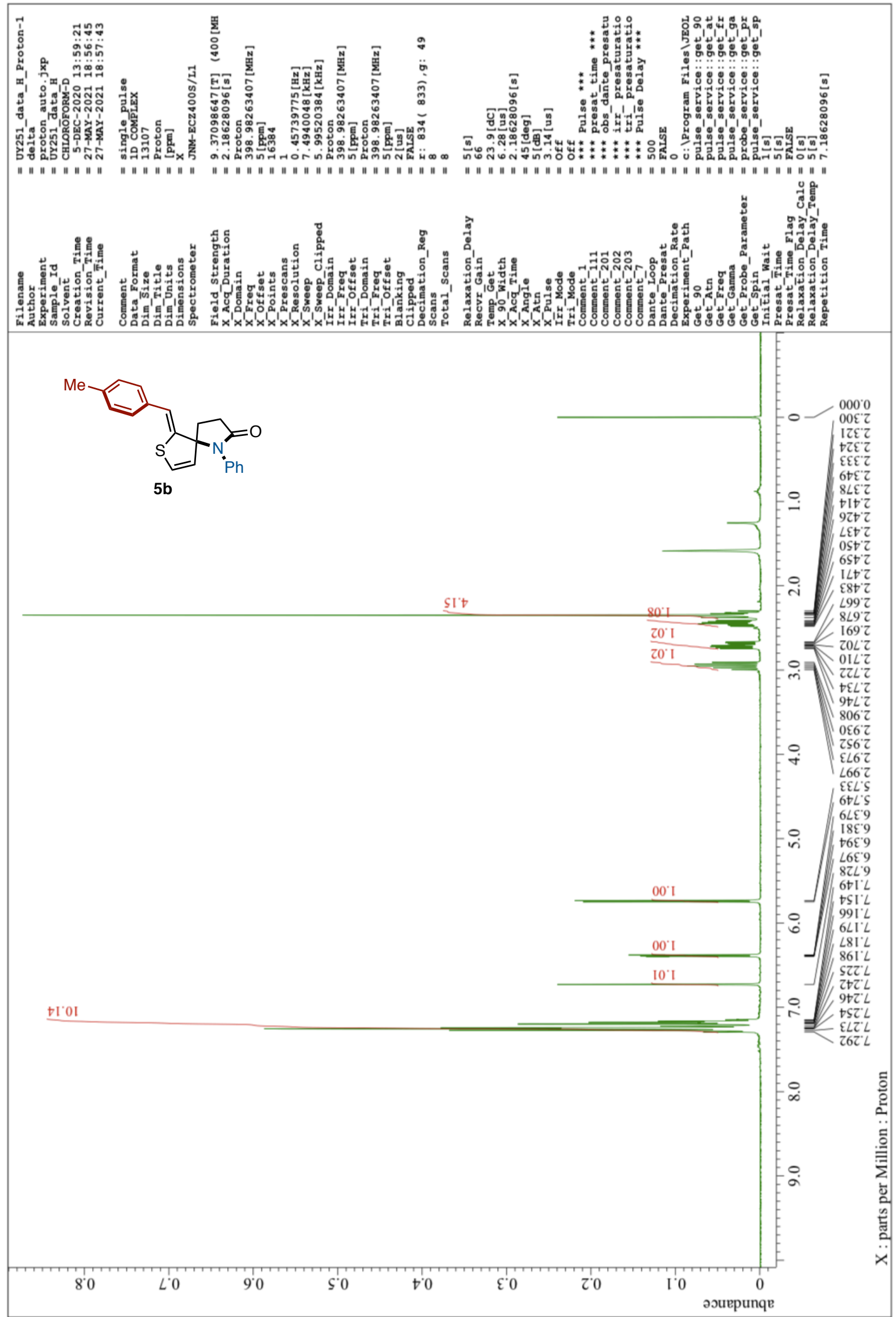


${ }^{13} \mathrm{C}$ NMR of 5b (101 MHz, $\left.\mathrm{CDCl}_{3}\right)$

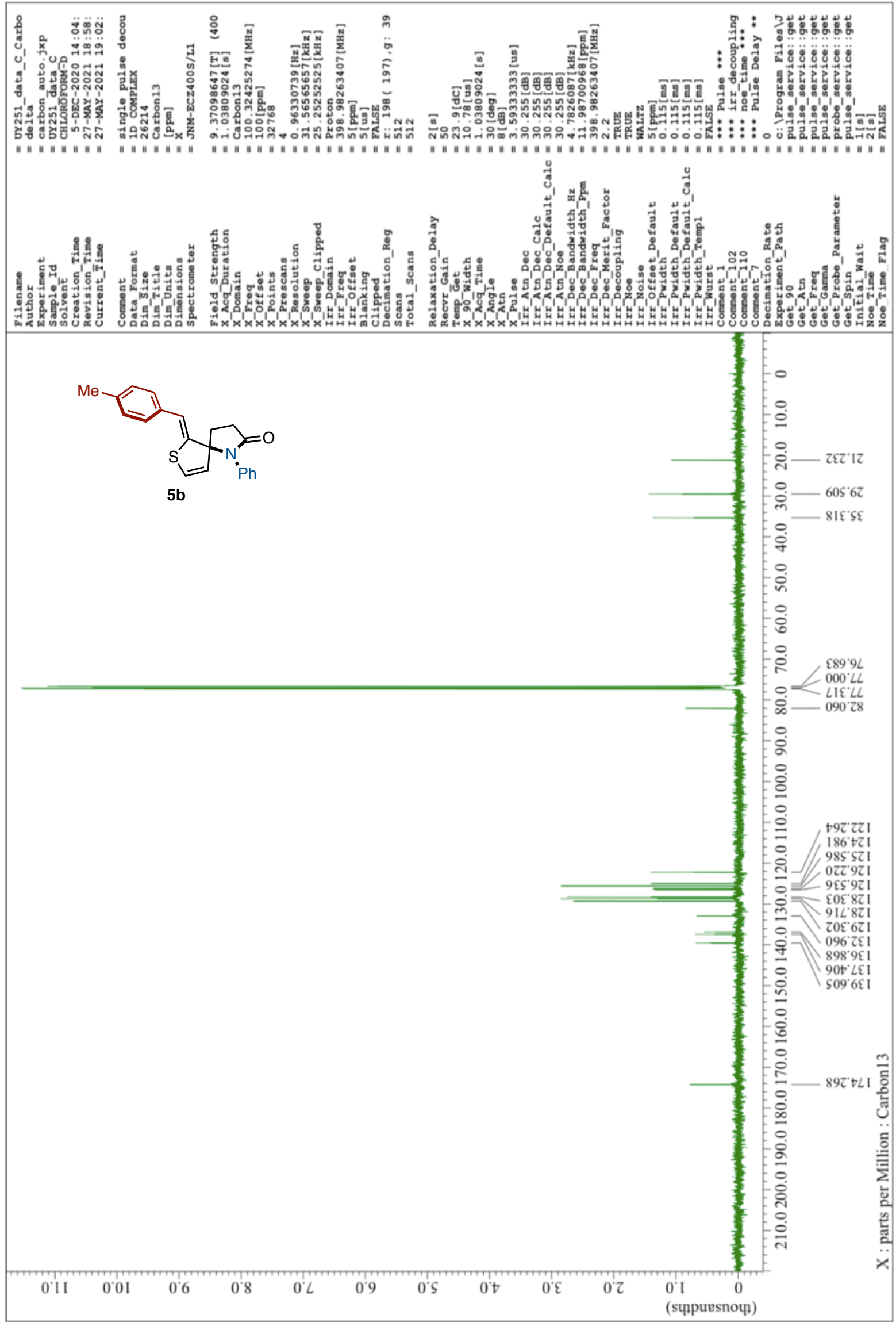


${ }^{1} \mathrm{H}$ NMR of $5 \mathrm{~m}\left(400 \mathrm{MHz}, \mathrm{CDCl}_{3}\right)$

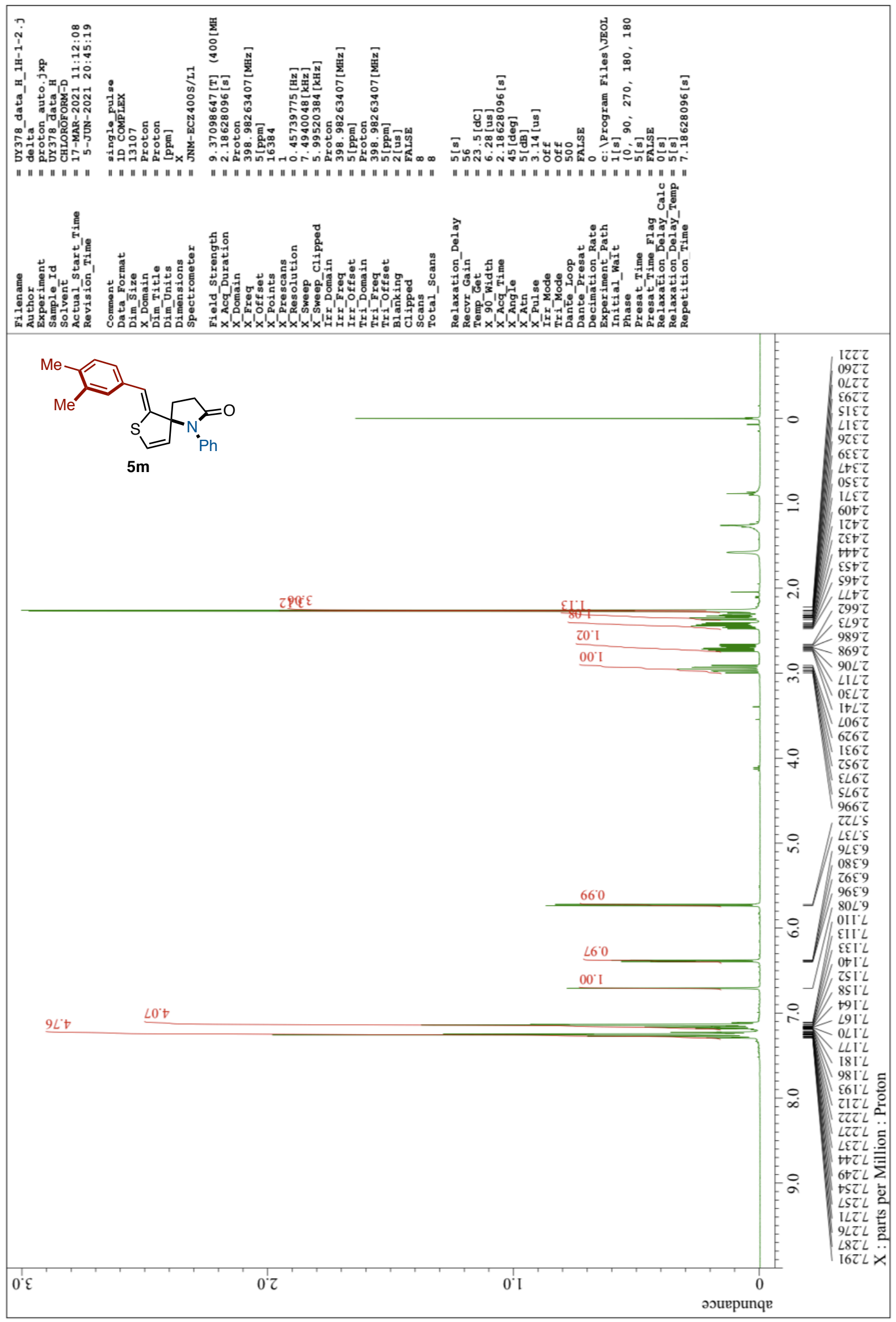


${ }^{13} \mathrm{C}$ NMR of $6 \mathrm{~m}\left(101 \mathrm{MHz}, \mathrm{CDCl}_{3}\right)$

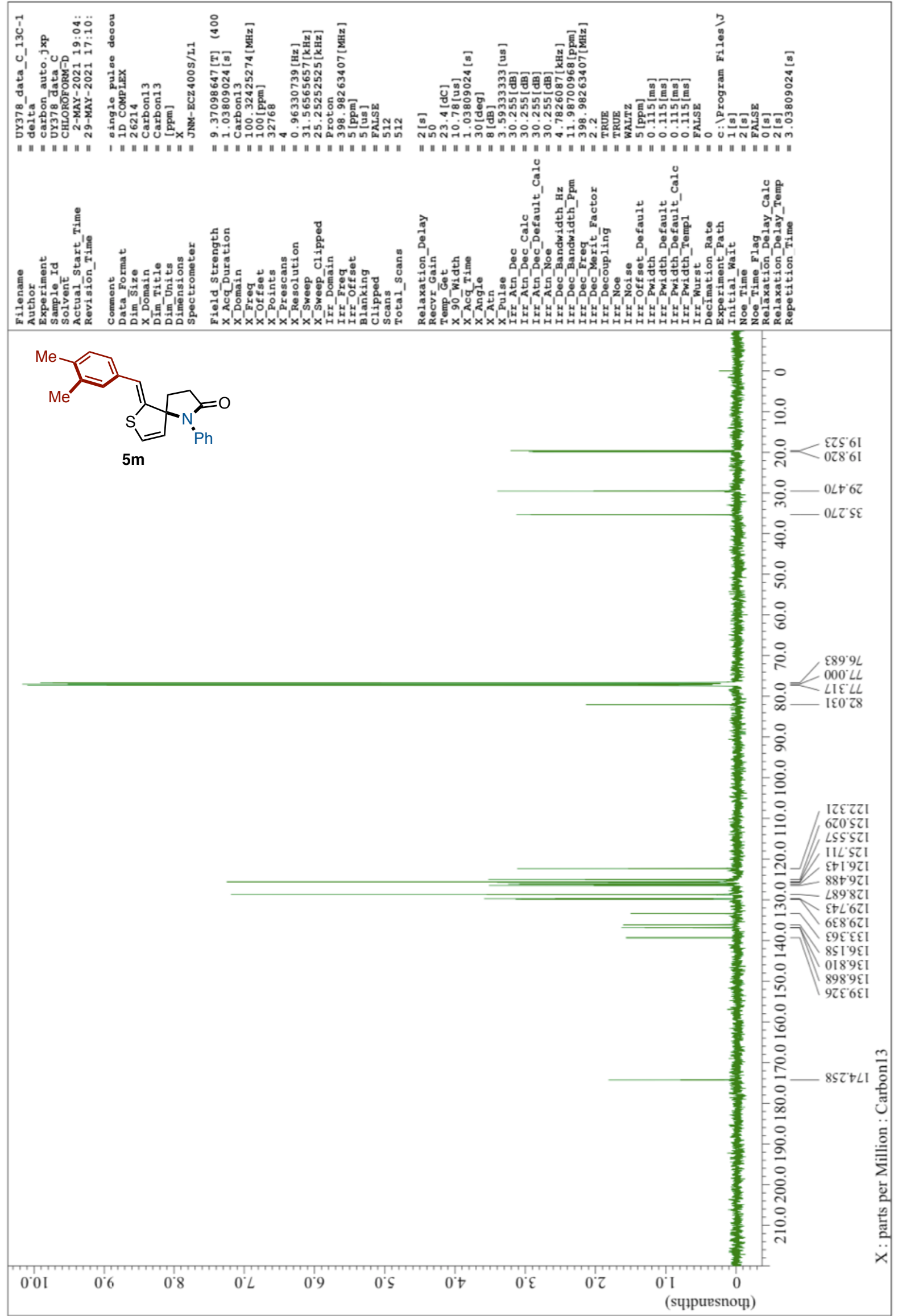


${ }^{1} \mathrm{H}$ NMR of $7\left(400 \mathrm{MHz}, \mathrm{CDCl}_{3}\right)$

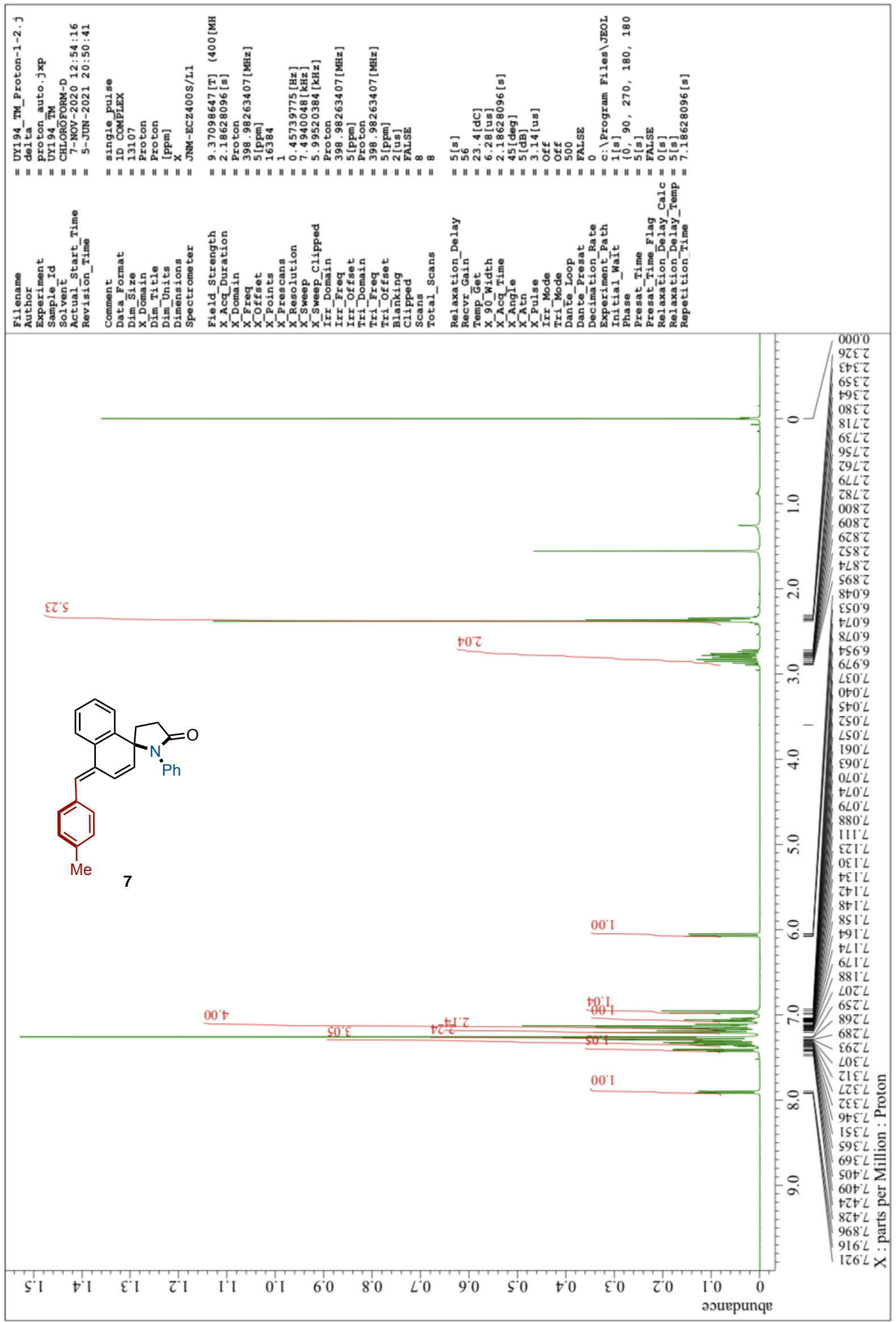


${ }^{13} \mathrm{C}$ NMR of 7 (101 MHz, $\left.\mathrm{CDCl}_{3}\right)$

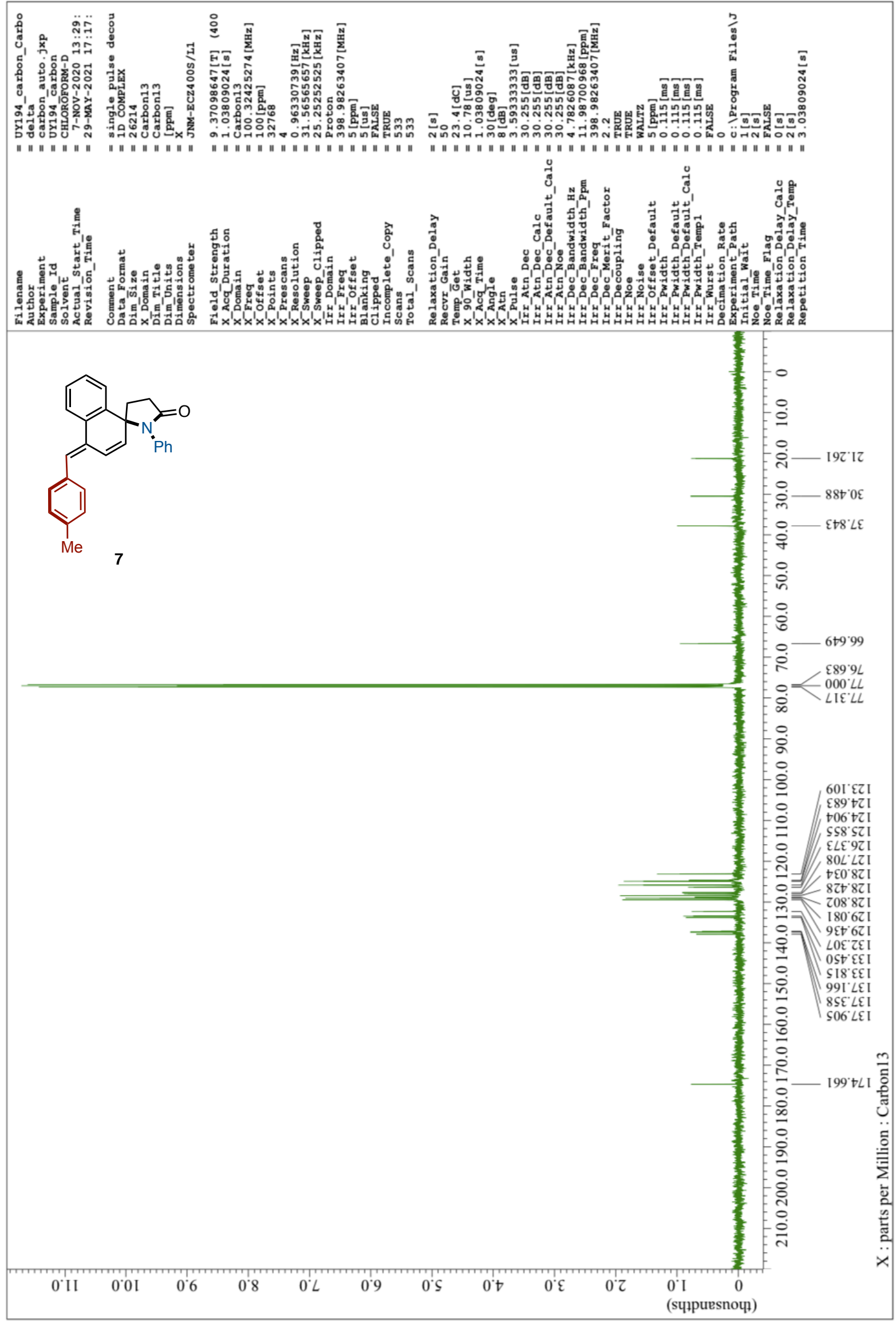


${ }^{1} \mathrm{H}$ NMR of 9Aa (400 MHz, $\mathrm{CDCl}_{3}$ )

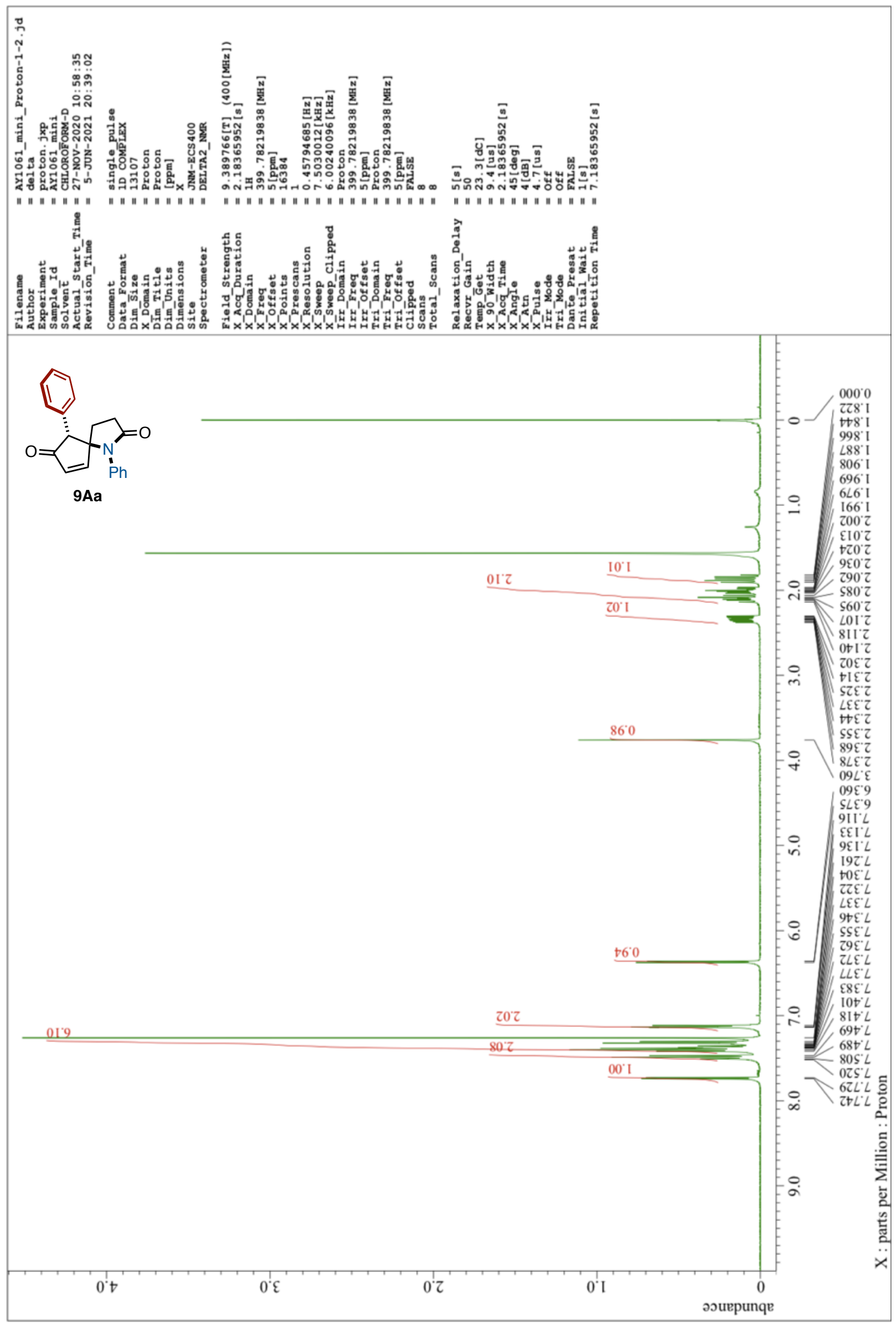


${ }^{13} \mathrm{C}$ NMR of 9Aa (101 MHz, $\left.\mathrm{CDCl}_{3}\right)$

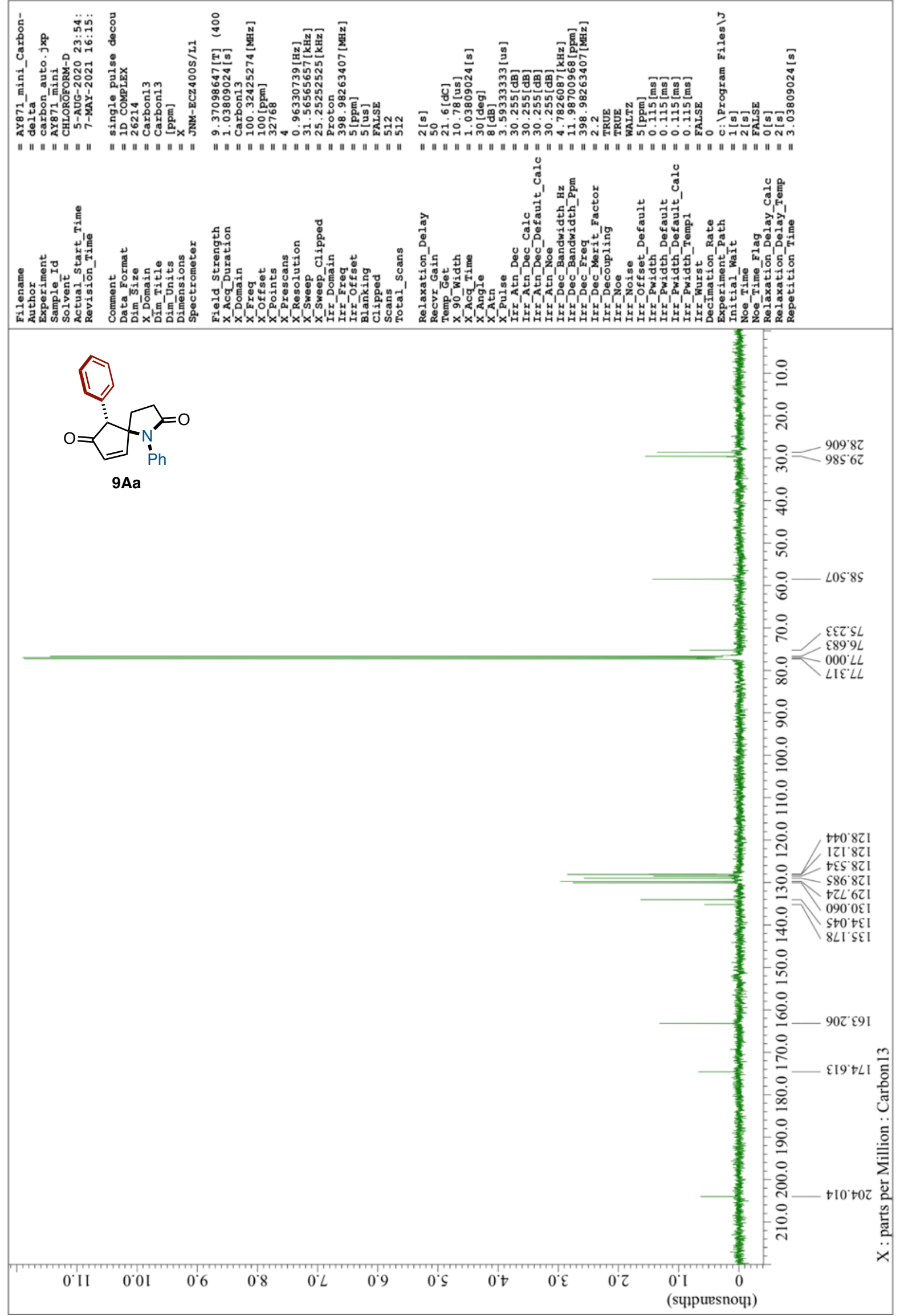


${ }^{1} \mathrm{H}$ NMR of 9Ba (400 MHz, $\left.\mathrm{CDCl}_{3}\right)$

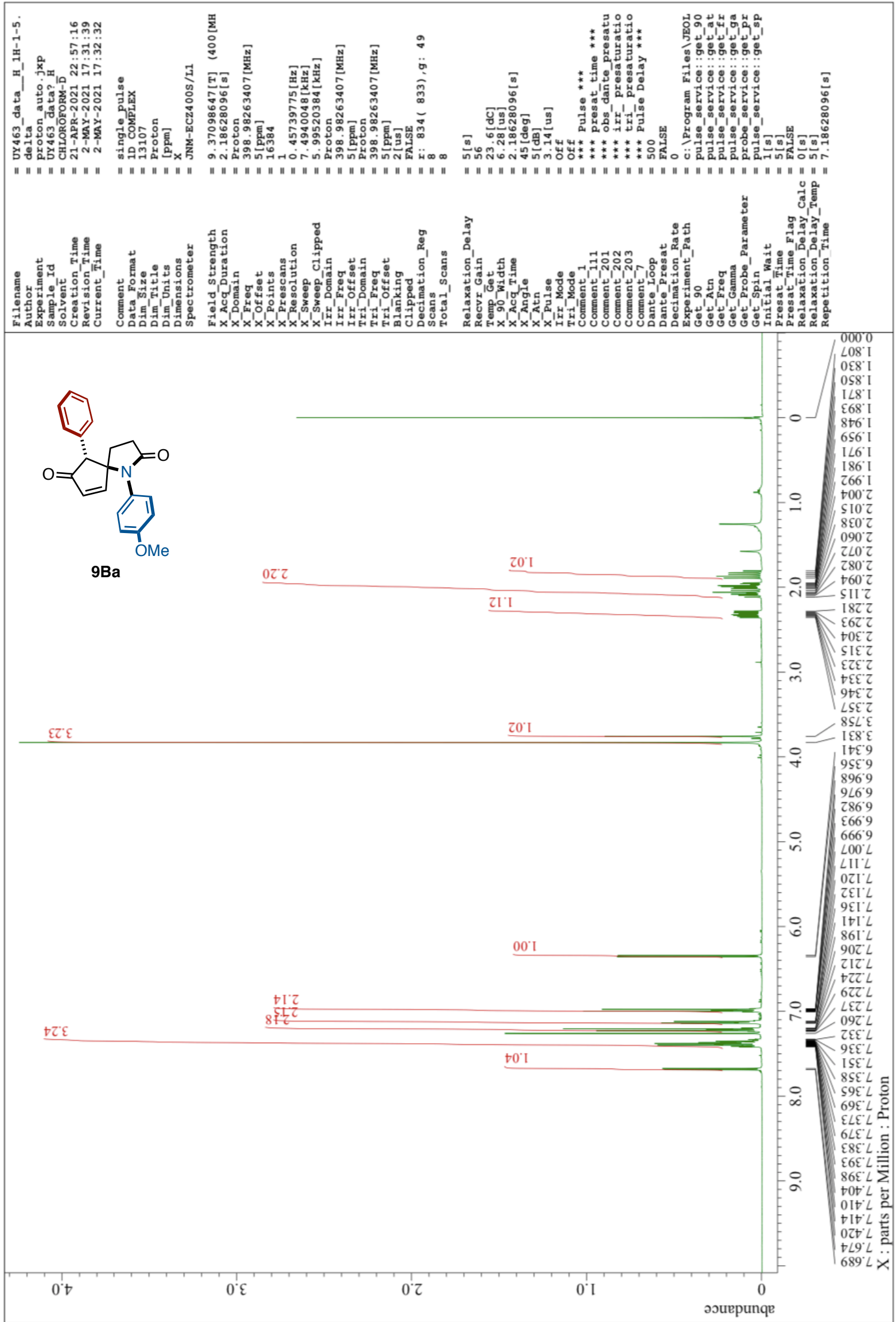


${ }^{13} \mathrm{C}$ NMR of 9Ba (101 MHz, $\left.\mathrm{CDCl}_{3}\right)$

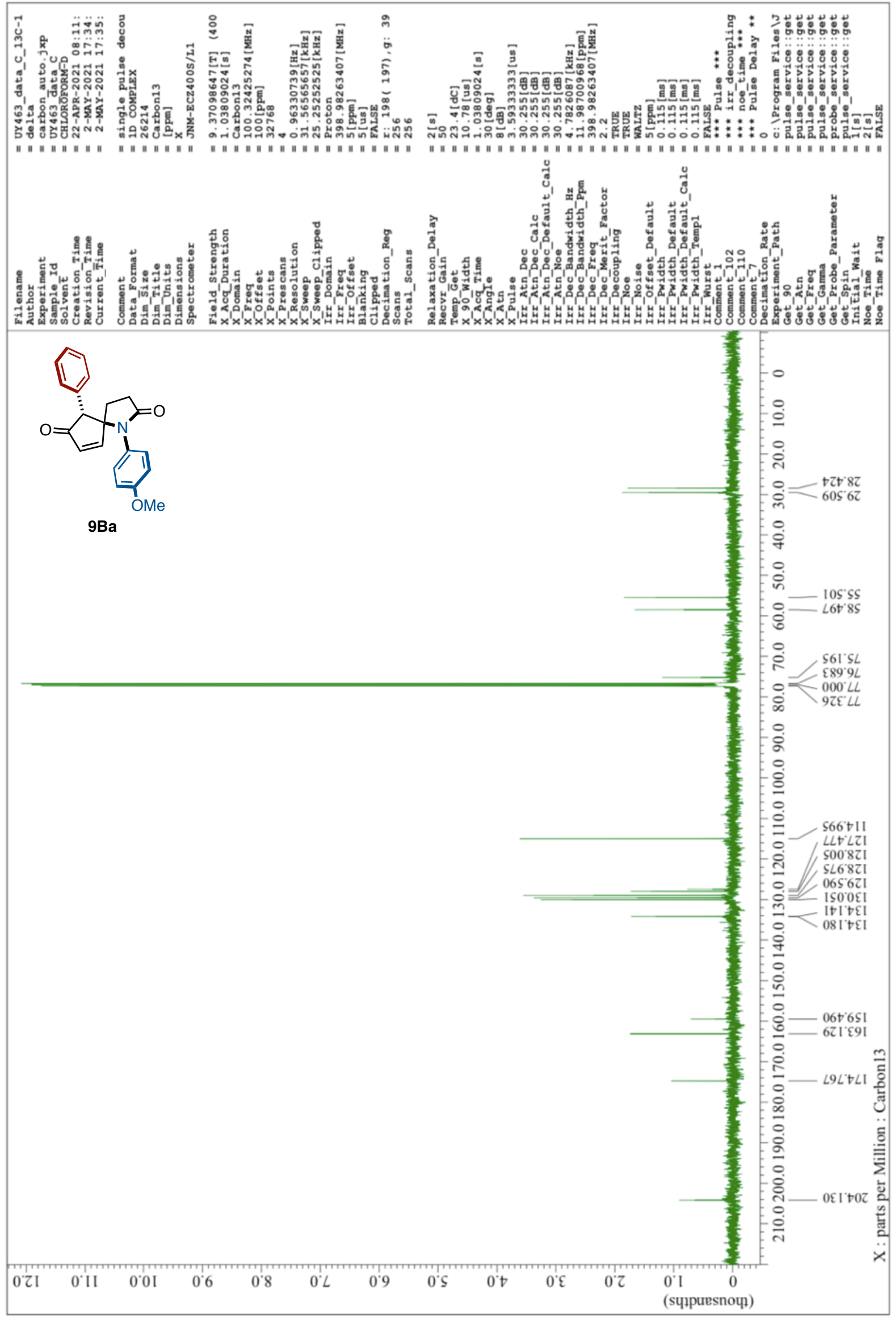


${ }^{1} \mathrm{H}$ NMR of 9Ca (400 MHz, $\left.\mathrm{CDCl}_{3}\right)$

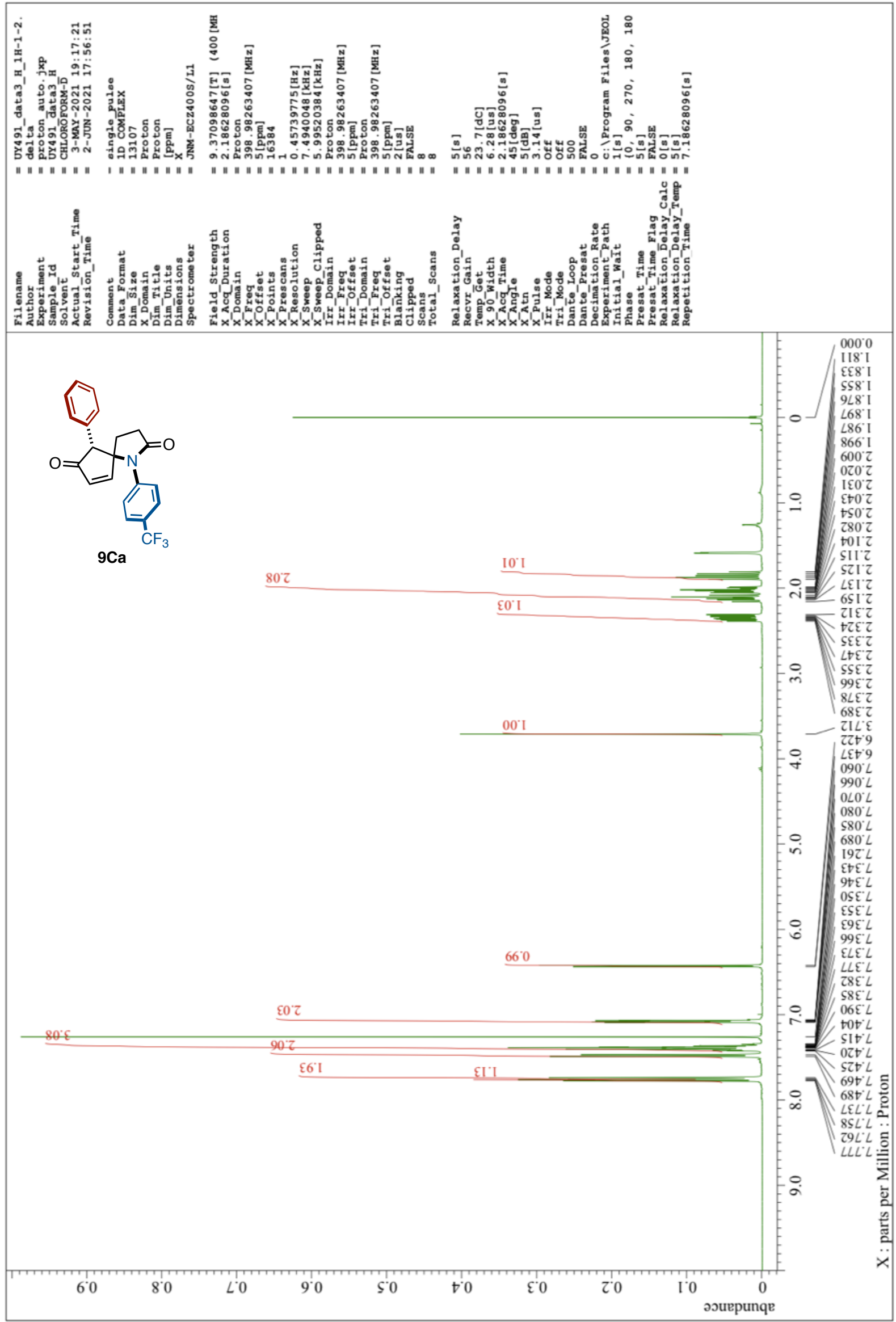


${ }^{13} \mathrm{C}$ NMR of $9 \mathrm{Ca}\left(101 \mathrm{MHz}, \mathrm{CDCl}_{3}\right)$

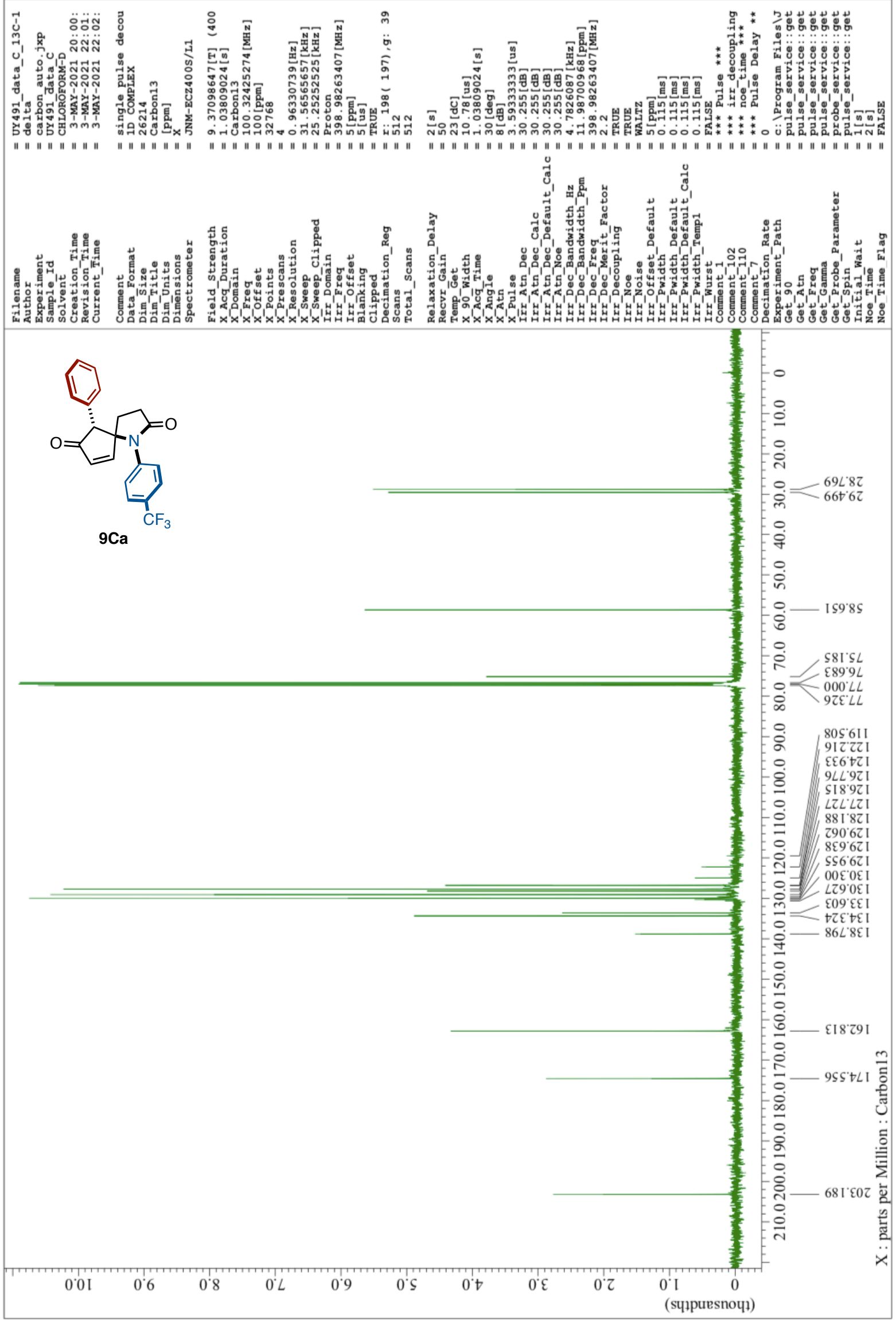


19F NMR of $9 \mathrm{Ca}\left(376 \mathrm{MHz}, \mathrm{CDCl}_{3}\right)$

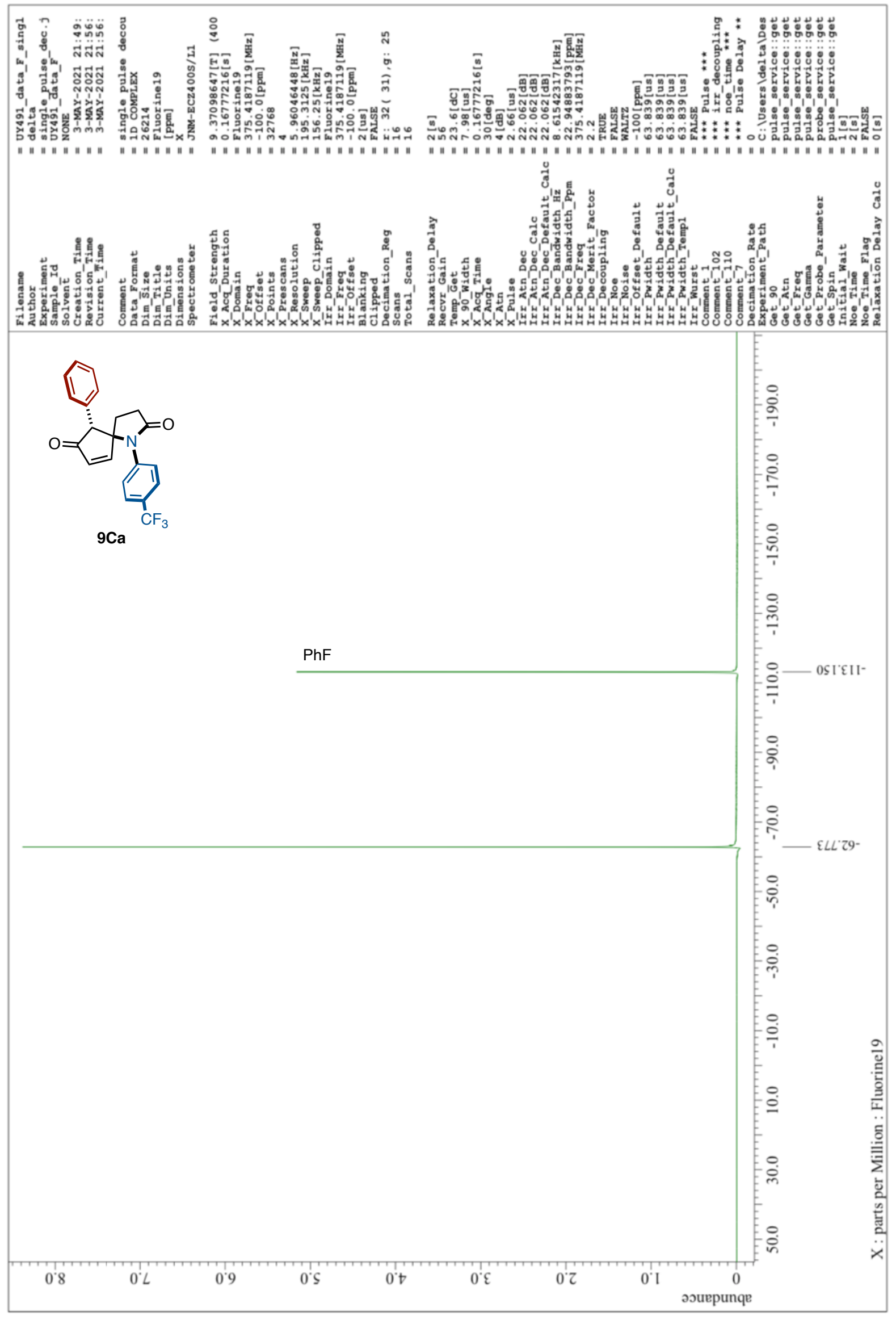


${ }^{1} \mathrm{H}$ NMR of 9Ab (400 $\left.\mathrm{MHz}, \mathrm{CDCl}_{3}\right)$

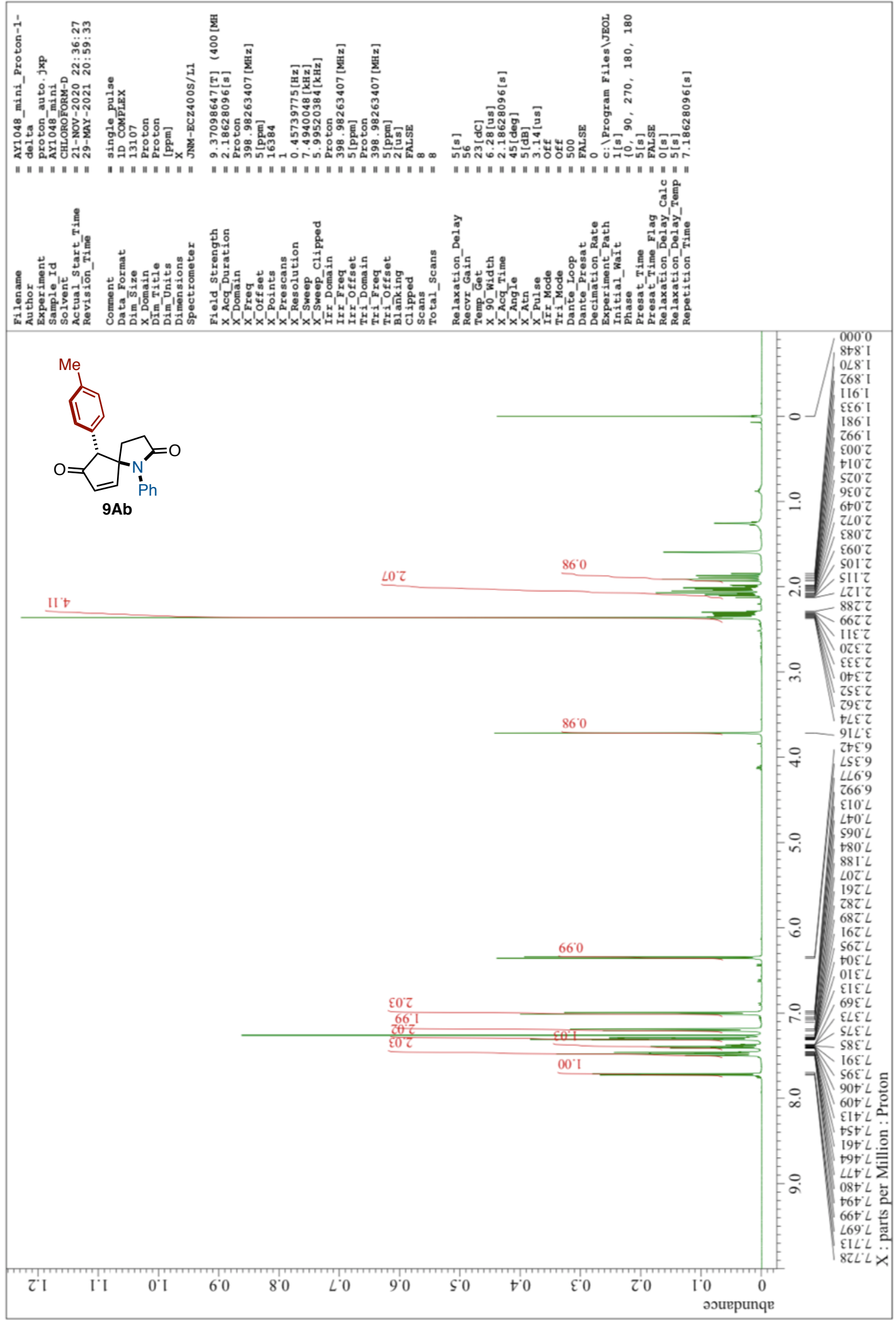


${ }^{13} \mathrm{C}$ NMR of 9Ab (101 MHz, $\left.\mathrm{CDCl}_{3}\right)$

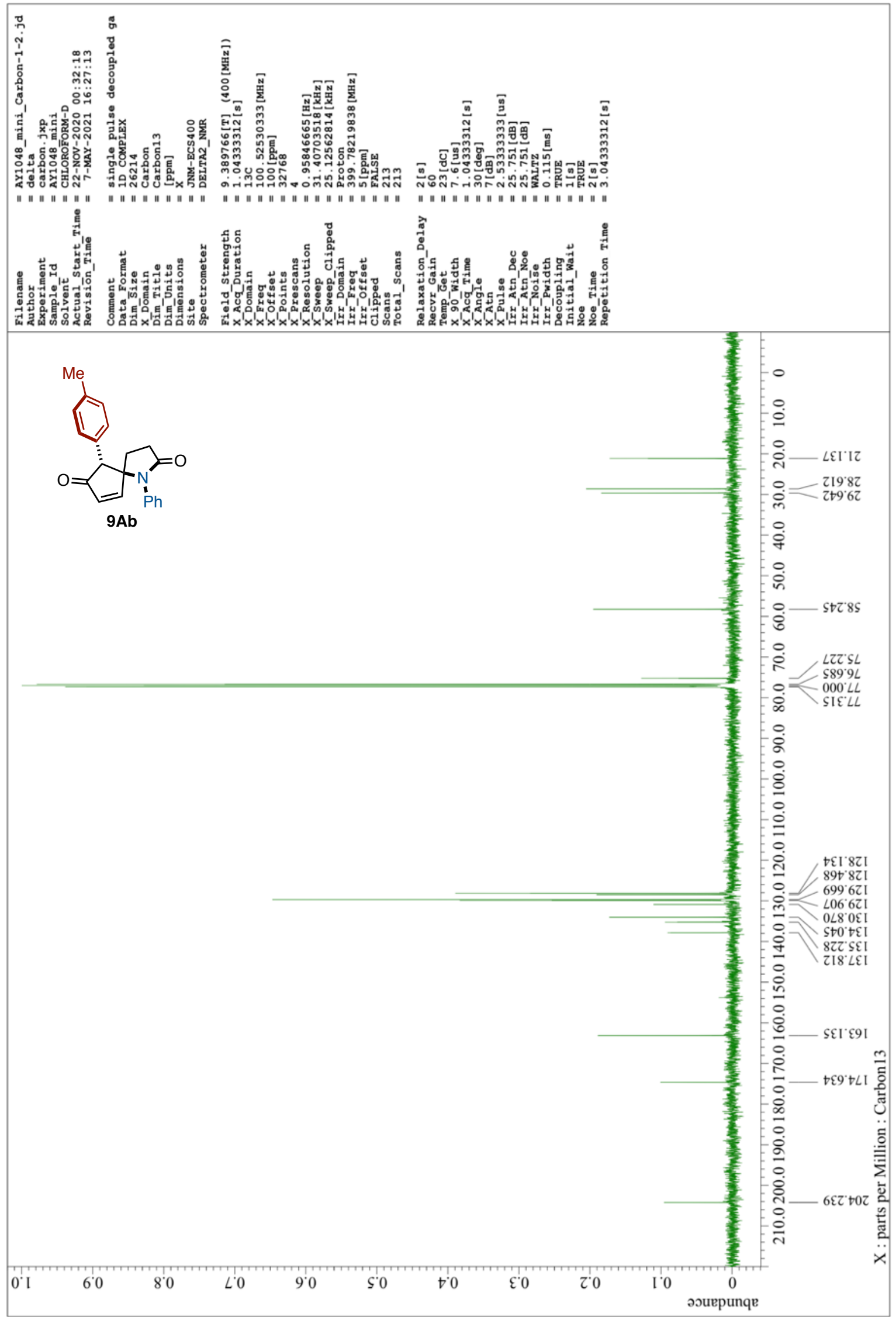


${ }^{1} \mathrm{H}$ NMR of 9Ac (400 MHz, $\left.\mathrm{CDCl}_{3}\right)$

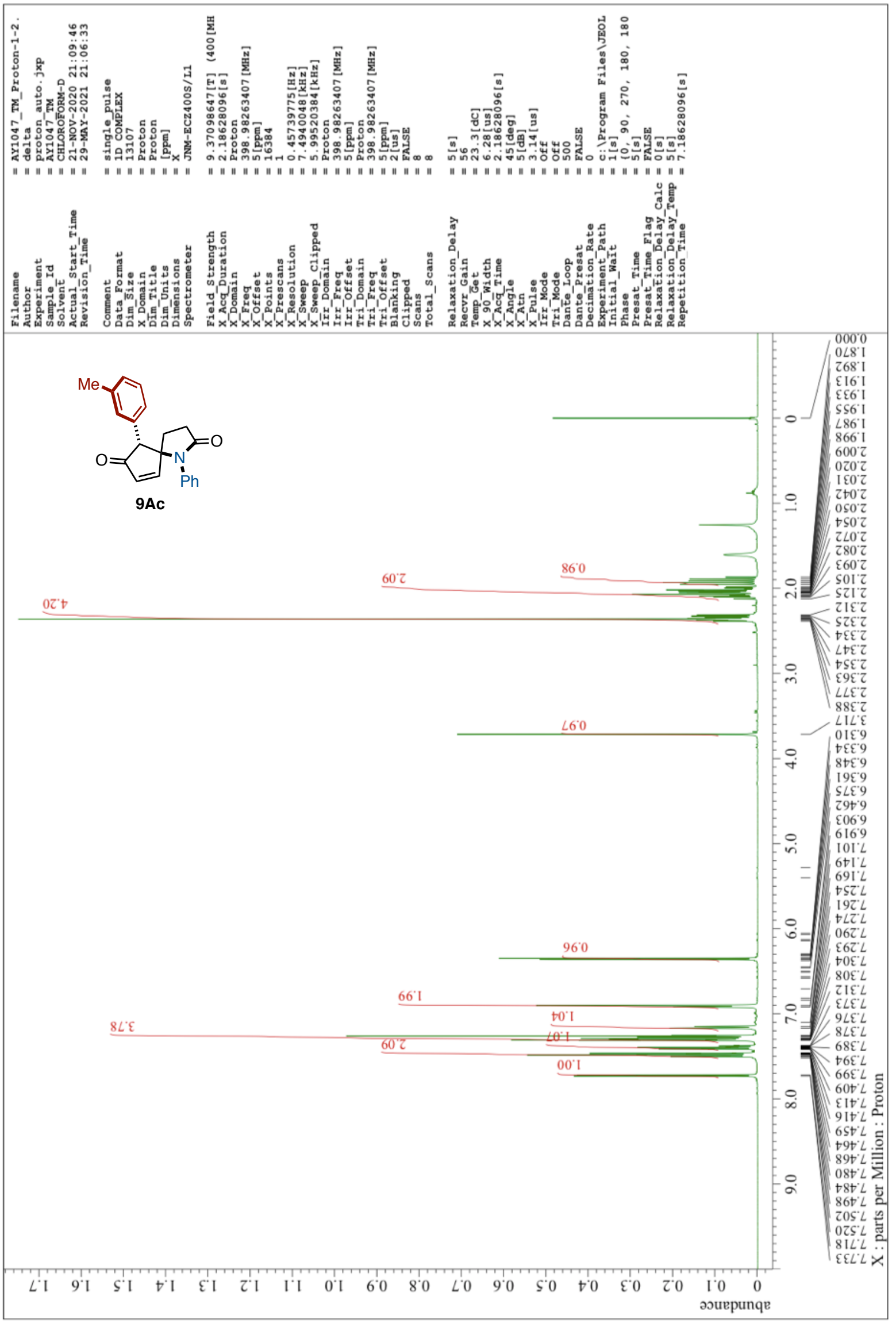


${ }^{13} \mathrm{C}$ NMR of 9Ac (101 MHz, $\left.\mathrm{CDCl}_{3}\right)$

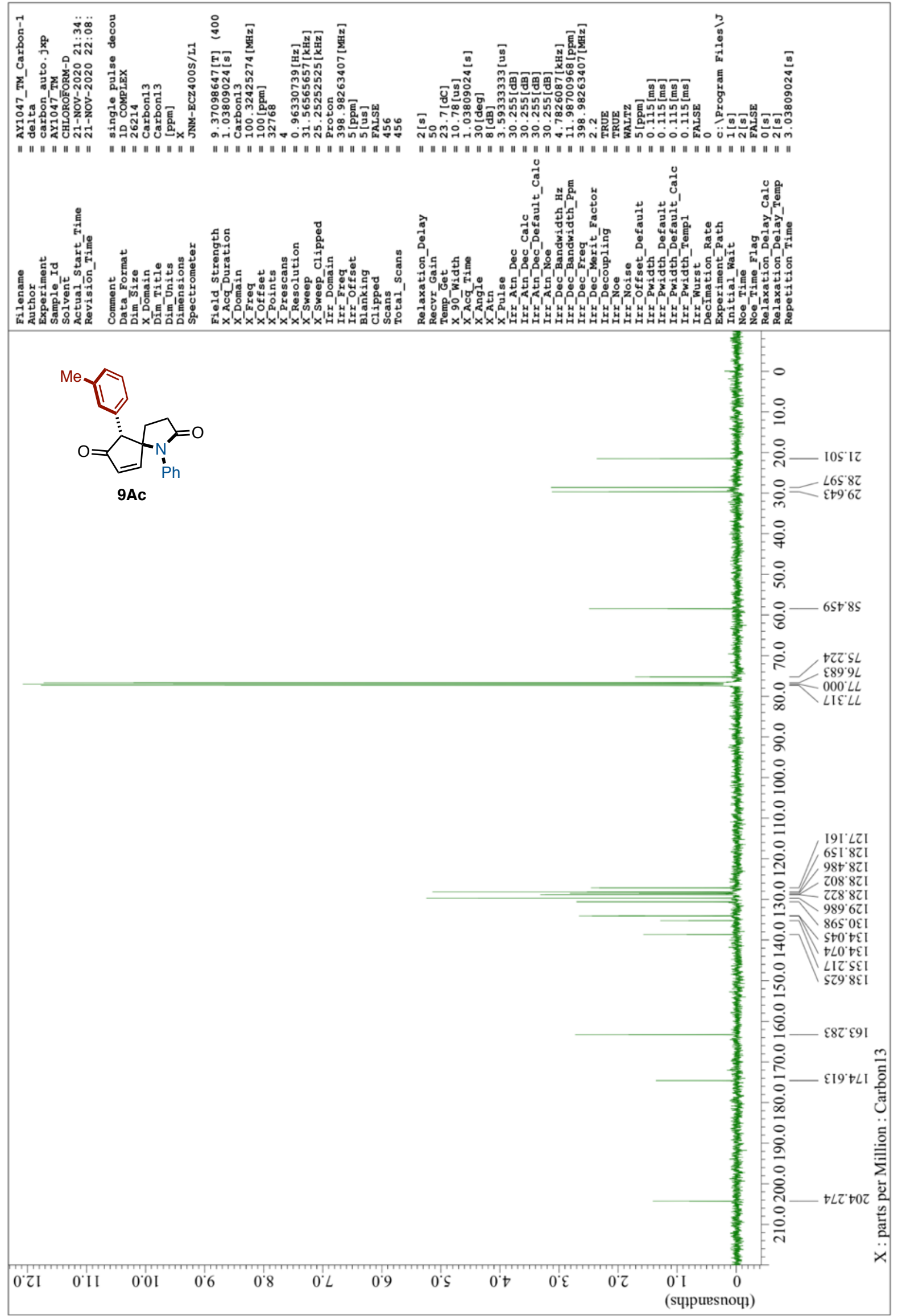


${ }^{1} \mathrm{H}$ NMR of 9Ae (400 MHz, $\left.\mathrm{CDCl}_{3}\right)$

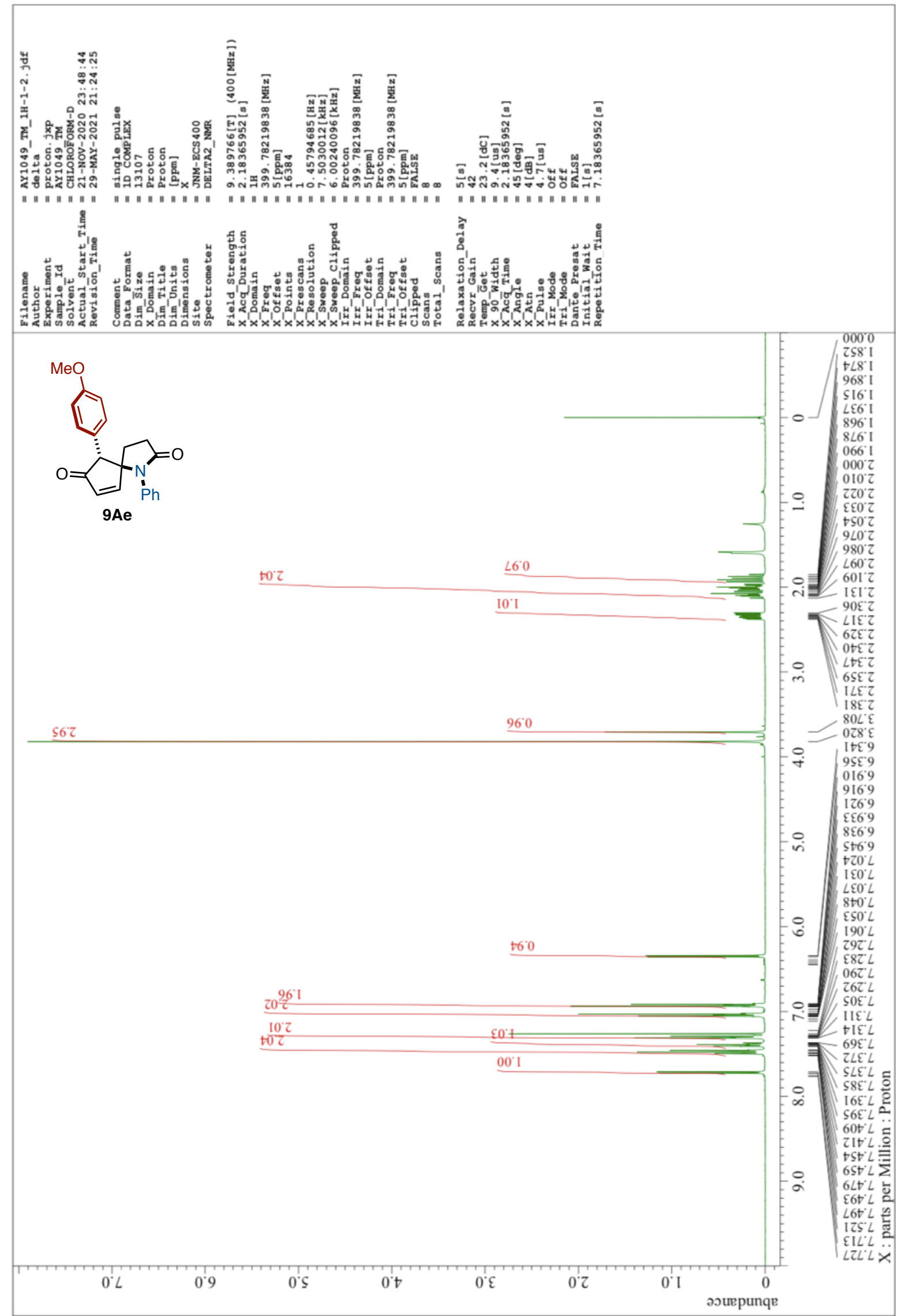


${ }^{13} \mathrm{C}$ NMR of 9Ae (101 MHz, $\left.\mathrm{CDCl}_{3}\right)$

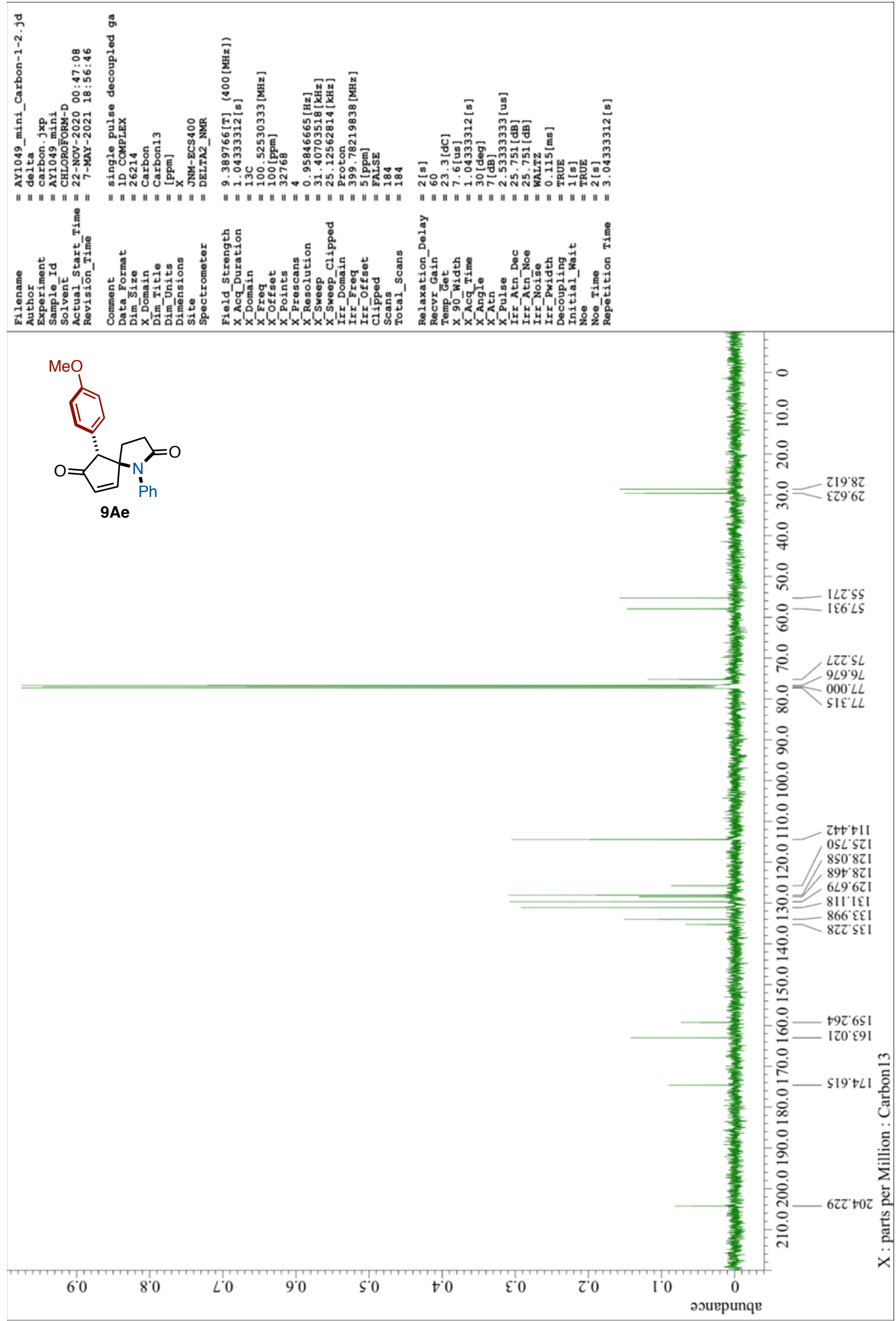


${ }^{1} \mathrm{H}$ NMRc of 9Af (101 MHz, $\left.\mathrm{CDCl}_{3}\right)$

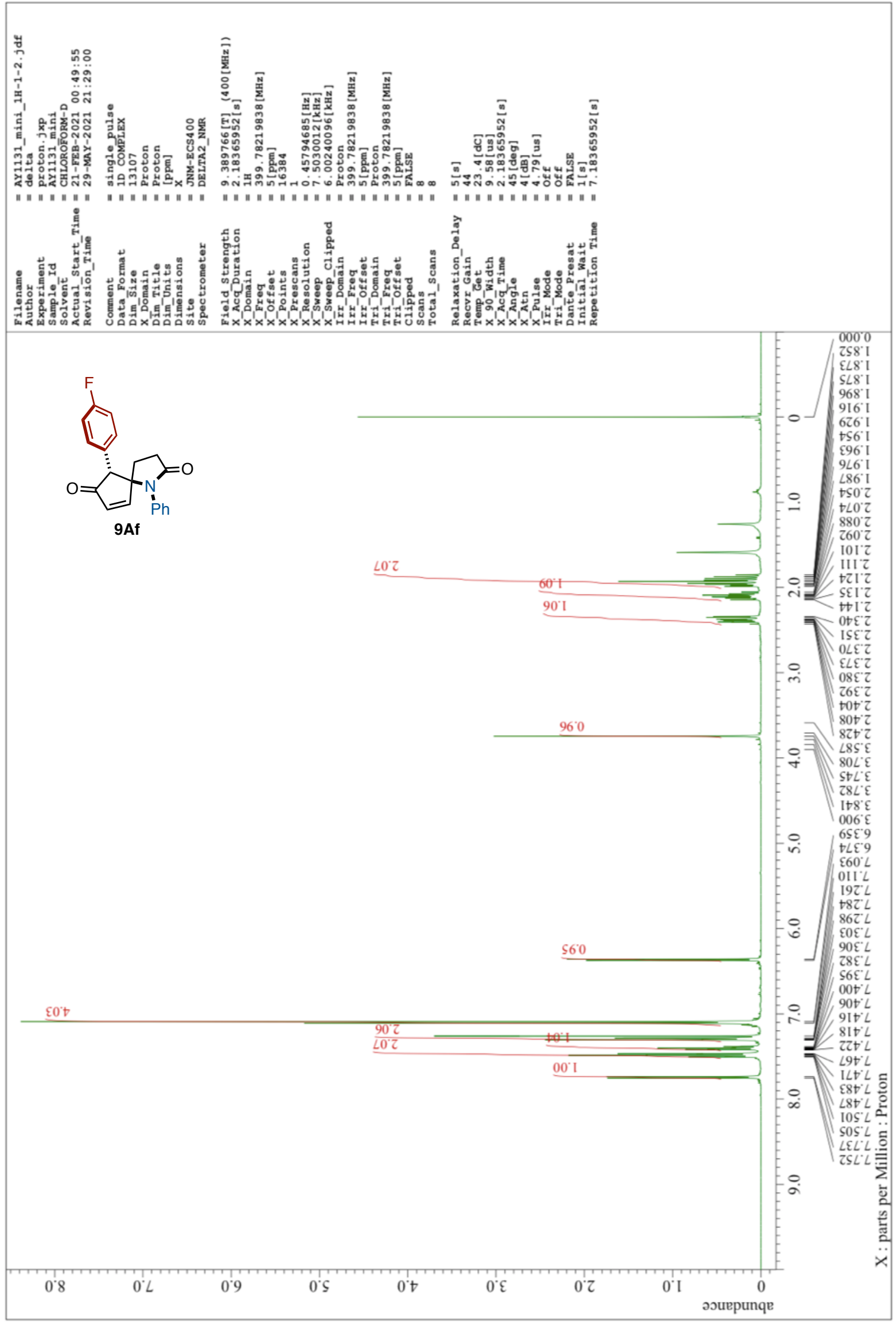


${ }^{13} \mathrm{C}$ NMR of 9Af (101 MHz, $\left.\mathrm{CDCl}_{3}\right)$
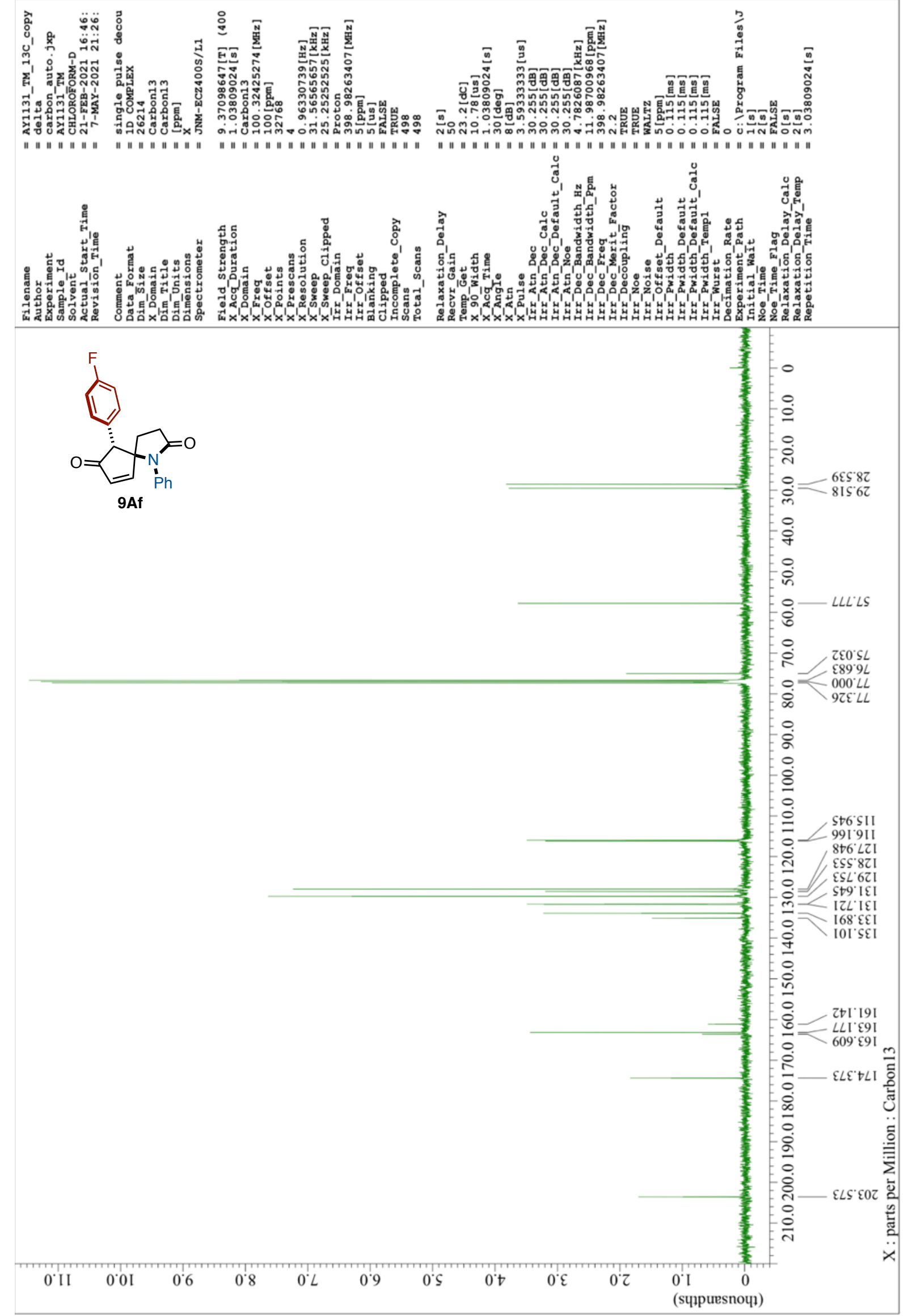
19F NMR of 9Af (376 MHz, $\left.\mathrm{CDCl}_{3}\right)$

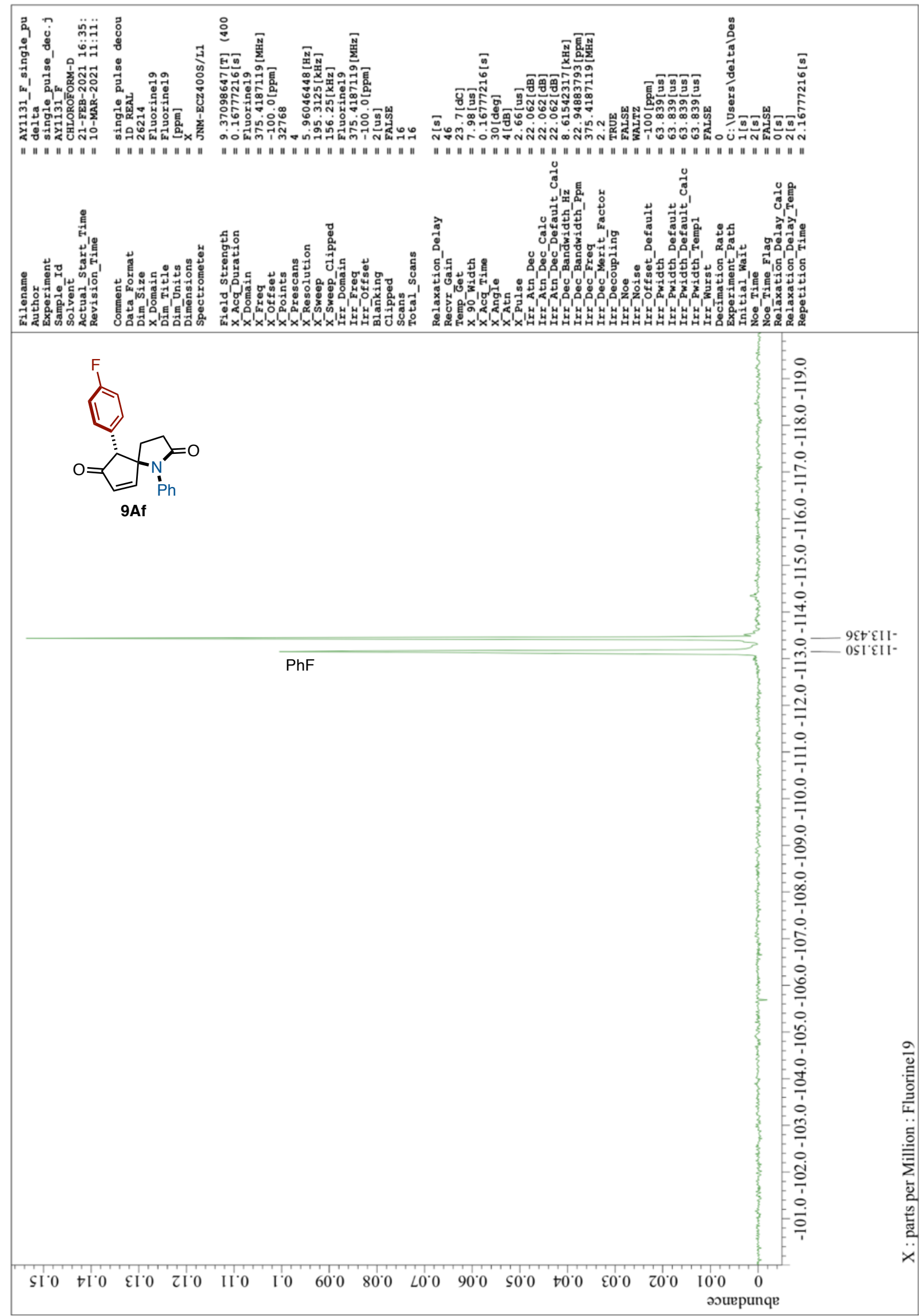


${ }^{1} \mathrm{H}$ NMR of 9Ah (400 MHz, $\left.\mathrm{CDCl}_{3}\right)$

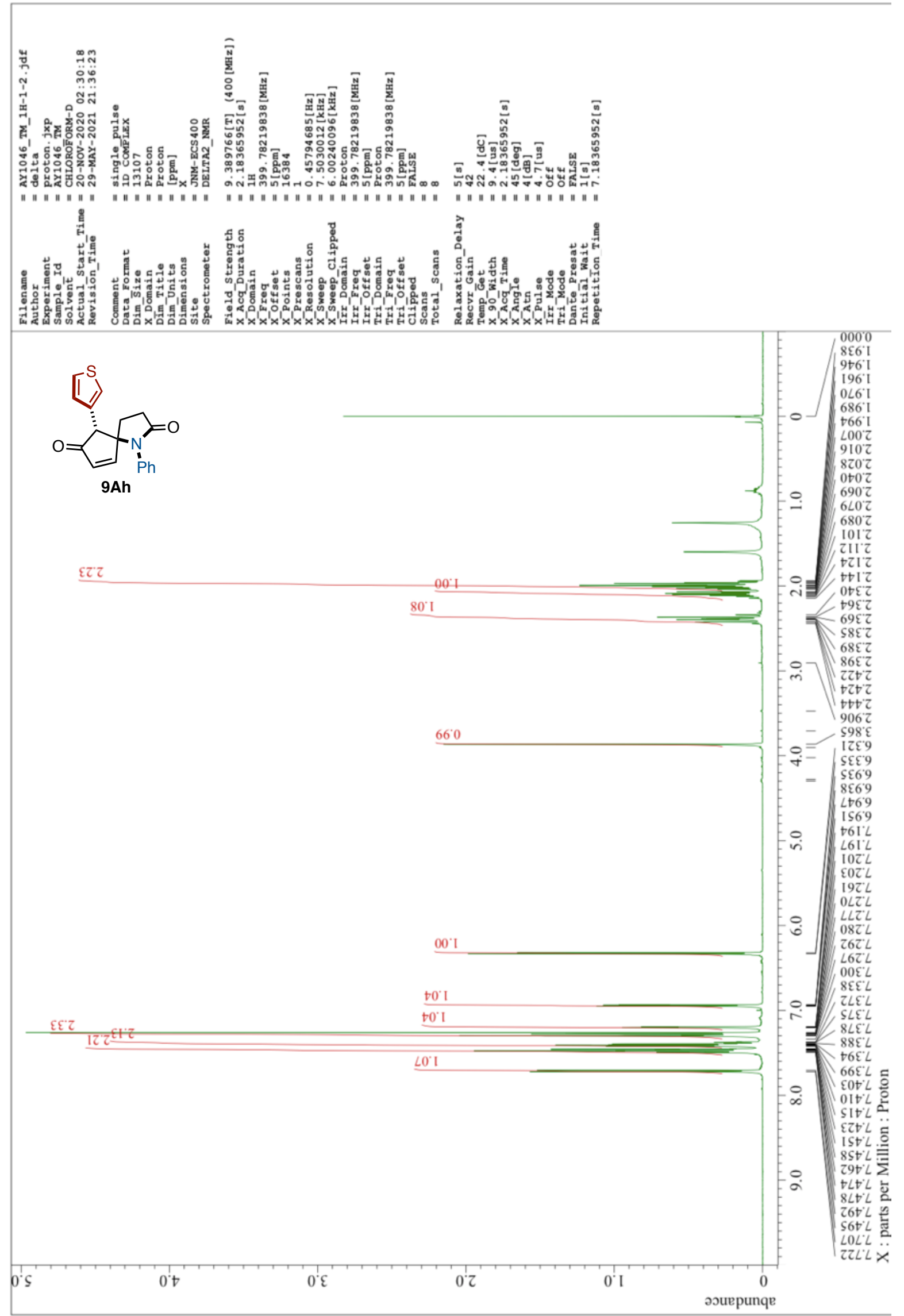


${ }^{13} \mathrm{C}$ NMR of 9Ah (101 MHz, $\left.\mathrm{CDCl}_{3}\right)$

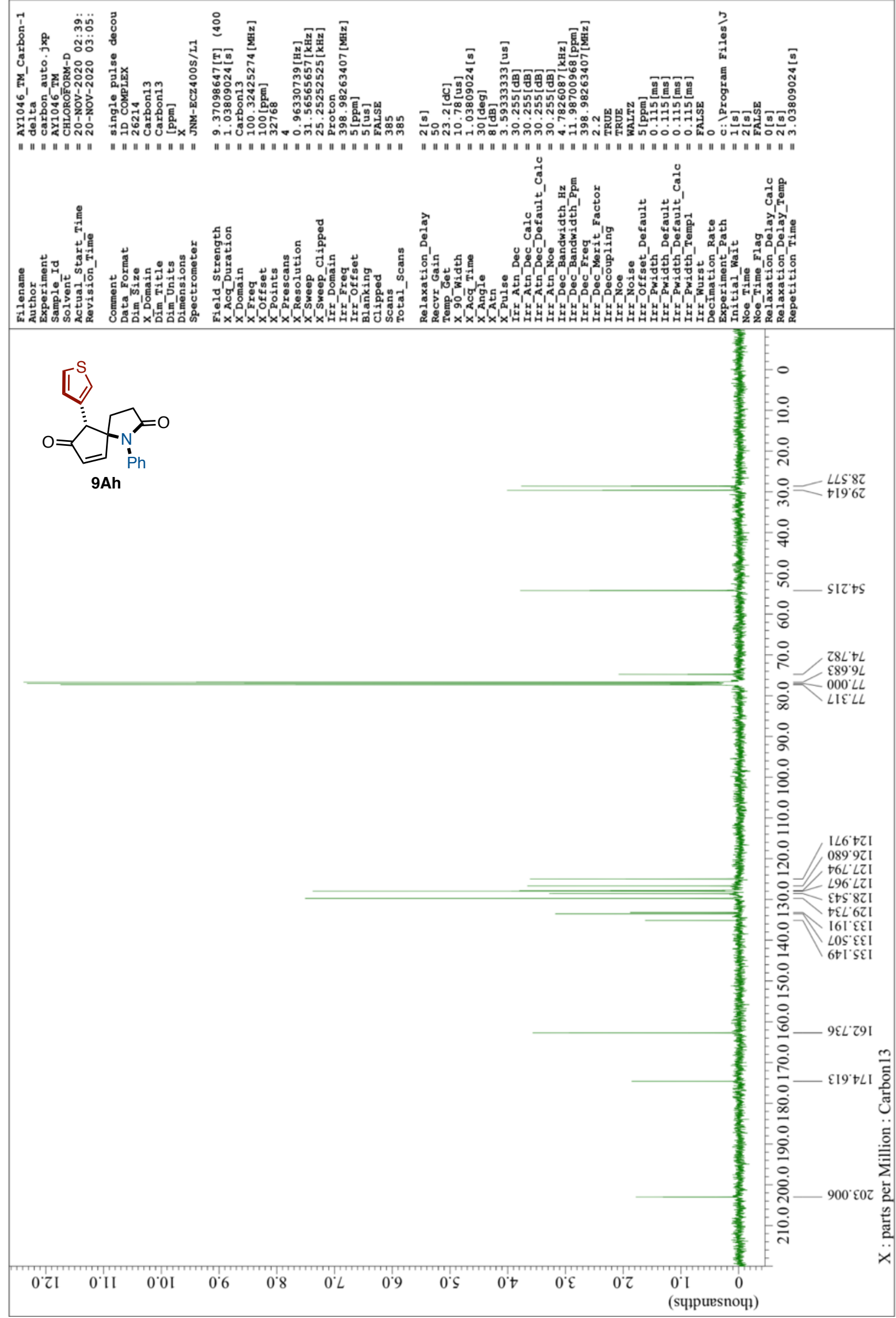


${ }^{1} \mathrm{H}$ NMR of 9Ai (400 MHz, $\left.\mathrm{CDCl}_{3}\right)$

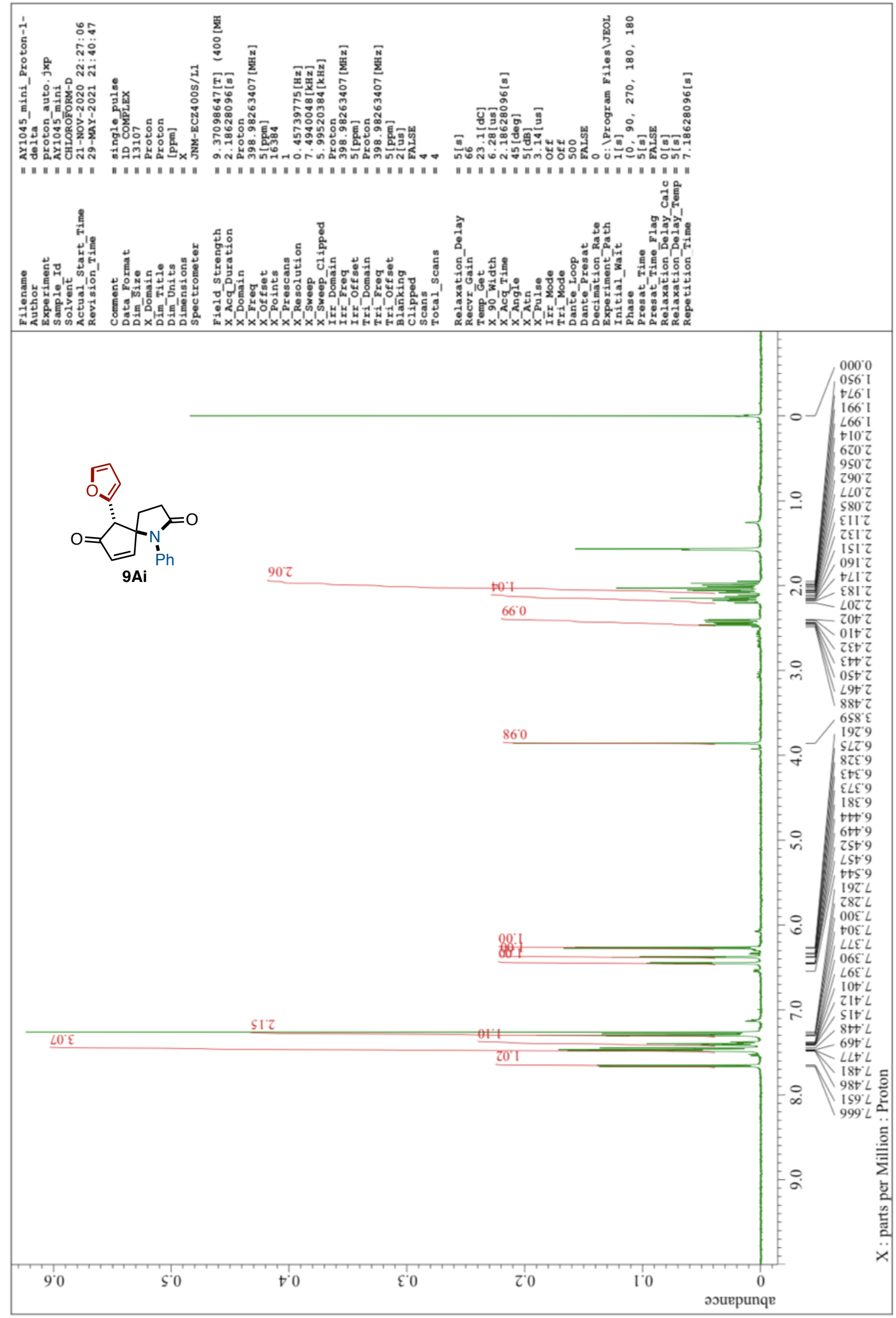


${ }^{13} \mathrm{C}$ NMR of 9Ai (101 MHz, $\left.\mathrm{CDCl}_{3}\right)$

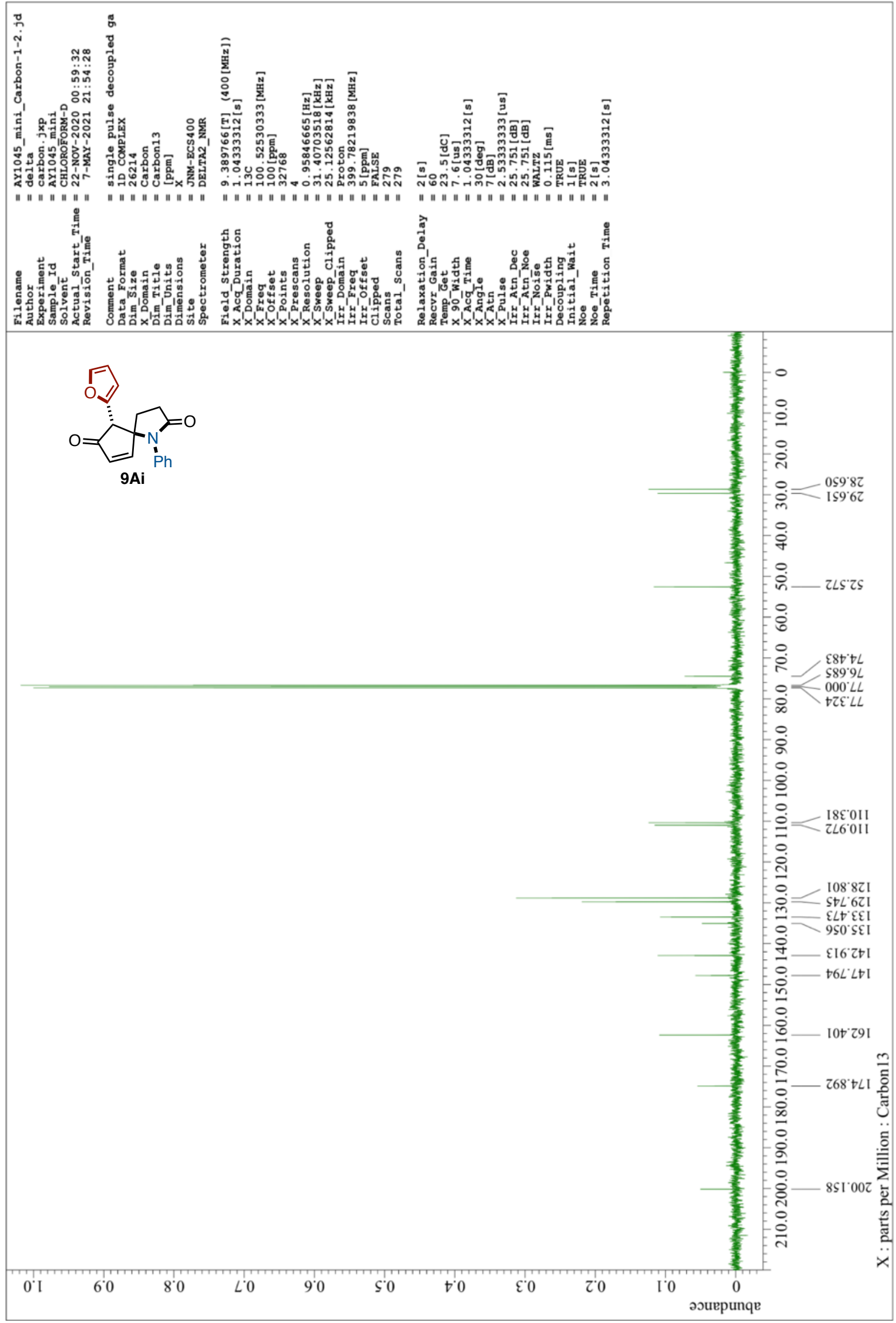


${ }^{1} \mathrm{H}$ NMR of 9Ak (400 $\left.\mathrm{MHz}, \mathrm{CDCl}_{3}\right)$

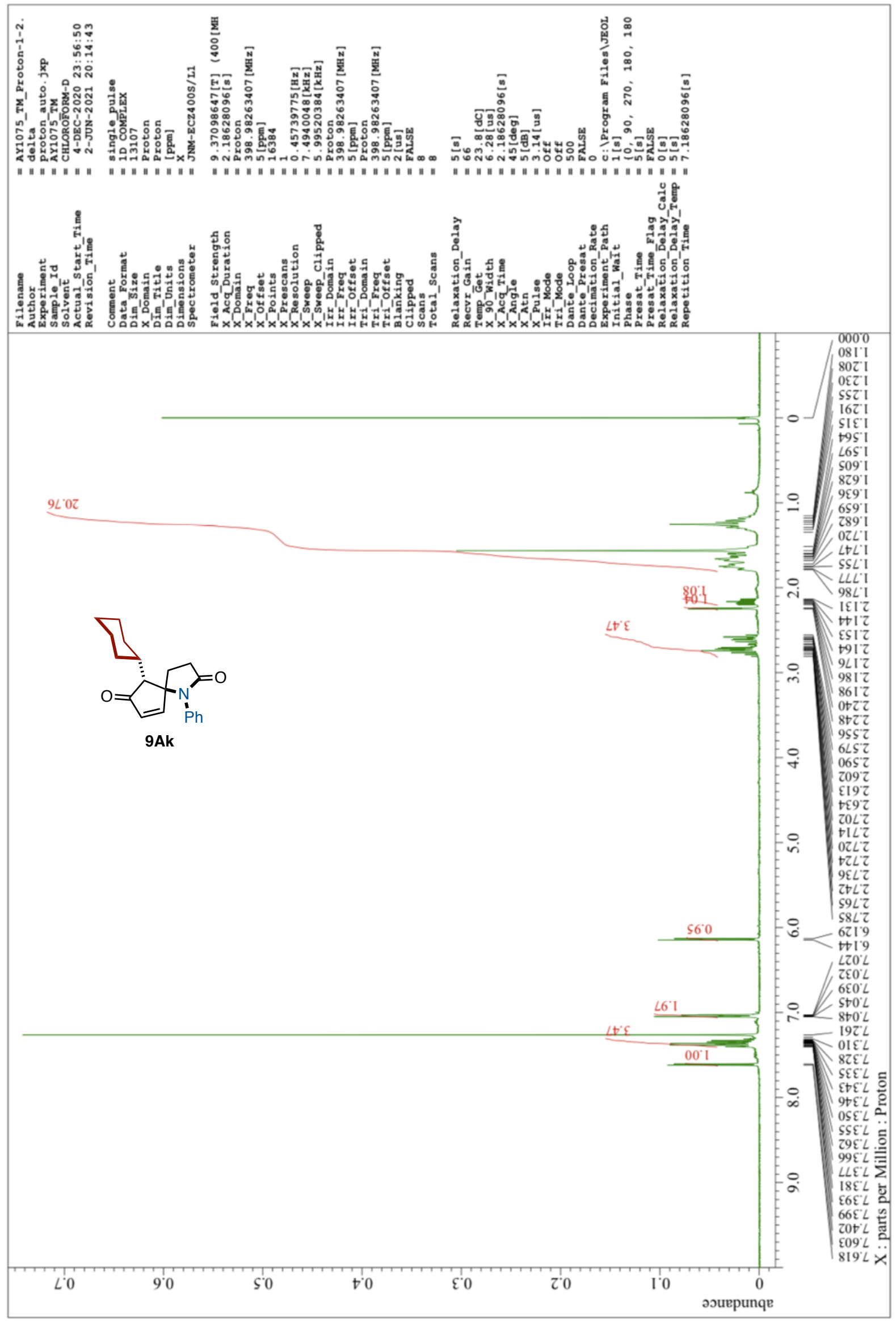


${ }^{13} \mathrm{C}$ NMR of 10Ak (101 MHz, $\left.\mathrm{CDCl}_{3}\right)$

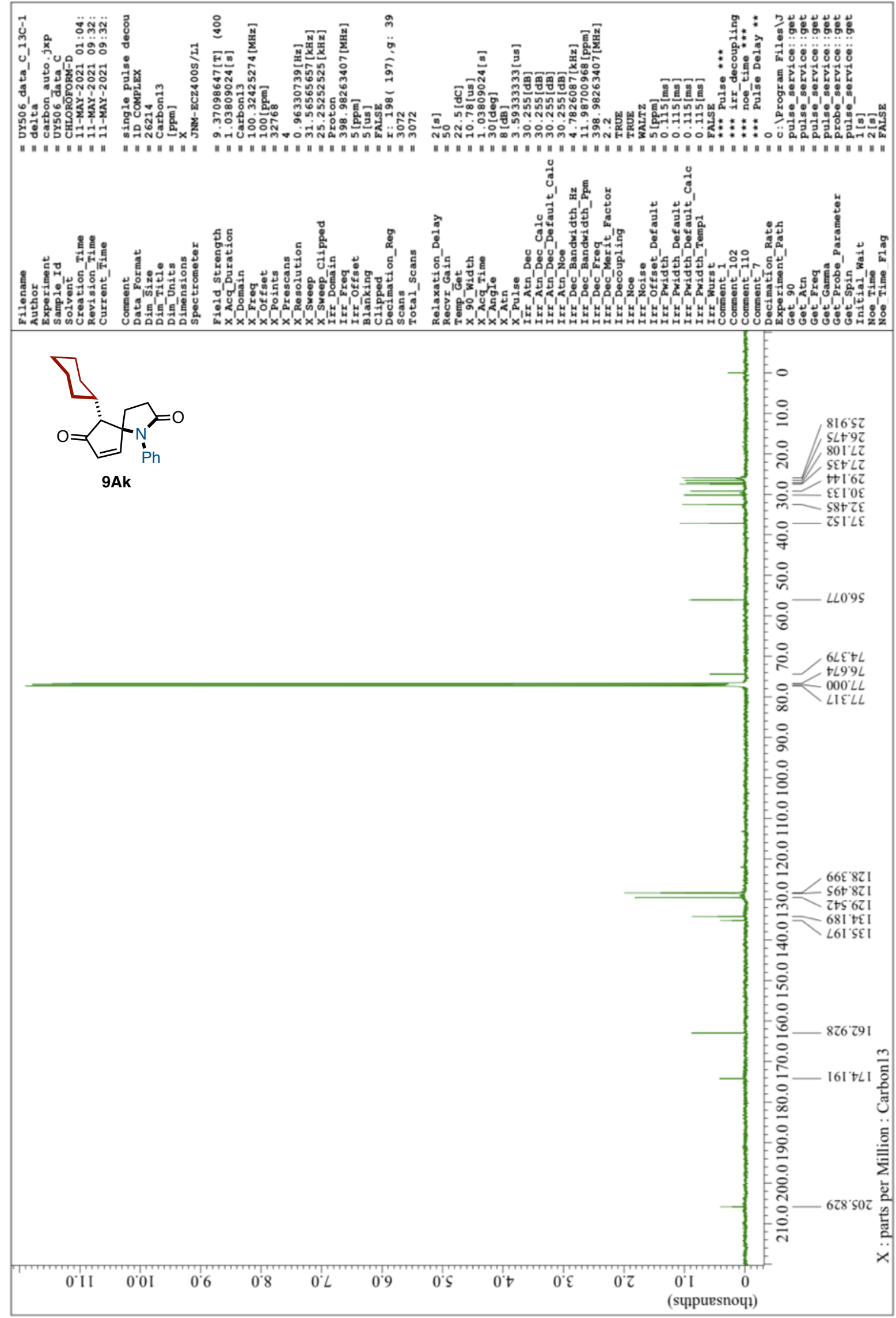


${ }^{1} \mathrm{H}$ NMR of 9Ea (400 $\left.\mathrm{MHz}, \mathrm{CDCl}_{3}\right)$

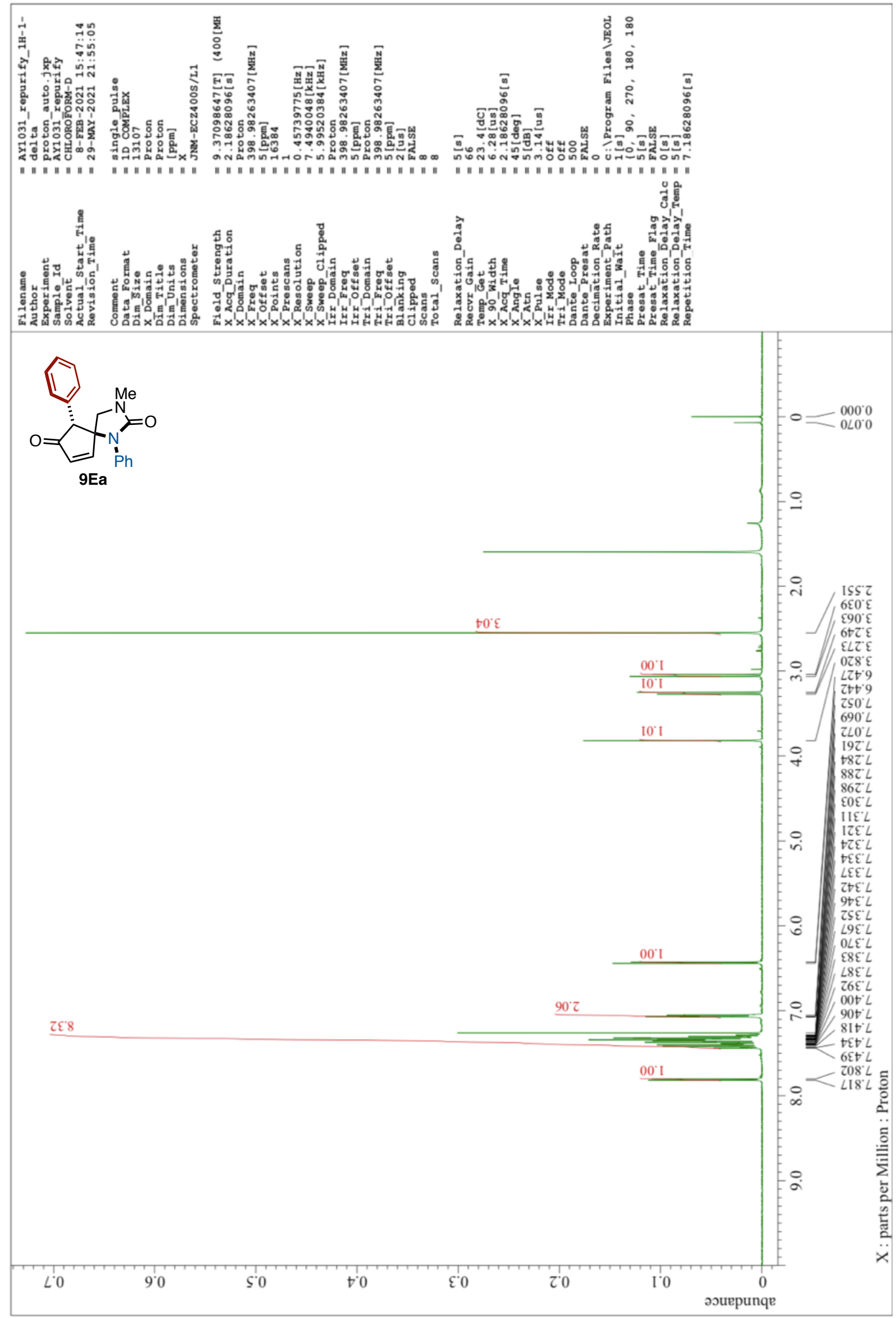


${ }^{13} \mathrm{C}$ NMR of 9Ea (101 MHz, $\left.\mathrm{CDCl}_{3}\right)$

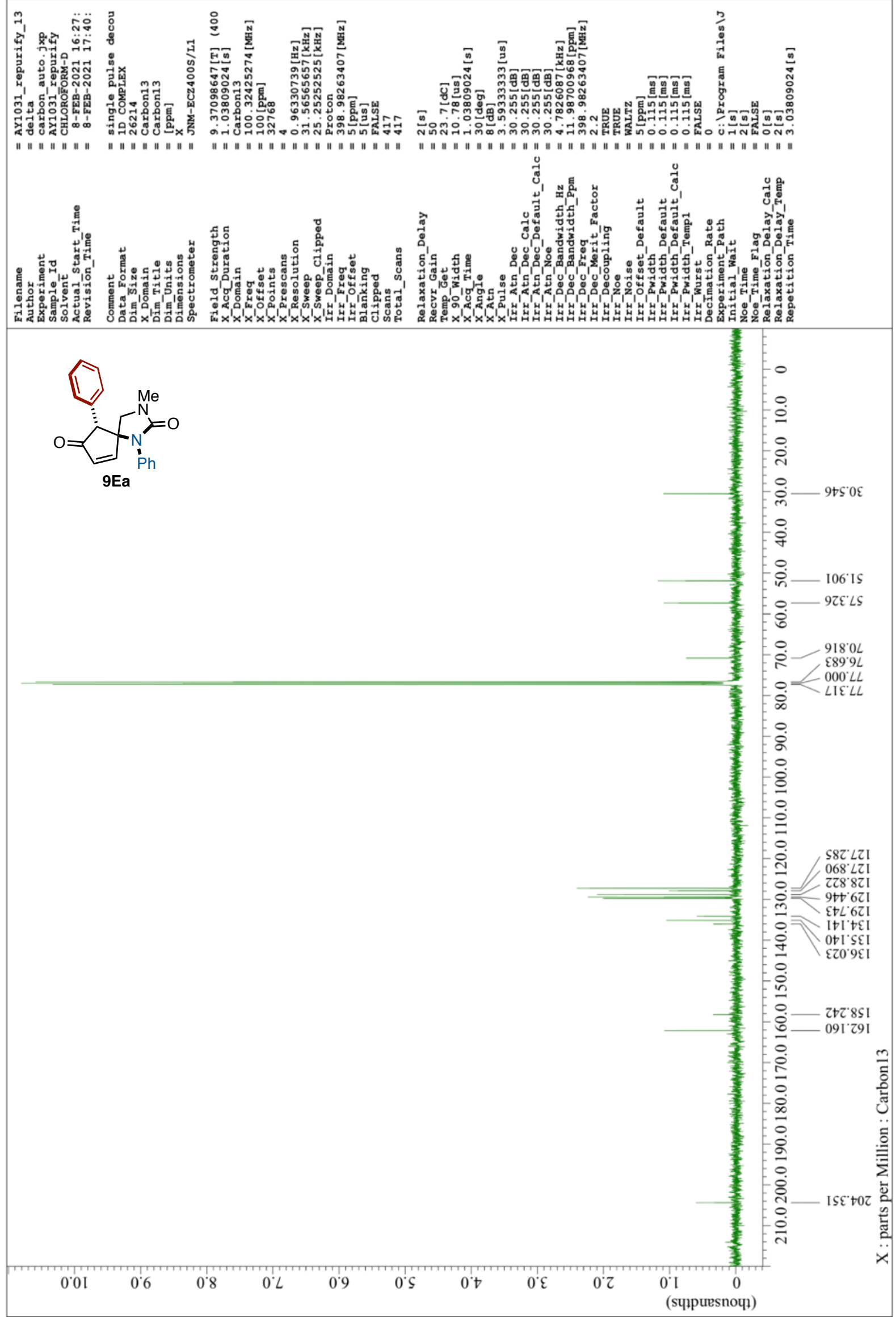


${ }^{1} \mathrm{H}$ NMR of 9Sa (400 MHz, $\left.\mathrm{CDCl}_{3}\right)$

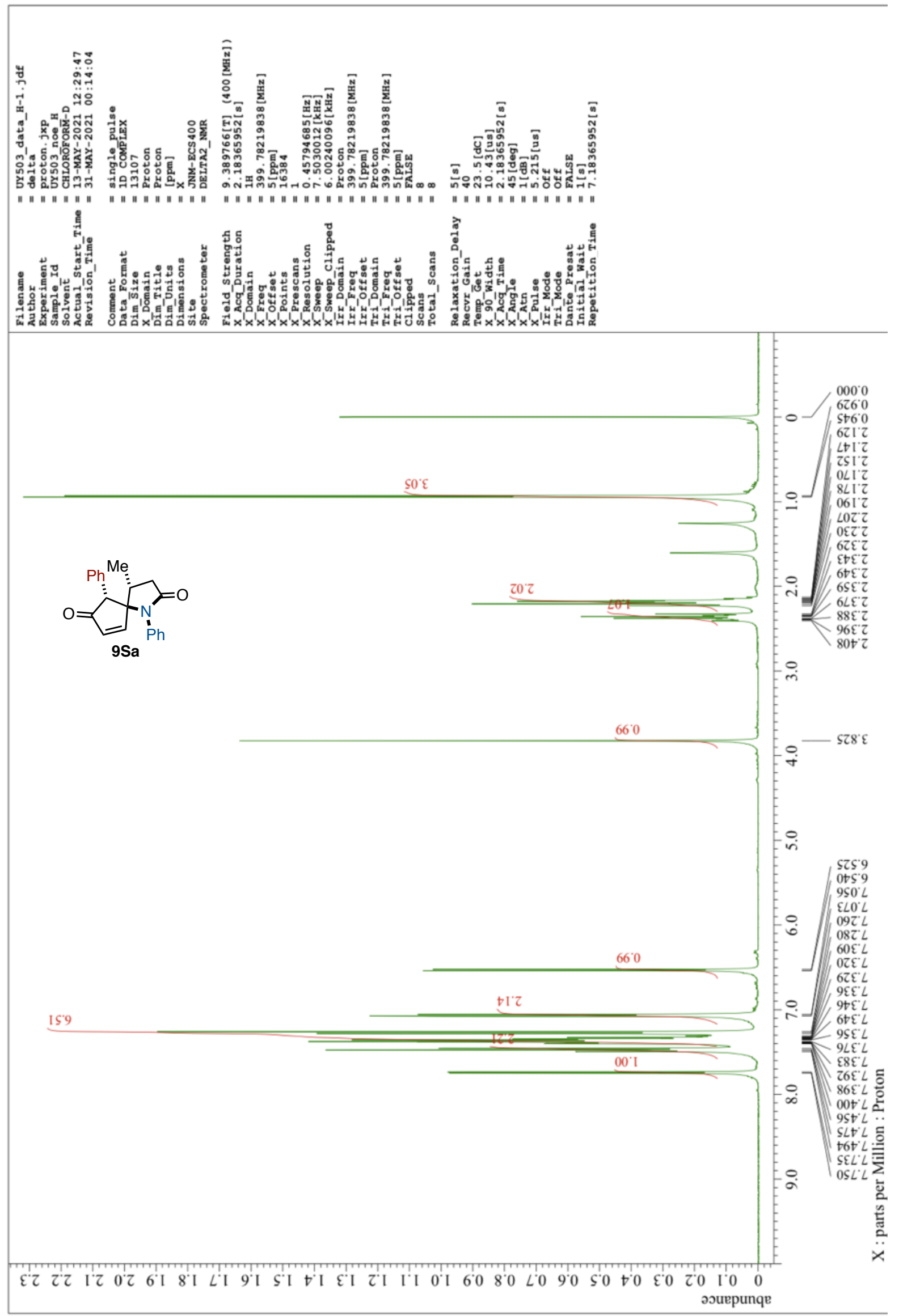


${ }^{13} \mathrm{C}$ NMR of 9Sa (101 MHz, $\left.\mathrm{CDCl}_{3}\right)$

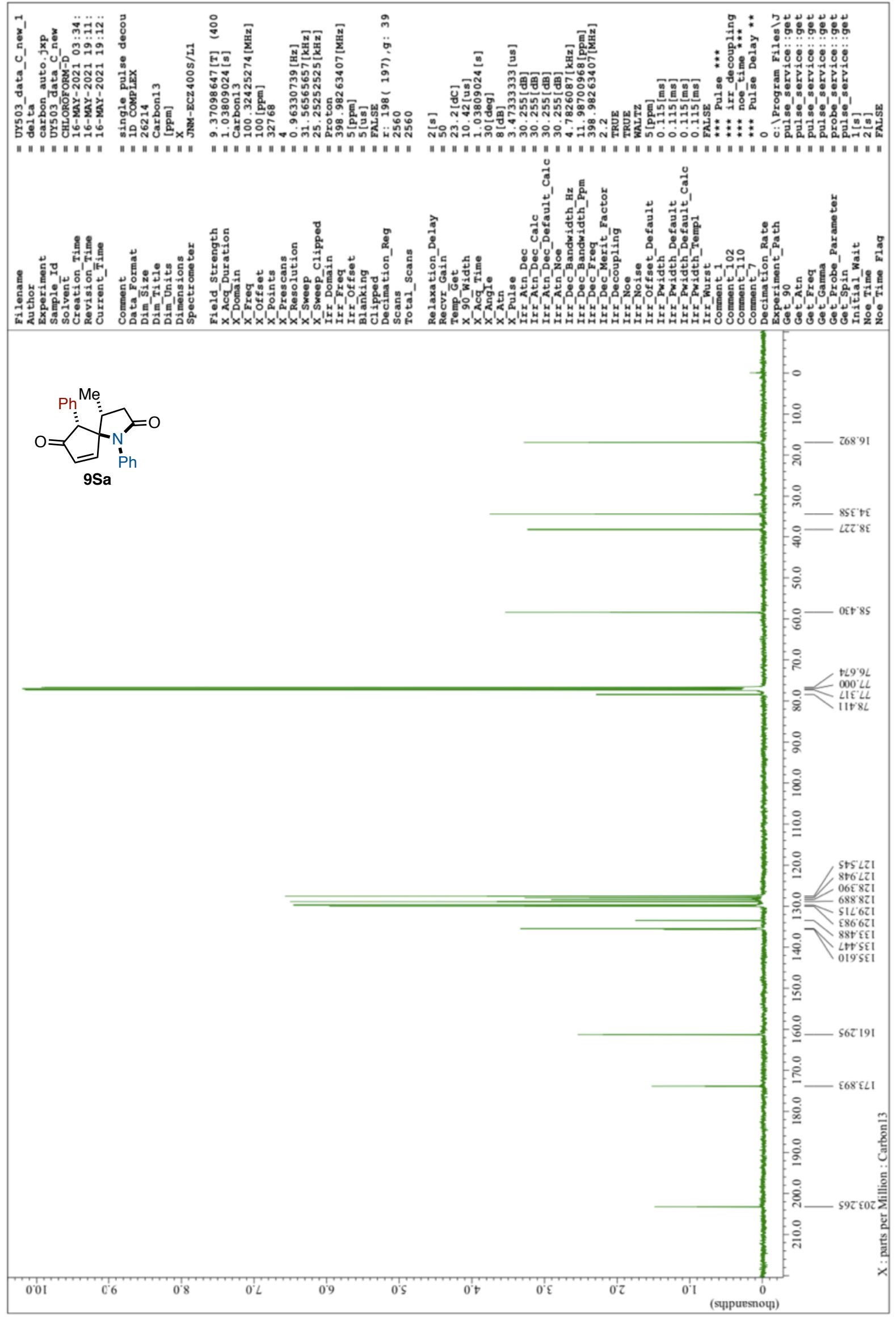


${ }^{1} \mathrm{H}$ NMR of 9Ta (400 MHz, $\left.\mathrm{CDCl}_{3}\right)$

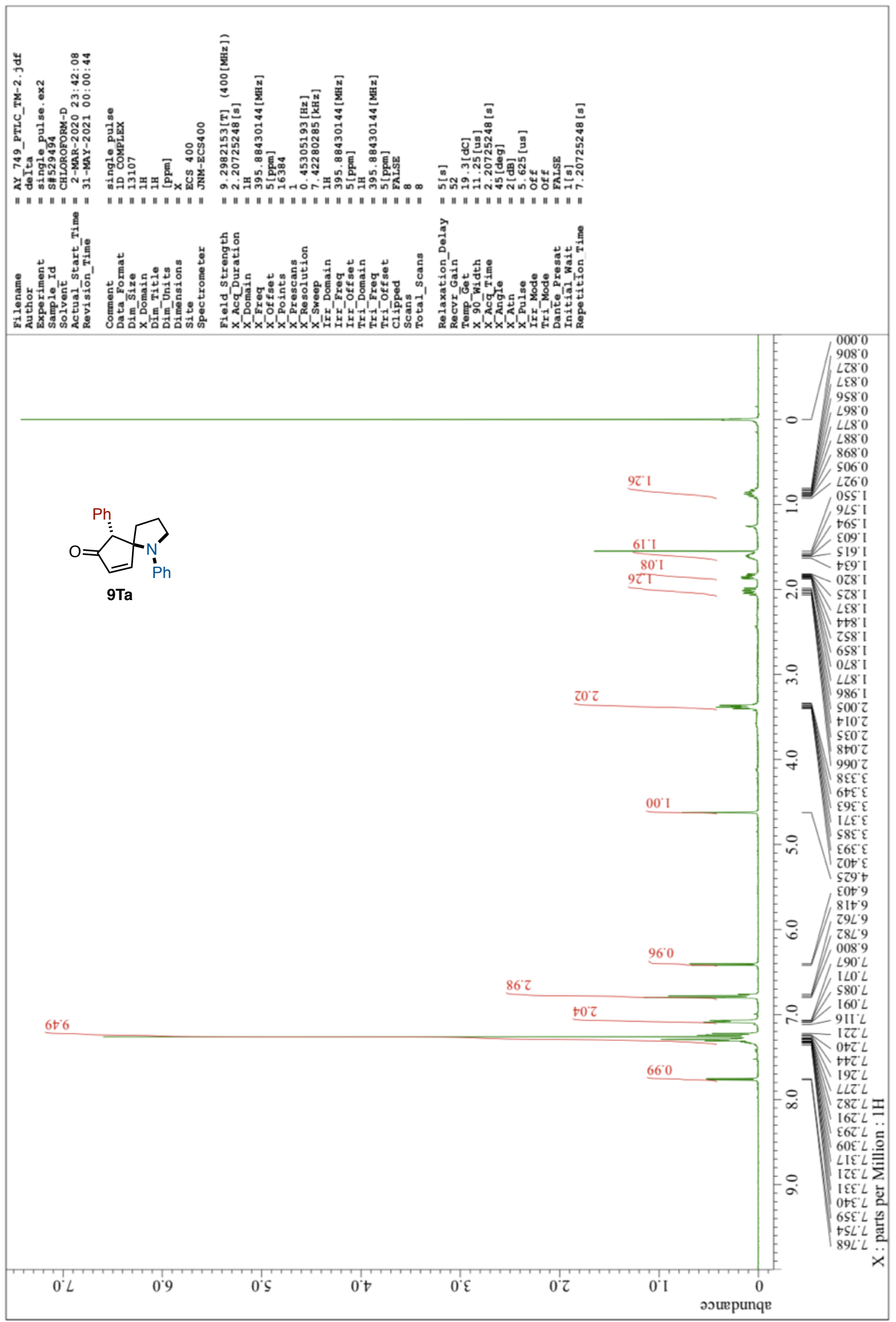


${ }^{13} \mathrm{C}$ NMR of 9Ta (101 MHz, $\left.\mathrm{CDCl}_{3}\right)$

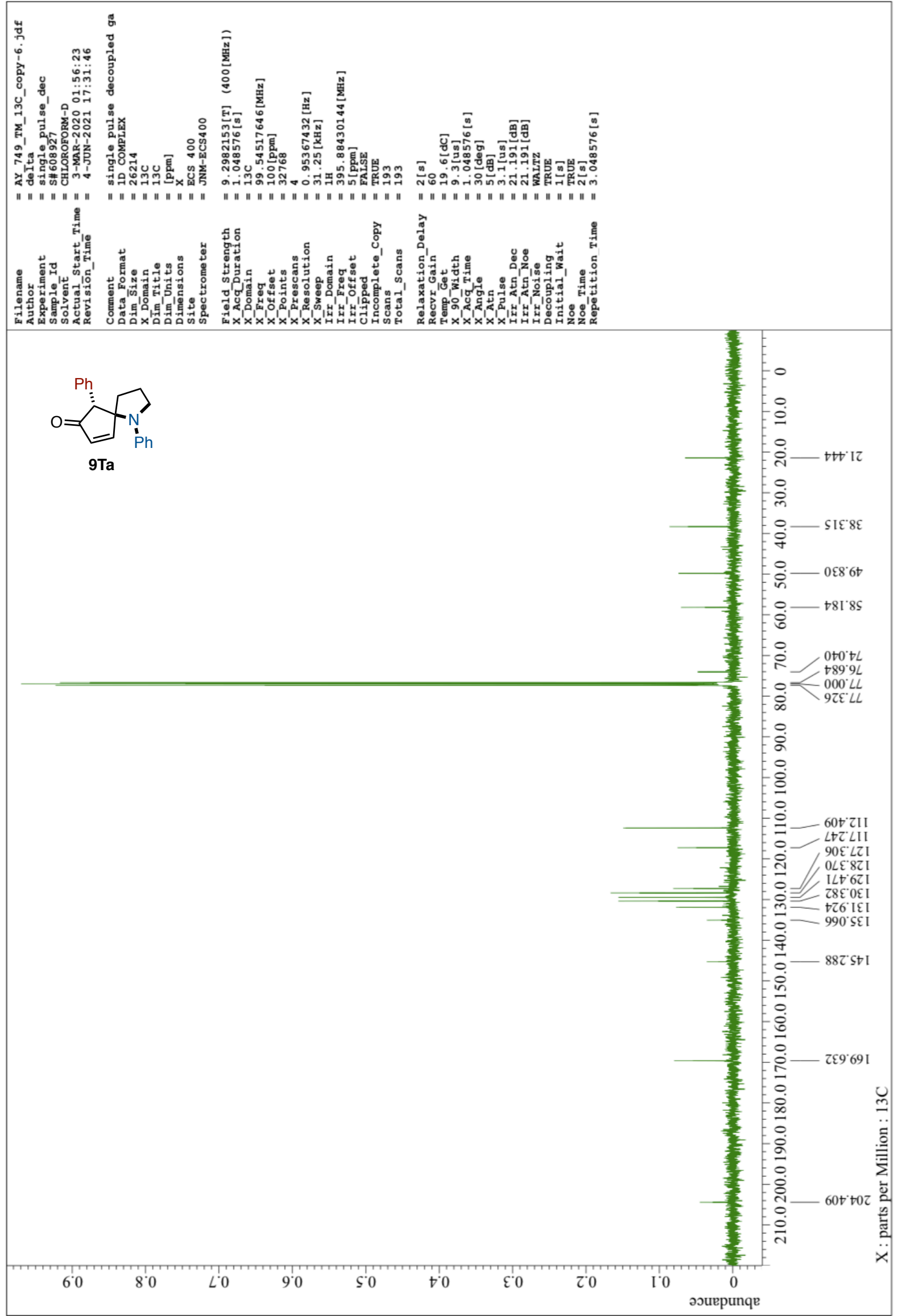


${ }^{1} \mathrm{H}$ NMR of 10 (400 $\left.\mathrm{MHz}, \mathrm{CDCl}_{3}\right)$

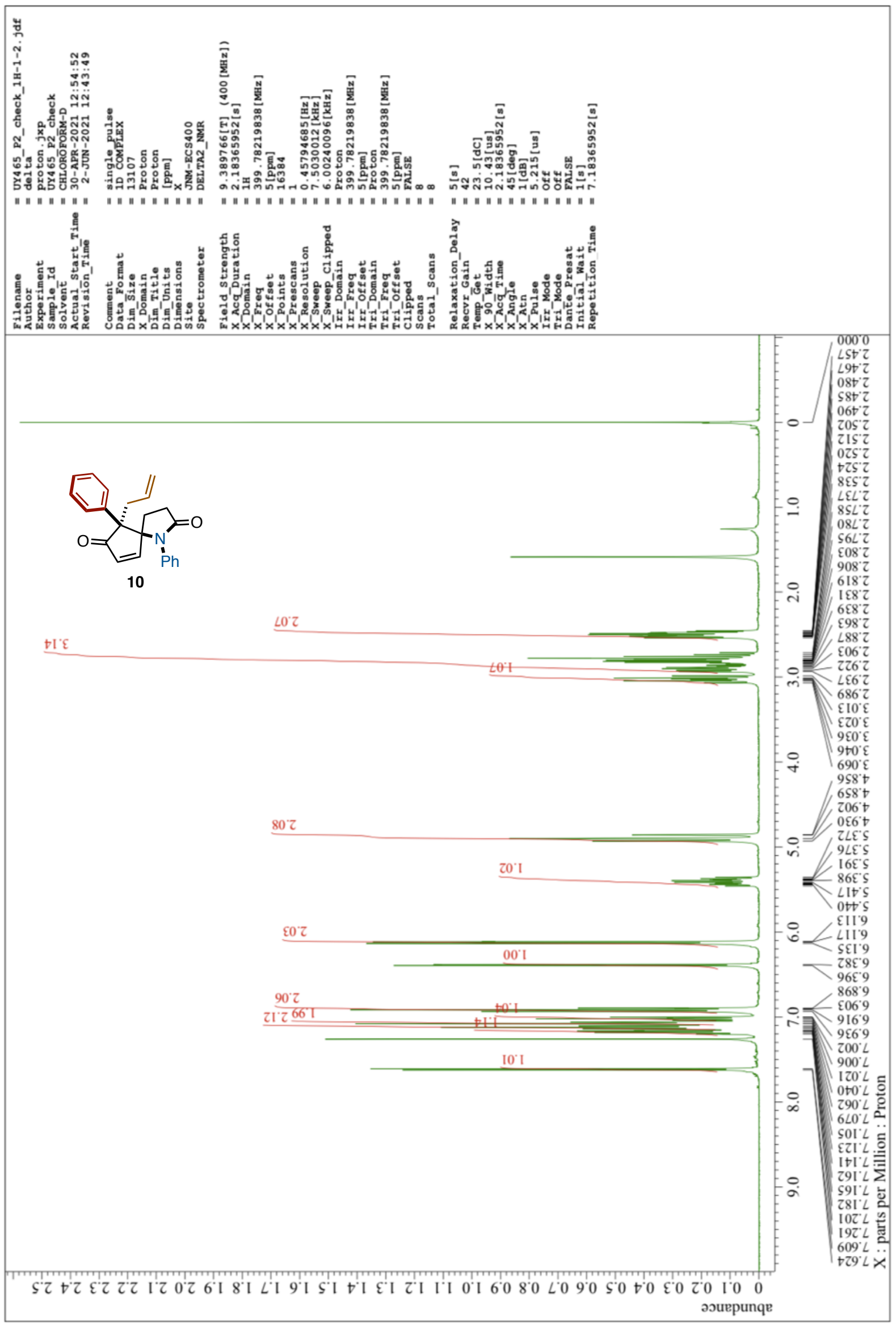


${ }^{13} \mathrm{C}$ NMR of 10 (101 MHz, $\left.\mathrm{CDCl}_{3}\right)$
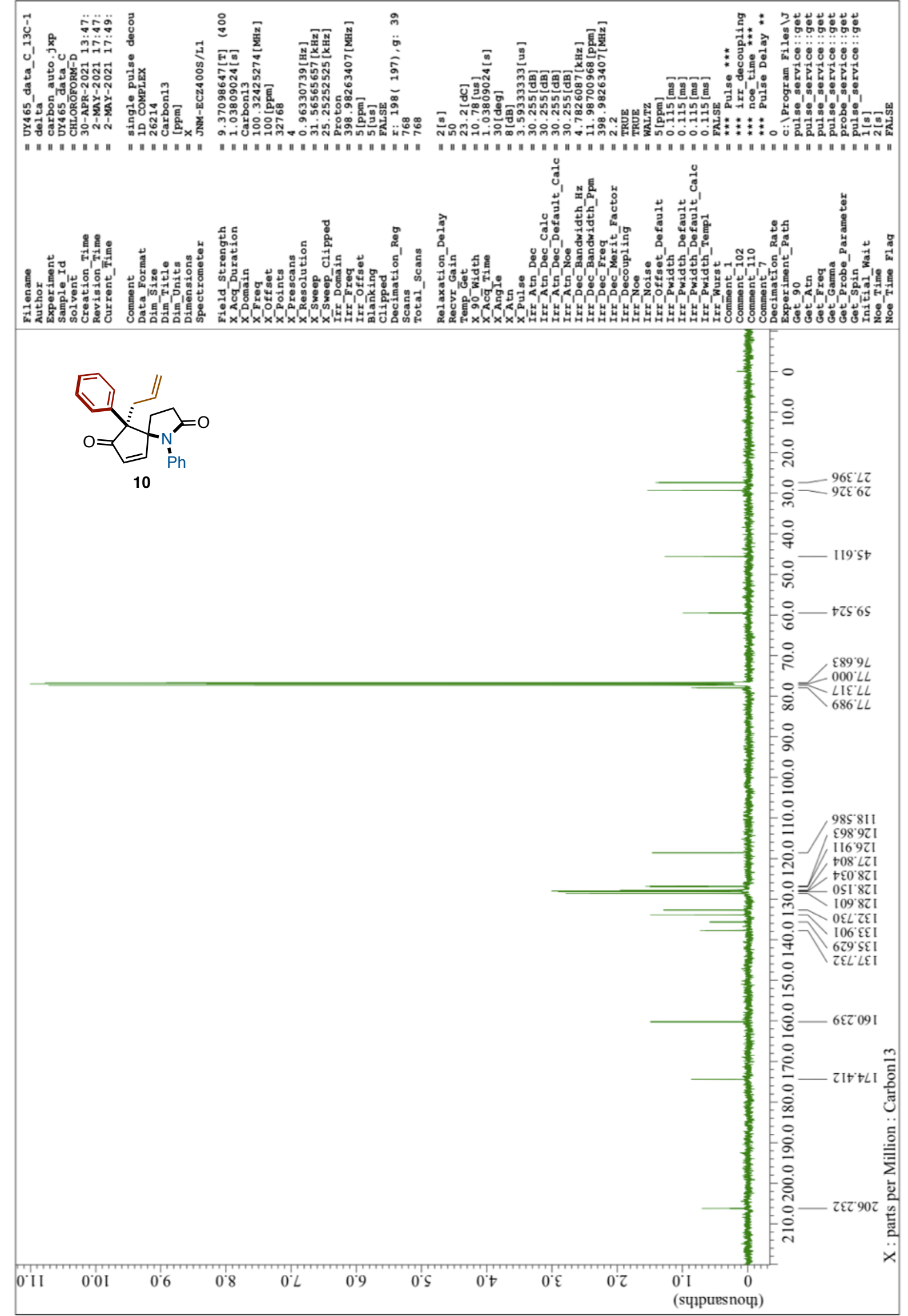
${ }^{1} \mathrm{H}$ NMR of $11\left(400 \mathrm{MHz}, \mathrm{CDCl}_{3}\right)$

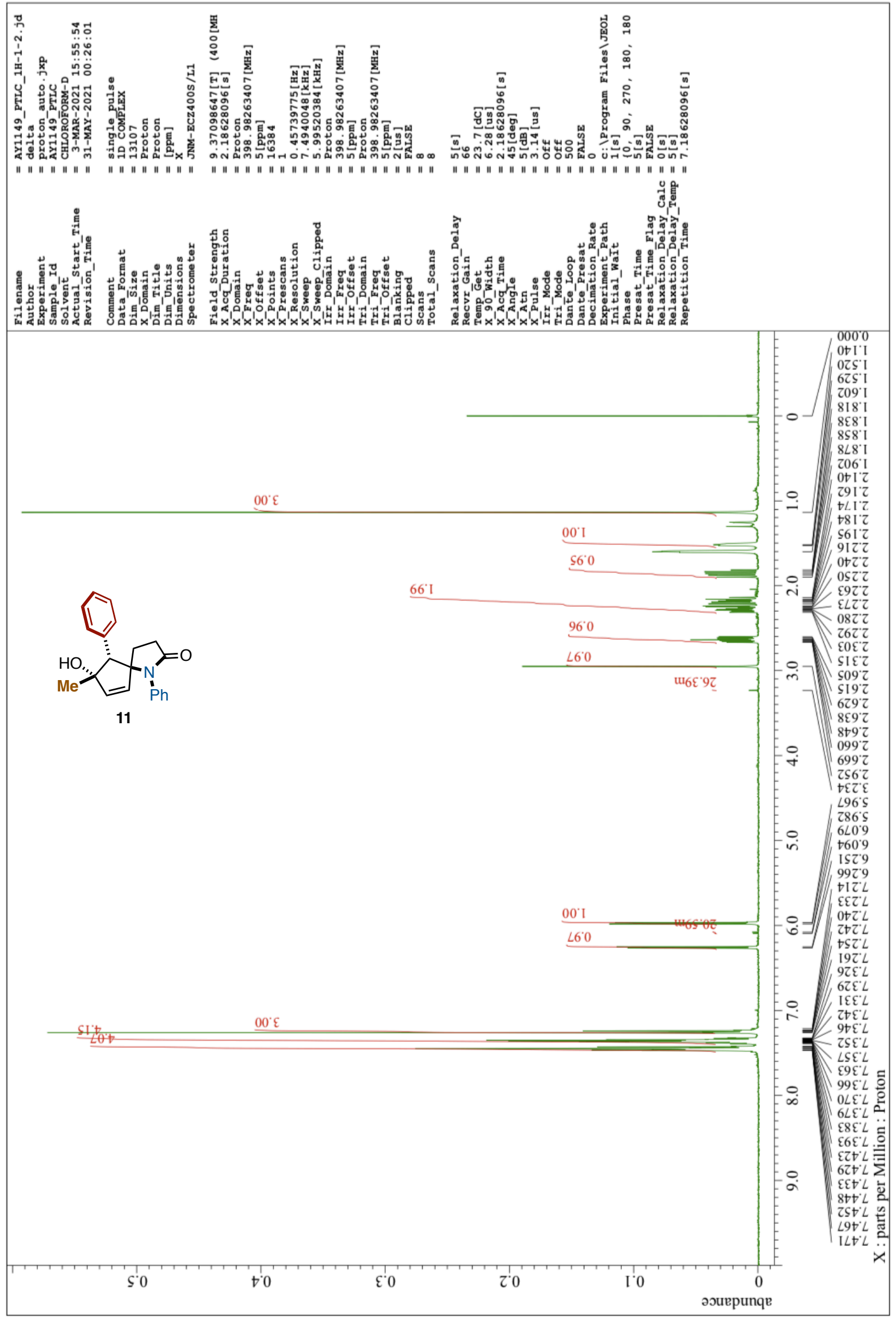


${ }^{13} \mathrm{C}$ NMR of $11\left(101 \mathrm{MHz}, \mathrm{CDCl}_{3}\right)$

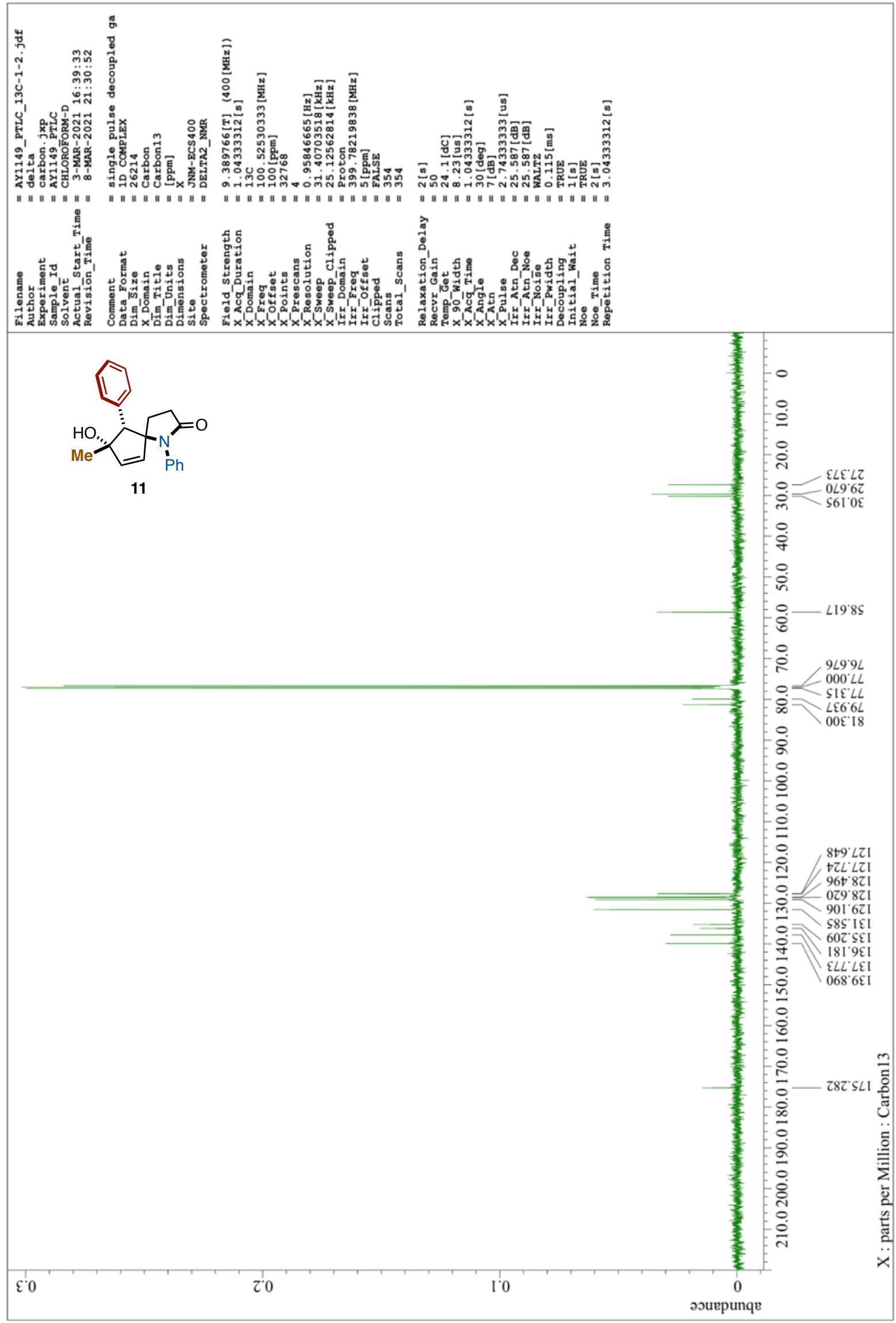


${ }^{1} \mathrm{H}$ NMR of $12\left(400 \mathrm{MHz}, \mathrm{CDCl}_{3}\right)$

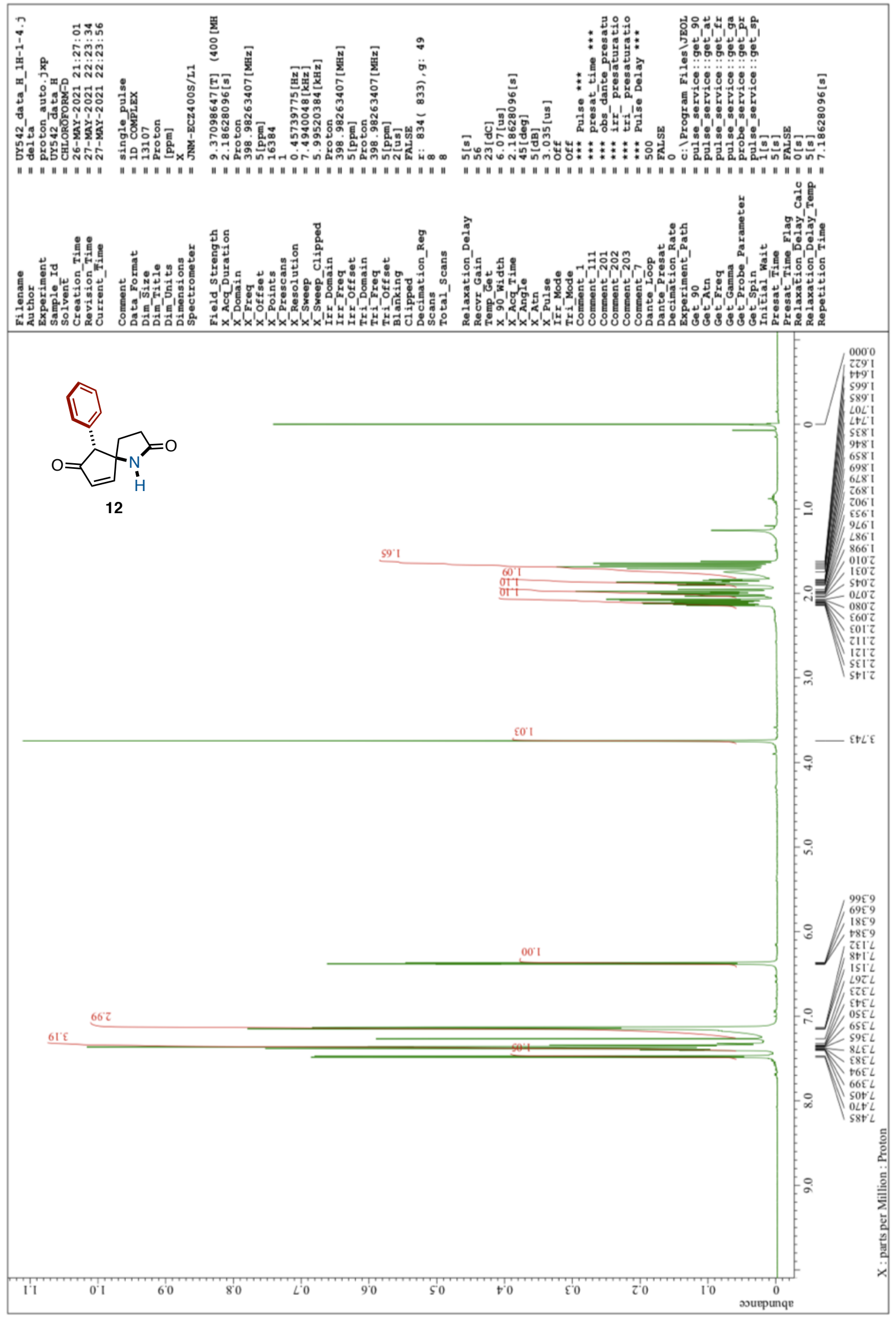


${ }^{13} \mathrm{C}$ NMR of $12\left(101 \mathrm{MHz}, \mathrm{CDCl}_{3}\right)$

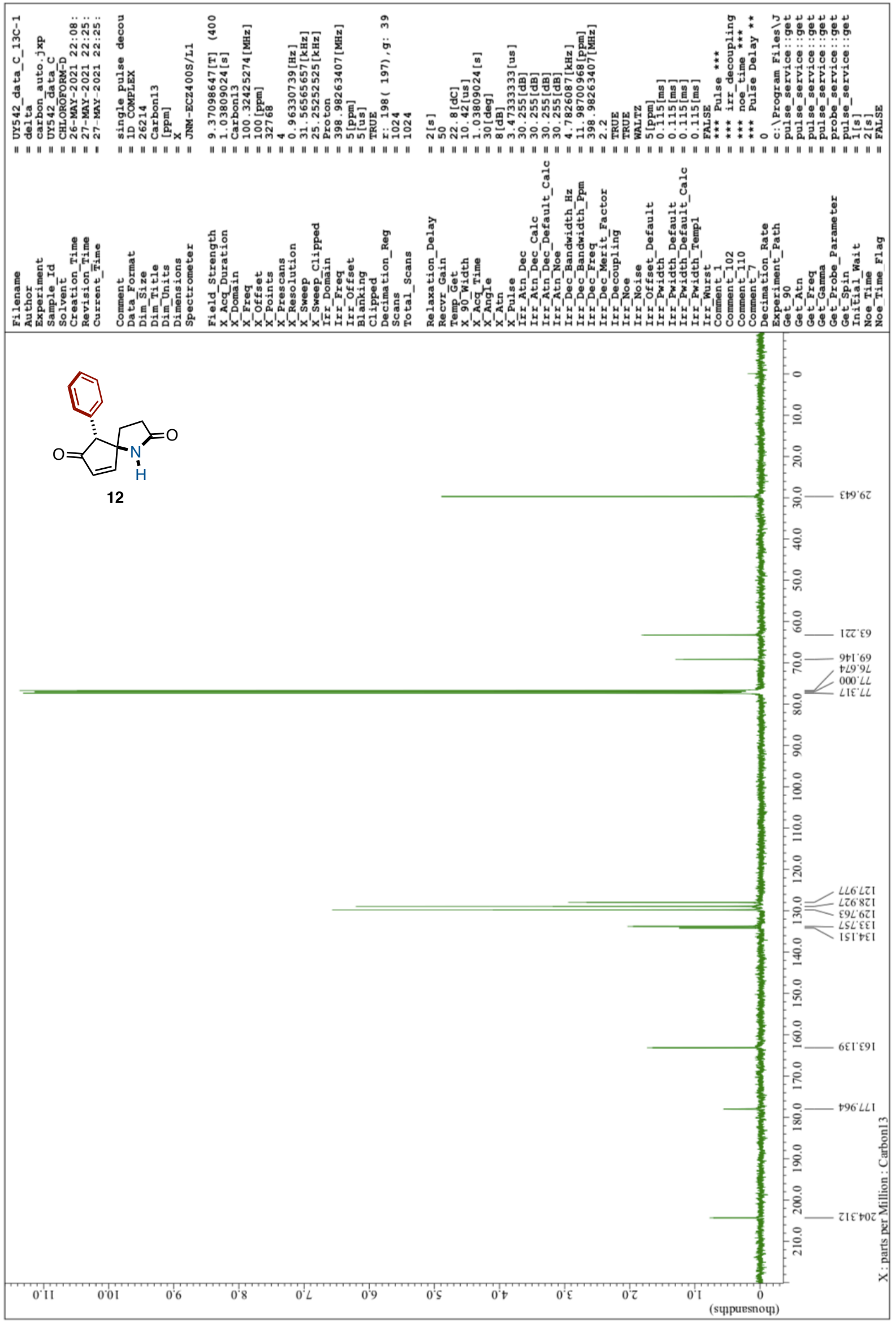


${ }^{1} \mathrm{H}$ NMR of 13 (400 $\left.\mathrm{MHz}, \mathrm{CDCl}_{3}\right)$

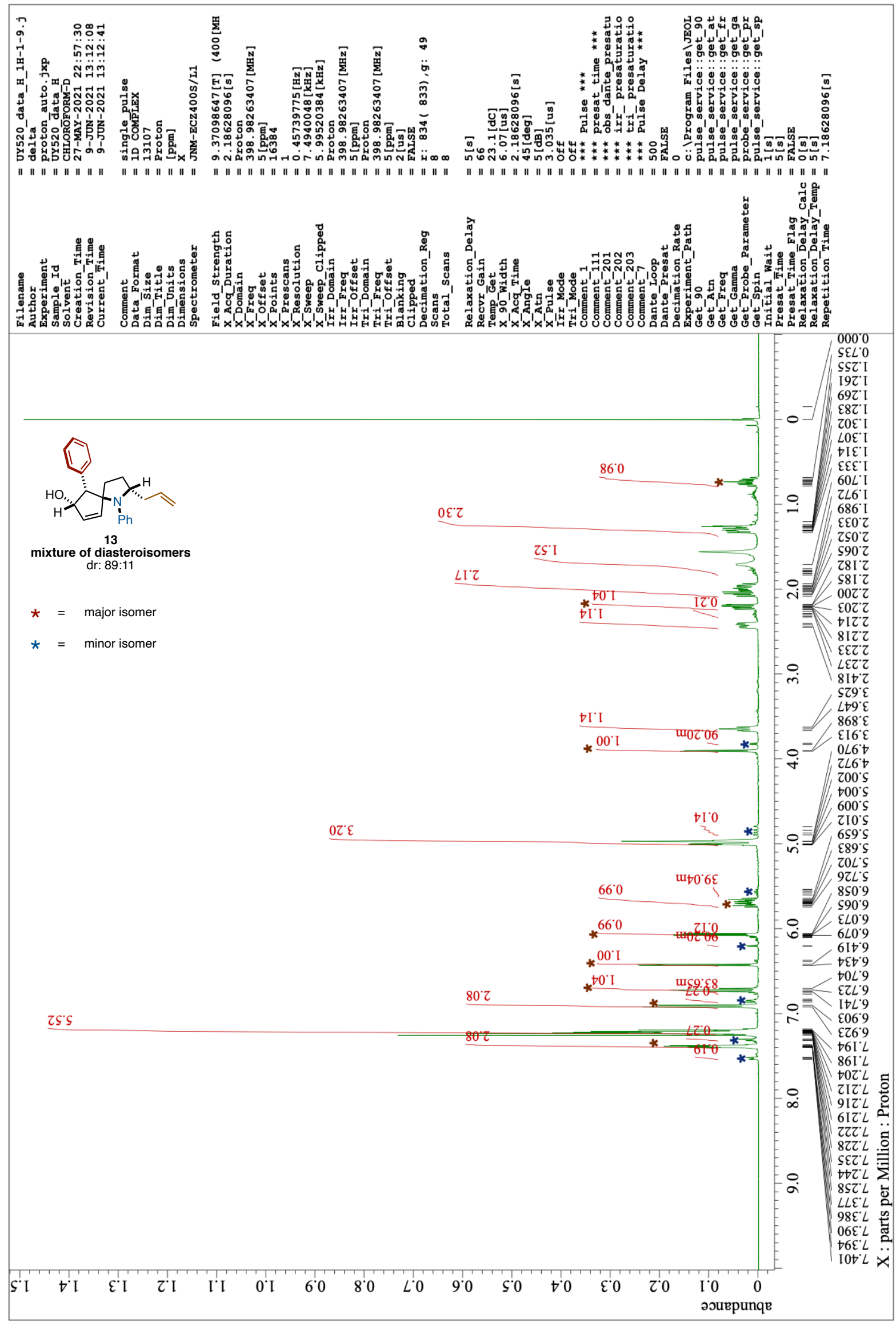


${ }^{1} \mathrm{H}$ NMR of $13\left(400 \mathrm{MHz}, \mathrm{C}_{6} \mathrm{D}_{6}, 333 \mathrm{~K}\right)$

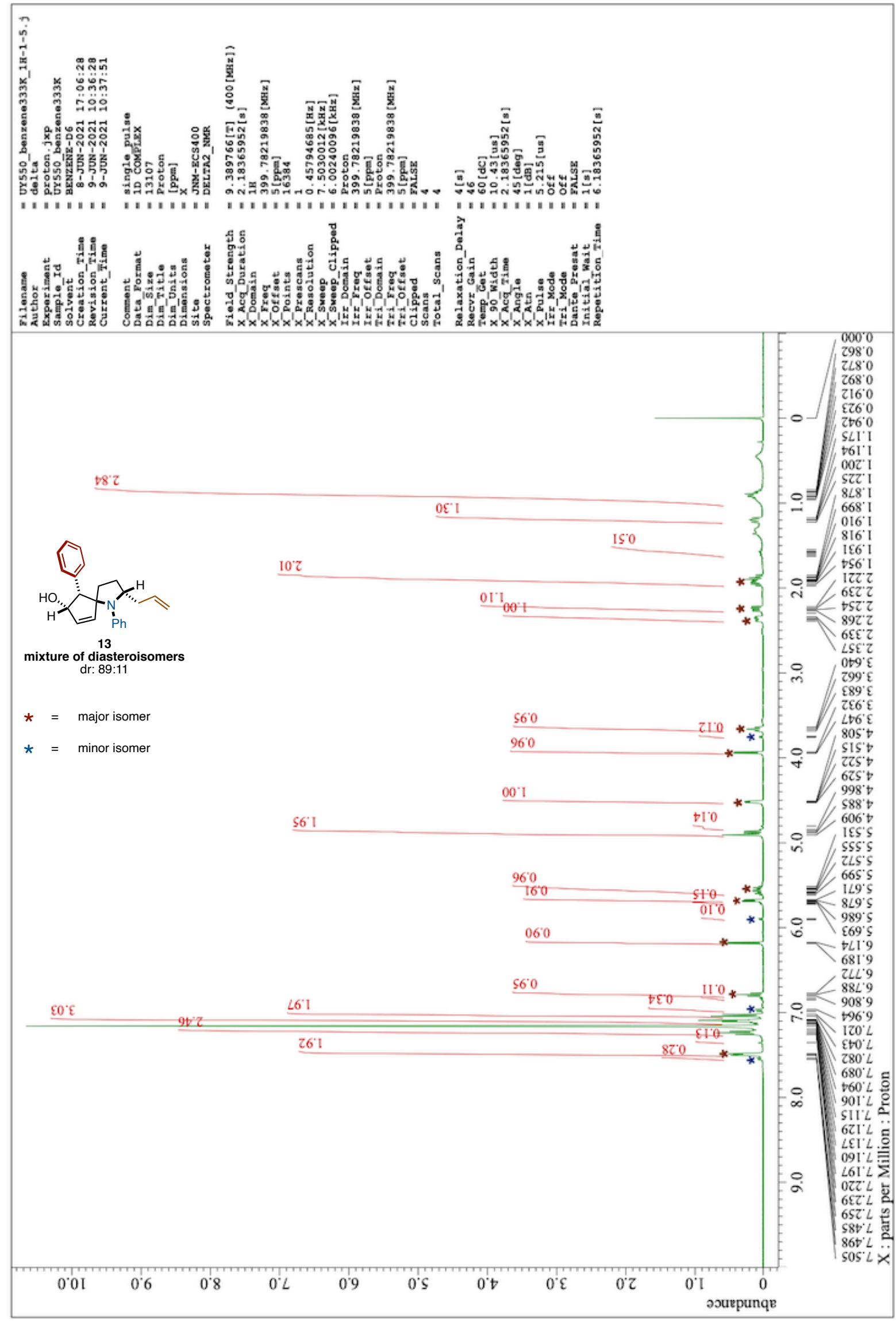


${ }^{13} \mathrm{C}$ NMR of 13 (101 MHz, $\left.\mathrm{CDCl}_{3}\right)$

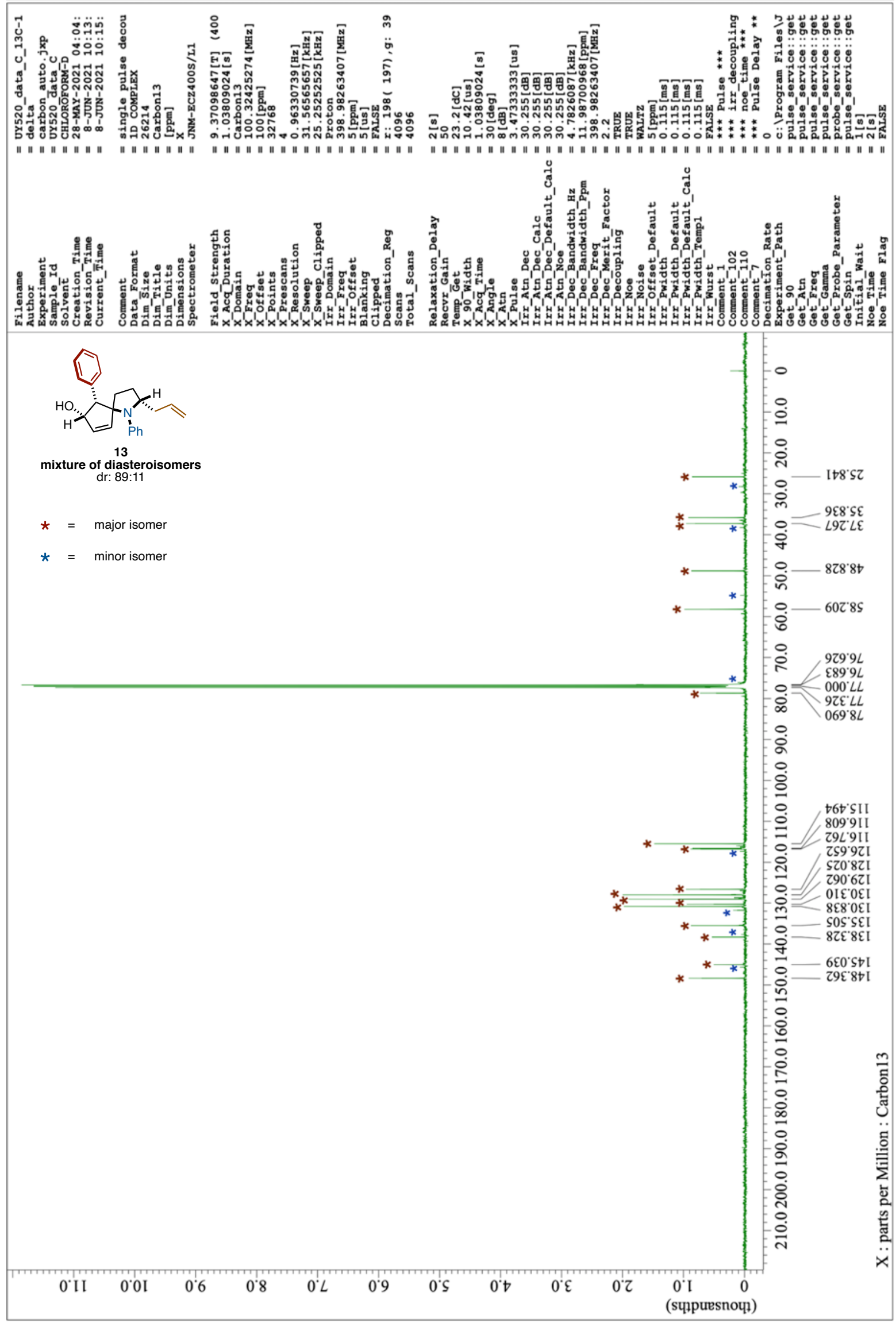

